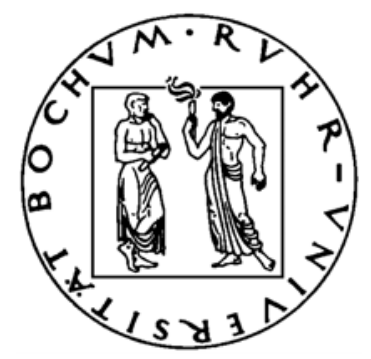

\title{
Zum Tragverhalten von Ankerschienenbefestigungen unter nichtruhenden Beanspruchungen
}

\author{
Von der Fakultät für Bauingenieurwesen \\ der Ruhr-Universität Bochum genehmigte
}

\section{Dissertation}

zur Erlangung des Grades

Doktor-Ingenieur (Dr.-Ing.)

von

Selçuk Güreş 
Doktorarbeit eingereicht am: 25. Januar 2005

Tag der mündlichen Prüfung: 10. Mai 2005

Berichter:

Prof. Dr.-Ing. R. Kindmann, Ruhr-Universität Bochum

Prof. Dr.-Ing. habil. W. Willems, Ruhr-Universität Bochum 


\section{Vorwort}

Die vorliegende Arbeit entstand während meiner Tätigkeit als wissenschaftlicher Mitarbeiter am Institut für Konstruktiven Ingenieurbau im Bereich Konstruktionsteilprüfung der Ruhr-Universität Bochum. Sie wurde von der Fakultät für Bauingenieurwesen als Dissertation angenommen.

Herrn Prof. Dr.-Ing. R. Kindmann danke ich für die Unterstützung dieser Arbeit, die äußerst wertvollen Ratschläge sowie Anregungen während der Bearbeitungsphase und die Übernahme des Referates.

Herrn Prof. Dr.-Ing. habil. W. Willems danke ich für das meiner Arbeit entgegengebrachte Interesse und die Übernahme des Koreferates.

Zum Gelingen dieser Arbeit trugen auch die Hilfsbereitschaft und die Unterstützung zahlreicher Mitarbeiter und Mitarbeiterinnen des Institutes für Konstruktionsteilprüfung bei, bei denen ich mich an dieser Stelle herzlichst bedanken möchte. Besonderer Dank gebührt Herrn Dr.-Ing. W. Hanenkamp für die äußerst wertvolle Unterstützung während der Durchführung dieser Arbeit. Meinem Kollegen Herrn Dipl.-Ing. F. Ensslen danke ich für die gute Zusammenarbeit und die schöne gemeinsame Zeit am Institut.

Bei den Firmen HaLfEN-DeHA, Wiernsheim, und Deutsche Kahneisen Gesellschaft (DKG / JORDAHL), Berlin, möchte ich mich ganz herzlich für die Bereitstellung der umfangreichen Versuchsmaterialien bedanken. Herrn Dr.-Ing. D. Lotze und Herrn Dr.-Ing. W. Neikes danke ich für die fachliche Unterstützung und stete Diskussionsbereitschaft.

Nicht zuletzt danke ich meiner Familie und insbesondere meiner Frau Neslihan und meiner Tochter Irem, die durch ihre liebevolle Nachsicht und Geduld mich zu jeder Zeit moralisch unterstützt haben. 



\section{Inhaltsverzeichnis}

\section{$1 \quad$ Einführung}

1.1 Problemstellung und Zielsetzung 1

1.2 Aufbau der Arbeit 2

2 Befestigungselement Ankerschiene 4

$2.1 \quad$ Stand der Forschung 4

2.2 Entstehungsgeschichte und Entwicklung 8

2.3 Herstellungsverfahren von Ankerschienen 11

2.4 Regelungen für Ankerschienen 13

$\begin{array}{lll}2.5 & \text { Vorteile von Ankerschienenbefestigungen } & 14\end{array}$

$\begin{array}{ll}2.6 \text { Anwendungsbereiche } & 15\end{array}$

3 Ermüdungsfestigkeit 18

3.1 Grundlegende Nachweiskonzepte 18

3.2 Beanspruchungen und Beanspruchbarkeiten 20

3.3 Ermüdungsfestigkeit von Stahl 26

3.3.1 Darstellung in Dauerfestigkeitsdiagrammen 26

3.3.2 Einflüsse auf das Ermüdungsverhalten 29

3.4 Ermüdungsfestigkeit von Beton 34

3.5 Schadensakkumulationshypothese 36

$4 \quad$ Experimentelle Untersuchungen 38

4.1 Überblick 38

4.2 Annahmen, Konventionen und Bezeichnungen 38

$\begin{array}{lll}4.3 & \text { Versuchskörper } & 40\end{array}$

4.3.1 Abmessungen der Ankerschienen 40

4.3.2 Materialkennwerte der Ankerschienen 43

4.3.3 Betoneigenschaften 44

4.3.4 Bestückung der Ankerschienen mit Dehnungsmeßstreifen 45

4.4 Versuchsaufbau und -durchführung 46

4.5 Ergebnisse und Auswertungen 51

4.5.1 Versagensfälle unter quasi-statischen Beanspruchungen 51

4.5.2 Versagensfälle unter schwingenden Beanspruchungen 52

4.5.2.1 Zentrische Zugbeanspruchung 53

4.5.2.2 Querbeanspruchung in Schienenlängsrichtung 55

4.5.2.3 Schrägbeanspruchung quer zur Schienenlängsrichtung 57

4.5.2.4 Schrägbeanspruchung in Schienenlängsrichtung 58

$\begin{array}{ll}\text { 4.5.3 Auswertung der Ermüdungsversuche } & 60\end{array}$

$\begin{array}{lll}\text { 4.5.3.1 } & \text { Festlegung der Bemessungsschwingspielzahl } & 60\end{array}$

$\begin{array}{lll}\text { 4.5.3.2 } & \text { Auswertemethodik } & 61\end{array}$ 
$\begin{array}{lll}\text { 4.5.4 Tragverhalten unter zentrischer Zugbeanspruchung } & 68\end{array}$

4.5.4.1 Statische Versuchsreihe 68

4.5.4.2 Schwingversuche 68

4.5.5 Tragverhalten unter Querbeanspruchungen $\quad 85$

4.5.5.1 Statische Versuchsreihe 85

4.5.5.2 Schwingversuche bei Beanspruchungen in Querrichtung 85

4.5.6 Kombinierte Zug- und Querbeanspruchungen 92

$\begin{array}{ll}\text { 4.5.6.1 Statische Versuchsreihe } & 92\end{array}$

4.5.6.2 Schwingversuche quer zur Schienenlängsrichtung 95

4.5.6.3 Schwingversuche in Schienenlängsrichtung 102

$\begin{array}{ll}\text { 4.5.6.4 Interaktion der Ermüdungstragfähigkeiten } & 104\end{array}$

$\begin{array}{lll}4.6 & \text { Zusammenfassung } & 108\end{array}$

5 Sicherheitskonzept 110

5.1 Sicherheitsanforderungen 110

5.2 Versagenswahrscheinlichkeit und Sicherheitsindex 111

$\begin{array}{lll}5.3 & \text { Bestimmung von Teilsicherheitsbeiwerten } & 113\end{array}$

5.4 Bemessung von Ankerschienenbefestigungen 116

5.4.1 Tragfähigkeit unter ruhenden Beanspruchungen 116

5.4.2 Tragfähigkeit unter nichtruhenden Beanspruchungen 120

5.4.3 Aktuelle Systemsicherheiten bei Ermüdungsbeanspruchungen 125

5.4.4 Ermittlung des Teilsicherheitsbeiwertes der Ermüdungsfestigkeit 126

5.4.5 Empfehlungen über die Mindestanzahl an Versuchen 129

$\begin{array}{lll}5.4 .6 & \text { Neues Bemessungskonzept } & 130\end{array}$

$\begin{array}{lll}5.5 & \text { Bemessungsbeispiel } & 137\end{array}$

5.6 Anwendungsbeispiel: Bodenverankerung von Maschinen 139

6 Zusammenfassung und Ausblick 144

$\begin{array}{ll}\text { Literaturverzeichnis } & 149\end{array}$

$\begin{array}{ll}\text { Anhang } & 160\end{array}$ 


\section{Kurzfassung}

In der vorliegenden Arbeit wird das Tragverhalten von Ankerschienen unter nichtruhenden, ermüdungsrelevanten Beanspruchungen untersucht. Anhand von experimentellen Untersuchungen werden maßgebende Einflüsse auf das Ermüdungsverhalten aufgezeigt. Der Einfluß des umgebenden Betons sowie die Größe der Abminderung der Ermüdungstragfähigkeit bei Laststellungen unter Ausnutzung von Mindestendüberständen wird dargestellt. Einen Schwerpunkt in den Untersuchungen bilden Versuche zum Einfluß des Lastniveaus auf die Ermüdungsfestigkeit, der unter Berücksichtigung von zusätzlich einwirkenden ruhenden Beanspruchungen ermittelt wird. In entsprechenden Ermüdungstragfähigkeitsdiagrammen wird das Tragverhalten dargestellt. Aus Schwingversuchen im Zugschwellbereich mit unterschiedlichen Lastrichtungen werden nichtlineare Interaktionsbeziehungen abgeleitet. Zugehörige Versagensmechanismen werden durch geeignete meßtechnische Methoden aufgezeigt.

Auf der Basis der experimentellen Untersuchungen wird unter Benutzung der Methode der Grenzzustände ein Bemessungskonzept für die Ermüdungstragfähigkeit von Ankerschienen bei nichtruhenden Beanspruchungen sowie unter Berücksichtigung verschiedener Beanspruchungsrichtungen erarbeitet. Für das System Ankerschiene werden Sicherheitsüberlegungen angestellt, welche aktuelleren Anforderungen, wie sie z.B. in den Europäischen Regelwerken (Eurocodes) angewendet werden, genügen. Dazu wird ein möglicher Teilsicherheitsbeiwert für die Ermüdungsfestigkeit abgeleitet und in das Bemessungskonzept eingeführt. Anhand eines Beispiels wird ein Vergleich des entwickelten Bemessungskonzeptes mit den derzeit gültigen Bemessungsregelungen vorgenommen. 


\section{Einführung}

\subsection{Problemstellung und Zielsetzung}

Ankerschienen aus Stahl stellen ein sicheres, universell einsetzbares und wirtschaftliches System für zahlreiche Anwendungen auf dem Gebiet der Befestigungstechnik dar. Für planmäßig nichtruhende und ermüdungsrelevante Belastungen, wie sie bei

- Führungsschienen im Aufzugsbau

- Abhängungen von Kranbahnen und Rohrleitungen

- Bodenverankerungen von Maschinen und Prüfständen

auftreten, sind besonders warmgewalzte, gezahnte Ankerschienen neuerer Generation als geeignete Problemlösung anzusehen.

Die Bestimmung der Tragfähigkeit der Ankerschienenbefestigungen unter nichtruhenden Beanspruchungen wird bisher auf der Basis von experimentellen Untersuchungen an nicht einbetonierten Ankerschienen unter zentrischer Zugschwellbelastung durchgeführt. Die Ergebnisse solcher Untersuchungen, kombiniert mit Erkenntnissen aus Versuchen unter ruhenden Belastungen, bilden derzeit die Grundlage für die Sicherheitsbetrachtung und das Bemessungskonzept bei Ankerschienen. Das aktuelle Sicherheitskonzept des Tragsystems Ankerschiene beruht darüber hinaus auf einer globalen Betrachtungsweise. Dies entspricht jedoch nicht den neueren Sicherheitsüberlegungen für Tragsysteme. Aus diesem Grunde ist es erforderlich, für das System Ankerschiene Sicherheitsbetrachtungen bereit zu stellen, die neueren Anforderungen, wie sie z.B. in den Europäischen Regelwerken angewendet werden, genügen. Diese Aufgabe kann nur mit Hilfe experimenteller Untersuchungen, die möglichst umfassende Ergebnisse zur Verfügung stellen, welche maßgebende Beanspruchungszustände bei ermüdungsrelevanten Einwirkungen abdecken, gelöst werden. Aus solchen versuchsgestützten Informationen sind dann Sicherheitsbetrachtungen unter Benutzung neuerer Methoden der Versagenstheorie zu entwickeln.

Ziel dieser Arbeit soll es deshalb sein, an warmgewalzten, gezahnten Ankerschienen zunächst experimentelle Untersuchungen zum Tragverhalten unter ermüdungsrelevanten Einwirkungen durchzuführen. Hier sind Fragen der Interaktion von verschiedenen Belastungsrichtungen, der Art der schwingenden Beanspruchung (Zugursprungs- bzw. Zugschwellbelastung), der Höhe der Lasthorizonte, der Einflüsse der Laststellung und des Einflusses des umgebenden Betons von Wichtigkeit für die umfassende Beurteilung des Tragsystems.

Die Ergebnisse der genannten experimentellen Untersuchungen dienen als Basis für die Erarbeitung eines umfassenden Sicherheitskonzeptes, welches auf der Methode der Grenzzustände beruht. Eine bedeutende charakteristische Eigenschaft dieser Me- 
thode spiegelt sich in der strengen Definition von Grenzzuständen wieder. Diese sind der Grenzzustand der

- Tragfähigkeit, z.B. Ermüdung

- Gebrauchstauglichkeit

- Dauerhaftigkeit

Jeder Zustand besitzt einen spezifischen Sicherheitsindex, welcher ein Maß für die Wahrscheinlichkeit des Versagens bzw. des Nichtversagens eines Bauteils angibt. Mit Hilfe dieser indikativen Werte gelingt es, ein einheitliches und vergleichbares Sicherheitsniveau zu erreichen. Die Methode der Grenzzustände bildet die Grundlage für die Nachweisgleichung der vereinheitlichten europäischen Normen. Dabei wird das Grundproblem der Sicherheitsanalyse $Z=R-S$ nach der Zuverlässigkeitstheorie 1. Ordnung in elementarer Form gelöst. Durch wahrscheinlichkeitstheoretische Ansätze (Verteilungstyp, Versagenswahrscheinlichkeit, Fraktilwert) in dieser Methode können mögliche Systemreserven erkannt und aktiviert werden.

Im Rahmen dieser Arbeit soll ein Sicherheitsnachweis zur Gewährleistung der Tragfähigkeit von Ankerschienenbefestigungen unter kombinierten ruhenden und nichtruhenden Zug-/ Querbeanspruchungen im Grenzzustand der Ermüdung erfolgen. Dazu sollen mögliche Teilsicherheitsbeiwerte für die Ermüdungsfestigkeit in Abhängigkeit von o. g. Einflüssen ermittelt werden, die ein angestrebtes Sicherheitsniveau gewährleisten sollen. Abschließend wird mit diesen Erkenntnissen ein neues Bemessungskonzept für Ankerschienen unter Ermüdungsbeanspruchung hergeleitet.

\subsection{Aufbau der Arbeit}

Die vorliegende Arbeit ist in 6 Kapitel unterteilt. Nach einer einleitenden Erläuterung der Problemstellung und der Zielsetzung der Arbeit wird in Kapitel 2 der Stand der Forschung von Ankerschienenbefestigungen unter vorwiegend ruhender und nichtruhender Beanspruchung dargestellt. Anschließend werden die historische Entwicklung der Ankerschiene und einige Vorteile ihrer Anwendung aufgezeigt. Das Kapitel schließt mit baupraktisch relevanten Anwendungsbeispielen ab.

Das Kapitel 3 stellt die Grundlagen der in dieser Arbeit verwendeten theoretischen Ansätze zur Beschreibung der Ermüdung vor. Nach der Erläuterung des Begriffes der nichtruhenden Beanspruchung wird auf die Ermüdungsfestigkeit von Stahl und dessen Darstellungsweisen im Dauerfestigkeitsdiagrammen (Wöhler, Goodman) näher eingegangen. Die Einflüsse auf das Ermüdungstragverhalten werden mit in der Literatur dokumentierten experimentellen Arbeiten erarbeitet. Sie dienen als Grundlage für die in Kapitel 4 wiedergegebenen Versuchsergebnisse. Nach einer grundlegenden Darstellung des Ermüdungsverhaltens von Beton wird auf die Schadensakkumulationshypothese eingegangen. Abschließend werden bruchmechanische Aspekte zur Beschreibung des Wachstums von Ermüdungsrissen aufgezeigt. 
Das Kapitel 4 umfaßt die durchgeführten experimentellen Untersuchungen. Zunächst werden in den ersten Abschnitten die Abmessungen der verwendeten Probekörper und deren Eigenschaften sowie die Versuchseinrichtung und -durchführung näher erläutert. Im Anschluß hieran erfolgt die Darstellung der erzielten Versuchsergebnisse in Abhängigkeit von einigen in Kapitel 3 dargestellten Einflußfaktoren. Nach einem umfassenden Überblick über vorhandene und mögliche Versagenszustände für ruhende und nichtruhende Beanspruchungen erfolgt die Darstellung der Versuchsergebnisse in Abhängigkeit von den Trageinflüssen aus dem umgebenden Beton, der Laststellung, dem Lastniveau und der Belastungsrichtung. Die erzielten statistischen Parameter werden in diesem Zusammenhang zusammengestellt und dienen als Grundlage für eine Sicherheitsbetrachtung im nachfolgenden Kapitel 5. Mit Hilfe geeigneter meßtechnischer Methoden wird der Ermüdungsmechanismus erfaßt und erläutert. Auf der Basis der erzielten Versuchsergebnisse werden mögliche Interaktionsdiagramme zur Beschreibung der Versuchsergebnisse bei unterschiedlichen Beanspruchungsrichtungen hergeleitet. Diese finden anschließend Eingang in eine für die Ankerschienen relevante Nachweisführung unter ermüdungsrelevanten Beanspruchungen. Das Kapitel schließt mit einem Vorschlag zur Bestimmung der charakteristischen Ermüdungstragfähigkeit ab.

Das zuvor experimentell untersuchte Tragverhalten von Ankerschienenbefestigungen stellt umfassende Informationen für eine Sicherheitsbetrachtung bzw. Nachweisführung in Kapitel 5 zur Verfügung. Nach einer Einführung in die Sicherheitstheorie wird die Ermittlung von Teilsicherheitsbeiwerten nach der im Eurocode 1 fest verankerten Zuverlässigkeitstheorie 1. Ordnung (engl. FORM, Stufe II) angestrebt. Da die Beanspruchungen nicht ausreichend bekannt sind, wird durch eine Vereinfachung die Sicherheitsbetrachtung bei der Herleitung von Bemessungsansätzen auf Basis von Versuchsergebnisse durchgeführt. Durch das Aufzeigen des aktuellen Bemessungsverfahrens von Ankerschienen werden vorhandene Mängel herausgestellt und die zur Zeit gültigen Auswerteverfahren zur Ermittlung von Bemessungswerten gegenübergestellt. Anschließend erfolgt hieraus die Ermittlung von Teilsicherheitsbeiwerten nach der o.g. Zuverlässigkeitstheorie, getrennt für jede Beanspruchungsrichtung. Der aus dieser Studie resultierende Teilsicherheitsbeiwert für die Ermüdungsfestigkeit findet Eingang in eine im Rahmen dieser Arbeit vorzuschlagenden Bemessung. Das Kapitel schließt mit einem Bemessungs- und einem Anwendungsbeispiel ab.

Die Arbeit endet in Kapitel 6 mit einer Zusammenfassung und einem Ausblick für weiterführende Arbeiten auf diesem Gebiet. 


\section{Befestigungselement Ankerschiene}

\subsection{Stand der Forschung}

Seit 1976 wird die Verwendung von Ankerschienen für tragende Konstruktionen in bauaufsichtlichen Zulassungen geregelt. Mit dem Zulassungsbescheid Z-21.4-34 [22] vom 19. Juli 1978 wurden Halfenschienen erstmalig auch für nichtruhende Lasten zugelassen. Dabei stützt sich die Bestimmung der Tragfähigkeit auf experimentelle Untersuchungen sowohl unter ruhender als auch unter nichtruhender Belastung. Die praktischen und theoretischen Erkenntnisse daraus sind bislang nicht veröffentlicht worden, weshalb in diesem Abschnitt der bisherige Kenntnisstand von Ankerschienenbefestigungen dargestellt werden soll.

In [4] stellen Rehm, Eligehausen, Mallée den Stand der Befestigungstechnik vor. Dabei werden lediglich mögliche Versagensursachen von Ankerschienen unter statischer Beanspruchung aufgezeigt und Hinweise zur deren Anwendung gegeben. Das Tragverhalten von Ankerschienen unter schwingender Beanspruchung wird in diesen Arbeiten nicht beschrieben.

Smeets [111] zeigt die historische Entwicklung der Befestigungstechnik mit Ankerschienen auf und geht dabei kurz auf das Trag- und Versagensverhalten unter statischer Beanspruchung sowie die Anwendungsbereiche und -bedingungen ein.

In [97], [98] werden durch Powell, Burdette, Oluokun erste theoretische Ansätze zur Beschreibung des Tragverhaltens von Ankerschienen unter zentrischer Zug- und Querbeanspruchung bei Betonausbruch gegeben. Dabei wird ein theoretisches Modell zur Beschreibung der Tragfähigkeit zugrunde gelegt, welches auf einer modifizierten Bestimmung der Betonausbruchslast beruht. Anschließend erfolgt ein Vergleich der experimentellen Untersuchungen mit den errechneten Tragfähigkeiten.

Nichtruhende Beanspruchungen sind nicht Gegenstand der Untersuchungen.

In [91] untersuchen Oluokun, Burdette das Tragverhalten von Ankerschienen unter statischer Beanspruchung bei kombinierter Zug- und Querbelastung. Dabei wird die Zuglast, bei einer aufgebrachten konstanten Querlast, bis zum Bruch der Verankerung quasistatisch gesteigert. Gegenstand der Untersuchungen sind dünne Stahlbetonplatten mit jeweils an den schmalen Seiten einbetonierten Ankerschienen.

Nichtruhenden Lasten werden im Rahmen dieser Untersuchungen nicht behandelt.

Wohlfahrt beschäftigt sich im Rahmen seiner Dissertation [122] mit dem Tragverhalten von Ankerschienen unter ruhenden Beanspruchungen, wobei die Beanspruchungsrichtungen variiert werden. Untersucht werden die Beanspruchungen in Richtung des Ankerbolzens (zentrischer Zug), quer zur Schienenlängsrichtung (Querzug) und eine Interaktion beider Richtungen (Schrägzug). Dabei stellt Wohlfahrt die wichtigsten Einflußfaktoren auf das Tragverhalten von Ankerschienen mit zwei bzw. drei Ankern dar. Zu den maßgebenden Parametern zählen hierbei die Achsabstände der Anker, die 
Werkstoffe der Schienenprofile sowie der Bolzen, der Überstand der Ankerschiene und die Lastposition. Die experimentell gewonnen Ergebnisse werden anschließend mit Hilfe der Finite Element Methode (FEM) verifiziert. Das Tragverhalten von Ankerschienen mit bis zu zwei Ankern wird mit dem Verhalten von Kopfbolzen verglichen. Demnach kann die zu erwartende Höchstlast der Ankerschiene mit dem für Kopfbolzen beschriebenen Concrete-Capacity-Verfahren [45], [46], [47] ermittelt werden. Ein Einfluß des einbetonierten Schienenprofils auf das Tragverhalten wird nicht festgestellt und daher vernachlässigt.

Nichtruhende Beanspruchungen sind nicht Gegenstand der Untersuchungen.

In [34] ist der aktuelle Stand in der Befestigungstechnik mit Ankerschienen dargestellt. Hierbei werden die wesentlichen Erkenntnisse aus Wohlfahrt [122] zusammenfassend beschrieben.

Hinsichtlich der nichtruhenden Beanspruchungen von Ankerschienen werden lediglich erste Informationen zu möglichen Versagensarten gegeben.

Kraus [79], [102] untersucht in seiner Dissertation ausschließlich das Tragverhalten von Ankerschienen unter vorwiegend ruhender zentrischer Zugbelastung. Dabei betrachtet er die Erkenntnisse aus [122] hinsichtlich der zentrischen Zugrichtung sehr kritisch. Gegenstand seiner Untersuchungen sind Einflüsse auf das Tragverhalten infolge unterschiedlicher Witterungsbedingungen (Frosteinwirkung und Austrocknen), der Nachbehandlung des Betons, der Schwindverformungen und verschiedener Einbausituationen. Untersucht werden Ankerschienen zunächst mit bis zu drei Ankerbolzen, welche anschließend auf Ankerschienen mit mehr als drei Ankerbolzen erweitert werden. Das Tragverhalten wird auf der Grundlage des Betonversagens analysiert. Dazu werden die experimentell erzielten Ergebnisse mittels FE-Berechnungen simuliert und anschließend in eine Bemessung für Ankerschienen umgesetzt. In Gegensatz zu den Erkenntnissen von [122] wird ein Einfluß der einbetonierten Ankerschiene auf das Tragverhalten festgestellt. Die Ankerschiene stellt im einbetonierten Zustand eine Störstelle im Beton dar und sollte somit in der Bemessung betrachtet werden.

Das Tragverhalten unter nichtruhenden Beanspruchungen wird nicht untersucht.

Nause [88] untersucht das Tragverhalten von einbetonierten Ankerschienen unter ruhender Belastung bei mehrseitiger Brandbeanspruchung. Dabei werden Einflußgrößen wie Achsabstände der Ankerbolzen unter Verwendung unterschiedlicher Schraubentypen untersucht. Weiterhin werden Auswirkungen der Störeinflüsse der Ankerschienen auf die Erwärmung verschiedener Stahlbetonbauteile aufgezeigt.

Das Tragverhalten unter nichtruhender Beanspruchung wird nicht behandelt.

In [43] sind erste Versuche zum Tragverhalten von Ankerschienen der Firma HALFEN vom Profils HTA 40/22 und 50/30 mit quergeschweißten I- bzw. T-Ankern unter nichtruhenden Beanspruchungen durchgeführt worden. Dabei wurde lediglich die Beanspruchung in zentrischer Zugrichtung untersucht. Die Versuche sollten Aufschluß über die Verwendungsmöglichkeit dieser Profile bei schwingender Beanspruchung im Aufzugs-, Kran- und Gleisbau geben. Sie erfolgten an nicht einbetonierten Ankerschienen mit einem bzw. zwei Ankerbolzen. Die Krafteinleitung erfolgte bei Anker- 
schienen mit einem Ankerbolzen direkt über dem Ankerbolzen, bei Ankerschienen mit zwei Ankerbolzen wurde die Belastung in Feldmitte eingeleitet. Ein Vergleich der aus unterschiedlichen Laststellungen resultierenden Ergebnisse zeigt jedoch, daß die Beanspruchung direkt über dem Bolzen die für eine Bemessung maßgebenden Werte liefert. Bei allen Versuchen wurde die Oberlast entsprechend der zulässigen statischen Last konstant gehalten und bei veränderlicher Schwingbreite die Schwingspielzahl ermittelt.

Die Erkenntnisse hinsichtlich des Ermüdungstragverhaltens sind nicht veröffentlicht worden. Die zulässigen Schwingbreiten können den Zulassungen entnommen werden.

In [3] wird die Durchführung der Schwingversuche im Rahmen der Zulassungen kritisch betrachtet. Die bis zu diesem Zeitpunkt in Versuchen konstant gehaltene Oberlast mit Variation der Schwingbreite zeigt eine steilere Neigung der Ermüdungstragfähigkeitsfunktion als bei Versuchen mit konstant gehaltener Mittellast. Der Einfluß aus der Mittellast spiegelt sich in der ertragbaren Schwingbreite bei einer fest definierten Schwingspielzahl wieder. Aus versuchstechnischen und wirtschaftlichen Gründen wird die Grenzschwingspielzahl auf $2 \cdot 10^{6}$ festgelegt.

Erste systematische Untersuchungen zum Tragverhalten von Ankerschienen der neuen Generation unter nichtruhenden Beanspruchungen wurden an der Ruhr-Universität Bochum durchgeführt. Aufbauend auf Versuchen an gezahnten Ankerschienen vom Typ 29/20 und 38/23 der Güte St und A4 im Rahmen der Zulassung [21], [23] ergaben sich Fragestellungen zum Tragverhalten bei Variation der Richtung unter nichtruhender Beanspruchung. Die Oberlast in den Versuchen wurde konstant gehalten und entsprach etwa dem 1,4-fachen der statisch zulässigen Last. Dieser Erhöhungsfaktor wurde gemäß des SVA [25] festgelegt.

[95] untersucht neben der bauaufsichtlich zugelassenen zentrischen Zugrichtung für die Profile vom Typ 29/20 St und 38/23 St zusätzlich das Tragverhalten von einbetonierten Ankerschienen bei Beanspruchungen quer zur Schienenlängsrichtung unter nichtruhender Belastung. Die Versuchsdurchführung erfolgt gemäß den Anforderungen des SVA [25] an einbetonierten Profilen mit einem angeordneten Ankerbolzen und Endüberständen von 25 und $50 \mathrm{~mm}$. Hieraus werden erste mögliche Ansätze zur Beschreibung der Interaktionen zwischen der zentrischen Zugrichtung und der Querrichtung dargestellt. Entgegen den Vorschriften der jeweiligen Zulassungen [21], [23] werden hierbei die Anbauteile gegen den Beton vorgespannt. Die zulässigen Lasten werden knapp erreicht. Auftretende Versagensarten (Stahlversagen) werden in Abhängigkeit von den Beanspruchungsrichtungen dargestellt.

[86] untersucht den Einfluß der Endüberstände und die Stützwirkung des umgebenden Betons auf die Tragfähigkeit von Ankerschienen des Profils 38/23 St unter nichtruhender zentrischer Zugschwellbelastung. Dabei werden Zugschwellversuche bei konstanter Oberlast entsprechend den Anforderungen des SVA [25] durchgeführt. Der Kraftangriff erfolgt in der Achse des Ankerbolzens. Aus den Untersuchungen wird gefolgert, daß für Ankerschienen im Fall der Einbetonierung durch die Stützwirkung des umgebenden Betons eine zusätzliche Erhöhung der Tragfähigkeit erreicht wird. 
Die Variation der Endüberstände von $25 \mathrm{~mm}$ nach den Vorgaben in [21], [23] auf 50 $\mathrm{mm}$, wie sie im Rahmen der Zulassungsversuche in [50], [52] an nicht einbetonierten Ankerschienen verwendet wurden, zeigt nur geringfügige Zunahmen in der Tragfähigkeit.

[8] stellt das Tragverhalten von einbetonierten Ankerschienen unter nichtruhender Zug- und Querbeanspruchung (quer zur Schienenlängsrichtung) dar. Die Lasteinleitung erfolgt dabei direkt über dem Ankerbolzen bei Endüberständen von 25 und 50 $\mathrm{mm}$. Durch Variation des Belastungswinkels von $0^{\circ}, 30^{\circ}, 45^{\circ}, 60^{\circ}$ relativ zur zentrischen Zugrichtung ergeben sich mögliche Interaktionen. Alle Versuche werden bei konstanter Oberlast entsprechend den Anforderungen des SVA [25] durchgeführt. Eine Zugschwellbeanspruchung quer zur Schienenlängsrichtung wird nicht durchgeführt. Mit geeigneten meßtechnischen Methoden wird der Ermüdungsmechanismus erfaßt und beschrieben. Die erzielten Ergebnisse unter nichtruhender Beanspruchung zeigen erste Erkenntnisse zur Erweiterung des Anwendungsbereiches auf Beanspruchungen in Querrichtung.

[80] stellt das Tragverhalten von nicht einbetonierten Ankerschienen des Profils 38/23 St unter nichtruhender Zugschwellbeanspruchung bei verschiedenen konstanten Unterlasten vor. Die Beanspruchung erfolgt in der Achse des Ankerbolzens bei Endüberständen von 25 und $50 \mathrm{~mm}$. Anschließend werden die erzielten Ergebnisse im Rahmen einer Bemessung nach Goodman dargestellt, um somit Aussagen über den Einfluß des Lastniveaus treffen zu können.

[119] führt Untersuchungen zur Interaktion von einbetonierten Ankerschienen unter nichtruhenden Zug- und Querbeanspruchungen durch. Hierbei erfolgt im Gegensatz zu [8] und [95] eine Belastung in Schienenlängsrichtung. Die Versuche erfolgen im Zugschwellbereich mit konstanter Unterlast entsprechend dem etwa 0,01-fachen der statisch zulässigen Last. Die Ergebnisse zeigen Erkenntnisse über eine mögliche Erweiterung des Bemessungsbereiches.

Auf der Basis von Schwingversuchen im Zugschwellbereich wird in [61] ein Vergleich der zur Zeit gültigen Bemessung für Ankerschienen bei ermüdungsrelevanter Einwirkungen mit einem Bemessungsvorschlag für Dübelbefestigungen vorgenommen. Dabei wir das Tragverhalten bei zentrischer Zugbeanspruchung und einer Beanspruchung unter verschiedenen Belastungswinkeln quer zur Schienenlängsrichtung untersucht. Als Fazit wird festgehalten, daß eine deutliche Abhängigkeit der ertragbaren Ermüdungstragfähigkeit vom Lastniveau vorhanden ist und bei einer Bemessung in Anlehnung an die Dübelbefestigungen mögliche Tragreserven im System aktiviert werden können.

Für nichtruhende Beanspruchungen beim Verankerungselement Dübel wird auf die wissenschaftlichen Arbeiten [7] und [82] hingewiesen. 


\subsection{Entstehungsgeschichte und Entwicklung}

Die unterschiedlichen Verfahren der modernen Befestigungstechnik werden anhand von Bild 2.1 wiedergegeben. Diese gliedern sich in den Bereich der Einlegemontage und den der nachträglichen Montage, welche durch die Bohrmontage und die Direktmontage gekennzeichnet ist. Die Ankerschiene ist dem Bereich der Einlegemontage zuzuordnen.

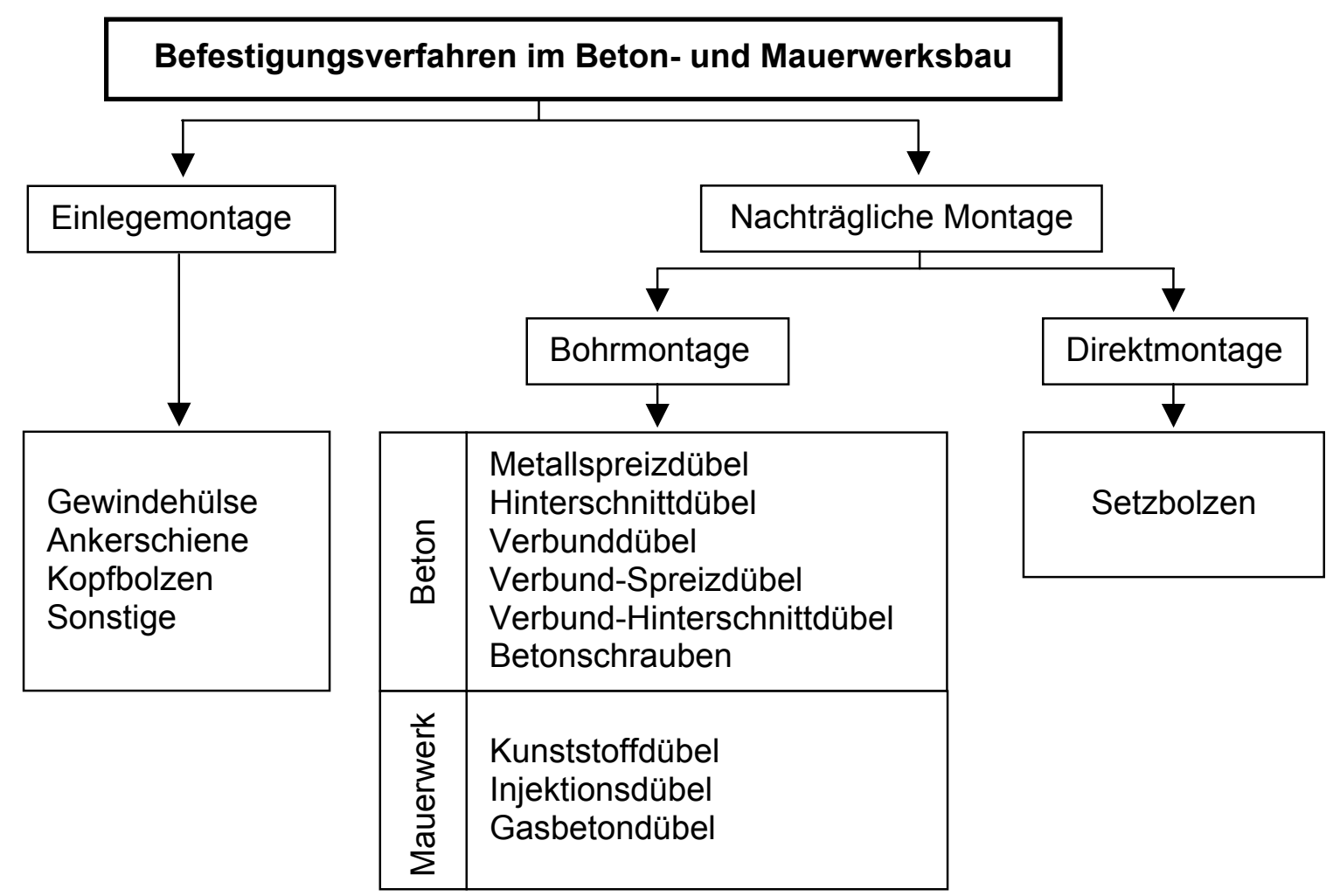

Bild 2.1 Übersicht der Befestigungsverfahren [34]

Erste Schritte bei der Einlegemontage an Betonkonstruktionen wurden ab 1850 realisiert. Als Einlegeteile verwendete man Holz-Trapezleisten, die zur Befestigung von kleinen Lasten dienten. Zusätzlich, zur Vergrößerung der Verankerungswirkung im Beton, wurden abgewinkelte Nägel in die Holzleisten eingeschlagen (Bild 2.2, links). Um jedoch höhere Lasten z.B. durch Kranbahnen u.ä. zu befestigen, mußten neue Möglichkeiten geschaffen werden. Die Lösung brachte der Eisenbetonbau zu Anfang des 20. Jahrhunderts mit sich. Mit Hilfe von klauenförmigen Stahlteilen konnten an den Flanschen von einbetonierten Eisenträgern hohe Lasten in das System eingeleitet werden (Bild 2.2, rechts).

Im Laufe der Zeit verdrängte die Stahlbetonbauweise den Eisenbetonbau, so daß neue Ideen für weitere Befestigungssysteme geschaffen werden mußten. Grundsätzlich gab es zwei Lösungsmöglichkeiten. Bei der ersten Möglichkeit wurde das Befestigungsmittel in die Betonkonstruktion einbetoniert. Die Position der Befestigung mußte hierbei vorgeplant sein. Die zweite Möglichkeit war die des nachträglich an bzw. in 
die Betonkonstruktion angebrachten Befestigungsmittels. Durch die Weiterentwicklung der bereits bestehenden Variante der einbetonierten Holz-Trapezleisten wurde der Weg für die heutige Ankerschiene vorgezeichnet. Jedoch wurden innovative Verbesserungsmöglichkeiten gesucht, um die Entwicklung hinsichtlich der Tragfähigkeit zu beschleunigen. Die trapezförmigen Holzleisten ersetzte man durch kaltverformte Stahlprofile, die über Bügel in der Stahlbetonkonstruktion verankert wurden. Mit Hilfe spezieller Schrauben konnten unterschiedliche Lasten an den Stahlprofilen befestigt werden.
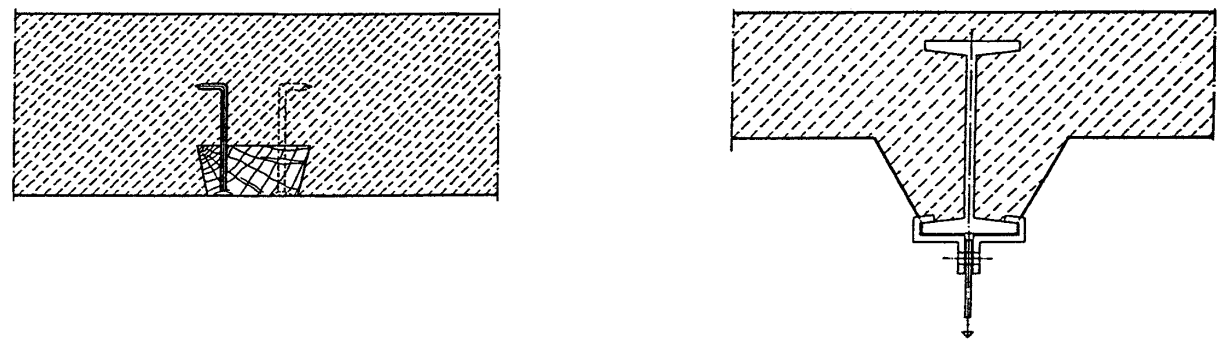

Bild 2.2 Holz-Trapezleiste (links), ausbetonierter Kappenträger (rechts) [111]

Der endgültige Übergang zu den Ankerschienen erfolgte 1913 durch das System Manz. Hierbei wurde die Last mit Hilfe einer Hakenschraube über eine S-förmige Schiene abgetragen, die zunächst von Beton freigelegt werden mußte. Die zeitlich parallel entstandene Bauer-Schiene war bereits ohne jeglichen Mehraufwand frei zugänglich.
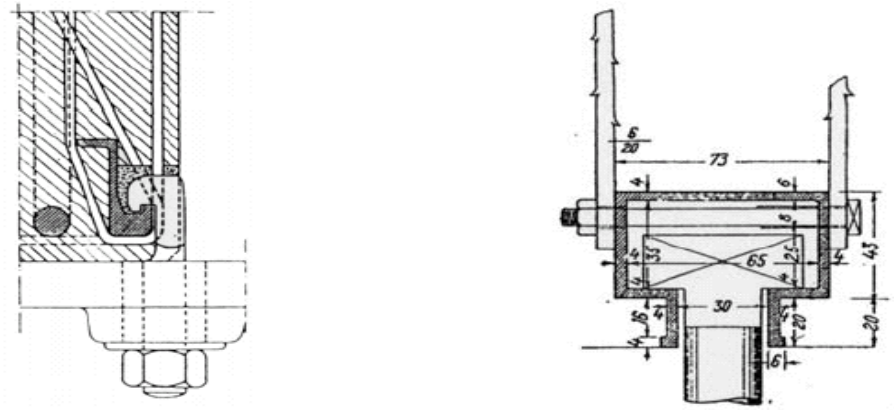

Bild 2.3 System Manz, 1913 (links) im Vergleich zu Bauer-Schiene, 1913 (rechts)

Im Laufe der Jahre widmeten sich immer mehr Unternehmen der Entwicklung der Ankerschienen. In Bild 2.4 sind die verschiedenen Modelle der unterschiedlichen Hersteller einander gegenübergestellt.

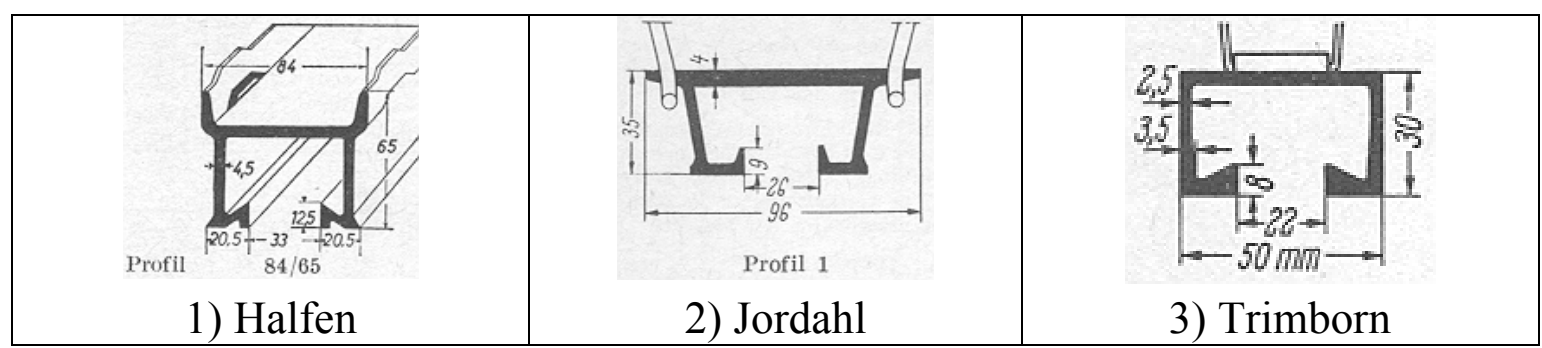




\begin{tabular}{|l|l|l|}
\hline 4) Meyn (System Moenus) & 5) KSV & Profil 2975 \\
\hline
\end{tabular}

Bild 2.4 Ankerschienenprofile verschiedener Hersteller

Die erste bauaufsichtliche Zulassung (ruhende Lasten) erfolgte am 24. Mai 1976 an Ankerschienen mit angeschweißten T-Ankern. Mit dem Zulassungsbescheid Z-21.434 vom 19. Juli 1978 wurden Ankerschienen erstmalig auch für nichtruhende Lasten zugelassen. Die Entwicklung des Befestigungselements Ankerschiene wurde von diesem Zeitpunkt an weiter vorangetrieben. Als Verankerungselement fanden neben den T-Ankern immer mehr Stauchanker Verwendung, wobei die Bügelverankerung nicht mehr ausgeführt wurde. Seit 1989 wird unter anderem die Verankerungswirkung mit Hilfe von Kopfbolzen erzielt, die durch den Schienenrücken gepreßt werden. Eine Zusammenstellung der unterschiedlichen Verankerungselemente zeigt Bild 2.5.

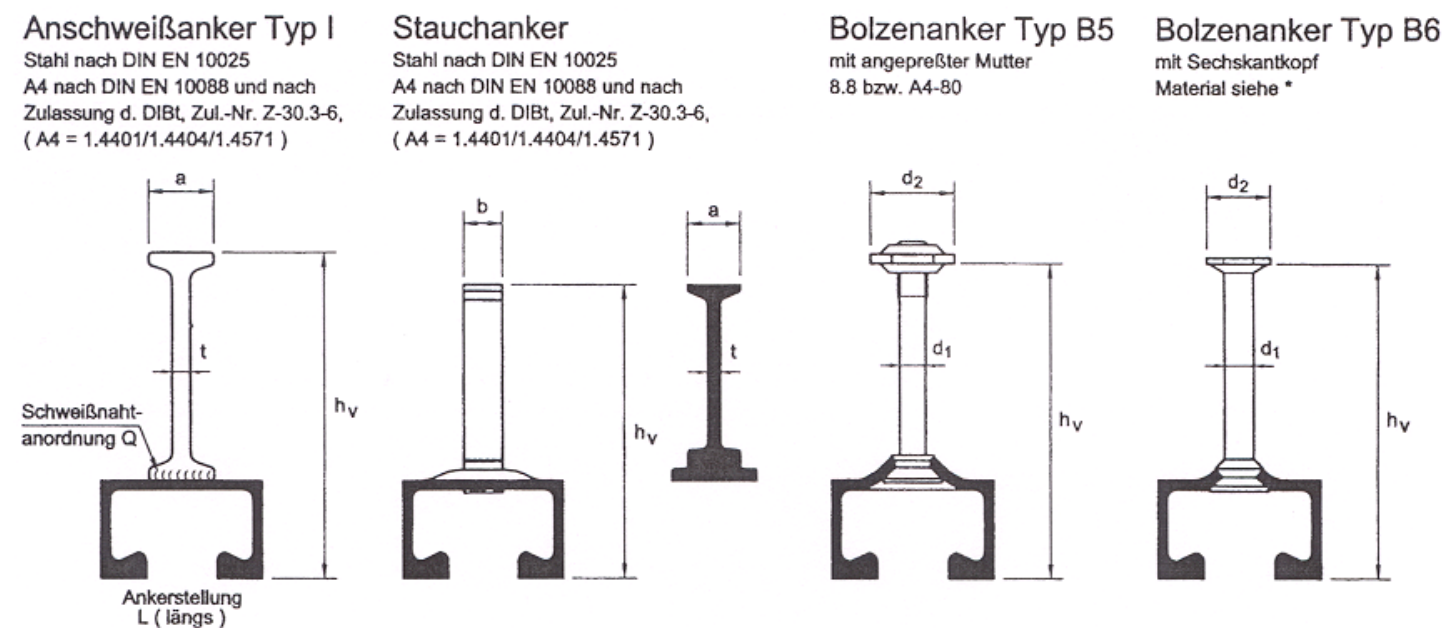

Bild 2.5 Unterschiedliche Verankerungselemente [79]

Bereits 1982 entstand die gezahnte Ankerschiene der Firma HALFEN. Zusätzlich zu der Belastung in zentrischer Zugrichtung konnten diese Typen, aufgrund von Verzahnungen an den Schienenlippen, auch Kräfte in Schienenlängsrichtung aufnehmen. Die zugehörigen Hammerkopfschrauben besaßen ebenfalls Verzahnungen an den Schraubenköpfen, weshalb eine gesicherte Lastübertragung gewährleistet werden konnte. 


\subsection{Herstellungsverfahren von Ankerschienen}

Die Herstellung von Ankerschienen wird grundsätzlich in zwei Verfahren unterteilt:

- Kaltwalzverfahren

- Warmwalzverfahren

Für die Herstellung der Ankerschienen wurde bis vor etwa 20 Jahren ausschließlich das Kaltwalzverfahren angewendet. Hierbei werden die Profile mit Hilfe mehrstufiger Profiliermaschinen aus Warmbandstahl angefertigt.

Auf dem Markt befinden sich Ankerschienen verschiedener Hersteller, die sehr geringe Abweichungen in der Geometrie und den zulässigen Lasten aufweisen. Diese Abweichungen sind neben den Materialeigenschaften der Schienen auf die unterschiedlichen Techniken der Befestigung der Ankerbolzen im Schienenrücken zurückzuführen. An dieser Stelle werden die Ankerschienen der Firmen HALFEN und DEUTSCHE KAHNEISEN GESELLSCHAFT genannt, die führend auf diesem Gebiet sind.

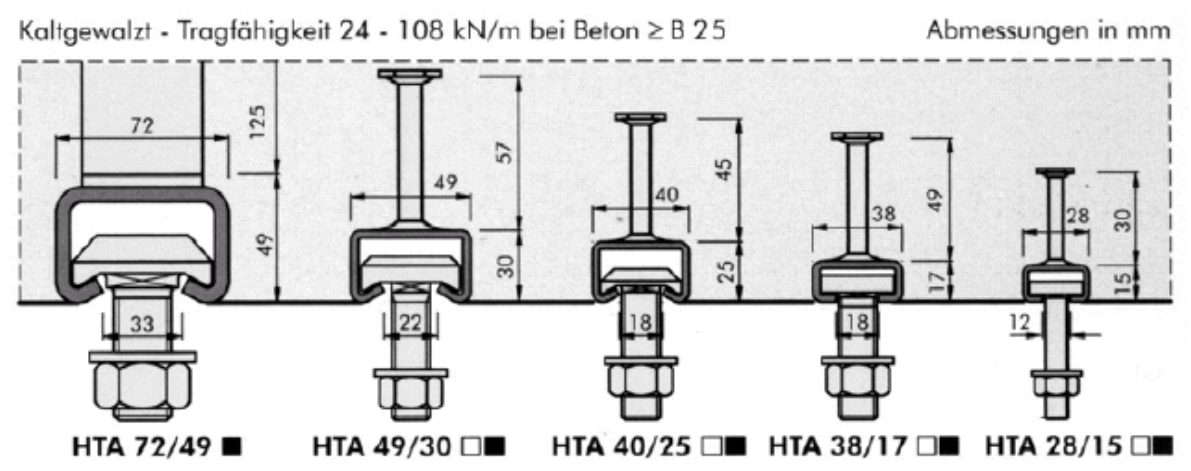

Bild 2.6 Verschiedene kaltgewalzte Ankerschienenprofile [60]

Für höhere statische Lasten und auch dynamische Anwendungsgebiete stellten diese Profile jedoch ein Problem in der Ermüdungstragfähigkeit und somit der Nutzungsdauer dar. Kaltgewalzte Profile sind nicht, bis auf einige Sonderfälle bestimmter Hersteller, für nichtruhende Beanspruchung zugelassen. Der Grund hierfür liegt, wie bereits angedeutet, in der schnellen Ermüdung infolge des spröden Materials und somit dem schnellen Rißwachstum im Schienenmaterial. Um Ankerschienen auch unter vorwiegend nichtruhenden Belastungen dauerhaft anwenden zu können, mußten neue Fertigungsverfahren entwickelt werden. Dadurch kam es zur Herstellung von Ankerschienen im Warmwalzverfahren.

Warmgewalzte Profile werden aus Normalstahl gemäß DIN 17100 und aus Edelstahl gemäß DIN 17440 hergestellt. Zunächst wird der Stahlblock im Walzwerk auf die erforderliche Walztemperatur gebracht und durchläuft danach verschiedene Stiche, auch Rohlinge genannt, die zur Formgebung der Ankerschienen dienen. Je nach Ankerschienentyp werden im Anschluß die Verzahnungen an den Schienenschenkeln eingeprägt. Für unterschiedliche Profile sind auch unterschiedliche Walzen notwendig. Im letzten Schritt werden die Ankerschienen kalt beigebogen und die Anker in 
den Schienenrücken angebracht. Die warmgewalzten Profile kennzeichnen sich durch eine gleichbleibende Zähigkeit und Härte über dem Querschnitt.

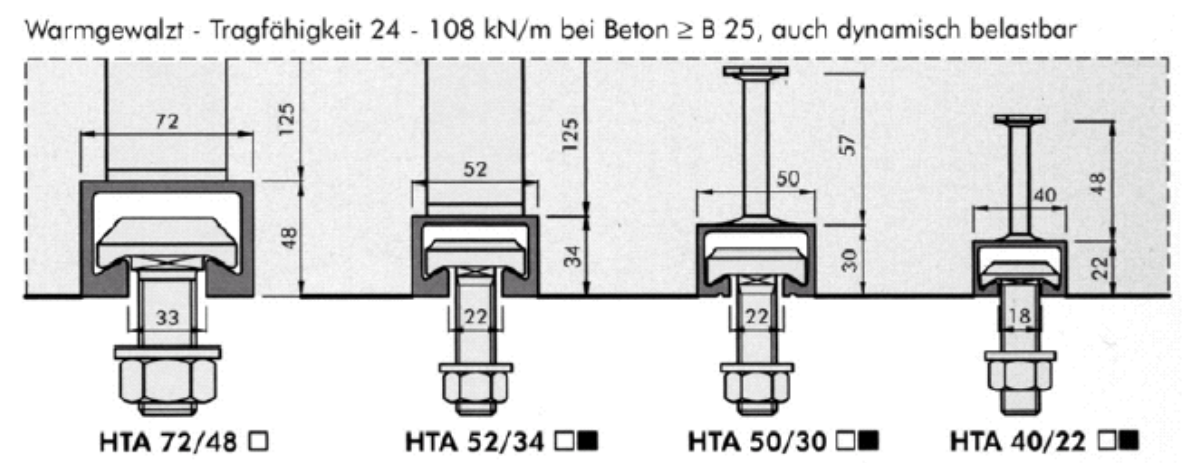

Bild 2.7 Verschiedene warmgewalzte Ankerschienenprofile [60]

Am 27.11.2000 wurde die erste bauaufsichtliche Zulassung [21], [23] für warmgewalzte, gezahnte Profile aus Normalstahl des Typs HZA (HALFEN) bzw. JXA-W (JORDAHL) 38/23 und 29/20 erteilt. Bild 2.8 zeigt die Formen und Abmessungen der unterschiedlichen Profile.
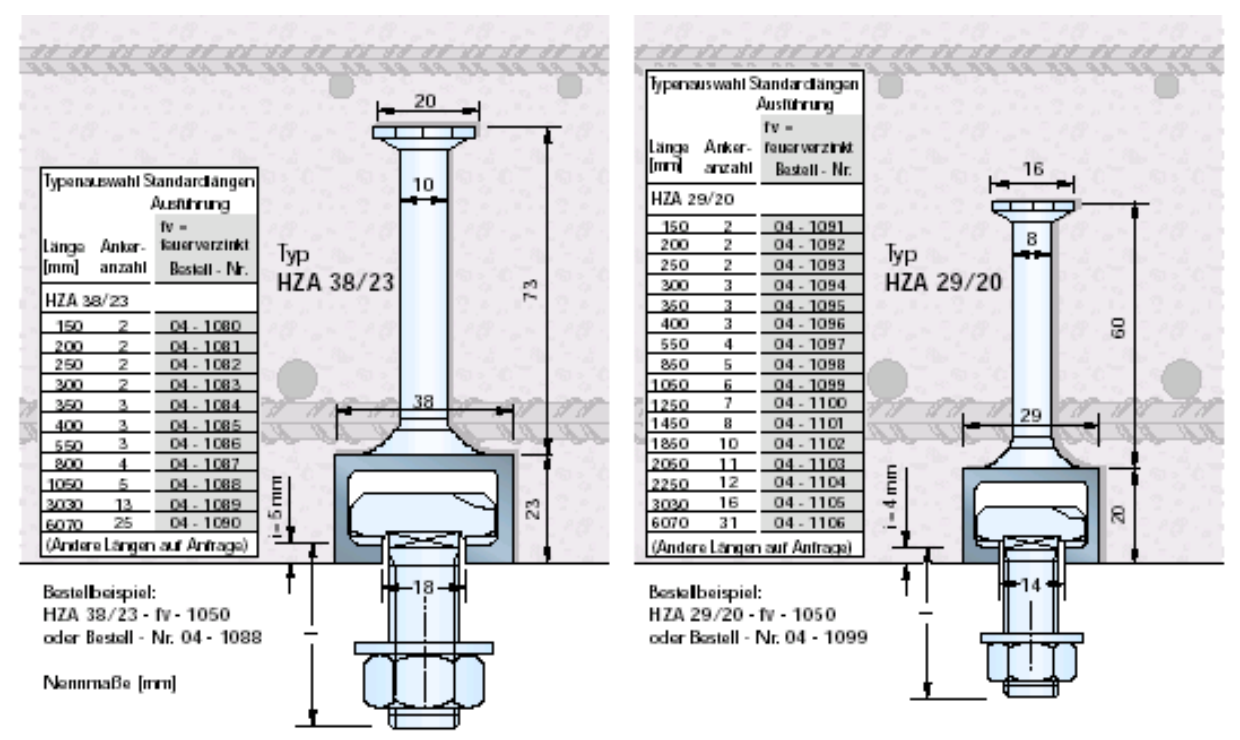

Bild 2.8 Verzahnte Ankerschienen des Profils 29/20 (rechts) und 38/23 (links) [58]

Die Beanspruchung der Ankerschiene darf bei vorwiegend ruhenden Lasten in allen Richtungen erfolgen, sofern eine allseitige Beanspruchung vorgesehen ist.

Für die Beanspruchung unter vorwiegend nichtruhenden Lasten kann zum heutigen Kenntnisstand keine allseitige Beanspruchung angesetzt werden. Untersuchungen zur Querbeanspruchung sowohl in, als auch quer zur Schienenlängsrichtung und der kombinierten Zug- und Querbeanspruchung bei nichtruhender Belastung sind Gegenstand der vorliegenden Arbeit. 


\subsection{Regelungen für Ankerschienen}

In tragenden Konstruktionen sind Ankerschienen nur dann zu verwenden, wenn bauaufsichtliche Zulassungen vorhanden sind. Diese beinhalten Grenzwerte für die Beanspruchungsrichtungen, Ankeranordnungen, Anker- und Lastabstände sowie Beanspruchungen (ruhend, nichtruhend) und zugehörige Schraubenabmessungen. Die Grenzwerte wurden aus Versuchen ermittelt, die globalen Sicherheitsfaktoren unterlagen. Die einzusetzenden Ankerschienen richten sich nach der Tragfähigkeit und den auftretenden Belastungsrichtungen. Die mit Verzahnungen versehenen Ankerschienen neuerer Generation dürfen bei nicht gezahnten Schrauben des Typs HS nur senkrecht (y-Richtung) zur Schienenlängsachse beansprucht werden, wobei eine allseitige Beanspruchung unter Verwendung des gezahnten Schraubentyps möglich ist (Bild 2.9).
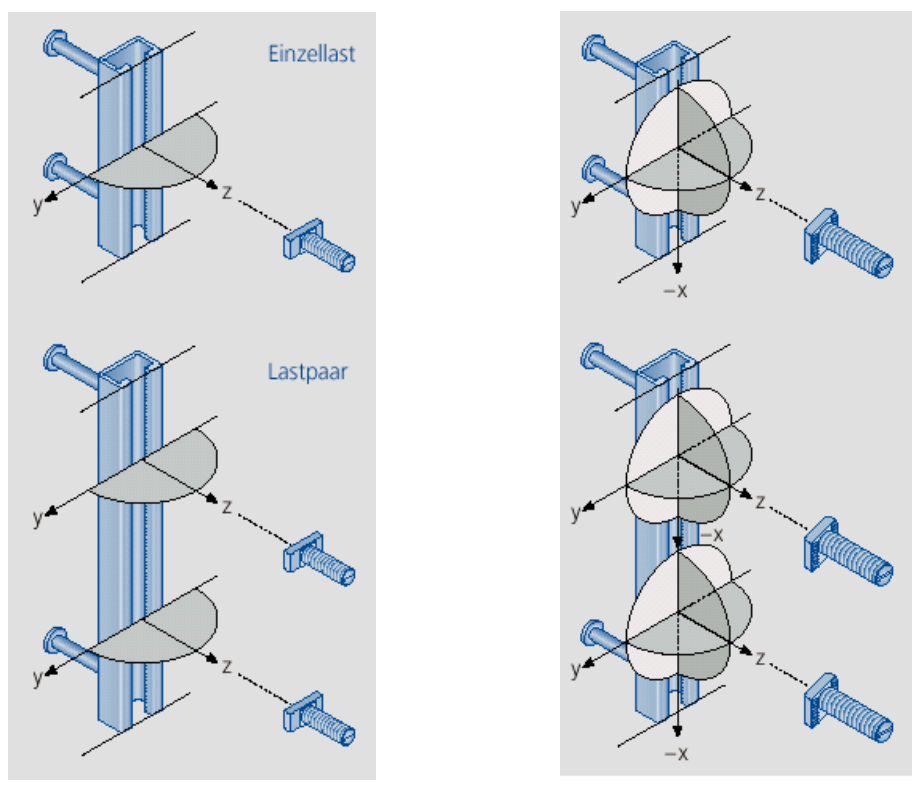

Bild 2.9 Beanspruchungsbereiche in Abhängigkeit vom Schraubentyp [21], [23], [69]

In Bild 2.10 sind die zugelassenen Regellängen der Ankerschienen aufgezeigt.

\begin{tabular}{|c|c|c|}
\hline \multirow{2}{*}{$\begin{array}{c}\text { Schienenlänge } \\
\text { [mm] }\end{array}$} & \multicolumn{2}{|c|}{ Achsabstand der Anker } \\
\hline & Austührung A und B (Anschweiß- und Bolzenanker) & Austihrung $S$ (Stauchanker) \\
\hline 100 & $25]_{50} \mathrm{~T}_{25}$ & \\
\hline 150 & $25 \quad 100 \quad T_{25}$ & 35] $80 \quad T_{35}$ \\
\hline 200 & $25]^{25} \quad 150 \quad T_{25}$ & $35]^{35} \quad 130 \quad T_{35}$ \\
\hline 250 & $25 \top_{200} \quad T_{25}$ & $\begin{array}{lll}35 & 180 & T_{35} \\
\end{array}$ \\
\hline$>250$ & 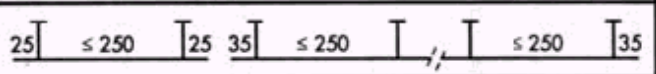 & 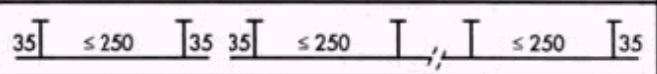 \\
\hline
\end{tabular}

Bild 2.10 Regellängen und Ankeranordnung [79] 


\subsection{Vorteile von Ankerschienenbefestigungen}

Die Einlegemontage mit Ankerschienen bietet gegenüber anderen Befestigungsmitteln eine Fülle von Vorteilen, die im Folgenden erläutert werden [109], [110]. Die aufgeführten Werte beziehen sich auf den Ankerschienentyp HZA 38/23.

- Die im Voraus geplante Anordnung der Ankerschiene gewährleistet bei richtiger Verlegung und Positionierung der Bewehrung eine sichere Kraftweiterleitung.

- Da die Ankerschienen Bestandteile des Betonbauteils darstellen, ist der Verbund zwischen Schiene und Beton spannungs- und spreizdruckfrei. Dadurch ist im Gegensatz zur nachträglichen Montage eine Beschädigung des Betons und der Bewehrung ausgeschlossen.

- Wegen ihrer geringen Abmessung und einzuhaltenden Achs- und Randabstände eignen sich Ankerschienen hervorragend für dünne bzw. schlanke Betonbauteile. Bei Verwendung von Zusatzbewehrung können die Randabstände von 15 bis auf 5 $\mathrm{cm}$ reduziert werden.

- Wegen ihrer hohen Flexibilität stellen Ankerschienen ein multiples Befestigungsmittel dar. Anbauteile können nachträglich ohne große Mühen demontiert, verschoben und wieder montiert werden. Laut den bauaufsichtlichen Zulassungen dürfen an den Ankerschienen auch Lastpaare, z.B. mit einem Abstand kleiner 5 $\mathrm{cm}$, befestigt werden.

- Die Einbaumontage mit Ankerschienen stellt im Gegensatz zur nachträglichen Montage eine geräuscharme, staubfreie und lohnkostensparende Befestigungsvariante dar.

- Bei mehrfach parallel befestigten Lasten können mit Hilfe der Ankerschienenmontage erhebliche Baukosten und Bauzeiten gegenüber der Bohrmontage eingespart werden.

- Bauteiltoleranzen können aufgrund der guten Justierbarkeit korrigiert werden.

- Ankerschienen dürfen laut den Zulassungsbescheiden in den Bauteilzugzonen eingebaut und verankert werden.

- Ankerschienen stellen für die verschiedenen Beanspruchungsbereiche ein weites Spektrum zur Verfügung. Besonders die gezahnten Schienen dürfen in Richtung der Schienenlängsachse mit einer zulässigen Last von $12 \mathrm{kN}$ beansprucht werden.

- Die im Warmwalzverfahren hergestellten Schienen dürfen auch bei nicht vorwiegend ruhenden zentrischen Zuglasten mit einer maximalen Schwingbreite von 3 $\mathrm{kN}$ angewendet werden. 


\subsection{Anwendungsbereiche}

Ankerschienen stellen ein vielseitiges Befestigungsmittel dar. Sie können nahezu für alle möglichen Befestigungen an Beton verwendet werden. Die nachfolgend aufgezeigten Beispiele sind aus einer großen Anzahl von Anwendungsmöglichkeiten herausgegriffen worden.

- Befestigungen von Aufzugsführungsschienen

Verwendung aufgrund der Aufnahmemöglichkeit von nichtruhenden Lasten, guter Justierbarkeit und Möglichkeit der Anordnung von Lastpaaren [5], [13].

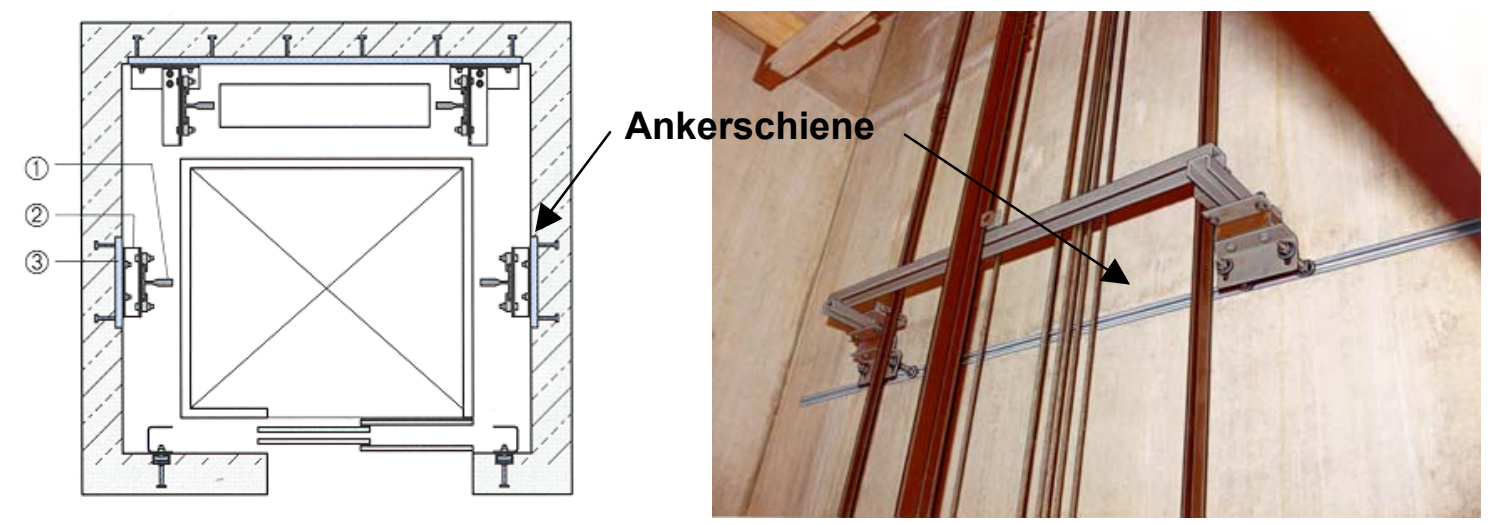

Bild 2.11 Aufzugsführungsschienen [57], [59]

- Befestigungen von Trägern und Stützen in Industrieanlagen

Verwendung aufgrund der guten Justierbarkeit, dadurch Ausgleich von Bautoleranzen und Vermeidung der Beschädigung von Bewehrung.
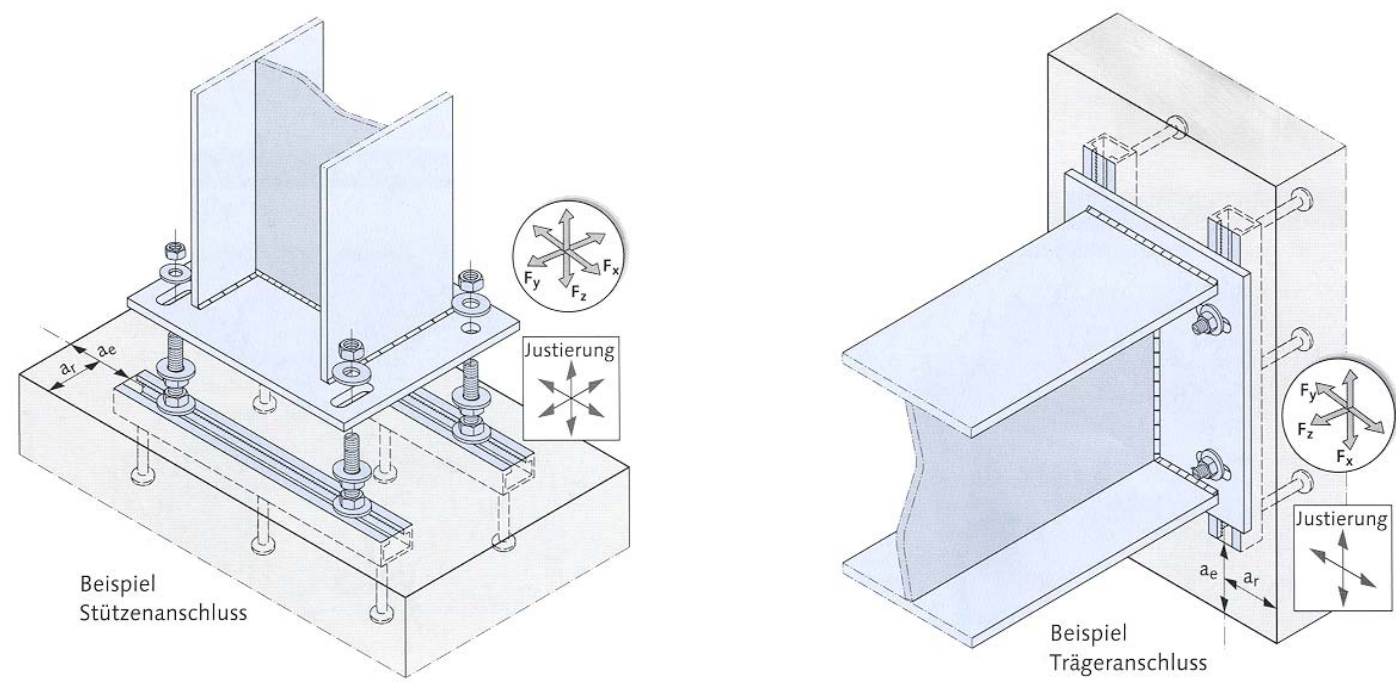

Bild 2.12 Verankerungen von Stützen und Trägern [58] 
- Verankerungen von Fassadenkonstruktionen

Verwendung aufgrund der guten Justierbarkeit und hohen Flexibilität. Hierbei stellt vor allem die Einlegemontage mit Ankerschienen eine schnelle, sichere und dauerhafte Befestigungsmethode dar. Die aus Edelstahl hergestellten Schienen bieten für diesen Anwendungsfall die idealen Voraussetzungen, da bei späteren Inspektionen die Befestigungsstellen nicht mehr zugänglich und so ungeschützt den Witterungsbedingungen ausgesetzt sind [54].
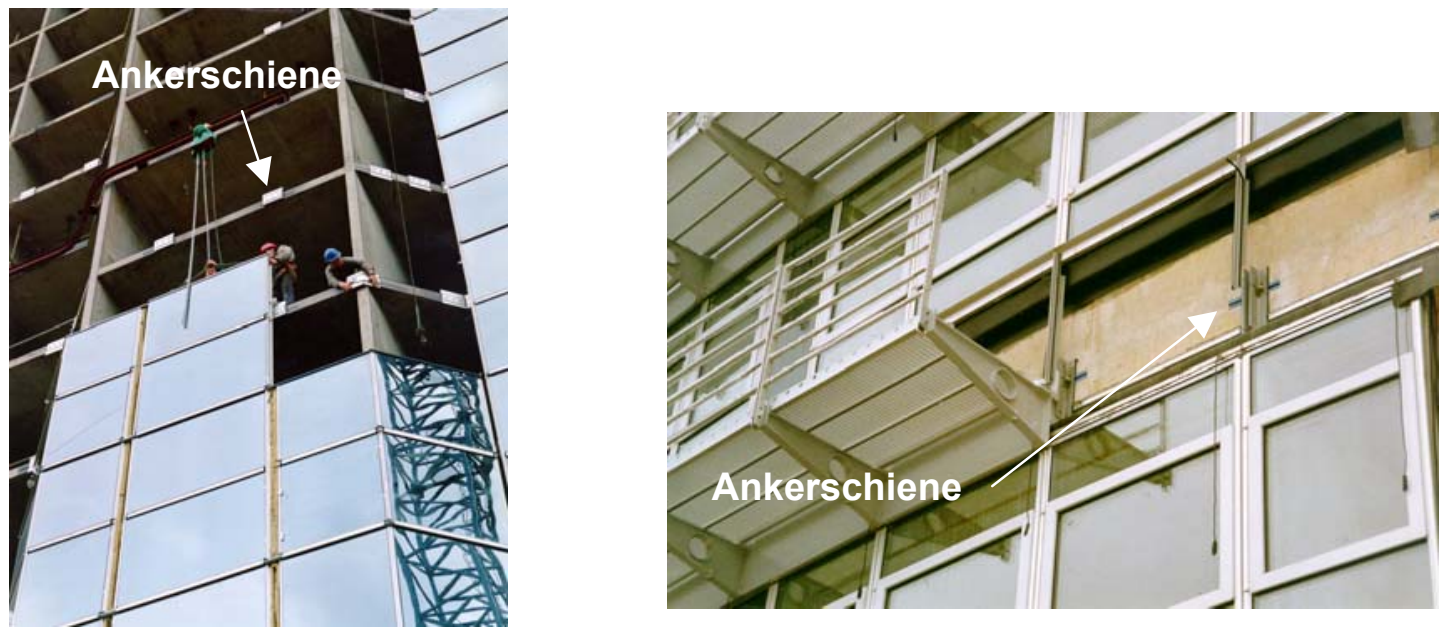

Bild 2.13 Fassadenkonstruktionen aus dem konstruktiven Glasbau [56]

- Befestigungenen von Kranbahnen

Verwendung aufgrund der guten Justierung der Kranbahn, der möglichen Abmessungstoleranzen sowie der nachträglichen Begehbarkeit bei Schadensfällen.
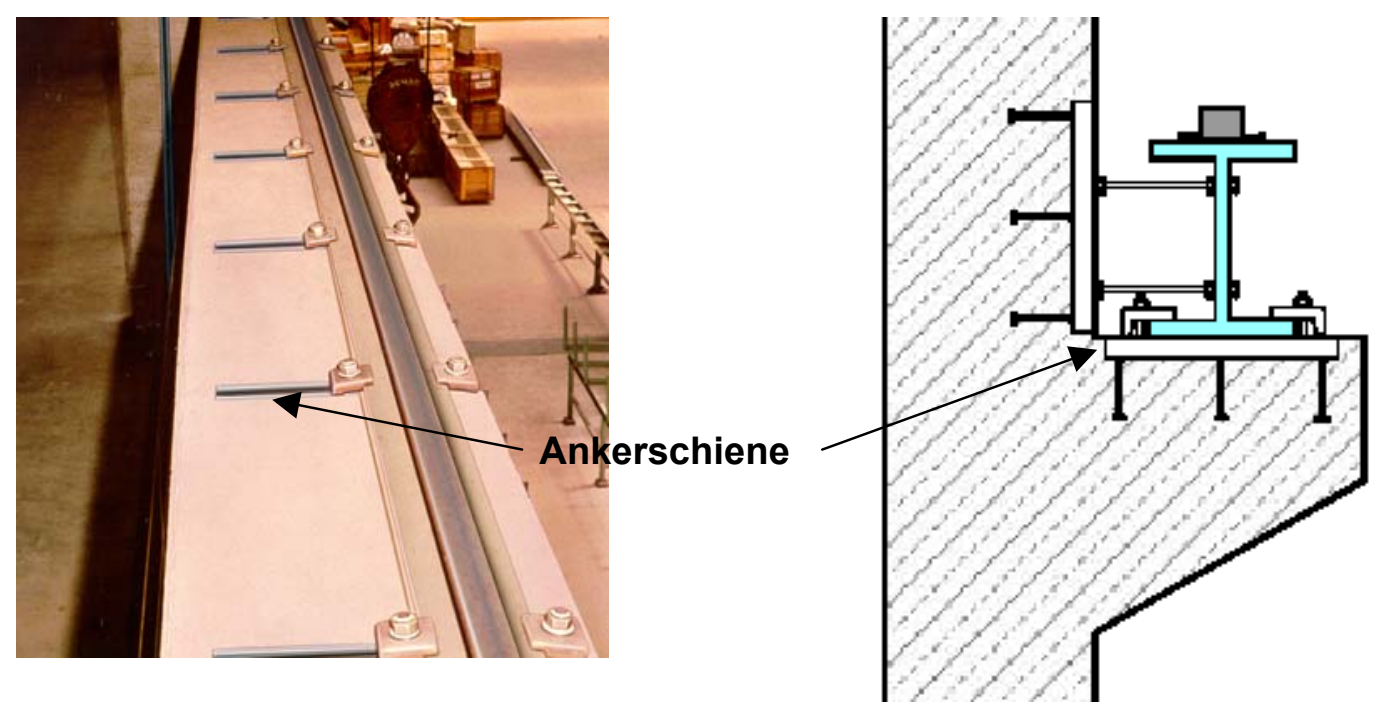

Bild 2.14 Kranbahn mit Hilfe von Ankerschienen [55] 
- Befestigungen von Maschinenelementen

Verwendung aufgrund der guten Justierbarkeit, Möglichkeit der Anordnung von Lastpaaren und Aufnahme von nichtruhenden Lasten.

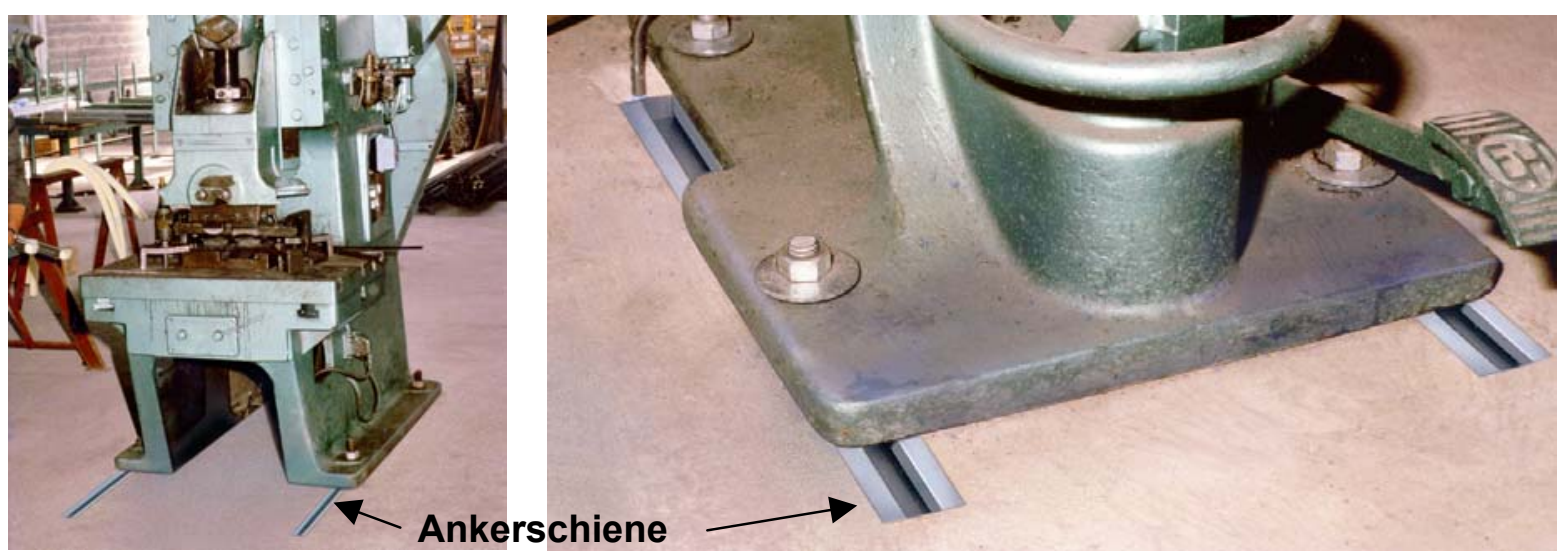

Bild 2.15 Bodenverankerung von Maschinen [55]

Durch das Einbetonieren der Ankerschienen ist eine langzeitig sichere Befestigung der angeschraubten Konstruktionen gegeben. Um eine sichere Weiterleitung der Kraft zu gewährleisten, sollten die Ankerschienen bei der Planung berücksichtigt werden.

\section{- Befestigungen in Verkehrs- und Versorgungstunneln}

Anwendung von gebogenen Ankerschienen (Sonderanfertigungen), mit Vorteilen in der einfachen Justierung und Kostenersparnissen aufgrund der Möglichkeit der schnelleren und sicheren Durchführung von Mehrfachinstallationen.
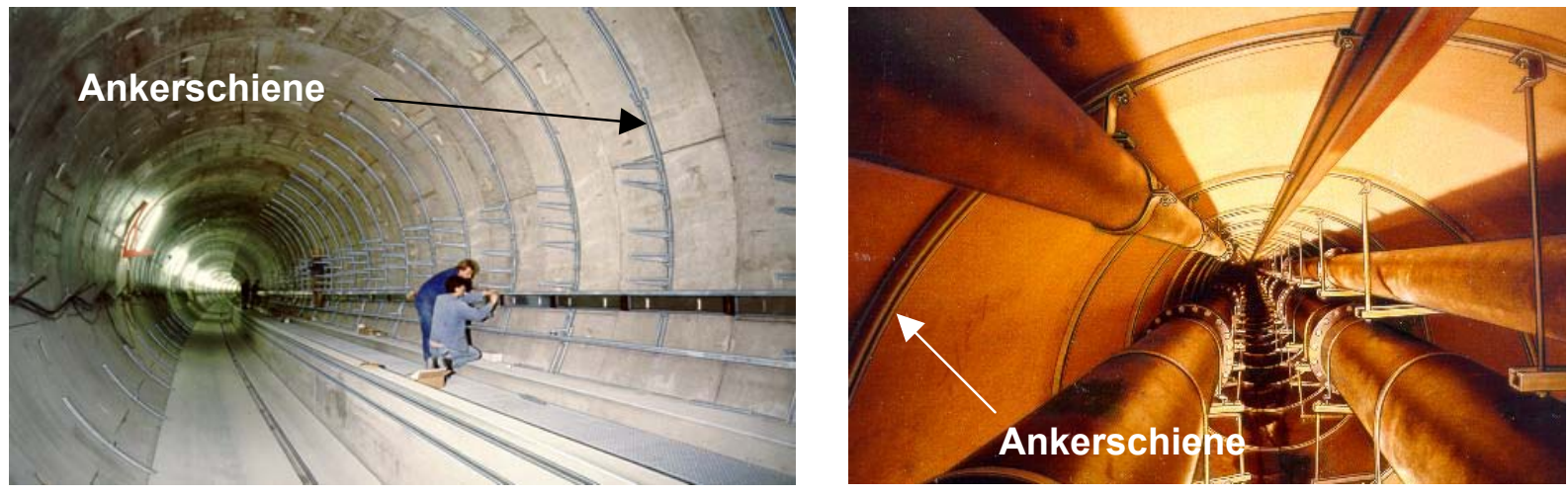

Bild 2.16 Befestigungen innerhalb von Tunneln und Versorgungsleitungen [55]

Für planmäßig nichtruhende und ermüdungsrelevante Einwirkungen, wie sie durchaus bei den vorgestellten Anwendungsbereichen auftreten können, sind besonders warmgewalzte, gezahnte Ankerschienen neuerer Generation als geeignete Problemlösungen anzusehen. Im folgenden Kapitel werden daher die Grundlagen zum Ermüdungstragverhalten dargestellt. 


\section{Ermüdungsfestigkeit}

\subsection{Grundlegende Nachweiskonzepte}

Für die Bestimmung der Ermüdungsfestigkeit bzw. Betriebsfestigkeit liegen derzeit sechs Konzepte vor. Diese können aus [70], [100] entnommen werden und sind im einzelnen:

- Die Experimentelle Ermittlung

- Das Nennspannungskonzept

- Das Strukturspannungskonzept

- Das Kerbspannungskonzept (Konzept der örtlich elastischen Beanspruchen)

- Das Kerbgrundkonzept (Konzept der örtlichen Beanspruchungen)

- Das Bruchmechanikkonzept (Rißfortschrittskonzept)

Es stellt sich hierbei die Frage, welches Nachweiskonzept für den konkreten Anwendungsfall $\mathrm{zu}$ wählen ist und welche Aussagen erwartet werden können. Eine rein experimentelle Ermittlung wird im Bauwesen aus Gründen der Wirtschaftlichkeit meistens ausgeschlossen. Da es sich bei den Konzepten jedoch nur um Hypothesen handelt, müssen diese experimentell verifiziert werden. In der Bemessungspraxis hat daher die rechnerische Abschätzung der ertragbaren Schwingspielzahl, auf der Basis von Versuchen an repräsentativen Bauteilen, größte Bedeutung.

Das Nennspannungskonzept ist aufgrund des relativ geringen Aufwandes zu bevorzugen. Es wird jedoch vorausgesetzt, daß geeignete und statistisch abgesicherte Funktionen der Ermüdungstragfähigkeit (Bild 3.5) vorliegen. In Kombination mit der linearen Schadensakkumulationshypothese (Abschnitt 3.5) kann mit einer entsprechenden Wahrscheinlichkeit die zulässige bzw. ertragbare Ermüdungsfestigkeit für eine vorgesehene Nutzungsdauer ermittelt werden. Bei Bauteilen mit einfacher Gestalt können die Nennspannungen nach der elementareren Festigkeitslehre ermittelt werden. Das Nennspannungskonzept bildet die Grundlage der Dimensionierung von Bauteilen und ist somit zur Bauteilauslegung in den Regelwerken verankert.

Bei komplexer gestalteten Bauteilen ist eine leichte Berechnung der Nennspannungen nicht mehr möglich. In diesem Fall erfolgt die Spannungsberechnung beispielsweise auf der Grundlage der Methode der Finiten Elemente. Die so berechneten Spannungen werden als Strukturspannungen bezeichnet (Strukturspannungskonzept). Durch eine weitere Verfeinerung des FE-Netzes können für den anzunehmenden Anrißort des Bauteils, unter Berücksichtigung von Spannungserhöhungen infolge Kerbwirkung, örtliche Kerbspannungen ermittelt werden (Kerbspannungskonzept). Struktur- und Kerbspannungen werden auch als ,verfeinerte“ Nennspannungen bezeichnet. Die Berechnung der ertragbaren Schwingspielzahlen in der vorgesehenen Nutzungsdauer erfolgt bei beiden Nachweiskonzepten anlog zum Nennspannungskonzept. 
Die Nenn-, Struktur- und Kerbspannungskonzepte basieren auf einem linear-elastischen Materialverhalten. Das Kerbgrundkonzept stellt darüber hinaus einen Zusammenhang zwischen äußeren Belastungen und elastisch-plastischen Beanspruchungen durch experimentell ermittelte oder sinnvoll abgeschätzte Last-Dehnungs-Kurven (Bauteilfließkurven) her. Sie bildet die Grundlage der Bauteildimensionierung, wenn Nennspannungen nicht definierbar sind. Aufgrund von verschiedenen Kennwerten, die Eingang in dieses Konzept finden, ist eine hohe Fehlerempfindlichkeit gegeben. Die Ermittlung der Eingangsparameter bedürfen daher wesentlich höherem Aufwand. Da beim Kerbgrundkonzept die Berechnung nur bis zum Versagen durch Anriß erfolgt, ist es zweckmäßig, eine Rißfortschrittberechnung hieran anzuschließen, um somit auch das Versagen durch Bruch zu erfassen.

Mit dem Auftreten eines ersten makroskopischen Anrisses verlieren örtliche Spannungen ihre Aussagekraft. In diesen Fällen werden unter Berücksichtigung von bruchmechanischen Gesetzmäßigkeiten die verbleibenden Schwingspielzahlen bis zum Bruch ermittelt. Das Rißfortschrittskonzept basiert auf einem Spannungsintensitätsfaktor, welcher den funktionalen Zusammenhang zwischen der Rißlänge und den Belastungszyklen angibt. Die Rißentwicklung ist wesentlich vom Werkstoff abhängig und kann durch die Gleichung nach Paris-Erdogan [94] für das stabile Rißwachstum beschrieben werden. Die Gleichung und deren konstanten Faktoren für unterschiedliche Werkstoffe können aus [53] entnommen werden.

Bei allen Verfahren ergibt sich das Problem hinsichtlich der Festlegung einer definierten Schadensumme, die von dem Werkstoff, dem Bauteil, der Fertigung, der Belastungsart und der Beanspruchungsfolge abhängt und nicht einheitlich ist. Sowohl beim Nennspannungskonzept, als auch beim Kerbgrundkonzept kann für einige Werkstoffe bei der Schadensakkumulationsrechnung sehr unsicher verfahren werden. Es sind daher derzeit intensive Untersuchungen zur Erfassung des Ermüdungstragverhaltens auf der Basis einer geeigneten Schadensakkumulation in der Entwicklung.

Um jedoch Absolutaussagen hinsichtlich der Festigkeit unter ermüdungsrelevanten Beanspruchungen treffen zu können, sind bei allen theoretischen Konzepten Versuche zur Verifikation der Ergebnisse unabdingbar. Im Rahmen der Untersuchungen in dieser Arbeit erfolgt die Bestimmung der Beanspruchbarkeit unter ermüdungsrelevanten Beanspruchungen ausschließlich auf experimenteller Basis. 


\subsection{Beanspruchungen und Beanspruchbarkeiten}

Der Begriff der Ermüdung (engl. fatigue) kennzeichnet das Versagen von Konstruktionen unter zeitlich veränderlichen Lasten. Häufige Lastwechsel einer Konstruktion reduzieren dessen Tragfähigkeit im Vergleich zu einer einmaligen, gleichbleibenden statischen Belastung.

Sämtliche auf dem Gebiet der Ermüdung existierenden Arbeiten zur Beschreibung der Ermüdungsproblematik greifen auf die grundlegenden Ansätze von August Wöhler [123] zurück. Im Rahmen experimenteller Untersuchungen von Eisenbahnwagenachsen unter konstanter Ermüdungslast entdeckte er 1860 den Zusammenhang zwischen der Beanspruchung und der Belastungsdauer (Schwingspielzahl) bis zum Bruch. Bis heute ist diese Beziehung die Grundlage der verwendeten Nachweisgleichungen gegen Ermüdung.

Unter dem Begriff der Ermüdungsfestigkeit werden folgende Begriffe zusammengefaßt:

- Schwingfestigkeit bei einstufiger Belastung

- Betriebsfestigkeit bei zufallsartiger, betriebsähnlicher Belastung

- Rißfortschritt bei einstufiger und zufallsartiger Belastung

Radaj [100] beschreibt die Ermüdungsfestigkeit als die Werkstoffschädigung, Rißeinleitung und Rißfortschritt unter zeitlich veränderlicher, häufig wiederholter Beanspruchung. Dabei ist die Art der Einwirkung von besonderem Interesse. Der Beanspruchungsablauf bzw. die nichtruhenden Einwirkungen können gemäß den in Bild 3.1 dargestellten Formen aufgeteilt werden. In Bild 3.2 werden zusätzlich die Ursachen für ruhende und nichtruhende Einwirkungen aufgezeigt.

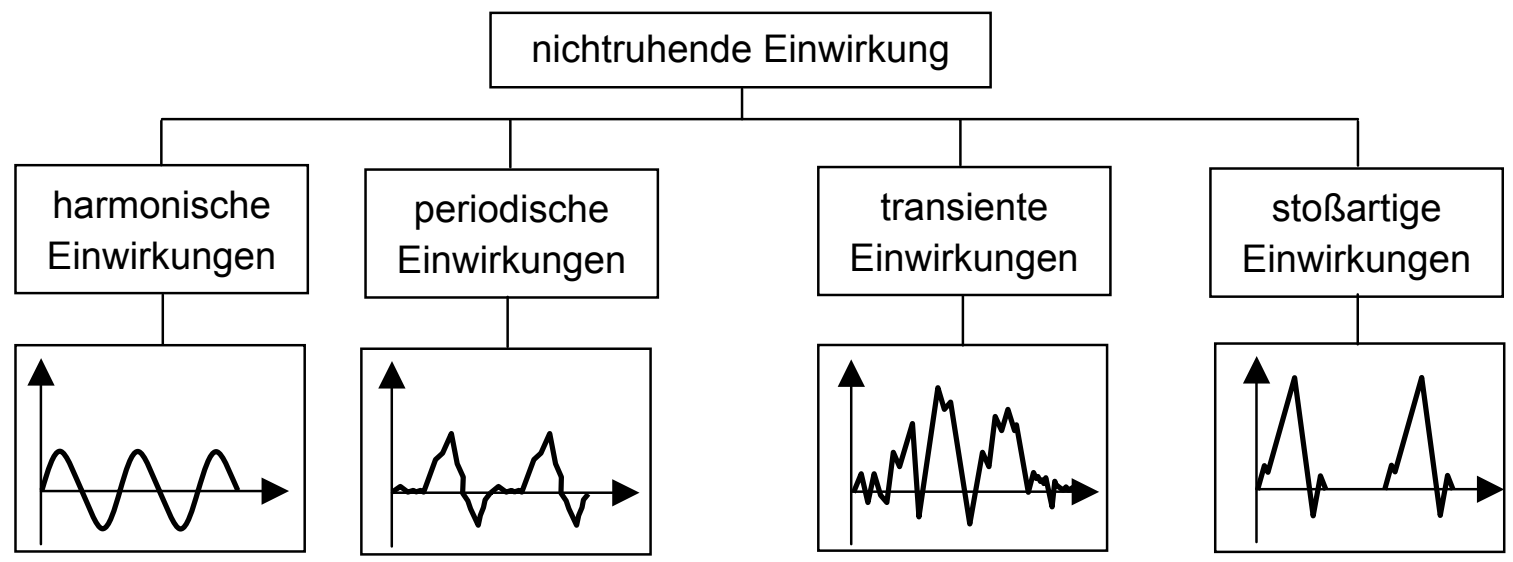

Bild 3.1 Ruhende und nichtruhende Einwirkungen und mögliche Ursachen 


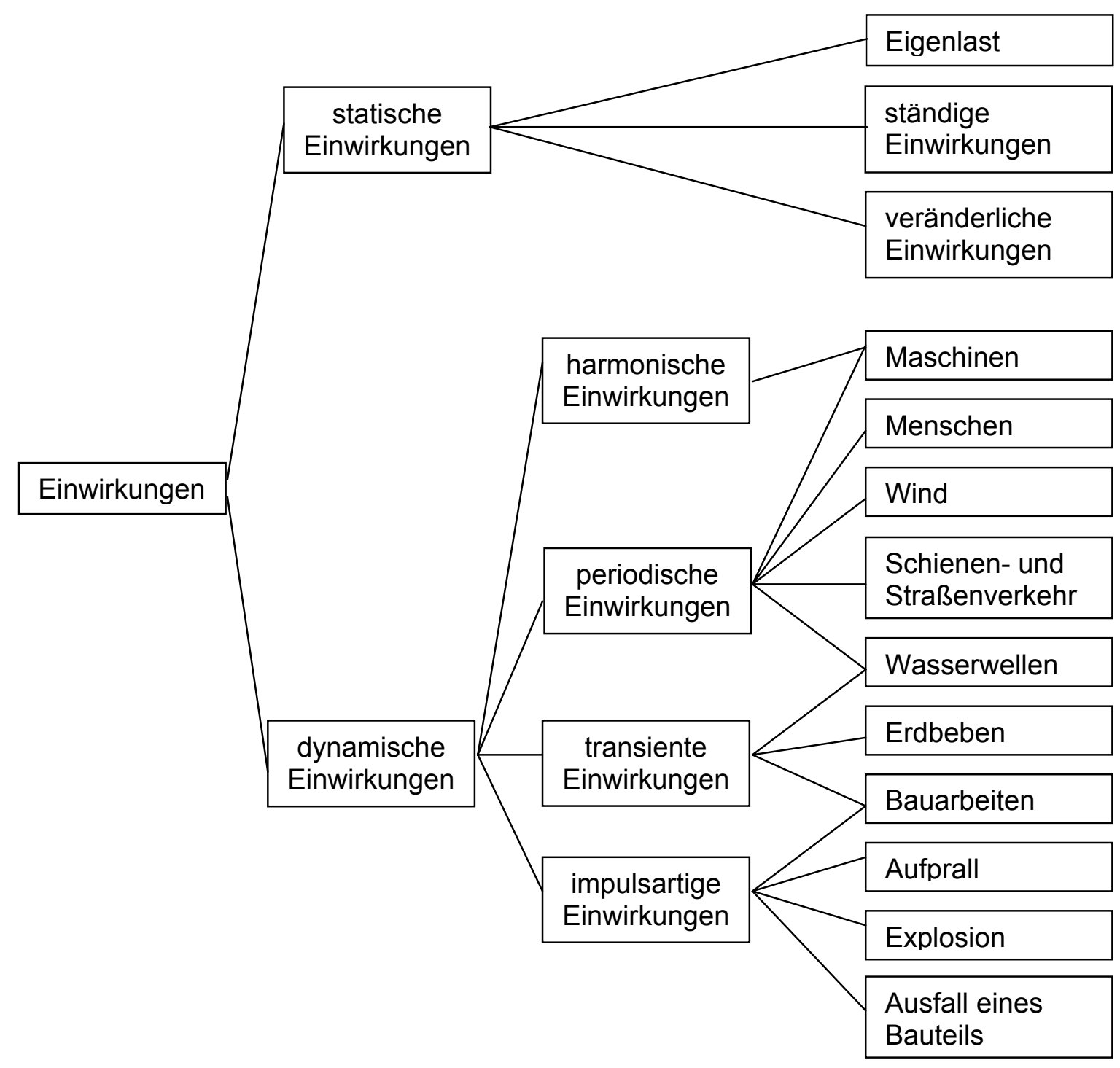

Bild 3.2 Ruhende und nichtruhende Einwirkungen und mögliche Ursachen [7]

Der zeitliche Verlauf der Beanspruchung ist für die Ermüdungsfestigkeit von sehr großer Bedeutung. Neben der Schwingfestigkeit bei zeitlich konstanter Beanspruchung (z.B. harmonisch, periodisch) ist auch die Erfassung betriebsähnlicher Belastungen (z.B. transient, stoßartig) sehr wichtig. Jedoch ist es nur schwer möglich, wirkliche betriebsähnliche Einwirkungen zu bestimmen. Diese bedürfen umfangreicher Messungen am realen Bauwerk, welche sehr aufwendig und kostspielig sein können. Deshalb werden bei experimentellen Untersuchungen periodische (harmonische) Beanspruchungsformen bevorzugt, bei der konstante Schwingbreiten relativ zu einer bereits wirkenden Belastung (z.B. aus ständiger Last) aufgebracht werden können. Diese Art der Schwingbeanspruchung wird im sog. Wöhlerversuch [123] durchgeführt und stellt den einfachsten Fall einer nichtruhenden Einwirkung dar, bei der eine sinusförmige Belastung zwischen zwei gleichbleibenden Lastgrenzen mit konstanter Belastungsfrequenz aufgebracht wird. Die mechanischen Beanspruchungs- 
kenngrößen zur Beurteilung der nichtruhenden Beanspruchungen sind in Bild 3.3 exemplarisch für ein Schwingspiel dargestellt.

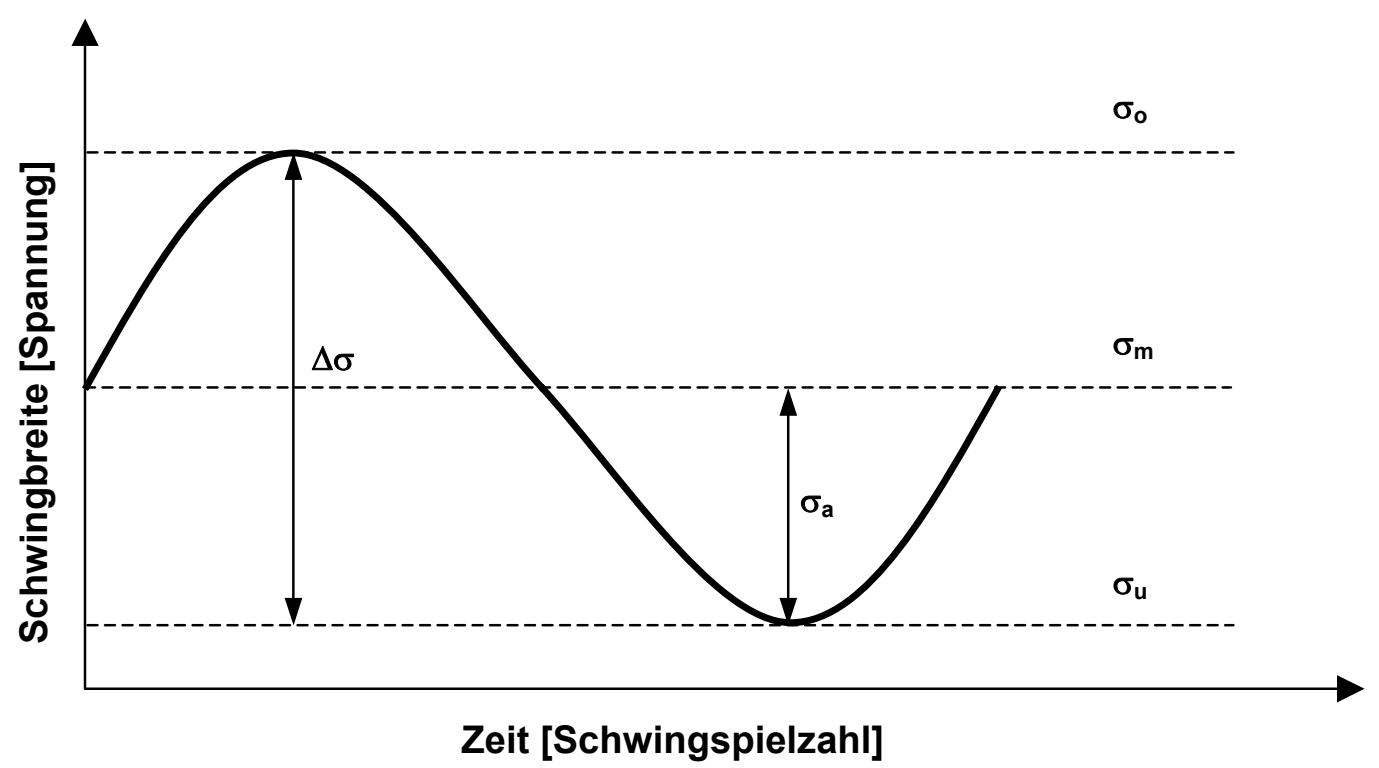

Bild 3.3 Mechanische Kenngrößen der periodischen Beanspruchung

Definition der allgemeinen Kennwerte:

$\sigma_{\mathrm{o}}: \quad$ Oberspannung

$\sigma_{\mathrm{u}}: \quad$ Unterspannung

$\sigma_{\mathrm{m}}=\left(\sigma_{\mathrm{o}}+\sigma_{\mathrm{u}}\right) / 2: \quad$ Mittelspannung

$\sigma_{\mathrm{a}}=\left(\sigma_{\mathrm{o}}-\sigma_{\mathrm{u}}\right) / 2: \quad$ Spannungsamplitude

$\Delta \sigma=\sigma_{\mathrm{o}}-\sigma_{\mathrm{u}}=2 \cdot \sigma_{\mathrm{a}}:$ Spannungsschwingbreite bzw. Spannungsdoppelamplitude

Eine weitere charakteristische Größe ist das Spannungsverhältnis R (auch $\kappa$ genannt), welches für die Durchführung der Betriebsfestigkeitsnachweise oft benötigt wird. Das Spannungsverhältnis ist folgendermaßen definiert:
$\mathrm{R}=\sigma_{\mathrm{u}} / \sigma_{\mathrm{o}}$
für $\left|\sigma_{u}\right| \leq\left|\sigma_{o}\right|$
$\mathrm{R}=\sigma_{\mathrm{o}} / \sigma_{\mathrm{u}}$
für $\left|\sigma_{u}\right|>\left|\sigma_{0}\right|$

Die auftretenden Belastungen sind mit Hilfe des Spannungsverhältnisses R drei unterschiedlichen Beanspruchungsbereichen zuzuordnen (Bild 3.4):

- Druckschwellbeanspruchung

- Wechselbeanspruchung

- Zugschwellbeanspruchung 


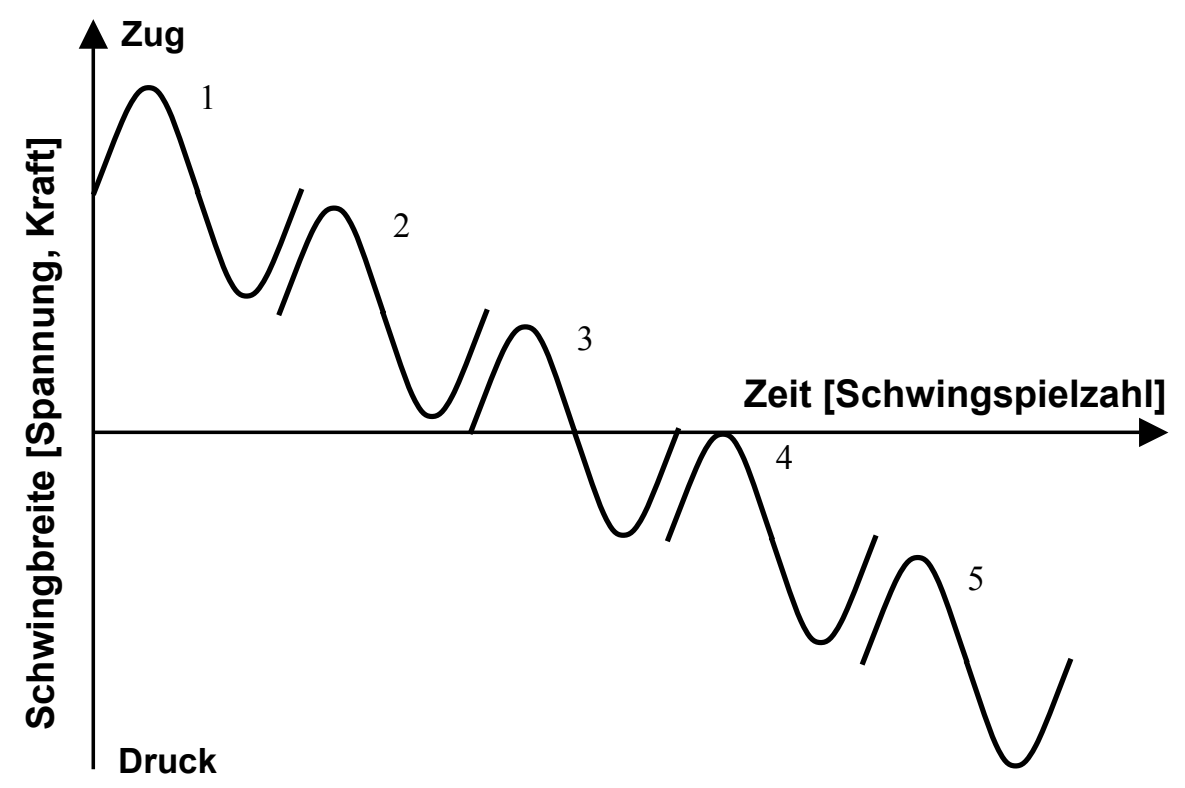

1. Zugschwellbeanspruchung: $\quad \sigma_{\mathrm{o}}>0 \quad$ und $\left.\quad \sigma_{\mathrm{u}}>0 \quad \mathrm{R}=\right] 0 ; 1[$

2. Zugursprungsbeanspruchung: $\sigma_{\mathrm{o}}>0$ und $\sigma_{\mathrm{u}}=0 \quad \mathrm{R}=0$

3. Reine Wechselbeanspruchung: $\sigma_{\mathrm{o}}=-\sigma_{\mathrm{u}} \quad \mathrm{R}=-1$

4. Druckursprungsbeanspruchung: $\sigma_{\mathrm{o}}=0 \quad$ und $\sigma_{\mathrm{u}}<0 \quad \mathrm{R}=0$

5. Druckschwellbeanspruchung: $\quad \sigma_{\mathrm{o}}<0 \quad$ und $\left.\quad \sigma_{\mathrm{u}}<0 \quad \mathrm{R}=\right] 0 ; 1[$

Bild 3.4 Arten der periodischen, harmonischen (hier sinusförmigen) Beanspruchung

Wie bereits erwähnt, hängt das Ermüdungstragverhalten eines Materials oder Systems von vielen Einflußgrößen ab, die material- oder lastspezifisch sein können. Eine genaue Erfassung dieser Einflüsse gelingt bei größter Anstrengung bis heute nicht vollständig, was zwangsläufig zur Forderung nach umfassenden experimentellen Untersuchungen führt. Das Ergebnis solcher Versuche ist bei Beanspruchung oberhalb der Dauerfestigkeit ein charakteristischer Ermüdungsbruch. Im Rahmen dieser Arbeit wird das Ermüdungstragverhalten unter periodischer Schwingbeanspruchung näher untersucht und dabei die Einflußfaktoren und deren Wirkung auf das Ermüdungstragverhalten ermittelt. Ein Ermüdungsnachweis wird nur bei vorwiegend nichtruhenden Einwirkungen notwendig.

Werden nun die bei konstanten Schwingbreiten, je nach Art der periodischen Beanspruchung, erzielten Schwingspielzahlen bis zum Versagen $\mathrm{N}_{\mathrm{f}}$ (Abszisse) in einem Diagramm aufgezeichnet, so läßt sich die bekannten Wöhlerlinie in Bild 3.5 darstellen (hier in halb logarithmischer Darstellung gemäß DIN 50100 [29]. Diese Beziehung ist die Grundlage der verwendeten Nachweise gegen Ermüdung. 


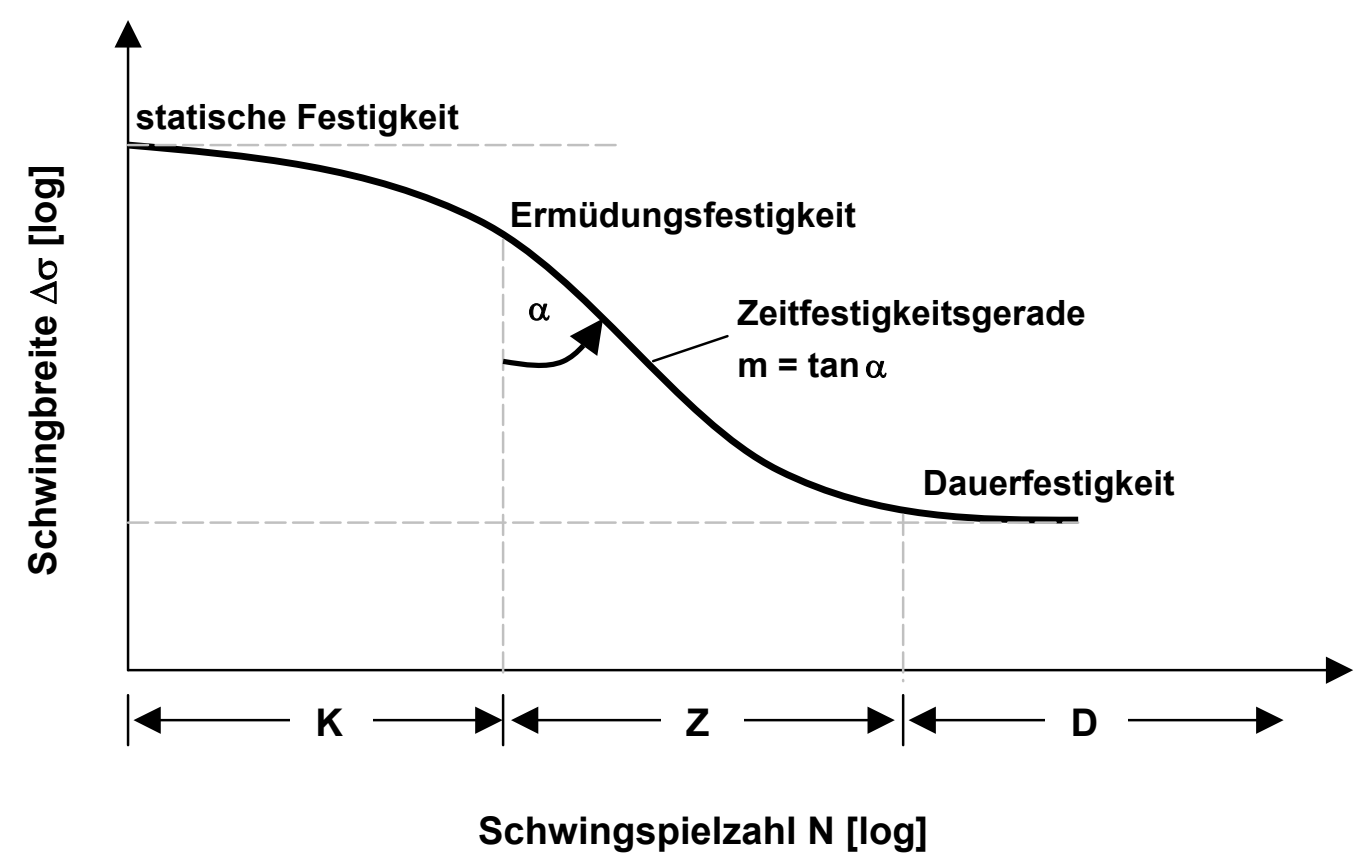

Bild 3.5 Darstellung der Ermüdungsfestigkeit in Form einer Wöhlerlinie

Beim Auftragen der Wöhlerlinie in der üblichen Darstellungsweise, mit der Abszisse im logarithmischen Maßstab, erhält man im charakteristischen Zeitfestigkeitsbereich einen zunächst steil abfallenden Verlauf mit einer zunehmenden Krümmung, der dann aber in eine Waagerechte übergeht. In diesem Fall entspricht der Wert an der Ordinate der Dauerfestigkeit. Die Wöhlerlinie wird, wie Bild $3.5 \mathrm{zu}$ entnehmen ist, für Stahl in folgende Bereiche unterteilt [113]:

- Kurzzeitfestigkeit K:

Ertragbare Schwingbreite etwa zwischen $\mathrm{N}=1$ und $\mathrm{N}=1 \cdot 10^{4}$

- Zeitfestigkeit Z:

Ertragbare Schwingbreite etwa Zwischen $\mathrm{N}=1 \cdot 10^{4}$ und $\mathrm{N}=2 \cdot 10^{6}$

- Dauerfestigkeit D:

Ertragbare Schwingbreite ab etwa $\mathrm{N}=2 \cdot 10^{6}$ bzw. $\mathrm{N}=5 \cdot 10^{6}$

Die Anzahl der Schwingspiele, ab der die Wöhlerlinie parallel zur Abszisse verläuft, kann unterschiedlich sein. Um den Übergang deutlich sichtbar zu machen, empfiehlt es sich, die ertragbare Schwingspielzahl im logarithmischem Maßstab darzustellen. Hierdurch wird ebenfalls der Bereich, in dem keine wesentliche Änderung in der Dauerfestigkeit erfolgt, stärker hervorgehoben. Als eine anerkannte Grenze im Stahlbau hat sich die sog. Stahlbaudauerfestigkeit bei $2 \cdot 10^{6}$ Schwingspielen als sinnvoll bestätigt. In [10] wird jedoch gezeigt, daß die Dauerfestigkeit als charakteristische Größe nur auf bestimmte Materialien beschränkt werden darf. Die Annahme einer unendlich langen Beanspruchbarkeit bei Spannungsamplituden unterhalb der Dauerfestigkeit lassen sich nach neueren Erkenntnissen nicht bestätigen. Weiterhin gibt es 
unterschiedliche Annahmen hinsichtlich des Verlaufes der Wöhlerlinie über die Dauerfestigkeit hinaus.

Corten und Dolan [20] setzen voraus, daß die Wöhlerlinie geradlinig bis zum Nullniveau der Schwingbreite verläuft. Dabei wird davon ausgegangen, daß der Schaden über die Dauerfestigkeit hinaus zunimmt. Nach der Hypothese von Haibach [53] existiert ein verringerter Schaden unterhalb der Dauerfestigkeit, d. h., daß die Wöhlerlinie ab der Dauerfestigkeit einen flacheren Verlauf aufweist. Nach der Hypothese von Miner [85] jedoch nimmt der Schaden unterhalb der Dauerfestigkeit nicht zu. In diesem Fall läuft die Wöhlerlinie in Höhe der Dauerfestigkeit horizontal. Somit erfolgt keine Schädigung über die Dauerfestigkeit hinaus. Bild 3.6 zeigt eine Zusammenstellung der o.g. Annahmen.

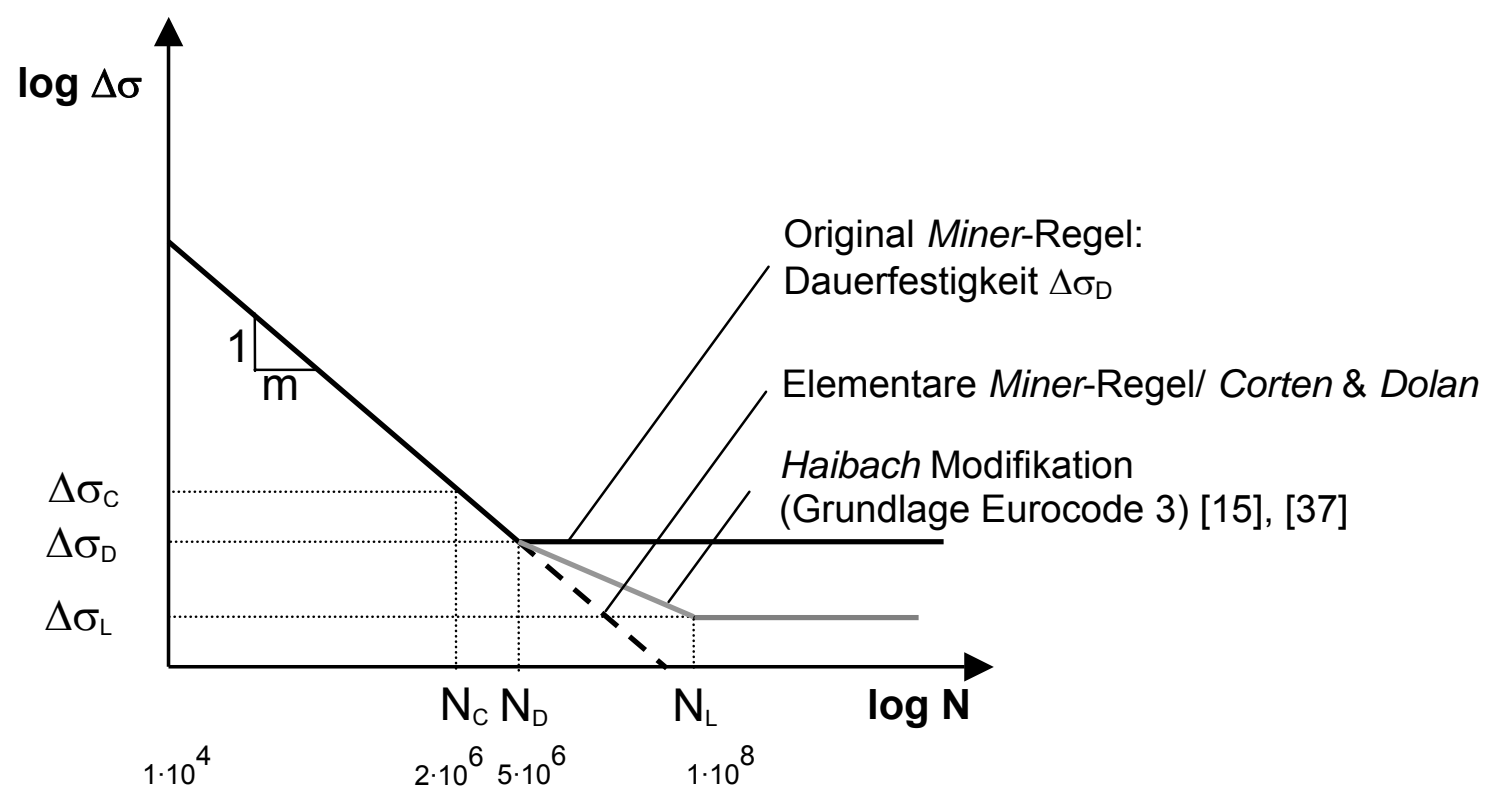

Bild 3.6 Modifizierte Ermüdungsfestigkeiten

Zur eindeutigen Charakterisierung einer Wöhlerlinie ist der Einfluß der unterschiedlichen Versuchsparameter von wichtiger Bedeutung. Wöhlerlinien können sowohl aus Versuchen mit konstanter Unterspannung $\sigma_{\mathrm{u}}$, konstanter Mittelspannung $\sigma_{\mathrm{m}}$, als auch mit konstanter Oberspannung $\sigma_{0}$ gewonnen werden. Jedoch liegt genau in diesen Parametern der Einfluß auf den Verlauf der Wöhlerlinie. Die Einflüsse aus diesen Parametern werden im experimentellen Teil dieser Arbeit näher untersucht.

Neben den Wöhlerlinien, die den zeitlichen Verlauf einer Beanspruchbarkeit in Form von Spannungen oder Lasten darstellen, sind weitere Darstellungen der Ermüdungsfestigkeit in Form von Dauerfestigkeitsdiagrammen vorhanden. Diese beziehen sich auf einen festen Zeitpunkt (z.B. $2 \cdot 10^{6}$ Schwingspiele). In den nachfolgenden Abschnitten werden zunächst Dauerfestigkeitsdiagramme von Stahl dargestellt und anschließend die Einflußfaktoren auf das Ermüdungstragverhalten aufgezeigt. 


\subsection{Ermüdungsfestigkeit von Stahl}

\subsubsection{Darstellung in Dauerfestigkeitsdiagrammen}

Eine sinnvolle Darstellung zur Verdeutlichung von Einflußfaktoren wie Schwingbreite, Mittellast, Grad der Kerbwirkung und Eigenspannungen auf die Ermüdungsfestigkeit wird mit Hilfe von Dauerfestigkeitsdiagrammen erreicht. Es gibt unterschiedliche Darstellungsformen, die je nach Art der Auswertung zweckmäßig sein können. Aus allen Dauerfestigkeitsdiagrammen können die Interaktionen zwischen der wirkenden Spannungen und den restlichen Kennwerten einer periodischen Beanspruchung ermittelt werden

\section{Darstellung nach Goodman}

Diese Darstellung geht zurück auf die Personen Goodman, Gerber, Launhardt-Weyrauch und Kommerell. Hierbei wird die ertragbare Spannungsamplitude (Dauerfestigkeit) direkt über die Unterspannung aufgetragen. Die Unterspannung bildet eine $45^{\circ}$ Linie, deren Verlauf durch die Streckgrenze begrenzt wird. Diese Darstellung ist dann besonders empfehlenswert, wenn zu einer ruhenden Last nichtruhende Zusatzlasten hinzukommen, wie z.B. im Brückenbau oder in vielen Bereichen der Befestigungstechnik [7].

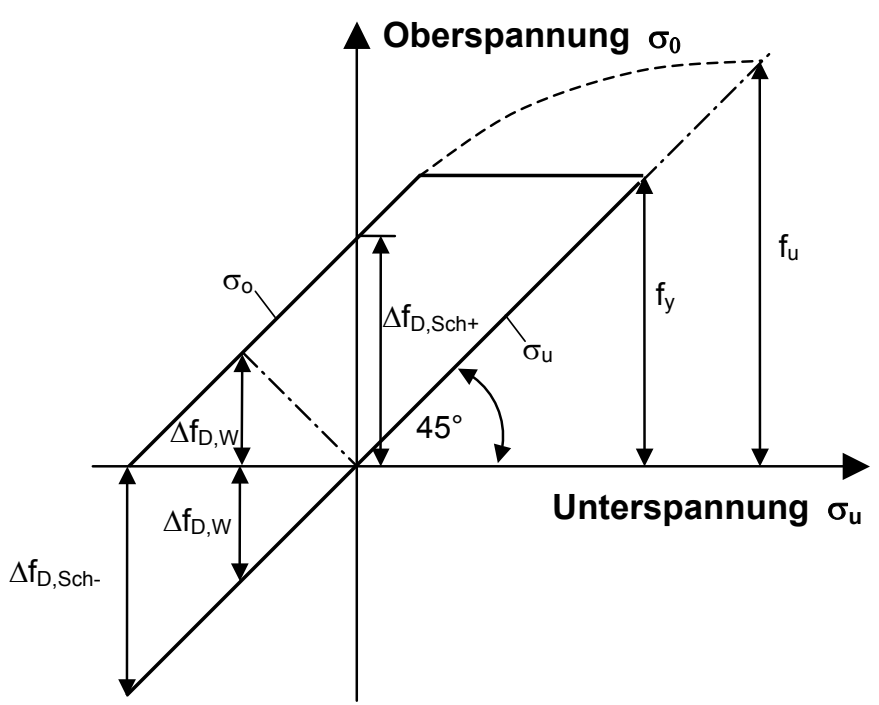

Bild 3.7 Dauerfestigkeitsdiagramm nach Goodman, Weyrauch [116]

Die Unterspannung $\sigma_{\mathrm{u}}$ bildet in einem Winkel von $45^{\circ}$ die Winkelhalbierende und läuft geradlinig bis zur Bruchgrenze $f_{u}$, wobei sich die Oberspannung $\sigma_{o}$ auf einer gekrümmten Linie dieser Grenze annähert. In Höhe der Streckgrenze $f_{y}$ wird das Diagramm durch eine Parallele zur Abszisse abgegrenzt. Verformungen oberhalb dieses Bereiches sind nicht Grundlage einer Bemessung, da sie meist stark plastische Verformungen aufweisen und nicht ohne weiteres behandelt werden können. Der Schnittpunkt der Oberspannung mit der Ordinate legt den Wert der Zugursprungsbeanspru- 
chung $\Delta f_{D, S c h}$ fest. Eine im Ursprung beginnende und im Winkel von $45^{\circ}$ steigende Gerade schneidet die Oberspannung bei der Wechselfestigkeit $\Delta f_{D, w}$. Wird nun eine Parallele zur Ordinate durch den Schnittpunkt der Oberspannung mit der Abszisse gezogen, so erhält man als Differenz zur Unterspannung die Druckursprungsbeanspruchung $\Delta \mathrm{f}_{\mathrm{D}, \mathrm{Sch} \text { - }}$

Ein weitere, sehr verbreitete und gebräuchliche Darstellung stellt das im nächsten Abschnitt beschriebene Smith-Diagramm dar.

\section{Darstellung nach Smith}

Dieses Dauerfestigkeitsdiagramm weist Ähnlichkeiten zur Darstellungsweise im Weyrauch \& Goodman-Diagramm auf. Im Smith-Diagramm wird die Oberspannung $\sigma_{\mathrm{o}}$ bzw. die Unterspannung $\sigma_{\mathrm{u}}$ als Funktion der Mittelspannung $\sigma_{\mathrm{m}}$ aufgetragen. Das Diagramm wird ebenfalls durch eine Parallele zur Abszisse in Höhe der Streckgrenze abgeschnitten, da Dauerbeanspruchungen über diesen Bereich hinaus nicht zulässig sind. Auf diese Weise werden plastische Verformungen verhindert.

Das Smith-Diagramm hat den Vorteil, daß neben der Bruchgrenze $f_{u}$ und der Streckgrenze $f_{y}$ nur drei weitere Dauerfestigkeitswerte erforderlich sind, um den Verlauf zu definieren. Diese sind die Wechselfestigkeit $\Delta \mathrm{f}_{\mathrm{D}, \mathrm{W}}$, die Zugschwellfestigkeit $\Delta \mathrm{f}_{\mathrm{D}, \mathrm{sch}+}$ und die Druckschwellfestigkeit $\Delta \mathrm{f}_{\mathrm{D}, \text { sch. }}$. In dem Diagramm lassen sich die Zusammenhänge zwischen Mittelspannung, Spannungsamplitude, Oberspannung und Unterspannung in anschaulicher Weise darstellen. Wie die Darstellung nach Goodman gilt das Smith-Diagramm nur für eine bestimmte Lastwechselzahl. Es stellt also einen Querschnitt in der Wöhlerlinie dar. Je stärker die Kerbwirkungen und Eigenspannungen eines Bauteils sind, desto mehr nähert sich das Smith-Diagramm zwei parallelen Geraden, d.h., daß in diesem Fall die Spannungsdifferenz $\Delta \sigma=2 \sigma_{\mathrm{a}}$ nahezu konstant ist.

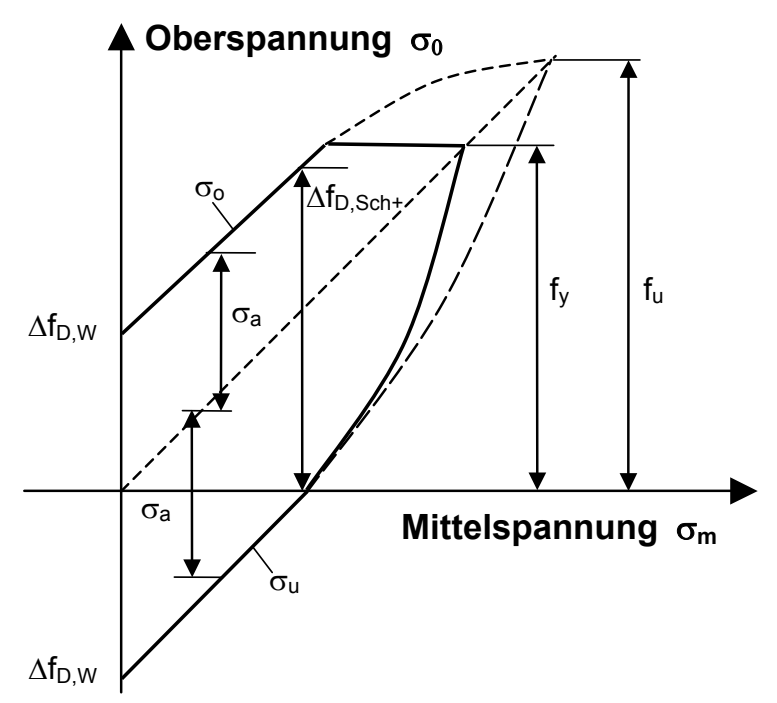

Bild 3.8 Dauerfestigkeitsdiagramm nach Smith 


\section{Darstellung nach Haigh}

Die Spannungsamplitude $\sigma_{\mathrm{a}}$ aufgetragen über die Mittelspannung $\sigma_{\mathrm{m}}$ liefert das Haigh-Diagramm. Die Abhängigkeit einer Spannungsamplitude von einer wirksamen Vorspannung, einer Schweißeigenspannung bzw. einer Mittelspannung kann hiermit erläutert werden. Es lassen sich Ursprungsgeraden einzeichnen, die die Werte für das gleichen Spannungsverhältnisses R kennzeichnen. Somit lassen sich gleichzeitig die Werte für die ertragbaren Spannungsamplituden bei gegebenen Spannungsverhältnissen ablesen. Es ist zu erkennen, daß nur der halbe Wert der dauerfest ertragbaren Spannungsamplitude abgelesen werden kann. Der Gültigkeitsbereich des Diagramms wird auch hier durch eine Streckgrenzengerade eingegrenzt, damit unzulässige Dehnungen ausgeschlossen sind.

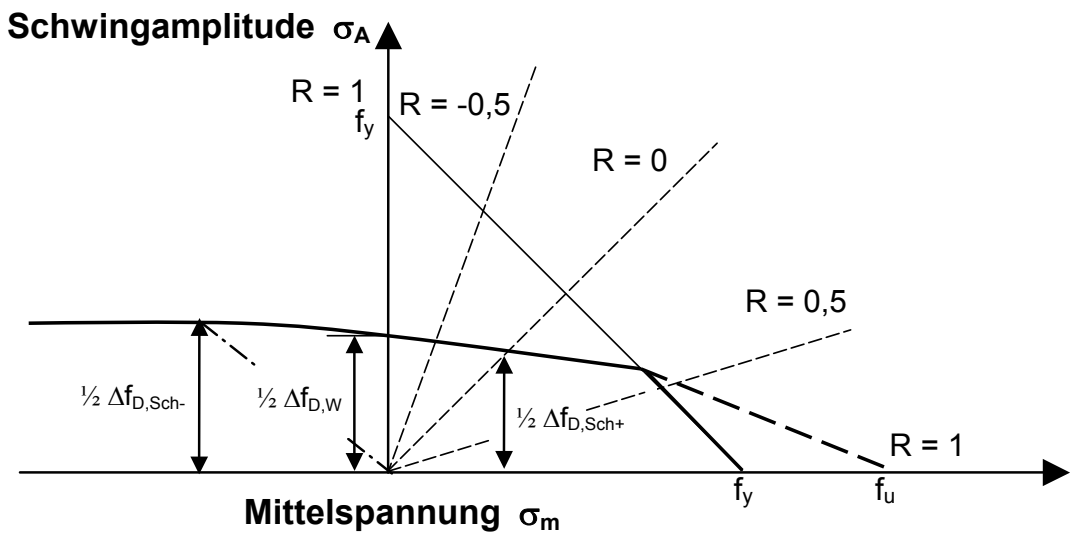

Bild 3.9 Dauerfestigkeitsdiagramm nach Haigh

\section{Darstellung nach Moore}

Im Stahlbau ist es üblich, die Darstellung nach Moore zu verwenden. Hierbei wird auf der Abszisse das Spannungsverhältnis R aufgetragen und auf der Ordinate die zugehörige Oberspannung. Wie in den Diagrammen zuvor findet auch hierbei eine Abgrenzung durch die Streckgrenze $\mathrm{f}_{\mathrm{y}}$ (Zugbereich) statt.

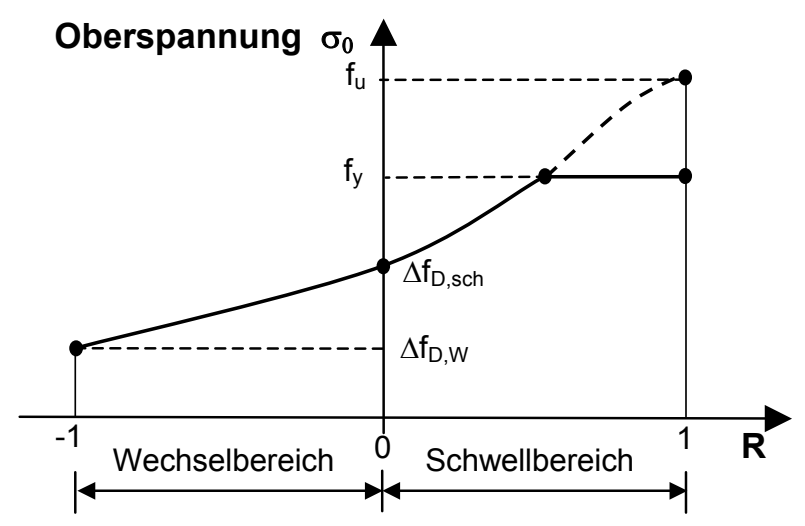

Bild 3.10 Dauerfestigkeitsdiagramm nach Moore [106] 


\subsubsection{Einflüsse auf das Ermüdungsverhalten}

Wie bereits einleitend in Anschnitt 3.2 erwähnt, wird das Ermüdungstragverhalten eines Bauteils von unterschiedlichen Einflußgrößen beeinflußt. Die wichtigsten und maßgebenden Einflüsse werden in [14] genannt:

- Herstellung und Verarbeitung

- Kerbwirkung, Eigenspannung

- Beanspruchungsintensität und -art (Spannungsverhältnis, Schwingbreite)

- Belastungsfrequenz (bei Stahl bis $1 \cdot 10^{3} \mathrm{~Hz}$ kein signifikanter Einfluß)

- Temperatur und Umgebungsmedium (Frost, Korrosion etc.)

In diesen Bereichen sind umfangreiche Untersuchungen durchgeführt worden. Alle möglichen Einflußfaktoren darzustellen wäre im Rahmen dieser Arbeit nicht zweckmäßig. Vielmehr soll anhand repräsentativer Darstellungen ein umfassender Überblick vermittelt werden. Generell werden in beanspruchungsunabhängige (werkstoffund fertigungsabhängig) und beanspruchungsabhängige Einflüsse unterschieden.

\section{Kerbwirkung}

Ausgehend von Fehlstellen, die Kerben in Form von Bohrungen, Absenkungen, Eindrücken etc. sein können, beginnen Anrisse, die bis zum Bruch fortschreiten können. Eine Rißentstehung an solchen Stellen ist auf eine ungleichmäßige Verteilung der Spannung zurückzuführen. Hierbei treten Spannungsspitzen auf, die zu örtlichen plastischen Verformungen führen. Kerben werden daher auch als Stellen mit hohen Spannungskonzentrationen bezeichnet. Bruchmechanische Gesichtspunkte bei gekerbten und ungekerbten Bauteilen sind von besonderer Wichtigkeit .

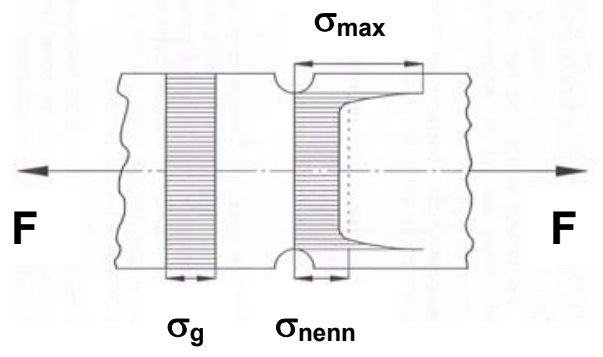

$\sigma_{\max }=$ maximale Spannung im Kerbgrund

$\sigma_{\text {nenn }}=$ Nennspannung im geschwächten Schnitt

$\sigma_{g}=$ Grundspannung im ungeschwächten Schnitt

Bild 3.11 Darstellung der Spannungen an einer Kerbe

Zur Beschreibung einer Kerbe wird die sog. Formzahl bzw. der Kerbfaktor $\alpha_{\mathrm{k}}$ als Verhältnis der Maximalspannung $\sigma_{\max } z u$ Nennspannung $\sigma_{\text {nenn }}$ eingeführt.

$$
\alpha_{\mathrm{k}}=\frac{\sigma_{\max }}{\sigma_{\text {nenn }}}
$$


Diese Beziehung gilt für den linear-elastischen Beanspruchungsbereich (Hook'sches Gesetz). Für eine nichtlineare Spannungskonzentration wird an dieser Stelle auf die Veröffentlichung von [89] verwiesen.

Es ist ersichtlich, daß die Maximalspannung von der Form der Kerbe abhängt. Aus diesem Grund existieren $\mathrm{zu}$ unterschiedlichen Kerbformen auch unterschiedliche Formzahlen. Die Formzahl bleibt unverändert, solange das Hook'sche Gesetz Gültigkeit besitzt. In diesem Fall ist sie unabhängig von der Nennspannung, vom Material und der Geometrie, auch wenn die Abmessungen proportional verändert werden. Jedoch zeigt sich ein von der Beanspruchungsart abhängiges Verhalten.

$\alpha_{\mathrm{k}, \text { Zug }}>\alpha_{\mathrm{k}, \text { Biegung }}>\alpha_{\mathrm{k}, \text { Torsion }}$

Die Größenordnung der jeweiligen Formzahlen unter reiner Zugbeanspruchung bzw. bei Biegung kann aus den Formzahldiagrammen in Bild 3.12 entnommen werden.
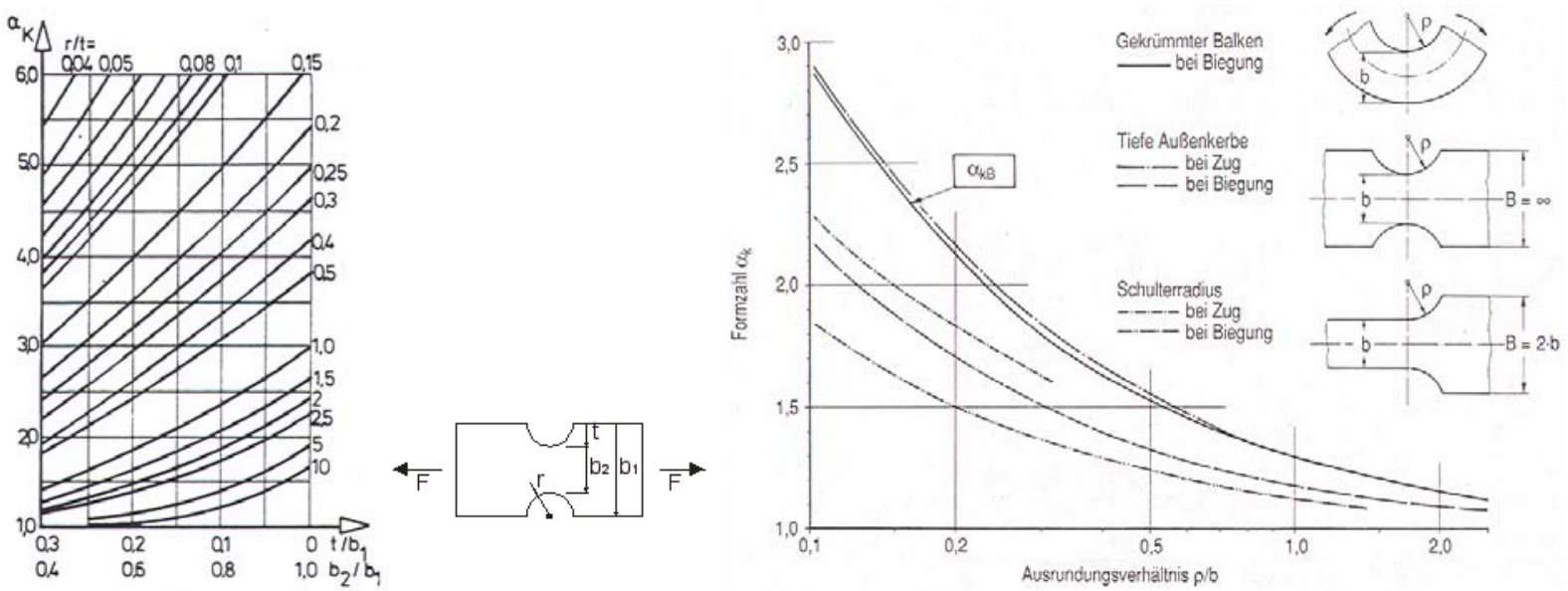

Bild 3.12 Formzahldiagramm nach [103] links, [53] rechts

Betrachtet man jedoch Schwingversuche, so stellt man fest, daß die ertragbare Schwingbreite sich nicht proportional mit der Formzahl ändert. Die Reduzierung der Schwingfestigkeit ist in der Regel geringer, als es nach der Formzahl zu erwarten wäre. Um das Verhältnis eines gekerbten zu einem ungekerbten Bauteil unter schwingender Beanspruchung aufzuzeigen, ist in Analogie zur Formzahl $\alpha_{k}$ die Kerbwirkungszahl $\beta_{\mathrm{k}}$ in der nachfolgenden Gleichung eingeführt worden. Dabei wird das Verhältnis der jeweiligen ertragbaren Schwingbreiten im ungekerbten Zustand zum gekerbten Zustand gebildet.

$\beta_{\mathrm{k}}=\frac{\Delta \sigma_{\text {ungekerbt }}}{\Delta \sigma_{\text {gekerbt }}}$

Die Differenz zwischen $\alpha_{\mathrm{k}}$ und $\beta_{\mathrm{k}}$ gibt kein festes Verhältnis an, sondern lediglich ein $\mathrm{Ma} ß$ für die Kerbempfindlichkeit. Je geringer die Differenz, desto unempfindlicher reagiert der Werkstoff bzw. das Bauteil auf die Kerbe. 
Eine Abhängigkeit der Formzahl von der Kerbwirkungszahl zeigt das linke Diagramm in Bild 3.13. Man erkennt eine einheitliches Verhalten der unterschiedlichen Vergütungsstähle. Zur Verdeutlichung des Kerbeinflusses in Abhängigkeit von den Schwingspielzahlen sind in [14] Versuche mit ungekerbten und gekerbten Proben dargestellt. Die zeitliche Abhängigkeit der Kerbwirkung zeigt das rechte Diagramm in Bild 3.13. Auch hierbei ist ein einheitliches Verhalten in Abhängigkeit von der Schwingspielzahl zu erkennen.

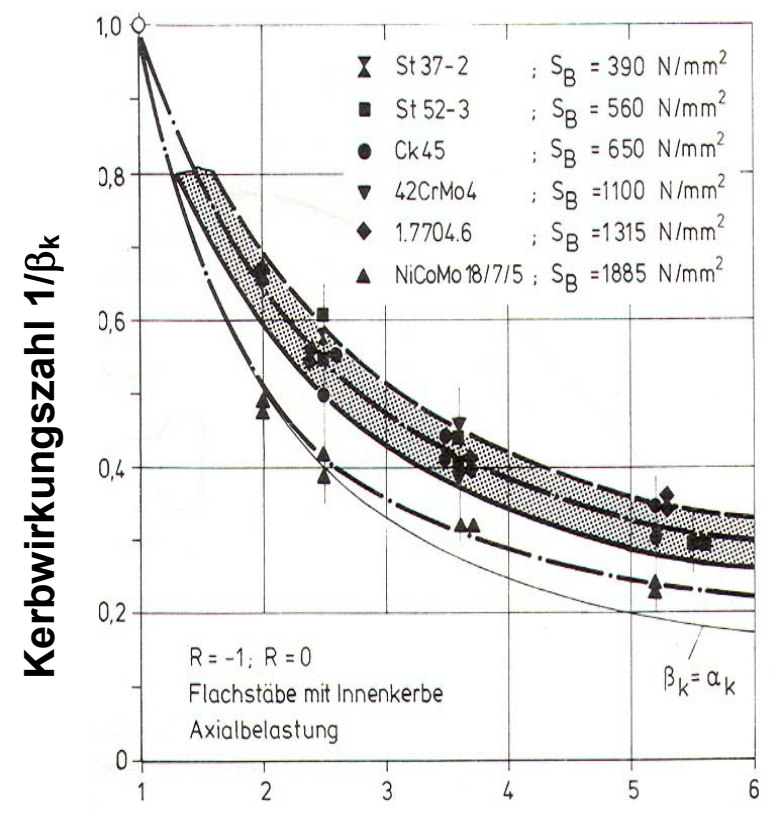

Formzahl $\alpha_{k}$

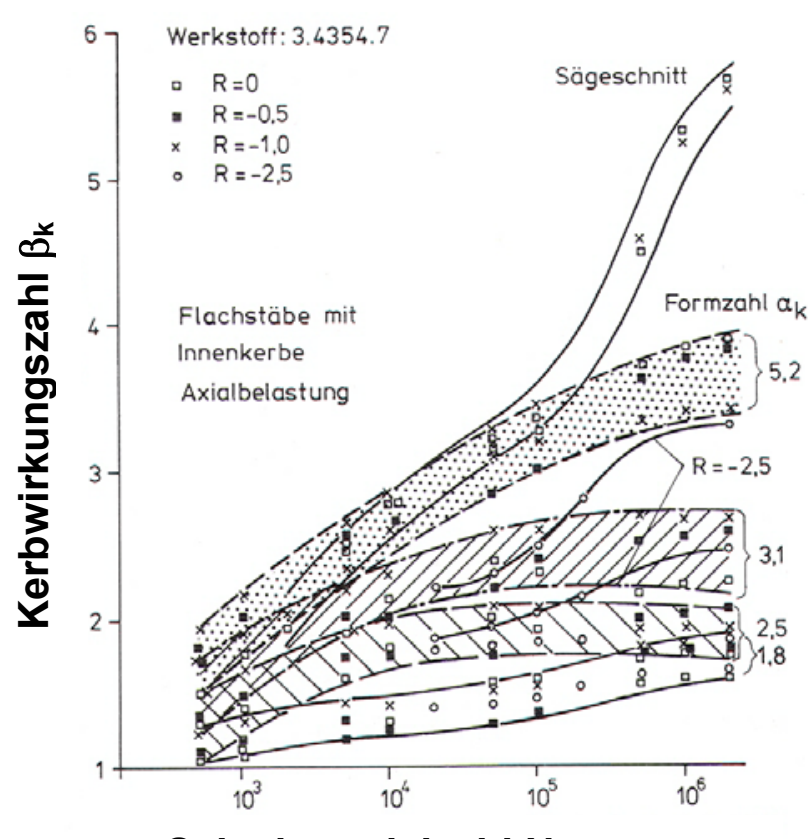

Schwingspielzahl $\mathbf{N}_{\mathrm{f}}$

Bild 3.13 Kerbwirkung über Formzahl (links) und Schwingspiele (rechts) [14]

Risse entstehen bei schwingender Beanspruchung hauptsächlich an Stellen höchster Spannungskonzentrationen, weshalb Kerben auch als Schwachpunkte anzusehen sind. Jedoch gibt es konstruktive Möglichkeiten, den Kerbeinfluß zu reduzieren. Zum einen ist es empfehlenswert, sogenannte Entlastungskerben anzuordnen, die durch Verwendung größerer Kerbgrundradien eine Reduzierung der Spannungen zur Folge haben. Eine weitere, jedoch aufwendigere Maßnahme zur Reduzierung der Spannungkonzentrationen sind mechanische bzw. thermische Verfahren, bei denen eine Druckeigenspannung an der Kerbe erzeugt wird. 


\section{Stahlgüte}

Die Güte des Stahls hat bei starker Kerbung einen vernachlässigbar kleinen Einfluß auf das Ermüdungsverhalten. Nachfolgendes Bild 3.14 zeigt die Abhängigkeit der Oberspannungen bei unterschiedlichen Materialfestigkeiten von der Schwingspielzahl für vier Betonstähle mit gleicher Rippenausbildung.

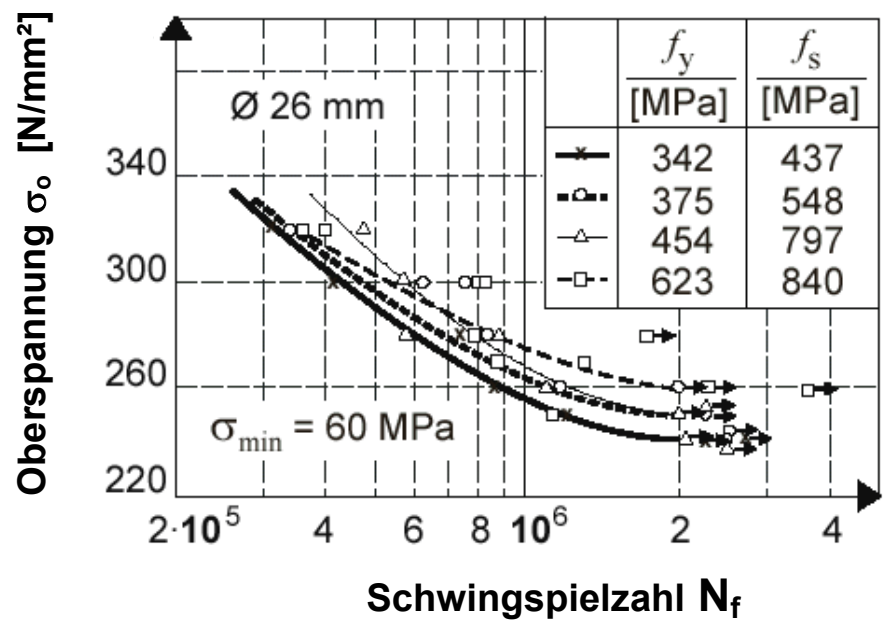

Bild 3.14 Wöhlerlinien verschiedener Materialfestigkeiten [120]

Es ist deutlich $\mathrm{zu}$ erkennen, daß es keine nennenswerten Einflüsse auf das Tragverhalten bei unterschiedlichen Stahlsorten gibt. Die Ermüdungsfestigkeit geht ab $2 \cdot 10^{6}$ Schwingspielen in die Dauerfestigkeit über. Sicherlich ist die Eigenschaft der Unabhängigkeit von der Stahlgüte, wie es in Bild $3.14 \mathrm{zu}$ sehen ist, auf die starke Kerbwirkung zurückzuführen. Wirken jedoch betriebsähnliche Belastungen, so gewinnt die Stahlsorte enorm an Bedeutung. Ebenfalls an Bedeutung gewinnt die Stahlgüte in Abhängigkeit von der Oberflächenbeschaffenheit. Betrachtet man polierte bzw. mit Walzhaut versehene Stähle, so zeigt sich eine mit der Streckgrenze des Stahls wachsende Ermüdungs- bzw. Dauerfestigkeit.

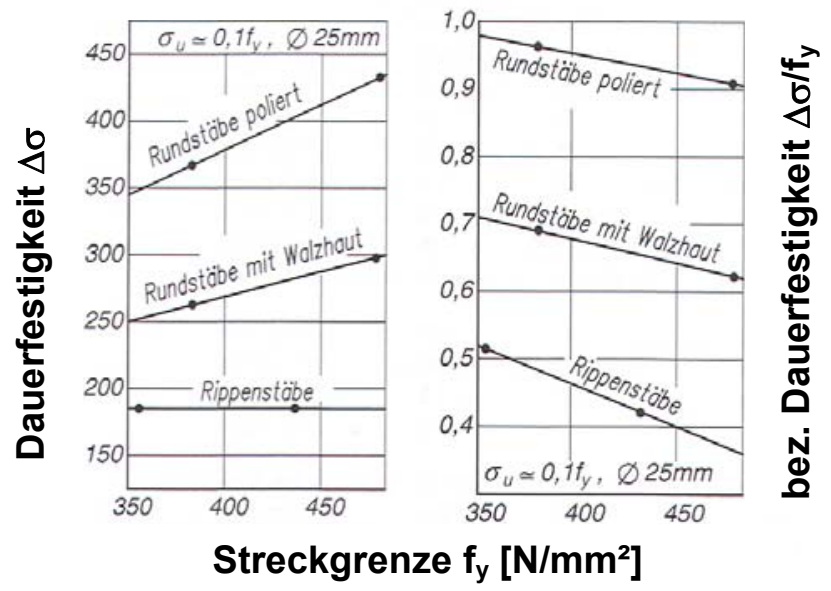

Bild 3.15 Einfluß der Oberflächenbeschaffenheit und Materialfestigkeit [7] 


\section{Mittellast bzw. Mittelspannung}

Der Einfluß des Lastniveaus zeigt einen nicht vernachlässigbaren Anteil an der Ermüdungsfestigkeit. Hierzu wurden in Abschnitt 3.3.1 Dauerfestigkeitsdiagramme vorgestellt, die es ermöglichen, lastabhängiges Verhalten bei einer charakteristischen Schwingspiezahl aufzuzeigen.

In [118] wird u.a. der Einfluß der Mittelspannung von axialbeanspruchten Betonstählen vergleichend dargestellt. In Bild 3.16 sind mehrere Wöhlerlinien bei konstanter Mittellast und variierender Schwingbreite dargestellt.

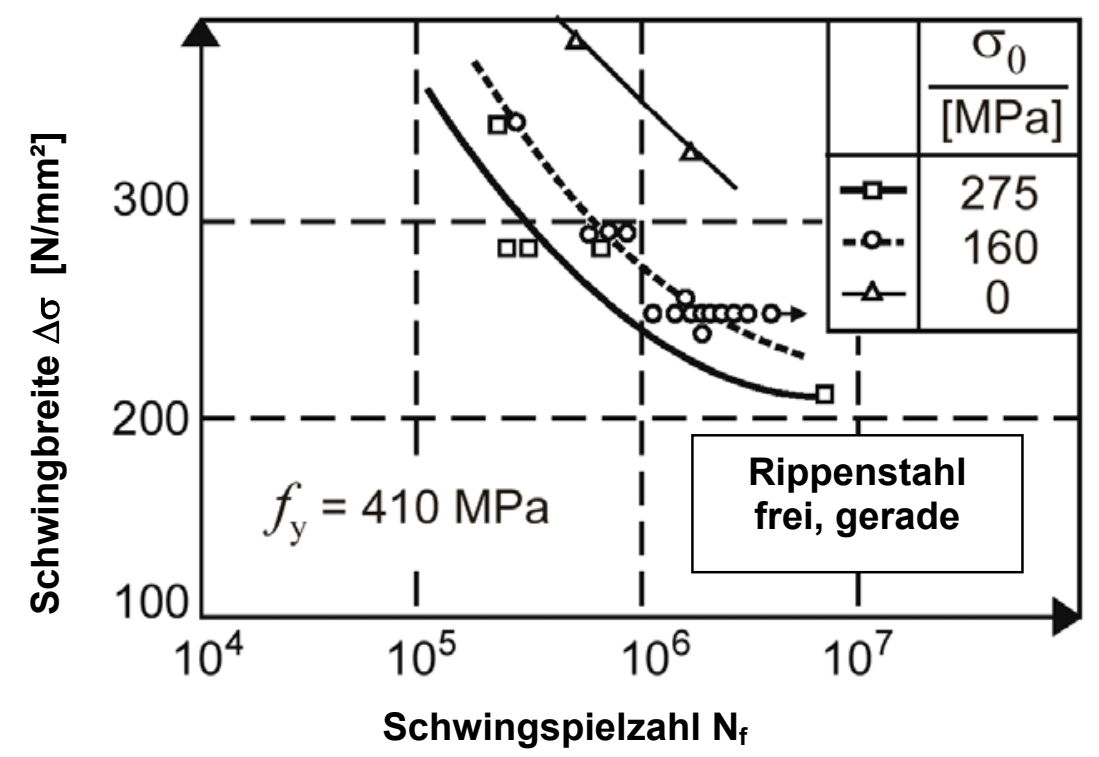

Bild 3.16 Einfluß der Mittelspannung auf die Wöhlerlinie nach [118]

Man erkennt deutlich, daß eine antiproportionale Abhängigkeit der Ermüdungsfestigkeit von der Mittelspannung vorhanden ist. In Bezug auf die Streckgrenze $f_{y}$ wird hierbei ein Abfall der Ermüdungsfestigkeit von $30 \%$ erreicht. Diese Versuche zeigen ebenfalls, daß der Betonstahl seine Dauerfestigkeiten zwischen $10^{6}$ und $10^{7}$ Schwingspielen erreicht. 


\subsection{Ermüdungsfestigkeit von Beton}

Zum Tragverhalten von Beton unter zyklischer Beanspruchungen erfolgten umfangreiche Untersuchungen auf folgenden Hauptgebieten, welche zur Zeit weiterhin durchgeführt werden:

- niederzyklische Ermüdungsversuche

- hochzyklische Ermüdungsversuche

Im Rahmen dieser Arbeit wird das Tragverhalten von Beton bei hochzyklischen Ermüdungsbeanspruchungen sowohl unter Druck als auch Zug dargestellt. Da ein Betonversagen unter ermüdungsrelevanten Beanspruchungen im Rahmen dieser Arbeit nicht beobachtet werden konnte, erfolgt eine knappe Darstellungsweise. Die Beanspruchungen beim Ermüdungstragverhalten können dem Abschnitt 3.2 entnommen werden und gelten für Stahl genauso wie für Beton.

Zur Ermüdungsfestigkeit im Zeitfestigkeitsbereich (hochzyklisch) können aus [75] folgende modifizierte Wöhlerlinien für den Beton entnommen werden.

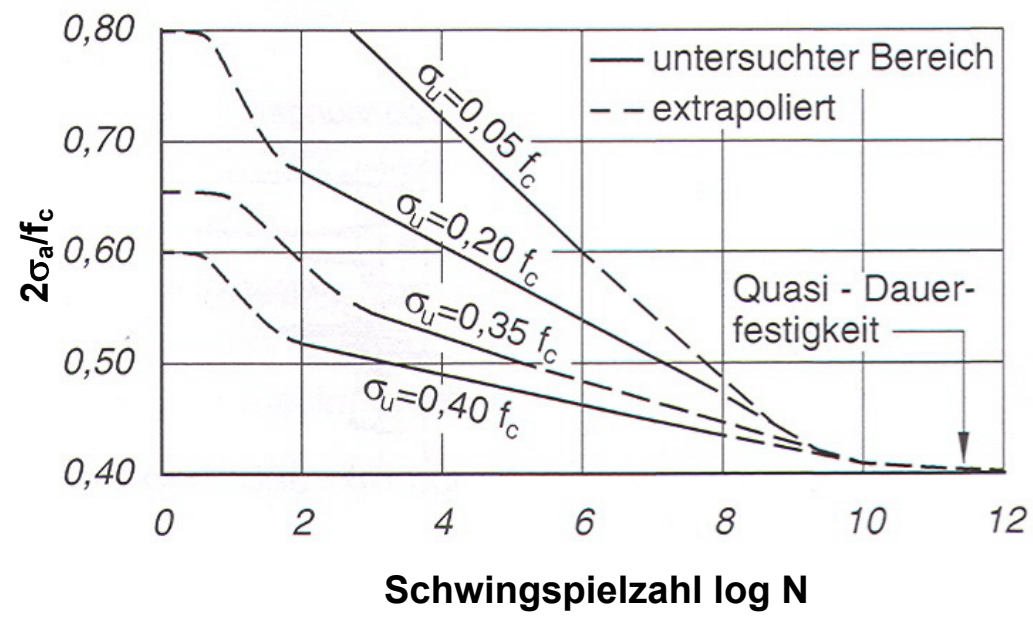

Bild 3.17 Wöhlerlinien des Beton unter Druckbeanspruchung

Dieses Diagramm zeigt eine Abhängigkeit der ertragbaren Schwingbreiten, bezogen auf die Druckfestigkeit $f_{c}$ des Betons, von der Unterlast bzw. Unterspannung $\sigma_{u}$ im Wöhlerversuch bei einer definierten Schwingspielzahl. Die Durchführung der Versuche erfolgte hierbei in einem Bereich zwischen $\mathrm{N}=10^{2}-10^{8}$ Schwingspielen. Es geht deutlich hervor, daß der Beton eine Abhängigkeit vom Lastniveau aufzeigt. Bei $2 \cdot 10^{6}$ Schwingspielen zeigt sich eine auf die Kurzzeitfestigkeit bezogene Ermüdungsfestigkeit zwischen $45 \%$ und $60 \%$. Die Dauerfestigkeit des Betons wird erst nach $10^{10}$ Schwingspielen erreicht und beträgt etwa $40 \%$ der Kurzzeitfestigkeit.

Eine weitere Darstellungsweise, um Lasteinflüsse darstellen zu können, wird in Form eines Smith-Diagramms in Bild 3.18 verwendet. Solch eine Darstellungsform der o.g. 
Ergebnisse wird für eine Schwingspielzahl gegen unendlich strebend im nachfolgenden Bild 3.18 dargestellt.

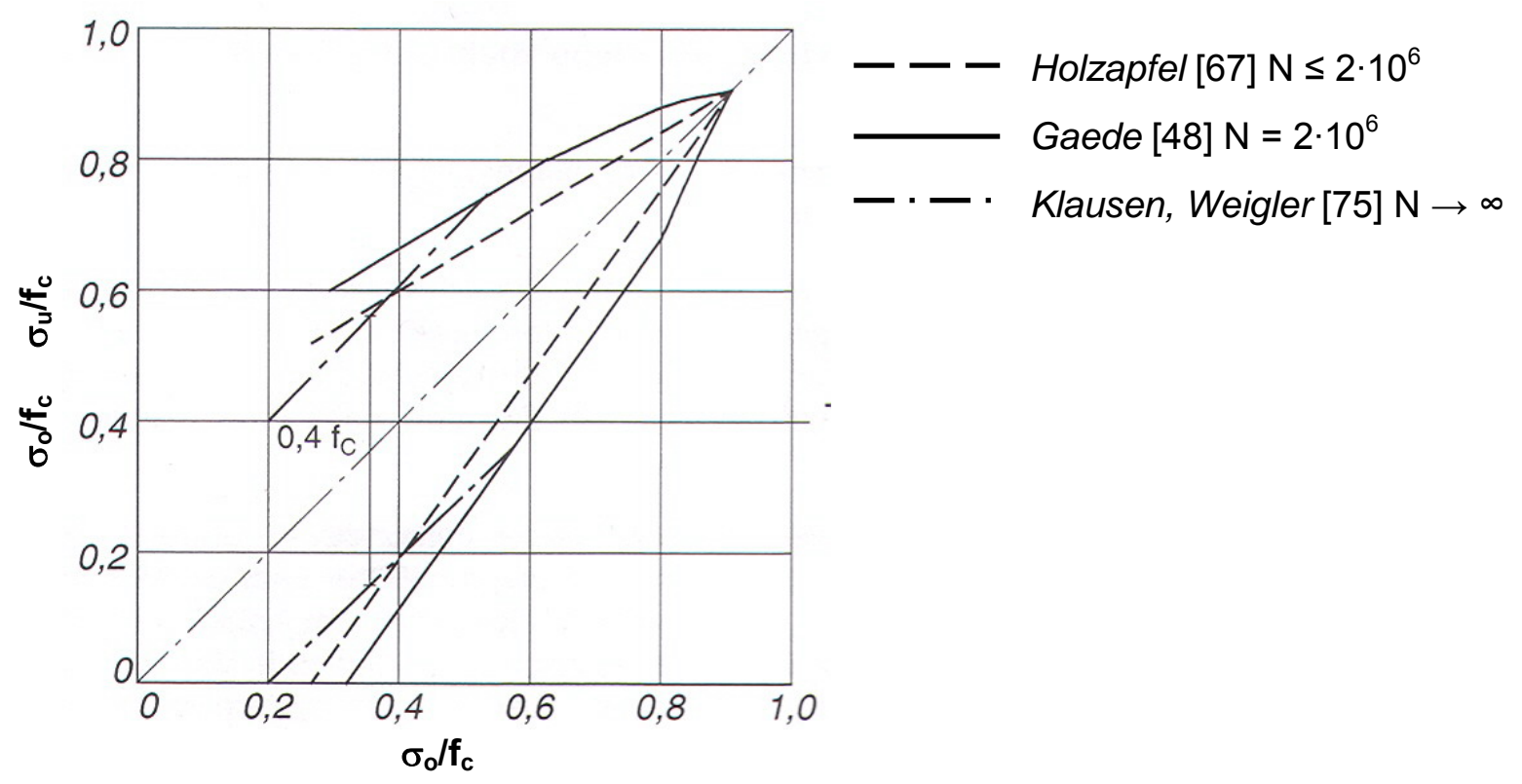

Bild 3.18 Smith-Diagramm für Beton

Für $2 \cdot 10^{6}$ Schwingspiele sind zwei weitere Möglichkeiten nach [48] und [67] dargestellt. Letzterer untersucht das Verhalten von Zementstein unter nichtruhender Beanspruchung. Hierbei ist mit einer $60 \%$-igen Reduzierung der Kurzzeitfestigkeit zu rechnen. Der Maximalwert liegt deutlich unterhalb von $50 \%$. Diese Erkenntnis ist für kraftschlüssige Verbindungen zwischen Stahl und Beton von besonderer Bedeutung, da die Festigkeit von Stahl im Vergleich zu Beton nach $2 \cdot 10^{6}$ Schwingspielen lediglich eine Festigkeit von etwa 20 bis $30 \%$ aufweist.

In Bezug auf Befestigungen schließt Rehm in [4] ein Betonausbruch für Befestigungssysteme aus, wenn die Oberlast geringer ist als $50 \%$ der statischen Bruchlast. Tritt kein Ermüdungsversagen auf, so wird davon ausgegangen, daß durch diese Vorbelastung keine Beeinflussung der anschließenden Beanspruchungen erfolgt. Ein eventueller Reihenfolgeeffekt wird ausgeschlossen. In [90] wird jedoch für Beton ein Einfluß aus der Reihenfolge der Beanspruchung festgestellt.

Die Zahl der experimentellen Untersuchungen für den Beton unter schwingender Zugbeanspruchung ist wesentlich geringer als bei Druckbeanspruchung. Dementsprechend sind die Entwicklung und der Wissensstand verhältnismäßig weniger fortgeschritten. Die Gründe hierfür sind zum einem auf die relativ komplizierte Versuchsdurchführung und zum anderen auf die untergeordnete Rolle des Betons bei der Bemessung zurückzuführen. Hier seien die Arbeiten [19], [117] genannt. 


\subsection{Schadensakkumulationshypothese}

Die Schadensakkumulationshypothese nach [85], [93] und ist das einfachste und zugleich effektivste Hilfsmittel zur Berechnung von Schäden an Bauteilen, welche unter betriebsähnlichen Beanspruchungen erfolgten. Die Grundidee dabei ist, daß jede schwingende Beanspruchung einen Schaden bewirkt, welcher sich im Laufe der Einwirkungen bis zu einem Grenzschaden akkumulieren. Grundvoraussetzung dieser Hypothese ist, daß die Reihenfolgen der Beanspruchungen keinen Einfluß auf die Entwicklung der Schädigung haben. Diese empirische Hypothese erlaubt es, komplizierte physikalische Zusammenhänge einfach darzustellen [65].

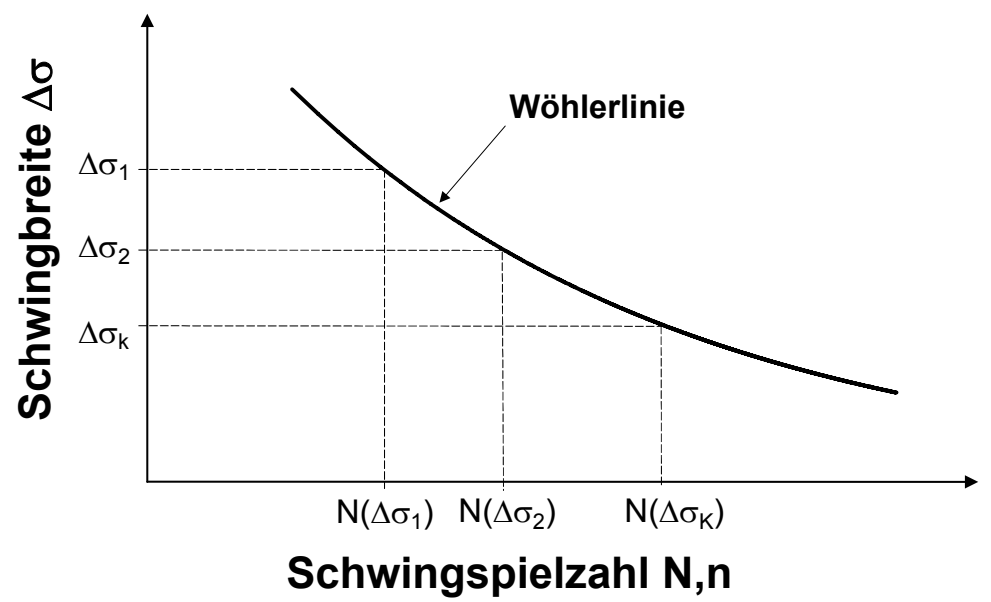

Bild 3.19 Lineare Schadensakkumulation (Miner-Regel)

Bei der linearen Schadensakkumulationshypothese (Palmgren-Miner Hypothese) wird davon ausgegangen, da $\beta$ jede Schwingbreite $\Delta \sigma_{i}$ eine Teilschädigung $\Delta \mathrm{D}_{\mathrm{i}}$ erzeugt und ein Bruch eintritt, wenn die Summe aller Teilschäden den Wert 1 erreicht.

Die Teil- bzw. Einheitsschädigung, verursacht durch ein Schwingspiel der Schwingbreite $\Delta \sigma_{\mathrm{k}}$, kann somit wie folgt erfaßt werden:

$$
\mathrm{d}_{\mathrm{k}}=\frac{1}{\mathrm{~N}\left(\Delta \sigma_{\mathrm{k}}\right)}
$$

Dabei ist $N\left(\Delta \sigma_{\mathrm{k}}\right)$ die zur Schwingbreite $\Delta \sigma_{\mathrm{k}}$ zugehörige Schwingspielzahl, ermittelt an der Wöhlerlinie. Die Anzahl von $n_{k}$ Schwingspielen bedeutet folgende Schädigung:

$\mathrm{D}_{\mathrm{k}}=\mathrm{n}_{\mathrm{k}} \cdot \mathrm{d}_{\mathrm{k}}=\frac{\mathrm{n}_{\mathrm{k}}\left(\Delta \sigma_{\mathrm{k}}\right)}{\mathrm{N}\left(\Delta \sigma_{\mathrm{k}}\right)}$

Die Gesamtschädigung aufeinander folgender $m$ Beanspruchungsblöcke mit $n_{k}$ Schwingspielen konstanter Schwingbreite $\sigma_{\mathrm{k}}$ beträgt: 
$\mathrm{D}=\sum_{\mathrm{i}=1}^{\mathrm{m}} \mathrm{D}_{\mathrm{k}} \leq 1$

Die Tragfähigkeit eines Systems ist mit dem Erreichen eines Grenzwertes der Schädigung von $\mathrm{D}_{\text {lim }}=1$ erreicht, und es tritt ein Bruch ein. Der Geltungsbereich der linearen Schadensakkumulationshypothese wird nach [103] wie folgt eingeschränkt :

- Es sollen keine Kaltverfestigungen und Trainiereffekte wirksam sein.

- Der Rißbeginn wird als Schaden betrachtet.

- Die Beanspruchungen sollen oberhalb der Dauerfestigkeit liegen.

- Die Mittelspannungen sollen möglichst konstant sein.

Zur Erfassung der Ermüdung unter Betriebsbeanspruchungen, die meist ein regellosen Verlauf aufweisen, sind verschiedene Methoden entwickelt worden, die sich durch unterschiedliche Definition eines Schwingspiels und der zugehörigen Schwingbreite kennzeichnen:

- Spektralmethode

- TNO-Verfahren

- Rainflow-Verfahren

- Reservoir-Zählmethode

Die Reservoir-Zählmethode ist im Vergleich zu den anderen Methoden sehr anschaulich und hat sich in weiten Bereichen des Ingenieurwesens durchgesetzt. Diese Methode ist beispielsweise in [77] erläutert. Weitere Methoden sind in [87] aufgeführt.

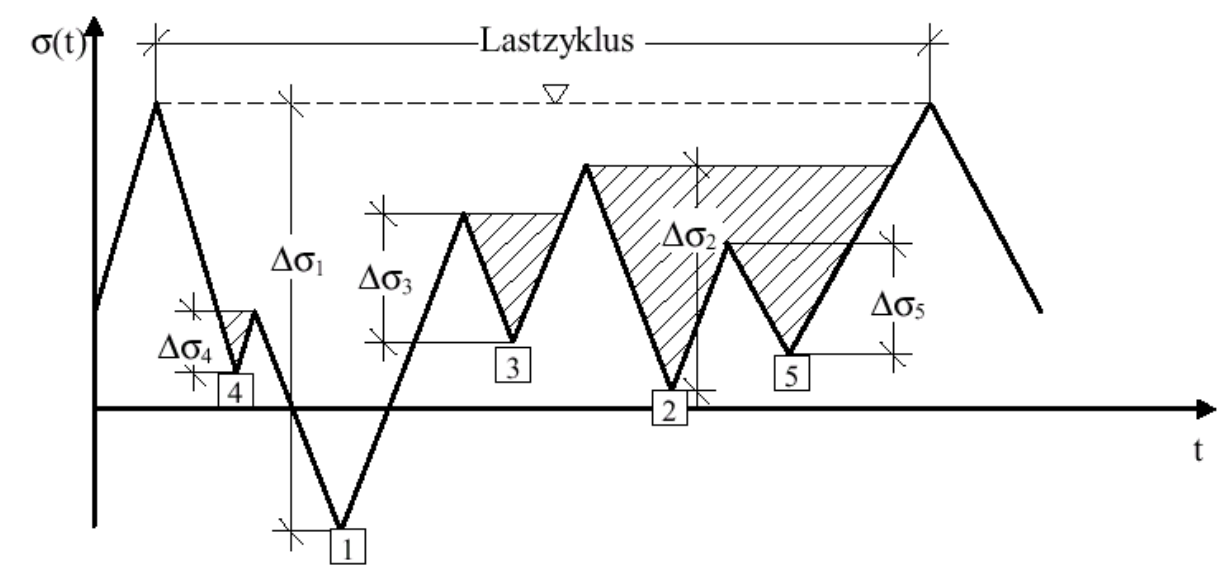

\section{Bild 3.20 Reservoir-Zählmethode}

Im Stahlbau haben sich ebenfalls bruchmechanische Methoden zur Bestimmung der Ermüdungsfestigkeit bewährt. Tiefergehende Informationen zum Phänomen der Ermüdung sind in [17] und [115] zu finden. 


\section{Experimentelle Untersuchungen}

\section{1 Überblick}

Experimentelle Untersuchungen zum Tragverhalten von warmgewalzten, gezahnten Ankerschienen unter nichtruhenden, ermüdungsrelevanten Einwirkungen bilden die Grundlage dieses Kapitels. Dabei werden unterschiedliche Einflußfaktoren auf das Ermüdungstragverhalten aufgezeigt und erläutert. Der Einfluß des umgebenden Betons, sowie der Einfluß unterschiedlicher Laststellungen werden dargestellt. Einen zentralen Schwerpunkt der experimentellen Untersuchungen bildet das Lastniveau bei Einstufenversuchen, welches durch das Verhältnis der Unterlast zur Oberlast angegeben wird. Bei Betrachtung des Goodman-Diagramms in Kapitel 3 (Abschnitt 3.3.1) zeigen sich ertragbare Schwingbreiten in Abhängigkeit vom Lastniveau. Zur Erfassung dieses Einflusses werden Versuche mit unterschiedlichen Unter- und Oberlasten durchgeführt. Weiterhin werden bei Zugschwellbeanspruchungen mit unterschiedlichen Lastrichtungen mögliche Interaktionsbeziehungen für nichtruhende Beanspruchung entwickelt. Für eine umfassende Beurteilung des Tragsystems Ankerschiene ist dabei die kombinierte Zug- und Querbeanspruchung ein zentraler Schwerpunkt, da sie die Form des Interaktionsdiagramms zwischen den jeweiligen Kraftgrößen maßgebend bestimmt. Durch geeignete meßtechnische Methoden wird das Tragverhalten erfaßt und in Abhängigkeit von der Schwingspielzahl dargestellt. Allen Untersuchungen liegt als maßgebendes Versagenskriterium das Stahlversagen zugrunde. Betonversagen wurde unter Ausnutzung von End- und Randabständen in keinem Fall beobachtet. Die experimentellen Untersuchungen dienen als Basis für die anschließende Erarbeitung einer umfassenden Sicherheitsbetrachtung in Kapitel 5.

\subsection{Annahmen, Konventionen und Bezeichnungen}

Im Rahmen dieser Arbeit werden in Hinblick auf bisher vorliegende Erkenntnisse, entgegen den allgemeinen Konventionen im Stahlbau [71], die Bezeichnungen aus der Befestigungstechnik übernommen. Das Koordinatensystem wird dabei in die Achse des Ankerbolzens, in Höhe der Schienenlippen gemäß Bild 4.1 angeordnet und soll somit lediglich die Stelle des Lastangriffes in den Versuchen kennzeichnen. Die Kraftgröße bei Beanspruchung in Schienenlängsrichtung ( $x$-Richtung) wird mit $V_{x}$, bei Beanspruchung quer zur Schienenlängsrichtung mit $\mathrm{V}_{\mathrm{y}}$ und axial in Richtung der Hammerkopfschraube mit $\mathrm{N}$ bezeichnet. Die resultierenden Weggrößen haben die Bezeichnungen $\mathrm{u}, \mathrm{v}$ und $\mathrm{w}$. Der Winkel der Lasteinleitung wird mit $\varphi$ bezeichnet und stets relativ zur Achse der Hammerkopfschraube gemessen. Die reine zentrische Zugrichtung entspricht somit einem Winkel von $0^{\circ}$, wobei der Winkel von $90^{\circ}$ der Beanspruchung in Querrichtung zuzuordnen ist. 


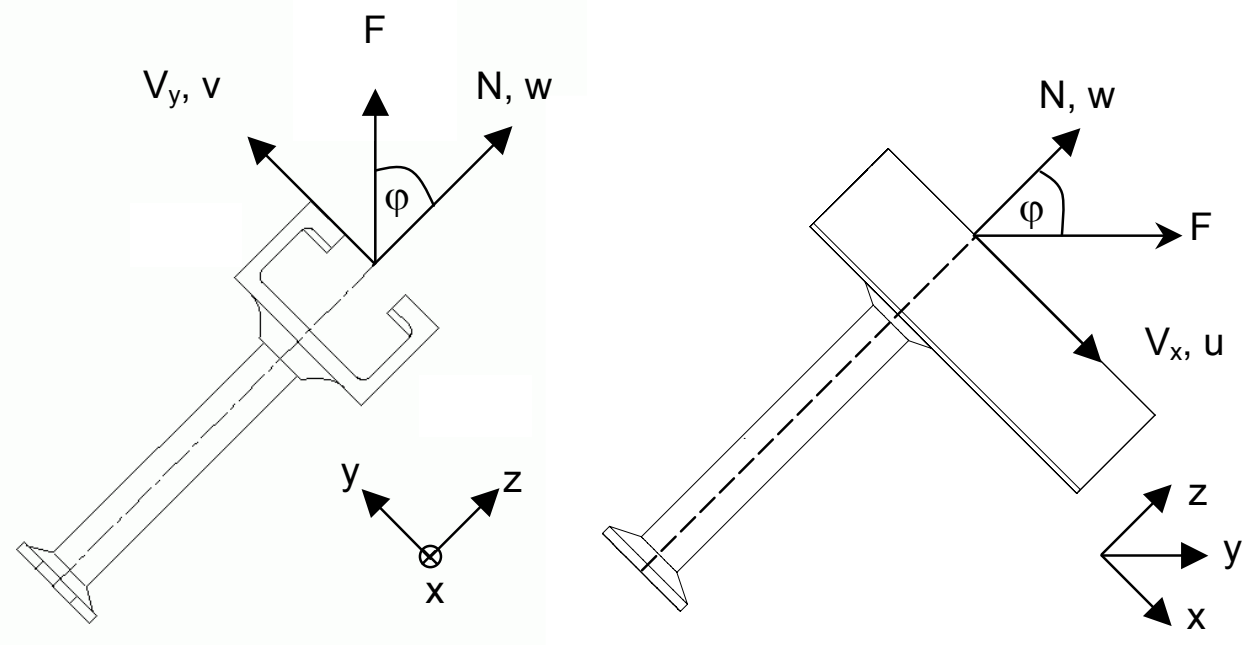

(3)

6

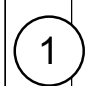

$1)$

6

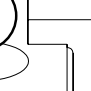

4

5

(7)

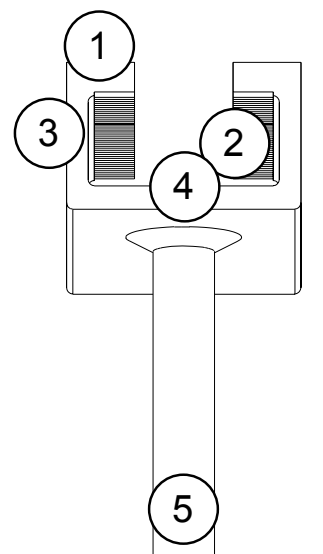

(7)
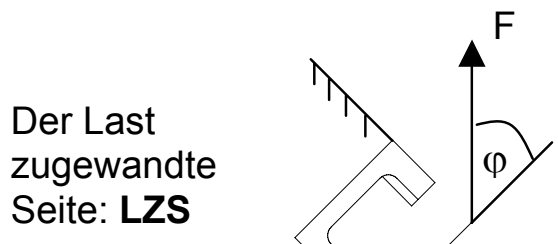

$\lambda$

Betonoberfläche

Der Last abgewandte Seite: LAS

$\mathrm{V}_{\mathrm{x}}$ : Kraftgröße für Querbeanspruchung in Schienenlängsrichtung (x-Richtung)

$\mathrm{V}_{\mathrm{y}}$ : Kraftgröße für Querbeanspruchung quer zur Schienenlängsrichtung (y-Richtung)

$\mathrm{N}$ : Kraftgröße für zentrische Zug- bzw. Axialbeanspruchung (z-Richtung)

$\mathrm{u}, \mathrm{v}, \mathrm{w}$ : Weggröße in $\mathrm{x}-, \mathrm{y}-, \mathrm{z}-\mathrm{Richtung}$

$\varphi$ : Beanspruchungswinkel

1: Schienenlippe

2: Schienenverzahnung

3: Schienenschenkel

4: Schienenrücken

5: Ankerbolzen

6: Ankerfuß

7: Ankerkopf

Bild 4.1 Bezeichnungen, Richtungskonvention, Kraft- und Weggrößen 


\subsection{Versuchskörper}

\subsubsection{Abmessungen der Ankerschienen}

Als Probekörper sind Ankerschienen vom Typ 38/23 aus Normalstahl der Güte St 44 untersucht worden. Dabei kamen die Ankerschienenprofile HZA 38/23 (Hersteller HALFEN-DeHA) und JXA-W 38/23 (Hersteller DKG/JORDAHL) zum Einsatz. Das Bild 4.2 zeigt exemplarisch die Sollabmessungen der verwendeten Profile. Es handelt sich hierbei um warmgewalzte Ankerschienen mit zusätzlicher Verzahnung an den Unterseiten der Schienenlippen, wodurch es gelingt, zusätzliche Kräfte in Schienenlängsrichtung zu übertragen. Die Verankerung der Schiene im Beton erfolgt mit einem eingepreßten Bolzen (Rundanker), dessen unteres Ende einen angestauchten Kopf besitzt und der oben im Rücken des Schienenprofils verpreßt ist. Die Besonderheit dieser neuen Ankerschienengeneration ist neben der Verzahnung der Schienenlippen, die spezielle Verpreßtechnik des Ankerbolzens, welcher in einem besonderen Verfahren mit dem Schienenrücken im Formschluß verbunden wird. Unterschiede der untersuchten Profile zwischen den verschiedenen Herstellern sind dabei gerade in der Art der Befestigung des Ankerbolzens zu finden.
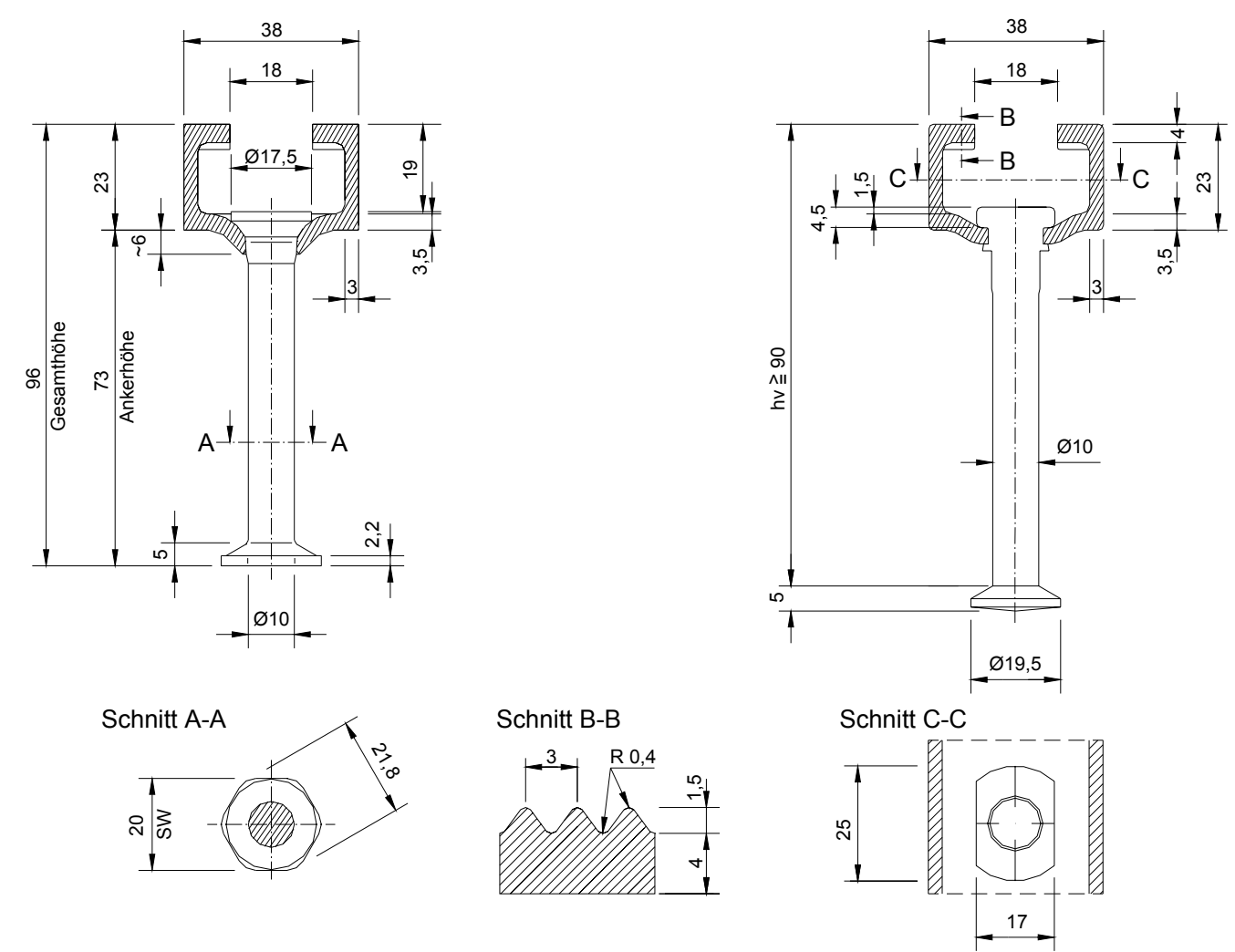

Angaben in [mm]

Bild 4.2 Ankerschienenprofil HZA 38/23 St (links) und JXA-W 38/23 St (rechts)

Die Ankerschienen waren hauptsächlich mit einem angeordneten Bolzen versehen. Die Endüberstände der Ankerschienen, gemessen vom Mittelpunkt des Rundankers, 
betrugen zum einen 25/50 und zum anderen 50/50 mm. Das Bild 4.3 zeigt die Abmessungen der untersuchten Ankerschienenabschnitte.

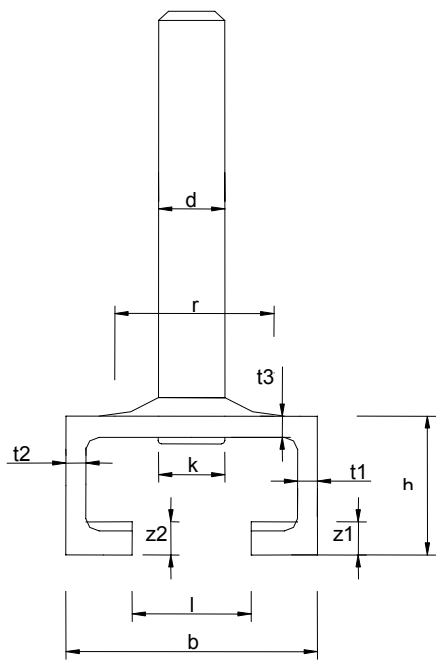

Bild 4.3 Untersuchte Ankerschienenabschnitte 25/50 (mittig), 50/50 (rechts)

Die geometrische Form des in Bild 4.3, mittig, dargestellten Systems ist unter Einhaltung der in [21], [23] geforderten Mindestabstände die ungünstigere Ausführung. Die Ankerschienen wurden als Meterware angeliefert und vor der Prüfung in die in Bild 4.3 dargestellten Formen gebracht. Anschließend wurden sie mit einem elektronischen Meßschieber vermessen. Tabelle 4.1 gibt eine Übersicht über die Querschnittsabmessungen der Ankerschienen wieder.

Tabelle 4.1 Querschnittsabmessungen

\begin{tabular}{|c|c|c|c|c|c|c|c|c|c|c|c|c|}
\hline Profil & & $\mathbf{h}$ & b & I & $t 1$ & t2 & t3 & $z 1$ & $\mathrm{z2}$ & k & d & $r$ \\
\hline & & \multicolumn{11}{|c|}{ [mm] } \\
\hline \multirow{4}{*}{$\begin{array}{l}\mathrm{HZA} \\
\text { Serie A }\end{array}$} & MW & 22,74 & 39,04 & 18,01 & 3,24 & 3,23 & 3,52 & 5,21 & 5,17 & 17,38 & 10,02 & 26,46 \\
\hline & $\min$. & 22,60 & 38,78 & 17,79 & 3,19 & 3,17 & 3,42 & 5,08 & 5,08 & 17,16 & 9,97 & 26,28 \\
\hline & max. & 22,84 & 39,22 & 18,22 & 3,30 & 3,33 & 3,61 & 5,33 & 5,25 & 17,60 & 10,08 & 26,61 \\
\hline & $s$ & 0,07 & 0,14 & 0,13 & 0,03 & 0,05 & 0,05 & 0,07 & 0,06 & 0,16 & 0,04 & 0,10 \\
\hline \multirow{4}{*}{$\begin{array}{l}\mathrm{HZA} \\
\text { Serie B }\end{array}$} & MW & 23,50 & 38,76 & 18,18 & 3,30 & 3,17 & 3,72 & - & - & - & - & 25,25 \\
\hline & $\min$. & 23,32 & 38,27 & 17,40 & 3,00 & 3,01 & 3,52 & - & - & - & - & 24,50 \\
\hline & max. & 23,81 & 39,10 & 18,62 & 3,80 & 3,33 & 3,96 & - & - & - & - & 26,00 \\
\hline & $s$ & 0,12 & 0,22 & 0,21 & 0,16 & 0,08 & 0,10 & - & - & - & - & 0,36 \\
\hline \multirow{4}{*}{$\begin{array}{l}\mathrm{HZA} \\
\text { Serie C }\end{array}$} & MW & 22,64 & 38,74 & 18,05 & 3,21 & 3,22 & 3,71 & 5,37 & 5,37 & 17,44 & 10,04 & 26,45 \\
\hline & $\min$. & 22,41 & 38,38 & 17,81 & 3,08 & 3,06 & 3,60 & 5,25 & 5,25 & 16,83 & 10,02 & 26,17 \\
\hline & max. & 22,89 & 39,06 & 18,45 & 3,36 & 3,38 & 3,80 & 5,53 & 5,46 & 18,38 & 10,06 & 26,68 \\
\hline & $\mathrm{s}$ & 0,11 & 0,16 & 0,13 & 0,09 & 0,11 & 0,05 & 0,07 & 0,05 & 0,34 & 0,01 & 0,11 \\
\hline \multirow{4}{*}{$\begin{array}{l}\text { JXA-W } \\
\text { Serie D }\end{array}$} & MW & 22,91 & 38,95 & 18,33 & 3,24 & 3,25 & 3,66 & 5,35 & 5,31 & - & 10,03 & - \\
\hline & $\min$. & 22,78 & 38,66 & 18,07 & 3,17 & 3,15 & 3,60 & 5,23 & 5,20 & - & 10,02 & - \\
\hline & max. & 23,06 & 39,16 & 18,65 & 3,31 & 3,47 & 3,72 & 5,63 & 5,62 & - & 10,04 & - \\
\hline & $s$ & 0,09 & 0,15 & 0,21 & 0,05 & 0,08 & 0,04 & 0,11 & 0,12 & - & 0,01 & - \\
\hline
\end{tabular}


Im weiteren Verlauf der Untersuchungen werden die zwei verschiedenen Varianten, gemäß ihren Abmessungen, vereinfachend mit 25/50 und 50/50 bezeichnet. Vereinzelt sind zusätzliche Untersuchungen an Ankerschienenkurzstücken mit zwei Ankerbolzen und einer Gesamtlänge von $200 \mathrm{~mm}$ durchgeführt worden, deren Endüberstände 25 $\mathrm{mm}$ betrugen. Die Ankerschienenkurzstücke sind sowohl im einbetonierten (B) als auch im nicht einbetonierten (OB) Zustand untersucht worden.

Die Befestigung von Anbauteilen an der Schiene und somit die Lasteinleitung in den Untergrund erfolgt mit speziell angepaßten Schrauben (Standardschrauben bzw. Hammerkopfschrauben Typ HS, Zahnschrauben Typ HZS), die stets mit einem für die Schraube spezifischen Drehmoment vorzuspannen sind. Bei diesen Schrauben der neueren Generation wurde die Kopfform im Vergleich zu älteren Varianten derart verändert, daß ein Durchdrehen der Schrauben auszuschließen ist. Dadurch ist eine nahezu optimale Montagesicherheit gegeben. In Bild 4.4 sind exemplarisch die verwendeten Schraubentypen passend für das Ankerschienenprofil 38/23 dargestellt. Es stehen Zahnschrauben und Standardschrauben verschiedener Abmessungen und Hersteller zur Wahl. Im Gegensatz zu Standardschrauben weisen Zahnschrauben Einprägungen an der Unterseite des Schraubenkopfes auf, wodurch in Verbindung mit der Verzahnung der Ankerschiene und der entsprechenden Vorspannung eine sichere Verbindung zur Lastabtragung auch in Schienenlängsrichtung ermöglicht wird. Weitere Spezifikationen der Schrauben können den zur Zeit gültigen bauaufsichtlichen Zulassungen [21], [23] entnommen werden.
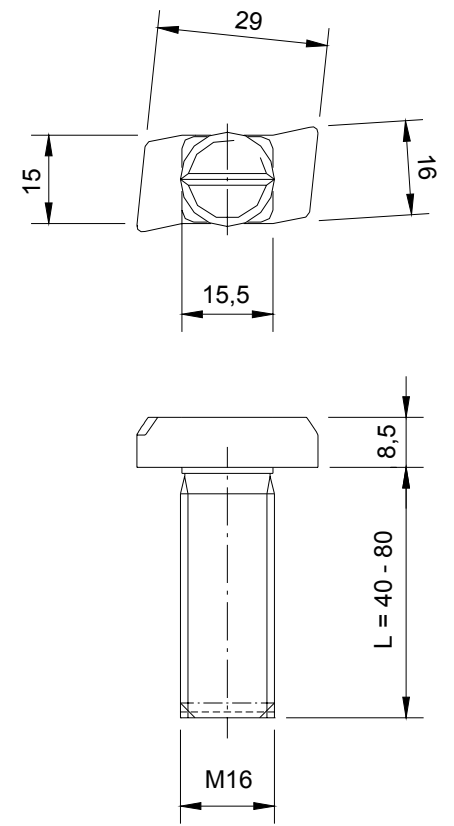

HS 38/23 M16
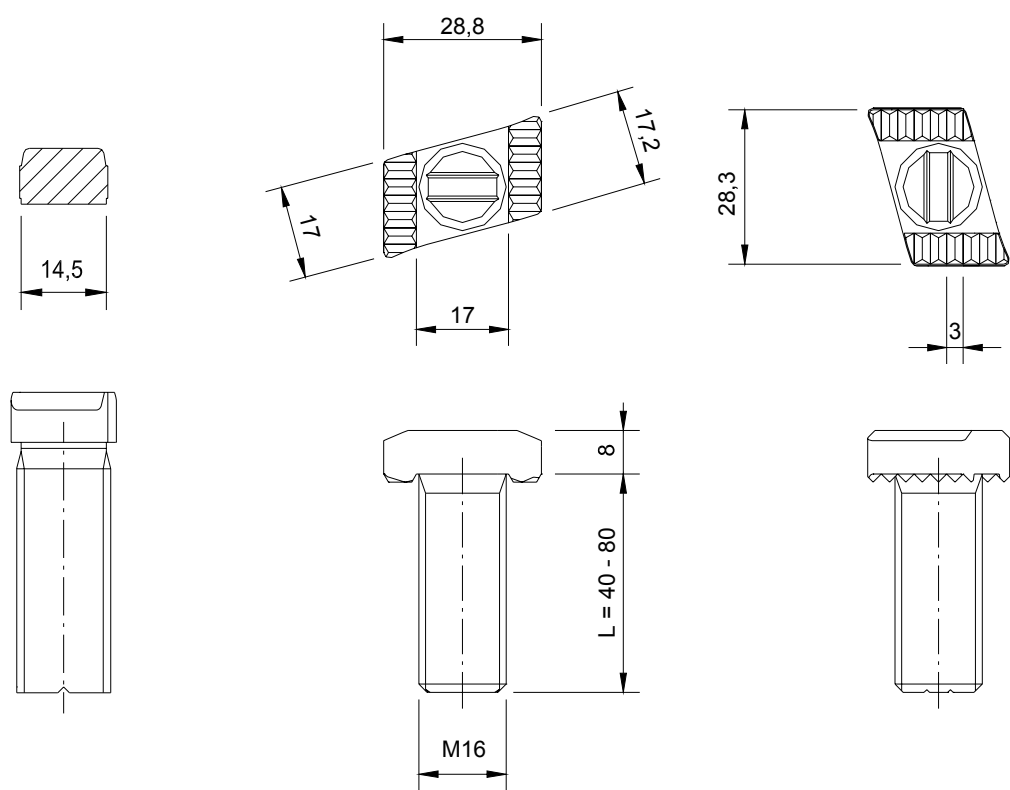

HZS 38/23 M16

Angaben in $[\mathrm{mm}]$

Bild 4.4 Verwendete Standardschrauben HS (links), Zahnschrauben HZS (rechts) 


\subsubsection{Materialkennwerte der Ankerschienen}

In der Regel bestehen die Ankerschienenprofile der neueren Generation aus Stahl der Güte St 44 und die jeweiligen Ankerbolzen aus der Stahlsorte St 37. Zur Bestimmung der aktuellen Festigkeitseigenschaften der vorhandenen Ankerschienenprofile sind an zusätzlichen Profilen aus den seitlichen Schenkeln, dem Rücken und dem Ankerbolzen der Ankerschienen Stahlproben nach DIN 50125 [30] entnommen und nach DIN EN 10002-1 [31] geprüft worden. Die Mittelwerte der Ergebnisse sind in der nachfolgenden Tabelle aufgeführt. Die Streuungen der Festigkeiten waren sehr gering. Der Variationskoeffizient ist kleiner als $10 \%$.

Tabelle 4.2 Ergebnisse der Materialprüfung

\begin{tabular}{|c||c|c|c|c|c|c|c|}
\hline \multicolumn{2}{|c|}{ Profil } & \multicolumn{2}{c|}{ Schenkel } & \multicolumn{2}{c|}{ Rücken } & \multicolumn{2}{c|}{ Bolzen } \\
\cline { 3 - 8 } \multicolumn{2}{|c|}{} & $R_{\mathrm{p} 0,2}$ & $\mathrm{R}_{\mathrm{m}}$ & $\mathrm{R}_{\mathrm{p} 0,2}$ & $\mathrm{R}_{\mathrm{m}}$ & $\mathrm{R}_{\mathrm{p} 0,2}$ & $\mathrm{R}_{\mathrm{m}}$ \\
\cline { 3 - 9 } & {$\left[\mathrm{N} / \mathrm{mm}^{2}\right]$} & {$\left[\mathrm{N} / \mathrm{mm}^{2}\right]$} & {$\left[\mathrm{N} / \mathrm{mm}^{2}\right]$} & {$\left[\mathrm{N} / \mathrm{mm}^{2}\right]$} & {$\left[\mathrm{N} / \mathrm{mm}^{2}\right]$} & {$\left[\mathrm{N} / \mathrm{mm}^{2}\right]$} \\
\hline \multirow{3}{*}{$\mathrm{HZA}$} & Serie A & 505 & 537 & 440 & 495 & 424 & 527 \\
\cline { 2 - 8 } & Serie B & 532 & 572 & 487 & 545 & --- & --- \\
\cline { 2 - 8 } & Serie C & 619 & 651 & 560 & 610 & 381 & 462 \\
\hline \multirow{2}{*}{ JXA-W } & Serie D & 560 & 592 & 526 & 575 & 448 & 516 \\
\hline
\end{tabular}

Die Ankerschienen im einbetonierten Zustand wurden in quadratische Betonkörper der Abmessungen $\mathrm{B} \times \mathrm{H} \times \mathrm{T}$ von $460 \times 460 \times 150 \mathrm{~mm}^{3}$, die unter Berücksichtigung der minimal zulässigen Randabstände aus [21], [23] gewählt wurden, eingebaut. Als konstruktive Bewehrung wurde direkt unterhalb des Schienenrückens eine Matte Q131-BSt 500 angeordnet. Eine Rückhängebewehrung wurde nicht vorgesehen. Die Schienenstücke waren auf der Schalung so positioniert, daß die Achse des Ankerbolzens zentrisch im Betonkörper lag. Das nachfolgende Bild 4.5 zeigt den Zustand des Versuchskörpers vor der Betonierung.

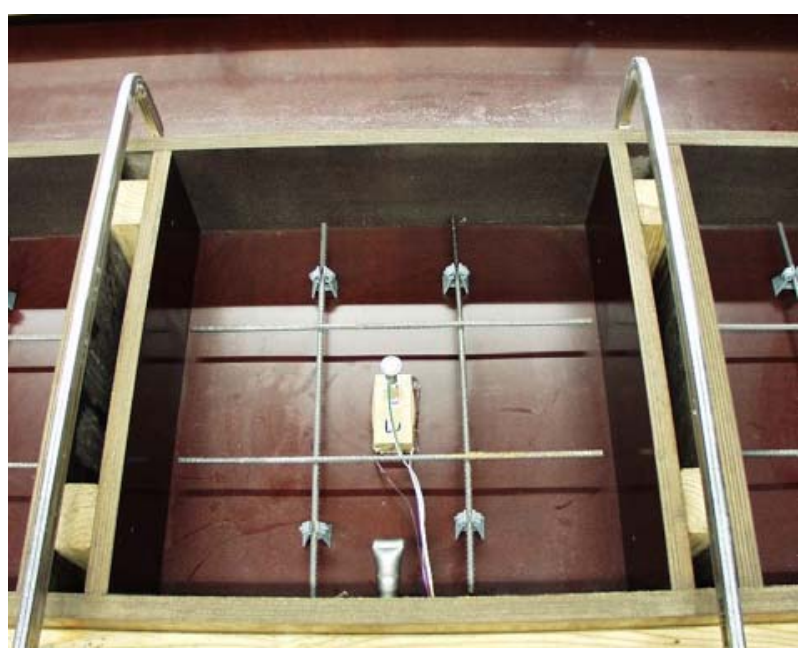

Bild 4.5 Versuchskörper vor der Betonierung

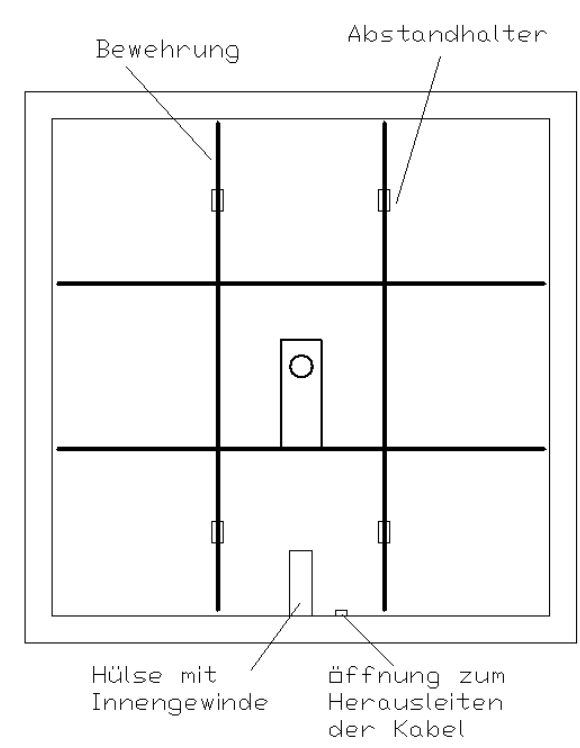


Die Schienenabschnitte wurden rundherum mit einer Folie beklebt, um eine Haftung an den umgebenden Beton und ein Einlaufen des Betons an den Stirnseiten des Profils auszuschließen, und somit einen günstigen Lastfall zu verhindern. Die Zusammensetzung des Betons war für die Festigkeitsklasse B25 ausgelegt.

\subsubsection{Betoneigenschaften}

In der Zulassung [21], [23] wird für Ankerschienen unter vorwiegend nichtruhender Zugbelastung bis $2 \cdot 10^{6}$ Lastspielen ein bewehrter Normalbeton von mindestens B15 nach DIN 1045 (1988-07 Beton und Stahlbeton, Bemessung und Ausführung) [26] gefordert, sofern keine Anforderungen hinsichtlich der Feuerwiderstandsdauer an die Gesamtkonstruktion einschließlich der Ankerschiene gestellt werden. Diese Bedingungen werden im Rahmen dieser Arbeit eingehalten.

Die Betonrezeptur ist im Folgenden aufgeführt:

Für den verwendeten Zement CEM I 42,5 R $\left(\mathrm{N}_{28}=58,4 \mathrm{~N} / \mathrm{mm}^{2}, \rho_{\mathrm{z}}=3,1 \mathrm{~kg} / \mathrm{dm}^{3}\right) \mathrm{er}-$ gibt sich hinsichtlich der angestrebten Festigkeitsklasse B25 und dem angestrebten Konsistenzbereich KR in Abhängigkeit von der Regelsieblinie B16 $\left(\mathrm{k}=3,66, \rho_{\mathrm{g}}=\right.$ $2,65 \mathrm{~kg} / \mathrm{dm}^{3}$ ) ein $\mathrm{w} / \mathrm{z}$-Wert von 0,70 . Unter der Annahme einer Wasserdichte $\rho_{\mathrm{w}}=$ $1,00 \mathrm{~kg} / \mathrm{dm}^{3}$ und einem Luftporengehalt von $p=20 \mathrm{dm}^{3} / \mathrm{m}^{3}$ resultieren je $\mathrm{m}^{3}$ Beton folgende Mengen:

Wasseranteil $\mathrm{w}=195 \mathrm{~kg}$

Zementanteil $\mathrm{z}=\mathrm{w} /(\mathrm{w} / \mathrm{z})=195 / 0,7=278,6 \mathrm{~kg}$

Zuschlaganteil g $=\left[1000-\mathrm{z} / \rho_{\mathrm{z}}-\mathrm{w} / \rho_{\mathrm{w}}-\mathrm{p}\right] \cdot \rho_{\mathrm{g}}=1842 \mathrm{~kg}$

Die Zusammensetzung des Zuschlags der Sieblinie B16 ist in Tabelle 4.3 angegeben.

Tabelle 4.3 Zusammensetzung des Zuschlags für Regelsieblinie B16

\begin{tabular}{|c||c|c|c|c|c|c|c|}
\hline $\begin{array}{c}\text { Siebloch- } \\
\text { weite }\end{array}$ & $0-0,25$ & $0,25-0,5$ & $0,5-1$ & $1-2$ & $2-4$ & $4-8$ & $8-16$ \\
\hline \hline$[\%]$ & 8 & 12 & 12 & 10 & 14 & 20 & 24 \\
\hline$[\mathrm{kg}]$ & 147,4 & 221,0 & 221,0 & 184,2 & 257,9 & 368,4 & 442,1 \\
\hline
\end{tabular}

Nach der Herstellung des Betons wurde dieser in einem Ausbreitversuch hinsichtlich seiner Konsistenz untersucht. Anhand eines Ausbreitversuchs wurde das Ausbreitmaß bestimmt. Alle Betonierserien entsprachen dem angestrebten Konsistenzbereich KR (Regelkonsistenz). Anschließend erfolgte die Messung des Luftporengehaltes und die Ermittlung der Frischbetonrohdichte. Die Tabelle 4.4 gibt die repräsentativen Werte der Frischbetonprüfung wieder. 
Tabelle 4.4 Ergebnisse der Frischbetonprüfung

\begin{tabular}{|l||c|c|c|c|}
\hline & Ausbreitmaß & Luftporengehalt & Frischbetonrohdichte & $\begin{array}{c}\text { Verwendung mit } \\
\text { Ankerschienen der }\end{array}$ \\
\cline { 2 - 4 } & {$[\mathrm{cm}]$} & {$[\mathrm{Vol}-\%]$} & {$\left[\mathrm{kg} / \mathrm{dm}^{3}\right]$} & Serie A \\
\hline Serie 1 & 49,0 & --- & 2,29 & Serie B \\
\hline Serie 2 & 46,4 & --- & 2,24 & Serie C \& D \\
\hline Serie 3 & 45,0 & 1,8 & 2,33 & \\
\hline
\end{tabular}

Aus herstellungstechnischen Gründen waren verschiedene Betonierserien notwendig. Die mittleren Betondruckfestigkeiten im Prüfzeitraum der jeweiligen Serie, ermittelt an Würfeln der Kantenlänge 150 mm, können der Tabelle 4.5 entnommen werden.

Tabelle 4.5 Mittelwerte der Festigkeitsprüfungen während des Prüfzeitraums

\begin{tabular}{|l||c|c|}
\hline & \begin{tabular}{c} 
Festigkeiten \\
zu Beginn \\
\cline { 2 - 3 }$\left[\mathrm{N} / \mathrm{mm}^{2}\right]$
\end{tabular} & $\begin{array}{c}\text { Festigkeiten } \\
\text { am Ende } \\
{\left[\mathrm{N} / \mathrm{mm}^{2}\right]}\end{array}$ \\
\hline Serie 1 & 36,1 & 38,9 \\
\hline Serie 2 & 27,8 & 37,1 \\
\hline Serie 3 & 35,5 & 44,6 \\
\hline
\end{tabular}

Zusätzlich zu Serie 3 wurden zwei weitere Serien aus Fertigbeton hergestellt. Diese waren für eine Festigkeitsklasse B25 bzw. B55 ausgelegt und zeigten nach 28 Tagen Druckfestigkeiten von $42 \mathrm{~N} / \mathrm{mm}^{2}$ bzw. $61 \mathrm{~N} / \mathrm{mm}^{2}$.

\subsubsection{Bestückung der Ankerschienen mit Dehnungsmeßstreifen}

Zur Beschreibung der Versagensmechanismen in der Ankerschiene sind metallische Dehnungsmeßstreifen (DMS) verwendet worden. Diese sind in unmittelbarer Nähe der zu erwartenden Versagensstellen positioniert worden. Voraussetzung für eine Übertragung der Dehnung mittels der DMS ohne Verluste ist eine innige Verbindung zum Bauteil. Die mit einem speziellen Kleber angebrachten Meßgitter aus dem Werkstoff Konstantan können gedehnt und gestaucht werden. Dementsprechend erhöht bzw. verringert sich der elektrische Widerstand, welcher einer bestimmten Dehnung entspricht. Die Meßstellen der Dehnungen sind exemplarisch an Ankerschienen der Ausführungen 25/50 in Bild 4.4 zu ersehen. Dabei sind DMS1 und DMS2 mittig über die Höhe der Seitenschenkel in der Achse des Ankerbolzens und DMS3 im unmittelbaren Stauchbereich des Ankerfußes positioniert worden. Zur Aufzeichnung der Dehnungen während einer kombinierten Zug- und Querbeanspruchung sind 3-axiale DMS, sog. Rosetten verwendet worden. Eine Übersicht der DMS-Applikationen gibt Bild 4.6 wieder. 

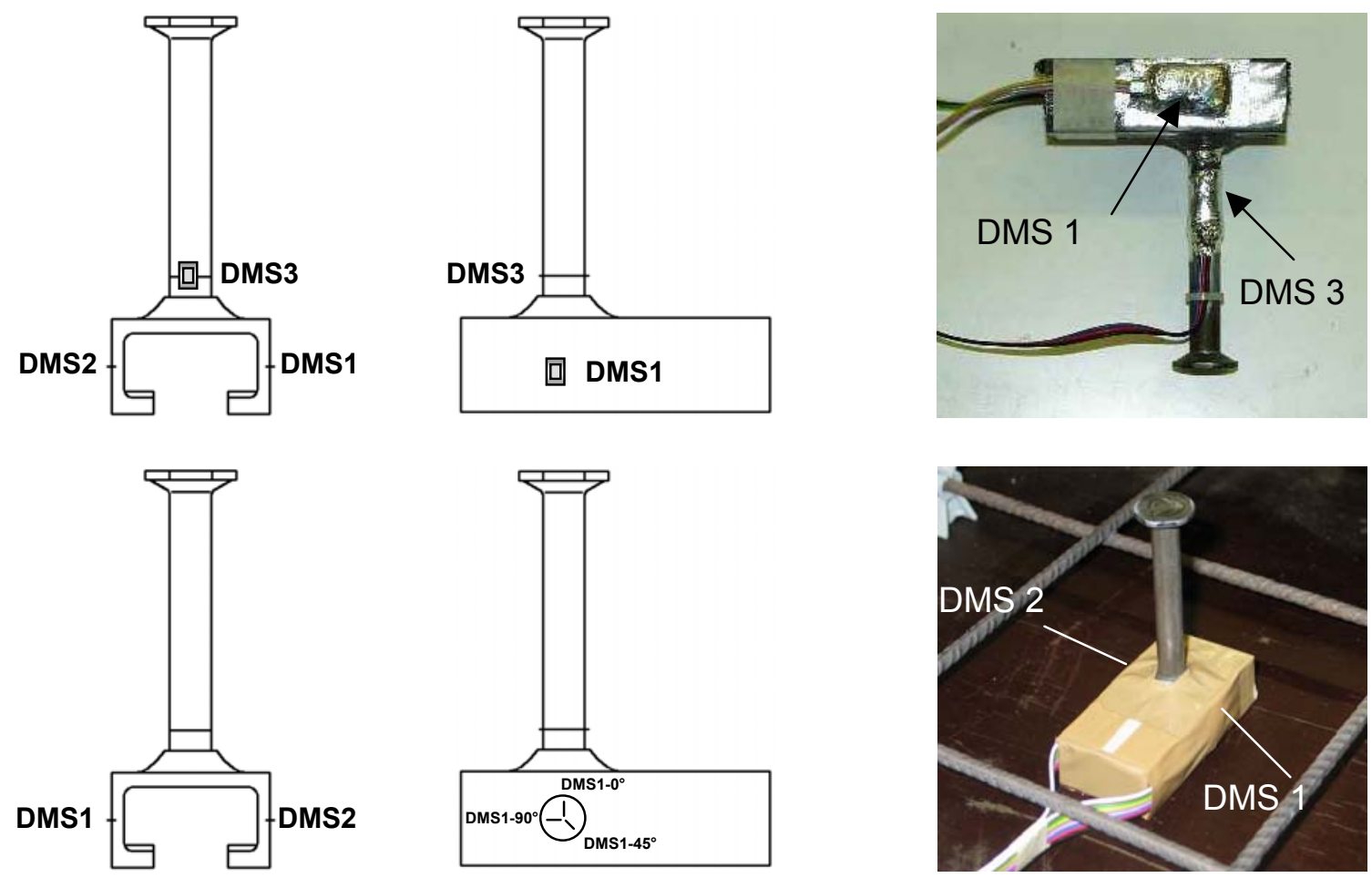

Bild 4.6 Bestückung mittels 1- und 3-achsialen DMS

\subsection{Versuchsaufbau und -durchführung}

Wie aus Abschnitt 4.3 hervorgeht, wurden Versuche an Kurzstücken des Profils 38/23 der Ausführungen 25/50 und 50/50, sowohl im einbetonierten, als auch im nicht einbetonierten Zustand durchgeführt. Die schematischen Versuchsaufbauten für die zentrischen Zugversuche einbetonierter und nicht einbetonierter Ankerschienen sind in Bild 4.7 dargestellt.

Die verwendete Schraube mit Unterlegscheibe und Mutter entsprechender Größe wurde zentrisch in der Achse des Ankerbolzens eingesetzt und die Mutter mit einem definierten Drehmoment von 60 Nm (Standardschraube der Güte 4.6) bzw. 120 Nm (Zahnschraube der Güte 8.8) gegen das Schienenprofil vorgespannt. Um die nicht einbetonierten Ankerschienen in die Spannbacken der Prüfmaschinen einspannen zu können, wurden zunächst die Köpfe der Ankerbolzen abgetrennt und anschließend der runde Anker in den Spannbacken der Prüfmaschine festgespannt. Auf diese Weise war eine sichere Befestigung der Ankerschienen gewährleistet. Die Krafteinleitung in die Ankerschiene erfolgte über eine in einer Gelenklagerung angeordneten Gewindehülse, durch die eine zentrische und biegearme Einspannung gesichert wurde (Bild 4.7 , rechts).

Für den Versuchsaufbau im Falle der einbetonierten Ankerschiene wurde eine zusätzliche Rahmenkonstruktion, bestehend aus vier Profilen L 80 x 40, vier Profilen U $60 \mathrm{x}$ 30 und einer Grundplatte 460 x 460 x 30, angefertigt. Diese waren über eine gelenkige 
Lagerung mit dem Querhaupt der Prüfmaschine verbunden. Die Krafteinleitung in den Versuchskörper erfolgte mit Hilfe eines Anbauteils, das ebenfalls gelenkig mit dem Prüfzylinder verbunden war. Die Vorspannung erfolgte ausschließlich gegen die Schenkel der Ankerschienen. Das vorliegende statische System als Pendelstütze konnte eine reine zentrische Zuglast ohne zusätzliche Momente sicherstellen. Somit waren auch Nebeneffekte infolge des Verkantens durch Rißbildung im Profil auszuschließen (Bild 4.7, links).

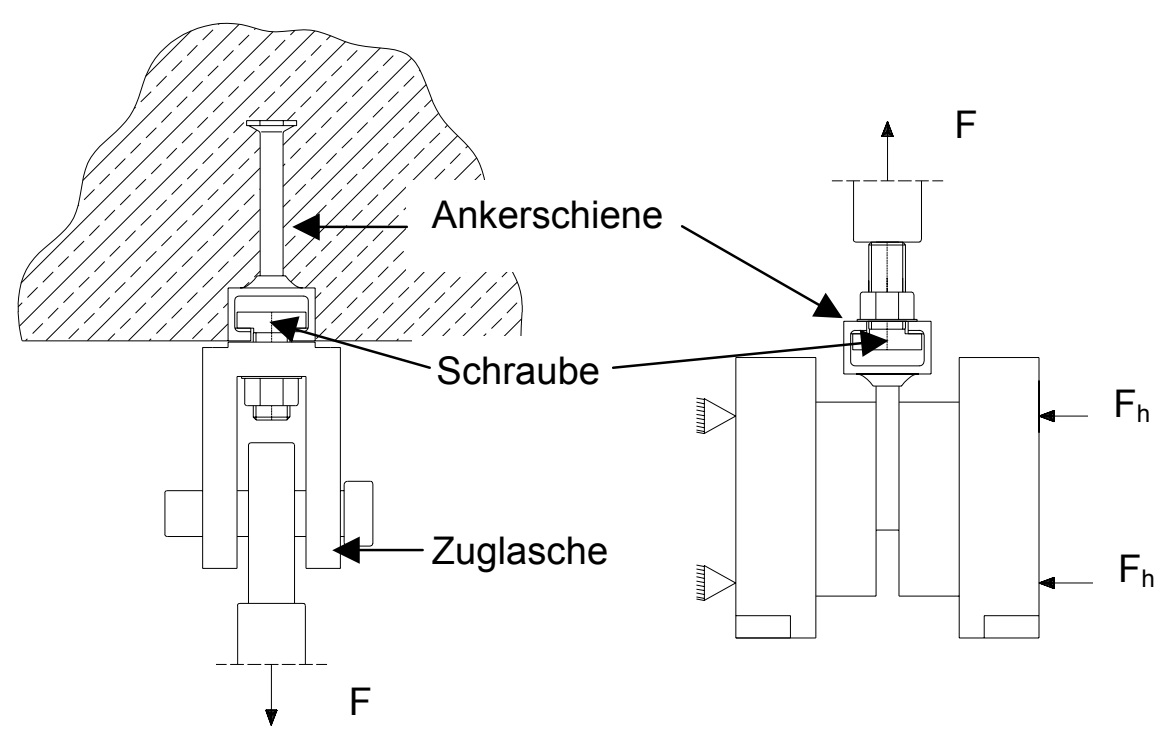

Bild 4.7 Aufbau einbetonierter (links) und nicht einbetonierter (rechts) Ankerschienen

Zusätzlich zu der reinen zentrischen Zugbeanspruchung waren auch Beanspruchungen unter variierenden Belastungswinkeln $\left(30^{\circ}, 45^{\circ}, 60^{\circ}\right.$ und $90^{\circ}$ bezogen auf die zentrische Zugrichtung) Bestandteil dieser Arbeit. Diese wurden aus versuchstechnischen Gründen ausschließlich an einbetonierten Ankerschienen durchgeführt. In Bild 4.8 ist der Versuchsaufbau schematisch für eine Schrägzugbeanspruchung quer zur Schienenlängsrichtung dargestellt. Die o.g. Rahmenkonstruktion wurde auf verschiedene Unterbauten mit unterschiedlichen Schrägneigungen, die aus Profilen (HEB 160, HEB 180) zusammengesetzt waren, montiert. Nachdem der Betonkörper zentriert und arretiert wurde, erfolgte die Krafteinleitung in den Versuchskörper über eine Gewindestange, die sowohl am Prüfzylinder, als auch am Anbauteil gelenkig angeschlossen war. Um die unterschiedlichen Belastungsrichtungen zu gewährleisten, wurde lediglich der Unterbau und das Anbauteil variiert. Bild 4.9 zeigt den schematischen Versuchsaufbau bei Querbeanspruchung. Dabei wurde der einbetonierte Versuchskörper zwischen zwei Profilen U-160 mittels vier Gewindestangen M16 unter Aufbringung eines Drehmomentes eingespannt. Die gesamte Konstruktion lagerte auf einer Unterkonstruktion, welches aus I-Profilen zu einem quadratischen Rahmen zusammengeschweißt wurde und mit dem Aufspanntisch verbunden war. 

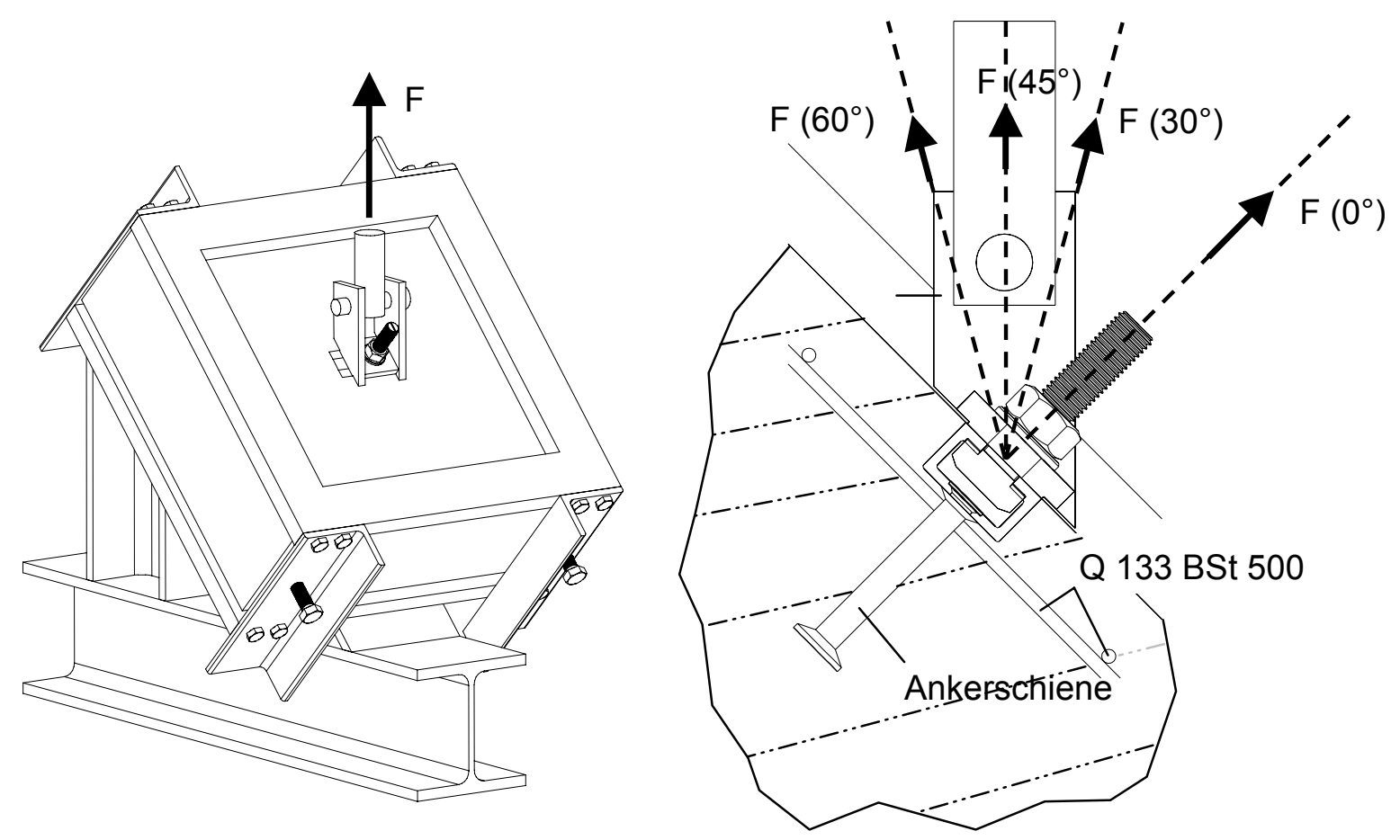

Bild 4.8 Aufbau Schrägzugversuche (exemplarisch quer zur Schienenlängsrichtung)

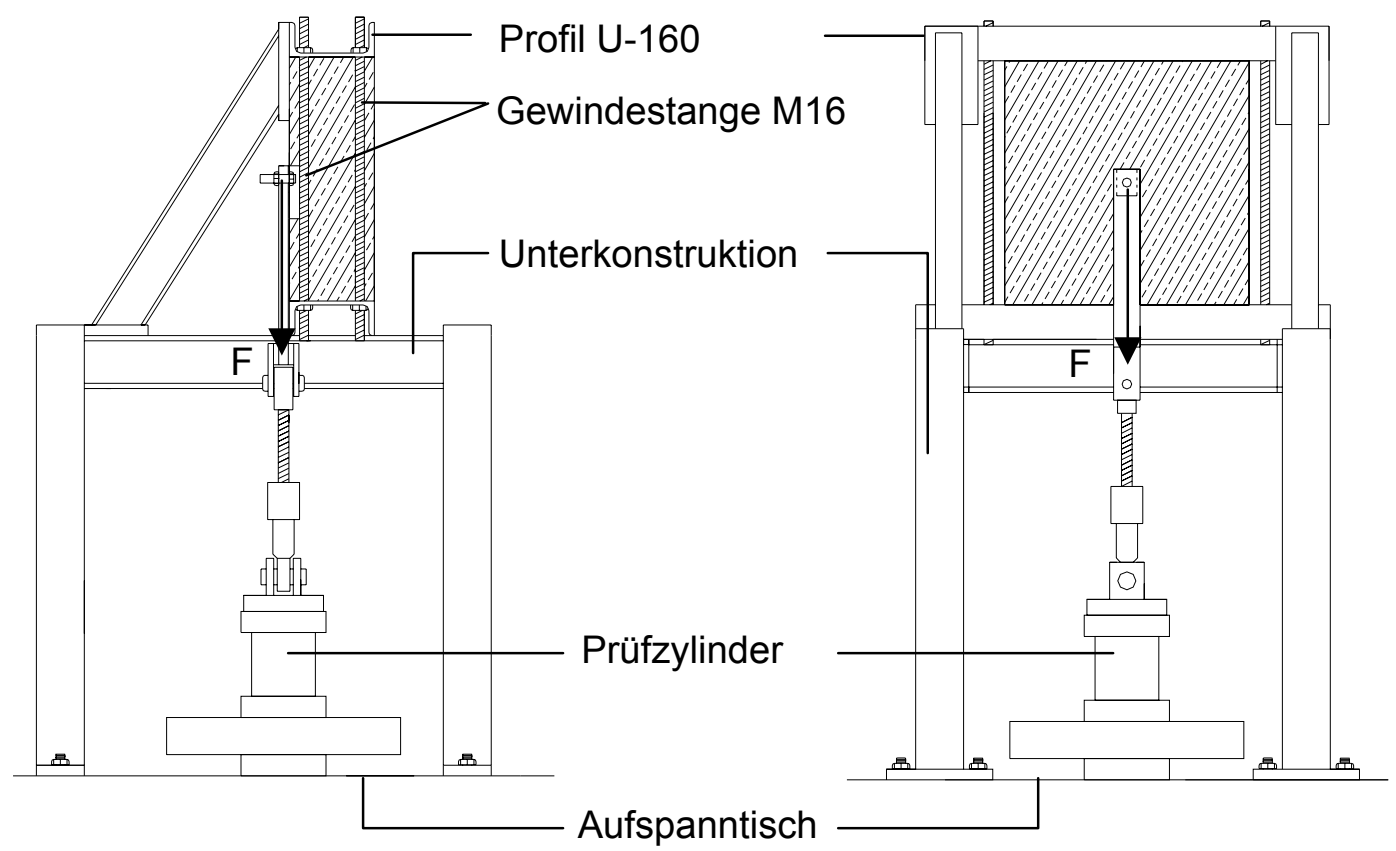

Bild 4.9 Versuchsaufbau für Querbeanspruchungen 
Zur Gewährleistung der variierenden Belastungswinkel sind Zuglaschen mit unterschiedlichen Neigungswinkeln für jede Lasteinleitung angefertigt worden. Diese wurden so hergestellt, daß an der Kontaktfläche zur Ankerschiene eine Erhöhung vorhanden war und somit ausschließlich eine planmäßige Vorspannung gegen die Ankerschiene erfolgen konnte.

\begin{tabular}{|c|c|c|}
\hline $0^{\circ}$ & $30^{\circ}, 45^{\circ}, 60^{\circ}$ & $90^{\circ}$ \\
\hline & &
\end{tabular}

Bild 4.10 Konstruktionen zur Lasteinleitung

Die Durchführung der Versuche erfolgte mit servohydraulischen Prüfmaschinen der Firma SCHENCK. Weitere Informationen zu den Prüfmaschinen sind in [62] gegeben. Zunächst erfolgten statische Zugversuche in Kolbenwegregelung mit einer Belastungsgeschwindigkeit von 1,25 $\mathrm{mm} / \mathrm{min}$. Die anschließenden Schwingversuche wurden unter Kraftregelung durchgeführt. Bei Versuchsbeginn erfolgte zunächst eine zügige Belastung bis $\mathrm{zu}$ einer definierten Mittellast $\mathrm{F}_{\mathrm{m}}$. Anschließend wurde die Schwingbeanspruchung langsam aufgeblendet, bis die eingestellte Oberlast $\mathrm{F}_{\mathrm{o}}$ bzw. Unterlast $\mathrm{F}_{\mathrm{u}}$ erreicht wurde. Durch die Spitzenwertregelung der Prüfeinrichtung wurden Ober- und Unterlasten auch bei sich verändernden Steifigkeiten des Prüfkörpers (z.B. infolge Rißbildung) stets konstant gehalten. Die Beanspruchung erfolgte sinusförmig mit einer Frequenz entsprechend der Arbeitslinie des Ventils der verwendeten Prüfmaschine. Diese betrug je nach Belastungsintensität und -amplitude zwischen 10 und $30 \mathrm{~Hz}$. Die Schwingbreite $\Delta \mathrm{F}$ wurde durch Einstellung der Ober- bzw. Unterlast variiert. Als Versuchsende wurde in Abhängigkeit vom Lastniveau eine Zunahme des Kolbenweges von 3 bis $5 \mathrm{~mm}$ gegenüber der Ausgangsposition definiert. 
Zur Aufnahme von Verschiebungen wurden induktive Wegaufnehmer vom Typ HOTTINGER W10-TK (Meßbereich $\pm 10 \mathrm{~mm}$ ) bzw. W5-TK (Meßbereich $\pm 5 \mathrm{~mm}$ ) mit einem Meßfehler $<1 \%$ verwendet. Die Erfassung der Dehnungen an den Ankerschienen (Profilschenkeln) erfolgte über Dehnungsmeßstreifen.
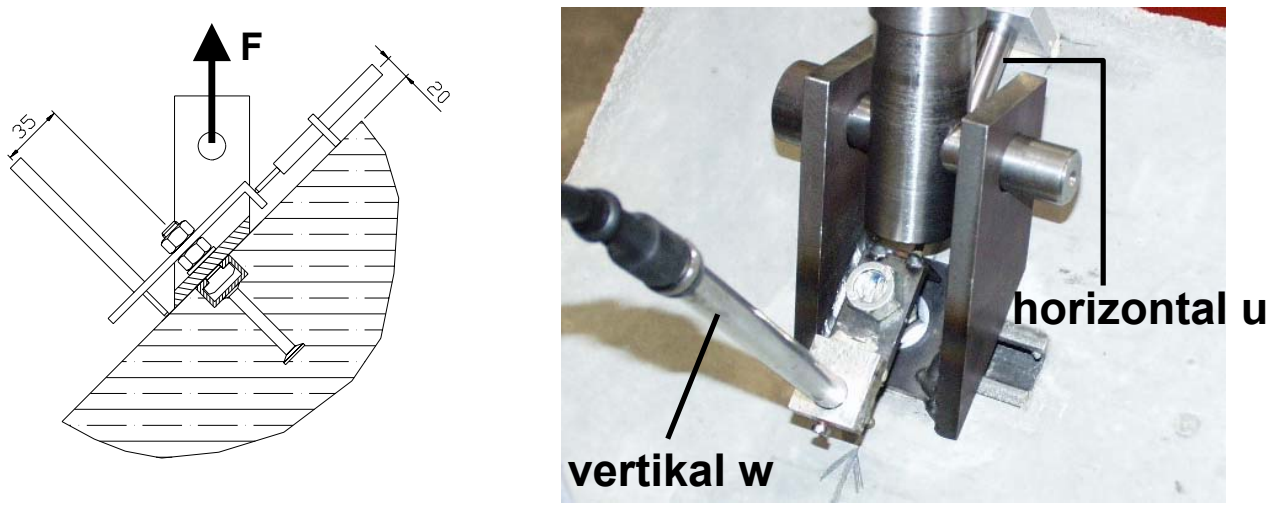

Bild 4.11 Wegaufnehmer bei Schrägbeanspruchung quer zur Schienenlängsrichtung
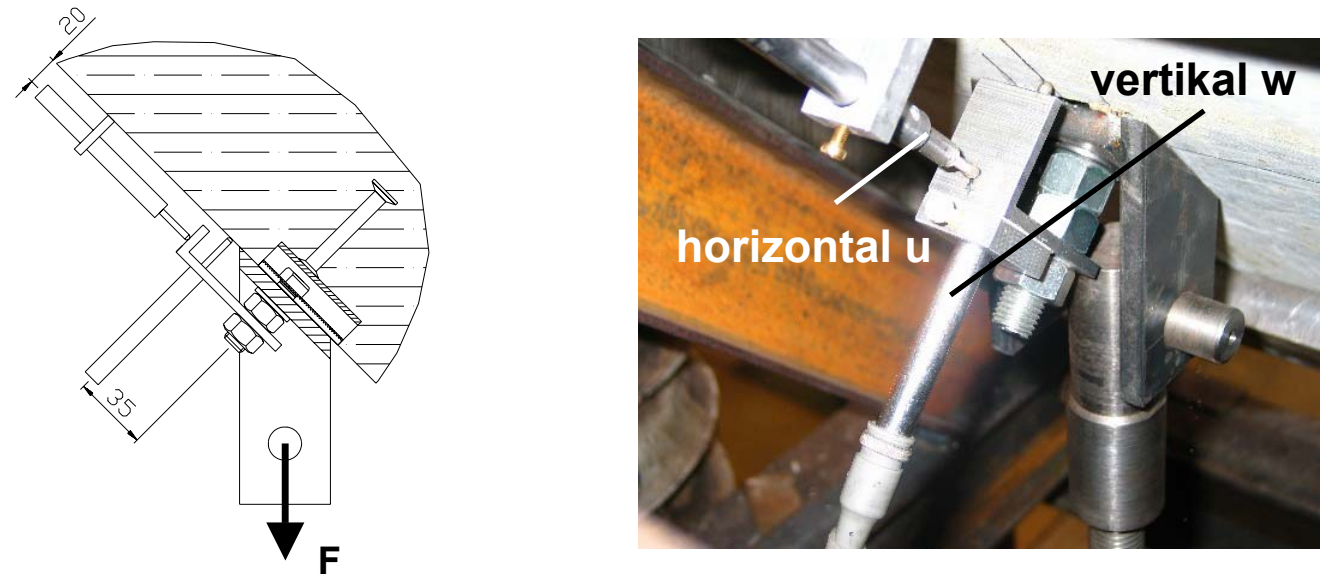

Bild 4.12 Anordnung der Wegaufnehmer bei Beanspruchung in Schienenlängsrichtung
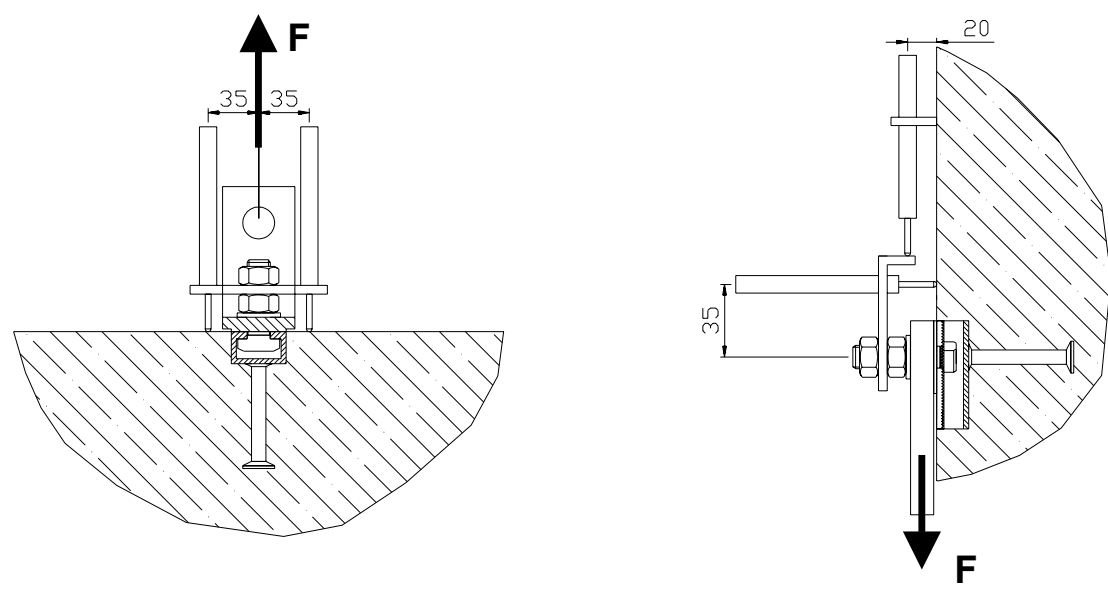

Bild 4.13 Wegaufnehmer zentrischer Zug (links) und in Schienenlängsrichtung (rechts) 


\subsection{Ergebnisse und Auswertungen}

\subsubsection{Versagensfälle unter quasi-statischen Beanspruchungen}

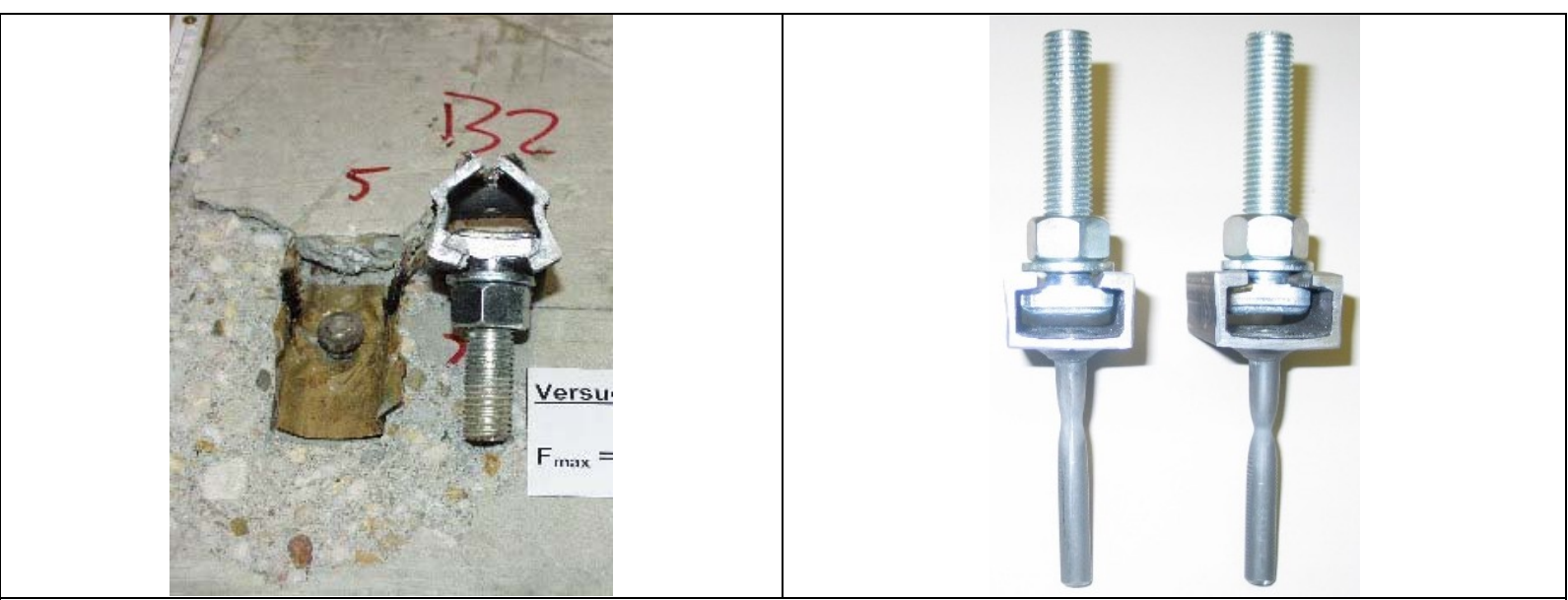

Bild 4.14 Ausreißen des Ankerfußes aus dem Rücken (Serie A und Serie B), links \& plastische Verformung am Ankerschaft (Serie C), rechts, unter Zuglast
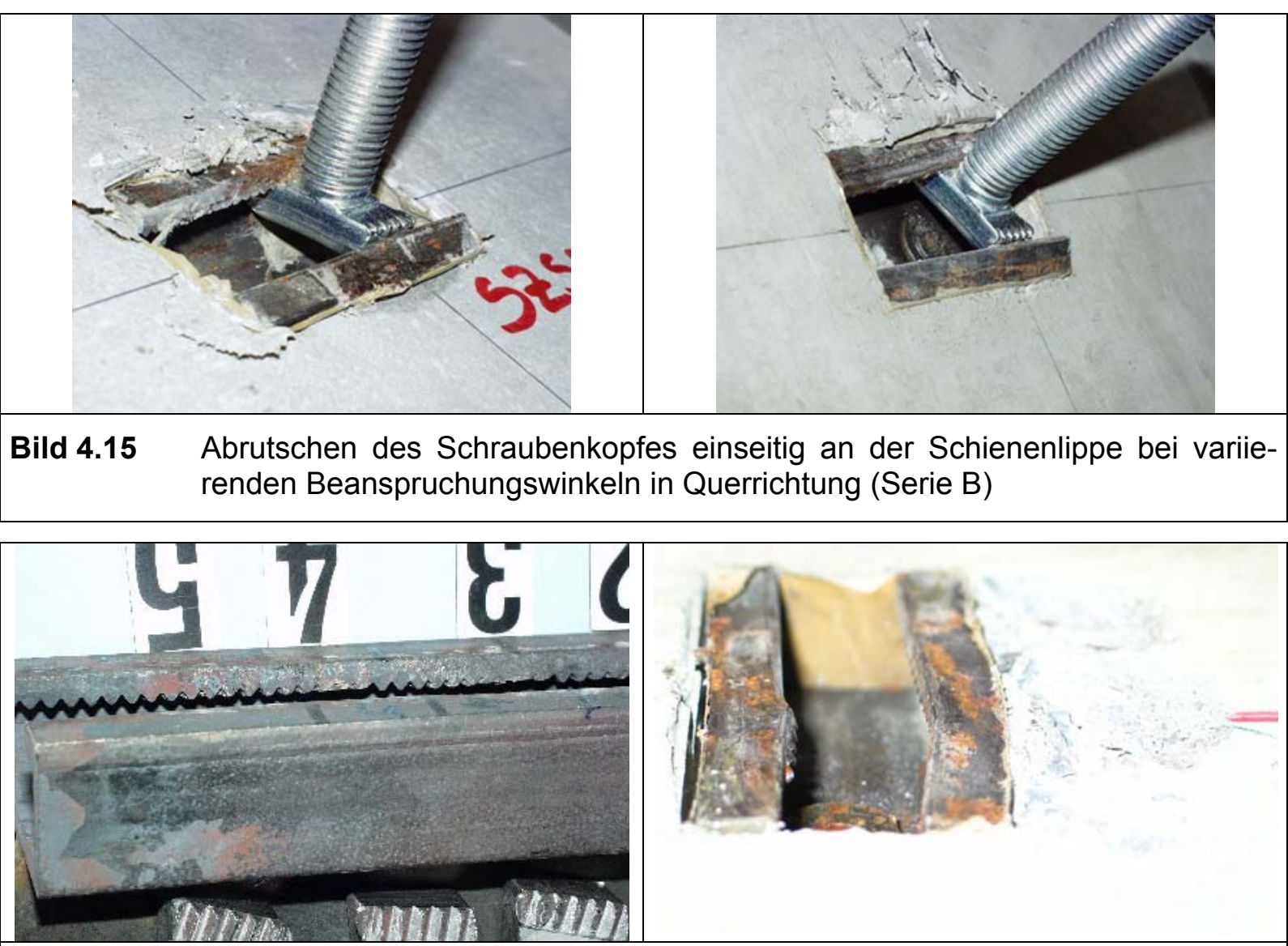

Bild 4.16 Schädigung der Verzahnung bei Beanspruchung in Schienenlängsrichtung, links, und Versagen bei Belastung quer zur Schienenlängsrichtung, rechts 


\subsubsection{Versagensfälle unter schwingenden Beanspruchungen}

Eine wichtige Grundlage für die Auswertung von Versuchen und speziell für die Bestimmung der charakteristischen Größen ist die Tatsache, daß ein tragendes Bauteil mehrere grundsätzlich unterschiedliche Versagensarten haben kann [36]. Ein System wird immer an der Stelle versagen, an der die Tragfähigkeit am geringsten ist.

Zur genaueren Kategorisierung der Versagensfälle in Abhängigkeit von den Beanspruchungsarten sind nach Abschluß der Versuche die Versagenszustände dokumentiert worden. Ein Charakteristikum für Versagenszustände unter schwingender Beanspruchung im Vergleich zu statisch beanspruchten Ankerschienen ist das Entstehen von Rissen an den stark beanspruchten Stellen des Profilquerschnitts. Betrachtet man eine Ankerschiene im einbetonierten Zustand, die durch schwingende Beanspruchung belastet wird, so muß zunächst grundsätzlich in zwei Versagensarten unterschieden werden:

- Betonversagen

- Stahlversagen

Ein Betonversagen bei schwingender Beanspruchung konnte wegen der Einhaltung der Anforderungen gemäß der bauaufsichtlichen Zulassung nicht beobachtet werden. Als Versagensart war stets das Stahlversagen maßgebend. Aufgrund der Ankerschienengeometrie sind wiederum unterschiedliche Versagensstellen im Profilquerschnitt möglich:

- Risse in Schienenlängsrichtung im Übergangsbereich Schienenrücken zum Schienenschenkel (einseitig, beidseitig)

- Risse in Schienenlängsrichtung im Übergangsbereich Schienenschenkel zur Schienenlippe (einseitig, beidseitig)

- Risse im Rückenbereich der Ankerschiene mit möglichem Ausreißen des Ankerbolzens aus dem Rücken

- Bruch des Ankerbolzens

Die Last wird über die Ankerschraube in die Verzahnung der Schienenlippen eingeleitet, welche dann über die Seitenschenkel in den Schienenrücken und anschließend in den Ankerbolzen und somit in den Beton weitergeleitet wird. Die Übergangsbereiche Schienenlippe - Schienenschenkel, Schienenschenkel - Schienenrücken und Schienenrücken - Ankerbolzen sind in jedem Fall die Stellen des Ankerschienenquerschnitts, an denen hohe Spannungskonzentrationen auftreten. Aus diesem Grund zeigen sich die meisten Riß- und Brucherscheinungen gerade in diesen Bereichen. Ein Versagen der Ankerschraube wurde kaum beobachtet. In vielen Fällen ist eine Kombination der o.g. Versagensarten möglich, welche infolge von Lastumlagerungen aufgrund von Rißbildung stattfinden. Je nach Belastungsart und -richtung stellten sich unterschiedliche Versagensfälle ein. Nachfolgend werden die beobachteten Hauptversagensarten in Abhängigkeit von der Beanspruchungsrichtung dargestellt und anschließend kategorisiert. 


\subsubsection{Zentrische Zugbeanspruchung}

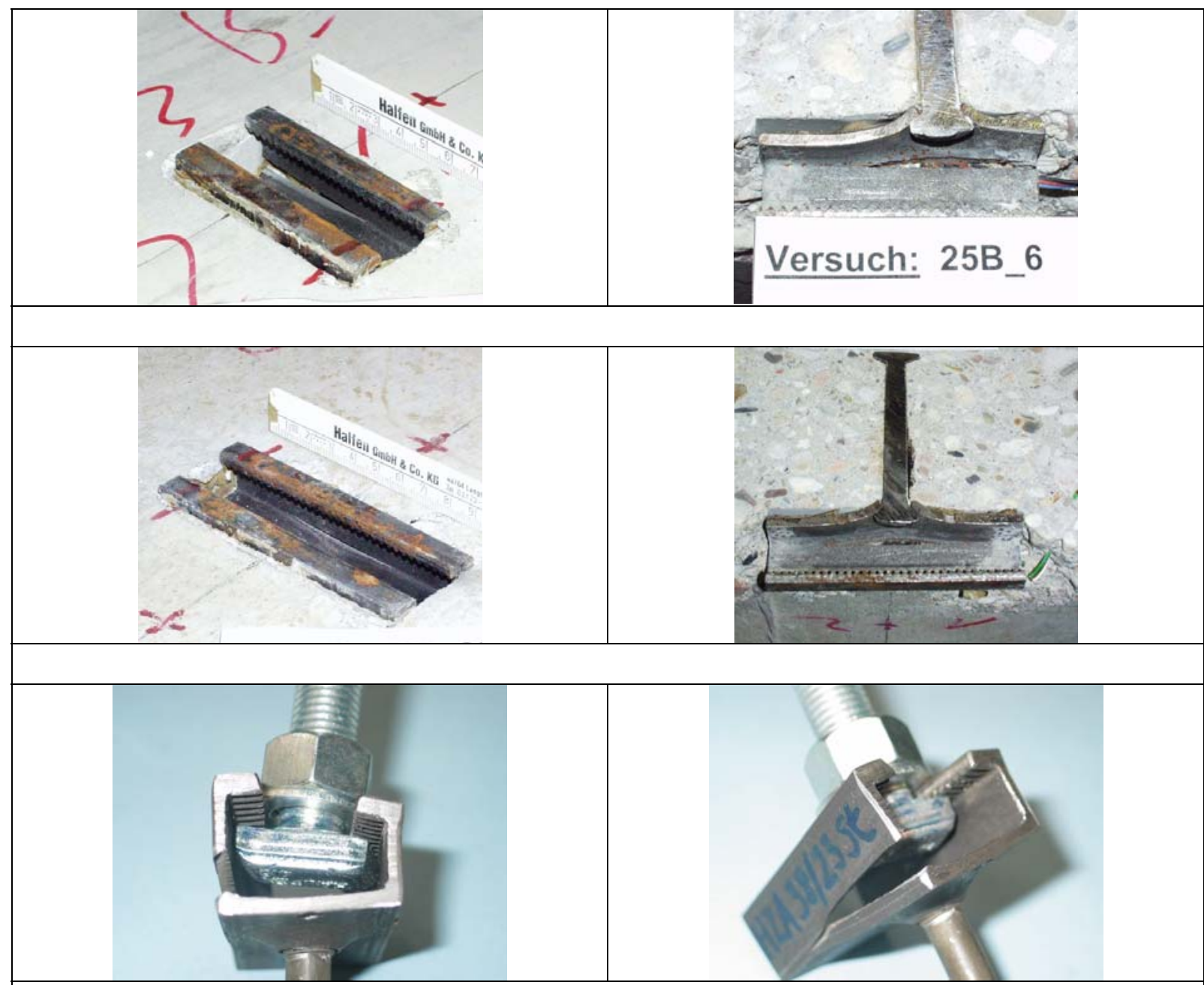

Bild 4.17 Längsriß im Übergangsbereich Rücken - Seitenschenkel, einseitig

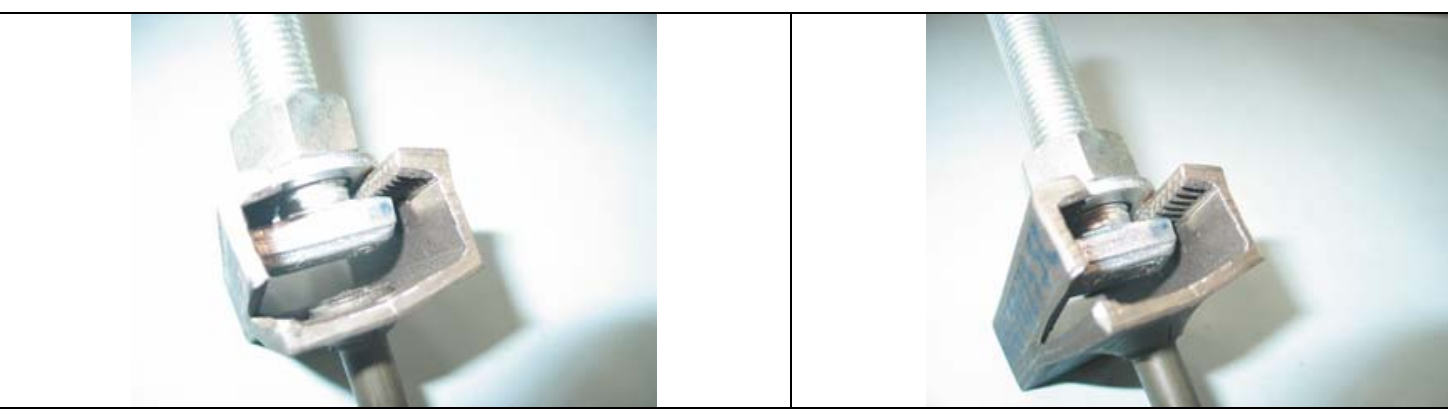

Bild 4.18 Anriß an der gegenüberliegenden Seite im Übergangsbereich Schienenlippe Seitenschenkel 


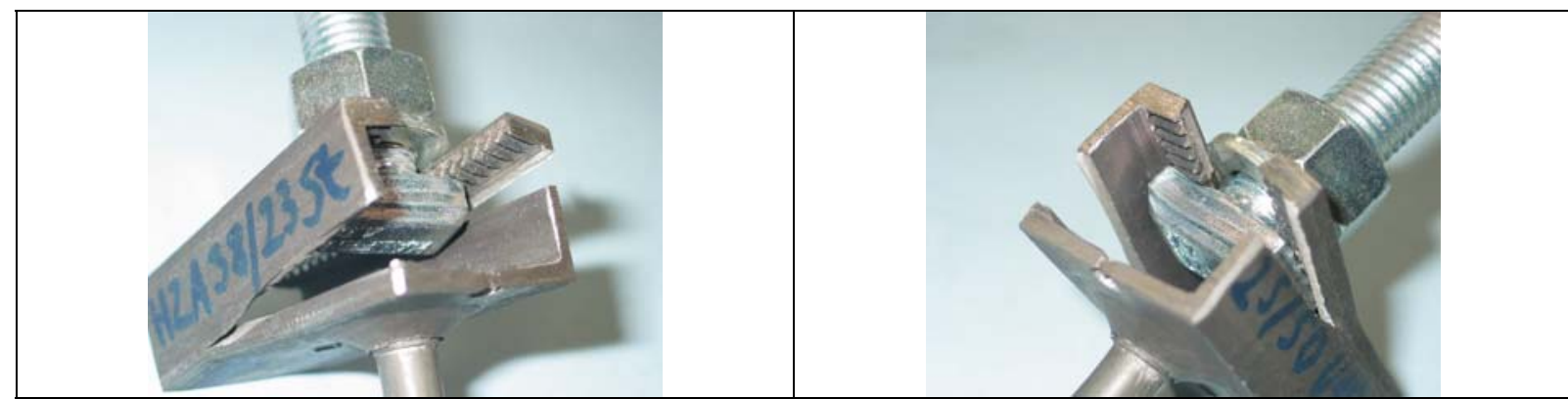

Bild 4.19 Riß an der gegenüberliegenden Seite im Übergangsbereich Seitenschenkel Schienenlippe

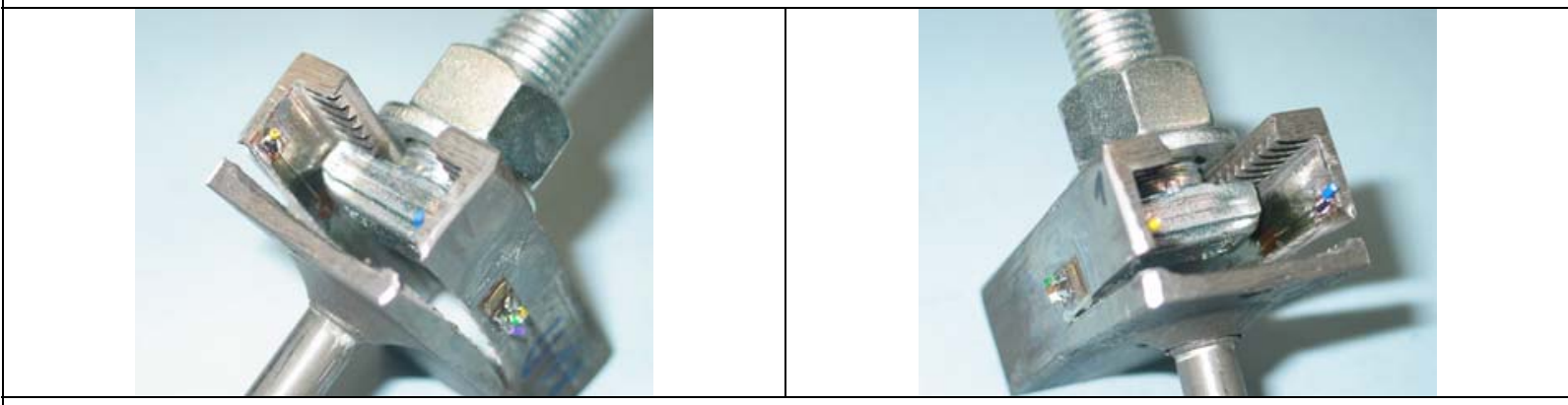

Bild 4.20 Riß im Übergangsbereich Schienenrücken - Schienenschenkel, beidseitig

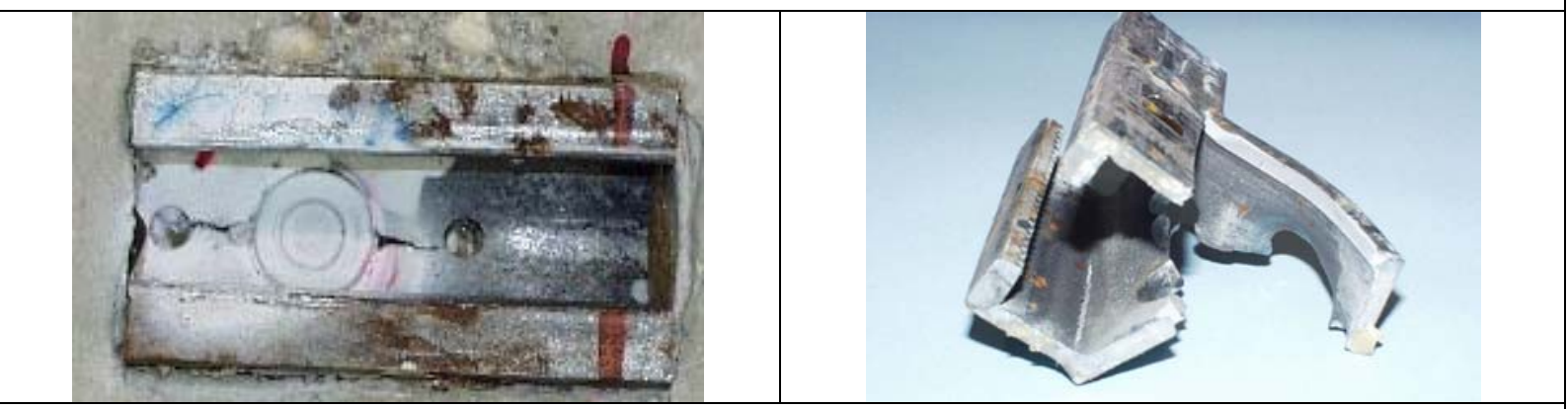

Bild 4.21 Risse im Rückenbereich, ausgehend vom Ankerfuß zu den Nagellöchern

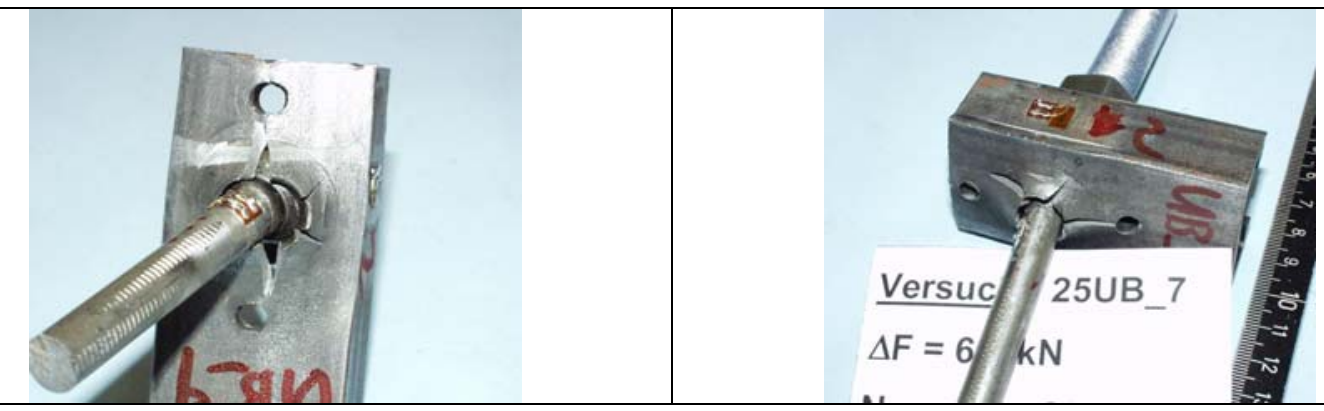

Bild 4.22 Sternförmige Rißausbildung im Rückenbereich mit verstärkter Ausbildung in Schienenlängsrichtung (in Richtung der Nagellöcher) 


\subsubsection{Querbeanspruchung in Schienenlängsrichtung}

Bild 4.23 Längsriß im Übergangsbereich Schienenschenkel - Schienenlippe, einseitig

Bild 4.24 Sekundär: kompletter Abriß des Schienenschenkels
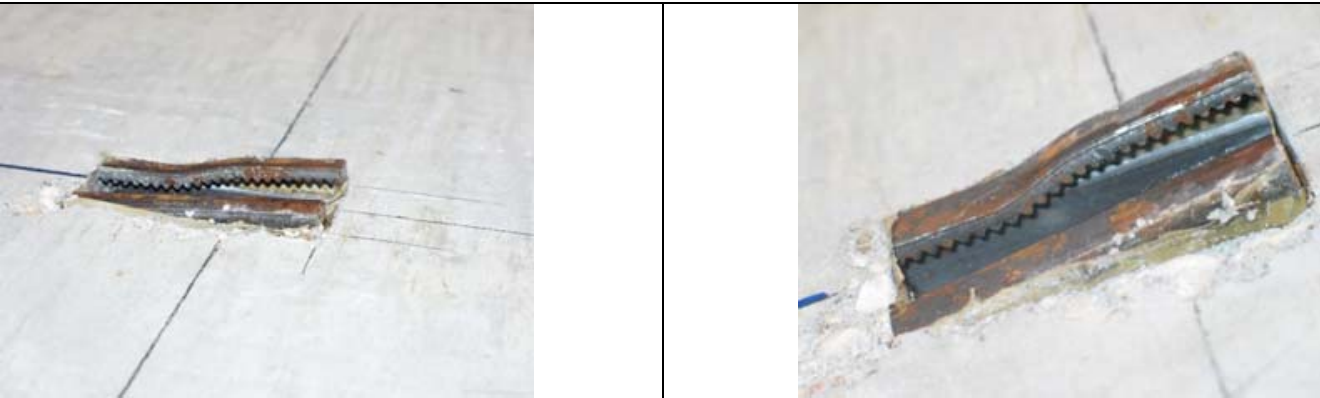

Bild 4.25 Längsriß im Übergangsbereich Schienenschenkel - Schienenlippe, beidseitig

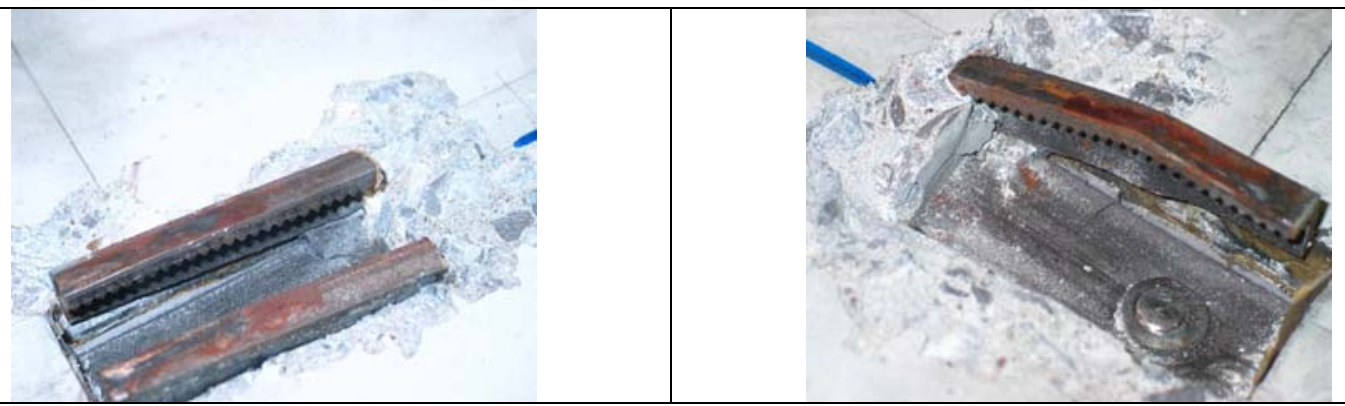

Bild 4.26 Längsriß im Übergang Schienenrücken - Schienenschenkel, beidseitig 


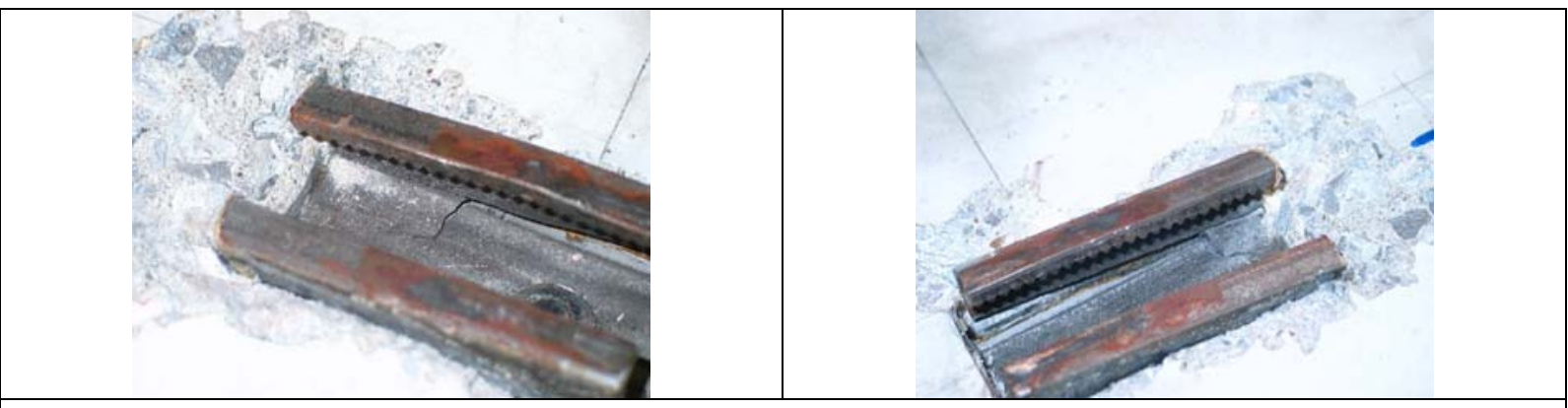

Bild 4.27 Sekundär: Rißausbreitung bis in den Rückenbereich

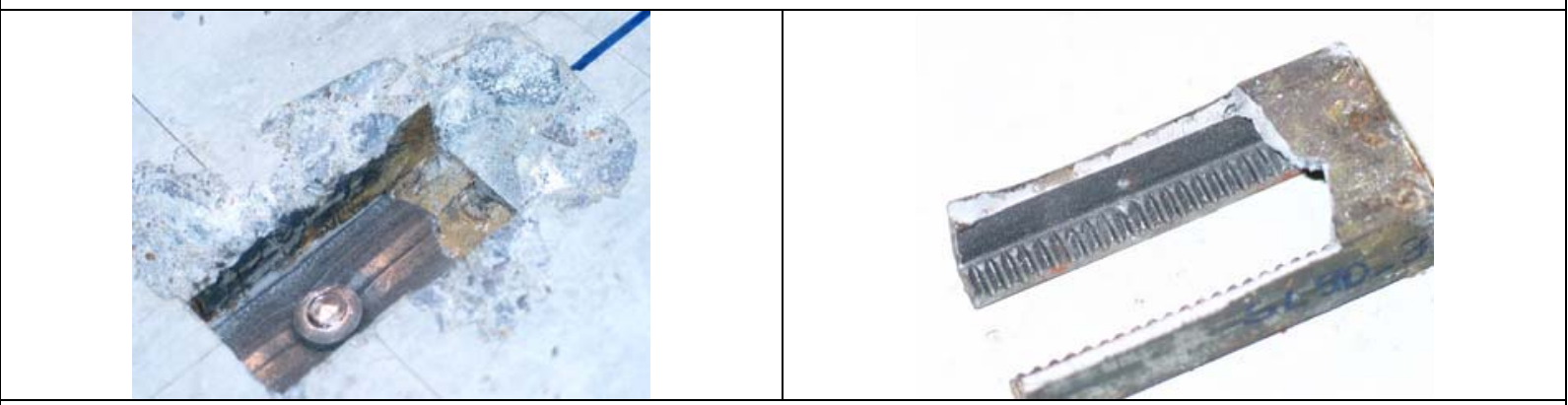

Bild 4.28 Sekundär: kompletter Abriß der Ankerschiene im Rückenbereich

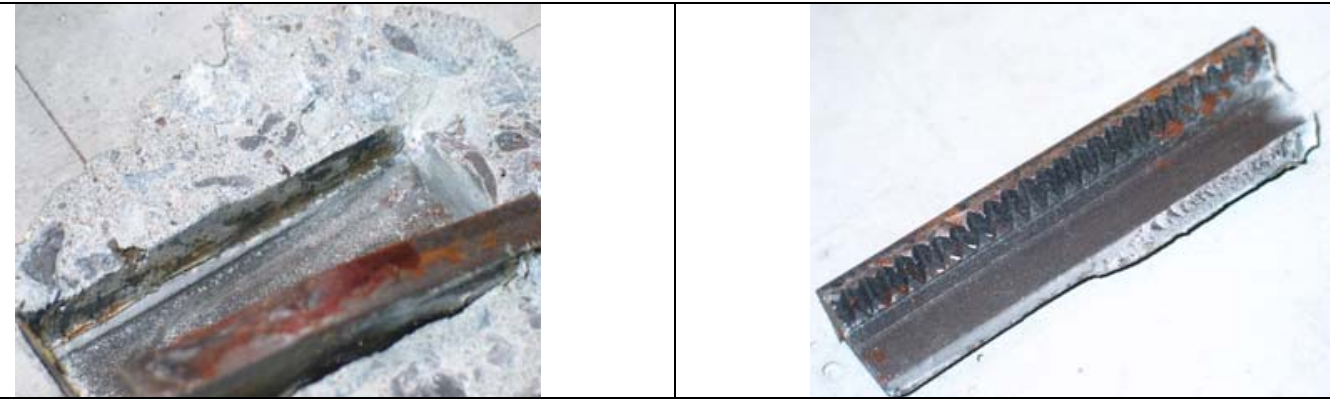

Bild 4.29 Sekundär: Abriß des Schienenschenkels, einseitig

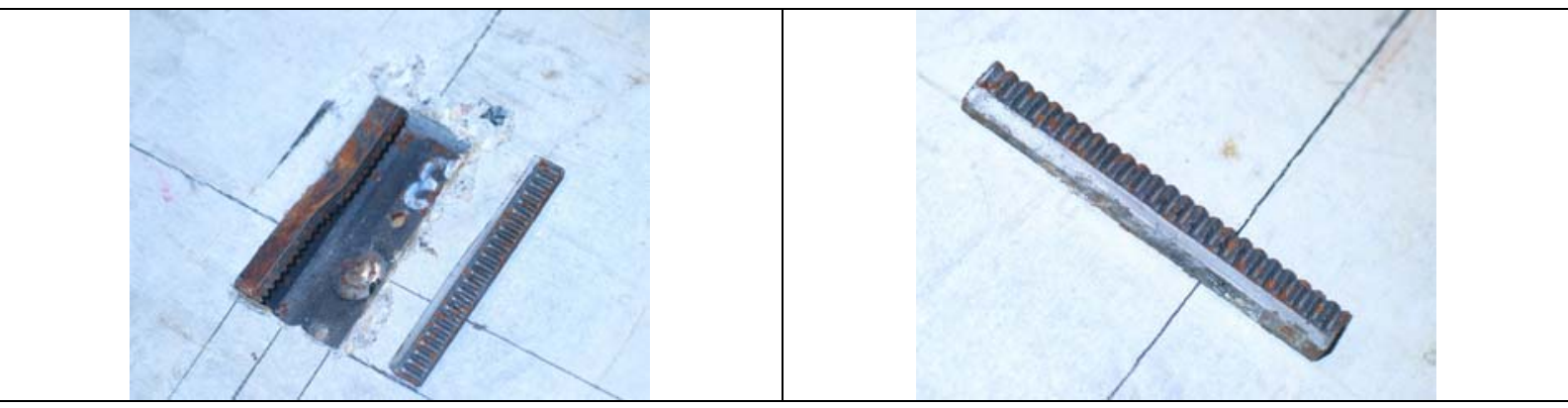

Bild 4.30 Sekundär: kompletter Abriß einer Schienenlippe, einseitig 


\subsubsection{Schrägbeanspruchung quer zur Schienenlängsrichtung}

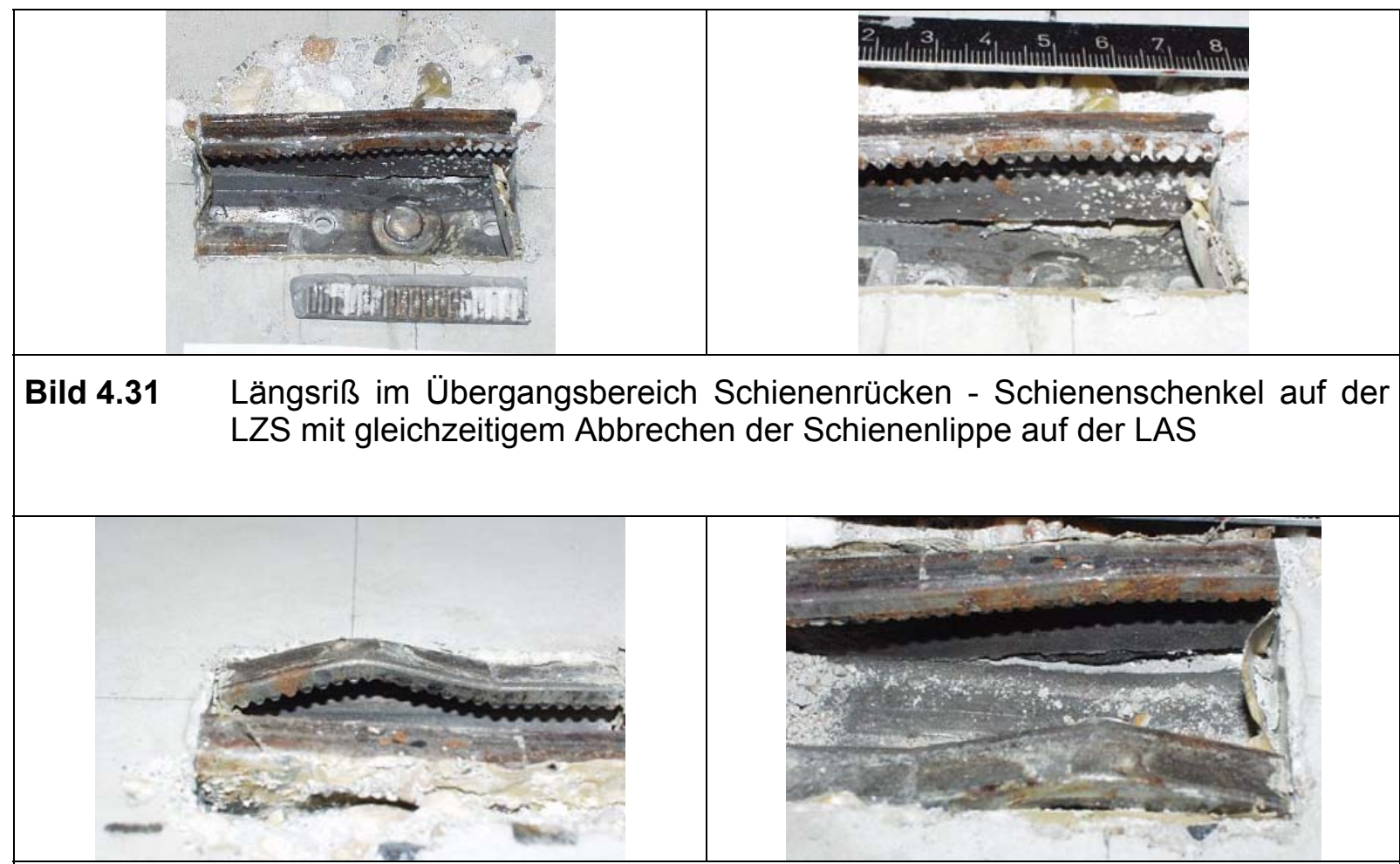

Bild 4.32 Längsrisse im Übergangsbereich Schienenrücken - Schienenschenkel auf der LZS mit gleichzeitigem Aufbiegen der Schienenlippe auf der LAS

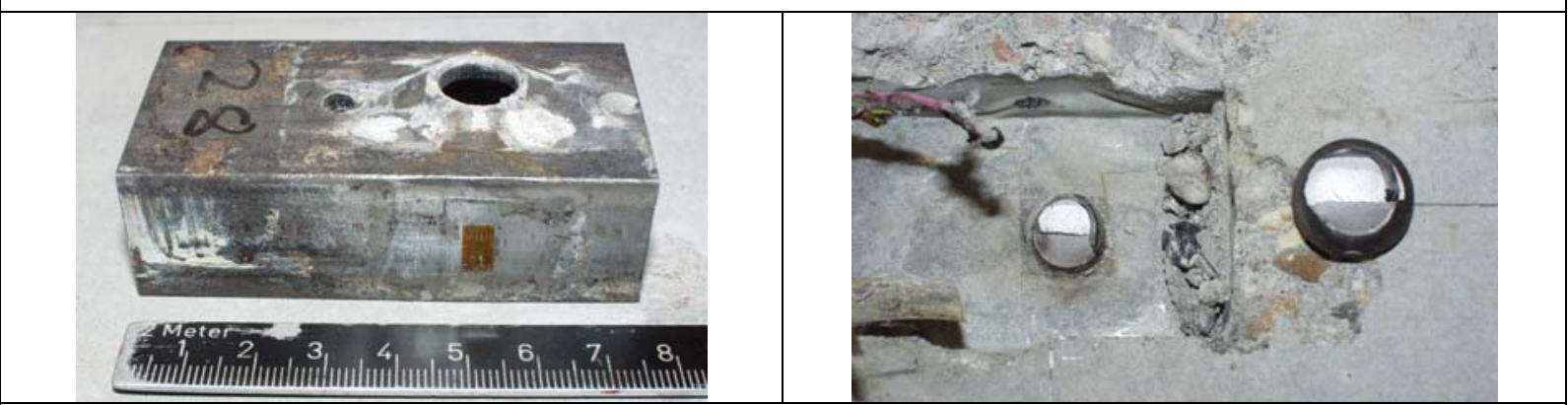

Bild 4.33 Abbruch des Ankerfußes
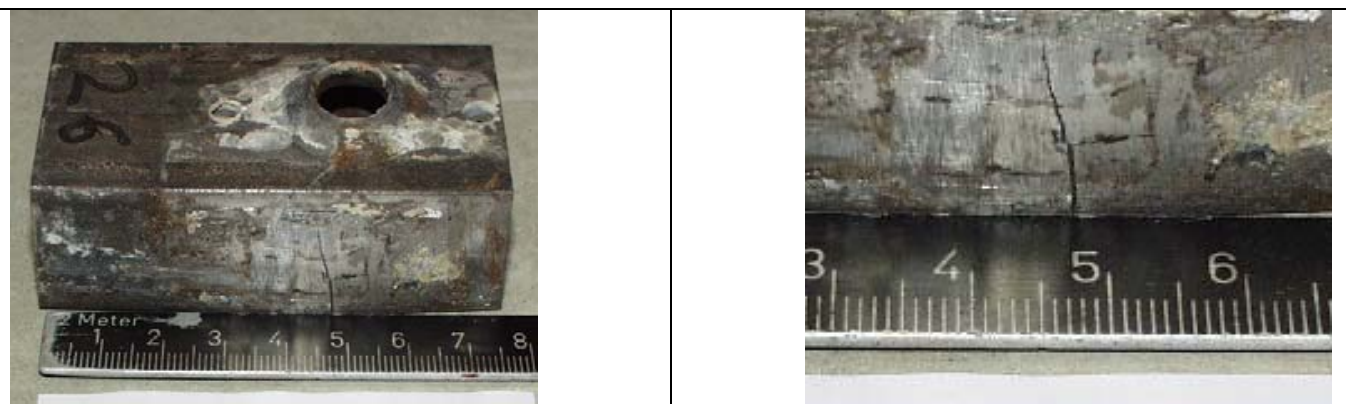

Bild 4.34 Vertikalriß am Schienenschenkel auf der LZS in Richtung Schienenlippen 


\subsubsection{Schrägbeanspruchung in Schienenlängsrichtung}

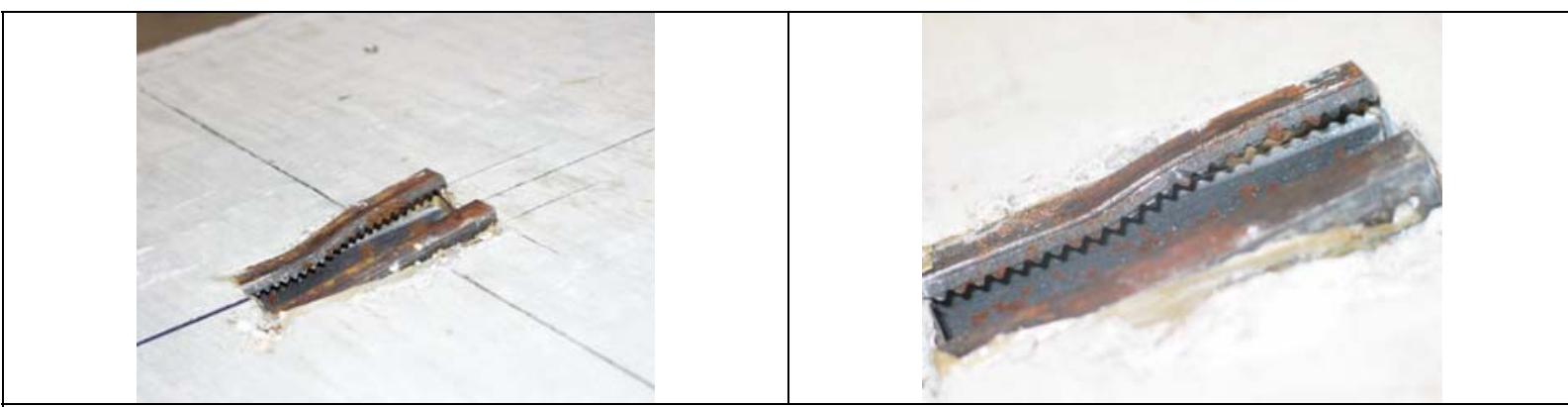

Bild 4.35 Längsriß im Übergangsbereich Seitenschenkel - Schienenlippe, einseitig

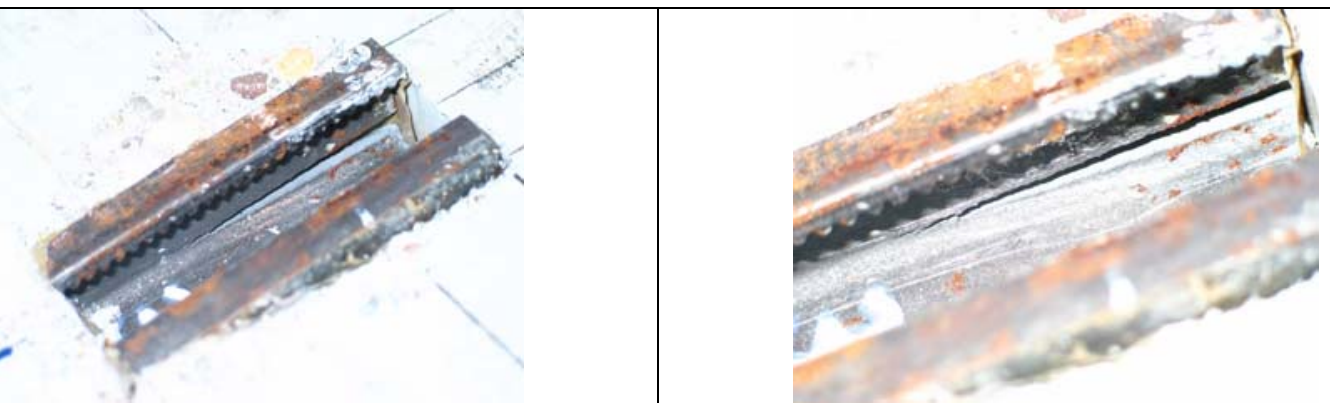

Bild 4.36 Längsriß im Übergangsbereich Schienenrücken - Schienenschenkel, einseitig

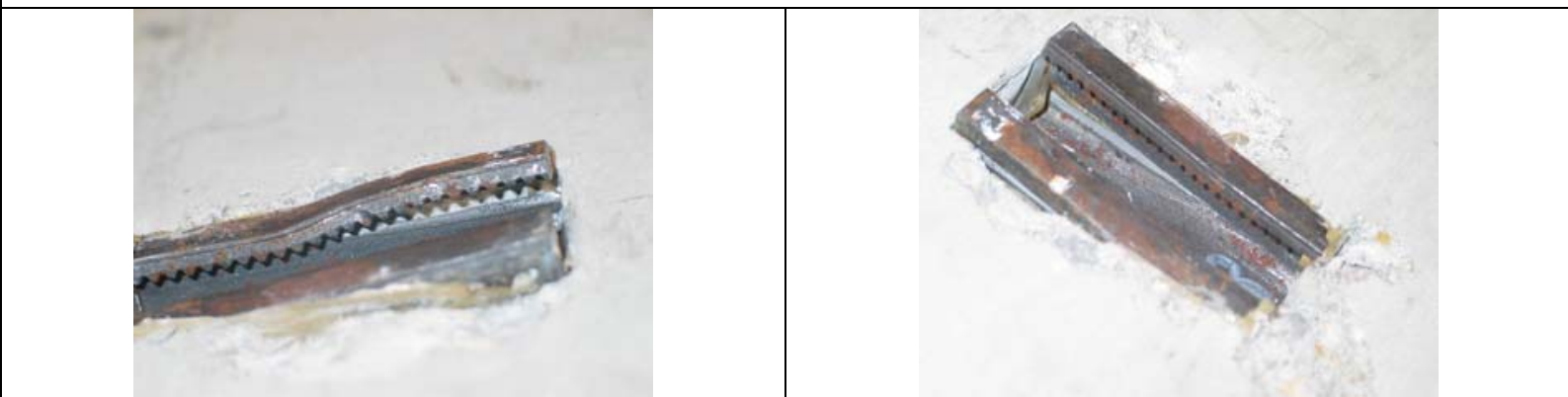

Bild 4.37 Längsriß im Übergangsbereich Schienenrücken - Schienenschenkel, einseitig, und Schienenschenkel - Schienenverzahnung auf der gegenüberliegenden Seite

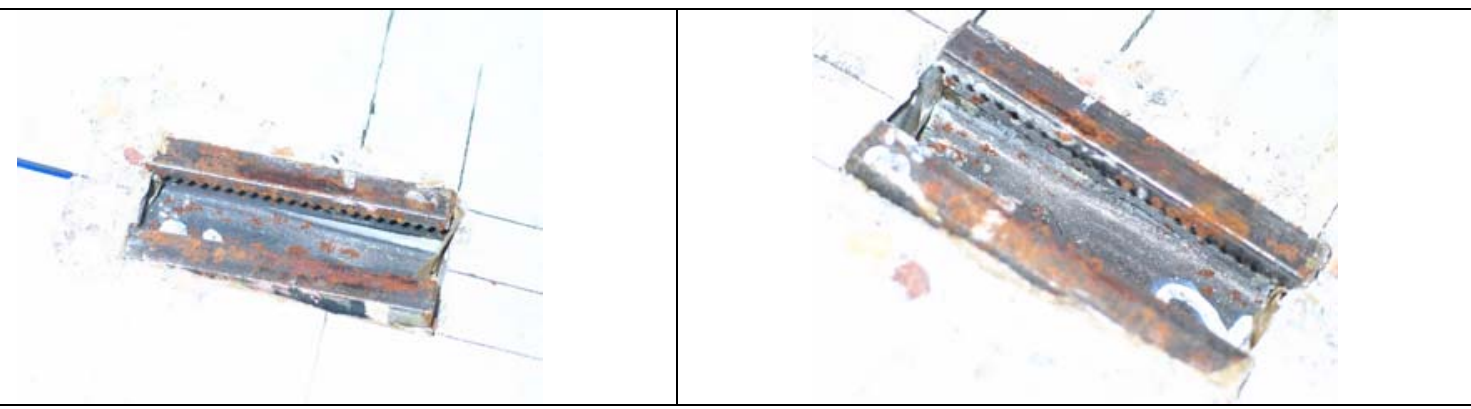

Bild 4.38 Längsriß im Übergang Schienenrücken - Schienenschenkel, beidseitig 
Aus den Darstellungen in Bild 4.17 bis Bild 4.38 gehen die unterschiedlichen Versagensfälle deutlich hervor und bestätigen die Annahme über die o.g. Schadensstellen. Diese zeigen teilweise Abhängigkeiten von der Belastungsrichtung. Der häufigste Versagensfall ist im Querschnitt der Schiene zu beobachten. In den meisten Fällen treten hier Risse einseitig bzw. beidseitig im Übergangsbereich des Schienenrückens zum Schienenschenkel auf. Im Falle eines stark einseitigen Risses konnten teilweise auch leichte Anrisse auf den gegenüberliegenden Schenkelseiten, im Übergangsbereich des Schienenrückens zum Schienenschenkel bzw. des Schienenschenkels zur Schienenlippe, beobachtet werden. Ein Versagen des Ankerfußes im Stauchbereich wurde hauptsächlich bei Beanspruchungen quer zur Schienenlängsrichtung beobachtet und ist somit charakteristisch für diese Beanspruchungsrichtung.

Zusammenfassend können die auftretenden Hauptversagensfälle in Bild 4.39 festgehalten werden. Diese werden nach der Häufigkeit ihres Auftretens geordnet. Die Erläuterung der Versagensmechanismen erfolgt im Rahmen der Beschreibung der Versuchsergebnisse.

\begin{tabular}{|l|l|l|l|}
\hline & \\
\hline
\end{tabular}

Bild 4.39 Übersicht der Hauptversagensarten 


\subsubsection{Auswertung der Ermüdungsversuche}

\subsubsection{Festlegung der Bemessungsschwingspielzahl}

Bisherige Auswertungen von Ermüdungsversuchen bei Befestigungselementen beziehen sich auf die Dauerfestigkeit bei $2 \cdot 10^{6}$ Schwingspielen. Für den Werkstoff Stahl ist diese Festigkeit ein allgemein anerkannter Grenzwert, welcher ebenfalls in [37] für die aufgeführten Betriebsfestigkeitsnachweise verwendet wird. Es ist somit zunächst zu klären, ob die Gültigkeit der Dauerfestigkeit bei $2 \cdot 10^{6}$ Schwingspielen auch für Ankerschienenbefestigungen gewährleistet ist. Zur Ermittlung dieses Schwellenwertes ist eine hohe Anzahl von Versuchen mit einem gemeinsamen Merkmal (beispielsweise konstante Unter- oder Mittellast) erforderlich. In [14] werden 20 bis 30 Versuche auf mindestens zwei Beanspruchungshorizonten empfohlen. Um im Rahmen dieser Arbeit eine aussagekräftige Anzahl an Versuchen bereitzustellen, wurde als gemeinsames Merkmal die Mittellast in den Versuchen betrachtet. Dadurch wurden insgesamt 48 Versuche bei acht unterschiedlichen Mittellasten (Streuband der Mittellast von 4,25 bis $6,5 \mathrm{kN}$ ) zur Verfügung gestellt. Eine Beurteilung der Dauerfestigkeit mit dieser Versuchsanzahl erscheint nach Ansicht des Verfassers als ausreichend. Der Verlauf der Ermüdungsfestigkeit wird unter Verwendung der Gleichung (4.1) nach [7] beschrieben. Damit gelingt es, den Übergang von der Zeitfestigkeit in die Dauerfestigkeit im Vergleich zur Formel nach Wöhler [96] viel exakter abzubilden und somit einen möglichst genauen Betrag der Dauerfestigkeit zu erreichen.

$\Delta \mathrm{F}=\Delta \mathrm{F}_{\mathrm{D}}+\left(\mathrm{F}_{\mathrm{m}}-\mathrm{F}_{\mathrm{u}}-\Delta \mathrm{F}_{\mathrm{D}}\right) \cdot \mathrm{a}^{(\mathrm{lgN})^{\mathrm{b}}}$

$\mathrm{a}, \mathrm{b}=$ positive, dimensionslose Zahlen mit $\mathrm{a}<1$

$\mathrm{N}=$ Anzahl der Schwingspiele

$\Delta \mathrm{F}=$ Schwingbreite der Ermüdungstragfähigkeit

$\Delta \mathrm{F}_{\mathrm{D}}=$ Schwingbreite der Dauerermüdungstragfähigkeit

$\mathrm{F}_{\mathrm{m}}=$ statische Tragfähigkeit

$\mathrm{F}_{\mathrm{u}}=$ untere Grenze der Beanspruchungszyklen

Die für eine rechnerische Analyse erforderliche und nach der Schwingspielzahl N umgestellte Funktion ist in Gleichung (4.2) dargestellt.

$(\lg \mathrm{N})^{\mathrm{b}}=\log _{\mathrm{a}}\left(\frac{\Delta \mathrm{F}-\Delta \mathrm{F}_{\mathrm{D}}}{\mathrm{F}_{\mathrm{m}}-\mathrm{F}_{\mathrm{u}}-\Delta \mathrm{F}_{\mathrm{D}}}\right)$

Unter Berücksichtigung eines Parameters $b$ wird im Vergleich zu den ähnlich aufgebauten Darstellungsweisen nach Weibull und Stüssi aus [53], der Übergang in den horizontalen Bereich der Wöhlerlinie besser angepaßt. Somit können qualitativ bessere Aussagen zur Ermüdungstragfähigkeit getroffen werden.

Im nachfolgenden Diagramm in Bild 4.40 ist der Verlauf der Ermüdungstragfähigkeit für die zentrischen Zugversuche im nicht einbetonierten Zustand für ein Streuband der Mittellast von 4,25 kN und 6,5 kN dargestellt. 


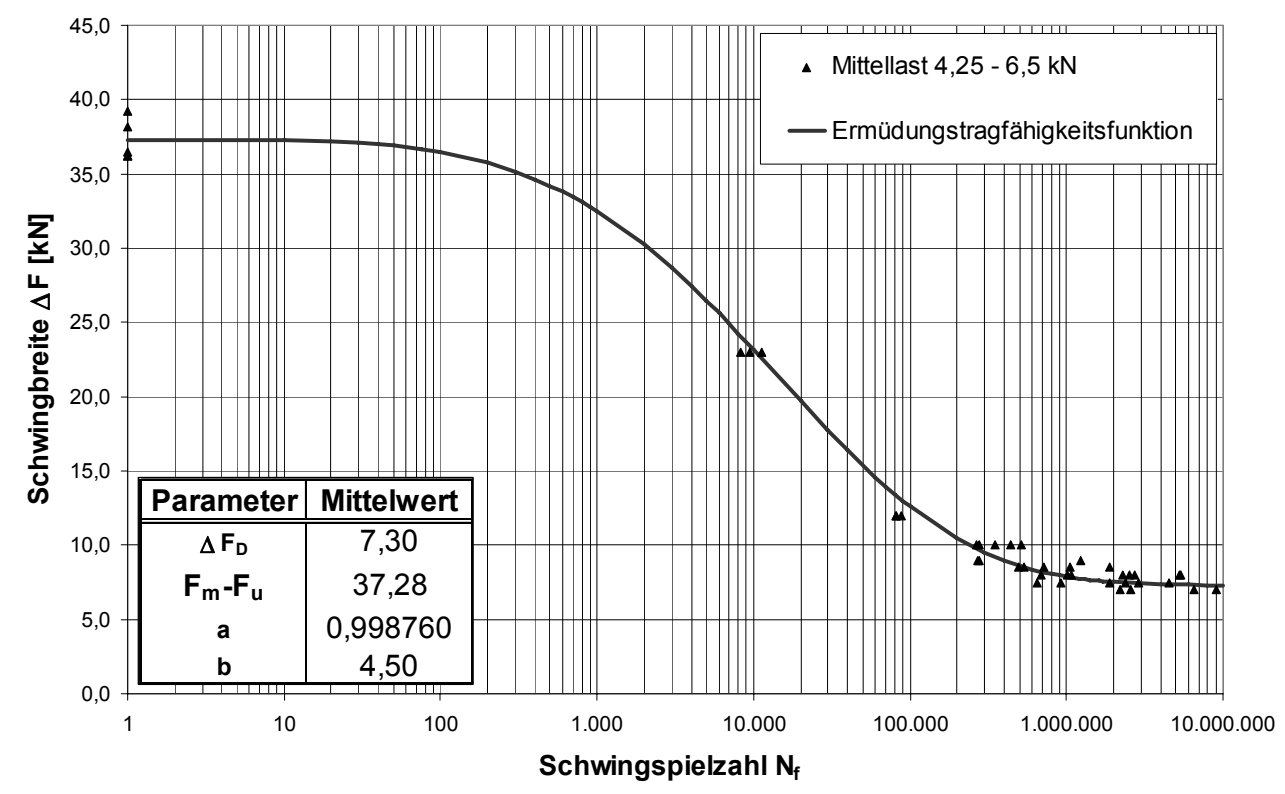

Bild 4.40 Ermüdungstragfähigkeit nach Gleichung (4.2) bezogen auf die Mittellasten

Dem Diagramm ist deutlich zu entnehmen, daß ab einer Schwingspielzahl von $1 \cdot 10^{6}$ die Kurve sehr auffällig abflacht und allmählich in den Bereich der Dauerfestigkeit übergeht. Die nach Gleichung (4.2) ermittelte rechnerische Dauerfestigkeit beträgt 7,3 $\mathrm{kN}$ und wird deutlich über $2 \cdot 10^{6}$ Schwingspielen erreicht. Die Ermüdungsfestigkeit bei $2 \cdot 10^{6}$ Schwingspielen im Vergleich beträgt $7,52 \mathrm{kN}$ und weist zur rechnerischen Ermüdungsfestigkeit eine Differenz von $0,22 \mathrm{kN}$ auf, die eine Reduzierung um 2,9\% bedeutet. Aufgrund dieser Erkenntnis ist die Ermittlung der ertragbaren Schwingbreite bei $2 \cdot 10^{6}$ Schwingspielen im Rahmen einer Sicherheitsbetrachtung als gerechtfertigt anzusehen. Eine Bemessungsschwingspielzahl von $2 \cdot 10^{6}$ wird aufgrund wirtschaftlicher und versuchstechnischer Aspekte zusätzlich im Rahmen dieser Arbeit empfohlen. In [6] wird gezeigt, daß die Dauerfestigkeit von Dübeln bei Stahlversagen ebenfalls bei $2 \cdot 10^{6}$ Schwingspielen nachgewiesen ist.

\subsubsection{Auswertemethodik}

Als Ergebnis von Ermüdungsversuchen unter periodischer Beanspruchung erhält man in Abhängigkeit von einer voreingestellten Schwingbreite $\Delta \mathrm{F}$ die zugehörige Versagensschwingspielzahl $\mathrm{N}_{\mathrm{f}}$. Werden nun mehrere Versuche auf mindestens drei Lastniveaus durchgeführt, so erhält man die in Bild 4.41 dargestellten funktionalen Verläufe der Schwingbreite (Mittelwert, 5\%-Quantil, 95\%-Quantil) in Abhängigkeit von der Schwingspielzahl im halblogarithmischen Maßstab. 


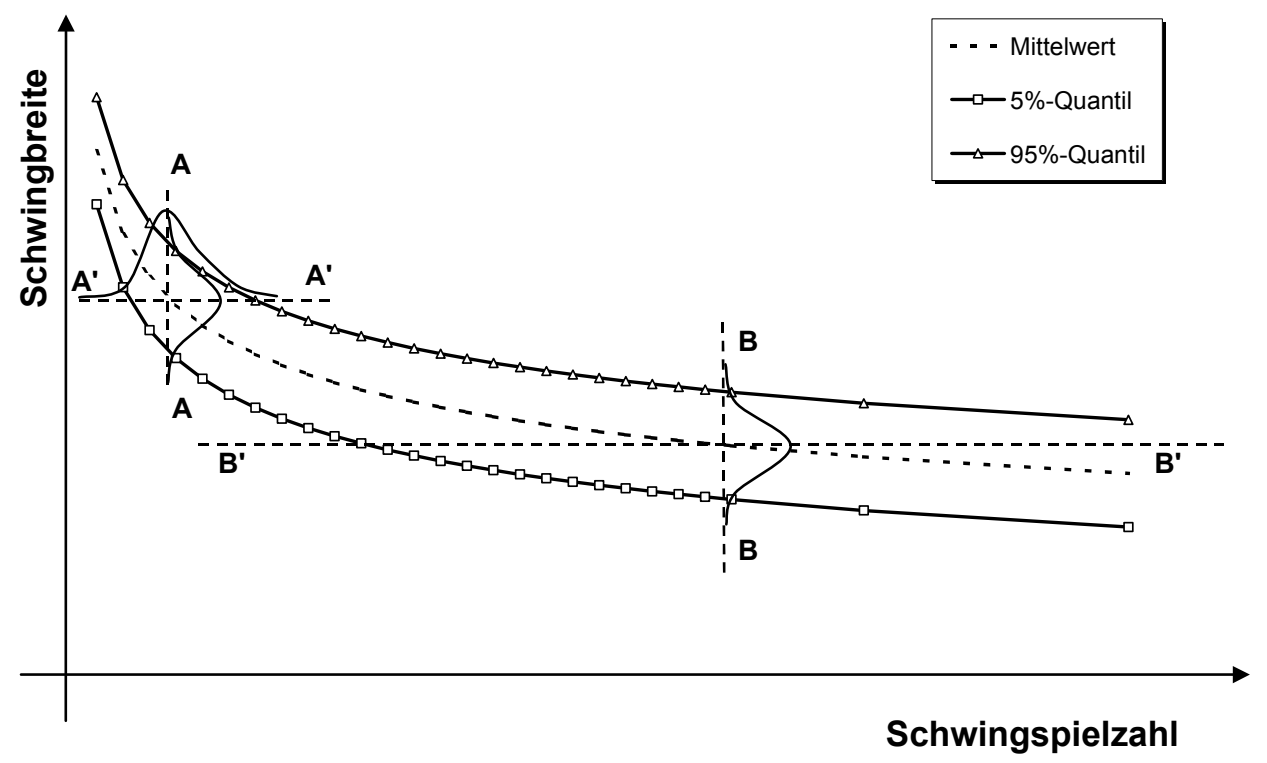

Bild 4.41 Verteilungen an unterschiedlichen Schnitten der Ermüdungstragfähigkeit [7]

In Bereichen der Zeitfestigkeit, in der die ertragbare Schwingbreite deutlich sinkt, diese sind der Schnitt A-A bzw. A'-A', führt die Auswertung der Versuchsdaten zu vergleichbaren Ergebnissen. In beiden Fällen können die Versuchsergebnisse ausreichend mit Hilfe eines geeigneten Verteilungstyps beschrieben werden. Im Übergangsbereich zur Dauerfestigkeit hingegen, im Schnitt B-B und B'-B', ergeben sich starke Unterschiede. Im Schnitt B'-B' zeigt sich eine Situation, in der die Beurteilung der Schwingspielzahl durch eine geeignete Verteilungsdichte nicht möglich ist. In diesem Schnitt gibt es zwar einen unteren Endpunkt, dieser ist das 5\%-Quantil, jedoch liegt der Schnittpunkt mit dem 95\%-Quantil unverhältnismäßig weit entfernt. Die Verteilungsdichte ist somit zur rechten Seite hin geöffnet. Gerade diese Tatsache erschwert es, eine sinnvolle Verteilung zur Beschreibung der Versuchsergebnisse anzunehmen und somit den charakteristischen Wert der Beanspruchbarkeit zu bestimmen. Diese Probleme ergeben sich bei der Betrachtung des Schnittes B-B nicht. Die zu wählende Verteilung besitzt sowohl eine untere als auch eine obere Begrenzung, so daß die Eindeutigkeit der Verteilung gegeben ist.

In Anlehnung an das bisherige Vorgehen bei der Auswertung von Schwingversuchen wird die Abhängigkeit zwischen der Schwingbreite $\Delta \mathrm{F}$ und der Schwingspielzahl $\mathrm{N}_{\mathrm{f}}$ bis zum Bruch betrachtet. Die Auswertung der Versuchsergebnisse erfolgt für den Schnitt B-B. Die Einzelergebnisse aus den Versuchen werden in einem Wöhlerdiagramm, im doppelt logarithmischen Maßstab, aufgetragen. Bei diesem Maßstab ergibt sich ein linearer Zusammenhang zwischen der Schwingbreite und Schwingspielzahl. Durch eine lineare Regressionsanalyse nach der Methode der „Minimierung der Fehlerquadrate" kann anschließend der funktionelle Zusammenhang zwischen $\mathrm{N}_{\mathrm{f}}$ und $\Delta \mathrm{F}$ angegeben werden. Die Schwingbreite $\Delta \mathrm{F}$ wird dabei als abhängige Variable betrachtet. Der funktionelle Zusammenhang in Form einer Regressions- bzw. Ausgleichsgeraden ist empirisch bestätigt und wird in Gleichung (4.3) angegeben: 
$\log \Delta F_{i}=a \cdot \log N_{f i}+b$

Soll die Schwingspielzahl $\mathrm{N}_{\mathrm{f}}$ als abhängige Variable definiert werden, so erhält man durch eine Umstellung folgenden Zusammenhang:

$\log N_{\text {fi }}=\frac{b}{a}-m \cdot \log \Delta F_{i}$

Im Eurocode 3 [37] wird folgende Gleichung für den funktionellen Zusammenhang zwischen der Schwingspielzahl und der Schwingbreite vorgegeben:

$\log \mathrm{N}_{\mathrm{fi}}=\log \mathrm{a}-\mathrm{m} \cdot \log \Delta \mathrm{F}_{\mathrm{i}}$

Vergleicht man nun Gleichung (4.4) und (4.5) miteinander, so unterscheiden sich beide Formen nur durch den konstanten Teil der Funktion. Für (loga) werden im Eurocode in Abhängigkeit vom Kerbfall und der Neigung an der betrachteten Schwingspielzahl unterschiedliche konstante Werte vorgegeben. Die Koeffizienten a und $b$ der Regressionsgeraden aus Gleichung (4.11) und (4.4) werden für eine Anzahl von $\mathrm{n}$ Versuchen wie folgt bestimmt:

$$
\begin{aligned}
& \mathrm{a}=\frac{\sum_{\mathrm{i}=1}^{\mathrm{n}}\left(\log \mathrm{N}_{\mathrm{fi}}-\log \mathrm{N}_{\mathrm{fm}}\right) \cdot\left(\log \Delta \mathrm{F}_{\mathrm{i}}-\log \Delta \mathrm{F}_{\mathrm{m}}\right)}{\sum_{\mathrm{i}=1}^{\mathrm{n}}\left(\log \mathrm{N}_{\mathrm{fi}}-\log \mathrm{N}_{\mathrm{fm}}\right)^{2}} \\
& \mathrm{~b}=\log \Delta \mathrm{F}_{\mathrm{m}}-\mathrm{a} \cdot \log \mathrm{N}_{\mathrm{m}}
\end{aligned}
$$

Dabei sind $\mathrm{N}_{\mathrm{fi}}$ und $\Delta \mathrm{F}_{\mathrm{i}}$ die jeweiligen Einzelwerte bzw. $\mathrm{N}_{\text {fim }}$ und $\Delta \mathrm{F}_{\mathrm{m}}$ die zugehörigen Mittelwerte bis zum Versagen. Der Kehrwert des Koeffizienten a gibt die Steigung $\mathrm{m}$ der Regressionsgeraden an.

$$
\mathrm{m}=\frac{1}{\mathrm{a}}
$$

Die Bestimmung des Mittelwertes der ertragbaren Schwingbreite $\Delta \mathrm{F}$, dessen Standardabweichung $\sigma$ und deren Variationskoeffizienten V erfolgt bei der Bemessungsschwingspielzahl von $2 \cdot 10^{6}$. Dieser Wert ist die Basis für die Ermittlung der zulässigen Schwingbreite nach der derzeitigen Bemessung. Hierzu werden die erzielten Versuchswerte $\Delta \mathrm{F}_{\mathrm{i}}$ und $\mathrm{N}_{\mathrm{fi}}$ über Parallelen zur ermittelten Regressionsgeraden auf die Bemessungsschwingspielzahl von $2 \cdot 10^{6}$ projiziert. Unter Berücksichtigung der Gleichung (4.3) mit dem Faktor b aus Gleichung (4.7), welcher für eine bestimmte Schwingspielzahl $\mathrm{N}_{\mathrm{fi}}$ und Schwingbreite $\Delta \mathrm{F}_{\mathrm{i}}$ ermittelt wird, kann der Wert der ertragbaren Schwingbreite bei der maßgebenden Bemessungsschwingspielzahl von $2 \cdot 10^{6}$ gemäß Gleichung (4.9) errechnet werden: 
$\Delta \mathrm{F}_{\mathrm{i}}\left(2 \cdot 10^{6}\right)=10^{\log \Delta \mathrm{F}_{\mathrm{i}}-\mathrm{a} \cdot \log \mathrm{N}_{\mathrm{i}}+\mathrm{a} \cdot \log \left(2 \cdot 10^{6}\right)}$

In Bild 4.42 ist die aktuelle Auswertemethodik schematisch veranschaulicht.

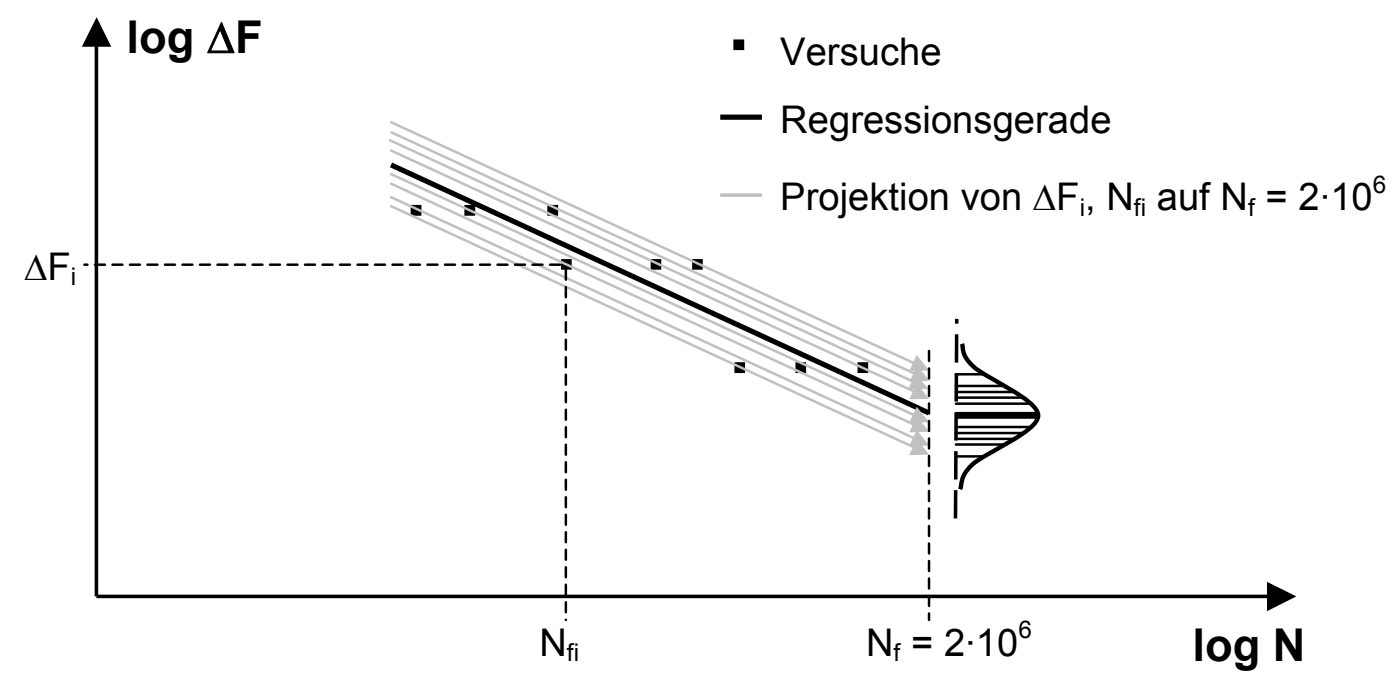

Bild 4.42 Auswertemethodik für Schwingversuche bei $2 \cdot 10^{6}$ Schwingspielen

Es ergibt sich eine Häufigkeitsverteilung der ertragbaren Schwingbreite bei $2 \cdot 10^{6}$, die mit statistischen Methoden behandelt werden kann. Die beschriebene Auswertemethode ermöglicht eine vergleichsweise einfache Ermittlung der Fraktile für die Schwingbreite $\Delta \mathrm{F}$. Sie liefert bei üblichen Streuungen Ergebnisse, die nicht wesentlich abweichen von der Methode, bei welcher die Schwingspielzahl N als abhängige Variable und nicht wie hier die Schwingbreite $\Delta \mathrm{F}$ betrachtet wird. Die beschriebene Auswertemethodik ist bei der Auswertung von Schwingversuchen üblich und wird bei vergleichbaren Versuchen in der Befestigungstechnik durchgängig angewendet.

Eine wichtige Fragestellung bei der oben beschriebenen Auswertemethodik ergibt sich hinsichtlich der Empfindlichkeit der Steigung $\mathrm{m}$ der Regressionsgeraden nach Gleichung (4.8) und dessen Auswirkung auf die ertragbare Schwingbreite bei der Bemessungsschwingspielzahl von $2 \cdot 10^{6}$. Die Steigung der Regressionsgeraden hängt wesentlich von dem Bereich ab, in dem sich die aus den Versuchen resultierenden Schwingspielzahlen $\mathrm{N}_{\mathrm{f}}$ befinden. Je umfassender der Zeitfestigkeitsbereich abgebildet wird, desto genauer stimmt die Steigung der Regressionsgeraden mit der Steigung der Wöhlerlinie überein. Liegen die Schwingspielzahlen aus den Versuchen jedoch in unmittelbarer Nähe der Bemessungsschwingspielzahl, so ist die resultierende Steigung nicht repräsentativ für die Wöhlerlinie. Um den Einfluß der Schwingspielzahlen auf die Steigung der Regressionsgeraden zu verdeutlichen, sind Versuche auf 5 unterschiedlichen Lastniveaus zwischen $5 \mathrm{kN}$ (etwa $12 \%$ der maximalen Zugkraft) und 16 $\mathrm{kN}$ (etwa $40 \%$ der maximalen Zugkraft) im Zeitfestigkeitsbereich durchgeführt worden. Die erzielten Schwingspielzahlen erstrecken sich dabei von $5 \cdot 10^{4}$ bis etwa $3 \cdot 10^{6}$. Die Versuchsergebnisse sind in Tabelle 4.6 dargestellt. 
Tabelle 4.6 Versuche zur Empfindlichkeit der Steigung $m$ der Regressionsgeraden

\begin{tabular}{|c|c|c|c|c|c|}
\hline & \multicolumn{5}{|c|}{ Schwingbreite $\Delta F[k N]$} \\
\hline & 5 & 5,5 & 6 & 9 & 16 \\
\hline \multirow{9}{*}{ 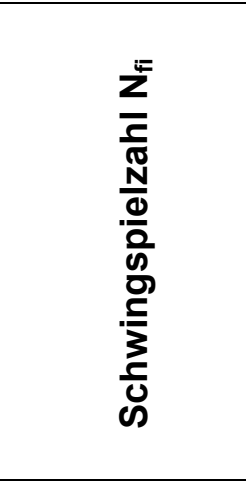 } & 1.850 .478 & 1.266 .907 & 975.178 & 304.869 & 50.000 \\
\hline & 1.450 .366 & 1.688 .024 & 862.635 & 300.085 & 54.085 \\
\hline & 2.069 .910 & 1.191 .057 & 989.042 & 337.810 & 50.685 \\
\hline & 2.238 .483 & 1.119 .242 & 1.122 .347 & 324.497 & 52.143 \\
\hline & 2.374 .720 & 1.424 .111 & 924.148 & 333.649 & 50.146 \\
\hline & 1.895 .100 & 1.759 .529 & 1.229 .577 & 244.362 & - \\
\hline & 2.023 .458 & 1.634 .384 & 1.004 .025 & 285.182 & - \\
\hline & 2.284 .641 & 1.239 .733 & 963.969 & 272.851 & - \\
\hline & 2.914 .178 & 1.672 .775 & 1.122 .849 & 358.977 & - \\
\hline Mittelwert & 2.122 .370 & 1.443 .974 & 1.021 .530 & 306.920 & 51.412 \\
\hline Standardabw. & 406.226 & 247.644 & 114.710 & 35.856 & 1.718 \\
\hline
\end{tabular}

Es werden insgesamt drei Grenzfälle betrachtet, aus denen dann die Steigungen der Regressionsgeraden abgeleitet werden. Die erste Regressionsgerade (Regression 1) berücksichtigt alle vorhandenen Versuchsergebnisse im Zeitfestigkeitsbereich aus Tabelle 4.6. Für die Regression 2 werden die erzielten Schwingspielzahlen bei der Schwingbreite von $\Delta \mathrm{F}=16 \mathrm{kN}$ (letzte Spalte in Tabelle 4.6) nicht in die Auswertung mit einbezogen. Bei der Regression 3 werden zusätzlich die erzielten Schwingspiele bei der Schwingbreite von $9 \mathrm{kN}$ außer Acht gelassen und befinden sich somit in unmittelbarer Nähe der Bemessungsschwingspielzahl. Der Verlauf der Regressionsgeraden ist in Bild 4.43 dargestellt.

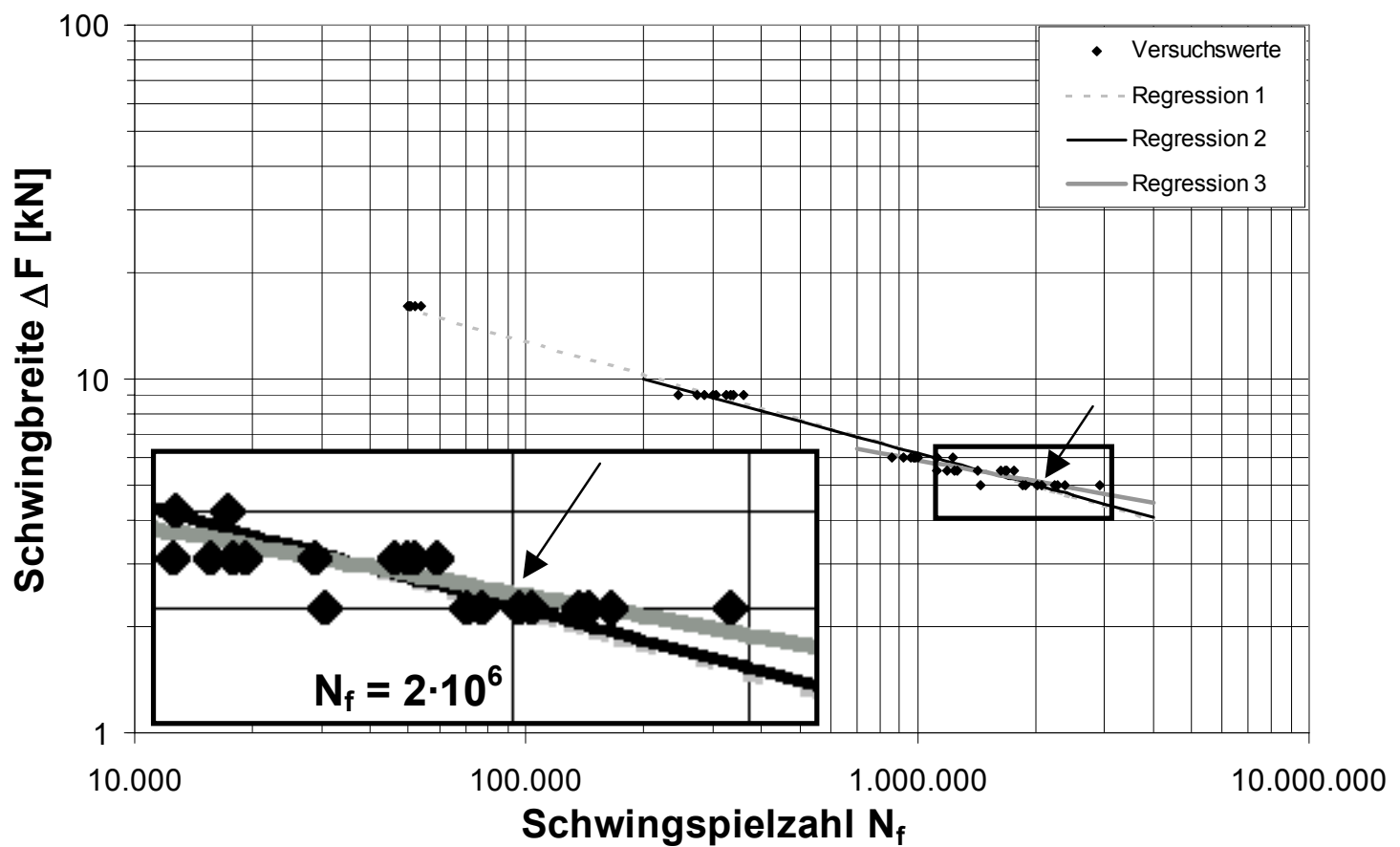

Bild 4.43 Regressionsgeraden in Abhängigkeit von der Schwingbreite $\Delta \mathrm{F}$ 
Aus dem Bild 4.43 geht hervor, da $\beta$ die Steigung der Regressionsgeraden für die Regression 3 im Vergleich zu den Steigungen der Regression 1 und Regression 2 deutlich kleiner ist Die charakteristischen Zahlenwerte der Versuchsergebnisse können der Tabelle 4.7 entnommen werden.

Tabelle 4.7 Parameter und Ergebnisse der Regressionsrechnung

\begin{tabular}{|c|c|c|c|}
\hline & Regression 1 & Regression 2 & Regression 3 \\
\hline & \multicolumn{3}{|c|}{ Schwingbreite $\Delta \mathrm{F}_{\mathrm{i}}[\mathrm{kN}]$} \\
\hline & $5,0-16,0$ & $5,0-9,0$ & $5,0-6,0$ \\
\hline Anzahl $\mathbf{n}$ & 41 & 36 & 27 \\
\hline $\mathbf{N}_{\mathrm{fi}}$ & $5 \cdot 10^{4}-3 \cdot 10^{6}$ & $2 \cdot 10^{5}-3 \cdot 10^{6}$ & $1 \cdot 10^{6}-3 \cdot 10^{6}$ \\
\hline Faktor a & $-0,32$ & $-0,30$ & $-0,20$ \\
\hline Faktor b & 2,67 & 2,58 & 1,96 \\
\hline Steigung $\mathbf{m}$ & 3,19 & 3,35 & 5,03 \\
\hline Abweichung $\Delta_{\mathrm{m}}$ & 1,00 & 1,05 & 1,58 \\
\hline$\Delta \mathrm{F}\left(2 \cdot 10^{6}\right)[\mathrm{kN}]$ & 4,98 & 5,01 & 5,14 \\
\hline Abweichung $\Delta_{\Delta \mathrm{F}}$ & 1,00 & 1,01 & 1,03 \\
\hline
\end{tabular}

Es ist zu erkennen, daß mit Abnahme der einwirkenden Schwingbreite $\Delta F_{i}$ die Steigung $\mathrm{m}$ der Regressionsgeraden deutlich zunimmt und der Verlauf somit flacher wird. Die ertragbare Schwingbreite $\Delta \mathrm{F}$ an der maßgebenden Bemessungsschwingspielzahl von $2 \cdot 10^{6}$ ebenfalls nimmt ab. Ein deutlicher Anstieg der Steigung der Regressionsgeraden um $58 \%$ führt $\mathrm{zu}$ einer eher unwesentlichen Zunahme der Schwingbreite an der maßgebenden Bemessungsschwingspielzahl um etwa 3\%.

Die Ursache für die stark unterschiedlichen Steigungen $\mathrm{m}$ der Regressionsgeraden liegt darin, daß die Versuche im Rahmen der Untersuchungen hauptsächlich in unmittelbarer Nähe der Bemessungsschwingspielzahl von $2 \cdot 10^{6}$ durchgeführt worden sind. Die charakteristischen Eigenschaften entsprechen somit der Regression 3 aus Tabelle 4.7. Um jedoch eine abgesicherte Aussage über die Steigung m der Wöhlerlinie für den gesamten Zeitfestigkeitsbereich treffen zu können, sind zusätzliche experimentelle Untersuchungen im Übergangsbereich zur Kurzzeitfestigkeit $\left(\mathrm{N}=1 \cdot 10^{4}\right)$ durchzuführen. In diesem Fall entsprechen die Eigenschaften der Wöhlerlinie der Regression 1 aus Tabelle 4.7. Es resultieren somit geringere ertragbare Schwingbreiten an der maßgebenden Bemessungsschwingspielzahl, welche jedoch im Vergleich zu den Ergebnissen der Regression 3 auf der sicheren Seite liegen. Dem Verlauf der Wöhlerlinie kommt im Rahmen der Untersuchungen keine wesentliche Bedeutung zu, da die Bestimmung der ermüdungsrelevanten Dauerbeanspruchbarkeit (ertragbare Schwingbreite) im Vordergrund steht. Je näher die Versuchsergebnisse um den Bemessungsbereich liegen, desto genauer ist die Ermittlung der ertragbaren Schwingbreite an der maßgebenden Bemessungsschwingspielzahl nach der in Bild 4.42 dargestellten Auswertemethodik. 
Eine weitere Fragestellung ergibt sich bezüglich der geeigneten Beschreibung der Häufigkeitsverteilung der ertragbaren Schwingbreite an der maßgebenden Bemessungsschwingspielzahl durch geeignete Verteilungstypen. Die in Bild 4.42 aufgezeigte Häufigkeitsverteilung ist von besonderer Wichtigkeit für eine statistische Beurteilung der Schwingbreite. Erst eine sinnvolle Abbildung der Versuchsergebnisse liefert auch eine zuverlässige Aussage über die Genauigkeit des charakteristischen Wertes, der anschließend die Grundlage für die Sicherheitsbetrachtung bildet. Bei der Annäherung von Versuchsergebnissen an Verteilungsfunktionen ist somit die Analogie der statistischen Theorie zum vorliegenden physikalischen Problem von größter Bedeutung [44], [107].

Zur Wahl des Verteilungstyps der Ermüdungsfestigkeit sind im Rahmen dieser Arbeit folgende Typen aus [64] untersucht worden:

- Normalverteilung

- Logarithmische Normalverteilung

- Weibullverteilung

- Gammaverteilung

- Gumbelverteilung

Keine der genannten Verteilungen besitzt einen entscheidenden Vorteil den anderen gegenüber, so daß eine Verteilungsfunktion vorrangig Anwendung finden kann. Zur Einschätzung der Anpassungsfähigkeit der Versuchsergebnisse an eine der oben genannten Verteilungstypen, wurde ein Programm auf der Basis der $X^{2}$-Verteilung in Anlehnung an [1], [2], der $X^{2}$-Test durchgeführt. Damit gelang es, eine Beurteilung der Anpassungsfähigkeit eines gewählten Verteilungstyps vorzunehmen. Eine weitere Alternative zum $X^{2}$-Test bildet der Anpassungstest nach Kolmogorow \& Smirnow [68], welcher zusätzlich in einigen Fällen angewendet wurde. Als Ergebnis dieser Untersuchungen wurde ermittelt, daß sowohl die logarithmische Normalverteilung, als auch die 3-parametrige Weibullverteilung [121] den Anpassungstest auf einem hohen Signifikanzniveau von $5 \%$ erfüllen und daher eine geeignete Beschreibung der Schwingbreite bei einer Schwingspielzahl von $2 \cdot 10^{6}$ gewährleisten. Der Nachteil der Weibullverteilung besteht jedoch darin, daß der zur Absicherung der experimentellen Daten durchzuführende statistische Aufwand enorm steigt. Daher wird in weiteren Untersuchungen in Kapitel 5, aufgrund der relativ unkomplizierten Handhabung, die Standard- bzw. logarithmische Normalverteilung als Grundlage zur Festlegung der charakteristischen Größen herangezogen. 


\subsubsection{Tragverhalten unter zentrischer Zugbeanspruchung}

\subsubsection{Statische Versuchsreihe}

Zur Ermittlung der statischen Tragfähigkeit der Ankerschienen des Typs 38/23 St sind statische Versuche an den in Bild 4.3 dargestellten Profilabschnitten im einbetonierten und nicht einbetonierten Zustand durchgeführt worden. Eine Zusammenstellung der Ergebnisse beinhaltet Tabelle 4.8.

Tabelle 4.8 Mittelwerte der zentrischen Zugbeanspruchung $\left(0^{\circ}\right)$

\begin{tabular}{|c|c|c|c|}
\hline \multirow{3}{*}{ Serie } & \multicolumn{3}{|c|}{ Beanspruchung N in zentrischer Zugrichtung } \\
\cline { 2 - 4 } & nicht einbetoniert & \multicolumn{2}{|c|}{ einbetoniert } \\
\cline { 2 - 4 } & $100 \%$ Vorspannung & $100 \%$ Vorspannung \\
\cline { 2 - 4 } & $\mathrm{N}_{\mathrm{u}}$ & $\mathrm{N}_{\mathrm{u}}$ & $\mathrm{f}_{\mathrm{cm}, \text { cube }}$ \\
\cline { 2 - 4 } & {$[\mathrm{kN}]$} & {$[\mathrm{kN}]$} & {$\left[\mathrm{N} / \mathrm{mm}^{2}\right]$} \\
\hline $\mathrm{A}(25 / 50)$ & --- & 34,47 & 34,15 \\
\hline $\mathrm{A}(50 / 50)$ & --- & 36,52 & 34,00 \\
\hline $\mathrm{B}(25 / 50)$ & --- & 41,79 & 35,80 \\
\hline $\mathrm{C}(25 / 50)$ & 37,26 & --- & --- \\
\hline
\end{tabular}

Die Materialfestigkeiten der jeweiligen Ankerschienen können Tabelle 4.2 entnommen werden. In der Zulassung [21], [23] der Ankerschiene 38/23 St wird bei einer zulässigen Einzellast von $12 \mathrm{kN}$ eine Mindestbruchlast von $30 \mathrm{kN}$ gefordert. Aus Tabelle 4.8 geht hervor, daß diese Anforderung stets erfüllt ist. Bei allen Versuchen war Stahlversagen die maßgebende Versagensursache. Die typischen Versagensbilder können dem Abschnitt 4.5.1 entnommen werden.

\subsubsection{Schwingversuche}

Es wurden Einstufenversuche an Ankerschienen 38/23 St mit Endüberständen von $25 / 50$ und 50/50 $\mathrm{mm}$ (Variante 25/50 und 50/50, siehe Bild 4.3) sowohl im einbetonierten (B), als auch im nicht einbetonierten Zustand (OB) bei konstanter Ober- und Unterlast geprüft. Zur Anwendung kamen Profile mit den in Tabelle 4.2 aufgeführten leicht unterschiedlichen Festigkeiten, die aufgrund von Streuungen aus der Herstellung resultieren. Bei periodischer Beanspruchung zwischen zwei konstanten Grenzen hat die Festigkeit keinen Einfluß auf die Tragfähigkeit (siehe Kapitel 3). Dieses kann in [72] ebenfalls nachvollzogen werden. Die Mittelwerte der Betondruckfestigkeiten bei Verwendung von Ankerschienen im einbetonierten Zustand können der Tabelle 4.5 entnommen werden. Eine Übersicht der erzielten Ergebnisse wird im Anhang dargestellt. Gegenstand der Untersuchungen war es, mögliche Einflußfaktoren auf das Tragverhalten zu verifizieren. Es sollte der Frage nachgegangen werden, welchen Einfluß unterschiedliche Endüberstände und der umgebende Beton auf die ruhende und nichtruhende Tragfähigkeit haben. Weiterhin werden die Einflüsse des Lastniveaus näher betrachtet. Zur Klärung dieser Fragen wurden entsprechende Versuche durchge- 
führt. Im folgenden sind die Einflußfaktoren auf das Ermüdungstragverhalten gesondert aufgelistet:

- Variation der Endüberstände der Ankerschienen

- umgebender Beton

- unterschiedliche Lastniveaus

Eine Kombination dieser Einflußfaktoren wird ebenfalls betrachtet.

\section{Einfluß bei Variation der Endüberstände}

Die Bestimmung der zulässigen Schwingbreite von Ankerschienen im Rahmen der bauaufsichtlichen Zulassung erfolgt auf der Grundlage von zentrischen Zugversuchen an Ankerschienen mit Endüberständen von 50/50 mm im nicht einbetonierten $\mathrm{Zu}-$ stand. Laut bauaufsichtlichen Zulassungen [21], [23] sind jedoch nur Einbauzustände mit Lasteinleitungen im Endabstand von $25 \mathrm{~mm}$ vom Profilende möglich. In diesem Zustand wird die Last direkt über dem Bolzen in den Untergrund eingeleitet, der den ungünstigsten Beanspruchungsfall darstellt. Untersucht wurden zunächst Profile der Serie A (siehe Tabelle 4.2) in der Ausführung 25/50 und 50/50. Die Versuche erfolgten unter konstanter Oberlast von $17 \mathrm{kN}$ und Variation der Schwingbreite. Die Schwingspielzahlen bis zum Bruch liegen je nach Spannungsniveau zwischen $2 \cdot 10^{4}$ und $4 \cdot 10^{6}$. Dieses Intervall ist folglich durch Versuche abgesichert, und es ist möglich, den Bereich der Zeitfestigkeit ausreichend gut abzubilden. Aufgrund einer übersichtlicheren Darstellungsweise wird die Darstellung nach DIN 50100 [29] bevorzugt. Dabei wird die Schwingspielzahl auf der Abszisse im logarithmischen Maßstab und die Schwingbreite auf der Ordinate im arithmetischen bzw. metrisch geteilten Maßstab aufgetragen.

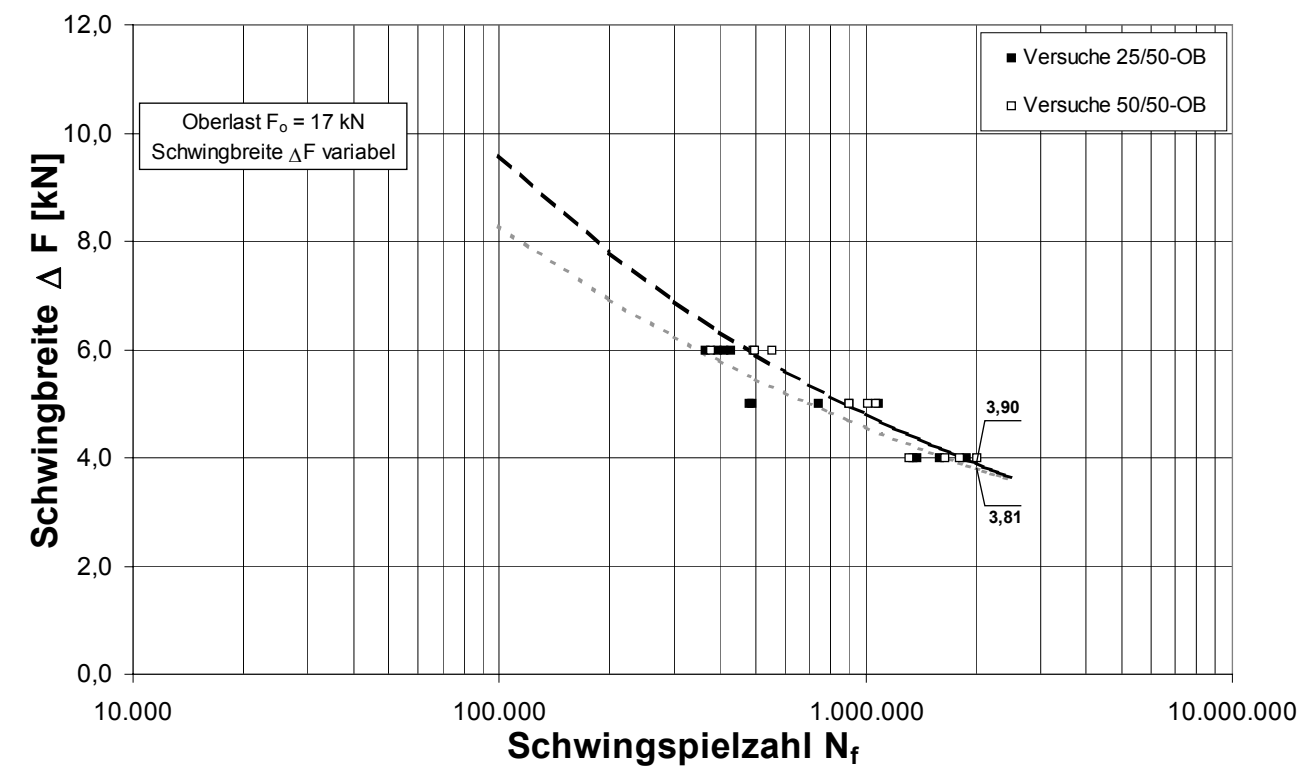

Bild 4.44 Ermüdungstragfähigkeiten der Serie A 
Als Versagen wurde eine sternförmige Rißausbildung im Schienenrücken mit Kombination von Längsrissen im Übergangsbereich des Schienenrückens zum Schienenschenkel beobachtet, welches in Bild 4.22 und Bild 4.39 unter Fall 1 und Fall 3 schematisch dargestellt ist. Es ist ersichtlich, daß die eingezeichneten Verläufe (als Kurven dargestellt) der Ermüdungstragfähigkeiten bei einer Schwingspielzahl von $2 \cdot 10^{6}$ Schwingspielen keine nennenswerten Unterschiede in der Ermüdungsfestigkeit zwischen der Variante 25/50 und 50/50 zeigen.

Tabelle 4.9 Ergebnisse an nicht einbetonierten Ankerschienen der Serie A

\begin{tabular}{|c|c|c|c|c|c|c|c|}
\hline Variante & $\begin{array}{c}\text { Mittelw. } \Delta \mathrm{F} \\
\text { bei } 2 \cdot 10^{6} \\
{[\mathrm{kN}]}\end{array}$ & $\begin{array}{c}\sigma \\
{[\mathrm{kN}]}\end{array}$ & $\begin{array}{l}\mathrm{V} \\
{[\%]}\end{array}$ & $\begin{array}{l}\text { Steigung } \mathrm{m} \\
\text { Regression }\end{array}$ & Anzahl n & $\begin{array}{c}\text { Schwingbr. } \\
\Delta \mathrm{F}_{\mathrm{i}} \\
{[\mathrm{kN}]}\end{array}$ & $\begin{array}{c}\text { Anhang } \\
\text { Tab. }\end{array}$ \\
\hline $25 / 50-\mathrm{OB}$ & 3,81 & 0,24 & 6,27 & 3,85 & 12 & $4,0-6,0$ & A2 \\
\hline $50 / 50-O B$ & 3,90 & 0,18 & 4,68 & 3,32 & 12 & $4,0-6,0$ & A9 \\
\hline Faktor $\mathrm{f}_{\mathrm{OB}}$ & 1,02 & & & & & & \\
\hline
\end{tabular}

Der Vergleichsfaktor $\mathrm{f}_{\mathrm{OB}}$ des nicht einbetonierten Zustandes, berechnet als Quotient der mittleren Ermüdungsfestigkeiten bei einer Schwingspielzahl von $2 \cdot 10^{6}$, zeigt eine relative Erhöhung um etwa $2 \%$.

Der oben beobachtete Versagensfall 3 deutet darauf hin, daß an der Verbindung zwischen Ankerfuß und Schienenrücken eine relativ hohe Kerbwirkungen vorhanden sein muß, welche in diesem Fall die ertragbare Schwingfestigkeit deutlich reduzieren kann. Die Ursache hierfür kann u.a. in der Fertigung liegen. Zur Klärung dieses Problems sind weitere Versuche an nicht einbetonierten Ankerschienen (Variante 25/50 und 50/50) der Serie C unter den gleichen Bedingungen durchgeführt worden. Im Diagramm in Bild 4.45 werden die Verläufe der Ermüdungstragfähigkeit für die Variante 25/50-OB gegenübergestellt.

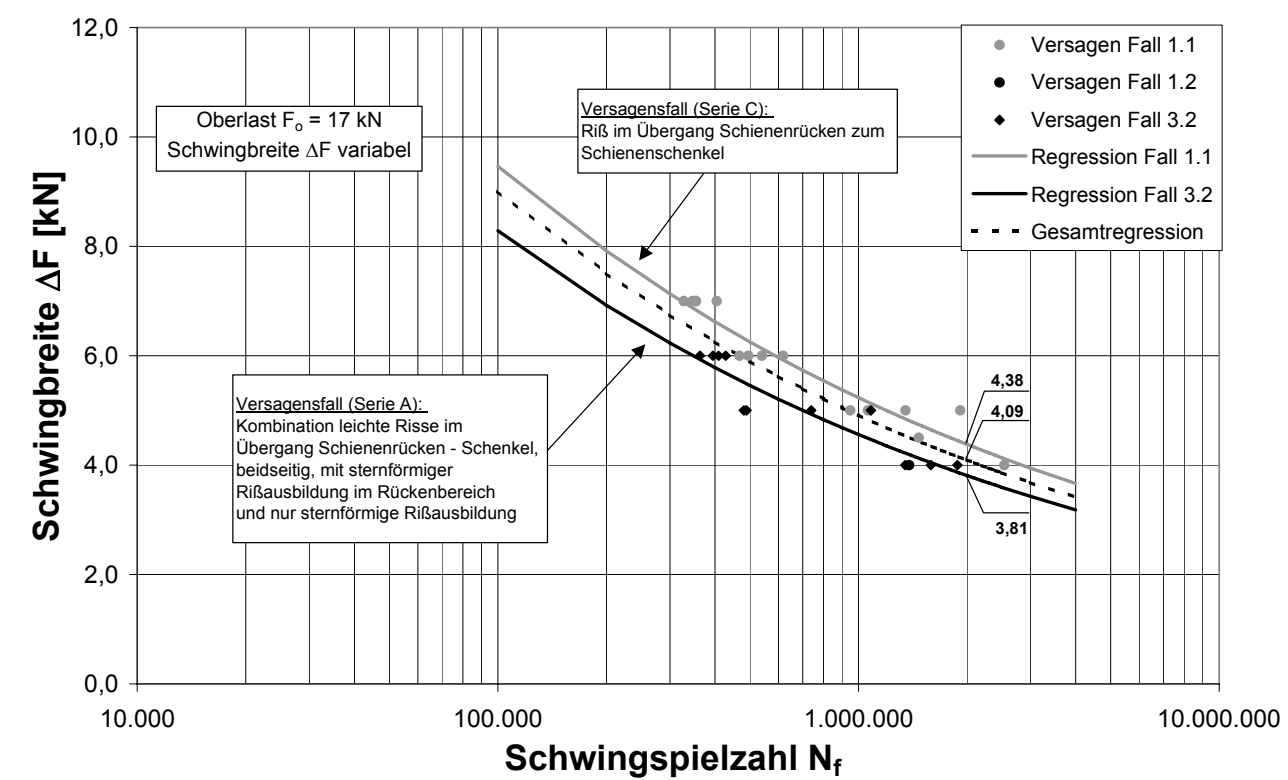

Bild 4.45 Ermüdungstragfähigkeiten der Serie A im Vergleich zur Serie C (25/50-OB) 
Der Streubereich der Schwingspielzahlen bis zum Bruch ist vergleichbar zur Serie A. Lediglich bei Schwingversuchen mit der Variante 50/50 der Serie C erstreckte sich der Bereich der Schwingspielzahl zwischen $5 \cdot 10^{4}$ und $3 \cdot 10^{6}$. Die Abmessungen der verwendeten Ankerschienen unterlagen ebenfalls dem gleichen Streubereich, so daß keine Einflüsse aus geometrischen Imperfektionen zu erwarten waren. In [73] ist der Festigkeitseinfluß auf die Ermüdungsfestigkeit ausgeschlossen worden.

Die Versagensart der Serie A unterscheidet sich grundsätzlich von den Versagenserscheinungen in der Serie C. In der letztgenannten Serie treten ausschließlich einseitige Rißerscheinungen im Übergangsbereich vom Schienenrücken zum Schienenschenkel (im Biegeradius) auf. Risse im Rückenbereich, ausgehend vom Ankerfuß bzw. Brüche im Ankerfuß, sind nicht beobachtet worden. Da die Ankerschiene als gekerbtes Befestigungselement angesehen wird, sind die unterschiedlichen Versagensarten nicht auf den Festigkeitsunterschied zurückzuführen. Vielmehr bestätigen die Versagenserscheinungen die Folge einer fertigungstechnischen Ursache.

Auch in Hinblick auf die Ermüdungstragfähigkeiten zeigen sich mit unterschiedlichen Versagensarten ebenfalls unterschiedliche Beträge der ertragbaren Schwingbreiten bei $2 \cdot 10^{6}$ Schwingspielen. Hier beträgt der Mittelwert der ertragbaren Schwingbreite 4,38 $\mathrm{kN}$ für die Serie C und ist somit um etwa $15 \%$ höher als der Wert an der gleichen Stelle für Serie A. Ein Vergleich der Variante 50/50-OB in Bild 4.46 zeigt prinzipiell ähnliche Relationen.

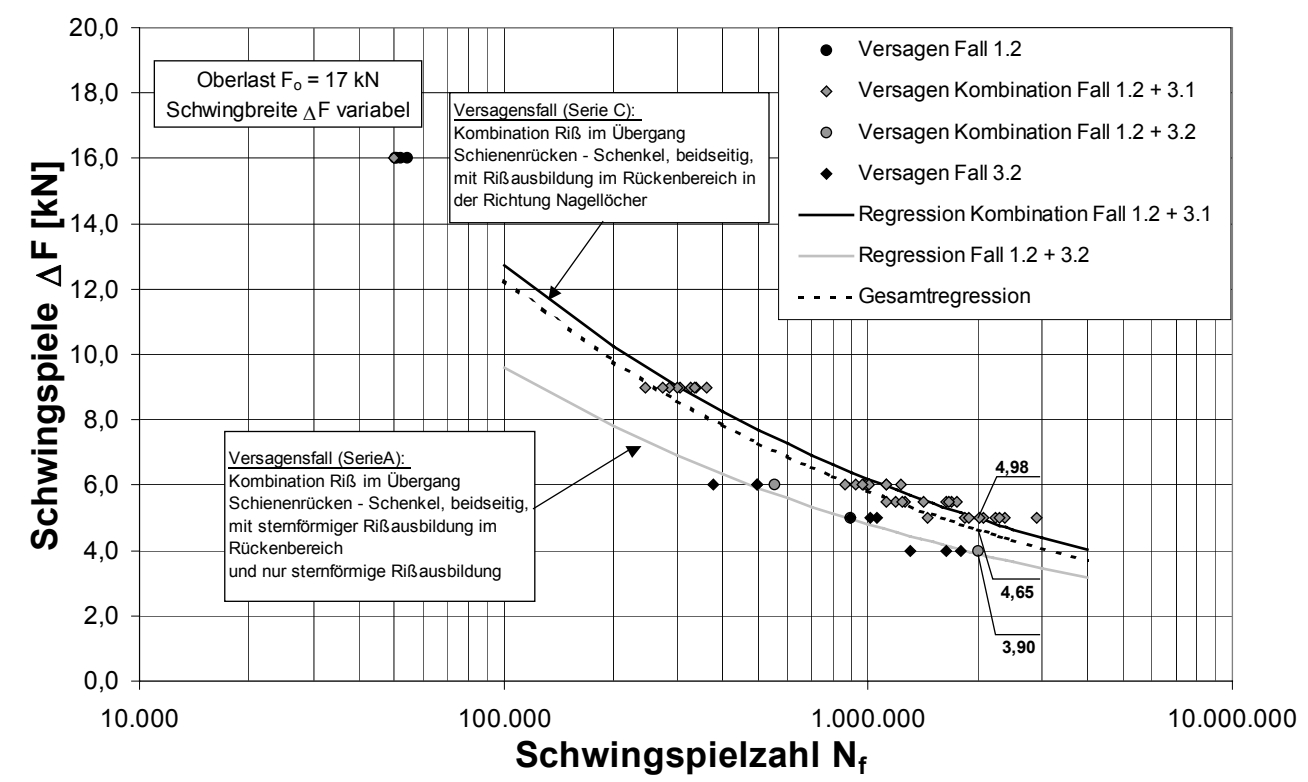

Bild 4.46 Ermüdungstragfähigkeiten der Serie A im Vergleich zur Serie C (50/50-OB)

Die ertragbaren Schwingbreiten der Serie C liegen bei 2 $10^{6}$ Schwingspielen mit 4,98 $\mathrm{kN}$ stets oberhalb der Ergebnisse der Serie A mit 3,9 kN. Führt man eine gemeinsame Auswertung beider Serien durch, dann ergibt sich ein Wert von 4,65 kN. Das Verhältnis der Mittelwerte der Ermüdungsfestigkeiten von Serie A und Serie B beträgt 1,28. Somit ist die ertragbare Schwingbreite der Serie C um ca. 28 \% höher. Wie bereits bei der Variante 25/50 sind auch hierbei unterschiedliche Versagensfälle zu beobachten. 
Als Hauptversagen in der Serie $\mathrm{C}$ trat eine Kombination von beidseitigen Rissen im Übergangsbereich des Schienenrückens zum Schienenschenkel (Fall 1.2) auf, welche durch leichte Rißausbildung im Rückenbereich der Ankerschienen, ausgehend vom Ankerfuß in Richtung der Nagellöcher (Fall 3.1), begleitet wurde (siehe Abschnitt 4.5.2.1).

Tabelle 4.10 Ergebnisse an nicht einbetonierten Ankerschienen der Serie C

\begin{tabular}{|c|c|c|c|c|c|c|c|}
\hline Variante & $\begin{array}{c}\text { Mittelw. } \Delta \mathrm{F} \\
\text { bei } 2 \cdot 10^{6} \\
{[\mathrm{kN}]}\end{array}$ & $\begin{array}{c}\sigma \\
{[\mathrm{kN}]} \\
\end{array}$ & $\begin{array}{l}\mathrm{V} \\
{[\%]} \\
\end{array}$ & $\begin{array}{l}\text { Steigung m } \\
\text { Regression }\end{array}$ & Anzahl n & $\begin{array}{c}\text { Schwingbr. } \\
\qquad \begin{array}{c}\Delta \mathrm{F}_{\mathrm{i}} \\
{[\mathrm{kN}]}\end{array}\end{array}$ & $\begin{array}{c}\text { Anhang } \\
\text { Tab. }\end{array}$ \\
\hline $25 / 50-\mathrm{OB}$ & 4,38 & 0,22 & 5,08 & 3,89 & 15 & $4,0-7,0$ & A3 \\
\hline $50 / 50-\mathrm{OB}$ & 4,98 & 0,23 & 4,62 & 3,19 & 41 & $5,0-16,0$ & A10 \\
\hline Faktor $\mathrm{f}_{\mathrm{OB}}$ & 1,14 & & & & & & \\
\hline
\end{tabular}

Die Tabelle 4.10 bestätigt den Trend des Faktors $f_{O B}$ im Vergleich zur Serie A. Bei dieser Versuchsserie, die durch eine grundsätzlich andere Versagensart im Vergleich zur Serie A gekennzeichnet ist, ergibt sich eine relative Erhöhung der Schwingbreite von etwa $14 \%$.

Versuche der Serie D, mit dem Profil JXA-W, bestätigen die erzielten Erkenntnisse bei vergleichbaren Versagensarten. Der Mittelwert der Versuchsergebnisse bei $2 \cdot 10^{6}$ Schwingspielen der Variante $25 / 50$ beträgt 4,78 kN und 5,36 kN für die Ausführung 50/50 (siehe Tabelle 4.11). Dies führt zu einer Erhöhung des Mittelwertes um $12 \%$, welcher im gleichen Rahmen wie die Ergebnisse der Serie C liegt (zum Vergleich siehe Tabelle 4.10).

Tabelle 4.11 Ergebnisse an nicht einbetonierten Ankerschienen der Serie D

\begin{tabular}{|c|c|c|c|c|c|c|c|}
\hline Variante & $\begin{array}{c}\text { Mittelw. } \Delta \mathrm{F} \\
\text { bei } 2 \cdot 10^{6} \\
{[\mathrm{kN}]}\end{array}$ & $\begin{array}{c}\sigma \\
{[\mathrm{kN}]} \\
\end{array}$ & $\begin{array}{l}\mathrm{V} \\
{[\%]} \\
\end{array}$ & $\begin{array}{l}\text { Steigung } \mathrm{m} \\
\text { Regression }\end{array}$ & Anzahl n & $\begin{array}{c}\text { Schwingbr. } \\
\qquad \mathrm{F}_{\mathrm{i}} \\
{[\mathrm{kN}]}\end{array}$ & $\begin{array}{c}\text { Anhang } \\
\text { Tab. }\end{array}$ \\
\hline $25 / 50-O B$ & 4,81 & 0,28 & 5,93 & 3,23 & 21 & $5,0-9,0$ & A7 \\
\hline 50/50-OB & 5,37 & 0,26 & 4,77 & 3,69 & 9 & $5,0-9,0$ & A12 \\
\hline Faktor $\mathrm{f}_{\mathrm{OB}}$ & 1,12 & & & & & & \\
\hline
\end{tabular}

Die leichte Erhöhung der Mittelwerte der ertragbaren Schwingbreite der Serie D im Vergleich zu Serie C bei $2 \cdot 10^{6}$ Schwingspielen kann auf die unterschiedlichen Ankerausführungen der beiden Hersteller und auf die vorhandenen Abmessungen des Schienenquerschnitts zurückgeführt werden.

Aus den Untersuchungen der Serie A und Serie B kann gefolgert werden, daß die relative Erhöhung der ertragbaren Ermüdungsfestigkeit zwischen den Varianten 25/50 und 50/50 stark von der Versagensart abhängt. Im äußerst ungünstigen Fall, d.h. bei sehr starker Kerbwirkung, liegt die Erhöhung bei etwa $2 \%$, wobei der Normalfall einer geringen Kerbwirkung eine Erhöhung um etwa $14 \%$ aufweist. Der Einfluß der Laststellung für den einbetonierten Fall wird nachfolgend geklärt. 


\section{Einfluß des umgebenden Betons}

Laut [21], [23] dürfen Ankerschienen für Verankerungen unter nicht vorwiegend ruhender zentrischer Zugbelastung bis $2 \cdot 10^{6}$ Schwingspielen in bewehrtem Beton der Festigkeitsklasse von mindestens B15 nach [26] verwendet werden, sofern keine Anforderungen hinsichtlich der Feuerwiderstandsdauer an die Gesamtkonstruktion einschließlich der Ankerschiene gestellt werden. Somit ergibt sich eine weitere und wichtige Frage nach dem Einfluß des umgebenden Betons auf die Ermüdungstragfähigkeit. Zur Klärung dieser Fragestellungen sind zusätzliche Ankerschienen der Serie A und Serie C im einbetonierten Zustand geprüft worden. Die Versuche erfolgten unter konstanter Oberlast von $17 \mathrm{kN}$ und Variation der einwirkenden Schwingbreite. Die Schwingspielzahlen, die zum vorzeitigen Versagen der Ankerschiene führten, lagen zwischen $4 \cdot 10^{4}$ und $4 \cdot 10^{6}$. Im Diagramm in Bild 4.47 ist die übliche Darstellungsweise der Ermüdungstragfähigkeiten von nicht einbetonierten im Vergleich zu einbetonierten Ankerschienen der Variante 25/50 und 50/50 für die Serie A dargestellt. Hier sind zusätzlich zu den bekannten Verläufen der nicht einbetonierten Ankerschienen der Serie A aus Bild 4.44 die Verläufe (als Kurven) der Ermüdungstragfähigkeiten im einbetonierten Zustand dargestellt. Es ist auffällig, daß die Ermüdungsfestigkeiten im einbetonierten Zustand stets über denen der nicht einbetonierten Ankerschienen liegen.

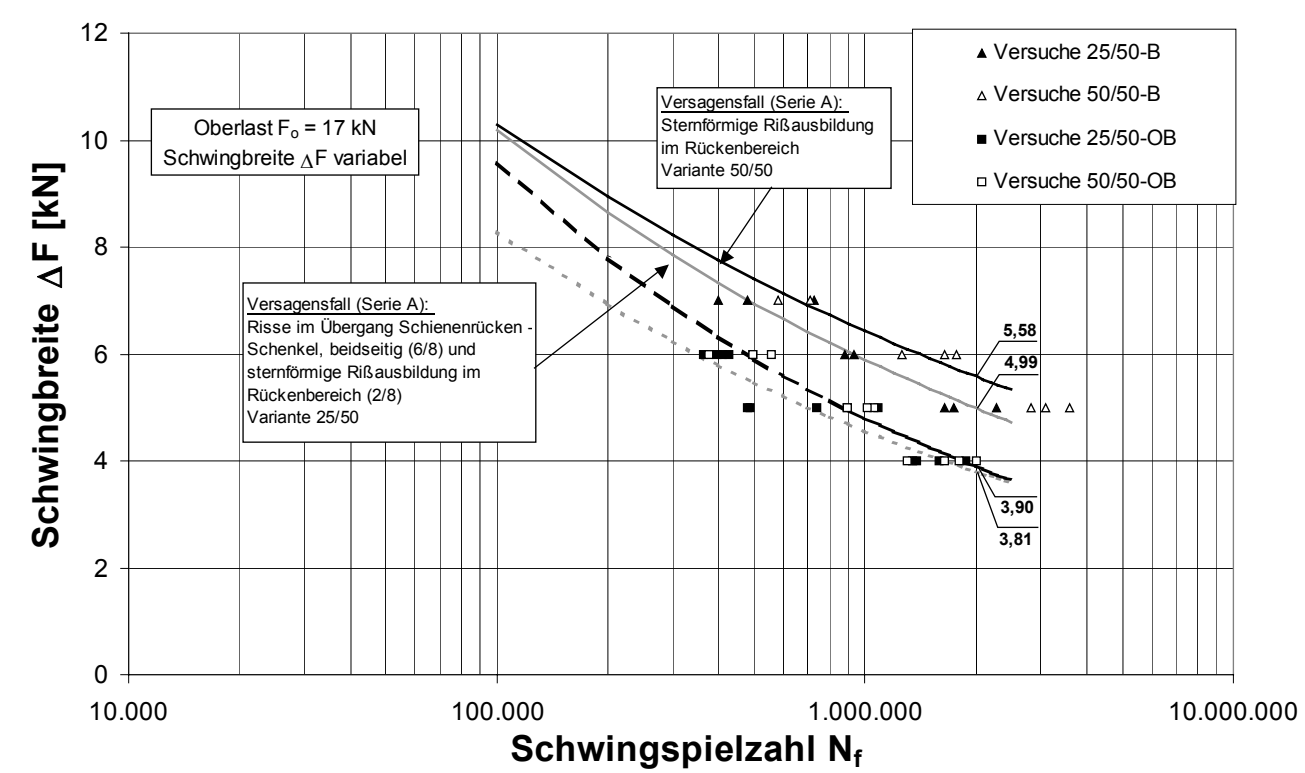

Bild 4.47 Ermüdungstragfähigkeiten der Serie A im einbetonierten Zustand

In Tabelle 4.12 sind die spezifischen Werte der Ermüdungsfestigkeit der Varianten 25/50 und 50/50 im einbetonierten Zustand dargestellt. Eine vergleichbare Untersuchung anhand von Ankerschienen der Serie $\mathrm{C}$ erfolgte nicht, da für diese Serie die Variante 50/50 nicht einbetoniert wurde. Jedoch sind hierfür ähnliche Relationen zu erwarten. 
4 Experimentelle Untersuchungen

Tabelle 4.12 Ergebnisse an einbetonierten Ankerschienen der Serie A

\begin{tabular}{|c|c|c|c|c|c|c|c|}
\hline Variante & $\begin{array}{c}\text { Mittelw. } \Delta \mathrm{F} \\
\text { bei } 2 \cdot 10^{6} \\
{[\mathrm{kN}]}\end{array}$ & $\begin{array}{c}\sigma \\
{[\mathrm{kN}]}\end{array}$ & $\begin{array}{l}\mathrm{V} \\
{[\%]}\end{array}$ & $\begin{array}{l}\text { Steigung } \mathrm{m} \\
\text { Regression }\end{array}$ & Anzahl n & $\begin{array}{c}\text { Schwingbr } \\
\Delta \mathrm{F}_{\mathrm{i}} \\
{[\mathrm{kN}]}\end{array}$ & $\begin{array}{c}\text { Anhang } \\
\text { Tab. }\end{array}$ \\
\hline $25 / 50-B$ & 4,99 & 0,24 & $\overline{~ 4,81}$ & 4,20 & 8 & $5,0-7,0$ & A14 \\
\hline $50 / 50-B$ & 5,58 & 0,18 & 3,13 & 4,89 & 8 & $5,0-7,0$ & A19 \\
\hline Faktor $f_{B}$ & 1,12 & & & & & & \\
\hline
\end{tabular}

Im Vergleich zum nicht einbetonierten Zustand, bei dem eine Erhöhung von $2 \%$ zwischen der Variante 25/50 und 50/50 vorlag, beträgt der Faktor für den einbetonierten Fall $\mathrm{f}_{\mathrm{B}}=12 \%$. Der höhere Faktor ist mit sehr hoher Wahrscheinlichkeit auf die im Vergleich zum nicht einbetonierten Fall ungleichmäßige Beanspruchung der kritischen Versagensstellen aufgrund des umgebenden Betons zurückzuführen. Die Versagensarten der Variante 25/50 sind vergleichbar mit dem nicht einbetonierten Zustand. Lediglich bei den Ankerschienen der Ausführung 50/50 gibt es eine Änderung in der Versagensform. Hierbei treten im Vergleich zu vorher ausschließlich sternförmige Rißerscheinungen um das Ankerloch auf. Die Begründung für ein solches Verhalten ist wahrscheinlich auf die Stützwirkung des umgebenden Betons zurückzuführen. Dadurch werden die vorher maßgebenden Stellen (Übergangsbereich Rücken - Schenkel) deutlich weniger beeinflußt und es findet eine Umlagerung der Kräfte bzw. Spannungen statt.

Um jedoch den Einfluß des umgebenden Betons zu verdeutlichen, werden die Ermüdungsfestigkeiten der vergleichbaren Versuchsserien gegenübergestellt. Eine Zusammenstellung der ermittelten Ergebnisse mit Bestimmung des Einflusses des umgebenden Betons, ausgedrückt durch die Faktoren $\mathrm{f}_{\mathrm{B}-\mathrm{OB} 25 / 50}$ (Variante 25/50) bzw. $\mathrm{f}_{\mathrm{B}-\mathrm{OB}}$ 50/50 (Variante 50/50), beinhaltet Tabelle 4.13 und Tabelle 4.14.

Tabelle 4.13 Ergebnisse an einbetonierten Ankerschienen der Serie A (Variante 25/50)

\begin{tabular}{|c|c|c|c|c|c|c|c|}
\hline Variante & $\begin{array}{c}\text { Mittelw. } \Delta \mathrm{F} \\
\text { bei } 2 \cdot 10^{6} \\
{[\mathrm{kN}]}\end{array}$ & $\begin{array}{c}\sigma \\
{[\mathrm{kN}]}\end{array}$ & $\begin{array}{l}\mathrm{V} \\
{[\%]}\end{array}$ & $\begin{array}{l}\text { Steigung } \mathrm{m} \\
\text { Regression }\end{array}$ & Anzahl n & $\begin{array}{c}\text { Schwingbr. } \\
\qquad \mathrm{F}_{\mathrm{i}} \\
{[\mathrm{kN}]}\end{array}$ & $\begin{array}{c}\text { Anhang } \\
\text { Tab. }\end{array}$ \\
\hline $25 / 50-O B$ & 3,81 & 0,24 & 6,27 & 3,85 & 12 & $4,0-6,0$ & $\mathrm{~A} 2$ \\
\hline $25 / 50-B$ & 4,99 & 0,24 & 4,81 & 4,20 & 8 & $5,0-7,0$ & A14 \\
\hline $\mathrm{f}_{\mathrm{B}-\mathrm{OB} 25 / 50}$ & 1,31 & & & & & & \\
\hline
\end{tabular}

Tabelle 4.14 Ergebnisse an einbetonierten Ankerschienen der Serie A (Variante 50/50)

\begin{tabular}{|c|c|c|c|c|c|c|c|}
\hline Variante & $\begin{array}{c}\text { Mittelw. } \Delta \mathrm{F} \\
\text { bei } 2 \cdot 10^{6} \\
{[\mathrm{kN}]}\end{array}$ & $\begin{array}{c}\sigma \\
{[\mathrm{kN}]}\end{array}$ & $\begin{array}{l}\mathrm{V} \\
{[\%]}\end{array}$ & $\begin{array}{l}\text { Steigung } \mathrm{m} \\
\text { Regression }\end{array}$ & Anzahl n & $\begin{array}{c}\text { Schwingbr. } \\
\qquad \mathrm{F}_{\mathrm{i}} \\
{[\mathrm{kN}]}\end{array}$ & $\begin{array}{c}\text { Anhang } \\
\text { Tab. }\end{array}$ \\
\hline 50/50-OB & 3,90 & 0,18 & 4,68 & 3,32 & 12 & $4,0-6,0$ & A9 \\
\hline 50/50-B & 5,58 & 0,18 & 3,13 & 4,89 & 8 & $5,0-7,0$ & A19 \\
\hline$f_{B-O B ~ 50 / 50}$ & 1,43 & & & & & & \\
\hline
\end{tabular}


Die Erhöhung des Mittelwertes der Ermüdungstragfähigkeit durch den umgebenden Beton der Variante 25/50 beträgt $31 \%$. Hieraus geht deutlich hervor, daß durch eine Betonierung die Ermüdungstragfähigkeit und somit auch die Zuverlässigkeit des Systems deutlich erhöht wird. Im Falle der Variante 50/50 wird sogar eine bemerkenswerte Erhöhung von etwa $43 \%$ erreicht, welche auch hier auf zusätzlich aktivierte Tragreserven im einbetonierten Zustand der Ankerschiene zurückzuführen sind. Dieses Verhalten ist durch die Stützwirkung des Betons auf die Seitenschenkel der Ankerschienen und der damit verbundenen Umlagerung der Spannungen im Querschnitt zu erklären. Die kritischen Stellen im Querschnitt werden wesentlich schwächer beansprucht als im einbetonierten Zustand.

Zusätzlich wurde der Einfluß des umgebenden Betons an den Ankerschienenabschnitten 25/50 mit Hilfe der Serie C verifiziert. Die Versuche erfolgten unter konstanter Oberlast von $17 \mathrm{kN}$ mit Variation der Schwingbreite. Durchgeführt wurden lediglich drei Versuche im einbetonierten Zustand bei unterschiedlichen Schwingbreiten. Die Schwingspielzahlen bis zum Bruch liegen in Abhängigkeit von der Schwingbreite zwischen $3 \cdot 10^{4}$ und $2 \cdot 10^{6}$. Die Mittelwerte der Betonfestigkeiten entsprechen der Serie 3 in Tabelle 4.5. Als Versagensart wurde ein beidseitiges Versagen im Übergang vom Schienenrücken zum Schienenschenkel beobachtet. Die prinzipielle Versagensart entspricht dem in Bild 4.39 dargestellten Fall 1.2.

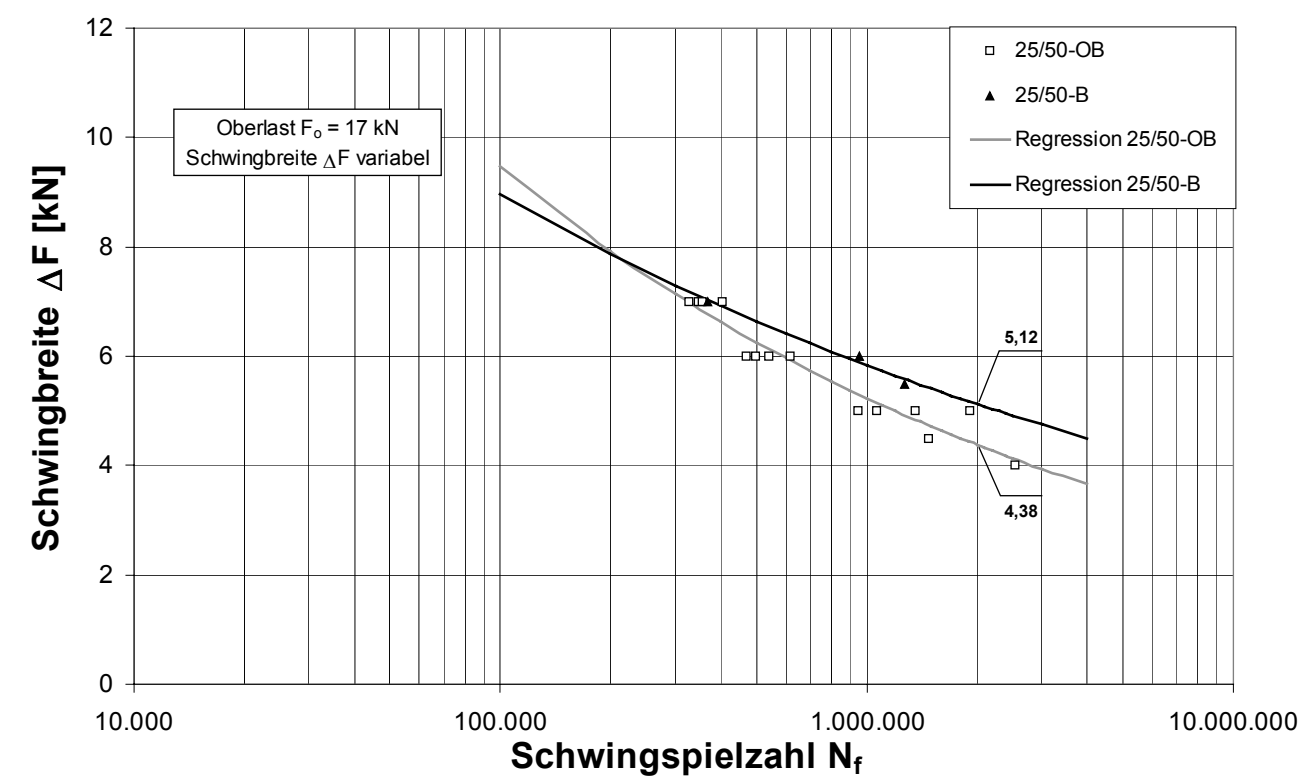

Bild 4.48 Einfluß des umgebenden Betons bei der Serie C (Variante 25/50)

Es stellt sich die Frage, inwiefern drei Versuche im einbetonierten Fall aussagekräftige Ergebnisse liefern. Bei Betrachtung des Mittelwertes kann jedoch davon ausgegangen werden, daß eine Anzahl von drei Versuchen ausreichend genaue Ergebnisse liefert. Dieses wurde mit Hilfe einer Simulationsrechnung kontrolliert und bestätigt. Dabei lag der auf $2 \cdot 10^{6}$ Schwingspielen projizierte Mittelwert aus 20 Simulationsläufen und einer Anzahl von 3 Versuchen auf unterschiedlichen Lastniveaus um etwa 9 $\%$ unter bzw. über dem Zielwert. Zur Verdeutlichung sind die Verläufe der bezogenen 
Schwingbreite über die Anzahl der Versuche bei 20 Simulationen für den Mittelwert, das Minimum und Maximum der Variante 50/50-OB der Serie C, in Bild 4.49 dargestellt. Nach dem Zufallsprinzip, mit Hilfe eines Zufallsgenerators, werden aus den vorliegenden Versuchsdaten Werte auf mindestens drei unterschiedlichen Lastniveaus ausgewählt. Mit Hilfe dieser Werte wird der Mittelwert der Regressionsgeraden bei $2 \cdot 10^{6}$ Schwingspielen errechnet. Als Bezugsbasis dient dabei der Wert der Schwingbreite bei maximaler Versuchsanzahl, welcher aufgrund der hohen Anzahl repräsentativ für den Mittelwert der Grundgesamtheit ist. Gewählt wurde die Variante 50/50-OB, da hierbei statistischen Gesichtspunkten eine relativ hohe Anzahl $(\mathrm{n}=41)$ an Versuchen auf unterschiedlichen Lastniveaus vorlag. Eine Übertragung der Ergebnisse auf die Variante 25/50-OB ist nach Ansicht des Verfassers prinzipiell möglich.

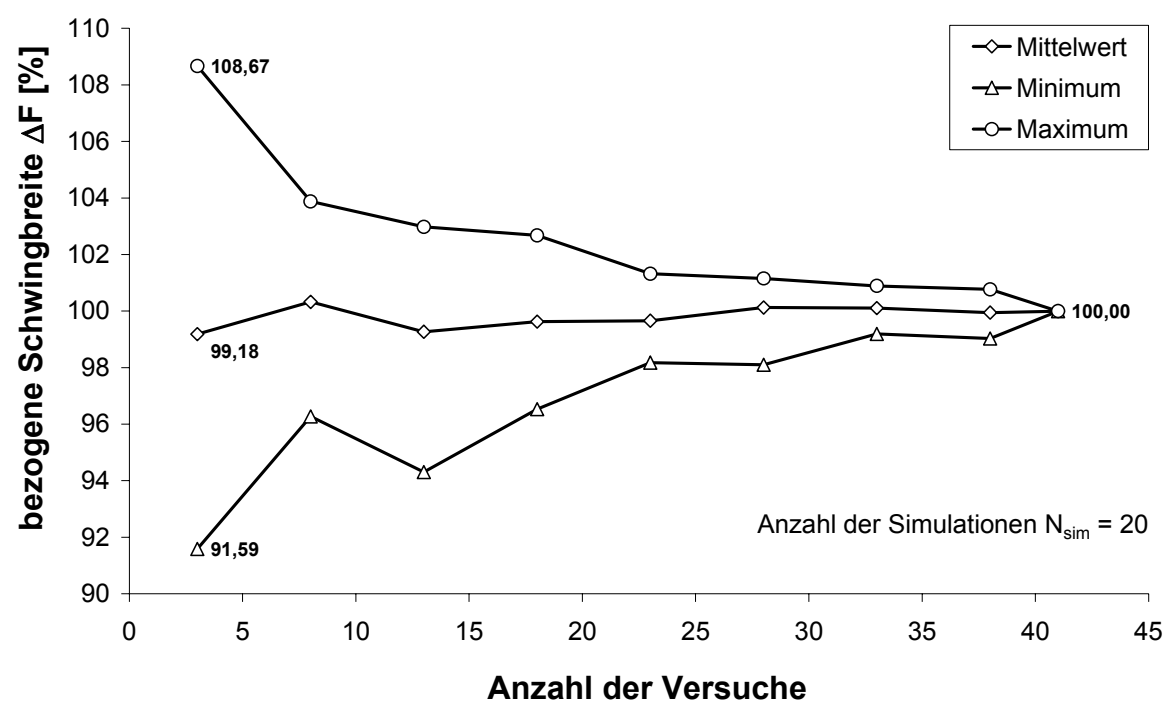

Bild 4.49 Bezogene Schwingbreite in Abhängigkeit von der Versuchsanzahl $\mathrm{N}_{\text {sim }}=20$

Die nachfolgende Tabelle 4.15 zeigt u.a. den Einfluß des Betons auf den Mittelwert der Ermüdungsfestigkeit der Serie C.

Tabelle 4.15 Einfluß des umgebenden Betons bei Ankerschienen der Serie C

\begin{tabular}{|c|c|c|c|c|c|c|c|}
\hline Variante & $\begin{array}{c}\text { Mittelw. } \Delta \mathrm{F} \\
\text { bei } 2 \cdot 10^{6} \\
{[\mathrm{kN}]}\end{array}$ & $\begin{array}{c}\sigma \\
{[\mathrm{kN}]}\end{array}$ & $\begin{array}{l}\mathrm{V} \\
\text { [\%] }\end{array}$ & $\begin{array}{l}\text { Steigung } \mathrm{m} \\
\text { Regression }\end{array}$ & Anzahl $n$ & $\begin{array}{c}\text { Schwingbr. } \\
\Delta \mathrm{F}_{\mathrm{i}} \\
{[\mathrm{kN}]}\end{array}$ & $\begin{array}{c}\text { Anhang } \\
\text { Tab. }\end{array}$ \\
\hline $25 / 50-O B$ & 4,38 & 0,22 & 5,08 & 3,89 & 15 & $4,0-7,0$ & A3 \\
\hline 25/50-B & 5,12 & 0,09 & 1,74 & 5,36 & 3 & $5,5-7,0$ & A16 \\
\hline $\mathrm{f}_{\mathrm{B}-\mathrm{OB} 25 / 50}$ & 1,17 & & & & & & \\
\hline
\end{tabular}

Unter Berücksichtigung von drei Versuchen ergibt sich eine Tragfähigkeitserhöhung durch den umgebenden Beton um etwa $17 \%$, welche deutlich unterhalb der Faktors von $31 \%$ (siehe Tabelle 4.13 Serie A) liegt. Berücksichtigt man jedoch eine Erhöhung der Ermüdungsfestigkeit der Variante 25/50-B bei einer ausreichenden Anzahl 
von Versuchen nach der Erkenntnis der Simulationsrechnung um max. $9 \%$, so würde mit dem Mittelwert der Schwingbreite $\Delta \mathrm{F}=5,58 \mathrm{kN}$ eine Erhöhung der Ermüdungstragfähigkeit durch den umgebenden Beton von etwa $27 \%$ resultieren, was vergleichbar zur Serie A wäre.

Eine Ermittlung des Betoneinflusses auf Basis der Versuche der Serie B kann nicht erfolgen, da für diese Serie keine Versuche im nicht einbetonierten Zustand vorliegen. Betrachtet man jedoch den Streubereich der ertragbaren Schwingbreiten und die resultierenden Versagensarten der Serie B im Vergleich zur Serie C, so kann durchaus eine gemeinsame Auswertung durchgeführt werden. Beim Vergleich der kritischen Abmessungen $\mathrm{t} 1$ bis $\mathrm{t} 3$ aus Tabelle 4.1 können nur sehr geringe Abweichungen der Mittelwerte festgestellt werden, wodurch eine gemeinsame Auswertung unterstützt wird.

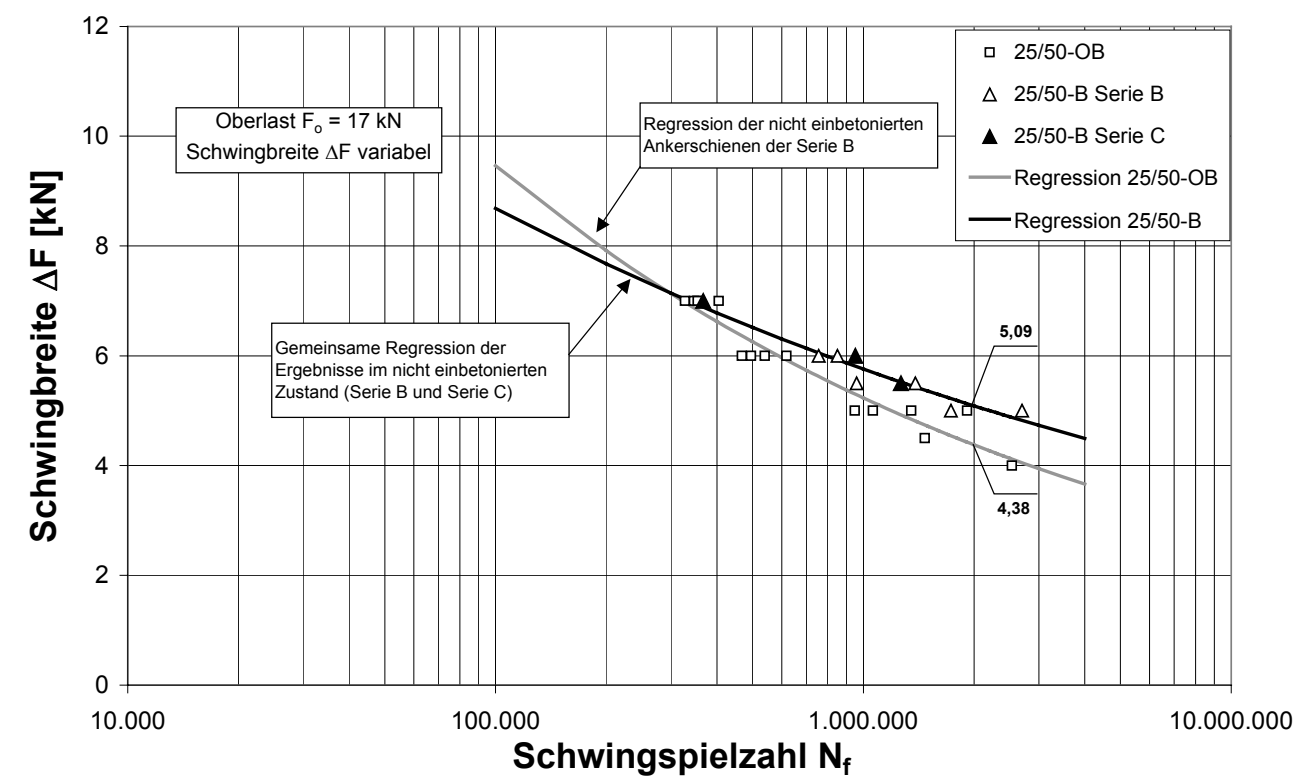

Bild 4.50 Schwingbreite in Abhängigkeit von der Schwingspielzahl (Serie B \& Serie C)

Es ist deutlich zu erkennen, daß die ertragbaren Schwingbreiten der Serien B und C im selben Streuband liegen. Die Betondruckfestigkeiten der Serie B entsprechen den Werten der Serie 2 aus Tabelle 4.5. Der Mittelwert der einbetonierten Variante ändert sich mit 5,11 kN kaum im Vergleich zur gemeinsamen Auswertung mit 5,09 kN.

Tabelle 4.16 Einfluß des umgebenden Betons bei Ankerschienen der Serie B

\begin{tabular}{|l||c|c|c|c|c|c|c|}
\hline \multicolumn{1}{|c|}{ Variante } & $\begin{array}{c}\text { Mittelw. } \Delta \mathrm{F} \\
\text { bei } 2 \cdot 10^{6} \\
{[\mathrm{kN}]}\end{array}$ & $\sigma$ & $\mathrm{V}$ & $\begin{array}{c}\text { Steigung } \mathrm{m} \\
\text { Regression } \\
{[\mathrm{kN}]}\end{array}$ & $\begin{array}{c}\text { Anz. } \mathrm{n} \\
{[\%}\end{array}$ & $\begin{array}{c}\text { Schwingbr. } \\
\Delta \mathrm{F}_{\mathrm{i}} \\
{[\mathrm{kN}]}\end{array}$ & $\begin{array}{c}\text { Anhang } \\
\text { Tab. }\end{array}$ \\
\hline $25 / 50-\mathrm{OB}$ & 4,38 & 0,22 & 5,08 & 3,89 & 15 & $4,0-7,0$ & $\mathrm{~A} 3$ \\
\hline $25 / 50-\mathrm{B}(\mathrm{B})$ & 5,11 & 0,17 & 3,24 & 6,50 & 6 & $5,0-6,0$ & $\mathrm{~A} 15$ \\
\hline $25 / 50-\mathrm{B}(\mathrm{B} \& \mathrm{C})$ & 5,09 & 0,16 & 3,08 & 5,60 & 9 & $5,0-7,0$ & $\mathrm{~A} 18$ \\
\hline $\mathrm{f}_{\mathrm{B}-\mathrm{OB} 25 / 50}$ & 1,16 & \multicolumn{7}{|l}{}
\end{tabular}


Die Ursache für die Erhöhung der ertragbaren Schwingbreite im einbetonierten Fall wird mittels der Verläufe der statischen Zugversuche der Serie A anhand von Bild 4.51 veranschaulicht. Aufgetragen ist der Mittelwert der Dehnungen, gemessen an den Außenseiten der beiden Schenkel des Schienenprofils, als Funktion der bezogenen statischen zentrischen Zugbelastung. Der Bezugswert $\mathrm{N}_{\mathrm{Rd}}$ ist der zur Zeit gültige Bemessungswert nach [21], [23] und beträgt $12 \mathrm{kN}$ für das verwendete Profil. Die Meßstellen der Dehnungen sind Bild 4.6 zu entnehmen.

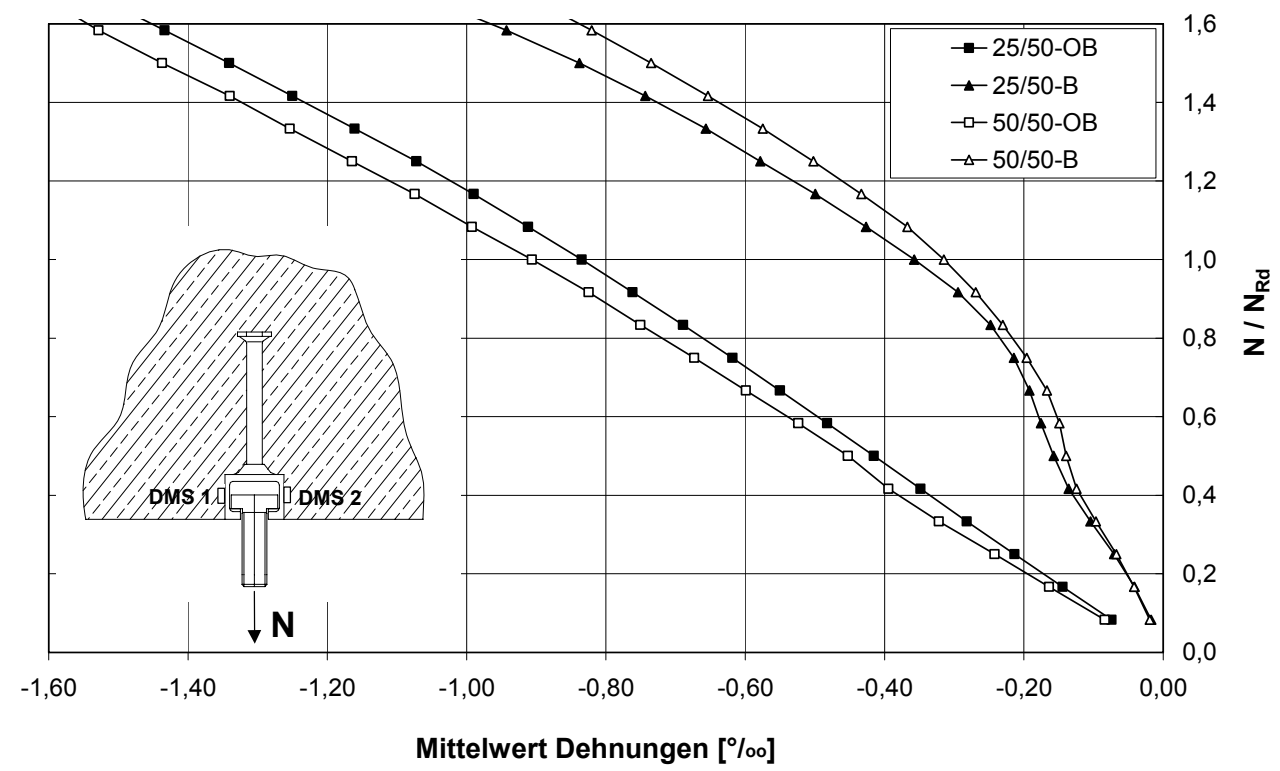

Bild 4.51 Normierte Zugkraft über mittlere Dehnungen an den Schienenschenkeln

Man erkennt, daß die Druckdehnung im maximal zulässigen statischen Bereich der Ankerschiene $\left(\mathrm{N} / \mathrm{N}_{\mathrm{Rd}}=1,24\right)$ im einbetonierten Fall nur etwa $55 \%$ der Dehnungen des nicht einbetonierten Zustandes ausmachen. Durch die Stützwirkung des umgebenden Betons wird die Dehnung bzw. Spannung in Bereichen des Schenkels reduziert, welche bei gleicher Belastung zu höheren Versagensschwingspielzahlen gegenüber dem nicht einbetonierten Fall führt. Zusätzlich findet eine Umlagerung der Kraft von den Schienenschenkeln in den Schienenrücken statt, welche dazu führen kann, daß Versagen auch in Bereichen des Ankerfußes im Rücken auftreten können.

Die in Versuchen mit konstanter Oberlast von $17 \mathrm{kN}$ gemessenen Dehnungen für die Ausführung 25/50, sowohl im einbetonierten als auch im nicht einbetonierten $\mathrm{Zu}$ stand, sind in Bild 4.52 für die vergleichbaren Schwingbreiten von $5 \mathrm{kN}$ grafisch dargestellt. Dabei ist die Dehnung, gemessen an den Außenschenkeln der Ankerschiene, über die bezogene Schwingbreite aufgetragen. Der Bezugswert stellt je Versuch die Schwingspielzahl im Versagenszustand $\mathrm{N}_{\mathrm{f}}$ dar.

Der prinzipielle Verlauf der Dehnungen für den einbetonierten Zustand ist vergleichbar mit denen der nicht einbetonierten Ankerschienen. Führt man den Vergleich bezüglich der Abnahme der Druckdehnungen durch, so wird erkennbar, daß diese in beiden Fällen etwa ab dem gleichen bezogenen Niveau $\left(\mathrm{N} / \mathrm{N}_{\mathrm{f}}\right)$ erfolgt. 

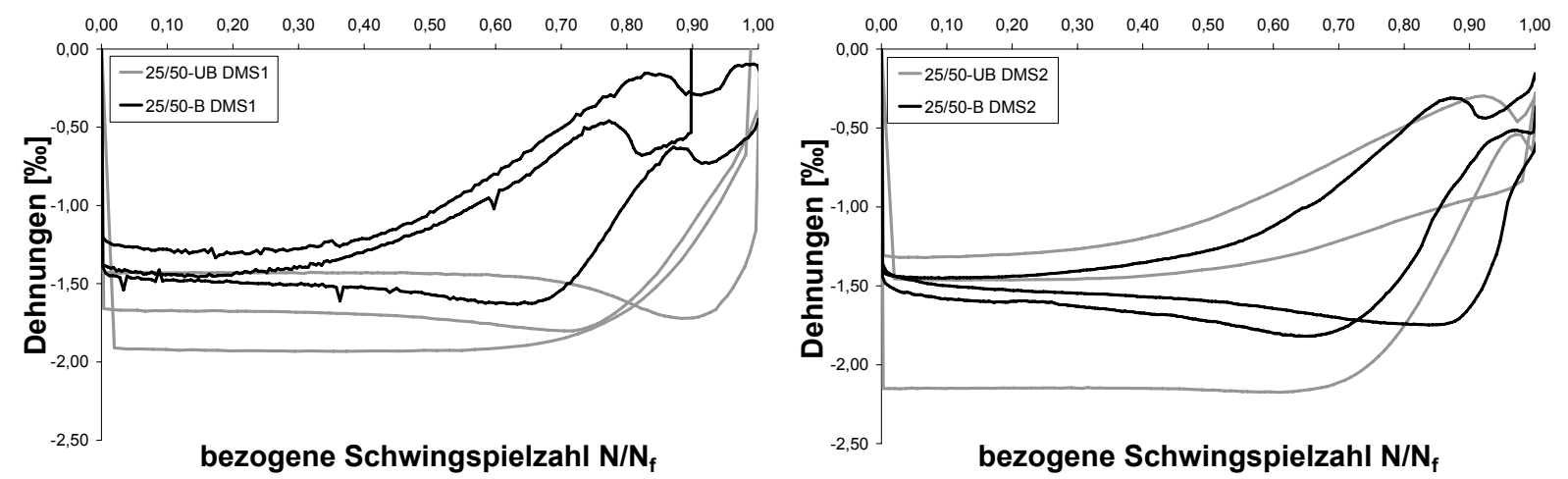

Bild 4.52 Dehnungen als Funktion der bezogenen Schwingbreiten $(\Delta F=5 k N)$ Serie $A$

Die Mittelwerte der Anfangsdehnungen für den nicht einbetonierten Zustand liegen etwa zwischen -1,3 \%o und -2,2 \%o (Ausreißer), wobei sich für den einbetonierten Fall nahezu gleichmäßige Werte um etwa $-1,4$ \%o einstellen. Bei einer Schwingbreite von 6 $\mathrm{kN}$ liegen diese Werte für den einbetonierten Fall zwischen -1,3 \%o und -1,8 \%o, und für den nicht einbetonierten Zustand bei ca. $-1,8 \%$.

Es wird auch hier deutlich, daß an den kritischen Versagensstellen der einbetonierten Ankerschienen geringere Spannungen vorherrschen, welche bei gleichem Lastniveau höhere ertragbare Schwingspielzahlen ergeben. Ab 40 - 60 \% der Versagensschwingspielzahl machen sich Risse in den Schenkeln der Ankerschiene durch Abnahme der Druckdehnungen bemerkbar, welche zu einer Umlagerung im Spannungsquerschnitt führen. Diese breiten sich anschließend bis etwa 70 bis $90 \%$ sehr stabil aus. Ab diesem Punkt tritt der instabile Restbruch ein. Für die Ausführung 50/50 ist ein prinzipiell ähnliches Verhalten zu beobachten.

Die Dehnungen der einbetonierten Ankerschienen sind vom Betrag kleiner als im einbetonierten Zustand. Die kritischen Stellen im Querschnitt der Ankerschiene werden im einbetonierten Zustand im Mittel um etwa $65 \%$ weniger beansprucht. Dieses Ergebnis deckt sich mit den Erkenntnissen aus statischen Versuchen, bei denen eine Reduzierung von $55 \%$ vom einbetonierten Zustand zum nicht einbetonierten Zustand erfolgte.

Somit kann gefolgert werden, daß hauptsächlich der umgebende Beton eine Erhöhung der Ermüdungstragfähigkeit verursacht.

Das Diagramm in Bild 4.53 zeigt den Effekt der Umlagerung anhand von Ankerschienen der Serie B. Es wurden Versuche mit konstanter Oberlast von $17 \mathrm{kN}$ und variierenden Schwingbreiten von 5 und $6 \mathrm{kN}$ durchgeführt. Die Verläufe der Dehnungen (DMS1, DMS2), gemessen an den Schienenschenkeln, zeigen eine gewisse Abhängigkeit voneinander. Dieses Verhalten ist auf die Umlagerungen der Spannungen im Querschnitt infolge Rißbildung zurückzuführen. 


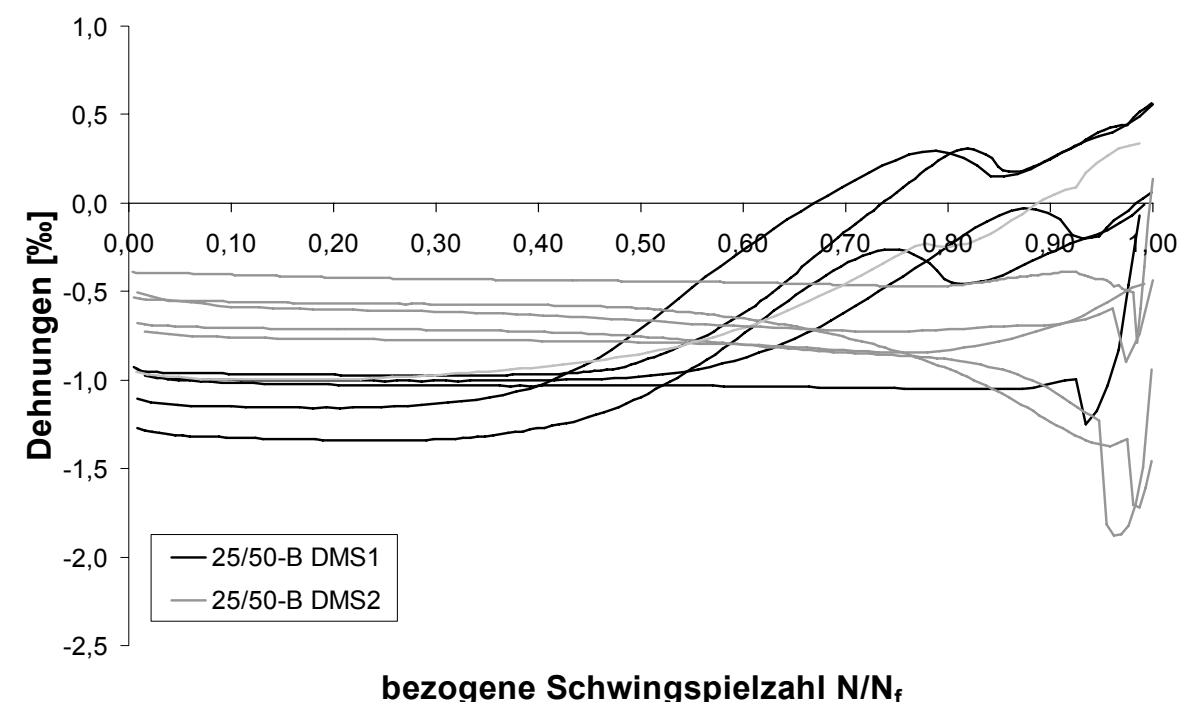

Bild 4.53 Dehnungen bei Schwingbreiten $\Delta \mathrm{F}=5$ bis $6 \mathrm{kN}$ (Serie $\mathrm{B}$ )

Es ist deutlich zu erkennen, daß mit der Abnahme der Druckdehnungen (DMS1), infolge einer anfänglichen Rißentstehung ab etwa $40 \%$, eine Erhöhung der Druckdehnungen auf der gegenüberliegenden Seite (DMS2) bis etwa $90 \%$ der bezogenen Schwingspielzahl erfolgt.

\section{Einfluß des Lastniveaus}

Ein weiterer Einflußfaktor auf das Ermüdungstragverhalten ist, wie bereits in Kapitel 3 dargestellt, die Variation des Lastniveaus. Schwingversuche zeigen bei nicht stark gekerbten Bauteilen eine Abhängigkeit zwischen dem Lastniveau und der ertragbaren Schwingspielzahl. Sind die Bauteile jedoch stark gekerbt, wie es z.B. bei Schweißverbindungen der Fall ist, dann ist lediglich die Schwingbreite maßgebend [101]. Zur Klärung des Einflusses des Lastniveaus sind Versuche an einbetonierten und nicht einbetonierten Ankerschienen der Variante 25/50 und 50/50 durchgeführt worden. Dabei sind lediglich Profile der Serie C und Serie D verwendet worden. In Bild 4.54 sind die Ermüdungstragfähigkeiten der Variante 25/50 der Serie C im nicht einbetonierten Zustand aufgetragen. Es zeigt sich, daß die ertragbare Schwingbreite mit zunehmendem Lastniveau (Unterlast) geringer wird. Auch die Charakteristik hinsichtlich der Streuungen der Ermüdungstragfähigkeiten ändert sich sehr stark. Die Versuche mit konstanter Unterlast von 10 und $15 \mathrm{kN}$ bzw. mit konstanter Oberlast von $17 \mathrm{kN}$ (nicht in Bild 4.54 dargestellt) weisen ertragbare Schwingbreiten auf, die über die Beanspruchungsdauer vergleichbare Werte liefern. Vermutlich ist der Einfluß des Lastniveuas bei höheren Beanspruchungsniveaus nicht markant. 


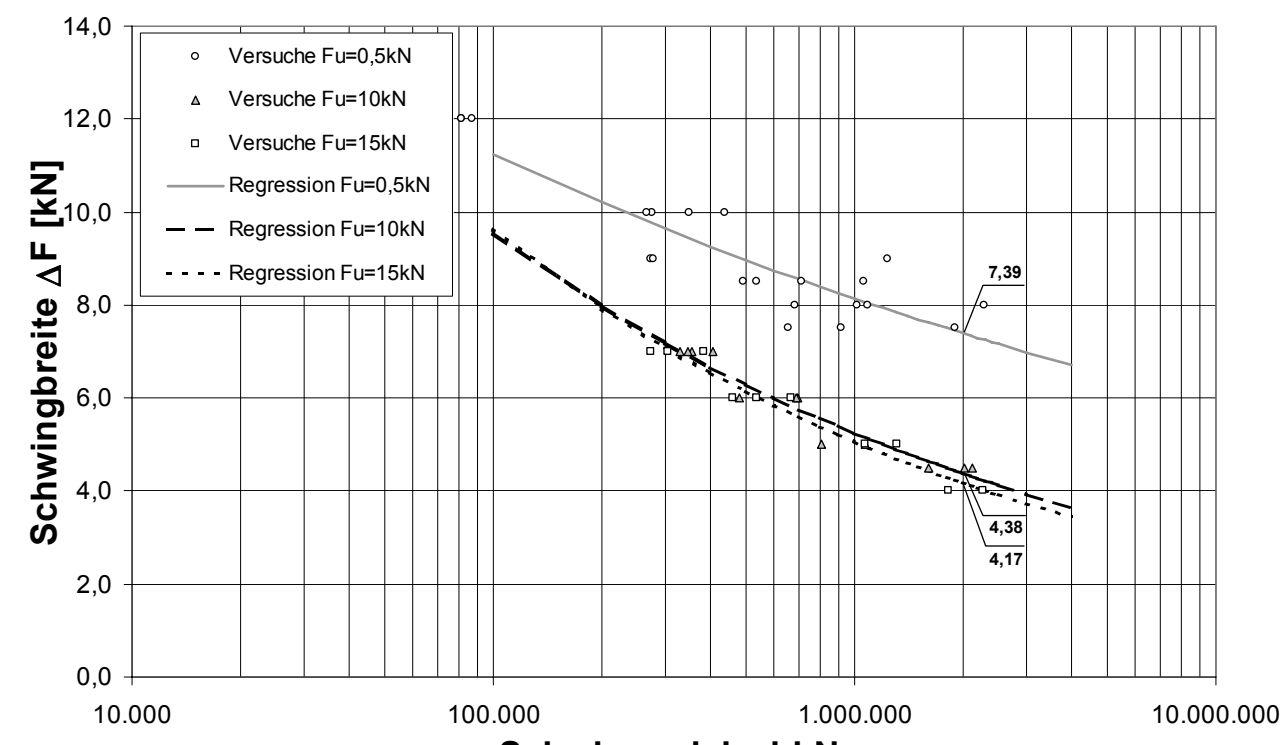

Schwingspielzahl $\mathbf{N}_{\mathrm{f}}$

Bild 4.54 Ermüdungstragfähigkeit in Abhängigkeit vom Lastniveau (Serie C)

Vergleicht man diese Versuche jedoch mit den Ergebnissen der Schwingversuche bei konstanter Unterlast von $0,5 \mathrm{kN}$, so zeigt sich eine deutlich höhere Ermüdungstragfähigkeit über die gesamte Beanspruchungsdauer (siehe Tabelle 4.17)

Tabelle 4.17 Schwingbreiten in Abhängigkeit von der Unterlast (Variante 25/50 - Serie C)

\begin{tabular}{|l||c|c|c|c|c|c|c|}
\hline Lastniveau & $\begin{array}{c}\text { Mittelw. } \Delta \mathrm{F} \\
\text { bei } 2 \cdot 10^{6} \\
{[\mathrm{kN}]}\end{array}$ & $\sigma$ & $\mathrm{V}$ & $\begin{array}{c}\text { Steigung } \mathrm{m} \\
\text { Regression }\end{array}$ & Anzahl $\mathrm{k}]$ & $\begin{array}{c}\text { Schwingbr. } \\
{[\%]}\end{array}$ & $\begin{array}{c}\text { Anhang } \\
{[\mathrm{kN}]}\end{array}$ \\
\hline $\mathrm{F}_{\mathrm{u}}=0,5 \mathrm{kN}$ & 7,41 & 0,52 & 6,99 & 7,13 & 21 & $7,5-12,0$ & $\mathrm{~A} 6$ \\
\hline $\mathrm{F}_{\mathrm{u}}=10 \mathrm{kN}$ & 4,38 & 0,20 & 4,64 & 3,83 & 12 & $4,5-7,0$ & $\mathrm{~A} 5$ \\
\hline $\mathrm{F}_{\mathrm{u}}=15 \mathrm{kN}$ & 4,17 & 0,19 & 4,58 & 3,57 & 10 & $4,0-7,0$ & $\mathrm{~A} 4$ \\
\hline
\end{tabular}

Eine gleiche Tendenz konnte auch bei den Varianten 25/50 der Serie D und 50/50 der Serie C und Serie D festgestellt werden. Um den Einfluß des Lastniveaus zu verdeutlichen, erfolgten hierbei zusätzlich Versuche mit konstanter Unterlast von 0,5 kN. Die Ergebnisse sind in tabellarischer Form dargestellt.

Tabelle 4.18 Kennwerte der Schwingversuche bei einer Unterlast von 0,5 kN

\begin{tabular}{|c||c|c|c|c|c|c|c|}
\hline Variante & $\begin{array}{c}\text { Mittelw. } \Delta \mathrm{F} \\
\text { bei } 2 \cdot 10^{6} \\
{[\mathrm{kN}]}\end{array}$ & $\sigma$ & $\mathrm{V}$ & $\begin{array}{c}\text { Steigung } \mathrm{m} \\
\text { Regression }\end{array}$ & Anz. $\mathrm{k}$ & $\begin{array}{c}\text { Schwingbr. } \\
\Delta \mathrm{F}_{\mathrm{i}} \\
{[\mathrm{kN}]}\end{array}$ & $\begin{array}{c}\text { Anhang } \\
\text { Tab. }\end{array}$ \\
\hline 25/50-OB (D) & 7,92 & 0,80 & 10,03 & 6,42 & 22 & $7,5-16,5$ & $\mathrm{~A} 8$ \\
\hline $50 / 50-\mathrm{OB}(\mathrm{C})$ & 7,59 & 0,36 & 4,80 & 5,08 & 16 & $8,0-16,0$ & $\mathrm{~A} 11$ \\
\hline $50 / 50-\mathrm{OB}(\mathrm{D})$ & 8,04 & 0,51 & 6,35 & 8,43 & 17 & $8,0-12,0$ & $\mathrm{~A} 13$ \\
\hline
\end{tabular}


Das Versagen der Serie D war hauptsächlich eine beidseitige Rißbildung im Übergangsbereich vom Schienenrücken zum Schienenschenkel (Fall 1.2). Bei Schwingbreiten von $6,5,7,5$ und $8,0 \mathrm{kN}$ trat bei insgesamt drei Versuche mit Schwingspielzahlen von ca. $4 \cdot 10^{6}$ über $7 \cdot 10^{6}$ und $1 \cdot 10^{7}$ keinen Bruch auf. Bei der Serie C konnten mit variierenden Unterlasten keine eindeutigen Versagensarten festgestellt werden. Eine graphische Übersicht der Versagensarten bei Durchführung der Versuche mit konstanter Unterlast von $0,5 \mathrm{kN}, 10 \mathrm{kN}, 15 \mathrm{kN}$ und mit konstanter Oberlast von $17 \mathrm{kN}$ zeigt das Bild 4.55.

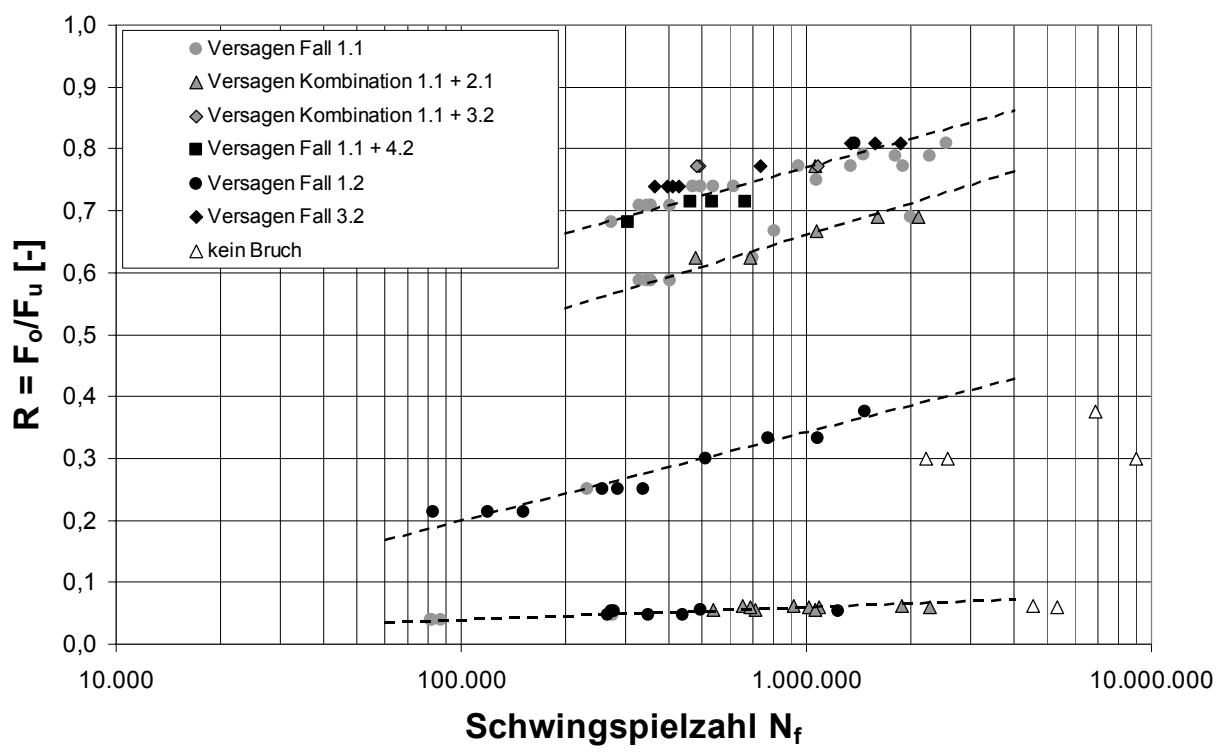

Bild 4.55 Versagensarten in Abhängigkeit vom Lastverhältnis R (Serie C)

Beidseitiges Versagen im Querschnitt der Ankerschiene stellt sich hauptsächlich bei geringen Lastniveaus ein, während stark einseitiges Versagen bzw. ein Ausriß des Ankerbolzens bei höheren Lastniveaus zu beobachten ist. Die ertragbaren Schwingbreiten bei $2 \cdot 10^{6}$ Schwingspielen weisen bei Versuchen mit konstanter Unterlast von 0,5 $\mathrm{kN}(\mathrm{R} \approx 0)$ stets höhere Tragfähigkeiten auf als bei Versuchen mit konstanter Oberlast von $17 \mathrm{kN}(\mathrm{R} \approx 0,75)$. Die Erhöhung der Ermüdungstragfähigkeit bei Durchführung der Versuche in einem Bereich von $\mathrm{R} \approx 0$ im Vergleich zu $\mathrm{R} \approx 0,75$ beträgt bei der Variante 25/50 (50/50) etwa $69 \%$ (51\%). In der Tabelle 4.19 wird der Einfluß des Lastniveaus auf die ertragbare Schwingbreite bei $2 \cdot 10^{6}$ Schwingspielen für den nicht einbetonierten Zustand der Ausführung 25/50 verdeutlicht.

Tabelle 4.19 Versuche mit konstanter Ober- und Unterlast (nicht einbetoniert, Serie C)

\begin{tabular}{|c|c|c|c|c|c|c|c|}
\hline Lastniveau & $\begin{array}{c}\text { Mittelw. } \Delta \mathrm{F} \\
\text { bei } 2 \cdot 10^{6} \\
{[\mathrm{kN}]}\end{array}$ & $\begin{array}{c}\sigma \\
{[\mathrm{kN}]}\end{array}$ & $\begin{array}{l}\mathrm{V} \\
{[\%]}\end{array}$ & $\begin{array}{l}\text { Steigung } \mathrm{m} \\
\text { Regression }\end{array}$ & Anzahl n & $\begin{array}{c}\text { Schwingbr. } \\
\Delta \mathrm{F}_{\mathrm{i}} \\
{[\mathrm{kN}]}\end{array}$ & $\begin{array}{c}\text { Anhang } \\
\text { Tab. }\end{array}$ \\
\hline $\mathrm{F}_{\mathrm{o}}=17 \mathrm{kN}$ & 4,38 & 0,22 & 5,08 & 3,89 & 15 & $4,0-7,0$ & A3 \\
\hline $\mathrm{F}_{\mathrm{u}}=0,5 \mathrm{kN}$ & 7,41 & 0,52 & 6,99 & 7,13 & 21 & $7,5-12,0$ & A6 \\
\hline $\mathrm{F}_{\mathrm{u}} / \mathrm{F}_{\mathrm{o}}$ & 1,69 & & & & & & \\
\hline
\end{tabular}


Somit kann gefolgert werden, daß es bei nicht einbetonierten, warmgewalzten Ankerschienen der neueren Generation einen Einfluß des Lastniveaus gibt.

Zusätzlich zum nicht einbetonierten Zustand sind Schwingversuche auch im einbetonierten Fall mit konstanter Unterlast von $0,5 \mathrm{kN}$ durchgeführt worden. Diese erfolgten an Ankerschienen der Serie C. Die zugehörigen Betondruckfestigkeiten entsprechen denen der Serie 3 in Tabelle 4.4.

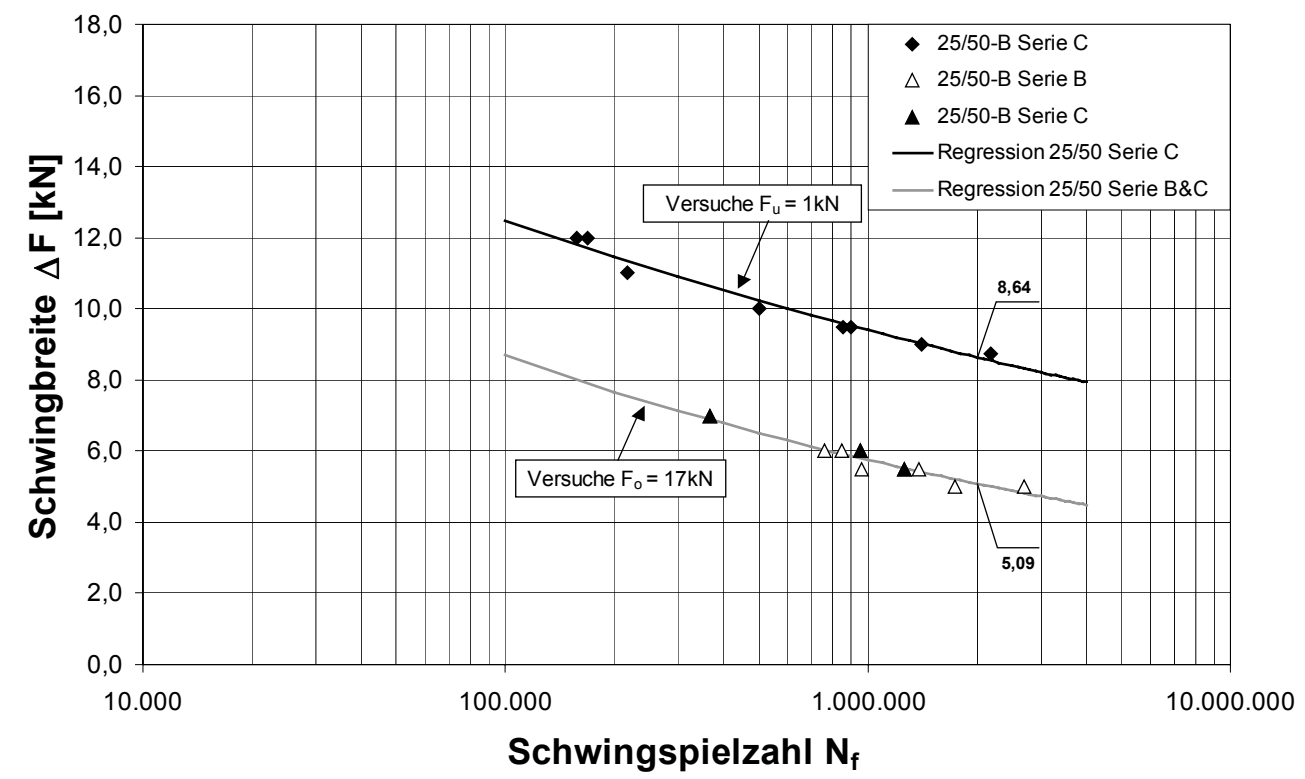

Bild 4.56 Ermüdungstragfähigkeit in Abhängigkeit vom Lastniveau (25/50-B Serie B\&C)

Wie zuvor in Bild 4.50 gezeigt, ist es möglich, eine gemeinsame Auswertung der Serien B und C vorzunehmen. Tabelle 4.20 zeigt die Ermüdungstragfähigkeit bei Variation des Lastniveaus für die Variante 25/50.

Tabelle 4.20 Versuche mit konstanter Ober- bzw. Unterlast (einbetoniert, Serie B und C)

\begin{tabular}{|c|c|c|c|c|c|c|c|}
\hline Lastniveau & $\begin{array}{c}\text { Mittelw. } \Delta \mathrm{F} \\
\text { bei } 2 \cdot 10^{6} \\
{[\mathrm{kN}]}\end{array}$ & $\begin{array}{c}\sigma \\
{[\mathrm{kN}]}\end{array}$ & $\begin{array}{l}\mathrm{V} \\
{[\%]}\end{array}$ & $\begin{array}{l}\text { Steigung } \mathrm{m} \\
\text { Regression }\end{array}$ & Anzahl $n$ & $\begin{array}{c}\text { Schwingbr. } \\
\Delta \mathrm{F}_{\mathrm{i}} \\
{[\mathrm{kN}]}\end{array}$ & $\begin{array}{c}\text { Anhang } \\
\text { Tab. }\end{array}$ \\
\hline$F_{u}=1 \mathrm{kN}$ & 8,64 & 0,18 & 2,09 & 8,18 & 8 & $8,75-12,00$ & A17 \\
\hline $\mathrm{F}_{\mathrm{o}}=17 \mathrm{kN}$ & 5,09 & 0,16 & 3,08 & 5,60 & 9 & $5,0-7,0$ & A18 \\
\hline $\mathrm{F}_{\mathrm{u}} / \mathrm{F}_{\mathrm{o}}$ & 1,70 & & & & & & \\
\hline
\end{tabular}

Bei Durchführung der Versuche mit konstanter Unterlast von etwa $1 \mathrm{kN}(\mathrm{R} \approx 0)$ erhält man für die einbetonierten Ankerschienen eine Erhöhung der Ermüdungstragfähigkeit im Vergleich zu den Versuchen mit konstanter Oberlast $(\mathrm{R} \approx 0,7)$ von etwa $70 \%$. Im nicht einbetonierten Fall entsprach diese Erhöhung etwa $69 \%$, welche somit vergleichbar ist und keine Abhängigkeit vom umgebenden Beton zeigt. Aus dem Vergleich dieser Werte kann gefolgert werden, daß die Erhöhung der Ermüdungstragfähigkeit infolge des Einflusses aus dem Lastniveau keine Abhängigkeit vom umgebenden Beton aufweist. 
Zur besseren Veranschaulichung des Einflusses des Lastniveaus eignet sich das Ermüdungsfestigkeitsdiagramm nach Goodman, welches bereits in Kapitel 3 erläutert wurde. Diese Darstellung erscheint bei Anwendungen, bei denen einer ruhenden eine nichtruhende Beanspruchung hinzugefügt wird, wie es in vielen Bereichen der Befestigungstechnik der Fall ist, sinnvoll. Bei Versuchen mit $\mathrm{R}>0$ kann stets von einer vorhandenen statischen Last ausgegangen werden. Anhand der Versuchsergebnisse an Ankerschienen der Serie C, sowohl im einbetonierten als auch im nicht einbetonierten Zustand, wird das Ermüdungsfestigkeitsdiagramm für eine definierte Schwingspielzahl von $2 \cdot 10^{6}$ in Bild 4.57 dargestellt. Die Mittelwerte der erzielten Schwingbreiten werden dabei auf den aktuellen Bemessungswert für die statische Tragfähigkeit des Profils 38/23 St von 12 kN bezogen.
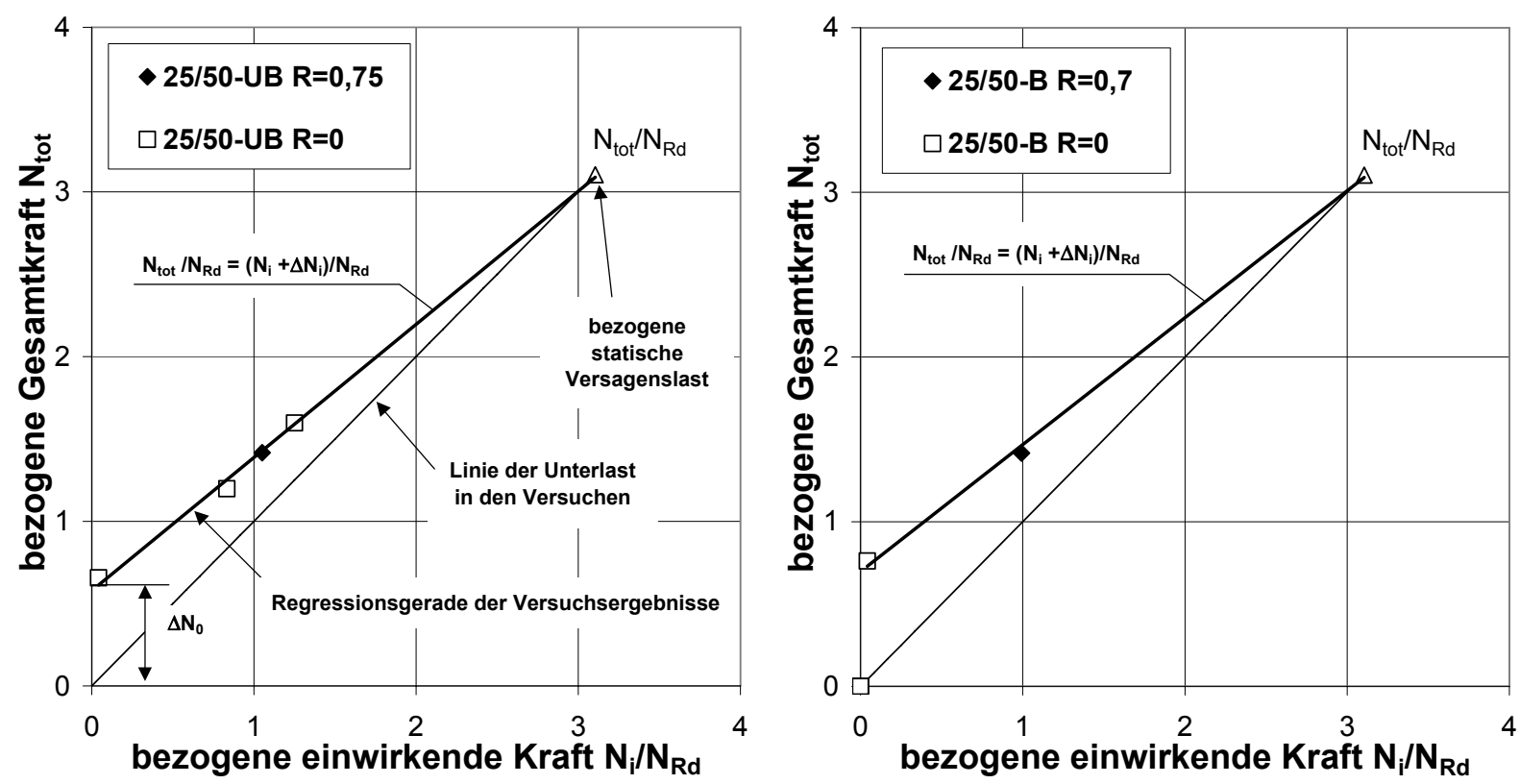

Bild 4.57 Ermüdungstragfähigkeit nach Goodman bei $2 \cdot 10^{6}$ Schwingspielen (Serie C)

Auf der Abszisse ist das Verhältnis der einwirkenden Kraft $\mathrm{N}_{\mathrm{i}}$ zur aktuellen Bemessungskraft $\mathrm{N}_{\mathrm{Rd}}$ laut [21], [23] aufgetragen. Auf der Ordinate ist die bezogene Gesamtkraft $\mathrm{N}_{\text {tot }}$ in zentrischer Zugrichtung dargestellt. Diese setzt sich aus der statisch wirkenden Last $\mathrm{N}_{\mathrm{i}}$ als Unterlast und der dazugehörigen Schwingbreite $\Delta \mathrm{N}_{\mathrm{i}}$ zusammen. Die Linie der ruhenden Last geht durch den Ursprung und verläuft im $45^{\circ}$ Winkel bis zur bezogenen statischen Versagenslast. Im Abstand zur ruhenden Last, entsprechend dem Mittelwert der ertragbaren Schwingbreite (parallel zur Ordinate), sind die einzelnen Versuchsergebnisse aufzufinden. Sind nur die statische Tragfähigkeit $\mathrm{N}_{\text {tot }}$ und die Schwingbreite $\Delta \mathrm{N}_{\mathrm{o}}$ bei ausschließlicher Ursprungsbeanspruchung bekannt, kann vereinfacht eine lineare Verbindung zwischen diesen Punkten angenommen werden, welche durch die Versuchsergebnisse in Bild 4.57 bestätigt wird. Es ist nun deutlich zu erkennen, daß mit Zunahme der Unterlast die Differenz zur bezogenen Gesamtkraft geringer wird und somit die ertragbare Schwingbreite abnimmt. 


\subsubsection{Tragverhalten unter Querbeanspruchungen}

\subsubsection{Statische Versuchsreihe}

Neben der reinen zentrischen Zugrichtung sind zusätzlich statische Versuche sowohl in als auch quer zur Schienenlängsrichtung $\left(\mathrm{V}_{\mathrm{x}}, \mathrm{V}_{\mathrm{y}}\right)$ durchgeführt worden. Für die Beanspruchung in Schienenlängsrichtung kamen einbetonierte Ankerschienen zur Anwendung. Die maßgebende Versagensart stellte sich in der Schienenverzahnung ein (Versagenszustand in Bild 4.16, links).

Die Beanspruchung quer zur Schienenlängsrichtung erfolgte ebenfalls im einbetonierten Zustand. Auch in diesem Fall war das Stahlversagen maßgebend, wobei der Beton wesentlich zum Tragverhalten betrug. Die resultierende Versagensart kann dem Bild 4.16 (rechts) entnommen werden.

Die Versuche wurden nur an Ankerschienen der Ausführung 25/50 durchgeführt.

Tabelle 4.21 Statische Tragfähigkeiten von Ankerschienen unter $90^{\circ}$-Querbeanspruchung

\begin{tabular}{|c||c|c|c|c|}
\hline \multicolumn{1}{|c|}{} & \multicolumn{2}{|c|}{$\begin{array}{c}\text { Beanspruchung } \mathrm{V}_{\mathrm{x}} \text { in } \\
\text { Schienenlängsrichtung }\end{array}$} & \multicolumn{2}{c|}{$\begin{array}{c}\text { Beanspruchung } \mathrm{V}_{\mathrm{y}} \text { quer zur } \\
\text { Schienenlängsrichtung }\end{array}$} \\
\cline { 2 - 5 } & \multicolumn{2}{|c|}{ nicht einbetoniert } & \multicolumn{2}{|c|}{ einbetoniert } \\
\cline { 2 - 5 } & $100 \%$ Vorspannung & \multicolumn{2}{c|}{$100 \%$ Vorspannung } \\
\cline { 2 - 5 } & $\mathrm{V}_{\mathrm{x}}$ & $\mathrm{f}_{\mathrm{cm}, \mathrm{cube}}$ & $\mathrm{V}_{\mathrm{y}}$ & $\mathrm{f}_{\mathrm{cm}, \mathrm{cube}}$ \\
\cline { 2 - 5 } & {$[\mathrm{kN}]$} & {$\left[\mathrm{N} / \mathrm{mm}^{2}\right]$} & {$[\mathrm{kN}]$} & {$\left[\mathrm{N} / \mathrm{mm}^{2}\right]$} \\
\hline 1 & 39,49 & 38,47 & 71,06 & 42 \\
\hline 2 & 40,22 & 38,47 & 76,81 & 42 \\
\hline 3 & 40,50 & 38,47 & 78,67 & 42 \\
\hline MW & 40,07 & 38,47 & 75,51 & \\
\hline
\end{tabular}

Weitere Versuchswerte in Kombination mit unterschiedlichen Schraubentypen und Vorspannungen der Schraube können [50], [51], [52] entnommen werden.

\subsubsection{Schwingversuche bei Beanspruchungen in Querrichtung}

Der aktuelle Anwendungsbereich von Ankerschienenbefestigungen sieht nichtruhende Beanspruchung lediglich in zentrischer Zugrichtung vor. Eine nichtruhende Beanspruchung in Querrichtung $\left(90^{\circ}\right)$ darf zur Zeit nicht angesetzt werden.

Zusätzlich zu der reinen zentrischen Zugrichtung sollten daher Versuche in Querrichtung Aufschluß über das Tragverhalten unter Querbeanspruchung geben. Die nichtruhende, periodische Beanspruchung erfolgte sowohl in Schienenlängsrichtung als auch quer dazu. Für die Beanspruchung quer zur Schienenlängsrichtung kamen Ankerschienen der Serie B zur Anwendung, wobei für eine Beanspruchung in Schienenlängsrichtung Ankerschienen der Serie $\mathrm{C}$ eingesetzt wurden. Die Versuche erfolgten ausschließlich an einbetonierten Ankerschienen der Ausführung 25/50. Der umgebende Beton der Ankerschienen der Serie B entsprach den Betondruckfestigkeiten der Serie 2 in Tabelle 4.5. Die Ankerschienen der Serie $\mathrm{C}$ waren in Beton eingebunden, 
dessen Festigkeit der Serie 3 und zusätzlich den Festigkeitsklassen B25 bzw. B55 aus der Lieferung des Fertigbetons entsprach. Die Versuche für die Ankerschienen der Serie B erfolgten ausschließlich unter konstanter Oberlast von $17 \mathrm{kN}$ und einer variablen Schwingbreite, wobei für die Serie C hauptsächlich konstante Unterlasten von 1 $\mathrm{kN}$ eingestellt wurden.

\section{Einfluß des umgebenden Betons bei Querbeanspruchung}

Bei Beanspruchung quer zur Schienenlängsrichtung $\mathrm{V}_{\mathrm{y}}$ war durch eine konstante Oberlast in den Versuchen der Beanspruchungsbereich mit $17 \mathrm{kN}$ nach oben begrenzt. Im reinen Zugschwellbereich waren somit Schwingbreiten lediglich kleiner als $17 \mathrm{kN}$ möglich. Diese reichten jedoch nicht aus, um ein Versagen hervorzurufen. Von insgesamt sechs untersuchten Versuchskörpern kam es nur einmal bei einer Schwingbreite von $15 \mathrm{kN}$ und einer Schwingspielzahl von etwa $1,9 \cdot 10^{6}$ zu einer Ermüdungserscheinung. Die restlichen Versuche, auch mehrmals bei einer Schwingbreite von $16 \mathrm{kN}$ getestet, führten bei Schwingspielzahlen deutlich über $4 \cdot 10^{6} \mathrm{zu}$ keiner Versagenserscheinung. Somit kann gefolgert werden, daß quer zur Schienenlängsrichtung die Oberlast von $17 \mathrm{kN}$ und die maximal mögliche Schwingbreite von etwa $17 \mathrm{kN}$ nicht ausreichend erscheint, um ein Versagen zu erzeugen. Auf der sicheren Seite liegend kann die Schwingbreite von $15 \mathrm{kN}$ als abgesicherter Mindestwert bei $2 \cdot 10^{6}$ Schwingspielen angesehen werden. Der Einfluß des umgebenden Betons wurde für diese Beanspruchung nicht untersucht, weshalb weitere systematische Untersuchungen notwendig sind.

Bei Beanspruchung in Schienenlängsrichtung $\mathrm{V}_{\mathrm{x}}$ hingegen, war es aufgrund der konstanten Unterlast möglich, die Oberlast variabel einzustellen und somit in jedem Fall ein Versagen zu erhalten. Als Versagensart unter Verwendung des Fertigbetons B55 wurden hauptsächlich Risse in den Übergangsbereichen des Schienenrückens zum Schienenschenkel festgestellt. Diese traten sowohl einseitig als auch beidseitig auf (Fall 1.1 bzw. Fall 1.2). Lediglich einmal trat Versagen infolge eines kompletten Ausrisses des Ankerbolzens aus dem Rücken auf. Eine Kombination von Rissen im Übergangsbereich des Schienenrückens zum Schienenschenkel und im Übergangsbereich von Schienenschenkel zur Schienenverzahnung auf der gegenüberliegenden Seite wurde ebenfalls nur einmal beobachtet. Für Ankerschienen im Beton der Festigkeitsklasse B25 konnte nur Versagensfall 1.2 festgestellt werden. Das nachfolgende Diagramm in Bild 4.58 zeigt die ertragbaren Schwingbreiten in Abhängigkeit von der Festigkeitsklasse. Dem Diagramm ist eindeutig zu entnehmen, daß die Druckfestigkeit des umgebenden Betons einen maßgebenden Einfluß auf die ertragbare Schwingbreite bei Beanspruchung in Schienenlängsrichtung hat. Beim Vergleich der Verläufe der Ermüdungstragfähigkeiten stellt man fest, daß diese für die Versagensart Fall 1.2 ähnliche Steigungen aufweisen. Die ertragbare Schwingbreite bei $2 \cdot 10^{6}$ Schwingspielen beträgt für den Beton B55 etwa 21,24 kN, während sie für den Beton der Festigkeitsklasse B25 etwa $16,71 \mathrm{kN}$ beträgt. Im Falle eines Festigkeitsverlustes von etwa $30 \%$ sind somit Einbußen der Ermüdungstragfähigkeit von etwa $27 \%$ im Mittel zu erwarten. 


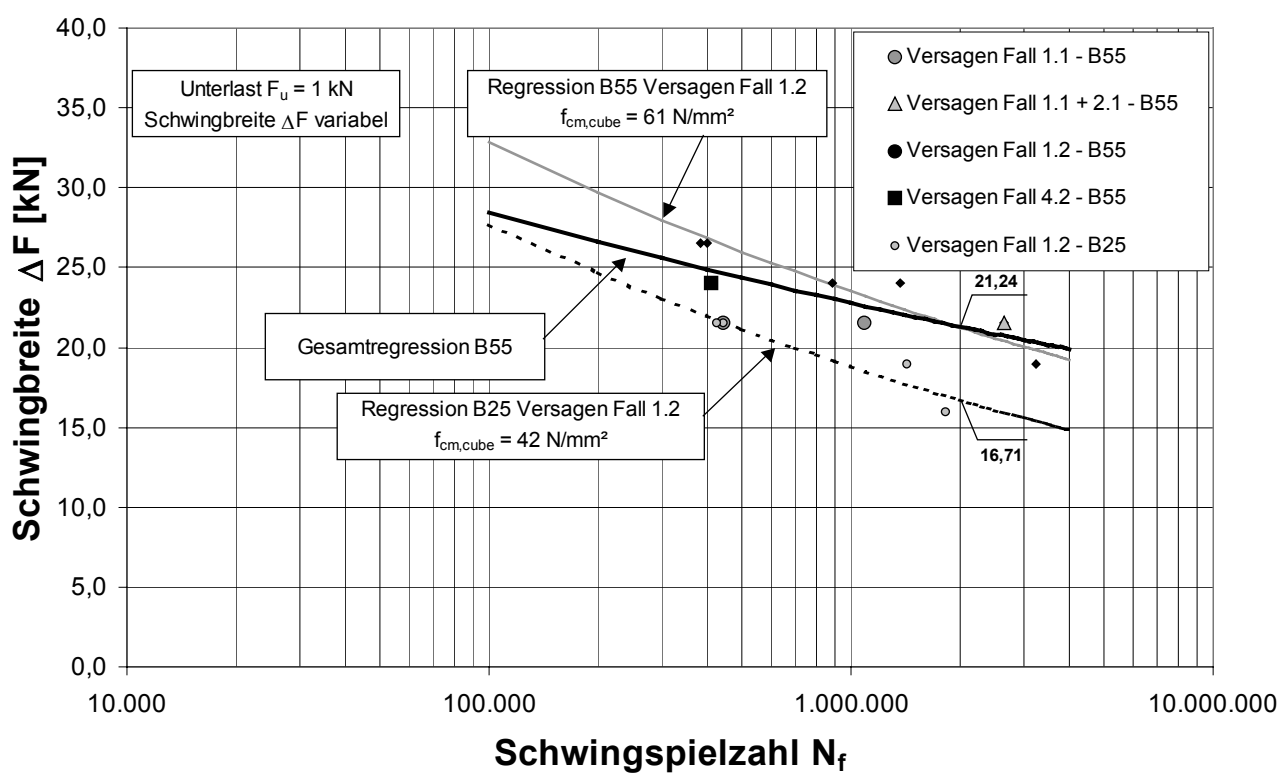

Bild 4.58 Schwingbreite als Funktion der Schwingspielzahl (Serie C)

In Tabelle 4.22 sind die Ergebnisse übersichtlich für das beidseitige Versagen im Übergangsbereich des Schienenrückens zum -schenkel (Fall 1.2) und eine Gesamtauswertung aller Versuchsergebnisse der Festigkeitsklasse B55 dargestellt.

Tabelle 4.22 Ergebnisse der Serie C für den Versagensfall 1.2

\begin{tabular}{|c|c|c|c|c|c|c|c|}
\hline Variante & $\begin{array}{c}\text { Mittelw. } \Delta \mathrm{F} \\
\text { bei } 2 \cdot 10^{6} \\
{[\mathrm{kN}]}\end{array}$ & $\begin{array}{c}\sigma \\
{[\mathrm{kN}]} \\
\end{array}$ & $\begin{array}{c}\mathrm{V} \\
{[\%]} \\
\end{array}$ & $\begin{array}{l}\text { Steigung } \mathrm{m} \\
\text { Regression }\end{array}$ & Anzahl n & $\begin{array}{c}\text { Schwingbr. } \\
\qquad \mathrm{F}_{\mathrm{i}} \\
{[\mathrm{kN}]}\end{array}$ & $\begin{array}{c}\text { Anhang } \\
\text { Tab. }\end{array}$ \\
\hline Serie C - B25 & 16,72 & 0,91 & 5,45 & 5,91 & 4 & $16,0-21,5$ & A20 \\
\hline Serie C - B55 & 21,26 & 0,88 & 4,13 & 6,89 & 5 & $19,0-26,5$ & A21 \\
\hline B55 Gesamt & 21,35 & 1,16 & 5,44 & 8,48 & 8 & $19,0-26,5$ & A22 \\
\hline$f_{B 55-B 25}$ & 1,27 & & & & & & \\
\hline
\end{tabular}

Bei gemeinsamer Betrachtung aller Versagensfälle der Serie C der Festigkeitsklasse B55 ergibt sich ein Mittelwert der ertragbaren Schwingbreite bei $2 \cdot 10^{6}$ Schwingspielen von $21,32 \mathrm{kN}$, welcher vergleichbar ist mit den Ergebnissen der Versuche, bei denen ausschließlich Versagensfall 1.2 betrachtet wird.

Diese Erkenntnis zeigt, daß die Betonfestigkeit bei dieser Beanspruchungsart in jedem Fall in Betracht gezogen werden muß. Unter der Annahme, daß sich das Verhalten der Ermüdungstragfähigkeit für den Fall 1.2 nicht ändert, würde nach einer Extrapolation für eine Betondruckfestigkeit von $25 \mathrm{~N} / \mathrm{mm}^{2}$ eine ertragbare Schwingbreite von etwa $12,66 \mathrm{kN}$ resultieren. Da jedoch die Rißentwicklung bei niedrigeren Schwingbreiten nach [94] auch langsamer fortschreitet, würden höhere Schwingspielzahlen und somit höhere ertragbare Schwingbreiten erreicht werden. Die Schwingbreite von 12, 66 kN stellt somit eine auf der sicheren Seite liegende Annahme dar. 


\section{Einfluß des Lastniveaus auf die Tragfähigkeit}

Neben dem Betoneinfluß bei Beanspruchung in Querrichtung ist auch das Lastniveau eine weitere Einflußgröße auf das Tragverhalten unter nichtruhender Beanspruchung. Im Rahmen dieser Arbeit sind für diese Belastungsrichtungen keine zusätzlichen Versuche mit kombinierter ruhender und nichtruhender Beanspruchung durchgeführt worden, um den Einfluß aus dem Lastniveau zu ermitteln. Jedoch kann auf der Grundlage des Goodman-Diagramms ein linearer Zusammenhang zwischen der vorwiegend ruhenden Beanspruchung $\mathrm{V}$ und der vorwiegend nichtruhenden Beanspruchung $(\mathrm{R} \approx 0)$ vereinfachend angenommen werden. Diese auf der sicheren Seite liegende Annahme wurde bereits bei der kritischen zentrischen Zugrichtung bestätigt und wird daher auf die Querrichtung übertragen.
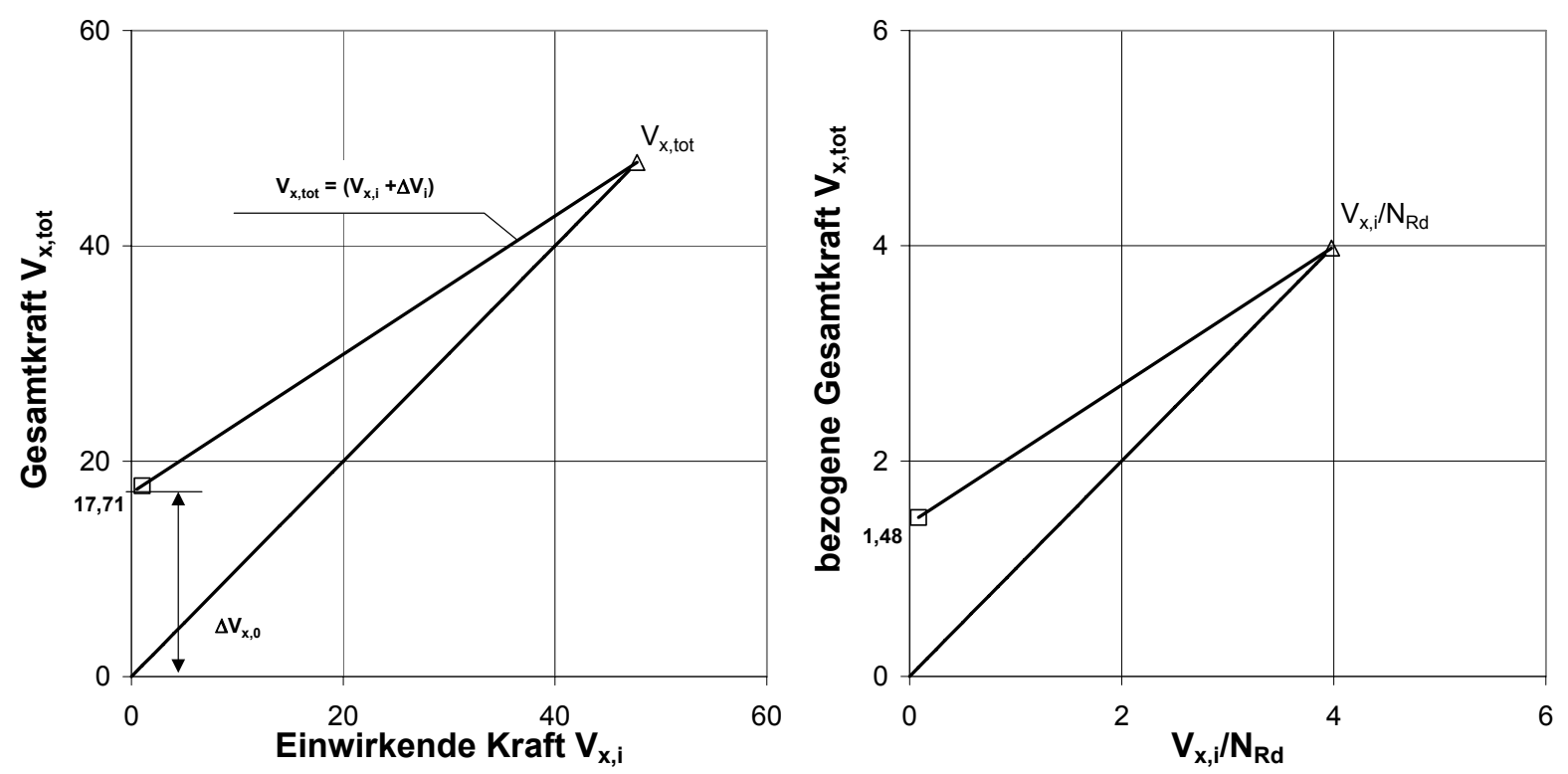

Bild 4.59 Ermüdungstragfähigkeit nach Goodman bei $N_{f}=2 \cdot 10^{6}$ für Beanspruchung $V_{x, \text { tot }}$

Bei Querbeanspruchung in Schienenlängsrichtung legt sich der Querschnitt der Ankerschiene an den Beton an und erzeugt somit hohe Spannungskonzentrationen an den Kontaktflächen, welche durchaus das Tragverhalten beeinflussen können. Bis zur Entstehung einer Schädigung an der Kontaktfläche zum Beton kann ein linearer Verlauf der Goodman-Geraden auf der sicheren Seite liegend angenommen werden. Schädigungen am Beton in Form von oberflächennahen Betonabplatzungen machten sich erst gegen Versuchsende bemerkbar.

In ähnlicher Weise ist das Ermüdungstragfähigkeitsdiagramm bei einer Schwingspielzahl von $2 \cdot 10^{6}$ für die Beanspruchung quer zur Schienenlängsrichtung $\mathrm{V}_{\mathrm{y}}$ in Bild 4.60 konstruiert worden. Auffällig sind hierbei die hohen statischen Tragfähigkeiten in Querrichtung. 

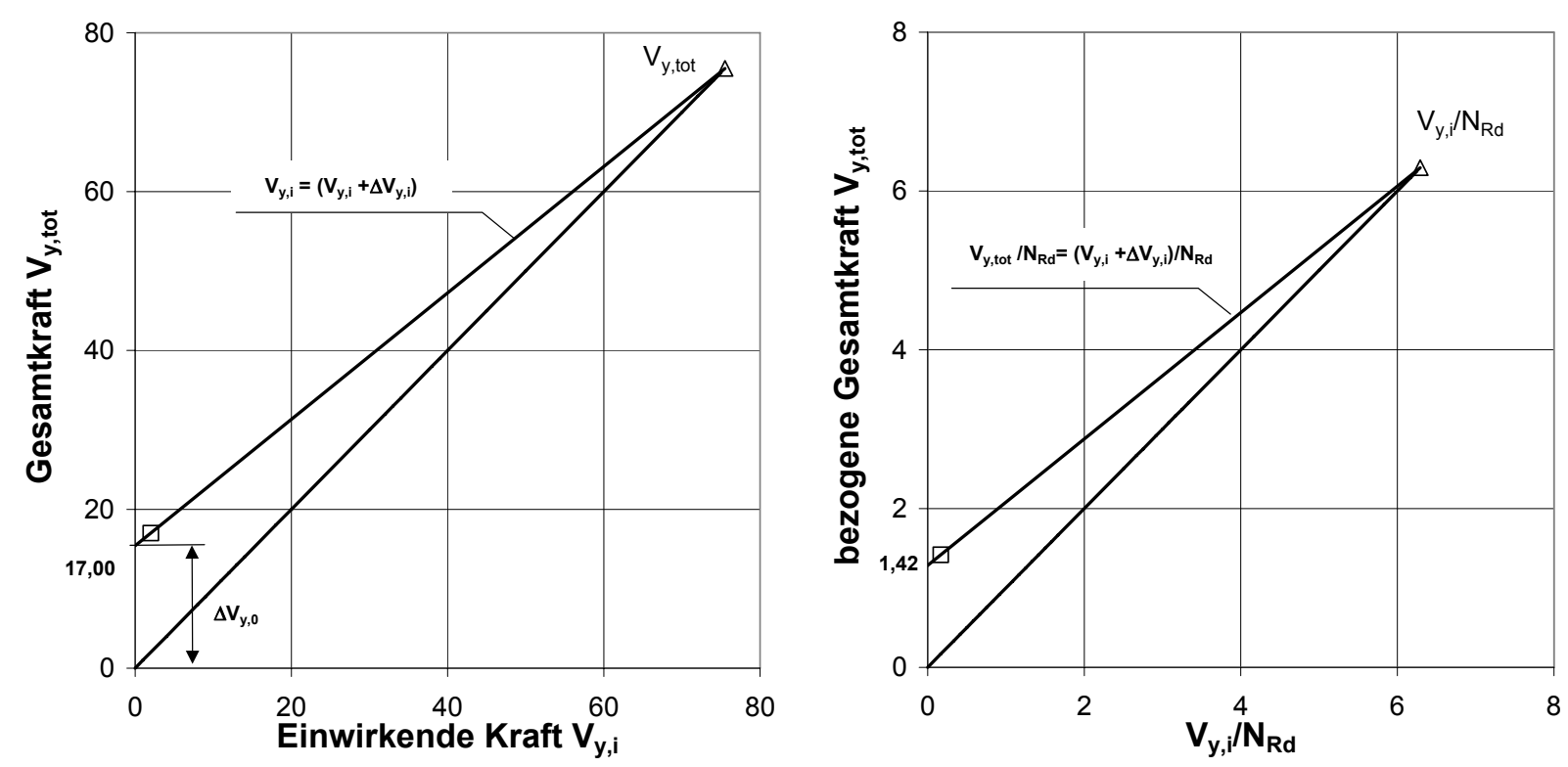

Bild 4.60 Ermüdungstragfähigkeit nach Goodman bei $N_{f}=2 \cdot 10^{6}$ für Beanspruchung $V_{y, t o t}$

Zur Verdeutlichung des Tragverhaltens bei Beanspruchung in Schienenlängsrichtung $\left(\mathrm{V}_{\mathrm{x}}\right)$ sind weiterhin die Dehnungen an den Außenflächen der Schienenschenkel gemessen worden. Die Positionierungen der Dehnungsmeßstreifen sind in Bild 4.6 abgebildet. Das Tragverhalten kann als repräsentativ für die Beanspruchung in Schienenlängsrichtung betrachtet werden. Bild 4.61 zeigt den Verlauf der Dehnungen in Schienenlängsrichtung über die Zeitpunkte der Einzelmessungen vor dem Beginn der Schwingbeanspruchung.

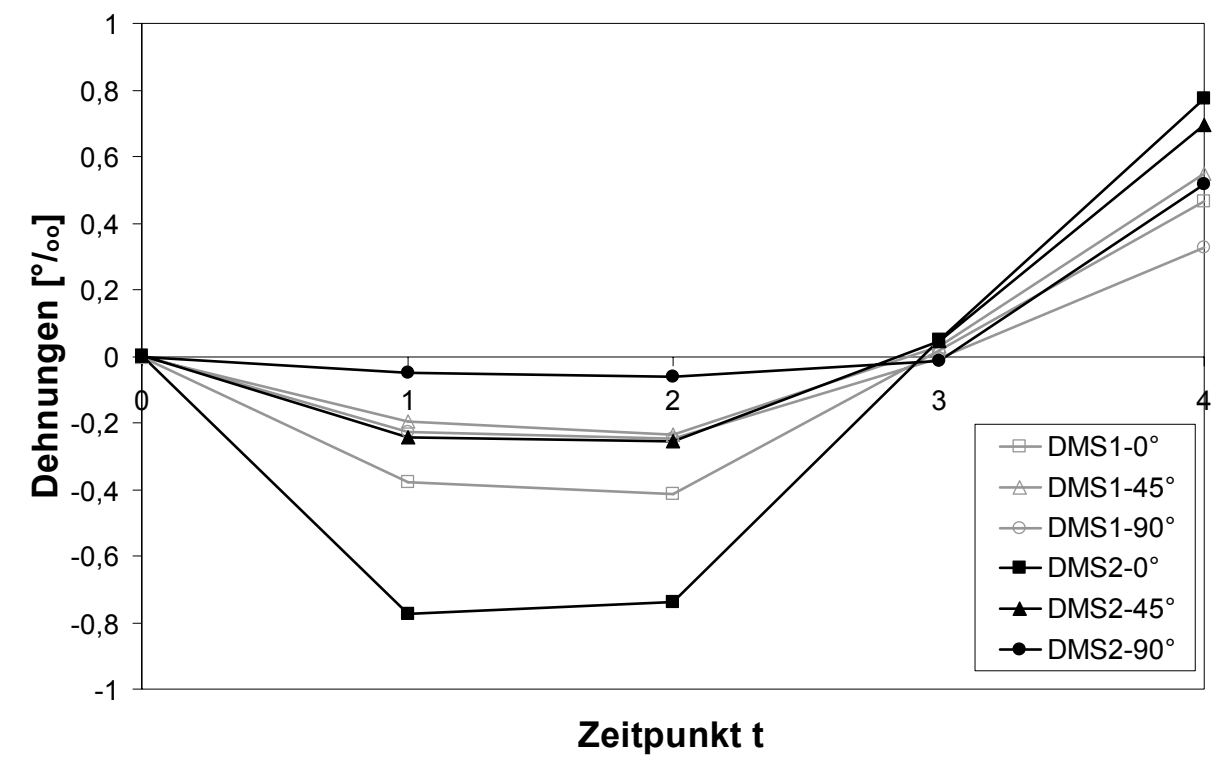

Bild 4.61 Mittelwert der Dehnungen vor Start der Schwingbeanspruchung 
Nachfolgend sind die einzelnen Meßvorgänge kurz erläutert.

- Zeitpunkt $\mathrm{t}=0$ : Nullabgleich der Meßstellen DMS1 und DMS2, keine Belastung.

- $\mathrm{t}=1$ : Aufbringen eines Anzugsdrehmomentes von $120 \mathrm{Nm}$, keine Belastung.

- $\mathrm{t}=2$ : Anbringen von horizontalen $\mathrm{u}$ und vertikalen $\mathrm{w}$ Wegaufnehmern mit gleichzeitigem Nullabgleich. Keine Belastung an der Ankerschiene.

- $\mathrm{t}=2-3$ : statische Beanspruchung bis zur Oberlast von $17 \mathrm{kN}$ und anschließender Entlastung bis auf $0 \mathrm{kN}$.

- $\mathrm{t}=4$ : statische Beanspruchung bis zur Mittellast von $9 \mathrm{kN}$. Anschließend erfolgt der Schwingversuch.

Sowohl DMS1 als auch DMS2 zeigen zwischen den Zeitpunkten $t=1-3$ durchweg negative Dehnungen an, die einer Druckbeanspruchung entsprechen. Auffällig ist der Abfall der Dehnungen zwischen dem Zeitpunkt $t=2-3$. Die zuvor durch das Anzugsdrehmoment aufgebaute Dehnung fällt nach Aufbringen der statischen Vorbelastung auf nahezu $0 \%$ ab, obwohl diese weiterhin im System vorhanden ist. Dieser Umstand ist auf die Entlastungen bzw. den Abbau der Dehnungen infolge von Druckbeanspruchungen des umgebenden Betons zurückzuführen. Nach dem Aufbringen der Mittellast und der anschließenden Schwingbeanspruchung stellt sich der in Bild 4.62 dargestellte Verlauf ein.

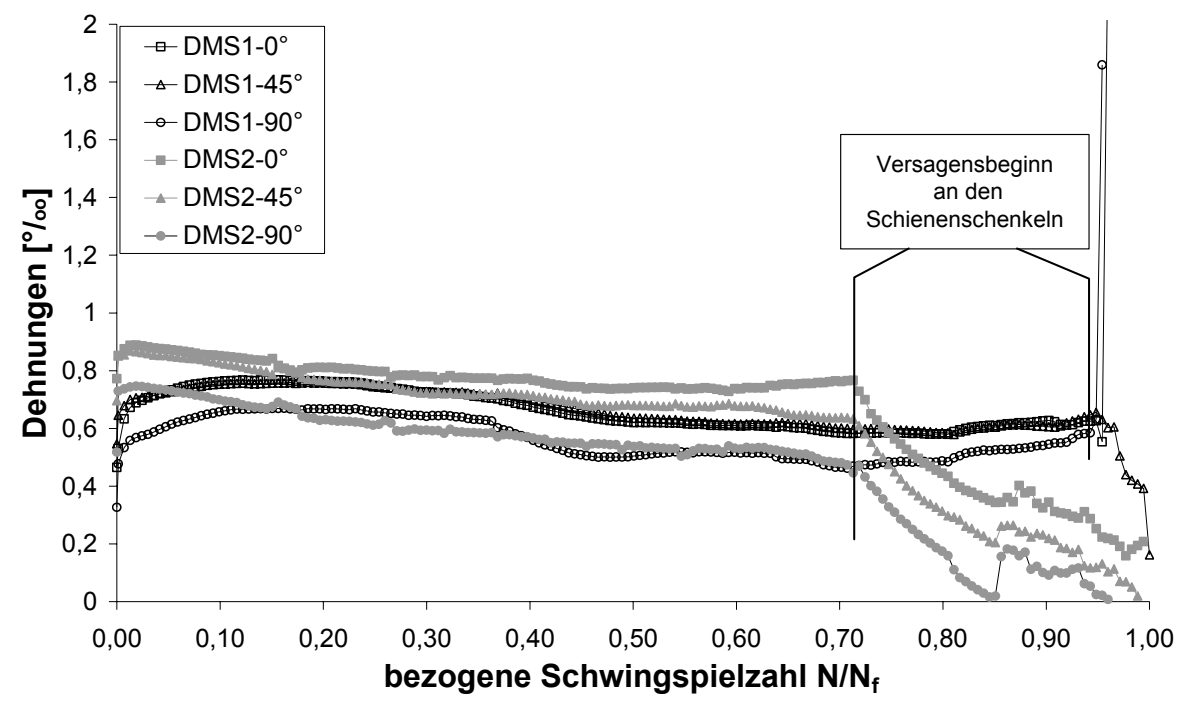

Bild 4.62 Mittelwert der Dehnungen während der Schwingbeanspruchung

$\mathrm{Zu}$ Beginn der Schwingbeanspruchung erfolgt eine leichte Erhöhung aller Dehnungen um etwa 10 bis $20 \%$, welche nach etwa $72 \%$ der Bruchschwingspielzahl wieder nahezu auf den Ausgangswert zurückfallen. Danach zeigt sich ein einseitiger Versagensbeginn am Schienenschenkel (DMS2) durch einen stabilen Abfall der Zugdehnungen bis etwa 85 bis $95 \%$ der bezogenen Bruchschwingspielzahl. Eine Umlagerung auf den gegenüberliegenden Schenkel macht sich in diesem Bereich nicht bemerkbar. Sie erfolgt vielmehr in den umgebenden Beton. Erst ab einer bezogenen Bruchschwingspielzahl von $95 \%$ zeigen sich starke Dehnungsänderungen auf der 
gegenüberliegenden Seite, welche auf die Entstehung von Rissen zurückzuführen sind.

Zusätzlich zum Dehnungsverhalten ist das Tragverhalten unter schwingender Beanspruchung infolge von Verformungen näher verifiziert worden. Dazu wurden diese in Schienenlängsrichtung $u$ und in Richtung der Schraubenachse w aufgezeichnet. Die Positionen der Wegaufnehmer können Bild 4.12 entnommen werden. Die Diagramme in Bild 4.63 und Bild 4.64 zeigen die Differenzen der Verformungen $\Delta \mathrm{u}$ und $\Delta \mathrm{w}$ über die bezogene Schwingspielzahl N/N .

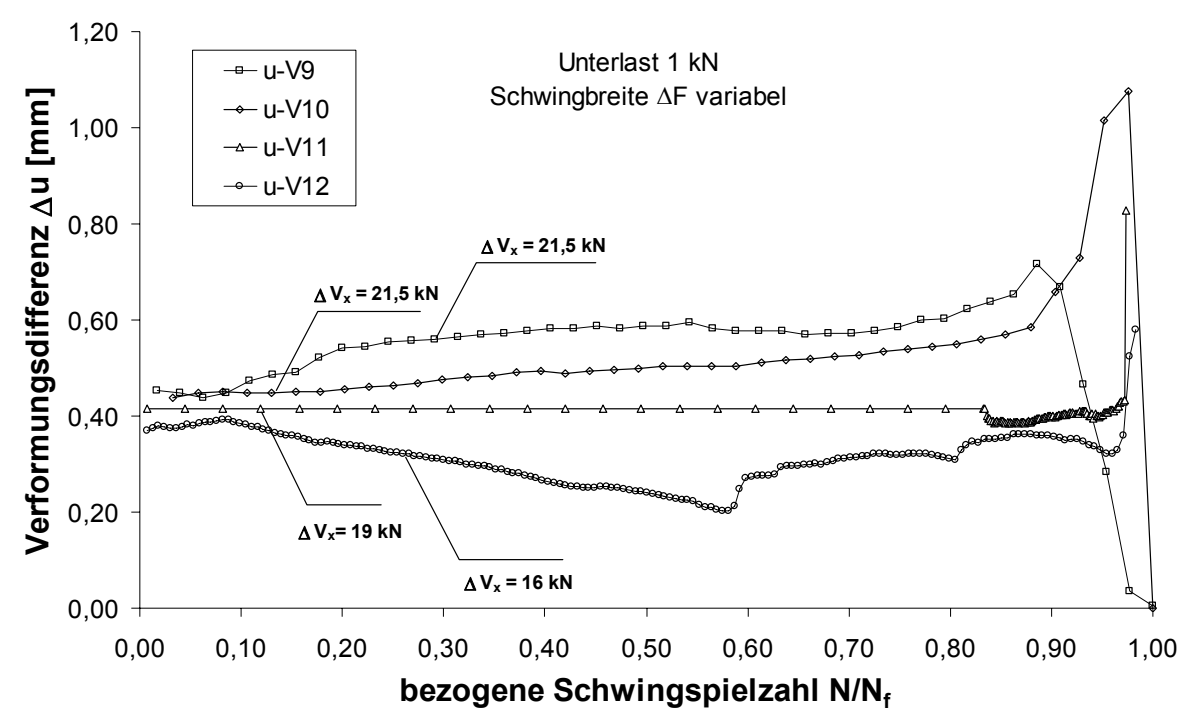

Bild 4.63 Verformungsdifferenz $\Delta u$ über bezogene Schwingspielzahl (Serie C - B25)

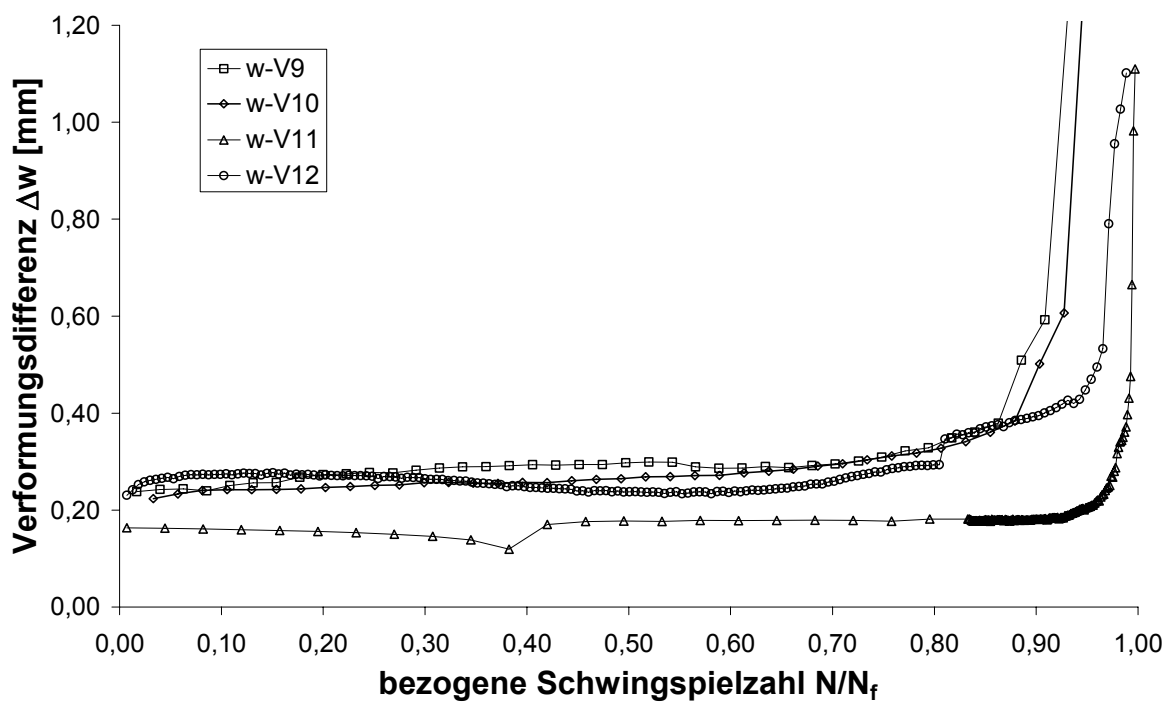

Bild 4.64 Verformungsdifferenz $\Delta \mathrm{W}_{2}$ über bezogene Schwingspielzahl (Serie C - B25)

Eine deutliche Zunahme der Verformung in horizontaler Richtung macht sich erst ab einer bezogenen Schwingspielzahl von etwa 80 bis $90 \%$ bemerkbar. Dieses Ergebnis bestätigt die Erkenntnisse aus den Dehnungsmessungen. 


\subsubsection{Kombinierte Zug- und Querbeanspruchungen}

\subsubsection{Statische Versuchsreihe}

Für die untersuchten Ankerschienen in Verbindung mit einer Zahnschraube ist laut [21], [23] eine ruhende Beanspruchung in der Größe entsprechend der zulässigen Last in allen Belastungsrichtungen möglich. Der Interaktionsverlauf zwischen der zentrischen Zugbelastung, einer Querbeanspruchung in Schienenlängsrichtung bzw. quer dazu, kann somit auf der Basis des Bemessungswertes kugelförmig angesetzt werden. Bei Verwendung einer Standardschraube ist eine Interaktion zwischen zentrischem Zug und Schub quer zur Längsrichtung erlaubt (Bild 2.9).

Um einen Zusammenhang zwischen der reinen zentrischen Zugrichtung und der reinen Querbeanspruchung herstellen zu können, sind Versuche unter variierenden Beanspruchungswinkeln durchgeführt worden. Somit gelingt es für den statischen Fall, ein Interaktionsdiagramm aufzubauen.

Tabelle 4.23 Mittelwerte der Tragfähigkeit unter variierenden Beanspruchungswinkeln

\begin{tabular}{|c|c|c|c|}
\hline \multirow{5}{*}{$\begin{array}{c}\text { Winkel } \varphi \\
{\left[{ }^{\circ}\right]}\end{array}$} & $\begin{array}{l}\text { Beanspruchung in } \\
\text { Schienenlängsrichtung }\end{array}$ & \multirow{2}{*}{\multicolumn{2}{|c|}{$\begin{array}{l}\text { Beanspruchung quer zur } \\
\text { Schienenlängsrichtung }\end{array}$}} \\
\hline & nicht einbetoniert & & \\
\hline & $50 \%$ Vorspannung & \multicolumn{2}{|c|}{$100 \%$ Vorspannung } \\
\hline & $\mathrm{V}_{\mathrm{x}-\mathrm{z}}$ & $V_{y-z}$ & $\mathrm{f}_{\mathrm{cm}, \text { cube }}$ \\
\hline & {$[\mathrm{kN}]$} & {$[\mathrm{kN}]$} & {$\left[\mathrm{N} / \mathrm{mm}^{2}\right]$} \\
\hline 30 & "--- & 37,69 & 32,62 \\
\hline 45 & 69,71 & 40,27 & 37,70 \\
\hline 60 & --- & 42,40 & 35,30 \\
\hline
\end{tabular}

Weitere Versuchsergebnisse in Abhängigkeit vom Schraubentyp, vom Drehmoment und von der Stahlsorte können [50], [51], [52] entnommen werden.

Zur grafischen Darstellung der gesamten Ergebnisse eignet sich die Darstellungsweise im Interaktionsdiagramm. Die nichtlineare Interaktionsbeziehung ist in Gleichung (4.10) aufgezeigt. Hierbei ist sowohl der Anteil aus der zentrischen Zugrichtung $\mathrm{N}_{\mathrm{i}}$, als auch der Anteil aus der Querrichtung $V_{i}$ auf den Wert der ertragbaren Last in zentrische Zugkraft $\mathrm{N}_{\mathrm{u}}$ bezogen worden. Somit wird in dieser Form zunächst eine von der zentrischen Zugrichtung abhängige Darstellungsweise erzielt.

$$
\left(\frac{1}{\mathrm{a}} \cdot \frac{\mathrm{N}_{\mathrm{i}}}{\mathrm{N}_{\mathrm{u}}}\right)^{\alpha_{\text {stat }}}+\left(\frac{1}{\mathrm{~b}} \cdot \frac{\mathrm{V}_{\mathrm{i}}}{\mathrm{N}_{\mathrm{u}}}\right)^{\alpha_{\text {stat }}} \leq 1,\left(\frac{1}{\mathrm{a}} \cdot \frac{\mathrm{V}_{\mathrm{x}, \mathrm{i}}}{\mathrm{N}_{\mathrm{u}}}\right)^{\alpha_{\text {stat }}}+\left(\frac{1}{\mathrm{~b}} \cdot \frac{\mathrm{V}_{\mathrm{y}, \mathrm{i}}}{\mathrm{N}_{\mathrm{u}}}\right)^{\alpha_{\text {stat }}} \leq 1
$$

Der Grund der Form diese Darstellungsweise aus [38] liegt darin, daß die zentrische Zugrichtung als kritisch betrachtet wird und somit eine zu dieser Richtung relative Abhängigkeit aufgezeigt werden kann. Die Werte a und b sind Kennwerte des Achsenabschnittes auf der Hauptachse x und der Nebenachse y. Der Formfaktor $\alpha_{\text {stat }}$ gibt die Form des Interaktionsdiagramms an. Ziel einer Interaktionsgleichung ist es, die 
ermittelten Versuchsergebnisse relativ genau abzubilden. Für den Formfaktor $\alpha_{\text {stat }}=1$ nimmt die Interaktionsgleichung die Form einer Geraden an. Bei einem Formfaktor kleiner als 1 stellt sich ein konkaver und bei Faktoren größer als 1 ein konvexer Kurvenverlauf ein. Bei einem Formfaktor von 2 wird für den Fall der auf die Einheitslänge 1 normierten Interaktionsgleichung ein Kreisverlauf erzielt, ansonsten resultiert eine Ellipse.
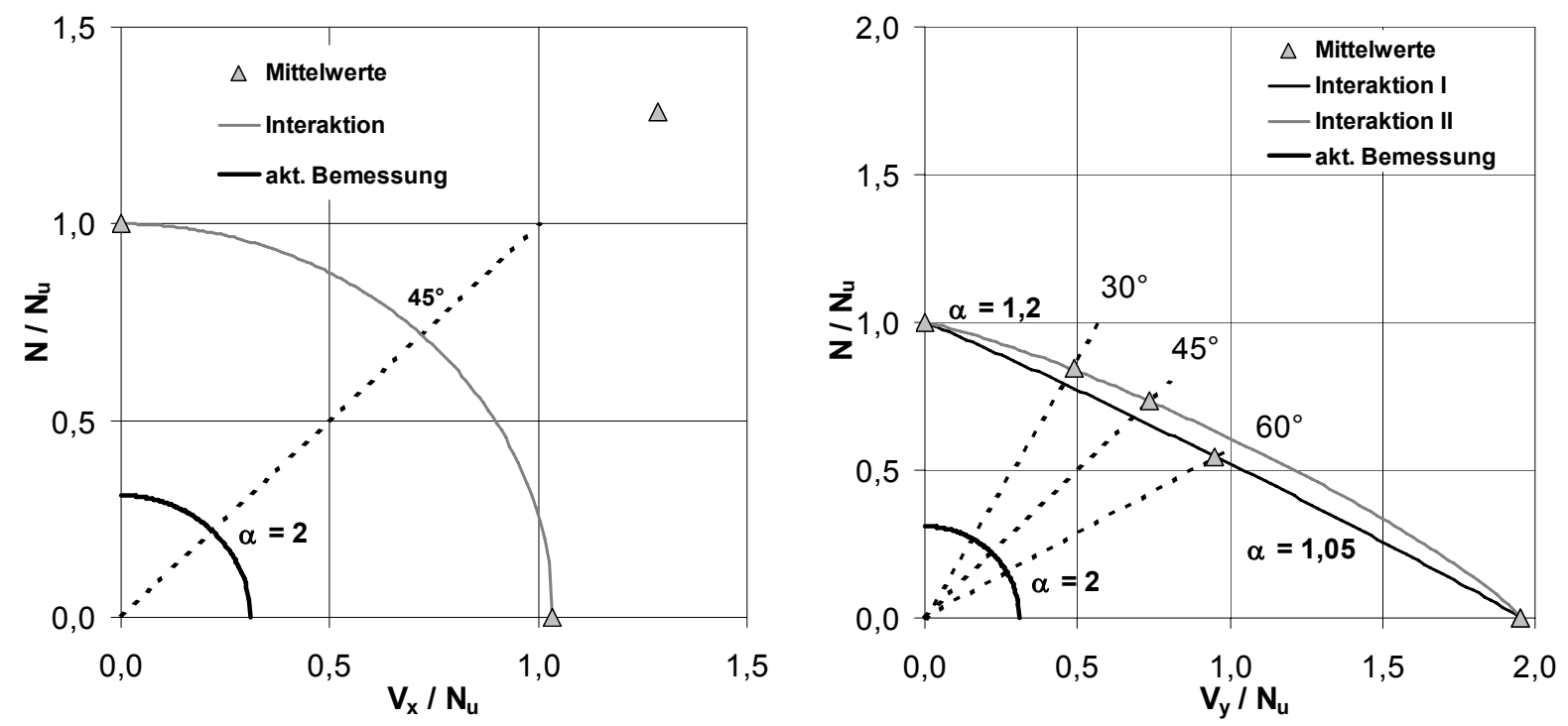

Bild 4.65 Interaktionsdiagramm für ruhende Zug- und Querbeanspruchung

Die Interaktionsbeziehung der Mittelwerte der statischen Tragfähigkeiten zwischen der zentrischen Zugkraft $\mathrm{N}$ und der Beanspruchung in Schienenlängsrichtung $\mathrm{V}_{\mathrm{x}}$ liefert für $\alpha=2$ eine mögliche Annäherung an die Versuchsergebnisse, wobei unter einem Beanspruchungswinkel von $45^{\circ}$ deutlich große Reserven vorhanden sind. Der aus den Versuchen erzielte Mittelwert liegt um den Faktor 2 über dem Wert, der aus der Interaktionsgleichung resultiert.

Bei Betrachtung der $\mathrm{N}-\mathrm{V}_{\mathrm{y}}$-Interaktion ergibt sich in Bezug auf den Mittelwert der maximalen Tragfähigkeiten ein Formfaktor von 1,05 (Bild 4.65, Interaktion I), welcher auf die hohe Tragfähigkeit in Querrichtung $\left(90^{\circ}\right)$ und zusätzlich auf die geringere Tragfähigkeit unter einem Beanspruchungswinkel von $60^{\circ}$ zurückzuführen ist. Vernachlässigt man jedoch den Wert der Tragfähigkeit bei $60^{\circ}$, so wird ein Formfaktor von 1,2 (Bild 4.65, Interaktion II) erreicht, welcher bei statischer Beanspruchbarkeit gering erscheint. Im Vergleich zur $\mathrm{N}-\mathrm{V}_{\mathrm{x}}$-Interaktion verläuft dieser deutlich flacher. Die Ursache hierfür liegt in den unterschiedlichen Versagensarten bei unterschiedlichen Beanspruchungsrichtungen. Zusätzlich bestimmt der umgebende Beton bei reiner Querbeanspruchung das Tragverhalten enorm. Die Versagenszustände können Bild 4.15 entnommen werden.

Zusätzlich ist in beiden Diagrammen in Bild 4.65 die Interaktion der aktuellen Bemessung im Bezug auf den Mittelwert der statischen Zuglasten aufgetragen worden, welche die vorhandenen Systemreserven unter statischer Beanspruchung verdeutlicht. 
Hierbei zeigen sich für eine Querbeanspruchungen $V_{\mathrm{y}}$ kritische ertragbare Lasten, die auf unterschiedliche Versagensarten zurückzuführen sind.

Zur Beschreibung der $\mathrm{V}_{\mathrm{x}}-\mathrm{V}_{\mathrm{y}}$-Interaktion liegen lediglich drei statische Versuche unter einem Beanspruchungswinkel von $45^{\circ}$ vor. Der Mittelwert beträgt etwa $48 \mathrm{kN}$. Somit kann auch für die statische Beanspruchung das in Bild 4.66 dargestellte Interaktionsdiagramm aufgebaut werden. Um eine Vergleichbarkeit zu den Diagrammen zuvor aufzeigen zu können, sind auch die Querlasten auf den Mittelwert der zentrischen Versagenslast bezogen worden.
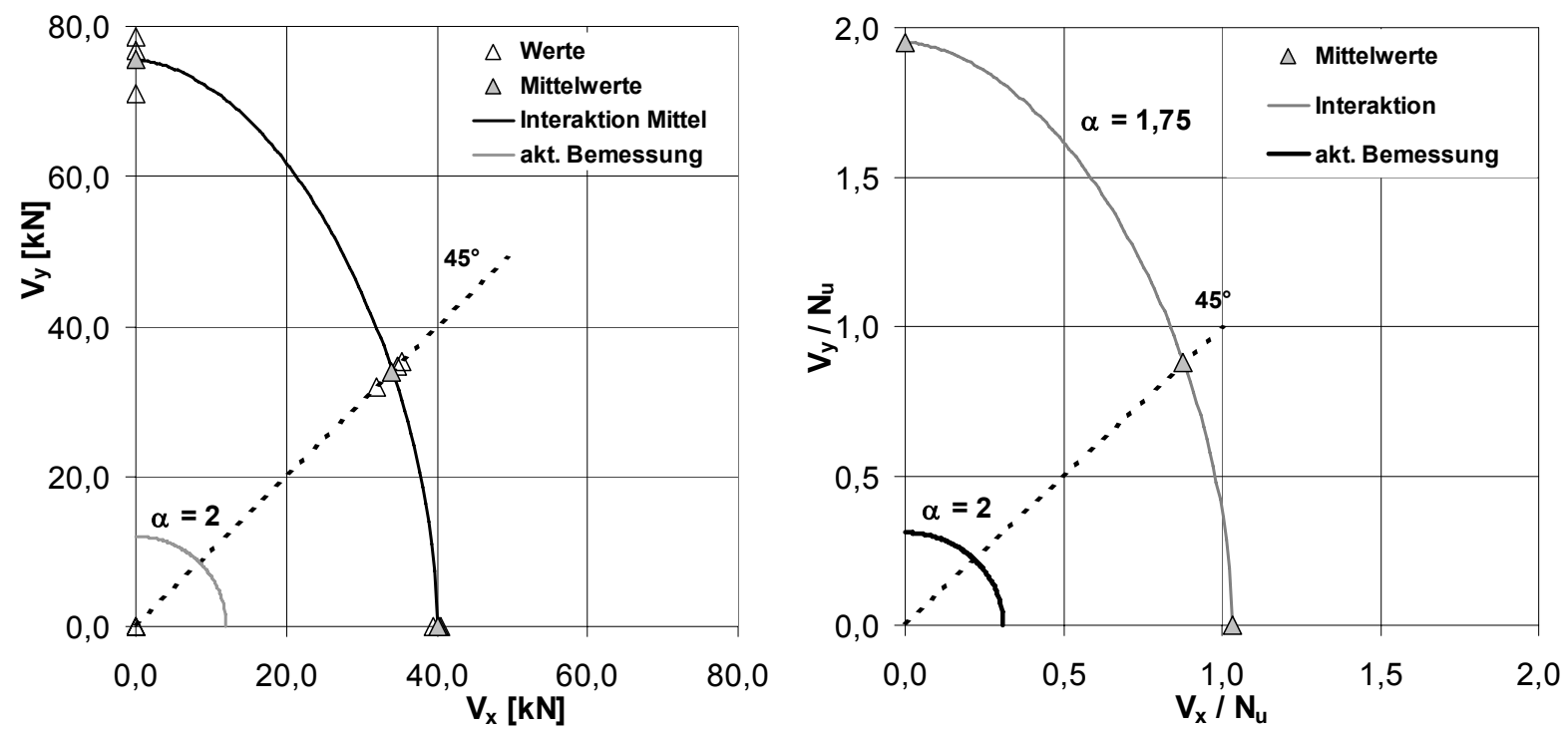

Bild 4.66 Interaktion der ruhenden Querbeanspruchungen $V_{x}$ und $V_{y}$

Die Interaktionsbeziehung der Mittelwerte der statischen Tragfähigkeiten zwischen der Querbeanspruchung in Schienenlängsrichtung $V_{x}$ und quer dazu $V_{y}$ zeigt für einen Formfaktor von $\alpha_{\text {stat }}=1,75$ die beste Annäherung an die Versuchsergebnisse. Um den vorhandenen Sicherheitsabstand zum aktuellen Bemessungswert der statischen Tragfähigkeit aufzeigen zu können, ist dieser ebenfalls eingezeichnet worden. Der auf den Mittelwert der statischen Tragfähigkeiten $V_{x}$ bezogene Sicherheitsfaktor liegt deutlich über einem Wert von 3. Quer zur Schienenlängsrichtung liegt er sogar deutlich über einem Wert von 6. Die hohe Tragfähigkeit ergibt sich aus der maßgebenden Beteiligung des Betons an der Tragfähigkeit, bis letztendlich das Stahlversagen eingeleitet wird. Die maßgebenden Versagensfälle in Abhängigkeit von der Beanspruchungsrichtung können dem Bild 4.16 entnommen werden. In beiden Fällen war Stahlversagen die maßgebende Versagensursache. 


\subsubsection{Schwingversuche quer zur Schienenlängsrichtung}

Um einen Querschnitt möglichst flexibel und optimal zu gestalten, müssen Kenntnisse über seine Tragfähigkeit vorliegen, wenn mehrere Schnittgrößen gleichzeitig wirken sollen. Das Tragverhalten eines Querschnitts unter Einwirkung von mehreren Schnittgrößen kann mit Hilfe von Interaktionen zwischen den jeweiligen Hauptrichtungen, wie sie bereits bei ruhender Beanspruchung vorliegen, dargestellt werden. Bei nichtruhenden Beanspruchungen werden keine kombinierten Zug- und Querbeanspruchungen für die Bemessung angesetzt. Lediglich die reine zentrische Zugrichtung wird als kritisch angesehen und bildet somit die Grundlage der Bemessung. Der Interaktionsverlauf zwischen der zentrischen Zugbelastung N, der Querbeanspruchung in Schienenlängsrichtung $V_{x}$ und quer dazu $V_{y}$ wird nicht angesetzt.

Um die Interaktion auch für nichtruhende Beanspruchungen zu untersuchen, wurden Versuche an einbetonierten Ankerschienen der Variante 25/50 der Serie B und C vorgenommen. Diese erfolgten für Ankerschienen der Serie B ausschließlich unter konstanter Oberlast von $17 \mathrm{kN}$ und für die Serie C zusätzlich noch unter Konstanthaltung der Unterlast von $1 \mathrm{kN}$. Die Betondruckfestigkeiten entsprachen denen der Serie 2 bzw. Serie 3 in Tabelle 4.4. Als Beanspruchungswinkel wurden $30^{\circ}, 45^{\circ}$ und $60^{\circ}$, relativ zur zentrischen Zugrichtung gemessen, gewählt. Um den Einfluß der Belastungsrichtung deutlich zu machen, sind zunächst die Ergebnisse für eine Schwingbeanspruchung quer zur Schienenlängsrichtung (y-Richtung) unter einem Beanspruchungswinkel von $30^{\circ}$ in Bild 4.67 dargestellt.

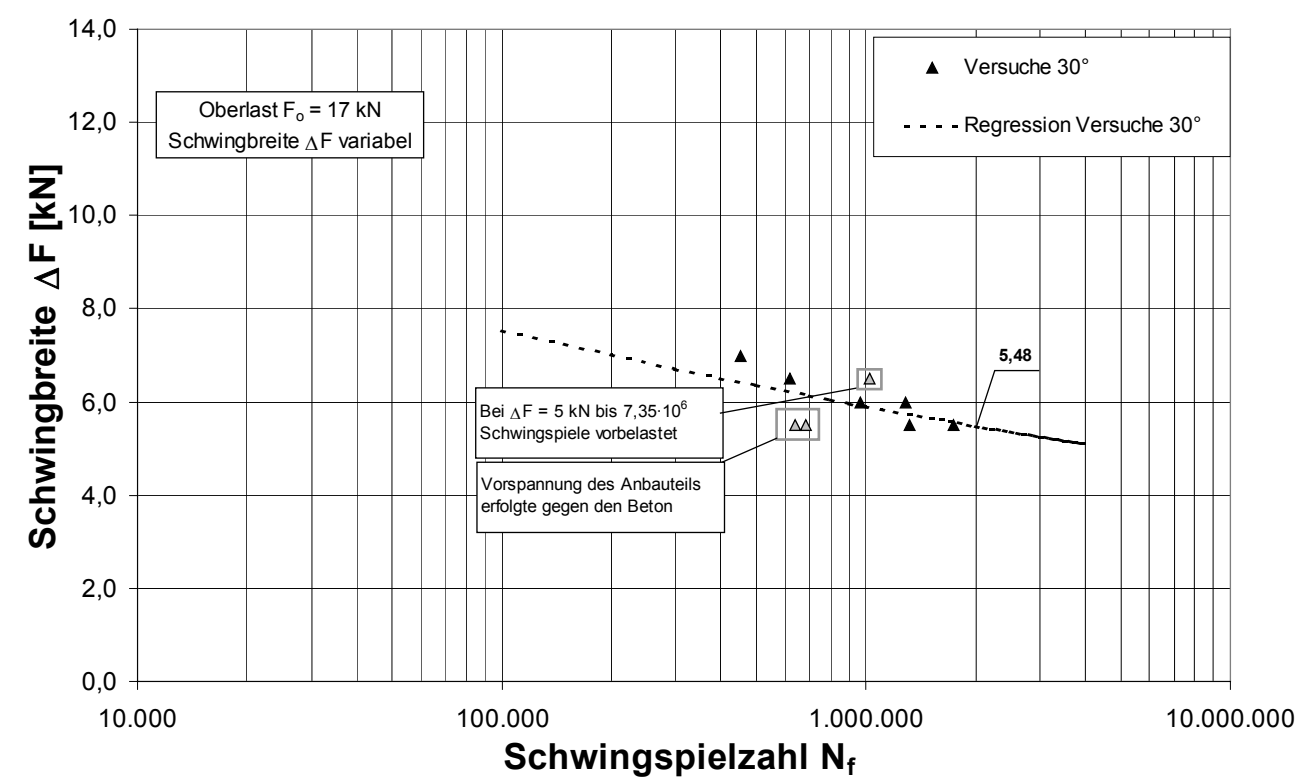

Bild 4.67 Ermüdungstragfähigkeiten unter einem Beanspruchungswinkel von $30^{\circ}$

Auffällig hierbei sind die gekennzeichneten Versuche, die gesondert betrachtet werden müssen. Zum einen handelt es sich um zwei Versuche, die auf einem niedrigen Lastniveau von $5,5 \mathrm{kN}$ und einer Oberlast von $17 \mathrm{kN}$ durchgeführt wurden und geringe Schwingspielzahlen erreichten. Hierbei lag das Problem vor, daß das Anbauteil gegen die Betonoberfläche vorgespannt wurde. In [21], [23] wird jedoch ausdrücklich 
auf eine Vorspannung gegen den Stahl hingewiesen. Somit dürfen diese Versuche nicht in einer gemeinsamen Auswertung betrachtet werden und sind daher nicht zu berücksichtigen. Zum anderen handelt es sich um einen bereits auf einem niedrigen Lastniveau vorbelasteten Versuch. Die ertragbare Schwingbreite liegt hierbei deutlich über den Mittelwert aller Versuche, aber immer noch im Streubereich der Gesamtergebnisse. Aus diesem Grunde wird dieser Versuch bei einer gemeinsamen Auswertung berücksichtigt. Die Vorbelastungen werden nicht berücksichtigt.

Als Versagen für die Beanspruchung unter einem Winkel von $30^{\circ}$ wurde hauptsächlich ein Riß im Übergangsbereich vom Schienenrücken zum Seitenschenkel (Fall 1.1) auf der lastzugewandten Seite (LZS, siehe Bild 4.1) beobachtet. Dieser trat in den meisten Fällen in Kombination mit einem Versagen im Übergangsbereich des Schienenschenkels zur Schienenlippe (Fall 2.1) auf der gegenüberliegenden, der Last abgewandten Seite (LAS) auf. In den meisten Fällen kam es dabei zur Aufbiegung der Schienenlippe (Fall 2.1 A) bzw. zum kompletten Abriß (Fall 2.1 B) und zum Bruch des Ankerfußes des Ankerbolzens (Fall 4.1) als sekundäre Erscheinung.

Nachfolgendes Diagramm in Bild 4.68 zeigt die Beanspruchung quer zur Schienenlängsrichtung unter einem Winkel von $45^{\circ}$ (Serie B und Serie C).

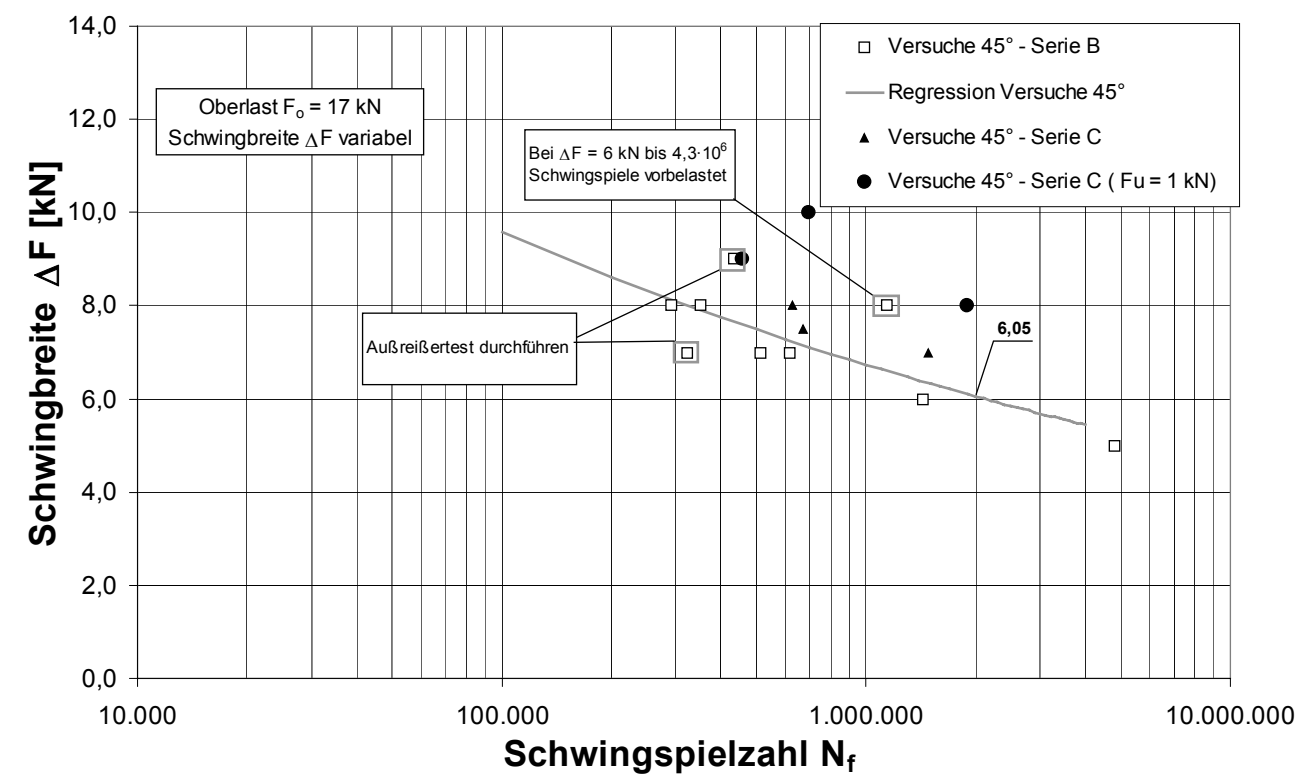

Bild 4.68 Ermüdungstragfähigkeiten unter einem Beanspruchungswinkel von $45^{\circ}$

Auffällig sind hierbei drei gekennzeichnete Versuche, die ebenfalls gesondert betrachtet werden müssen. Wie bereits bei den Versuchen unter $30^{\circ}$ wurde auch unter $45^{\circ}$ der bei einer Schwingbreite von $8 \mathrm{kN}$ markierte Versuch zuvor auf einem niedrigeren Lastniveau von $\Delta \mathrm{F}=6 \mathrm{kN}$ bis $4,3 \cdot 10^{6}$ Schwingspielen vorbelastet. Im Vergleich $\mathrm{zu}$ den anderen, nicht vorbelasteten Versuchen auf diesem Lastniveau, deren ertragbare Schwingspielzahl zwischen $3 \cdot 10^{4}$ und $4 \cdot 10^{4}$ liegen, stellt sich für den vorbelasteten Versuch eine Schwingspielzahl von $1 \cdot 10^{6}$ ein. Offensichtlich erlag dieser Versuch dem sog. „Trainier-Effekt", der dann auftritt, wenn die Einwirkung im Streubereich der Dauerfestigkeit liegt und ein allmählicher Ausgleich der Mikrospannungsspitzen 
zur Verfestigung des Bauteils ohne Mikrorisse führt. Demzufolge wird dieser Versuch aus der Auswertung ausgeschlossen. Die restlichen zwei Versuche unterlagen dem sog. Ausreißertest nach Grubbs [96], wobei sich der auf dem höheren Lastniveau getestete Versuch als Ausreißer und der auf dem niedrigeren Lastniveau nicht als Ausreißer herausstellte.

Als Versagen zeigten sich bei fast allen Versuchen der Serie B Risse im Übergangsbereich des Schienenrückens zum Seitenschenkel (Fall 1.1) auf der LZS in Kombination mit Rissen im Übergangsbereich des Schienenschenkels zur Schienenlippe (Fall 2.1, Fall 2.1 A+B) auf der LAS. Lediglich einmal trat Versagen als Kombination von Fall 2.1 (LZS), Fall 3.1 und Fall 4.1 auf. Die ertragbaren Schwingbreiten der Serie C zeigen Versagensarten entsprechend Fall 1.2 auf, welche vermutlich der Grund für eine leicht höhere Tragfähigkeit gegenüber Serie B sind. Eine separate Auswertung dieser Serie ist aufgrund der geringen Versuchsanzahl nicht aussagekräftig. Die Durchführung der Versuche beim niedrigen Lastniveau (konstanter Unterlast von $1 \mathrm{kN}$ ) zeigt deutlich höhere ertragbare Schwingbreiten. Die Versagensarten sind jedoch sehr unterschiedlich.

Nachfolgendes Diagramm zeigt die Ermüdungstragfähigkeit für einen Belastungswinkel von $60^{\circ}$.

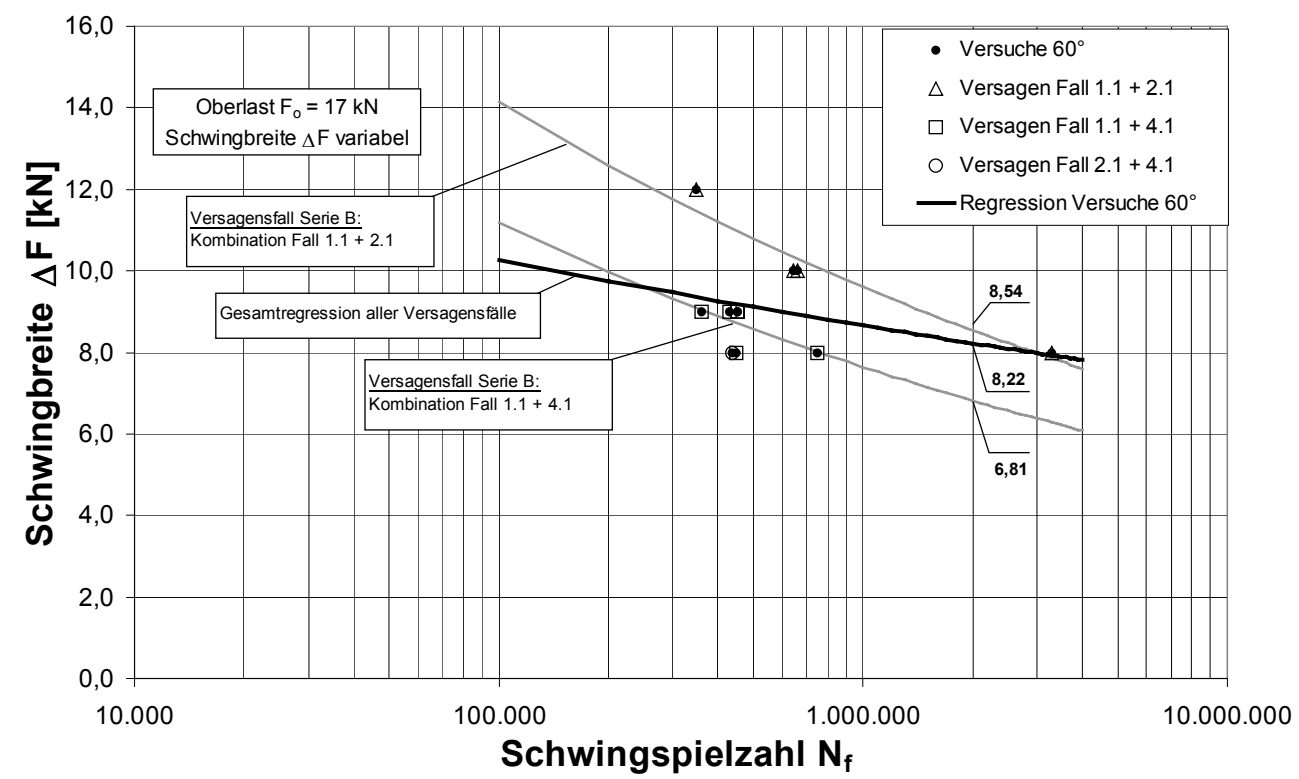

Bild 4.69 Ermüdungstragfähigkeiten unter einem Beanspruchungswinkel von $60^{\circ}$

Aus dem Ermüdungstragfähigkeitsdiagramm in Bild 4.69 geht eine sehr große Streuung der Versuchsergebnisse hervor. Mit Hilfe der Versagensfälle läßt sich dieses Verhalten jedoch erklären. Es liegen zwei unterschiedliche Versagensarten vor, deren Ursachen grundsätzlich unterschiedlich sind. Zum einen sind es Risse im Querschnitt der Schiene, die im Übergangsbereich des Schienenrückens zum Schienenschenkel auf der LZS und des Schienenschenkel zur Schienenlippe auf der gegenüberliegenden LAS entstehen (Fall 1.1 +2.1). Zum anderen sind es Brüche am Ankerfuß, die sich in Kombination mit Rissen im Übergangsbereich des Schienenschenkels zur Schienen- 
lippe einstellen (Fall $1.1+4.1$ ). Bei separater Betrachtung der versagensfallspezifischen Ermüdungstragfähigkeiten zeigen sich die in Bild 4.69 dargestellten Verläufe. Die ertragbaren Schwingbreiten sind im letztgenannten Versagensfall geringer, weil ein vorzeitiger Bruch des Ankerfußes das Hauptversagen einleitet.

Für alle Beanspruchungswinkel liegen die erzielten Schwingspielzahlen zwischen $3 \cdot 10^{4}$ und $2 \cdot 10^{6}$. Mit Zunahme des Winkels stellt sich auch eine höhere ertragbare Schwingbreite ein. Die nachfolgende Tabelle 4.24 zeigt eine Zusammenstellung der Ergebnisse bei Betrachtung der gesamten auftretenden Versagensfälle.

Tabelle 4.24 Gesamtergebnisse 25/50-B bei Beanspruchung quer zur Schiene (Serie B)

\begin{tabular}{|c|c|c|c|c|c|c|c|}
\hline $\begin{array}{c}\text { Winkel } \\
\left.{ }^{\circ}\right] \\
\end{array}$ & $\begin{array}{c}\text { Mittelw. } \Delta \mathrm{F} \\
\text { bei } 2 \cdot 10^{6} \\
{[\mathrm{kN}]}\end{array}$ & $\begin{array}{c}\sigma \\
{[\mathrm{kN}]}\end{array}$ & $\begin{array}{l}\mathrm{V} \\
{[\%]} \\
\end{array}$ & $\begin{array}{l}\text { Steigung } \mathrm{m} \\
\text { Regression }\end{array}$ & Anzahl $\mathrm{n}$ & $\begin{array}{c}\text { Schwingbr. } \\
\Delta \mathrm{F}_{\mathrm{i}} \\
{[\mathrm{kN}]} \\
\end{array}$ & $\begin{array}{c}\text { Anhang } \\
\text { Tab. }\end{array}$ \\
\hline 30 & 5,50 & 0,43 & 7,75 & 9,41 & 9 & $5,5-7,0$ & A23 \\
\hline 45 & 6,08 & 0,69 & 11,32 & 6,51 & 9 & $5,0-9,0$ & A25 \\
\hline 60 & 8,28 & 1,02 & 12,28 & 13,46 & 11 & $8,0-12,0$ & A30 \\
\hline
\end{tabular}

Neben der ertragbaren Schwingbreite nimmt auch der Variationskoeffizient mit $\mathrm{Zu}$ nahme des Beanspruchungswinkels zu. Die Ursache hierfür ist auf die starke Streuung der Versagensfälle zurückzuführen, die sich mit Variation des Beanspruchungswinkels einstellt.

Nachdem Entfernen von Ausreißern und der versagensfallspezifischen Betrachtung ergeben sich die in Tabelle 4.25 aufgeführten Ergebnisse.

Tabelle 4.25 Bereinigte Ergebnisse 25/50-B (Serie B)

\begin{tabular}{|c|c|c|c|c|c|c|c|}
\hline $\begin{array}{c}\text { Winkel } \\
\left.{ }^{\circ}\right]\end{array}$ & $\begin{array}{c}\text { Mittelw. } \Delta \mathrm{F} \\
\text { bei } 2 \cdot 10^{6} \\
{[\mathrm{kN}]}\end{array}$ & $\begin{array}{c}\sigma \\
{[\mathrm{kN}]}\end{array}$ & $\begin{array}{l}\mathrm{V} \\
{[\%]}\end{array}$ & $\begin{array}{l}\text { Steigung } \mathrm{m} \\
\text { Regression }\end{array}$ & Anzahl $n$ & $\begin{array}{c}\text { Schwingbr. } \\
\Delta \mathrm{F}_{\mathrm{i}} \\
{[\mathrm{kN}]}\end{array}$ & $\begin{array}{c}\text { Anhang } \\
\text { Tab. }\end{array}$ \\
\hline 30 & 5,32 & 0,15 & 2,74 & 5,62 & 6 & $5,5-7,0$ & A24 \\
\hline 45 & 5,74 & 0,26 & 4,52 & 6,35 & 7 & $5,0-8,0$ & A26 \\
\hline $60^{* 1}$ & 8,55 & 0,33 & 3,82 & 5,94 & 4 & $8,0-12,0$ & A31 \\
\hline $60^{* 2}$ & 6,82 & 0,30 & 4,40 & 6,06 & 6 & $8,0-9,0$ & A32 \\
\hline
\end{tabular}

${ }^{1}$ Versagen Fall $1.1+2.1$

$*^{2}$ Versagen Fall $1.1+4.1$

Die in Tabelle 4.25 zusammengestellten Versuchsergebnisse sind für alle Belastungsrichtungen in grafischer Form dem Bild 4.70 zu entnehmen. Es zeigen sich nun Funktionen der Ermüdungstragfähigkeiten, welche in Abhängigkeit vom Belastungswinkel einen nahezu parallelen Verlauf aufzeigen. Die Mittelwerte der ertragbaren Schwingbreiten bei $2 \cdot 10^{6}$ Schwingspielen steigen weiterhin mit Zunahme des Belastungswinkels. Während die Zunahme zwischen $30^{\circ}$ und $45^{\circ}$ etwa $7,7 \%$ beträgt, beläuft sie sich zwischen den Belastungswinkeln von $45^{\circ}$ und $60^{\circ}$ auf ungefähr $50 \%$ bei vergleichbaren Versagensfällen. 


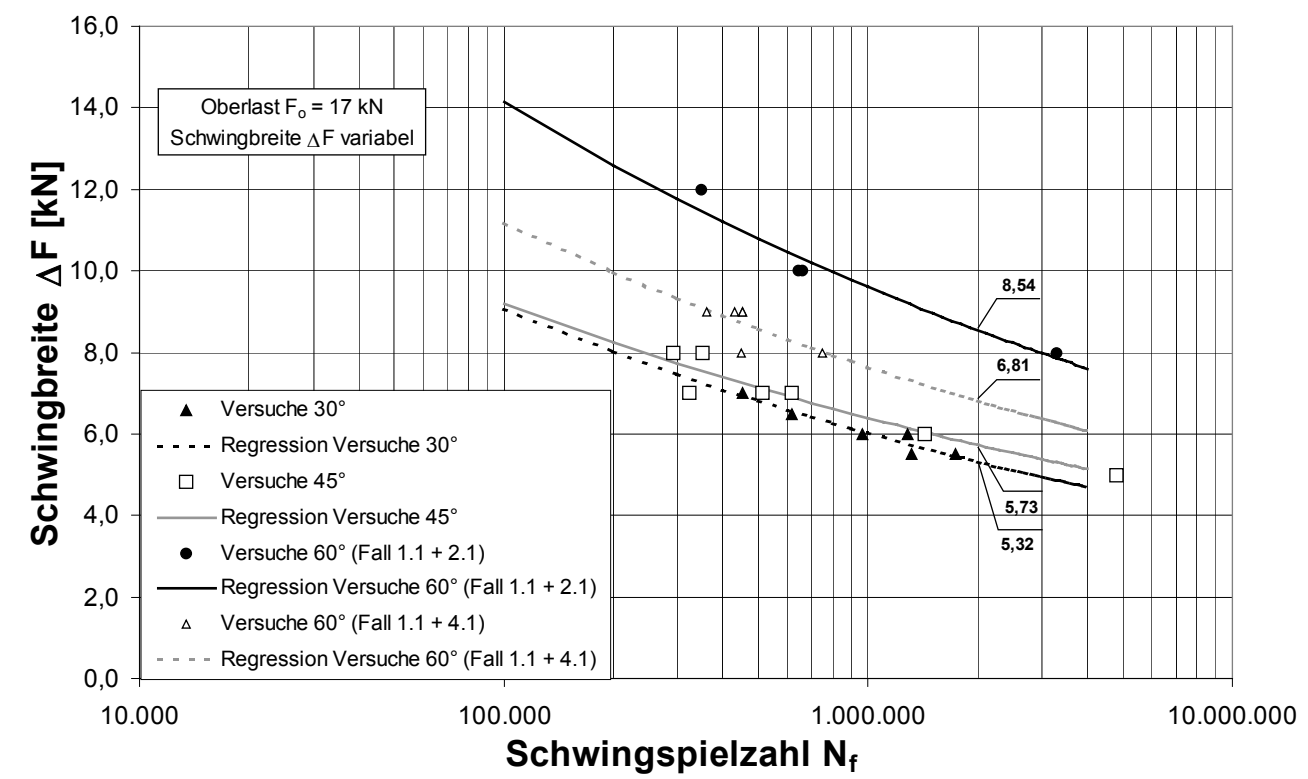

Bild 4.70 Ermüdungstragfähigkeiten unter variierenden Beanspruchungswinkeln

Aus Gründen der Vergleichbarkeit zwischen den Belastungsrichtungen und zur Veranschaulichung von Relationen sollten die ertragbaren Schwingbreiten bei $2 \cdot 10^{6}$ Schwingspielen für den Versagensfall 1.1 in Kombination mit Fall 2.1 betrachtet werden. Jedoch wird darauf hingewiesen, daß das Versagen des Ankerfußes (Fall 4.1) bei $60^{\circ}$ auf der unsicheren Seite liegt und somit für eine Bemessung maßgeblich wäre.

Zur Darstellung der Lastverteilung mit Hilfe von DMS (siehe Bild 4.6, oben) sind die Beanspruchungszustände dargestellt worden. Die Abhängigkeiten der Dehnungen von den Belastungswinkeln $\left(0^{\circ}\right.$ bis $\left.60^{\circ}\right)$ sind in den nachfolgenden Diagrammen in Bild 4.71 bis Bild 4.73 deutlich zu sehen. Zur besseren Übersicht sind die Maßstäbe der Ordinaten und Abszissen gleich gewählt worden. Der DMS1 befand sich stets auf der lastzugewandten Seite (LZS) und DMS2 auf der lastabgewandten Seite (LAS).

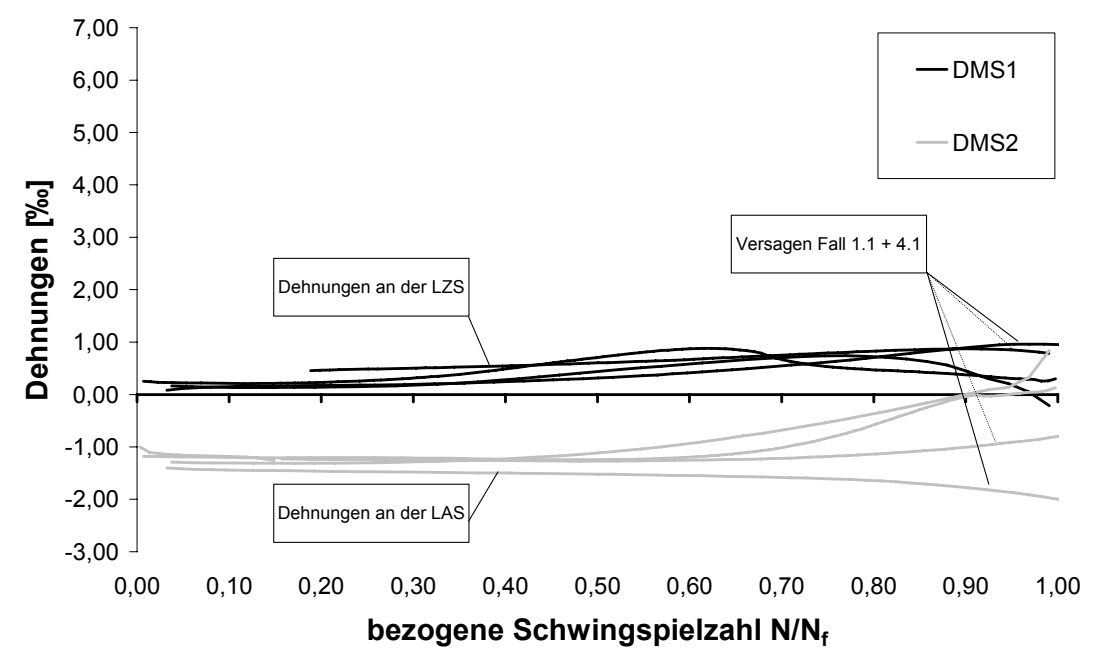

Bild 4.71 Dehnungen bei Schwingbreiten $\Delta \mathrm{F}=5-7 \mathrm{kN}\left(30^{\circ}\right)$ 


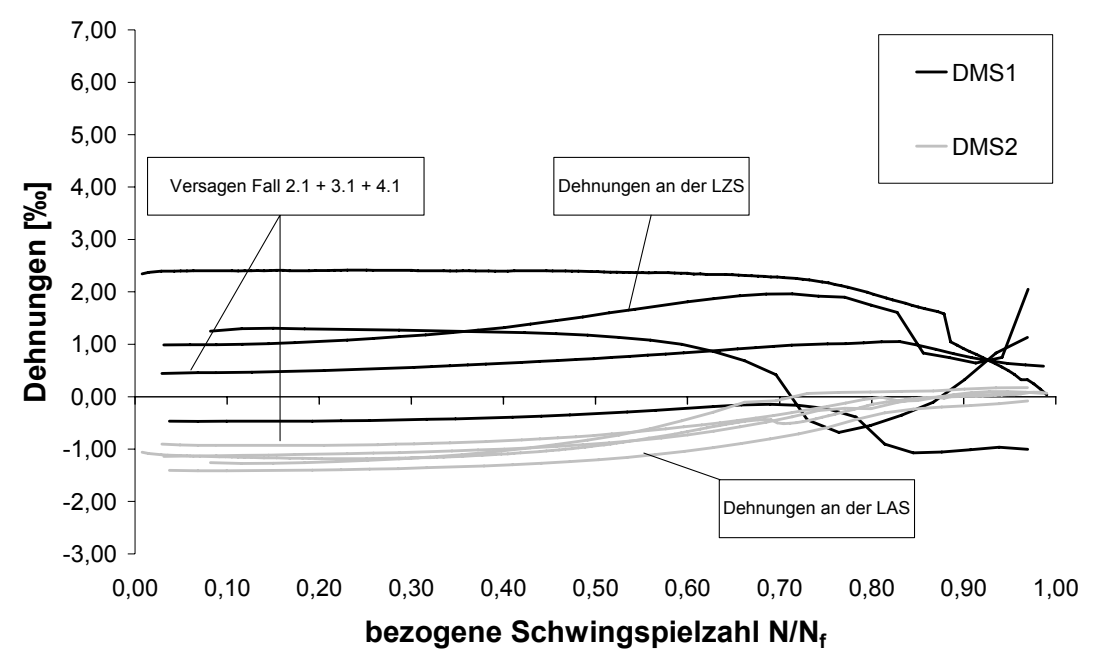

Bild 4.72 Dehnungen bei Schwingbreiten $\Delta \mathrm{F}=6-9 \mathrm{kN}\left(45^{\circ}\right)$

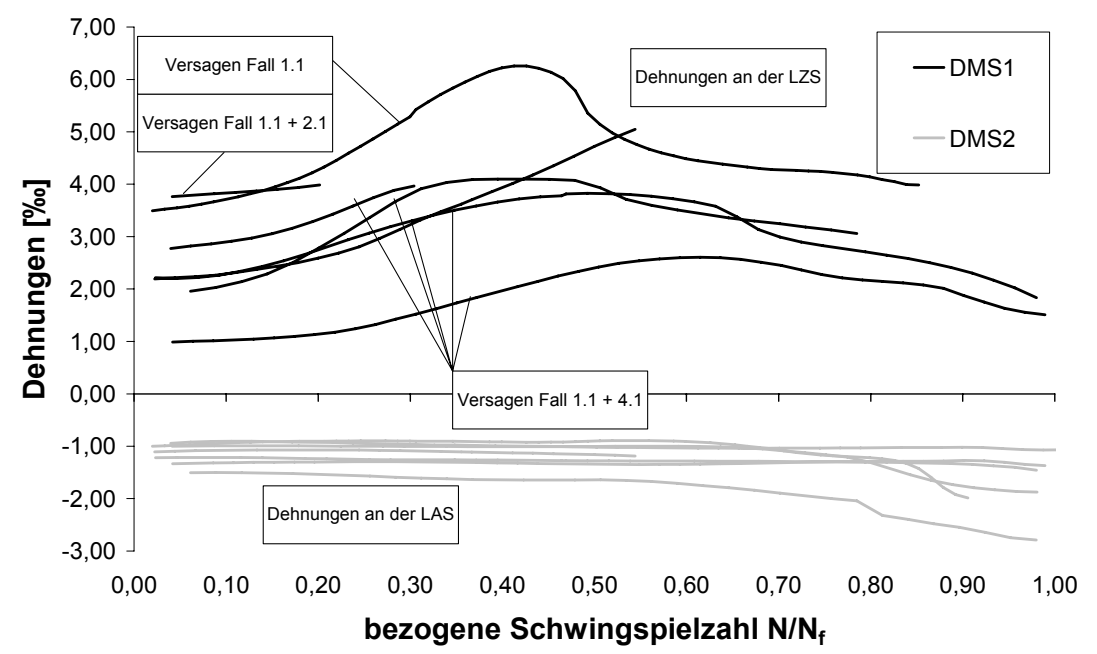

Bild 4.73 Dehnungen bei Schwingbreiten $\Delta \mathrm{F}=8-12 \mathrm{kN}\left(60^{\circ}\right)$

Die in den Diagrammen in Bild 4.71 bis Bild 4.73 dargestellten Verläufe der Dehnungen sind lediglich an einer begrenzten Versuchsanzahl durchgeführt worden. Diese geben aber umfassende Informationen zum Tragverhalten und zum Versagensmechanismus wieder. Bei Betrachtung der Belastungsrichtung $30^{\circ}$ zeigen sich erste Anzeichen für eine Dehnungsabnahme ab 60 bis $70 \%$ der bezogenen Schwingspielzahl, was auf eine allmähliche Rißentstehung zurückzuführen ist. Im Falle des Ankerversagens weisen die Dehnungen an den Schienenschenkeln am Versuchsende sehr hohe Restdehnungen auf. Durch eine Rißbildung im Übergangsbereich des Schienenrüc??kens zum Schienenschenkel auf der LAS findet eine Kraftumlagerung im Querschnitt statt und damit eine höhere Belastung des Ankerfußes und des Schienenschenkels auf der LZS. Je nachdem, wie stark bereits die Rißentwicklung auf der LZS fortgeschritten ist, kann ein Bruch des Ankerfußes resultieren. 
So einheitlich wie sich die Anfangsdehnungen bei den Versuchen unter einem Belastungswinkel von $30^{\circ}$ gestalten, erkennt man bei $45^{\circ}$ eine sehr große Streuung der Dehnungen auf der LZS. Die Ursache hierfür kann mit der stark einseitigen Belastung der Ankerschiene, bedingt durch das Rutschen der Schraube, erläutert werden. Ab etwa $50 \%$ der bezogenen Bruchschwingspielzahl reduzieren sich die Dehnungen auf der LAS und verlaufen stabil bis etwa $80 \%$. Fast alle Körper weisen eine einheitliche Schädigung auf. Diese stellt sich am Übergang vom Rücken der Ankerschiene zum Schienenschenkel (LZS) und vom Schienenschenkel zur Schienenlippe (LAS) ein (Versagen Fall $1.1+2.1$ ). Im Falle des Versagens im Ankerfuß stellen sich geringe anfängliche Dehnungen in den Schienenschenkeln ein (siehe Bild 4.72), die auf eine stärkere Belastung des Ankerfußes zurückzuführen sind. Folglich entsteht das Hauptversagen am Ankerfuß.

Beim Belastungswinkel von $60^{\circ}$ nimmt die Differenz der Dehnungen und die Streuung auf der LZS und LAS, bedingt durch die große Exzentrizität der Beanspruchung, weiterhin zu. Die Werte, gemessen an der LAS, bleiben in fast allen Fällen über die gesamte Beanspruchungsdauer nahezu konstant. Aufgrund der großen Lastkomponente parallel zur Betonoberfläche findet hier ein Rutschen der Schraube statt, woraus eine exzentrische Belastung der Schienenschenkel folgt und was somit zu einer höheren Belastung auf der LZS führt. Hier nehmen bereits ab einer bezogenen Schwingspielzahl von $10 \%$ die Dehnungen zu und erreichen je nach Schwingbreite zwischen 40 und $60 \%$ ihr Maximum. Die im Diagramm in Bild 4.73 dargestellten Dehnungsverläufe beziehen sich hauptsächlich auf das Hauptversagen im Ankerfuß. Zweimal konnte ein Versagen in Kombination von Fall $1.1+2.1$ beobachtet werden. Dabei sieht man deutlich, daß die anfänglichen Dehnungen auf der LZS im Vergleich zu den übrigen Dehnungswerten deutlich höher sind, obwohl die im Versuch eingestellte Schwingbreite dem Minimalwert von $\Delta \mathrm{F}=8 \mathrm{kN}$ (siehe Tabelle 4.25) entspricht. Danach erfolgt eine Abnahme der Dehnung, bedingt durch die Kraftumlagerung infolge von Rißbildung und Verformung, bis zum Bruch. Aufgrund der hohen Lastkomponente in Querrichtung wird der Schraubenkopf gegen die Schienenschenkel auf der LZS gedrückt, weshalb die Dehnung durch die hohe Formänderung stark beeinflußt wird.

Im Diagramm in Bild 4.74 ist zu sehen, daß mit Zunahme des Belastungswinkels die Verformung $\mathrm{v}$ in Richtung der Querbeanspruchung zunimmt. Die Ursache hierfür liegt in einer nicht ausreichenden Vorspannung, wenn neben reinen zentrischen Zugkräften zusätzlich Querkräfte übertragen werden müssen. Umfassende Erläuterungen bezüglich des konstruktiven Problems des Schraubenrutschens sind in [8] dargestellt. 


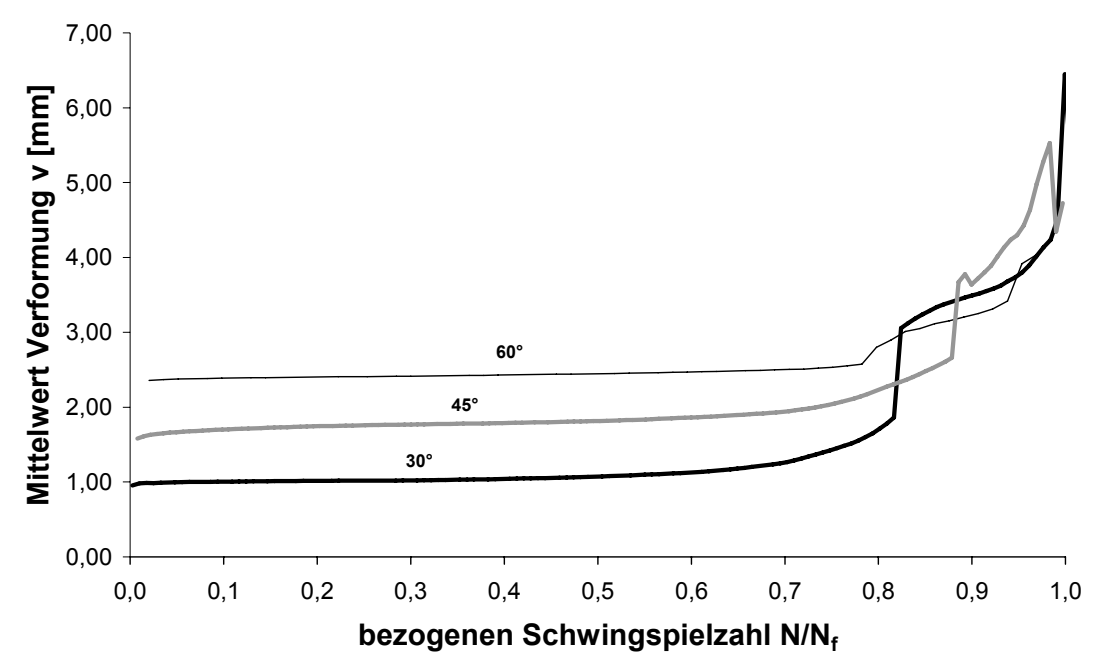

Bild 4.74 Mittelwert der Verformung $v$ in Abhängigkeit vom Belastungswinkel

Erst in einem Bereich zwischen 80 und 90 \% machen sich Änderungen in der Verformung bemerkbar. Die Phase der stabilen Rißentwicklung wird bereits abgeschlossen sein und in die instabile Rißentwicklung übergehen.

\subsubsection{Schwingversuche in Schienenlängsrichtung}

Untersuchungen in Schienenlängsrichtung unter einer Querbeanspruchung im Winkel von $45^{\circ}$ sind im folgenden an Ankerschienen der Serie C dargestellt.

Zur Anwendung kamen Ankerschienen der Ausführung 25/50. Als Beanspruchungswinkel wurde lediglich $45^{\circ}$ gewählt. Die Versuche erfolgten systematisch mit konstanter Unterlast von $1 \mathrm{kN}$. Die Betondruckfestigkeiten entsprechen der Serie 3 in Tabelle 4.4. In Tabelle 4.26 sind die erzielten Ergebnisse in Abhängigkeit von der Versagensart dargestellt.

Tabelle 4.26 Kennwerte bei $45^{\circ}$ Beanspruchung in Schienenlängsrichtung

\begin{tabular}{|c|c|c|c|c|c|c|c|}
\hline Versagen & $\begin{array}{c}\text { Mittelw. } \Delta \mathrm{F} \\
\text { bei } 2 \cdot 10^{6} \\
{[\mathrm{kN}]}\end{array}$ & $\begin{array}{c}\sigma \\
{[\mathrm{kN}]}\end{array}$ & $\begin{array}{l}\mathrm{V} \\
{[\%]}\end{array}$ & $\begin{array}{l}\text { Steigung } \mathrm{m} \\
\text { Regression }\end{array}$ & Anzahl n & $\begin{array}{c}\text { Schwingbr. } \\
\qquad \mathrm{F}_{\mathrm{i}} \\
{[\mathrm{kN}]}\end{array}$ & $\begin{array}{c}\text { Anhang } \\
\text { Tab. }\end{array}$ \\
\hline Fall 1.2 & 8,31 & 0,16 & 1,88 & 4,24 & 4 & $9,0-17,0$ & A27 \\
\hline Fall $1.1+2.1$ & 10,41 & 0,69 & 6,62 & 7,91 & 5 & $11,0-17,0$ & A28 \\
\hline Gesamt & 9,37 & 0,72 & 7,66 & 5,82 & 10 & $9,0-17,0$ & A29 \\
\hline
\end{tabular}

Die grafische Darstellung der Ergebnisse erfolgt im nachfolgenden Diagramm in Bild 4.75 . 


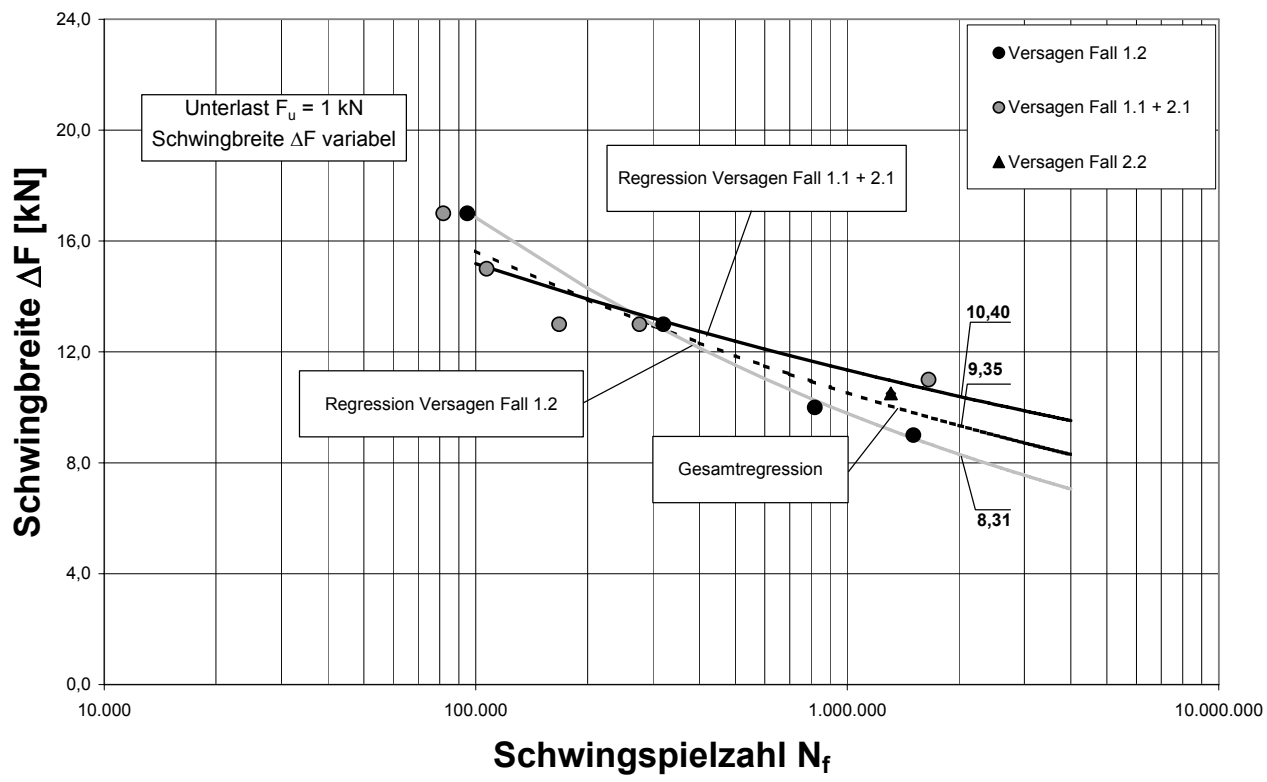

Bild 4.75 Ermüdungstragfähigkeit unter $45^{\circ}$-Beanspruchung in Schienenlängsrichtung

Auffällig ist, daß je nach Versagensart die ertragbare Schwingbreite zwischen einem Minimalwert von 8,31 kN (Versagen Fall 1.2, kritisch) und 10,40 kN (Versagen Fall $1.1+2.1$ ) liegt. Läßt man den Versagensfall unbeachtet, so kann der Mittelwert der ertragbaren Schwingbreite von $9,35 \mathrm{kN}$ bei $2 \cdot 10^{6}$ Schwingspielen angesetzt werden.

In Bild 4.76 sind die Verformungen $\Delta \mathrm{u}$ und $\Delta \mathrm{w}$ für einen Belastungswinkel von $45^{\circ}$ dargestellt. Die Anordnung der Wegaufnehmer ist in Bild 4.12 ersichtlich.

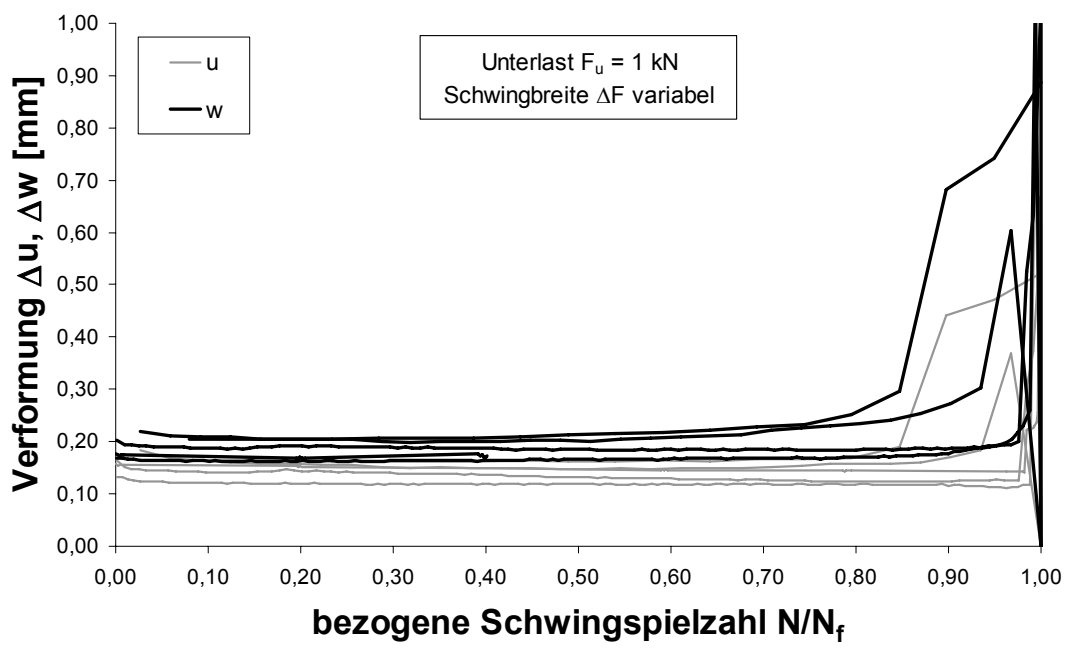

Bild 4.76 Zunahme der Verformungsdifferenzen $\Delta \mathrm{u}$ und $\Delta \mathrm{w}$ unter $45^{\circ}$

Die parallel zur Betonoberfläche aufgezeichnete Zunahme der Verformungsdifferenz $\Delta \mathrm{u}$ liegt stets unterhalb des Wertes von $\Delta \mathrm{w}$. Auch bei dieser Beanspruchungsrichtung zeigt sich, daß das Hauptversagen erst ab etwa 90 bis $95 \%$ der bezogenen Schwingspielzahl eintritt. 


\subsubsection{Interaktion der Ermüdungstragfähigkeiten}

Es stellt sich nun die Frage, welche Form der Interaktionsverlauf der Schwingbreite zwischen der reinen zentrischen Zugrichtung und der Querbeanspruchung sowohl in Schienenlängsrichtung $\left(\mathrm{V}_{\mathrm{x}}\right)$, als auch quer dazu $\left(\mathrm{V}_{\mathrm{y}}\right)$ annimmt.

Die nichtlineare Form der Interaktionsbeziehung gibt Gleichung (4.11) wieder.

$\left(\frac{1}{\mathrm{a}} \cdot \frac{\Delta \mathrm{N}_{\mathrm{i}}}{\Delta \mathrm{N}_{\mathrm{u}}}\right)^{\alpha_{\mathrm{dyn}}}+\left(\frac{1}{\mathrm{~b}} \cdot \frac{\Delta \mathrm{V}_{\mathrm{i}}}{\Delta \mathrm{N}_{\mathrm{u}}}\right)^{\alpha_{\mathrm{dyn}}} \leq 1$

In Bild 4.77 (links) ist die resultierende $\mathrm{N}-\mathrm{V}_{\mathrm{x}}$-Interaktion auf Basis der mittleren ertragbaren Schwingbreite dargestellt. Bei dieser Beanspruchungsebene zeigen sich ertragbare Schwingbreiten, die deutlich über dem Wert der reinen zentrischen Zugbeanspruchung liegen. Somit kann gefolgert werden, daß bei einer Beanspruchung in der $\mathrm{N}-\mathrm{V}_{\mathrm{x}}$-Ebene die reine zentrische Zugrichtung als kritische Belastungsrichtung angesehen werden kann. In Bild 4.77, rechts, ist das Interaktionsdiagramm, bezogen auf die ertragbare Schwingbreite für die kritische Belastungsrichtung bei konstanter Unterlast von $1 \mathrm{kN}$, dargestellt.

Die einwirkenden Schwingbreiten $\Delta \mathrm{N}_{\mathrm{i}}$ und $\Delta \mathrm{V}_{\mathrm{i}}$ sind variabel, wobei $\Delta \mathrm{N}_{\mathrm{u}}$ der Mittelwert der ertragbaren Schwingbreite der zentrischen Zugrichtung ist. Die in Bild 4.77 zu sehenden Interaktionsdiagramme weisen Formfaktoren von $\alpha=1,3$ und 1,7 (Versuche mit konstanter Unterlast von etwa $12 \mathrm{kN}$ ) auf. Zusätzlich ist hierbei der aktuelle Bemessungswert aus der zentrischen Zugrichtung auf die gesamte Belastungsrichtung projiziert worden. Die ertragbare Schwingbreite in Schienenlängsrichtung ist bei reiner Zugschwellbeanspruchung $(\mathrm{R} \approx 0)$ etwa um das 1,79-fache größer als in zentrischer Zugrichtung.
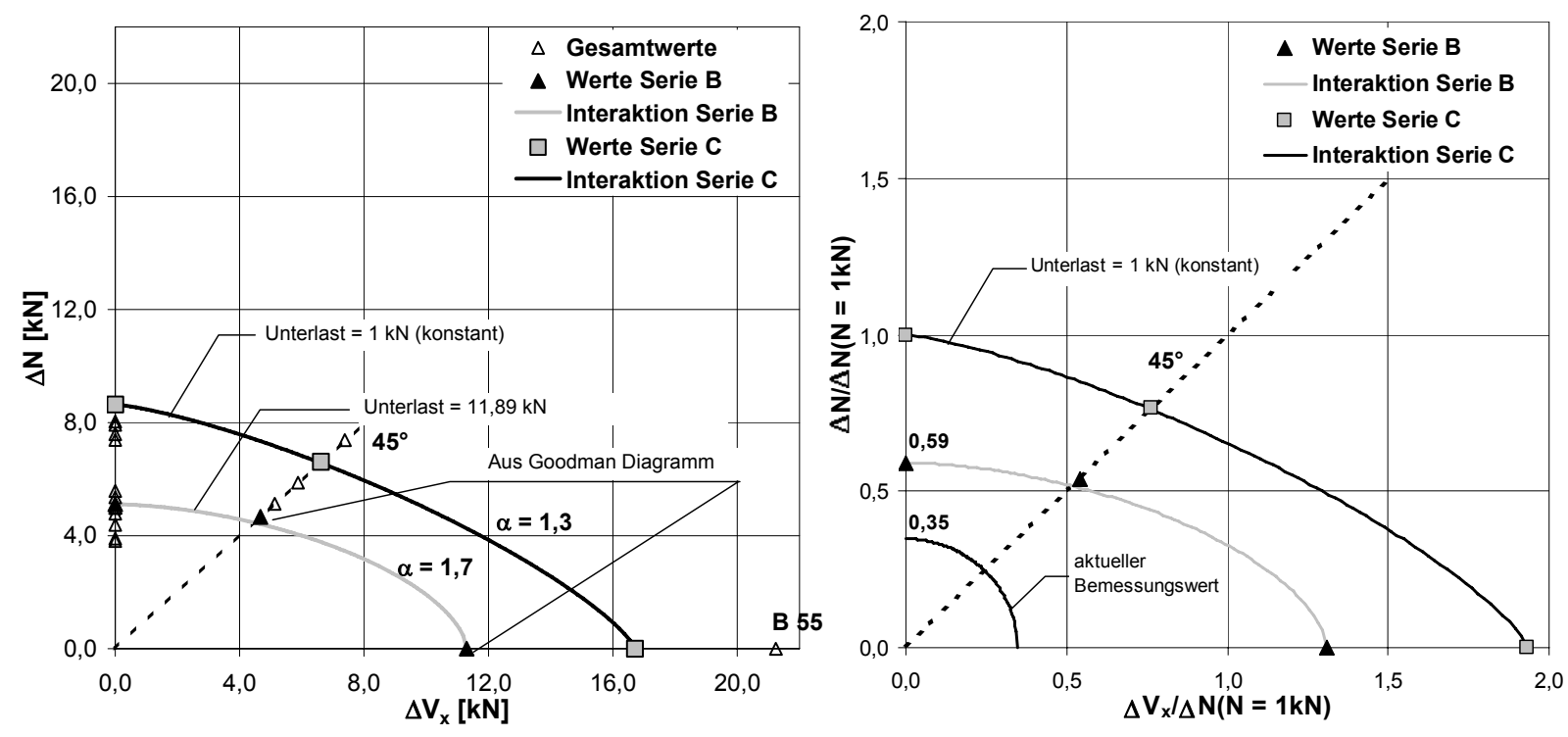

Bild 4.77 $\quad \Delta \mathrm{N}-\Delta \mathrm{V}_{\mathrm{x}}$-Interaktion 
Im Falle einer reinen Zugschwellbeanspruchung $(\mathrm{R} \approx 0)$ mit konstanter statischer Unterlast in allen Richtungen gelingt es, einen auf Versuchsdaten basierenden Formfaktor herzuleiten. Da jedoch nur Versuche für die zentrische Zugrichtung im Zugschwellbereich $(\mathrm{R} \approx 0,7)$ durchgeführt wurden, sind für $\mathrm{R}>0$ keine Versuche für eine kombinierte Zug- und Querbeanspruchung in Schienenlängsrichtung vorhanden. Jedoch können unter Verwendung des Goodman-Diagramms diese Werte näherungsweise auf der sicheren Seite liegend bestimmt werden. Das Goodman-Diagramm wurde auf der Basis der vorliegenden Versuchsergebnisse erstellt.

Die Interaktionsbeziehung bei Beanspruchung quer zur Schienenlängsrichtung ist in Bild 4.78 mit Bezug auf die Gleichung (4.11) dargestellt worden. Auch hierbei stellte sich die zentrische Zugbeanspruchung als kritisch heraus. Die Interaktionsgleichung nimmt für die Versuche bei konstanter Unterlast von $1 \mathrm{kN}$ den Formfaktor 1,2 an. Dieser beträgt bei einer Unterlast von etwa $12 \mathrm{kN} \mathrm{1,15}$. Bei der Durchführung der Versuche mit konstanter Oberlast von $17 \mathrm{kN}$ stellt sich ein Formfaktor von 1,1 ein. Es sei an dieser Stelle darauf hingewiesen, daß bei Beanspruchung quer zur Schienenlängsrichtung nur Versuche mit konstanter Oberlast von $17 \mathrm{kN}$ durchgeführt worden sind. Da es hierbei nur einmal zum Versagen bei einer Schwingbreite von $15 \mathrm{kN}$ und einer Schwingspielzahl von $1,9 \cdot 10^{6}$ kam und mehrere Versuche auf höheren Lastniveaus deutlich über $4 \cdot 10^{6}$ Schwingspielen ohne Bruch endeten, wird ein Mindestwert der Schwingbreite von $15 \mathrm{kN}$ zugrunde gelegt. Es sind jedoch ertragbare Schwingbreiten größer als $15 \mathrm{kN}$ in Querrichtung zu erwarten, die in dieser Interaktionsbeziehung nicht betrachtet werden können.
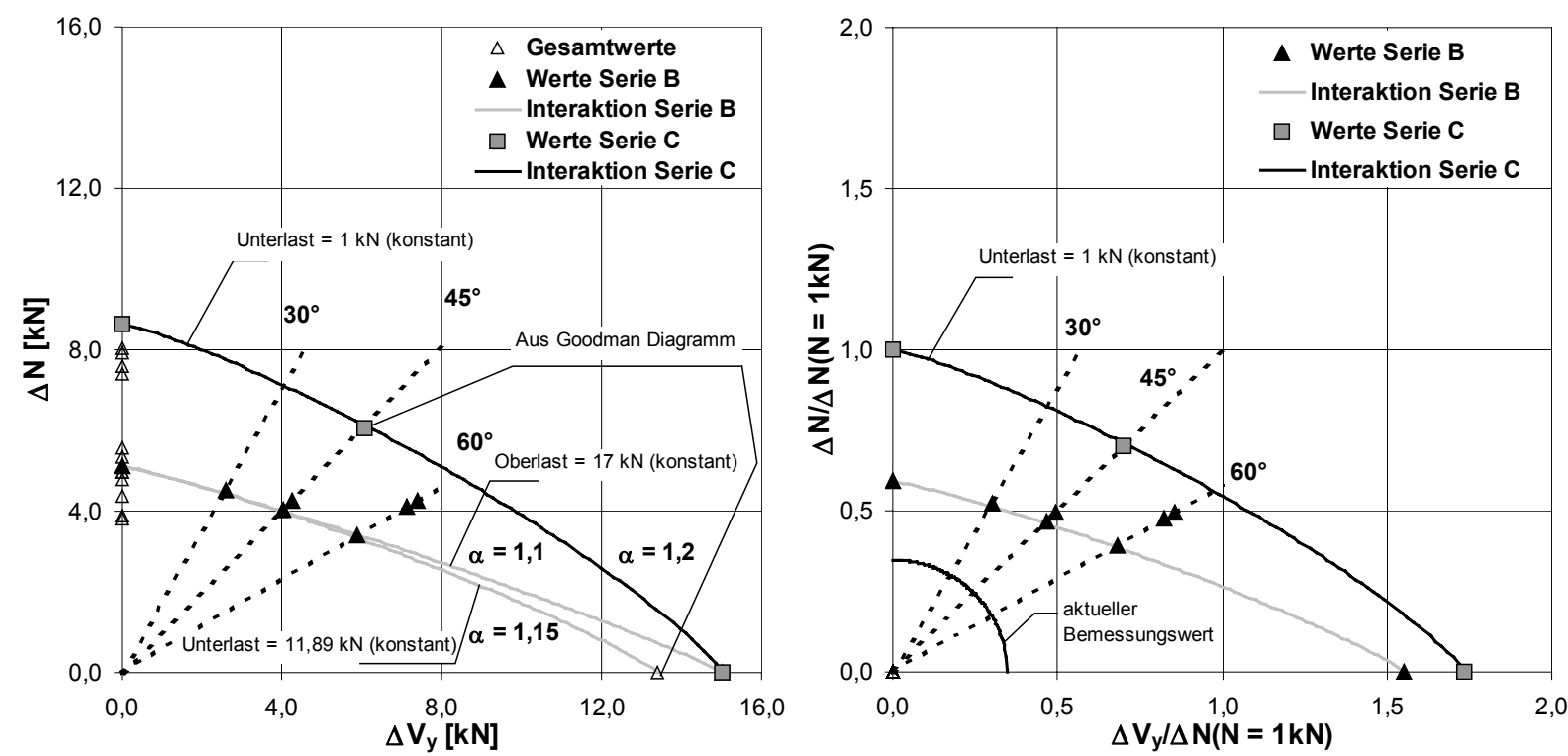

Bild 4.78 $\quad \Delta \mathrm{N}-\Delta \mathrm{V}_{\mathrm{y}}$-Interaktion

Weiterhin kann der Interaktionsbeziehung entnommen werden, daß die ertragbaren Schwingbreiten quer zur Schienenlängsrichtung etwa um den Faktor 1,7 größer sind als bei reiner zentrischer Zugschwellbeanspruchung. 
Versuche zur Interaktion zwischen nicht ruhenden Querkräften $\Delta \mathrm{V}_{\mathrm{x}}$ und $\Delta \mathrm{V}_{\mathrm{y}}$ sind nicht durchgeführt worden. Auf der sicheren Seite liegend wird hierfür jedoch ein Formfaktor von $\alpha=1,0$ (lineare Beziehung) angenommen. Vorhandene Tragreserven im System können bei dieser Annahme eventuell nicht ausgenutzt werden.

Mit den erzielten ertragbaren Schwingbreiten in Abhängigkeit von den Einflußgrößen und den Interaktionsdiagrammen zur Berücksichtigung der Lastrichtungen sind nun grundlegende Erkenntnisse für eine umfassende Bemessung gegeben. Ein Vergleich der erzielten Versuchsergebnisse zur aktuellen Bemessung in Bild 4.77 und Bild 4.78 zeigt deutlich, daß eine Erweiterung des Bemessungswertes auf alle Lastrichtungen durchaus gerechtfertigt ist und somit Systemreserven im Bauteil ab einem Belastungswinkel von $45^{\circ}$ ausgenutzt werden können. Diese Erkenntnisse bildet somit die Grundlage einer variablen und umfassenden Bemessung.

Durch den Bezug der Achsen auf die jeweiligen maximalen Tragfähigkeiten $\left(\mathrm{N}_{\mathrm{u}}, \mathrm{V}_{\mathrm{x}, \mathrm{u}}\right.$ und $\mathrm{V}_{\mathrm{y}, \mathrm{u}}$ bzw. $\Delta \mathrm{N}_{\mathrm{u}}, \Delta \mathrm{V}_{\mathrm{x}, \mathrm{u}}$ und $\Delta \mathrm{V}_{\mathrm{y}, \mathrm{u}}$ ) können Interaktionsdiagramme in normierter Darstellung angegeben werden. Die nicht linearen Beziehungen nach [38] sind in Gleichung (4.12) und (4.13) angegeben.

$$
\begin{aligned}
& \left(\frac{\mathrm{N}_{\mathrm{i}}}{\mathrm{N}_{\mathrm{u}}}\right)^{\alpha_{\text {stat }}}+\left(\frac{\mathrm{V}_{\mathrm{i}}}{\mathrm{V}_{\mathrm{u}}}\right)^{\alpha_{\text {stat }}} \leq 1 \\
& \left(\frac{\Delta \mathrm{N}_{\mathrm{i}}}{\Delta \mathrm{N}_{\mathrm{u}}}\right)^{\alpha_{\text {dyn }}}+\left(\frac{\Delta \mathrm{V}_{\mathrm{i}}}{\Delta \mathrm{V}_{\mathrm{u}}}\right)^{\alpha_{\text {dyn }}} \leq 1
\end{aligned}
$$

Zur Verdeutlichung der Formfaktoren als Funktion der einwirkenden Lasten (kombinierte ruhende und nichtruhende Beanspruchungen) werden diese in Bild 4.79 dargestellt.
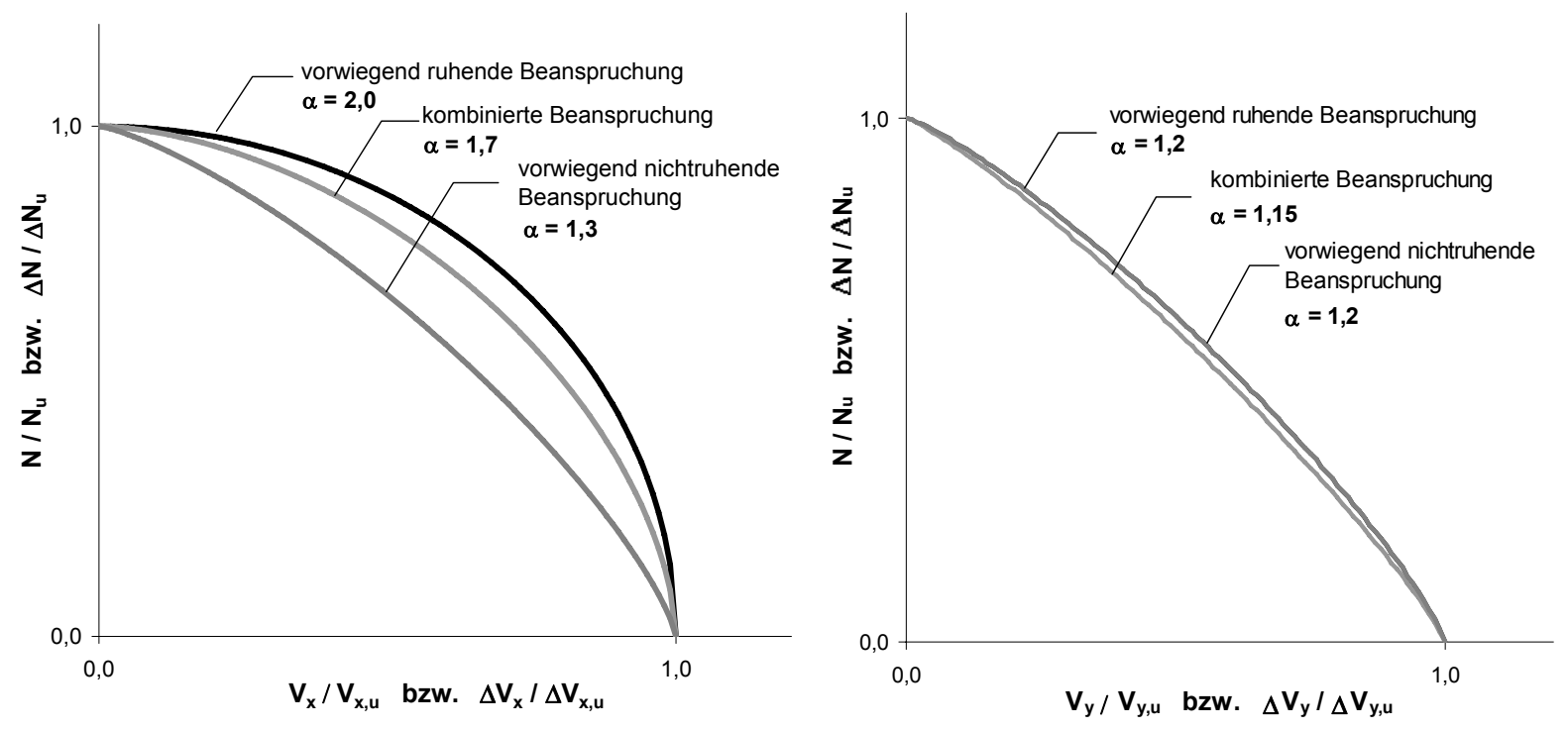

Bild 4.79 Normierte Interaktionsdiagramme $N-V_{x},\left(\Delta N-\Delta V_{x}\right)$ und $N-V_{y},\left(\Delta N-\Delta V_{y}\right)$ 


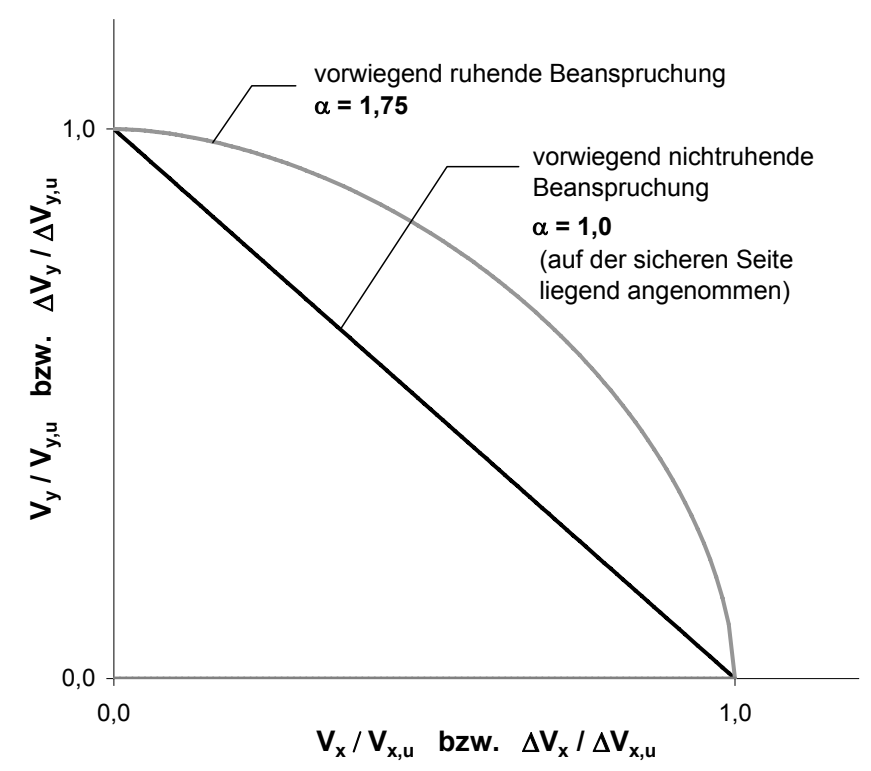

Bild 4.80 Normierte Interaktionsdiagramme $\mathrm{V}_{\mathrm{x}}-\mathrm{V}_{\mathrm{y}},\left(\Delta \mathrm{V}_{\mathrm{x}}-\Delta \mathrm{V}_{\mathrm{y}}\right)$

Auf der Grundlage der normierten Interaktionsdiagramme kann der Zusammenhang zwischen der einwirkenden statischen Last und dem Formfaktor hergeleitet werden.

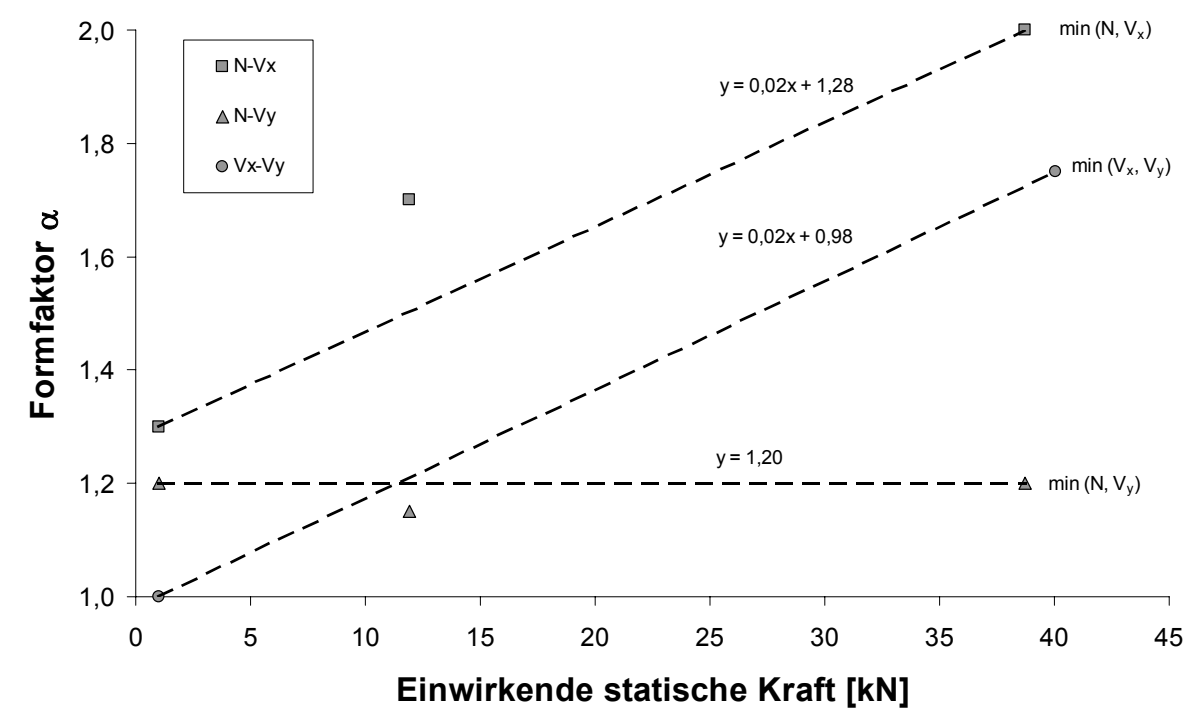

Bild 4.81 Abhängigkeit des Formfaktors von der einwirkenden Kraft

Die Diagramme in Bild 4.81 zur Beschreibung der Interaktion zwischen den jeweiligen Kraftgrößen zeigen, daß eine lineare Verbindung der Formfaktoren in Abhängigkeit von der einwirkenden statischen Kraft (Unterlast in den Versuchen) angenommen werden kann. Für die N-V $\mathrm{y}_{\mathrm{y}}$-Interaktion wird dabei der Formfaktor bei kombinierter ruhender und nichtruhender Beanspruchung unterschätzt, während dieser Faktor bei einer $\mathrm{N}-\mathrm{V}_{\mathrm{x}}$-Interaktion leicht überschätzt wird. 


\subsection{Zusammenfassung}

An warmgewalzten, gezahnten Ankerschienen aus Stahl sind experimentelle Untersuchungen zum Tragverhalten unter ermüdungsrelevanten Einwirkungen durchgeführt worden. Dabei sind Einflüsse auf die Tragfähigkeit aus dem umgebenden Beton, der Laststellung, der Höhe der Lasthorizonte und den unterschiedlichen Beanspruchungsrichtungen die Grundlage für die Versuchsdurchführung gewesen.

Als maßgebende Versagensursache unter den aufgebrachten Beanspruchungen ist in den überwiegenden Fällen eine Rißbildung mit Rißwachstum im Übergang vom Schienenrücken zum Schienenschenkel zu beobachten gewesen. Zusätzlich haben sich Risse im Übergang zur Verzahnung eingestellt. Unter Schrägbeanspruchungen bzw. kombinierten Zug- und Schubbeanspruchungen quer zur Schienenlängsrichtung sind ferner Brüche im Ankerfuß beobachtet worden. Ein Versagen der Schraube ist nicht aufgetreten. Ein Betonversagen ist in keinem Fall maßgebend gewesen.

Die Ermittlung der Schwingbreite bei Ankerschienenbefestigungen erfolgt in der Regel an nicht einbetonierten Schienenstücken mit symmetrischen Endüberständen für eine Einzelbefestigung unter zentrischer Zugrichtung. Als maßgebende Bemessungsschwingspielzahl wird dabei die ertragbare Schwingbreite bei $2 \cdot 10^{6}$ Schwingspielen zugrunde gelegt. Bei Versuchen im einbetonierten Zustand werden durch die Stützwirkung des umgebenden Betons höhere Schwingbreiten erwartet als im nicht einbetonierten Zustand. Bei den im Rahmen dieser Arbeit untersuchten Profilen liegt die auf den Mittelwert der ertragbaren Schwingbreite bezogene Erhöhung unter Ausnutzung von Mindestendüberständen etwa zwischen 17 und $30 \%$. Bei günstigeren Lasteinleitungskonfigurationen ist unter gleichen Randbedingungen eine Erhöhung um $43 \%$ erzielt worden.

Untersuchungen zur Beanspruchung in Schienenlängsrichtung sind ausschließlich an einbetonierten Ankerschienen durchgeführt worden. Dabei sind Ankerschienen in Beton mit der mittleren Druckfestigkeit von $42 \mathrm{~N} / \mathrm{mm}^{2}$ und $61 \mathrm{~N} / \mathrm{mm}^{2}$ eingelegt worden. Eine Zunahme der Betondruckfestigkeit von $30 \%$ bewirkt eine auf den Mittelwert der ertragbaren Ermüdungsfestigkeit bezogene Erhöhung von etwa $27 \%$.

Eine signifikante Abhängigkeit von den ertragbaren Schwingbreiten zeigen die Versuchsergebnisse bei unterschiedlichen Lastniveaus für den zentrischen Zug. Bei Durchführung der Versuche mit einer sehr geringen Unterlast zwischen 0,5 und $1 \mathrm{kN}$ $(\mathrm{R} \approx 0)$ sind mittlere ertragbare Schwingbreiten erreicht worden, die sowohl im einbetonierten als auch im nicht einbetonierten Zustand etwa um $70 \%$ höher liegen als die Schwingbreiten bei Lastverhältnissen von $\mathrm{R} \approx 0,7$. Durch die zusätzliche Ermittlung der statischen Tragfähigkeit können alle erzielten Ergebnisse durch eine linearisierte Darstellungsweise im Goodman-Diagramm aufgezeigt werden. Dabei ist der Wert der vorwiegend ruhenden statischen Tragfähigkeit mit der ertragbaren Schwingbreite bei Zugursprungsbeanspruchung (vorwiegend nichtruhend) linear zu verbinden. Für eine nicht zentrische Zugbeanspruchung sind Versuche ausschließlich mit sehr geringen Unterlasten $(R \approx 0)$ betrachtet worden. Zusätzlich sind für jede Belastungs- 
richtung die statischen Tragfähigkeiten ermittelt worden. Aufbauend auf den Erkenntnissen der zentrischen Zugrichtung werden auch für nicht zentrische Beanspruchungen linearisierte Goodman-Diagramme zugrunde gelegt.

Die experimentellen Untersuchungen zur Interaktion unter ermüdungsrelevanten Einwirkungen auf der Basis von Schwingversuchen an einbetonierten Ankerschienen und Laststellungen unter Ausnutzung von Mindestendüberständen zeigen sowohl lineare als auch nichtlineare Abhängigkeiten zwischen den Kraftgrößen. Dabei liegen alle aus den Versuchen ermittelten Werte außerhalb des von der Interaktion beschriebenen Bereiches. Bei der Annahme einer kreisförmigen Interaktionsbeziehung mit der nach aktueller Bemessungsrichtlinie anzusetzenden nichtruhenden Bemessungslast aus zentrischer Zugrichtung als Radius liegen die Versuchsergebnisse auf der sicheren Seite. Die zentrische Zugrichtung hat sich in allen Fällen als kritische Beanspruchungsrichtung erwiesen.

Im folgenden werden die erzielten Ergebnisse für Sicherheitsbetrachtungen herangezogen. Aufbauend auf diesen Erkenntnissen wird ein umfassendes Bemessungskonzept für kombinierte ruhende und nichtruhende Zug- und Querbeanspruchungen in Anlehnung an das aktuelle Sicherheitskonzept im Bauwesen hergeleitet. 


\section{Sicherheitskonzept}

\subsection{Sicherheitsanforderungen}

Die Bemessung und Konstruktion von Tragwerken unter ermüdungsrelevanten Beanspruchungen sollte so ausgelegt sein, daß sie den auftretenden Beanspruchungen während der vorgesehenen Nutzungsdauer mit einer angemessenen Sicherheit standhalten. Durch die Verwendung von Teilsicherheitsbeiwerten wird ein angestrebtes Sicherheitsniveau erreicht, so daß ein Versagen oder reparaturbedürftige Schäden durch Materialermüdung mit einer bestimmten Wahrscheinlichkeit ausgeschlossen werden kann. Es sind Teilsicherheitsbeiwerte für die Beanspruchung $\mathrm{S}$ und die Beanspruchbarkeit R zu ermitteln. Dabei sind aufgrund der relativ großen Streuungen in der Ermüdungsfestigkeit statistische Hilfsmittel anzuwenden. Den Schwerpunkt der sicherheitstheoretischen Betrachtungen bilden die Beanspruchbarkeiten, dessen charakteristische Werte aus den experimentellen Untersuchungen in Kapitel 4 abgeleitet werden. Die Annahmen für die Beanspruchungen könne in Anlehnung an [84], [112] erfolgen.

In Hinblick auf mögliche Folgen von Gefährdungen werden in den Grundlagen zur Festlegung der Sicherheitsanforderungen für bauliche Anlagen [49] in Sicherheitsklassen unterschieden. Dabei ist den Sicherheitsbedürfnissen der Öffentlichkeit und der Wirtschaftlichkeit Rechnung zu tragen. Die Tabelle 5.1 gibt eine Klassifizierung der Klassen unter Berücksichtigung der Trag- und Gebrauchsfähigkeit.

Tabelle 5.1 Sicherheitsklassen nach [51]

\begin{tabular}{|c|c|c|}
\hline \multicolumn{2}{|c|}{ Mögliche Folgen von Gefährdungen, die } & \multirow[b]{2}{*}{ Klasse } \\
\hline $\begin{array}{l}\text { vorwiegend die Tragfähigkeit } \\
\text { betreffen }\end{array}$ & $\begin{array}{l}\text { vorwiegend die Gebrauchsfähigkeit * }) \\
\text { betreffen }\end{array}$ & \\
\hline $\begin{array}{l}\text { Keine Gefahr für Menschenleben } \\
\text { und geringe wirtschaftliche Folgen }\end{array}$ & $\begin{array}{l}\text { Geringe wirtschaftliche Folgen, geringe } \\
\text { Beeinträchtigung der Nutzung }\end{array}$ & 1 \\
\hline $\begin{array}{l}\text { Gefahr für Menschenleben und/oder } \\
\text { beachtliche wirtschaftliche Folgen }\end{array}$ & $\begin{array}{l}\text { Beachtliche wirtschaftliche Folgen, be- } \\
\text { achtliche Beeinträchtigung der Nutzung }\end{array}$ & 2 \\
\hline $\begin{array}{l}\text { Große Bedeutung der baulichen } \\
\text { Anlage für die Öffentlichkeit }\end{array}$ & $\begin{array}{l}\text { Große wirtschaftliche Folgen, große Be- } \\
\text { einträchtigung der Nutzung }\end{array}$ & 3 \\
\hline \multicolumn{3}{|c|}{$\begin{array}{l}\text { *) Besteht bei Verlust der Gebrauchsfähigkeit Gefahr für Leib und Leben (z.B. Undichtig- } \\
\text { keit von Behältern und Leitungen mit gefährlichen Stoffen), so wird dieser wie ein Verlust } \\
\text { der Tragfähigkeit behandelt }\end{array}$} \\
\hline
\end{tabular}

Für die Mehrzahl der baulichen Anlagen ist die Sicherheitsklasse 2 maßgebend. Verschiedene Anwendungsbeispiele zur Sicherheitsproblematik können aus [76] und [105] entnommen werden. 


\subsection{Versagenswahrscheinlichkeit und Sicherheitsindex}

Der Sicherheitsabstand zwischen der Beanspruchung $\mathrm{S}$ (ermüdungsrelevante Beanspruchung $\Delta \mathrm{F}_{\mathrm{S}}$ ) und der Beanspruchbarkeit $\mathrm{R}$ (Ermüdungsfestigkeit $\Delta \mathrm{F}_{\mathrm{R}}$ ) stellt einen Grenzzustand dar und wird wie folgt definiert:

$\mathrm{Z}=\mathrm{R}-\mathrm{S}$

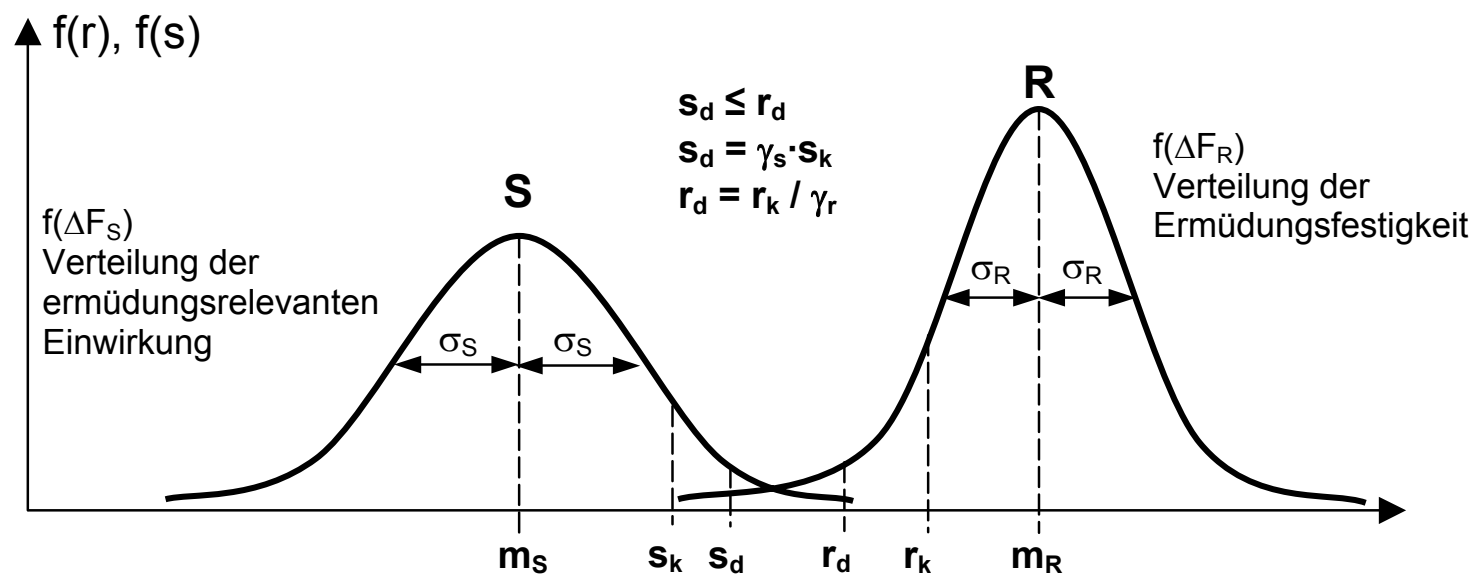

Bild 5.1 Beanspruchung S und Beanspruchbarkeit R

Der formulierte Sicherheitsabstand $Z$ unterteilt den Raum der Basisvariablen in zwei Bereiche. Zum einen ist es der sichere Bereich mit $Z \geq 0$, in dem mit hoher Wahrscheinlichkeit kein Versagen auftritt und zum anderen der unsichere Bereich $Z<0$, in dem mit einer einzuhaltenden Wahrscheinlichkeit das System versagt (siehe Bild 5.2). Zur Einhaltung eines bestimmten Sicherheitsniveaus am Ende der planmäßigen Nutzungsdauer eines Bauwerks wird eine Versagenswahrscheinlichkeit $p_{f} b z w$. ein hierzu äquivalenter Sicherheitsindex $\beta$ verwendet.

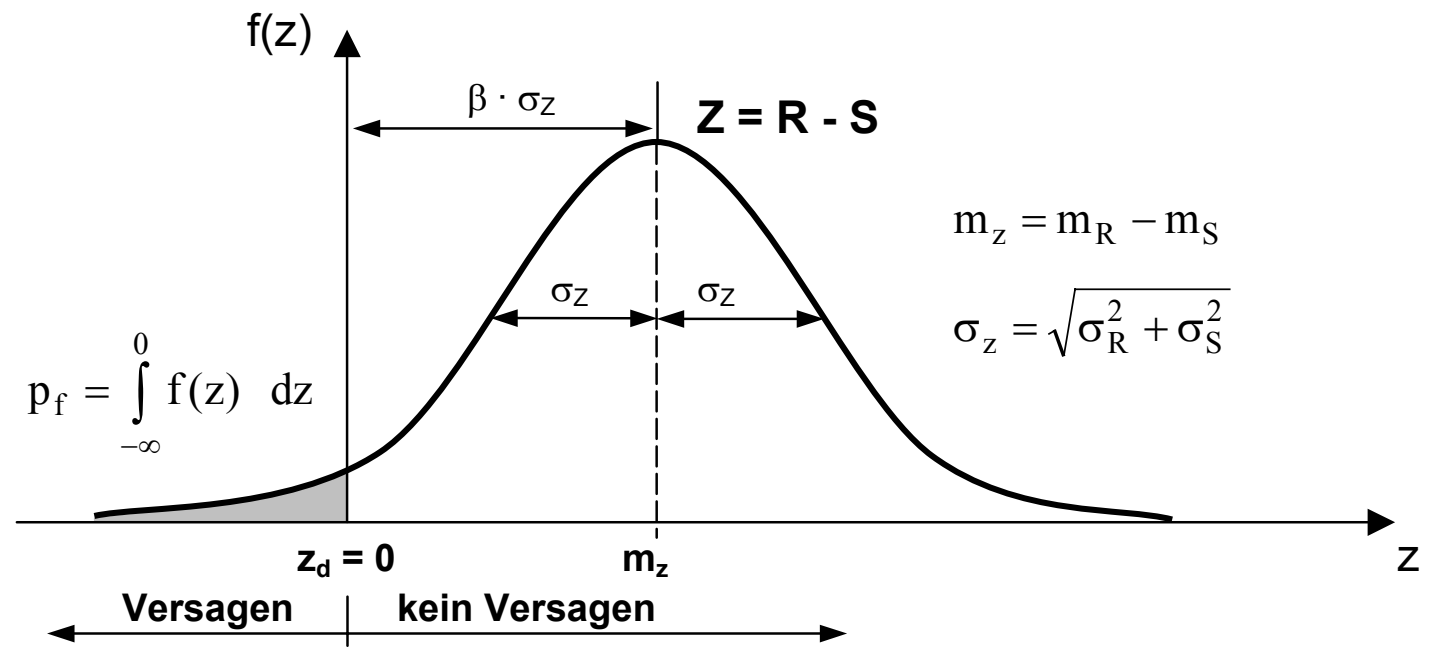

Bild 5.2 Verteilungsdichte $f(z)$, Versagenswahrscheinlichkeit $p_{f}$ und Sicherheitsindex $\beta$ 
In Bild 5.2 ist anschaulich dargestellt, daß die Differenz der Maxima der Verteilungsdichten $\mathrm{f}\left(\mathrm{s}=\Delta \mathrm{F}_{\mathrm{S}}\right)$ und $\mathrm{f}\left(\mathrm{r}=\Delta \mathrm{F}_{\mathrm{R}}\right)$ als $\beta$-fache Standardabweichung definiert ist. Der Sicherheitsindex $\beta$ ergibt sich somit zu:

$\beta=\frac{m_{Z}}{\sigma_{Z}}=\frac{m_{R}-m_{S}}{\sqrt{\sigma_{R}^{2}+\sigma_{S}^{2}}}$

Für den Bemessungszustand ist das Versagen im Grenzzustand der Tragfähigkeit bzw. der Gebrauchstauglichkeit mit einer einzuhaltenden Versagenswahrscheinlichkeit $\mathrm{p}_{\mathrm{f}}$ zu tolerieren. Die Versagenswahrscheinlichkeit gibt somit an, mit welcher Wahrscheinlichkeit ein Versagen eintritt. Der funktionelle Zusammenhang zwischen $\mathrm{p}_{\mathrm{f}}$ und $\beta$ ist über die Standardnormalverteilung $\Phi$ gegeben.

$\mathrm{p}_{\mathrm{f}}=\Phi(-\beta)$

Der Sicherheitsindex $\beta$ kann in Bereichen des Bauwesens Zahlenwerte annehmen, die in Abhängigkeit von der Sicherheitsklasse zwischen 1 und 6 liegen können. Aufgrund der besseren Handhabung im Vergleich zur Versagenswahrscheinlichkeit wird in der Regel der Sicherheitsindex $\beta$ verwendet. In Tabelle 5.2 sind die Sicherheitsindizes und die Versagenswahrscheinlichkeiten für den baupraktisch relevanten Bereich gegenübergestellt.

Tabelle 5.2 Sicherheitsindex $\beta$ und Versagenswahrscheinlichkeit $p_{f}$

\begin{tabular}{|l|l|l|l|l|l|l|}
\hline$\beta$ & 1 & 2 & 3 & 4 & 5 & 6 \\
\hline$p_{f}$ & $1,58610^{-1}$ & $2,27510^{-2}$ & $1,35010^{-3}$ & $3,16910^{-5}$ & $2,87110^{-7}$ & $9,90110^{-10}$ \\
\hline
\end{tabular}

In den europäischen Normen erfolgt eine Aufteilung der Bemessungskriterien in folgende Grenzzustände:

- Grenzzustand der Gebrauchstauglichkeit -SLS-

- Grenzzustand der Tragfähigkeit, z.B. Ermüdung -ULS-

Dabei sind in Abhängigkeit vom Grenzzustand und der vorgesehenen Nutzungsdauer indikative Werte für den angestrebten Sicherheitsindex $\beta$ bzw. Versagenswahrscheinlichkeit $\mathrm{p}_{\mathrm{f}}$ gegeben.

Tabelle 5.3 Sicherheitsindex $\beta$ und Versagenswahrscheinlichkeit $p_{f}$ nach [36]

\begin{tabular}{|l|c|c|c|c|}
\hline \multirow{2}{*}{ Grenzzustand } & \multicolumn{2}{|c|}{ grenz $\beta$} & \multicolumn{2}{c|}{ grenz $\mathrm{p}_{\mathrm{f}}$} \\
\cline { 2 - 5 } & $1 \mathrm{Jahr}$ & $50 \mathrm{Jahre}$ & 1 Jahr & 50 Jahre \\
\hline Tragfähigkeit -ULS- & 4,7 & 3,8 & $1,3 \cdot 10^{-6}$ & $7,2 \cdot 10^{-5}$ \\
\hline Ermüdung & - & $1,5-3,8$ & & \\
\hline Gebrauchstauglichkeit -SLS- & 3,0 & 1,5 & $1,3 \cdot 10^{-3}$ & $6,7 \cdot 10^{-2}$ \\
\hline
\end{tabular}

Für den Grenzzustand der Ermüdung ist der Sicherheitsindex in Abhängigkeit vom Grad der Überwachungs- und Instandsetzungsmöglichkeiten bzw. der Schadensto- 
leranz zu wählen. Die Anforderungen an die Sicherheit für den Grenzzustand der Gebrauchstauglichkeit sind geringer als im Grenzzustand der Tragfähigkeit. Die Gewährleistung der Gebrauchs- bzw. Tragfähigkeit eines Tragwerkes bzw. Bauteils wird nach mathematischer Betrachtung als notwendiger Sicherheitsabstand zwischen der Beanspruchung $\mathrm{S}$ und der Beanspruchbarkeit $\mathrm{R}$ verstanden. Bei einer Mißachtung dieser Bedingung ist nicht nur die Systemsicherheit gefährdet, sondern auch das Leben von Menschen. Die Einhaltung eines bestimmten, vorgegebenen Sicherheitsindizes $\beta$ wird somit durch die Vergrößerung des Abstandes zwischen der Beanspruchbarkeit R und der Beanspruchung S erreicht. Dieses ist gleichbedeutend damit, daß ein größerer Abstand beider Verteilungsdichten eine kleinere Versagenswahrscheinlichkeit $p_{f}$ als Ursache hat, wobei zu beachten ist, daß es sich um jeweils gleiche Verteilungstypen bei der Beschreibung der Zufallsgrößen R und $\mathrm{S}$ handelt.

\subsection{Bestimmung von Teilsicherheitsbeiwerten}

Das Sicherheitskonzept im Bauwesen wird in [11], [42] erläutert. Die Bestimmung von Sicherheitsbeiwerten erfolgt nach der in [36] aufgeführten Zuverlässigkeitstheorie 1. Ordnung (Stufe II), welche in sehr enger Beziehung zu den Namen Rackwitz [99], Lind [81] und Fießler [39] steht. Unter den Näherungsverfahren der Stufe II werden diejenigen Methoden verstanden, die zur Durchführung des Sicherheitsnachweises eine angenäherte Abbildung der Grenzzustandsgleichung benötigen. Für verschiedene Anwendungsfälle im konstruktiven Ingenieurbau hat sich die Genauigkeit dieser Theorie als ausreichend erwiesen und somit Eingang in Bemessungsnormen gefunden. Bei der Beschreibung der Zufallsgrößen werden dabei lediglich der Mittelwert und die Standardabweichung verwendet. Durch die Vorgabe einer einzuhaltenden Zielsicherheit $\beta$ nach Tabelle 5.3 und einer aus Versuchen bekannten Streuung ist der Teilsicherheitsbeiwert der jeweiligen Zufallsgrößen zu ermitteln. Das Ziel jeder Sicherheitsbetrachtung besteht im Nachweis, daß der untersuchte Grenzzustand mit einer vorgegebenen Zuverlässigkeit, ausgedrückt durch einen Sicherheitsindex $\beta$, nicht eintritt. Für $\beta$ werden in Abhängigkeit vom angestrebten Grenzzustand und der Nutzungsdauer die in Tabelle 5.3 aufgeführten indikativen Werte verwendet. Für den Nachweis der Tragfähigkeit im Grenzzustand der Ermüdung ist nach Gleichung (5.1) zu gewährleisten, daß die ermüdungsrelevanten Beanspruchungen $s_{d}=\Delta F_{S d}$ die Ermüdungsfestigkeit $r_{d}=\Delta F_{R d}$ des Bauteils nicht überschreiten.

$\mathrm{s}_{\mathrm{d}} \leq \mathrm{r}_{\mathrm{d}} \Rightarrow \Delta \mathrm{F}_{\mathrm{Sd}} \leq \Delta \mathrm{F}_{\mathrm{Rd}}$

Bei der Annahme einer Normalverteilung der Beanspruchung S und der Beanspruchbarkeit R können die charakteristischen Werte $\mathrm{s}_{\mathrm{k}}$ und $\mathrm{r}_{\mathrm{k}}$ nach Bild 5.1 unter Verwendung der Mittelwerte $m_{S}$ und $m_{R}$, der Standardabweichungen $\sigma_{S}$ und $\sigma_{R}$, sowie der Fraktilwerte $\mathrm{k}_{\mathrm{S}}$ und $\mathrm{k}_{\mathrm{R}}$ wie folgt beschrieben werden:

$\mathrm{s}_{\mathrm{k}}=\mathrm{m}_{\mathrm{S}}+\mathrm{k}_{\mathrm{S}} \cdot \sigma_{\mathrm{S}}=\mathrm{m}_{\mathrm{S}} \cdot\left(1+\mathrm{k}_{\mathrm{S}} \cdot \mathrm{V}_{\mathrm{S}}\right)$ 
$\mathrm{r}_{\mathrm{k}}=\mathrm{m}_{\mathrm{R}}-\mathrm{k}_{\mathrm{R}} \cdot \sigma_{\mathrm{R}}=\mathrm{m}_{\mathrm{R}} \cdot\left(1-\mathrm{k}_{\mathrm{R}} \cdot \mathrm{V}_{\mathrm{R}}\right)$

Dabei sind die Variationskoeffizienten $V_{S}$ und $V_{R}$ der Beanspruchung $S$ und der Beanspruchbarkeit $\mathrm{R}$ wie nachfolgend aufgeführt zu bestimmen:

$\mathrm{V}_{\mathrm{S}}=\frac{\sigma_{\mathrm{S}}}{\mathrm{m}_{\mathrm{S}}}$ bzW. $\quad \mathrm{V}_{\mathrm{R}}=\frac{\sigma_{\mathrm{R}}}{\mathrm{m}_{\mathrm{R}}}$

In den Gleichungen (5.5) und (5.6) sind $\mathrm{k}_{\mathrm{S}}$ und $\mathrm{k}_{\mathrm{R}}$ Fraktilwerte, die als Funktion von der Aussagewahrscheinlichkeit W, der Fraktile $\mathrm{p}$ und der Versuchsanzahl n ermittelt werden.

$\mathrm{k}_{\mathrm{R}}=\mathrm{f}_{\mathrm{R}}(\mathrm{p}, \mathrm{W}, \mathrm{n})$ bzw. $\mathrm{k}_{\mathrm{S}}=\mathrm{f}_{\mathrm{S}}(\mathrm{p}, \mathrm{W}, \mathrm{n})$

Die Bestimmung der Fraktilwerte k wird in [41], [42] erläutert und erfolgt nach Gleichung (5.9). Weitere Tabellenwerte sind in [92] aufgeführt.

$\mathrm{k}(\mathrm{p}, \mathrm{W}, \mathrm{n})=\sqrt{1+\frac{1}{\mathrm{n}}} \cdot\left(-\mathrm{t}_{\mathrm{n}-1, \mathrm{p}}+\mathrm{t}_{\mathrm{n}-1,1-\alpha} \cdot \sqrt{\frac{1+0,5 \cdot \mathrm{K}_{\mathrm{T}}^{2}}{\mathrm{n}}}\right)$

mit: $\quad \mathrm{K}_{\mathrm{T}}=\Phi^{-1}(1-\mathrm{p})$

$t_{n-1, p} t-V e r t e i l u n g$ mit n-1 Freiheitsgraden für das Quantil $p$

Tabelle $5.4 \quad$ Fraktilwerte $\mathrm{k}(\mathrm{p}, \mathrm{W}, \mathrm{n})$

\begin{tabular}{|c|c|c|c|c|}
\hline nach & SVA & EC 3 & ETAG 001 & EC 1 \\
\hline $\begin{array}{c}\text { Anzahl der } \\
\text { Versuche n }\end{array}$ & $\begin{array}{c}\mathrm{p}=10 \% \\
\mathrm{~W}=90 \%\end{array}$ & $\begin{array}{c}\mathrm{p}=5 \% \\
\mathrm{~W}=95 \%\end{array}$ & $\begin{array}{c}\mathrm{p}=5 \% \\
\mathrm{~W}=90 \%\end{array}$ & $\begin{array}{c}\mathrm{p}=5 \% \\
\mathrm{~W}=50 \%\end{array}$ \\
\hline \hline 3 & 3,874 & 6,358 & 5,300 & 3,372 \\
\hline 4 & 3,067 & 4,649 & 4,035 & 2,631 \\
\hline 5 & 2,693 & 3,937 & 3,487 & 2,335 \\
\hline 6 & 2,472 & 3,539 & 3,175 & 2,177 \\
\hline 7 & 2,324 & 3,282 & 2,970 & 2,077 \\
\hline 8 & 2,217 & 3,099 & 2,823 & 2,010 \\
\hline 9 & 2,135 & 2,962 & 2,713 & 1,960 \\
\hline 10 & 2,070 & 2,855 & 2,626 & 1,923 \\
\hline 20 & 1,771 & 2,3795 & 2,239 & 1,772 \\
\hline 30 & 1,662 & 2,2109 & 2,101 & 1,727 \\
\hline 40 & 1,602 & 2,1195 & 2,026 & 1,706 \\
\hline$\infty$ & 1,282 & 1,645 & 1,645 & 1,645 \\
\hline
\end{tabular}


Eine Aussagewahrscheinlichkeit von $50 \%$ für die 5\%-Fraktile ist in der Tabelle D.1 (Anhang D) von prEN 1990 [35] verwendet worden. Die Werte in der letzten Spalte der Tabelle 5.4 stimmen somit mit den Werten von Tabelle D.1 überein.

Die Bemessungswerte ergeben sich wie folgt:

$$
\begin{aligned}
& s_{d}=m_{S}+\alpha_{S} \cdot \beta \cdot \sigma_{S}=m_{S} \cdot\left(1+\alpha_{S} \cdot \beta \cdot v_{S}\right) \\
& r_{d}=m_{R}-\alpha_{R} \cdot \beta \cdot \sigma_{R}=m_{R} \cdot\left(1-\alpha_{R} \cdot \beta \cdot V_{R}\right)
\end{aligned}
$$

Die Faktoren $\alpha_{S}$ und $\alpha_{R}$ sind Wichtungsfaktoren. Auf der Zuverlässigkeitstheorie 1. Ordnung basierend werden in [27], [36], [49] auf der sicheren Seite liegend, Wichtungsfaktoren für die Beanspruchung von $\alpha_{S}=-0,7$ und für die Beanspruchbarkeit von $\alpha_{R}=0,8$ festgelegt. In [78] wird gezeigt, daß diese Annahme zu einer $6 \%$ Überschätzung führen kann.

Die Teilsicherheitsbeiwerte $\gamma_{R}$ und $\gamma_{S}$ können somit wie nachfolgend aufgeführt bestimmt werden:

$$
\begin{aligned}
& \gamma_{S}=\frac{s_{d}}{s_{k}}=\frac{1+\alpha_{S} \cdot \beta \cdot V_{S}}{1+k_{S} \cdot V_{S}} \\
& \gamma_{R}=\frac{r_{k}}{r_{d}}=\frac{1-k_{R} \cdot V_{R}}{1-\alpha_{R} \cdot \beta \cdot V_{R}}
\end{aligned}
$$

Für eine logarithmische Normalverteilung wird vorausgesetzt, daß die Logarithmen der Zufallsgrößen S und R normalverteilt sind. Um den Unterschied zur Normalverteilung zu verdeutlichen, werden alle Werte, die sich auf die natürlichen Logarithmen beziehen mit einem Querstrich gekennzeichnet.

$$
\begin{aligned}
& \overline{\mathrm{s}}_{\mathrm{k}}=\ln \mathrm{s}_{\mathrm{k}}=\overline{\mathrm{m}}_{\mathrm{S}}+\mathrm{k}_{\mathrm{S}} \cdot \bar{\sigma}_{\mathrm{S}} \Rightarrow \mathrm{s}_{\mathrm{k}}=\mathrm{e}^{\overline{\mathrm{m}}_{\mathrm{S}}+\mathrm{k}_{\mathrm{S}} \cdot \bar{\sigma}_{\mathrm{S}}} \\
& \overline{\mathrm{r}}_{\mathrm{k}}=\ln \mathrm{r}_{\mathrm{k}}=\overline{\mathrm{m}}_{\mathrm{R}}-\mathrm{k}_{\mathrm{R}} \cdot \bar{\sigma}_{\mathrm{R}} \Rightarrow \mathrm{r}_{\mathrm{k}}=\mathrm{e}^{\overline{\mathrm{m}}_{\mathrm{R}}-\mathrm{k}_{\mathrm{R}} \cdot \bar{\sigma}_{\mathrm{R}}}
\end{aligned}
$$

Die Standardabweichungen der logarithmischen Normalverteilung können durch die Variationskoeffizienten aus Gleichung (5.7) ausgedrückt werden:

$$
\bar{\sigma}_{\mathrm{S}}=\sqrt{\ln \left(1+\mathrm{V}_{\mathrm{S}}^{2}\right)} \text { bzw. } \quad \bar{\sigma}_{\mathrm{R}}=\sqrt{\ln \left(1+\mathrm{V}_{\mathrm{R}}^{2}\right)}
$$

Die Mittelwerte bei dieser Verteilung ergeben sich zu:

$$
\overline{\mathrm{m}}_{\mathrm{S}}=\ln \left(\mathrm{m}_{\mathrm{S}}\right)-\frac{1}{2} \sigma_{\mathrm{S}}^{2} \quad \text { bzw. } \overline{\mathrm{m}}_{\mathrm{R}}=\ln \left(\mathrm{m}_{\mathrm{R}}\right)-\frac{1}{2} \sigma_{\mathrm{R}}^{2}
$$


Die Bemessungswerte können analog zur Gleichung (5.10) und (5.11) bestimmt werden und lauten:

$$
\begin{aligned}
& \bar{s}_{d}=\ln s_{d}=\bar{m}_{S}+\alpha_{S} \cdot \beta \cdot \bar{\sigma}_{S} \Rightarrow s_{d}=e^{\bar{m}_{S}+\alpha_{S} \cdot \beta \cdot \bar{\sigma}_{S}} \\
& \bar{r}_{d}=\ln r_{d}=\bar{m}_{R}-\alpha_{R} \cdot \beta \cdot \bar{\sigma}_{R} \Rightarrow r_{d}=e^{\bar{m}_{R}-\alpha_{R} \cdot \beta \cdot \bar{\sigma}_{R}}
\end{aligned}
$$

Für die Teilsicherheitsbeiwerte erhält man anschließend analog zur Gleichung (5.12) und (5.13) folgende Ausdrücke:

$$
\begin{aligned}
& \gamma_{S}=\frac{s_{d}}{s_{k}}=\frac{e^{\bar{m}_{S}+\alpha_{S} \cdot \beta \cdot \bar{\sigma}_{s}}}{e^{\bar{m}_{S}+k_{s} \cdot \bar{\sigma}_{s}}}=e^{\alpha_{S} \cdot \beta \cdot \bar{\sigma}_{s}-k_{S} \cdot \bar{\sigma}_{s}} \\
& \gamma_{R}=\frac{r_{k}}{r_{d}}=\frac{e^{\bar{m}_{R}-k_{R} \cdot \bar{\sigma}_{R}}}{e^{\bar{m}_{R}-\alpha_{R} \cdot \beta \cdot \bar{\sigma}_{R}}}=e^{\alpha_{R} \cdot \beta \cdot \bar{\sigma}_{R}-k_{R} \cdot \bar{\sigma}_{R}}
\end{aligned}
$$

Betrachtet man zusätzlich zu den Wichtungsfaktoren $\mathrm{a}_{\mathrm{R}}$ und $\mathrm{a}_{\mathrm{S}}$ den höchsten empfohlenen Sicherheitsindex $\beta=3,8$ für den Grenzzustand der Ermüdung aus Tabelle 5.3, so können die Bemessungswerte der Beanspruchbarkeit gemäß Gleichung (5.10) und (5.11) für eine normalverteilte Zufallsgröße sowie nach Gleichung (5.18) und (5.19) für eine logarithmisch normalverteilte Zufallsgröße bestimmt werden. In [70], [74] und [124] werden die Teilsicherheitsbeiwerte auf diesem Wege bestimmt. Die Ermittlung von Teilsicherheitsbeiwerten auf dem Gebiet der Befestigungstechnik wird in [12] bzw. [33] behandelt.

\subsection{Bemessung von Ankerschienenbefestigungen}

\subsubsection{Tragfähigkeit unter ruhenden Beanspruchungen}

Das bisherige Bemessungskonzept von Ankerschienenbefestigungen beruht auf dem Vergleich der zulässigen Lasten $\mathrm{F}_{\text {zul }}$ mit vorhandenen Lasten $\mathrm{F}_{\text {vorh }}$. Diese Sicherheitsphilosophie beruht auf einer globalen Betrachtungsweise und entspricht nicht dem aktuellen Stand der Befestigungstechnik. Dabei ist die Gleichung (5.22) stets einzuhalten.

$$
\mathrm{F}_{\text {vorh }} \leq \mathrm{F}_{\mathrm{zul}}
$$

Die derzeitige Bemessung von Ankerschienen ist in den jeweiligen bauaufsichtlichen Zulassungen des DIBt geregelt. Die Tragfähigkeit unter ruhender Beanspruchung wird hierbei auf der Basis experimenteller Untersuchungen an nicht einbetonierten Ankerschienen unter zentrischer Zugbelastung ermittelt. Die damit verbundene Untersuchungsprozedur wurde 1986 in einem Sitzungsprotokoll des zuständigen Sachverständigenausschusses für Dübel und Ankerschienen (SVA) festgelegt und bildet im 
Grundsatz bis heute die Basis der Zulassungsverfahren für nichtruhende Beanspruchungen. Die zulässige Beanspruchbarkeit wird gemäß der Gleichung (5.23) bestimmt:

$\mathrm{F}_{\mathrm{zul}}=\frac{\mathrm{F}_{\mathrm{m}}-\mathrm{k} \cdot \sigma}{\gamma}$

mit:

$\mathrm{F}_{\mathrm{m}}=$ Mittelwert der Versagenslasten aus Versuchen

$\sigma=$ Streuung der Versagenslasten

$\gamma=\gamma_{\mathrm{S}} \cdot \gamma_{\mathrm{R}}=$ globaler Sicherheitsfaktor

Folgende Parameter werden bei der statistischen Auswertung zugrunde gelegt:

- 5\%-Fraktile der Bruchlasten

- $75 \%$ Aussagewahrscheinlichkeit nach Eurocode 1 [36]

- $90 \%$ Aussagewahrscheinlichkeit nach ETAG 001 [38]

Die zulässige statische Last ergibt sich somit aus der 5\%-Fraktile der Versagenslasten unter einer definierten Aussagewahrscheinlichkeit, welche durch einen globalen Sicherheitsbeiwert $\gamma$ dividiert wird. In den aktuellen bauaufsichtlichen Zulassungen der neuen, gezahnten Ankerschienengeneration wird für die Beanspruchbarkeit ein globaler Sicherheitsbeiwert von 2,5 angesetzt. Dieser setzt sich aus dem Produkt der Teilsicherheitsbeiwerte für die Beanspruchung $\gamma_{\mathrm{S}}$ bzw. $\gamma_{\mathrm{F}}$ und der Beanspruchbarkeit $\gamma_{\mathrm{R}}$ bzw. $\gamma_{\mathrm{M}}$ zusammen.

$\gamma=\gamma_{S} \cdot \gamma_{R}$

Die Teilsicherheitsbeiwerte für den Bemessungswert der Beanspruchung $\mathrm{s}_{\mathrm{d}}$ sind allgemein anerkannt. Sie betragen für den ungünstigen Fall nach [37]:

$\gamma_{\mathrm{G}}=1,35 \quad$ für eine ständige Einwirkung $\mathrm{G}$

$\gamma_{\mathrm{Q}}=1,50 \quad$ für eine veränderliche Einwirkung $\mathrm{Q}$

Für ein Verhältnis der ständigen Einwirkung G zur veränderlichen Einwirkung Q von $1 / 2$ ergibt sich ein Teilsicherheitsbeiwert für die Beanspruchung $\mathrm{zu} \gamma_{\mathrm{S}}=1,4$ [33].

Der Teilsicherheitsbeiwert für die Beanspruchbarkeit R ermittelt sich aus der maßgebenden Versagensart Betonversagen und setzt sich wie folgt zusammen [34], [38]:

$\gamma_{\mathrm{Mc}}=\gamma_{\mathrm{c}} \cdot \gamma_{1} \cdot \gamma_{2}$

mit:

$\gamma_{\mathrm{c}}=$ Teilsicherheitsbeiwert für Beton auf Druck

$=1,5$

$\gamma_{1}=$ Teilsicherheitsbeiwert zur Berücksichtigung der Streuung der Zugfestigkeit von Baustellenbeton

$$
=1,2
$$


$\gamma_{2}=$ Teilsicherheitsbeiwert zur Berücksichtigung der Montagesicherheit eines Systems

Zuglast:

$\gamma_{2}=1,0$ für Systeme mit hoher Montagesicherheit

$=1,2$ für Systeme mit normaler Montagesicherheit

$=1,4$ für Systeme mit geringer, aber noch ausreichender Montagesicherheit

Querlast:

$\gamma_{2}=1,0$

Bei Ankerschienenbefestigungen wird von einer hohen Montagesicherheit ausgegangen und somit $\gamma_{2}$ zu 1,0 gesetzt. Der Teilsicherheitsbeiwert für die Beanspruchbarkeit bei Betonversagen $\gamma_{\mathrm{Mc}}$ nach (5.25) ergibt sich somit zu 1,8.

Der globale Sicherheitsfaktor $\gamma$ kann nun als Produkt der jeweiligen Teilsicherheitsbeiwerte für die Beanspruchung und Beanspruchbarkeit abgeleitet werden und beträgt nach Gleichung (5.24) $\gamma=1,4 \cdot 1,8=2,5$.

Für die im Rahmen dieser Arbeit untersuchten Profile hat sich unter ruhender Beanspruchung in allen Fällen ein Stahlversagen als maßgebende Versagensart ergeben. Für diese Versagensart ist der Teilsicherheitsbeiwert $\gamma_{\text {Ms }}$ nach [38] zu ermitteln, welcher in Abhängigkeit von der maximalen Festigkeit $\mathrm{f}_{\mathrm{uk}}$ und der Fließspannung $\mathrm{f}_{\mathrm{yk}} \mathrm{zu}$ bestimmen ist. Für eine reine Zugbeanspruchung ergibt sich somit:

$\gamma_{\mathrm{Ms}}=\frac{1,2}{\mathrm{f}_{\mathrm{yk}} / \mathrm{f}_{\mathrm{uk}}} \geq 1,40$

Für eine reine Querbeanspruchung wird der Teilsicherheitsbeiwert über eine Fallunterscheidung der maximalen Festigkeit $\mathrm{f}_{\mathrm{uk}}$ bestimmt:

$\gamma_{\mathrm{Ms}}=\frac{1,0}{\mathrm{f}_{\mathrm{yk}} / \mathrm{f}_{\mathrm{uk}}} \geq 1,25$

für $\mathrm{f}_{\text {uk }} \leq 800 \mathrm{~N} / \mathrm{mm}^{2}$ und $\mathrm{f}_{\mathrm{yk}} / \mathrm{f}_{\mathrm{uk}} \leq 0,8$

bzw.

$\gamma_{\mathrm{Ms}}=\frac{1,2}{\mathrm{f}_{\mathrm{yk}} / \mathrm{f}_{\mathrm{uk}}} \geq 1,50$

für $\mathrm{f}_{\mathrm{uk}}>800 \mathrm{~N} / \mathrm{mm}^{2}$ oder $\mathrm{f}_{\mathrm{yk}} / \mathrm{f}_{\mathrm{uk}}>0,8$

Bei Betrachtung der kritischen Zugbeanspruchung beträgt der Teilsicherheitsbeiwert $\gamma_{\mathrm{Ms}}$ für Stahlversagen mindestens 1,4 nach Gleichung (5.26). Aus der Tabelle $4.2 \mathrm{kann}$ ein Quotient $\mathrm{f}_{\mathrm{uk}} / \mathrm{f}_{\mathrm{yk}}$ (entspricht $\mathrm{R}_{\mathrm{m}} / \mathrm{R}_{\mathrm{p} 0,2}$ ) für das Material der Ankerschiene (Schenkel, Rücken) von etwa 1,1 ermittelt werden. Da sich somit ein Beiwert von 1,32 
ergibt, welcher jedoch kleiner ist als die Mindestanforderung von 1,4, ist der Mindestwert zu berücksichtigen. Im Gegensatz zum Teilsicherheitsbeiwert aus dem Stahlbau mit $\gamma_{M}=1,1$, welcher im Bezug auf die Fließgrenze ermittelt wird, wird in der Befestigungstechnik der Teilsicherheitsbeiwert aus den maximalen Bruchlasten abgeleitet.

Der globale Sicherheitsfaktor $\gamma$ unter Berücksichtigung des Stahlversagens als maßgebende Versagensursache kann nun aus den jeweiligen Teilsicherheitsbeiwerten für die Beanspruchung bzw. Beanspruchbarkeit abgeleitet ebenfalls nach Gleichung (5.24) bestimmt werden und beträgt etwa $\gamma=1,4 \cdot 1,4=2,0$.

In einer derzeit nicht veröffentlichten Bemessungsvorschrift für Verankerungen im Beton, der CEN/TC 250 [16], werden zusätzlich zu den Gleichungen (5.26) bis (5.28) für das Stahlversagen bei Ankerschienen Teilsicherheitsbeiwerte in Abhängigkeit von der Versagensart angegeben. Für ein Versagen in der Verbindungsstelle zum Ankerbolzen im Rückenbereich wird der maximale Teilsicherheitsbeiwert von $\gamma_{\mathrm{Ms}, \mathrm{c}}$ (aktuelle Bezeichnung) von 1,8 angegeben. Somit ergibt sich auch hierbei ein globaler Sicherheitsfaktor von 2,5 .

Die aktuelleren Bemessungskonzepte von Befestigungselementen, wie sie bereits in den neuen europäischen Vorschriften

- Deutsches Institut für Bautechnik 6/1993 [24]

- Comité Euro-International du Béton 6/1995 [18]

- European Organisation for Technical Approvals 6/1997 [38]

für die Verankerung von Dübeln im Beton verwendet werden, basieren auf der Methode der Grenzzustände bzw. Teilsicherheitsbeiwerte, die zuvor in den Abschnitten 5.1 bis 5.3 vorgestellt worden ist. Dabei wird der Bemessungswert der Beanspruchbarkeit auf der Grundlage des 5\%-Fraktils der Bruchlasten in Abhängigkeit von der Versagensart (Betonversagen, Stahlversagen, Herausziehen) bei unterschiedlichen Belastungsrichtungen ermittelt. Je nach Versagensart sind unterschiedliche Teilsicherheitsbeiwerte anzusetzen. In [34] bzw. den o.g. Vorschriften können in Abhängigkeit von den relevanten Versagensarten und Belastungsrichtungen die charakteristischen Teilsicherheitsbeiwerte nachvollzogen werden. 


\subsubsection{Tragfähigkeit unter nichtruhenden Beanspruchungen}

Bei Bauteilen, die ständigen Schwingspielen ausgesetzt sind, kann der Grenzzustand der Ermüdung durchaus maßgebend werden. Dabei ist der Nachweis analog zur Gleichung (5.4) durchzuführen. Die Bestimmung der Tragfähigkeit von Ankerschienen unter nichtruhenden Beanspruchungen wird bisher auf der Basis von experimentellen Untersuchungen an nicht einbetonierten Ankerschienen unter zentrischer Zugschwellbelastung durchgeführt. Dabei wird mit einer konstanten Oberlast gearbeitet, die dem 1,4-fachen der zulässigen statischen Last $F_{z u l}$ nach Gleichung (5.23) entspricht. Die Oberlast entspricht somit dem Bemessungswert der ruhenden Beanspruchung nach [32]. Durch die Variation der Schwingbreite $\Delta \mathrm{F}$ wird folglich der funktionelle Zusammenhang der Schwingbreite über die Schwingspielzahl im Zugschwellbereich mit Hilfe einer Regressiongeraden erreicht (siehe Kapitel 4). Maßgebender Bemessungswert ist die ertragbare Schwingbreite bei der Bemessungsschwingspielzahl von 2 $10^{6}$ (Abschnitt 4.5.3.1). Der Bemessungswert der Schwingbreite wird unter der Annahme einer Normalverteilung bzw. logarithmischen Normalverteilung, behaftet mit einem Sicherheitsbeiwert bestimmt. Zum aktuellen Zeitpunkt wird bei der Ermittlung des ermüdungsrelevanten Bemessungswertes von Ankerschienensystemen zusätzlich zu den Anforderungen des SVA [25] mit

- $10 \%$ - Fraktile der Schwingbreiten im Versagensfall

- $90 \%$ Aussagewahrscheinlichkeit

- Teilsicherheitsbeiwert $\gamma=1,5$

die Auswertemethode des Eurocode 3 (siehe Tabelle 5.5) angewendet. Weiterhin werden in der Befestigungstechnik zur Vereinheitlichung der verschiedenen europäischen Normen durch die EOTA (European Organisation for Technical Approvals) sog. ETAG Richtlinien (European Technical Approval Guideline) erteilt. Für Ankerschienen gibt es zur Zeit keine ETAG Zulassung. Mit der ETAG 001 [38] sind Metalldübel als Verankerungselement im Beton zugelassen worden.

Tabelle 5.5 Parameter verschiedener Auswerteverfahren

\begin{tabular}{|c|c|c|c|}
\hline & SVA & Eurocode 3 & ETAG 001 \\
\hline \hline Quantile p [\%] & 10 & 5 & 5 \\
\hline Aussagewahrsch. W [\%] & 90 & 95 & 90 \\
\hline Sicherheitsbeiwert $\gamma$ & 1,50 & 1,35 & 1,35 \\
\hline
\end{tabular}

Die ETAG 001 stellt das aktuellste Regelwerk dar. Der Unterschied zum Eurocode 3 liegt in der geringeren Aussagewahrscheinlichkeit von 90 \%. Die Auswertung nach den Vorgaben des SVA sieht eine 10\%-Fraktile bei einer Aussagewahrscheinlichkeit von $90 \%$ vor. Zur besseren Veranschaulichung der unterschiedlichen Auswerteverfahren sind die charakteristischen Schwingbreiten und die daraus resultierenden Bemessungslasten in Abhängigkeit vom Variationskoeffizienten auf der Abszisse in Bild 5.3 dargestellt. Auf der Ordinate sind die auf den charakteristischen Wert des aktuellen Auswerteverfahrens nach SVA bezogenen prozentualen Schwingbreiten aufgetragen. 
Dem Diagramm wurde eine Anzahl von sieben Versuchen, die einen Mittelwert von $10 \mathrm{kN}$ aufweisen, zugrunde gelegt. Diese Annahme entspricht realistischen Versuchswerten.

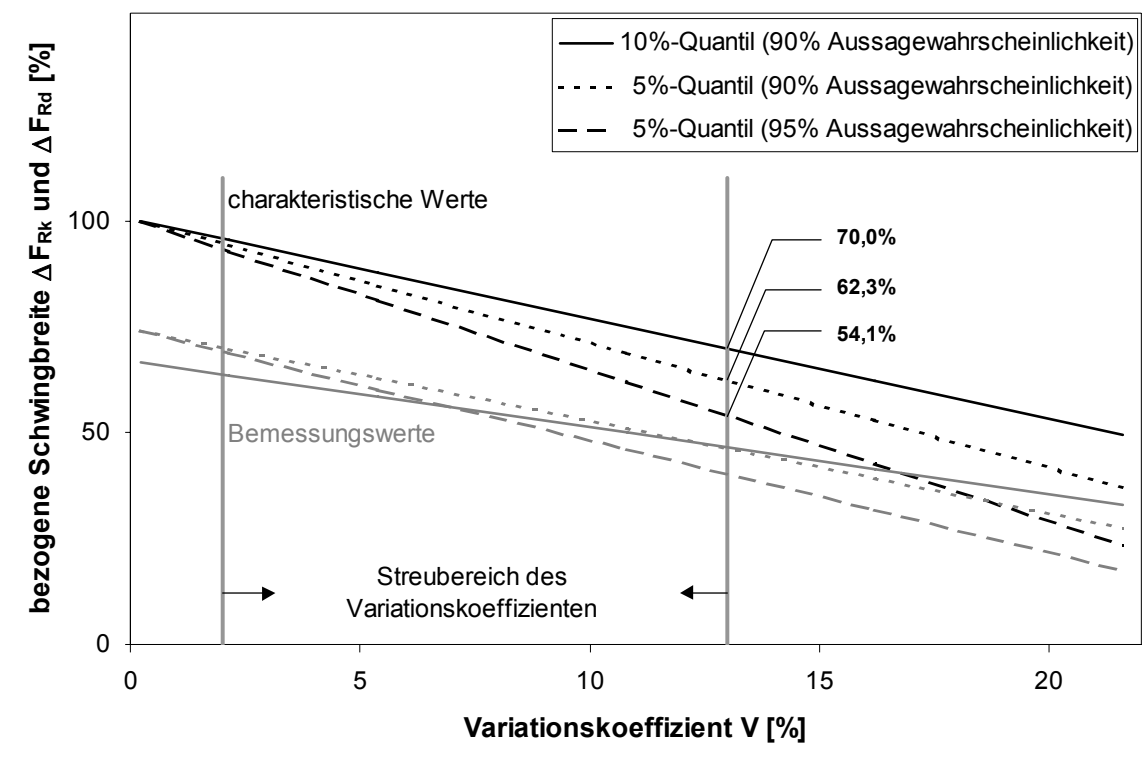

Bild 5.3 Bezogene Schwingbreiten in Abhängigkeit vom Variationskoeffizienten

Es wird deutlich, daß im Streubereich der in Kapitel 4 erzielten Versuchsergebnisse (etwa zwischen $2 \%$ und $13 \%$ ) die Bemessungswerte in Bild 5.3 keine starken Differenzen zueinander aufweisen. In diesem Bereich liegen die Schnittpunkte aller Bemessungswerte, weshalb alle Auswerteverfahren ähnliche Werte ergeben. Den allgemeinen Regeln der Sicherheitsanforderungen entsprechend sollte für die Bemessung die 5\%-Fraktile als charakteristischer Wert festgelegt werden. Eine Aussagewahrscheinlichkeit von $90 \%$ bei Ankerschienenbefestigungen wird in Anlehnung an die Vorgaben der ETAG 001 nach Ansicht des Verfassers als angemessen angesehen.

Die Bestimmung der ermüdungsrelevanten Beanspruchungen $\Delta \mathrm{F}_{\mathrm{Sd}}$ erfordert sehr umfangreiche statistische Informationen aus Messungen oder Beobachtungen von bereits durchgeführten Untersuchungen. Gerade diese Tatsache führt dazu, die Beanspruchungen vereinfacht darzustellen oder gar nur Maximalwerte anzugeben. Lastmodelle zur theoretischen Beschreibung der Einwirkung sind beispielsweise in [36] dargestellt. Eine analytische Beschreibung der Last liegt nur in speziellen Fällen (Kollektive bei Kranbahnen nach DIN 4132 [28]) vor. Um die Unsicherheiten bei der Bestimmung der Beanspruchbarkeit des Tragwerks zu kompensieren, sind die einwirkenden Schwingbreiten, behaftet mit einem Teilsicherheitsbeiwert $\gamma_{\mathrm{Ff}}$, zu bestimmen.

$\Delta \mathrm{F}_{\mathrm{Sd}}=\gamma_{\mathrm{Ff}} \cdot \Delta \mathrm{F}_{\mathrm{Sk}}$

In Anlehnung an europäische Normen wird der Teilsicherheitsbeiwert für die Ermüdungsbeanspruchung $\gamma_{\mathrm{Ff}}$ nach dem Eurocode 3 [37] mit 1,0 angesetzt werden, sofern 
keine zusätzlichen Festlegungen erfolgen. Die Grundlage für die empfohlenen Teilsicherheitsbeiwerte ist die Einstufung der Konstruktionsdetails in Kerbgruppen.

Im Falle der Beanspruchbarkeit jedoch ist die Bestimmung der Ermüdungsfestigkeit $\Delta \mathrm{F}_{\mathrm{RD}}$ im Vergleich zur Beanspruchung wesentlich unkomplizierter und kann deshalb auch genauer untersucht werden. Eventuell vorhandene Einflüsse auf das Tragverhalten können hervorgehoben und exakter beschrieben werden. Um Unsicherheiten bei der Bestimmung der Ermüdungsfestigkeit abzudecken, wird diese beim Nachweis gegen Ermüdung mit einem Teilsicherheitsbeiwert $\gamma_{\mathrm{Mf}}$ behaftet. Der Bemessungswert der ertragbaren Schwingbreite errechnet sich wie folgt:

$$
\Delta \mathrm{F}_{\mathrm{Rd}}=\frac{\Delta \mathrm{F}_{\mathrm{Rk}}}{\gamma_{\mathrm{Mf}}}
$$

Der Teilsicherheitsbeiwert für die Ermüdungsfestigkeit $\gamma_{\mathrm{Mf}}$ wird in Abhängigkeit von der Überprüfung und Unterhaltung sowie der Zugänglichkeit der Kerbstelle und der Schadensfolgen nach [37] festgelegt. Dabei wird von einer regelmäßigen Überprüfung ausgegangen. Der Teilsicherheitsbeiwert $\gamma_{\mathrm{Mf}}$ beträgt für schadenstolerante und einfach zugängliche Kerbstellen 1,00 bzw. 1,35 für nicht schadenstolerante und schlecht zugängliche Kerbstellen. Bei einer Ankerschienenbefestigung kann davon ausgegangen werden, daß aufgrund von Anbauteilen, welche bündig mit der Ankerschiene abschließen, eine schlechte Zugänglichkeit der möglichen Versagensstellen gegeben ist und vermutlich Schäden an den Kerbstellen nicht frühzeitig erkannt werden können. Im Aufzugsbau sind regelmäßige Kontrollen notwendig, bei denen die Kerbstellen genauer inspiziert werden müssen und somit auch eine leichte Zugänglichkeit vorausgesetzt wird. Kerbstellen bei Ankerschienen sind zum einen die Verbindungsstellen zwischen dem Ankerfuß und dem Schienenrücken und zum anderen die Übergangsbereiche vom Schienenrücken zum Schienenschenkel bzw. vom Schienenschenkel zur Schienenlippe. Betrachtet man hierzu die Versagensfälle in Abschnitt 4.5, so können die Kerbstellen bestätigt werden. Zusätzlich sollten Befestigungen mit Ankerschienen als nicht-schadenstolerante Bauteile betrachtet werden, weil im Falle eines Versagens keine Verteilung der Lasten gewährleistet werden kann. Um auf der sicheren Seite zu liegen sollte nach [37] der größte vorgegebene Sicherheitsbeiwert angesetzt werden.

Zur Veranschaulichung der Bemessungssituation wird eine leicht modifizierte Darstellungsweise im Dauerfestigkeitsdiagramm nach Goodman in Bild 5.4 verwendet. Diese ist vor allem bei einer kombinierten Beanspruchung durch ruhende und nichtruhende Lasten bevorzugt anzuwenden. Dabei wird der Bemessungswert der Gesamtbeanspruchbarkeit $F_{\text {tot }}=F_{\text {vorh }}+\Delta F_{R d}$ als Funktion der einwirkenden statischen Last $F_{\text {vorh }}$ bei einer fest definierten Schwingspielzahl aufgetragen. Diese Darstellung gilt nur für einen bestimmten Vertikalschnitt im Ermüdungstragfähigkeitsdiagramm. In der bisherigen Bemessung wird eine Bemessungsschwingspielzahl von $2 \cdot 10^{6}$ angesetzt und ist nach den Erkenntnissen aus Kapitel 4 gerechtfertigt. Die einwirkende statische Last $\mathrm{F}_{\mathrm{vor}}$ entspricht der Unterlast und bildet in diesem Diagramm die Winkelhalbierende. 
Die Oberlast verläuft parallel zur Unterlast mit einem Abstand entsprechend dem Bemessungswert der Schwingbreite $\Delta \mathrm{F}_{\mathrm{Rd}}$ aus der aktuellen Zulassung [21], [23]. Es wird deutlich, daß unabhängig vom Lastniveau dieselbe Schwingbreite $\Delta F_{R d}$ wirkt. Dieser Bemessungswert ist unabhängig von der Größe der statisch wirkenden Last $F_{\text {vorh }}$ und wird über die gesamte Beanspruchungsdauer bis $\mathrm{zu}$ einem Grenzwert, entsprechend der zulässigen Last $F_{\text {zul }}$, als eine Konstante angesetzt. Das Bemessungskonzept sieht somit eine obere Begrenzung des Bemessungsbereiches vor, die der zulässigen statischen Last entspricht.

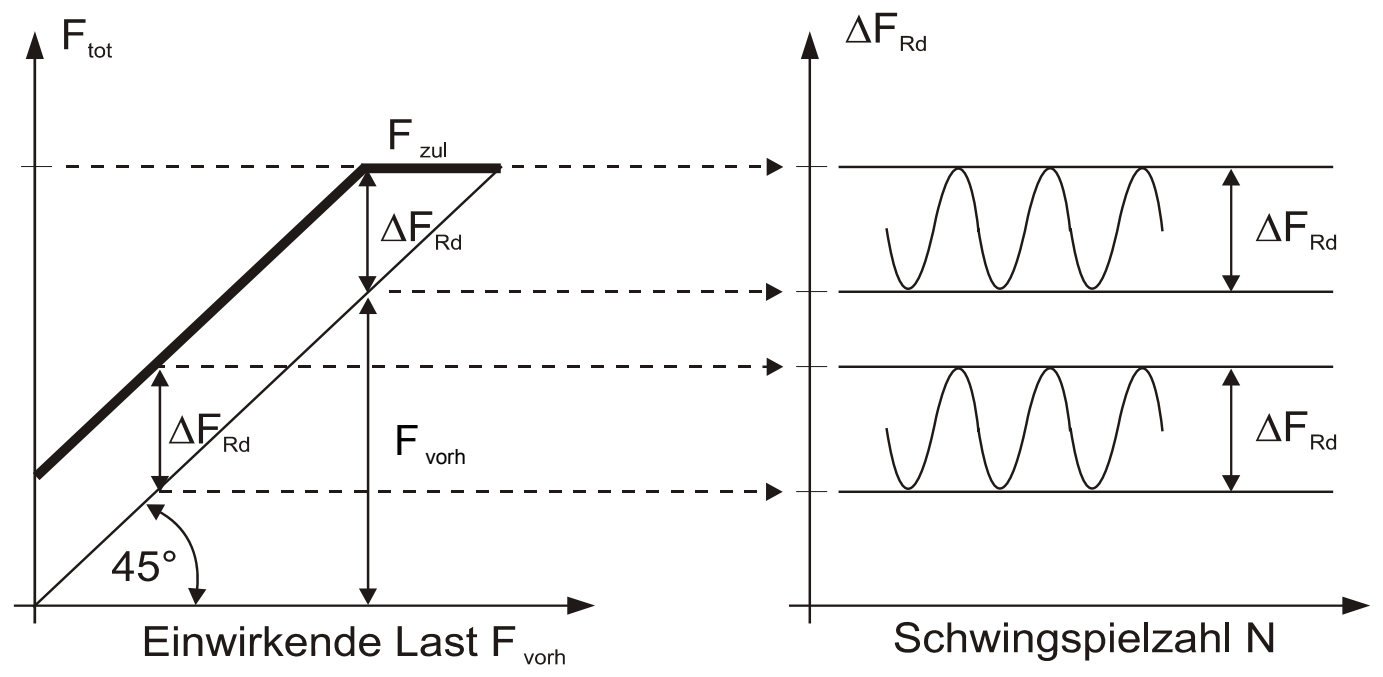

Bild 5.4 Bisheriges Bemessungskonzept

Treten ermüdungsrelevante Beanspruchungen in verschiedene Richtungen auf, so ist die Kenntnis einer Interaktion der Kraftgrößen von Notwendigkeit. Für eine Beanspruchung quer zur bzw. in Richtung der Schienenlängsachse liegen zur Zeit jedoch keine Ergebnisse unter nichtruhenden Beanspruchungen vor. Eine Interaktionsbeziehung zwischen der zentrischen Zugrichtung und einer Querrichtung ist folglich nicht bekannt. In [63] werden Ermüdungsnachweise für Kopfbolzendübel unter kombinierter Zug- und Schubbeanspruchungen gemäß der Bemessungsrichtlinie des Eurocode 3 [37] durchgeführt.

Als Fazit aus dem bisherigen Sicherheits- bzw. Bemessungskonzept können folgende Punkte gefolgert werden:

- Die Grundlage zur Ermittlung des Bemessungswertes der ertragbaren Schwingbreite basiert auf einer globalen Betrachtungsweise. Dabei dienen die nach der Methode der globalen Sicherheitsbeiwerte ermittelten zulässigen statischen Lasten als Eingangsparameter für das Lastniveau der nichtruhenden Beanspruchung. Diese bewirken eine sehr konservative Bemessung, weshalb mögliche Systemreserven auf der Materialseite nicht vollständig ausgenutzt werden können.

- Experimentelle Untersuchungen erfolgen auf einem $40 \%$ höheren Lastniveau als statisch zugelassen ist.

- Es zeigt sich keine Abhängigkeit der bereits einwirkenden Last von der Schwingbreite, welche jedoch nach dem Goodman-Diagramm zu erwarten wäre. 
- Die zentrische Zugrichtung wird bisher mangels weitergehender Untersuchungen als kritisch angesehen.

- Von der zentrischen Zugrichtung abweichende nichtruhende Beanspruchungen sind zur Zeit nicht zugelassen. Durch eine Interaktion verschiedener Beanspruchungsrichtungen ist eine bessere Ausnutzung des Querschnitts möglich.

Diese wesentlichen Punkte müssen eindeutig geklärt werden, um somit eine umfassende, einheitliche und variable Sicherheitsbetrachtung gewährleisten zu können.

Um in Einklang mit den in den Eurocodes verankerten Annahmen im Bereich des Bauwesens zu stehen, wird als charakteristische Größe das 5\%-Fraktile als Grundlage für eine Sicherheitsbetrachtung angestrebt. Eine Aussagewahrscheinlichkeit von $90 \%$ kann nach Ansicht des Verfassers, die nachfolgend dargestellt und erläutert wird, als ausreichend angesehen werden. In [40] wird in Abhängigkeit von der Aussagewahrscheinlichkeiten W der erforderliche Umfang der Stichprobe vorgeschlagen, welcher zusätzlich mit dem Variationskoeffizienten verknüpft wird. Dabei wird zwischen einem kleinen $(<10 \%)$ und einem großen $(>10 \%)$ Variationskoeffizienten unterschieden. Stahl weist in Hinblick auf seine Tragfähigkeit geringe Variationskoeffizienten auf, weshalb eine Aussagewahrscheinlichkeit von $90 \%$ in [40] vorgeschlagen wird. Je größer die Aussagewahrscheinlichkeit ist, desto größer ist der Bereich in dem die Stichproben liegen können und desto höher die Einbußen bei der Bestimmung des charakteristischen Wertes. Um diesem entgegenzuwirken, sollte die Aussagewahrscheinlichkeit möglichst den Anforderungen entsprechend gewählt werden. Für Dübelverbindungen wird in [38] eine Aussagewahrscheinlichkeit von $90 \%$ festgelegt. Der Anwendungsbereich von Ankerschienen und Dübeln ist identisch. Dübelbefestigungen haben im Vergleich zu Ankerschienen ein weites Versagensspektrum und somit größere Streuungen in der Tragfähigkeit. Es ist also nicht ersichtlich, weshalb Ankerschienen mit einer Aussagewahrscheinlichkeit von $95 \%$ nach [37] strengeren Kriterien unterliegen. Somit wird für Befestigungen von Ankerschienen, in Anlehnung an die Bemessung von Dübeln, eine Aussagewahrscheinlichkeit von $90 \%$ empfohlen. In Hinblick auf aktuelle Normen sollte ein Fraktile von $5 \%$ (statt $10 \%$ nach [25]) angestrebt werden. 


\subsubsection{Aktuelle Systemsicherheiten bei Ermüdungsbeanspruchungen}

Eine nichtruhende Beanspruchung abweichend von der zentrischen Zugrichtung ist zur Zeit bei Ankerschienensystemen nicht zugelassen. Nachfolgende Diagramme zeigen jedoch, daß durchaus eine Beanspruchung auch abweichend von der reinen zentrischen Zugrichtung angesetzt werden darf. Die zentrische Zugrichtung wird in Hinblick auf die Tragfähigkeit und den daraus resultierenden Sicherheitsbeiwerten auf Seiten der Beanspruchbarkeit als kritisch angesehen, da die Sicherheit in Bezug auf den charakteristischen Wert in diesem Fall am kleinsten ist. Betrachtet man den vorhandenen Sicherheitsbeiwert in Bild 5.5 in Bezug auf den aktuellen, in der Zulassung [21], [23] festgesetzten Zulassungswert von $3 \mathrm{kN}$ für das Profil 38/23 St, so zeigen sich vorhandene Sicherheiten bei Betrachtung des 5\%-Fraktils und $\mathrm{W}=90 \%$, die um den Wert 1,35 liegen. Lediglich vereinzelte Versuchsserien zeigen Beiwerte, die geringer sind. Die Ursache hierfür kann auf eventuelle Versagenszustände infolge von Herstellungsmängel (siehe Kapitel 4) zurückgeführt werden. Somit kann gefolgert werden, daß im Rahmen einer Bemessung der Teilsicherheitsbeiwert für die Ermüdungsbeanspruchung von mindestens 1,35 für die zentrische Zugrichtung anzustreben ist, um damit eine möglichst optimale Ausnutzung der Tragfähigkeit zu gewährleisten und mit den Vorgaben des Eurocode 3 [37] zu übereinstimmen.

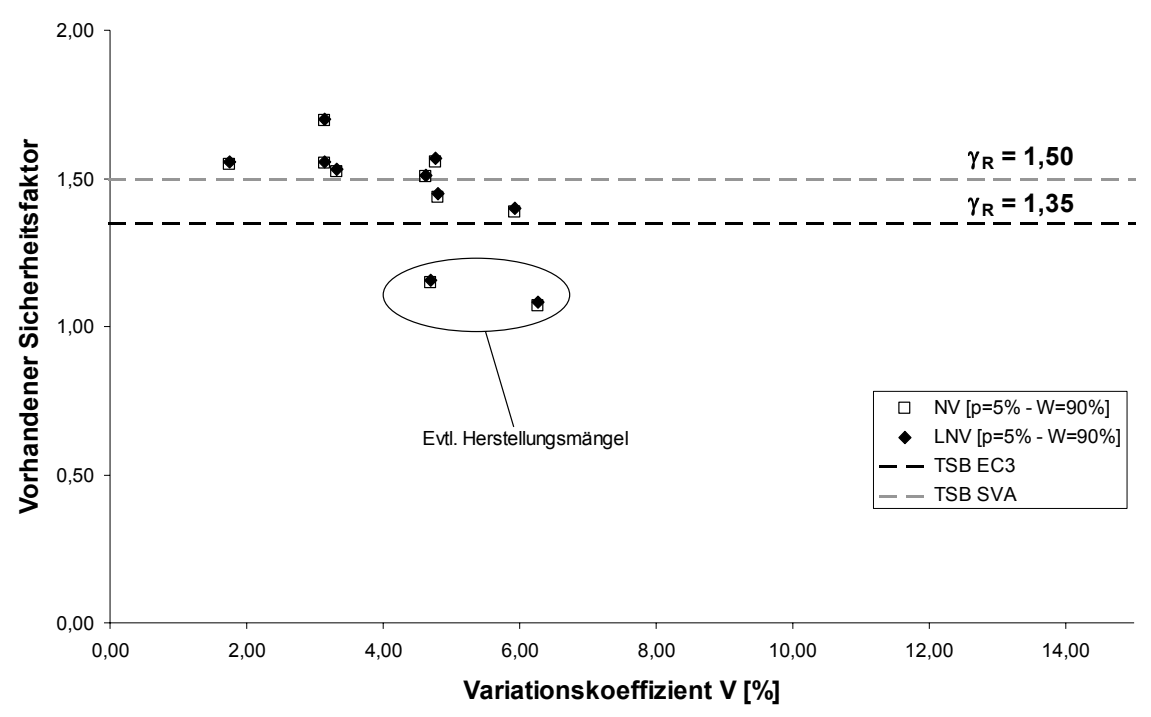

Bild 5.5 Vorhandene Sicherheit nach aktueller Bemessung (zentrischer Zug)

Eine auf den aktuellen Zulassungswert bezogene Sicherheitsbetrachtung für eine nicht zentrische Zugrichtung zeigt die vorhandenen Beiwerte in Bild 5.6, die ebenfalls oberhalb von 1,35 liegen und somit die möglichen Tragreserven im System zeigen. Der größte Sicherheitsabstand in Bezug auf den aktuellen in [21], [23] angegebenen Zulassungswert der zentrischen Zugrichtung stellt sich bei einer Querbeanspruchung in Schienenlängsrichtung $V_{x}$ ein. Hier werden Sicherheiten um den Wert 6 erreicht. 


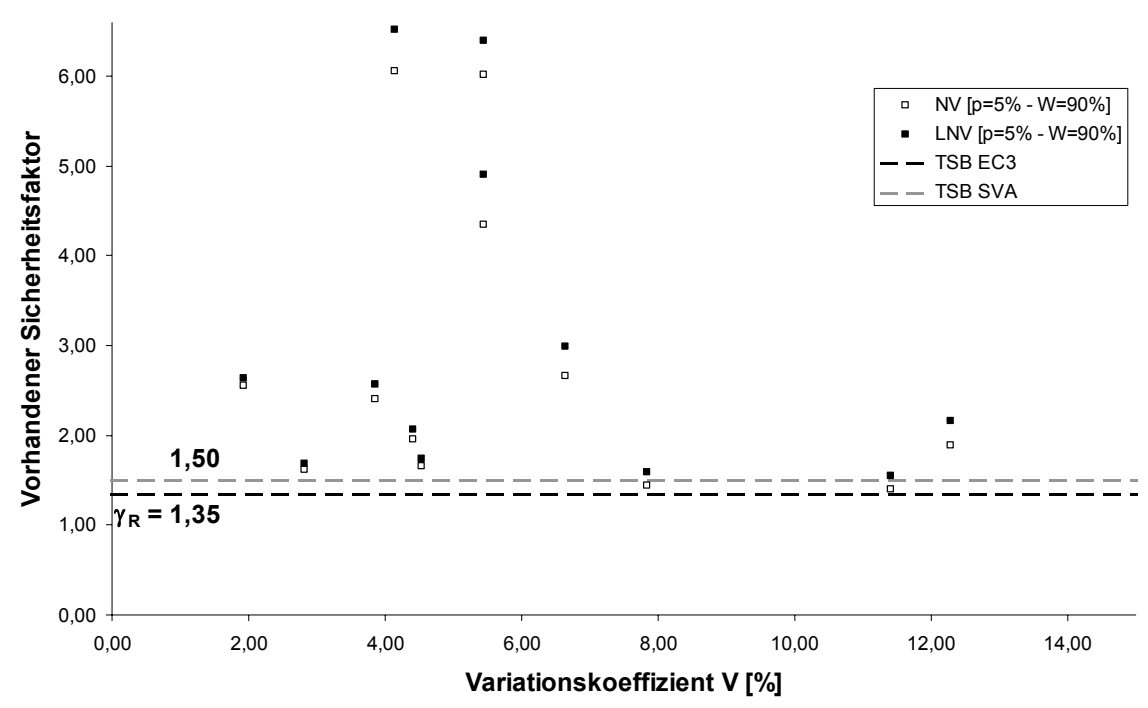

Bild 5.6 Sicherheit bei konstanter Beanspruchbarkeit $\Delta \mathrm{F}_{\mathrm{Rd}}=3 \mathrm{kN}$ in allen Richtungen

Es kann somit gefolgert werden, daß bei Ankerschienenbefestigungen eine allseitige nichtruhende Beanspruchung angesetzt werden darf. In allen Fällen ist ein Sicherheitsbeiwert von mindesten 1,35 im Bezug auf den aktuellen Zulassungswert gegeben. Im nachfolgenden Abschnitt werden die Teilsicherheitsbeiwerte gemäß des Anhanges $\mathrm{D}$ aus [35] für o.g. unterschiedliche Auswerteverfahren bestimmt.

\subsubsection{Ermittlung des Teilsicherheitsbeiwertes der Ermüdungsfestigkeit}

Es soll nachfolgend gezeigt werden, welche Teilsicherheitsbeiwerte $\gamma_{\mathrm{Mf}}$ in Abhängigkeit von den Belastungsrichtungen bei den zur Zeit gültigen Bemessungsverfahren aus Tabelle 5.5 resultieren. Zur Veranschaulichung der Teilsicherheitsbeiwerte für die Beanspruchbarkeit R wird das Verfahren $b$ aus dem Eurocode 1 zur direkten Abschätzung des Bemessungswertes zugrunde gelegt. Bei diesem Verfahren wird der Bemessungswert aus den Versuchsergebnissen ermittelt. Dabei wird in Abhängigkeit von der Anzahl der Versuche, der angestrebten Fraktile, der Aussagewahrscheinlichkeit und der Kenntnis des Variationskoeffizienten (ausgedrückt durch ein Wert $\mathrm{k}_{\mathrm{n}}$ ) der Bemessungswert ermittelt. Die in [36] angegebenen Tabellenwerte beziehen sich lediglich auf die 5\%- Fraktile bei einer Aussagewahrscheinlichkeit von $50 \%$. Da im Rahmen dieser Arbeit Aussagewahrscheinlichkeiten von mindestens $90 \%$ gefordert werden (siehe Abschnitt 5.4.2), ist es notwendig, die Fraktilwerte für diese Aussagewahrscheinlichkeiten zu ermitteln. Das Vorgehen zur Bestimmung dieser Werte wird in [42], [66], [108], [114] beschrieben. Der Bemessungswert der Beanspruchbarkeit kann dann, wie bereits in Abschnitt 5.3 beschrieben worden ist, für den Grenzzustand der Ermüdung mit einem maximal anzusetzenden Zuverlässigkeitsindex von $\beta=3,8$ direkt aus den Versuchsergebnissen bestimmt werden. 
Hinsichtlich der geeigneten Beschreibung der Versuchsergebnisse durch Verteilungsfunktionen empfiehlt [41], in Abhängigkeit von der Stichprobe $\mathrm{n}$ und des Variationskoeffizienten $\mathrm{V}$, den Verteilungstyp festzulegen. Zur Beschreibung der Versuchsergebnisse mit Hilfe einer Normalverteilung werden nachfolgende Parameter in Abhängigkeit von der Anzahl der Versuche und des Variationskoeffizienten empfohlen:

- $(4<\mathrm{n}<9), \mathrm{V}<10 \%$

- $(9<\mathrm{n}<29), \mathrm{V}<15 \%$

- $(\mathrm{n}>30), \mathrm{V}<20 \%$

Da im Rahmen dieser Arbeit die Variationskoeffizienten V für alle Belastungsrichtungen kleiner als $12 \%$ sind und die vorhandene Versuchsanzahl zwischen 3 und 40 liegt, würde sich nach der o.g. Empfehlung die Beschreibung der Versuchsergebnisse durch eine Normalverteilung (NV) gut eignen. Jedoch hat der $X^{2}$-Anpassungstest [1], [2] ergeben, daß die logarithmische Normalverteilung (LNV) eine bessere Anpassung liefert. Zur Bestimmung von Teilsicherheitsbeiwerten werden im weiteren Verlauf beide Verteilungstypen zugrunde gelegt.

Bei der direkten Abschätzung des Bemessungswertes bestimmt die Anzahl der Stichproben wesentlich die Höhe des Teilsicherheitsbeiwertes. Die Diagramme in Bild 5.7 und Bild 5.8 zeigen die Teilsicherheitsbeiwerte (TSB) in Abhängigkeit von dem Verteilungstyp, der Anzahl der Versuche und des Variationskoeffizienten.
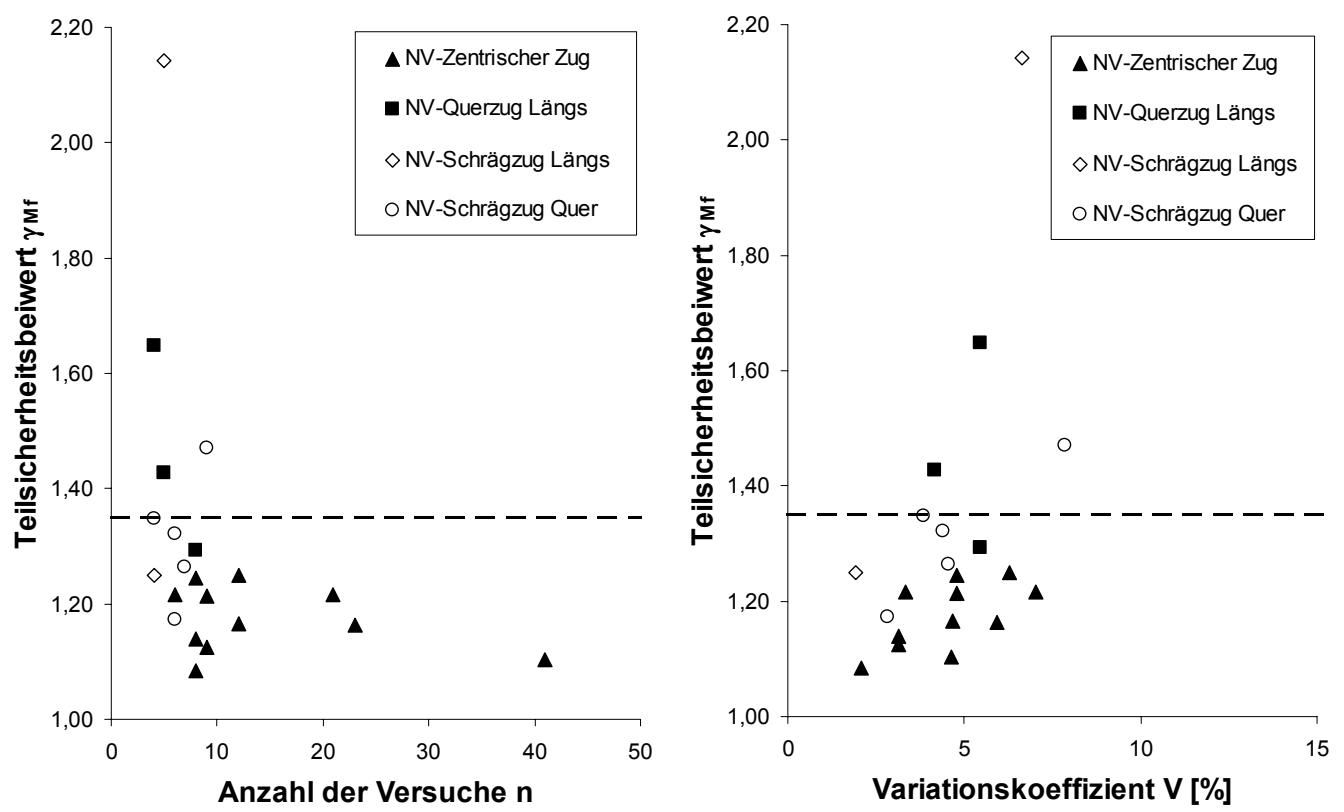

Bild 5.7 TSB als Funktion der Versuchsanzahl und Variationskoeffizienten (NV) 

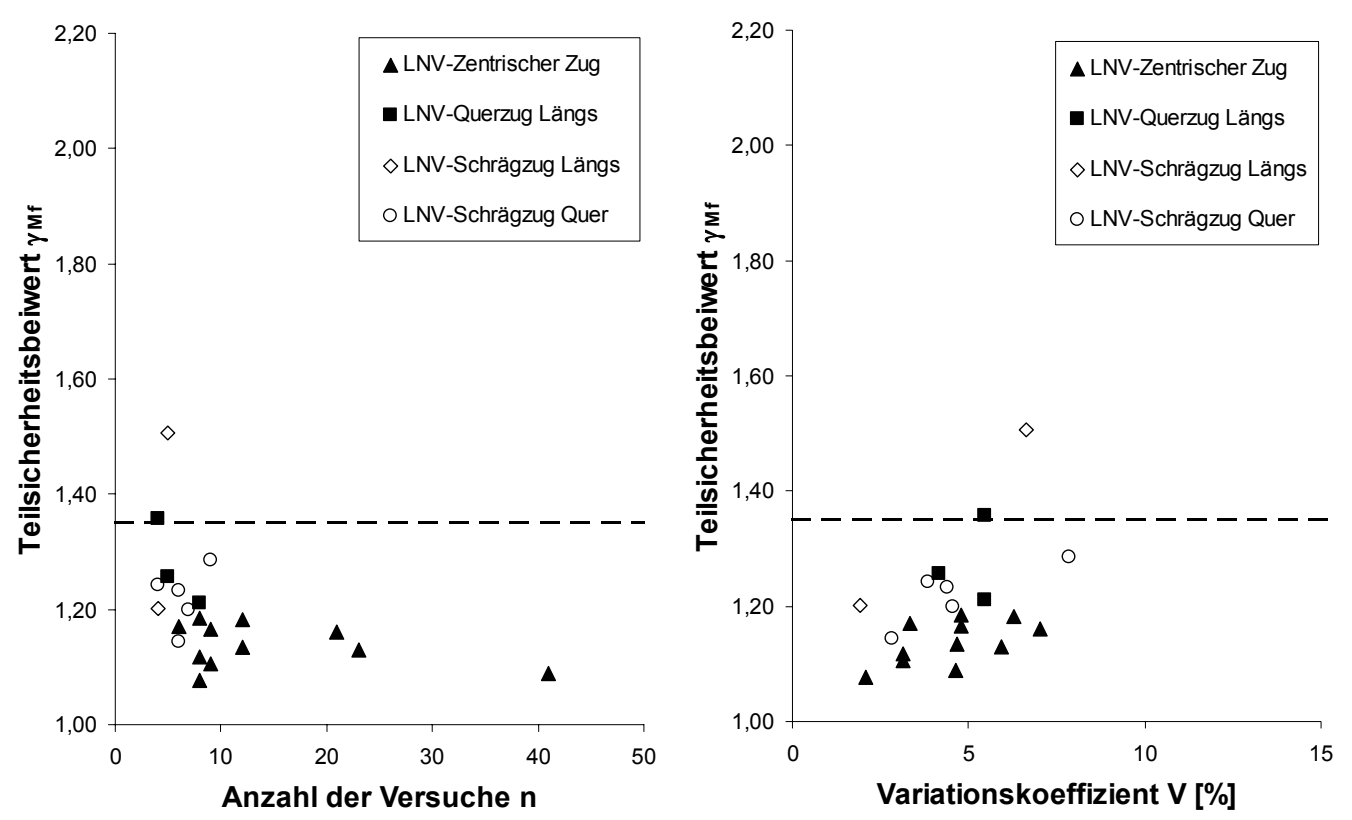

Bild 5.8 TSB als Funktion der Versuchsanzahl und Variationskoeffizienten (LNV)

Die Teilsicherheitsbeiwerte werden nach Abschnitt 5.3 ermittelt. Mit zunehmender Versuchsanzahl nimmt der Teilsicherheitsbeiwert für Ermüdungsbeanspruchung $\gamma_{\mathrm{Mf}}$ ab. Im Falle einer Normalverteilung beträgt der absolute Teilsicherheitsbeiwert für eine Schrägzugbeanspruchung in Schienenlängsrichtung und einer Anzahl von 5 Versuchen 2,14. Bei einer logarithmischen Normalverteilung beträgt dieser Wert 1,51. Für Schrägzugbeanspruchungen zeigen sich die größten Streuungen der Versuchsergebnisse. Die Annahme einer Normalverteilung oder einer logarithmischen Normalverteilung zur Beschreibung der Beanspruchbarkeit zeigt für die im Rahmen dieser Arbeit untersuchten Variationskoeffizienten deutliche Unterschiede in der Höhe des Teilsicherheitsbeiwertes, in Abhängigkeit vom Stichprobenumfang und vom Variationskoeffizienten. Eine wichtige Fragestellung bei der Auswertung ergibt sich hinsichtlich einer geeigneten Versuchsanzahl zur Beschreibung der Versuchsergebnisse im ausreichenden Maße. Ab etwa 8 Versuchen ergeben sich bei der Annahme einer Normalverteilung Teilsicherheitsbeiwerte unter 1,35. Werden die Versuchsergebnisse durch eine logarithmische Normalverteilung beschrieben, dann wird bereits ab einer Versuchsanzahl von 4 dieser Wert unterschritten.

Als Schlußfolgerung der in diesem Abschnitt erzielten Teilsicherheitsbeiwerte ergibt sich, daß zur Bestimmung des Bemessungswertes ein Teilsicherheitsbeiwert von 1,35, in Abhängigkeit von der Versuchsanzahl, für jede Beanspruchungsrichtung empfohlen werden kann. Durch die Wahl eines höheren Teilsicherheitsbeiwertes werden wertvolle Systemreserven nicht ausgenutzt. 


\subsubsection{Empfehlungen über die Mindestanzahl an Versuchen}

Die Versuchsanzahl ist bei Schwingfestigkeitsversuchen in der Regel relativ klein, weshalb der Mittelwert der Stichprobe den Mittelwert der Grundgesamtheit, der einer Bemessung zugrunde gelegt werden sollte, unter- bzw. überschätzt. Zur Abdeckung dieses Risikos sind im Laufe der Zeit Hilfsmittel entwickelt worden, die möglichst aussagekräftige Informationen hinsichtlich der statistischen Parameter in Abhängigkeit von der Versuchsanzahl erlauben. Diese sind in Abschnitt 5.3 vorgestellt worden. Aus bereits vorliegenden Versuchsdaten der zentrischen Zugrichtung mit einer relativ hohen Anzahl von 41 Versuchen auf 5 Lastniveaus (siehe Anhang Tabelle A10) sind nach einem Zufallsprinzip, mit Hilfe eines Zufallszahlengenerators, Versuchswerte auf mindestens drei unterschiedlichen Lastniveaus ausgewählt worden. Anschließend ist der Mittelwert der Regressionsgeraden $\Delta \mathrm{F}$ bei $2 \cdot 10^{6}$ Schwingspielen und der Variationskoeffizient V bestimmt worden. Die Mindestversuchsanzahl betrug dabei 3. Die Anzahl der Zufallsläufe bzw. der Simulation je Versuchsanzahl wurde nach Untersuchung der Güte mit $\mathrm{N}_{\text {sim }}=20$ festgelegt. Ab dieser Anzahl zeigten sich keine markanten Änderungen in den Zielwerten. Dabei wurde untersucht, ab welcher Simulationsanzahl $\mathrm{N}_{\text {sim }}$ es keinen bedeutenden Einfluß auf Ergebnisse, in diesem Fall die Schwingbreite und den Variationskoeffizienten, gibt und somit die Qualität der Ergebnisse akzeptabel ist. In Bild 5.9 zeigt sich eine deutliche Abhängigkeit der Schwingbreite bzw. dessen Variationskoeffizienten von der Anzahl der Versuche.
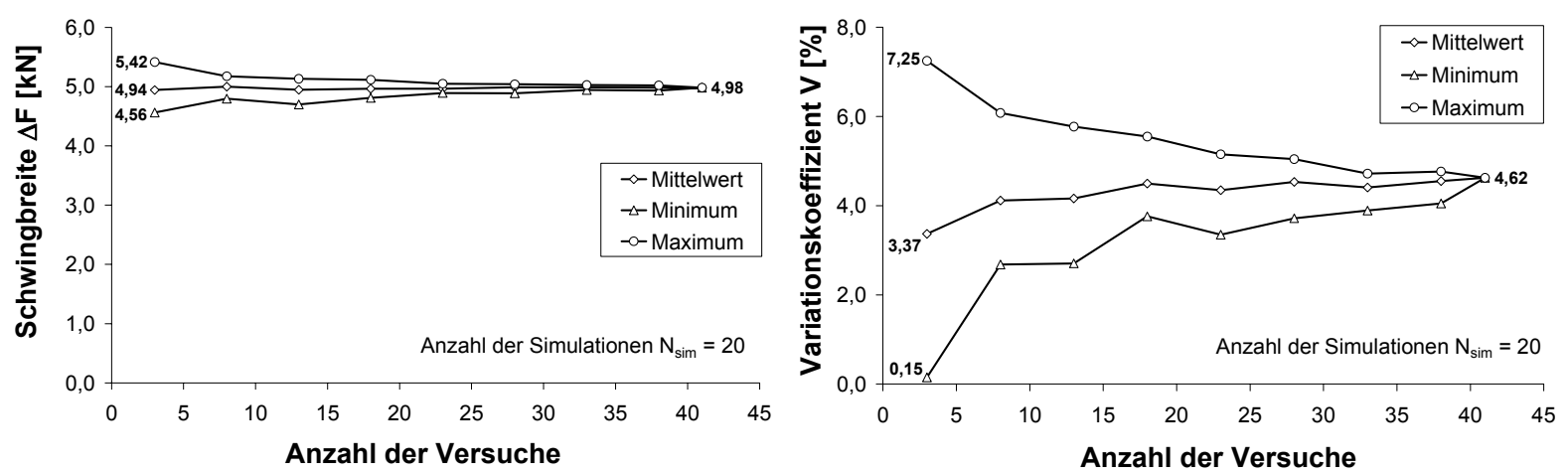

Bild 5.9 Schwingbreiten und Variationskoeffizienten über die Versuchsanzahl

Bei 8 Versuchen wird der Mittelwert der ertragbaren Schwingbreite im Vergleich zum Mittelwert der gesamten Versuche (Zielwert) um etwa $4 \%$ verfehlt. Der Variationskoeffizient streut zwischen 3 und $6 \%$. Ab einer Anzahl von 23 Versuchen ist die Differenz zum Zielwert der Schwingbreite kleiner als $2 \%$. Der Variationskoeffizient nimmt in diesem Punkt Werte von mindestens 3,5 \% und maximal $5 \%$ an.

Es kann gefolgert werden, daß für die im Rahmen dieser Arbeit vorgestellten Auswertemethode eine Anzahl von 8 Versuchen auf drei Lastniveaus als Mindestanforderung angestrebt werden sollte. Sind jedoch die bezogenen Streuungen größer als $10 \%$, empfiehlt es sich weitere Versuche durchzuführen. Methoden zur Untersuchungen der Versuchsanzahl werden in [7], [83] vorgestellt. 


\subsubsection{Neues Bemessungskonzept}

In den vorangegangenen Abschnitten sind die grundlegenden Zusammenhänge dargestellt und diskutiert worden, die nun eine umfassende und variable Bemessung für die Befestigung von Ankerschienen ermöglichen. Im Folgenden wird ein Bemessungsverfahren für Ankerschienen vorgeschlagen, welches auf den Ermüdungsdiagrammen nach Goodman beruht. Jedoch werden vorerst die grundlegenden Voraussetzungen und Annahmen zur Anwendung dieses Verfahrens genannt:

- Beanspruchungen und Beanspruchbarkeiten liegen in Form von Bemessungswerten vor.

- Diesem Bemessungsverfahren werden ruhende und nichtruhende Beanspruchungen zugrunde gelegt. Ruhende Zugbeanspruchungen können in allen Beanspruchungsrichtungen auftreten. Druckbeanspruchungen in Axialrichtung werden vom Beton aufgenommen und in diesem Bemessungsverfahren nicht berücksichtigt. Nichtruhende, ermüdungsrelevante Beanspruchungen können ebenfalls in allen Beanspruchungsrichtungen auftreten. Diese erfolgen jedoch lediglich im Zugschwellbereich. Eine Kombination aus ruhenden und nichtruhenden Beanspruchungen wird in diesem Bemessungsverfahren ebenfalls berücksichtigt.

- Der Nachweis der Tragfähigkeit erfolgt zunächst für eine ruhende Beanspruchung, bei dem die Summe der einwirkenden statischen Lasten und die maximale Schwingbreite der einwirkenden nichtruhenden Beanspruchung als ruhend angesetzt werden.

- Der Nachweis der nichtruhenden Belastung erfolgt für eine schadensäquivalente Schwingbreite bei einer repräsentativen Anzahl von $2 \cdot 10^{6}$ Schwingspielen, die durch Ermüdungseinwirkung hervorgerufen werden. Ermüdungseinwirkungen sind häufig auftretende und ständig veränderliche Lasten.

- Treten unterschiedliche Belastungsrichtungen auf, so werden diese mit Hilfe einer Interaktionsbeziehung nachgewiesen.

- Die Lasteinleitung in die Ankerschiene erfolgt direkt über dem Ankerbolzen.

- Die Beanspruchung erfolgt für eine Einzelbefestigung (ungünstigster Fall). Es wird vorausgesetzt, daß das Tragverhalten bei einer Mehrfachbefestigung aufgrund der Lastverteilung zu höheren Tragfähigkeiten führt und somit auf der sicheren Seite liegt.

- Der Nachweis der Tragfähigkeit für ermüdungsrelevante Einwirkungen erfolgt ausschließlich für das Stahlversagen.

- Ein Betonversagen ist unter Einhaltung der Mindestabmessungen nicht relevant und somit auch nicht Gegenstand dieser Bemessung.

Durch die Bestimmung der Ermüdungstragfähigkeit von Ankerschienenbefestigungen bei Zugursprungsbeanspruchung $\Delta \mathrm{F}_{\mathrm{Rd}, 0}$ und der maximalen statischen Tragfähigkeit $\mathrm{F}_{\mathrm{Rd}}$, sowie der Interaktionsbeziehung unter Berücksichtigung unterschiedlicher Belastungsrichtungen ist es nun möglich, eine umfassende Bemessung für Ankerschienen bereitzustellen. Um das derzeitige Bemessungskonzept zu überarbeiten und in abgesicherter Form zu präsentieren, ist es notwendig, sowohl die experimentellen 
Untersuchungen als auch die Nachweisführung zur Gewährleistung der Tragfähigkeit zu modifizieren.

Betrachtet man das Ermüdungsfestigkeitsdiagramm nach Goodman (siehe Kapitel 4), so wird deutlich, daß eine Abhängigkeit der Tragfähigkeiten zwischen ruhenden und nichtruhenden Lasten vorhanden sein muß. Dieses Verhalten wurde bereits im vorangegangenen Kapitel 4 durch Versuche bestätigt. Das Bild 5.10 veranschaulicht die Anteile der ruhenden und nichtruhenden Belastung zu einem bestimmten Zeitpunkt $\mathrm{t}$.
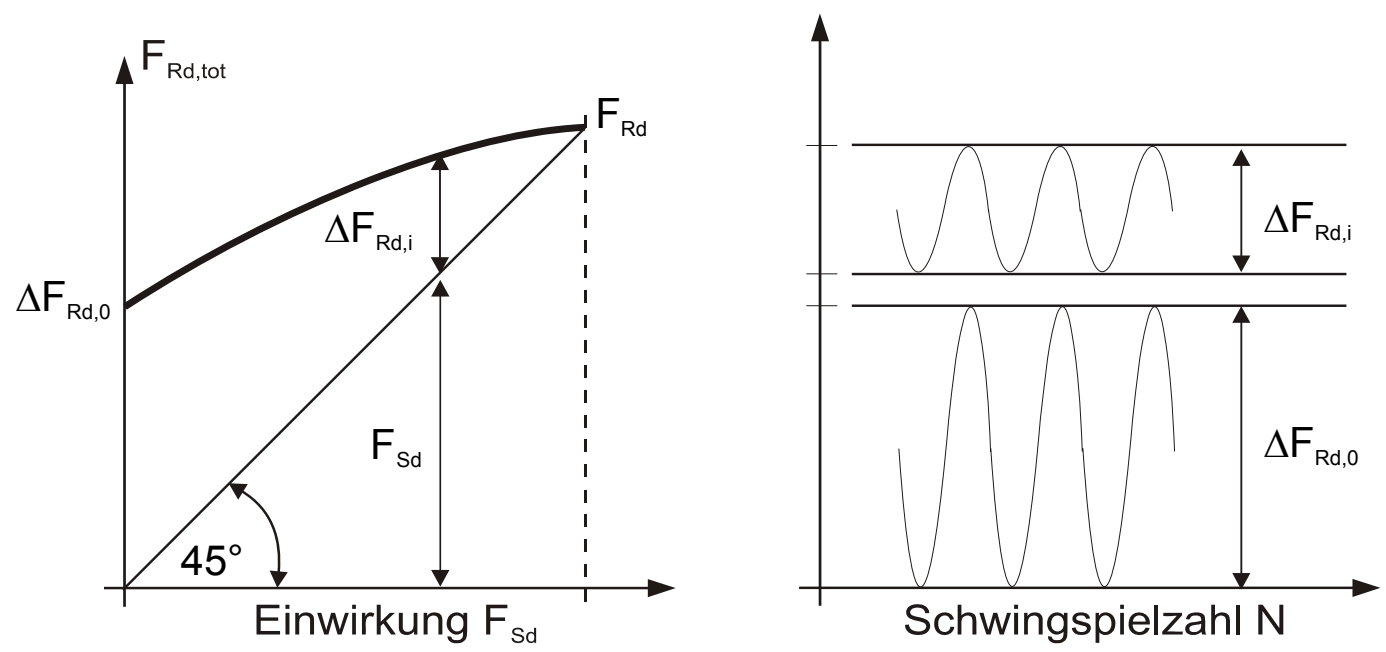

Bild 5.10 Bemessungsdiagramm nach Goodman

Es geht deutlich hervor, daß ein antiproportionaler Zusammenhang zwischen der ruhenden und nichtruhenden Beanspruchung vorliegen muß. Mit Zunahme der statisch wirkenden Last $\mathrm{F}_{\mathrm{Sd}}$ wird der Anteil der nichtruhenden Belastbarkeit geringer, bis schließlich nach Erreichen der Maximallast $F_{S d}=F_{R d}$ keine zusätzliche Beanspruchung mehr möglich ist. Eine ständig wirkende statische Dauerlast kann durch eine in Versuchen eingestellte konstante Unterlast erzeugt werden. Gerade solche Zusammenhänge müssen in „moderneren“ Sicherheitsbetrachtungen verstärkt berücksichtigt werden, um das eigentliche Tragverhalten genauer erfassen und umfassend darstellen zu können. Aufbauend auf den Bemessungsdiagrammen nach Goodman sollte ein umfassendes und variables Sicherheitskonzept eingeführt werden. Die Tragfähigkeitsnachweise können dann auf dieser Basis erfolgen. Um das in Bild 5.10 vorgestellte Bemessungsdiagramm nach Goodman aufbauen zu können, sind Versuchsserien auf unterschiedlichen Lastniveaus mit konstanten Unterlasten notwendig. Jedoch ist diese Art der Bemessung sehr aufwendig, unwirtschaftlich und nicht effizient. Deshalb behilft man sich mittels einer Linearisierung zwischen der statischen Tragfähigkeit $F_{R d}$ und der Schwingbreite bei Ursprungsbeanspruchung $\Delta \mathrm{F}_{\mathrm{Rd}, 0}$, welche auf der sicheren Seite liegt. Diese Darstellungsweise kann durch die Versuchsergebnisse bestätigt werden. Das Vorgehen wird bereits für Dübel in [7] angewendet. Für Ankerschienen ist in [61] ein Vergleich der Bemessungsverfahren für die zentrische Zugrichtung dargestellt.

Für das Problem der Ankerschienenbefestigung ergibt sich somit der nachfolgend in Bild 5.11 schematisch dargestellte Zusammenhang. 


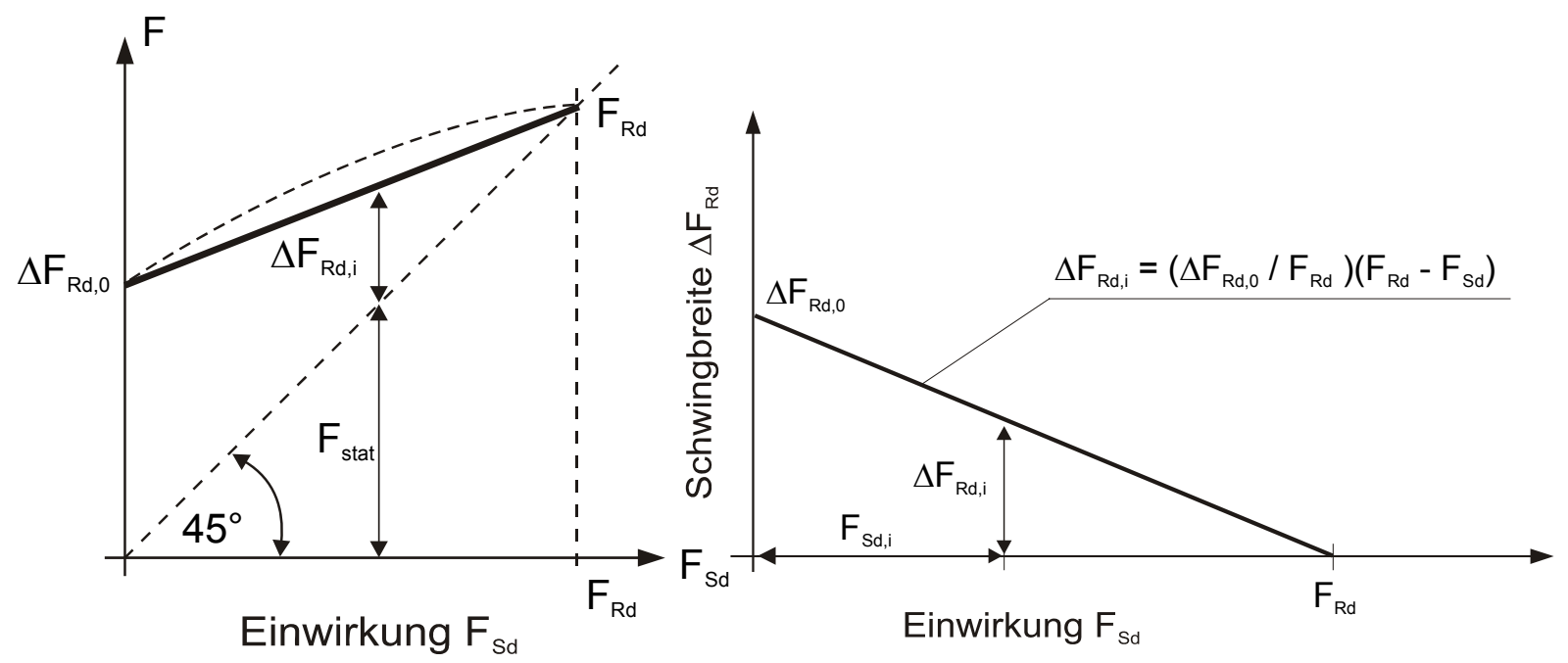

Bild 5.11 Linearisiertes Ermüdungstragfähigkeitsdiagramm nach Goodman

Durch die Linearisierung erhält man nun ein einfaches und variables Ingenieurmodell, welches die Beanspruchbarkeit auf der sicheren Seite liegend wesentlich exakter abbildet. Ruhende und nichtruhende Lasten können gemeinsam auftreten und werden in diesem Modell bei gemeinsamer Wirkung betrachtet. Es ergeben sich somit zwei durchzuführende Nachweise, die der üblichen Nachweisform im Eurocode entsprechen. Stellt man den funktionellen Verlauf der ertragbaren Schwingbreite über die statische Beanspruchung auf, so ergibt sich aus Bild 5.11, links, abgeleitet, die rechte Darstellungsweise. Eine wichtige Einflußgröße auf die ertragbare Schwingbreite ist das Verhältnis der Schwingbreite bei Ursprungsbeanspruchung $\Delta \mathrm{F}_{\mathrm{Rd}, 0}$ und der maximalen Tragfähigkeit $F_{R d}$ unter statischer Beanspruchung. Unter Einführung eines Faktors $\lambda_{0}$ zur Beschreibung dieses Verhältnisses kann die Geradengleichung aus Bild 5.11 wie folgt dargestellt werden.

$$
\Delta \mathrm{F}_{\mathrm{Rd}, \mathrm{i}}=\lambda_{0} \cdot\left(\mathrm{F}_{\mathrm{Rd}}-\mathrm{F}_{\mathrm{Sd}}\right), \quad \lambda_{0}=\frac{\Delta \mathrm{F}_{\mathrm{Rd}, 0}}{\mathrm{~F}_{\mathrm{Rd}}}
$$

Die Differenz der einwirkenden ruhenden Last $\mathrm{F}_{\mathrm{Sd}}$ zur statischen Beanspruchbarkeit $\mathrm{F}_{\mathrm{Rd}}$, behaftet mit dem Faktor $\lambda_{0}$, bestimmt somit die ertragbare Schwingbreite des Systems. Eine Möglichkeit zur Bemessung von vorwiegend ruhenden und nichtruhenden Beanspruchungen kann mit Hilfe der Formel (5.31) auf der sicheren Seite liegend beschrieben werden.

Eine wichtige Grundlage bei der Herleitung einer umfassenden Bemessung bildet die Beanspruchung unter verschiedenen Belastungswinkeln. Hierzu sind Interaktionsdiagramme notwendig, die kombinierte Zug- und Querbeanspruchungen möglichst ideal beschreiben. In Kapitel 4 sind mögliche Interaktionsdiagramme zwischen den Kraftgrößen $\mathrm{N}, \mathrm{V}_{\mathrm{x}}$ und $\mathrm{V}_{\mathrm{y}}$ auf Basis der Versuchsergebnisse dargestellt worden. Diesen Diagrammen ist zu entnehmen, da $\beta$ die Formfaktoren $\alpha$ zur Beschreibung der Interaktion zwischen der zentrischen Zugrichtung und der Querbeanspruchung eine Abhängigkeit von der statisch wirkenden Last aufweisen. Bei nichtruhender Beanspru- 
chung sind diese kleiner als im Falle der ruhenden Beanspruchung. Um jedoch das Tragverhalten unter kombinierter Beanspruchung abbilden zu können, sollte ein Formfaktor angegeben werden, der die Kombination aus ruhender und nichtruhender Beanspruchung beschreibt. Mit Bezug auf die Versuchsergebnisse kann ein linearer Zusammenhang zwischen den Formfaktoren und der statischen Beanspruchung angenommen werden. Die lineare Interpolation der Formfaktoren, aufgetragen über die einwirkende Kraft $\mathrm{F}_{\mathrm{Sd}}$, wird in Bild 5.12 veranschaulicht. Der Koordinatenursprung kennzeichnet dabei den Fall einer nichtruhenden Beanspruchung, dem ein $\alpha_{\text {dyn }}$ zugeordnet wird. Beträgt die einwirkende Last nahezu $F_{R d}$, so ist keine Schwingbreite möglich. In diesem Fall liegt eine vorwiegend ruhende Beanspruchung mit dem Formfaktor $\alpha_{\text {stat }}$ vor. Analog kann eine auf die maximale statische Tragfähigkeit bezogene Schwingbreite, aufgetragen auf der Abszisse, in Abhängigkeit vom Formfaktor aufgezeigt werden. Dieser nimmt im Falle der vorwiegend ruhenden Beanspruchung, also bei $\Delta \mathrm{F}_{\mathrm{Rd}}=0$, den Wert 0 und bei nichtruhender Beanspruchung den Faktor $\lambda_{0}$ an.

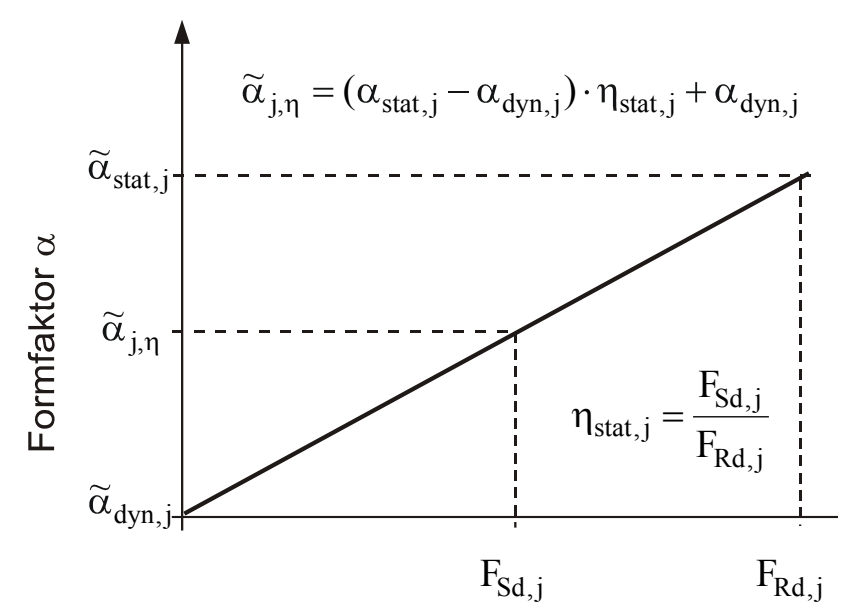

Einwirkung $F_{S d}$

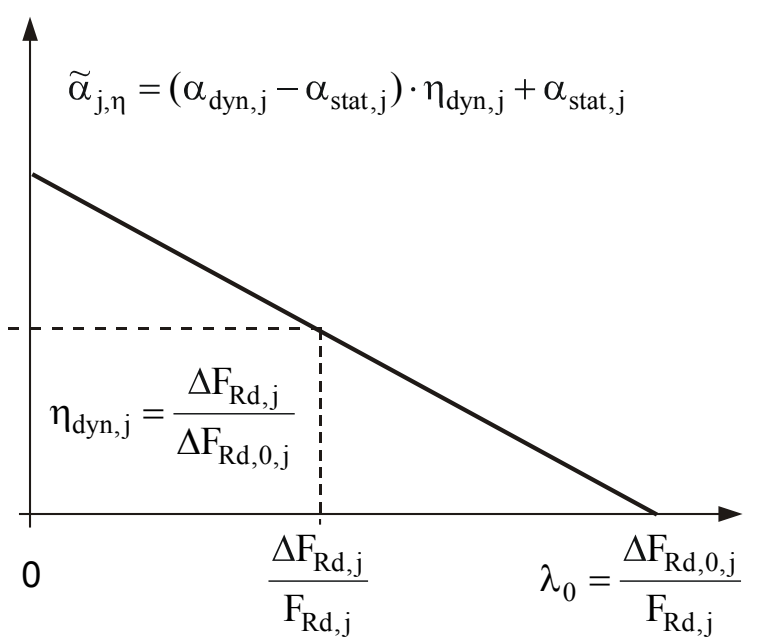

Bezogene Schwingbreite $\lambda$

Bild 5.12 Formfaktor bei kombinierter ruhender und nichtruhender Beanspruchung

Da bei Ankerschienen drei verschiedene Beanspruchungsrichtungen vorhanden sind, nämlich $\mathrm{N}, \mathrm{V}_{\mathrm{x}}$ und $\mathrm{V}_{\mathrm{y}}$, die unterschiedliche Trageigenschaften aufweisen, sind die Formfaktoren für jede Beanspruchungsebene $\mathrm{j}\left(\mathrm{N}-\mathrm{V}_{\mathrm{x}}, \mathrm{N}-\mathrm{V}_{\mathrm{y}}\right.$ sowie $\left.\mathrm{V}_{\mathrm{x}}-\mathrm{V}_{\mathrm{y}}\right)$ separat $\mathrm{zu}$ ermitteln. Dabei ist die einwirkende Gesamtlast im Raum auf jede o.g. Beanspruchungsebene zu projizieren. In Abhängigkeit vom ruhenden Anteil der einwirkenden Kraft $\mathrm{F}_{\mathrm{Sd}, \mathrm{j}}$ (entspricht der Unterlast in den Versuchen) sind dann zunächst die Formfaktoren $\widetilde{\alpha}_{N-V x, \eta}, \widetilde{\alpha}_{N-V y, \eta}$ und $\widetilde{\alpha}_{V x-V y, \eta}$ der jeweiligen Beanspruchungsebenen für eine kombinierte ruhende und nichtruhende Beanspruchung zu bestimmen. Anschließend sind die Kraftkomponenten auf die jeweiligen Hauptachsen zu beziehen. Mit Hilfe der drei ermittelten Formfaktoren und den einwirkenden Kräften für jede Beanspruchungsrichtung, kann somit der Nachweis der Tragfähigkeit für jede Beanspruchungsebene separat erfolgen. Jedoch ist durch diese Nachweisführung das Tragverhalten bei gleichzeitiger Wirkung von drei Kraftgrößen nicht sichergestellt. Um dieses 
zu gewährleisten, sind Interaktionsdiagramme für drei Kraftgrößen, wie sie in Bild 5.13 für den Grenzzustand der Ermüdung dargestellt sind, notwendig.

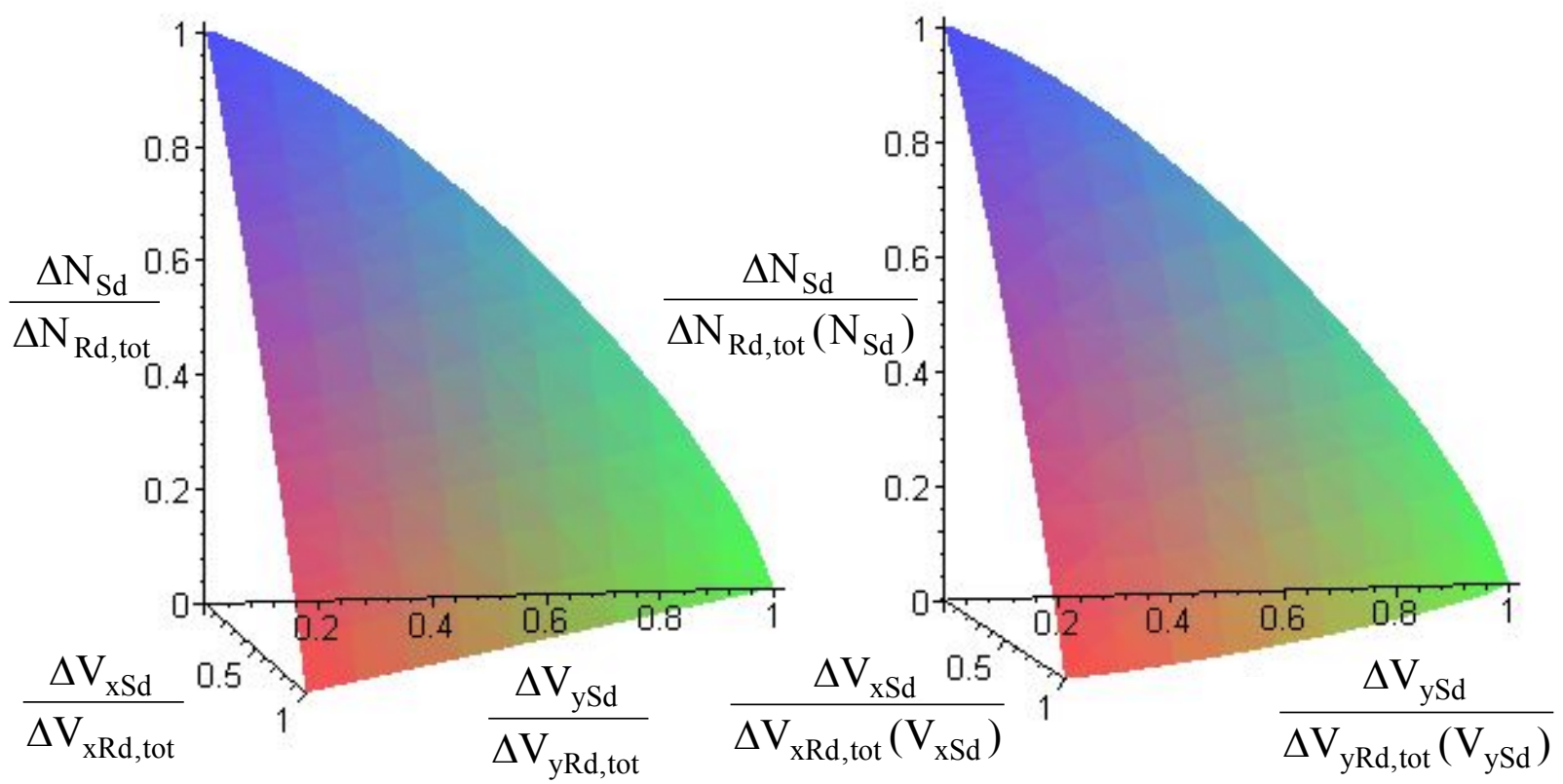

Bild 5.13 Interaktionsbeziehung für den Nachweis der Tragfähigkeit bei drei Kraftgrößen

Die zugehörige Funktionsgleichung gibt der Ausdruck in (5.32) wieder.

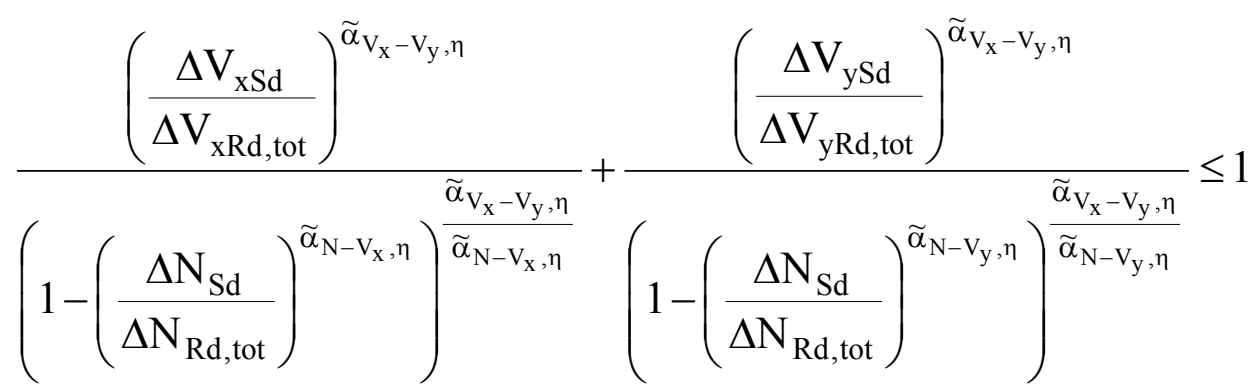

mit den folgenden Bemessungswerten gemäß Bild 5.11:

$$
\begin{aligned}
& \Delta \mathrm{N}_{\mathrm{Rd}, \text { tot }}=\Delta \mathrm{N}_{\mathrm{Rd}, \text { tot }}\left(\mathrm{N}_{\mathrm{Sd}}\right)=\frac{\Delta \mathrm{N}_{\mathrm{Rk}, 0} \cdot \gamma_{\mathrm{M}}}{\gamma_{\mathrm{Mf}} \cdot \mathrm{N}_{\mathrm{Rk}}} \cdot\left(\frac{\mathrm{N}_{\mathrm{Rk}}}{\gamma_{\mathrm{M}}}-\frac{\mathrm{N}_{\mathrm{Sk}}}{\gamma_{\mathrm{F}}}\right) \\
& \Delta \mathrm{V}_{\mathrm{xRd} \text {,tot }}=\Delta \mathrm{V}_{\mathrm{xRd,tot}}\left(\mathrm{V}_{\mathrm{xSd}}\right)=\frac{\Delta \mathrm{V}_{\mathrm{xRk}, 0} \cdot \gamma_{\mathrm{M}}}{\gamma_{\mathrm{Mf}} \cdot \mathrm{V}_{\mathrm{xRk}}} \cdot\left(\frac{\mathrm{V}_{\mathrm{xRk}}}{\gamma_{\mathrm{M}}}-\frac{\mathrm{V}_{\mathrm{xSk}}}{\gamma_{\mathrm{F}}}\right) \\
& \Delta \mathrm{V}_{\mathrm{yRd}, \text { tot }}=\Delta \mathrm{V}_{\mathrm{yRd}, \text { tot }}\left(\mathrm{V}_{\mathrm{xSd}}\right)=\frac{\Delta \mathrm{V}_{\mathrm{yRk}, 0} \cdot \gamma_{\mathrm{M}}}{\gamma_{\mathrm{Mf}} \cdot \mathrm{V}_{\mathrm{yRk}}} \cdot\left(\frac{\mathrm{V}_{\mathrm{yRk}}}{\gamma_{\mathrm{M}}}-\frac{\mathrm{V}_{\mathrm{ySk}}}{\gamma_{\mathrm{F}}}\right)
\end{aligned}
$$

und $\quad \gamma_{M}=\gamma_{M f}=1,35$, Teilsicherheitsbeiwert der Beanspruchbarkeit bzw. $\quad \gamma_{\mathrm{F}}, \gamma_{\mathrm{Ff}}$, Teilsicherheitsbeiwert der Beanspruchung, z.B. aus [37] 
Für den Fall, daß die Kraftkomponente der zentrischen Zugrichtung

$\left(\frac{\Delta \mathrm{N}_{\mathrm{Sd}}}{\Delta \mathrm{N}_{\mathrm{Rd}, \text { tot }}}\right)=1$ wird,

ist die Gleichung (5.32) nicht definiert und muß daher gesondert betrachtet werden. Für diesen Fall gilt dann:

$\left(\frac{\Delta \mathrm{V}_{\mathrm{xSd}}}{\Delta \mathrm{V}_{\mathrm{xRd}, \text { tot }}}\right)=\left(\frac{\Delta \mathrm{V}_{\mathrm{ySd}}}{\Delta \mathrm{V}_{\mathrm{yRd}, \text { tot }}}\right)=0$

Mit Hilfe der in Gleichung (5.32) dargestellten Interaktionsbeziehung gelingt nun der Nachweis der Tragfähigkeit von Ankerschienenbefestigungen bei kombinierter ruhender und nichtruhender Zug- und Querbeanspruchungen in allen Richtungen. Die aufgespannte Oberfläche des Volumens stellt die Grenztragfähigkeit dar und dient der Nachweisführung bei Ermüdungsbeanspruchung an der maßgebenden Bemessungsschwingspielzahl von $2 \cdot 10^{6}$ Schwingspielen. Das linke Volumen in Bild 5.13 ergibt sich bei der Annahme einer linearen Interaktionsbeziehung zwischen den Querbeanspruchungen für vorwiegend nichtruhende Belastungen. Im Rahmen dieser Arbeit ist die Interaktion von nichtruhenden Querbeanspruchungen jedoch nicht experimentell untersucht worden, weshalb diese sinnvoll angenommen wurde. Für den vorwiegend statischen Fall liegen Versuchsergebnisse vor, die dem Kapitel 4 entnommen werden können. Dabei zeigt sich eine nichtlineare Form der Interaktion. Folglich wird bei einer kombinierten ruhenden und nichtruhenden Beanspruchung der Formfaktor in Abhängigkeit von der einwirkenden Last nach Bild 5.12 größer als der Mindestwert 1 sein. Den nichtlinearen Fall zeigt die rechte Interaktionsbeziehung in Bild 5.13.

Bisher erfolgt der Nachweis der Ermüdungstragfähigkeit lediglich für den zentrischen Zug. Eine nichtruhende Beanspruchung, abweichend von der zentrischen Zugrichtung, ist nach aktueller Bemessung nicht zugelassen.

Zur Gewährleistung der Tragfähigkeit von Ankerschienenbefestigungen bei kombinierten ruhenden und nichtruhenden Zug- und Querbeanspruchungen sind zunächst die einwirkenden statischen Lasten nach den Gleichungen (5.36) bis (5.38) nachzuweisen. Dabei wird die Summe der ruhenden Last und der größten Schwingbreite als vorwiegend ruhend angesetzt.

$$
\begin{aligned}
& \left(\frac{\mathrm{N}_{\mathrm{Sd}, \text { tot }}}{\mathrm{N}_{\mathrm{Rd}}}\right)^{\alpha_{\text {stat }, \mathrm{N}-\mathrm{V}_{\mathrm{x}}}}+\left(\frac{\mathrm{V}_{\mathrm{xSd}, \text { tot }}}{\mathrm{V}_{\mathrm{xRd}}}\right)^{\alpha_{\mathrm{stat}, \mathrm{N}-\mathrm{V}_{\mathrm{x}}}} \leq 1 \\
& \left(\frac{\mathrm{N}_{\mathrm{Sd}, \text { tot }}}{\mathrm{N}_{\mathrm{Rd}}}\right)^{\alpha_{\text {stat }, \mathrm{N}-\mathrm{V}_{\mathrm{y}}}}+\left(\frac{\mathrm{V}_{\mathrm{ySd}, \text { tot }}}{\mathrm{V}_{\mathrm{yRd}}}\right)^{\alpha_{\text {stat }, \mathrm{N}-\mathrm{V}_{\mathrm{y}}}} \leq 1 \\
& \left(\frac{\mathrm{V}_{\mathrm{xSd}, \text { tot }}}{\mathrm{V}_{\mathrm{xRd}}}\right)^{\alpha_{\mathrm{stat}, \mathrm{V}_{\mathrm{x}}-\mathrm{V}_{\mathrm{y}}}}+\left(\frac{\mathrm{V}_{\mathrm{ySd}, \text { tot }}}{\mathrm{V}_{\mathrm{yRd}}}\right)^{\alpha_{\mathrm{stat}, \mathrm{V}_{\mathrm{x}}-\mathrm{V}_{\mathrm{y}}}} \leq 1
\end{aligned}
$$


Dabei sind

$\mathrm{N}_{\mathrm{Sd}, \mathrm{tot}}=\mathrm{N}_{\mathrm{Sd}}+\Delta \mathrm{N}_{\mathrm{Sd}}$,

$\mathrm{V}_{\mathrm{xSd}, \text { tot }}=\mathrm{V}_{\mathrm{xSd}}+\Delta \mathrm{V}_{\mathrm{xSd}}$,

$\mathrm{V}_{\mathrm{ySd}, \text { tot }}=\mathrm{V}_{\mathrm{ySd}}+\Delta \mathrm{V}_{\mathrm{ySd}}$

die Bemessungswerte der Beanspruchungen

und

$\mathrm{N}_{\text {Rd }}, \mathrm{V}_{\text {xRd }}, \mathrm{V}_{\text {yRd }}$.

die Bemessungswerte der Beanspruchbarkeiten.

Die Formfaktoren $\alpha_{\text {stat,j }}$ der jeweiligen Beanspruchungsebenen werden mit Hilfe der Resultierenden der einwirkenden Kraftgrößen $\mathrm{F}_{\text {Sd,j }}$ (ohne schwingenden Anteil) wie bereits beschrieben ermittelt.

$\mathrm{F}_{\mathrm{Sd}, \mathrm{N}-\mathrm{V}_{\mathrm{x}}}=\sqrt{\mathrm{N}_{\mathrm{Sd}}^{2}+\mathrm{V}_{\mathrm{xSd}}^{2}}$

$\mathrm{N}-\mathrm{V}_{\mathrm{y}}$ und $\mathrm{V}_{\mathrm{x}}-\mathrm{V}_{\mathrm{y}}$ sind in gleicher Weise zu bestimmen.

Anschließend wird der Quotient $\eta_{\text {stat }}$ nach Bild 5.12 zwischen den einwirkenden Kraftgrößen und dem kleinsten Bemessungswert der statischen Tragfähigkeit der jeweiligen Beanspruchungsebenen gebildet. Bei einer Beanspruchung durch die Kraftgrößen $N$ und $V_{x}$ wäre der kleinste Bemessungswert das Minimum zwischen $N_{R d}$ und $\mathrm{V}_{\mathrm{xRd}}$. Mit Hilfe des Faktors $\eta_{\text {stat }}$ kann schließlich aus dem Diagramm in Bild $5.12 \mathrm{der}$ relevante Formfaktor $\widetilde{\alpha}_{\mathrm{N}-\mathrm{Vx}, \eta}$ abgeleitet werden. Dieses Vorgehen ist in analoger Weise für die Formfaktoren der übrigen Beanspruchungsebenen durchzuführen. Unter Betrachtung aller wirkenden Kraftgrößen ergibt sich die Nachweisführung gemäß Gleichung (5.32) zur Gewährleistung der Sicherheit von Ankerschienenbefestigungen unter kombinierten ruhenden und nichtruhenden Zug- und Querbeanspruchungen.

In den nachfolgenden Abschnitten werden die erzielten Erkenntnisse an zwei Beispielen zur Bemessung von Ankerschienenbefestigungen dargelegt. Im ersten Beispiel erfolgt zunächst eine Gegenüberstellung der derzeitig gültigen Bemessung und des im Rahmen dieser Arbeit entwickelten Bemessungsvorschlages. Anschließend wird auf der Grundlage des neuen Bemessungsvorschlags ein möglicher Anwendungsfall behandelt, und hieran das Vorgehen erläutert. 


\subsection{Bemessungsbeispiel}

Um ein Vergleich zwischen dem derzeitigen Bemessungskonzept und der im Rahmen dieser Arbeit vorgestellten Bemessung durchführen zu können, wurden statische Zugversuche und Dauerschwingversuche im Zugschwellbereich an einbetonierten Ankerschienen unter Einhaltung der geforderten Mindestabmessungen nach [21] durchgeführt. Die Auswertung der statischen Zugversuche und der Schwingversuche in Hinsicht auf die Ermittlung der Schwingbreiten bei einer Versagensschwingspielzahl von $\mathrm{N}_{\mathrm{f}}=2 \cdot 10^{6}$ erfolgt nach den Vorgaben der ETAG $(\mathrm{p}=5 \%, \mathrm{~W}=90 \%)$.

\begin{tabular}{|c|c|}
\hline \multicolumn{1}{|c|}{ aktuelle Bemessung } & \multicolumn{1}{|c|}{ neuer Bemessungsvorschlag } \\
\hline Kennwerte der statischen Tragfähigkeit \\
\hline \multicolumn{1}{|c|}{$\mathrm{N}_{\mathrm{Rm}}=38,4 \mathrm{kN}$} \\
$\begin{array}{r}\sigma_{\text {stat }}=2,32 \mathrm{kN} \\
\mathrm{V}=6 \%\end{array}$ \\
Anzahl der Versuche $\mathrm{n}=4$
\end{tabular}


Die aus Versuchsdaten in Anlehnung an die aktuelle Bemessung ermittelte ertragbare Schwingbreite beträgt 3,46 kN, welche um ca. $15 \%$ höher ist als der Zulassungswert in der aktuellen Zulassung. Um einen Vergleich zwischen der aktuellen Bemessung und dem neuen Bemessungsvorschlag durchführen zu können, werden die im Rahmen des neuen Bemessungsvorschlags ermittelten Schwingbreiten um $15 \%$ reduziert. Zur Veranschaulichung beider Bemessungsverfahren werden diese gemeinsam im Diagramm in Bild 5.14 dargestellt.

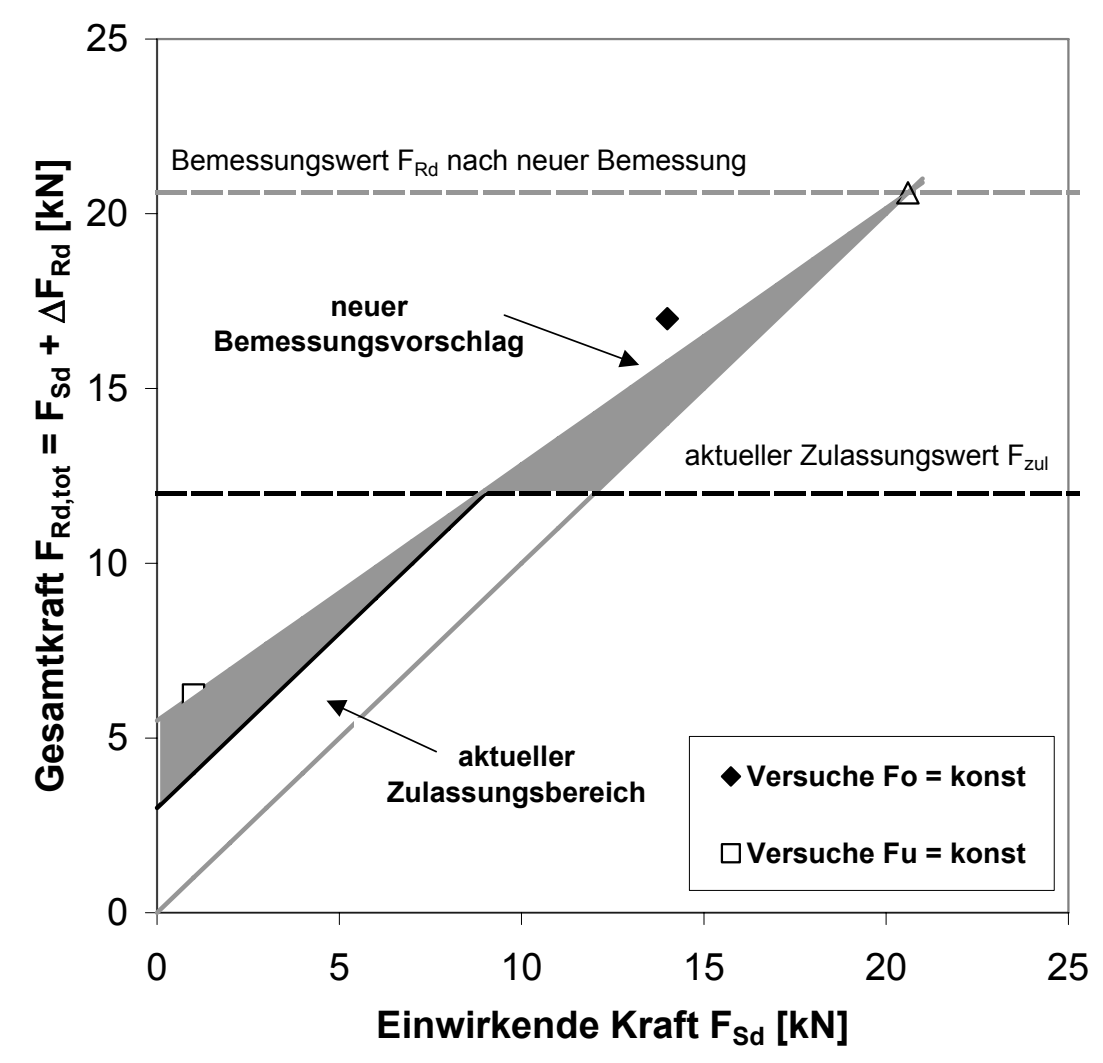

Bild 5.14 Aktuelle Zulassung im Vergleich zum neuen Bemessungsvorschlag

Der aktuelle Zulassungswert der ertragbaren Schwingbreite beträgt $\Delta \mathrm{N}_{\mathrm{Rd}}=3 \mathrm{kN}$ und gilt bis zur zulässigen statischen Tragfähigkeit $\mathrm{N}_{\mathrm{Rd}}=12 \mathrm{kN}$. Der aktuelle Zulassungsbereich liegt stets unterhalb des neuen, im Rahmen dieser Arbeit entwickelten Bemessungsvorschlags, erlaubt jedoch keine Beanspruchung oberhalb des aktuellen Zulassungswertes der ruhenden Last. Sowohl unterhalb als auch oberhalb dieses Wertes werden im Vergleich zum neuen Bemessungsvorschlag vorhandene Tragreserven nicht aktiviert. Im Falle einer Zugbeanspruchung entsprechend dem Zulassungswert der ruhenden Beanspruchung ist keine zusätzliche Schwingbeanspruchung mehr möglich, wo hingegen bei der neuen Bemessung noch deutliche ertragbare Schwingbreiten vorhanden sind. 
Anhand eines Zahlenbeispiels sollen die Grenzfälle „keine ruhende Last“ und „ruhende Last" entsprechend dem aktuellen Zulassungswert der ruhenden Beanspruchung verdeutlicht werden.

\begin{tabular}{|l|l|}
\hline \multicolumn{1}{|c|}{ aktuelle Bemessung } & \multicolumn{1}{|c|}{ neuer Bemessungsvorschlag } \\
\hline $\begin{array}{l}\mathrm{F}_{\text {vorh }}=0 \mathrm{kN} \\
\text { mögliche Schwingbreite } \Delta \mathrm{N}_{\mathrm{zul}}=\mathbf{3} \mathbf{~ k N} \\
\text { verbleibende Zuglast } \mathrm{N}_{\text {vorh }}=\mathbf{9} \mathbf{k N} \\
\text { Bedingung: } \mathrm{F}_{\text {vorh }}+\mathrm{N}_{\text {vorh }}+\Delta \mathrm{N}_{\mathrm{zul}} \leq 12 \mathrm{kN}\end{array}$ & $\begin{array}{l}\mathrm{F}_{\mathrm{Sd}}=0 \mathrm{kN} \\
\text { mögliche Schwingbreite } \Delta \mathrm{N}_{\mathrm{Rd}}=\mathbf{5 , 5} \mathbf{k N} \mathbf{N}^{1)} \\
\text { verbleibende Zuglast } \mathrm{N}_{\mathrm{Rd}}=\mathbf{1 5 , 1} \mathbf{k N} \\
\text { Bedingung: } \mathrm{F}_{\mathrm{Sd}}+\mathrm{N}_{\mathrm{Rd}}+\Delta \mathrm{N}_{\mathrm{Rd}} \leq 20,6 \mathrm{kN}\end{array}$ \\
\hline $\begin{array}{l}\mathrm{F}_{\text {vorh }}=12 \mathrm{kN} \\
\text { mögliche Schwingbreite } \Delta \mathrm{N}_{\text {zul }}=\mathbf{0} \mathbf{k N} \\
\text { verbleibende Zuglast } \\
\text { Bedingrh }\end{array}$ & $\begin{array}{l}\mathrm{F}_{\mathrm{Sd}}=12 \mathrm{kN} \\
\text { mögliche Schwingbreite } \Delta \mathrm{N}_{\mathrm{Rd}}=\mathbf{2 , 3} \mathbf{k N} \\
\text { verbleibende Zuglast } \mathrm{N}_{\mathrm{Rd}}=\mathbf{6 , 3} \mathbf{k N} \\
\text { Bedingung: } \mathrm{F}_{\mathrm{Sd}}+\mathrm{N}_{\mathrm{Rd}}+\Delta \mathrm{N}_{\mathrm{Rd}} \leq 20,6 \mathrm{kN}\end{array}$ \\
\hline $\begin{array}{l}{ }^{1)} \text { Beachte: um } 15 \% \text { reduzierter Wert aus Gründen der Vergleichbarkeit (s.o.). Die } \\
\text { Ermittlung der Schwingbreite erfolgt nach Gleichung (5.31). }\end{array}$ \\
\hline
\end{tabular}

Ganz besonders auffällig ist die ertragbare Schwingbreite nach dem neuen Bemessungsverfahren für den Fall einer ruhende Last $\mathrm{F}_{\mathrm{Sd}}$ entsprechend dem aktuellen Zulassungswert der ruhenden Beanspruchung von $12 \mathrm{kN}$. Wo bei der aktuellen Bemessung keine zusätzliche Schwingbeanspruchung mehr möglich ist, kann nach der neuen Bemessung noch eine ertragbaren Schwingbreite von etwa 2,3 $\mathrm{kN}$ als zusätzliche Schwingbeanspruchung aufgenommen werden.

\subsection{Anwendungsbeispiel: Bodenverankerung von Maschinen}

Aufgrund der guten Justierbarkeit, der Möglichkeit der Anordnung von Lastpaaren und der Aufnahme von nichtruhenden Lasten werden Ankerschienen zur Befestigung von Maschinenelementen an Fundamenten aus Stahlbeton bevorzugt verwendet. Um eine sichere Kraftleitung in den Untergrund zu gewährleisten werden Ankerschienen bereits in der Planung berücksichtigt.

Der im letzten Kapitel hergeleitete Sicherheitsnachweis, welcher den Einfluß von drei Kraftgrößen, einer zentrischen Zugkraft $N$ und den Querkräften $V_{x}$ und $V_{y}$, beschreibt, soll im nachfolgenden der Nachweisführung bei einer allseitigen Beanspruchung einer gezahnten Ankerschiene dienen. Anhand dieses Anwendungsbeispiels soll die Funktionsweise und Praxisnähe dieses Nachweisverfahrens demonstriert werden. 
Systemskizze

Ansicht:

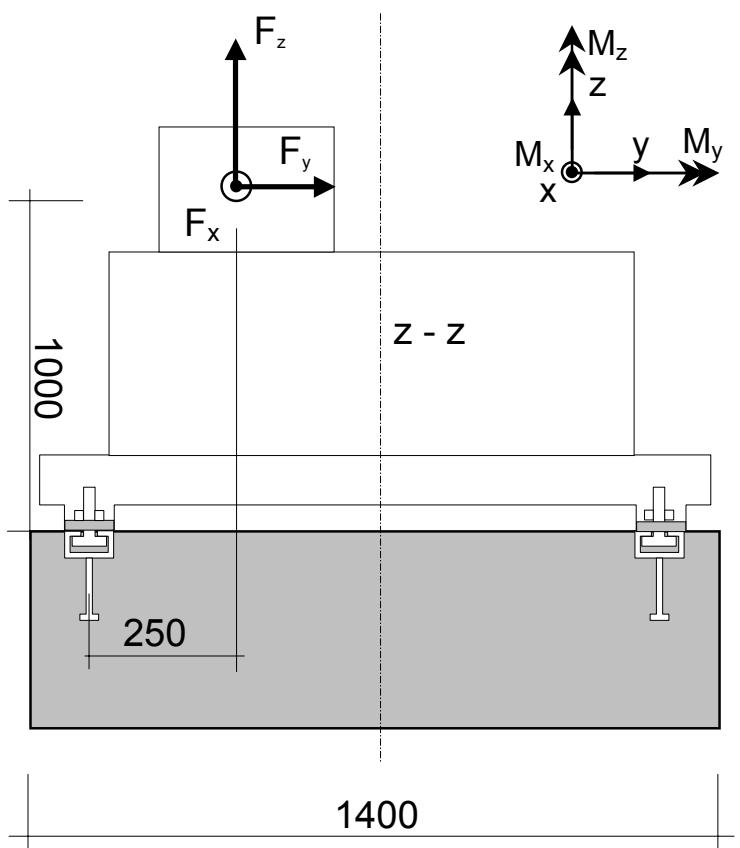

Draufsicht:

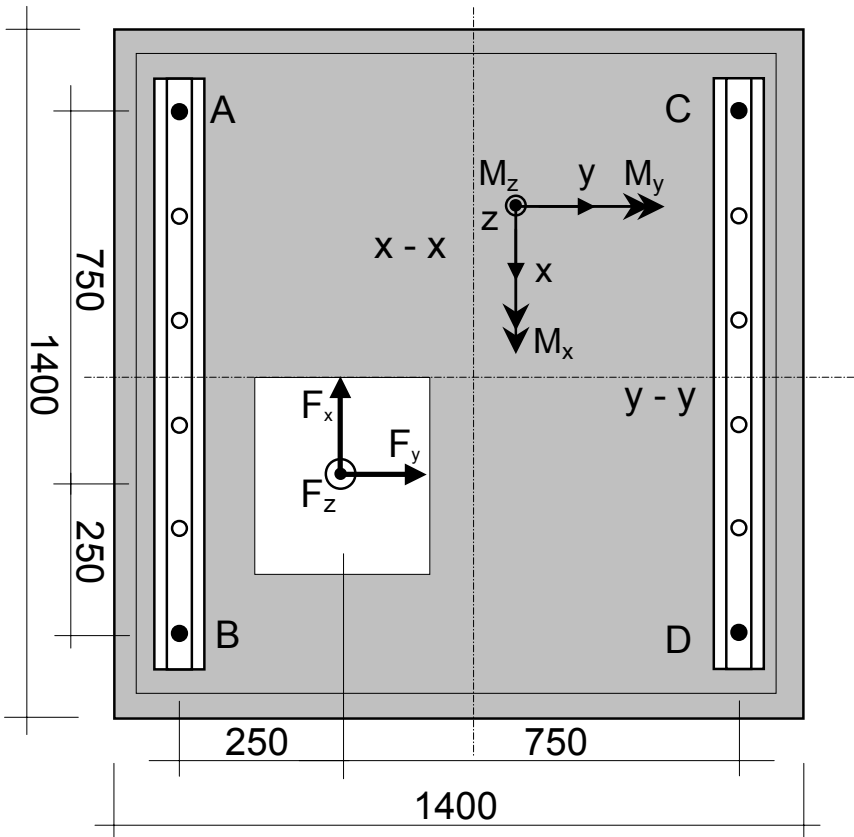

Angaben in [mm]

Bild 5.15 Befestigung eines Maschinensockels

Die Befestigung der Ankerschiene vom Profil 38/23 St erfolgt an den Befestigungspunkten A bis D (Bild 5.15) unter Verwendung von Zahnschrauben des Typs 38/23 der Güte 8.8 mit einem Anzugsmoment von $120 \mathrm{Nm}$. Anhand der einwirkenden Lasten muß zunächst die maßgebende Bemessungsstelle ermittelt werden. Während der Nutzungsdauer werden folgende Bemessungswerte der Beanspruchungen erwartet:
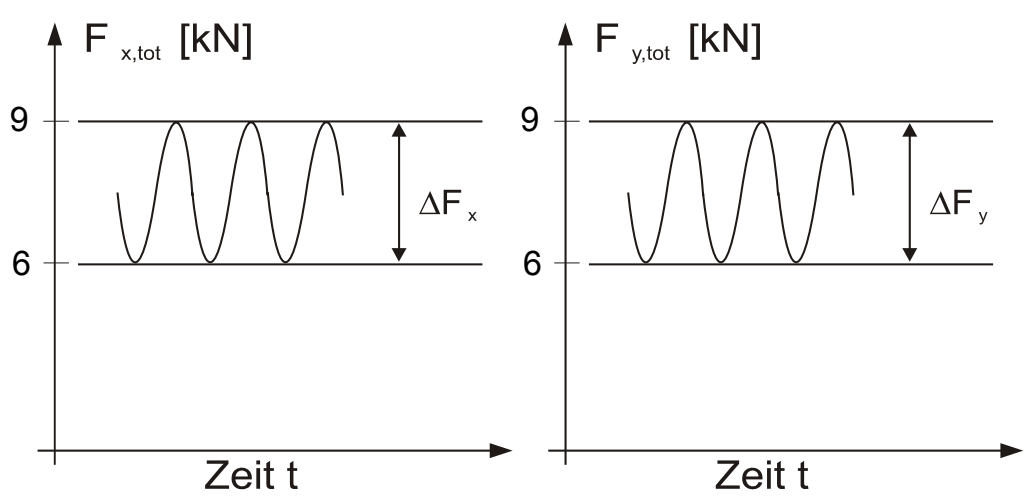

$\mathrm{F}_{\mathrm{xSd}}=6 \mathrm{kN}$

$\Delta \mathrm{F}_{\mathrm{XSd}}=3 \mathrm{kN}$
$\mathrm{F}_{\mathrm{y}, \mathrm{Sd}}=6 \mathrm{kN}$

$\Delta \mathrm{F}_{\mathrm{y}, \mathrm{Sd}}=3 \mathrm{kN}$

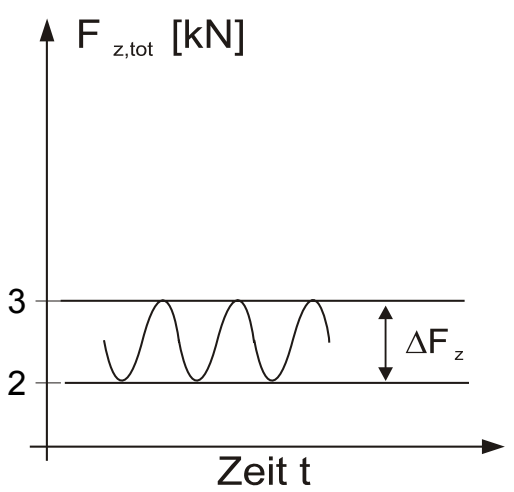

$\mathrm{F}_{\mathrm{zSd}}=2 \mathrm{kN}$

$\Delta \mathrm{F}_{\mathrm{zSd}}=1 \mathrm{kN}$

Bild 5.16 Einwirkende Kraftgrößen 
Die maßgebende Bemessungsstelle ergibt sich im Punkt B. Zur Bestimmung der Kraftkomponenten wird ein statisch bestimmtes System gewählt, wobei der Punkte B in allen Richtungen gelenkig gelagert wird. Die einwirkenden Kräfte verursachen somit folgende Reaktionskräfte an der Befestigungsstelle B:

$\mathrm{N}_{\mathrm{Sd}}=6 \mathrm{kN}$

$$
\begin{array}{lll}
\mathrm{N}_{\mathrm{Sd}}=6 \mathrm{kN} & \Delta \mathrm{N}_{\mathrm{Sd}}=3 \mathrm{kN} & \mathrm{N}_{\mathrm{Sd}, \text { tot }}=9 \mathrm{kN} \\
\mathrm{V}_{\mathrm{xSd}}=6 \mathrm{kN} & \Delta \mathrm{V}_{\mathrm{xSd}}=3 \mathrm{kN} & \mathrm{V}_{\mathrm{xSd} \text {,tot }}=9 \mathrm{kN}
\end{array}
$$$$
\mathrm{V}_{\mathrm{ySd}}=7,5 \mathrm{kN} \quad \Delta \mathrm{V}_{\mathrm{ySd}}=3,75 \mathrm{kN} \quad \mathrm{V}_{\mathrm{ySd}, \text { tot }}=11,25 \mathrm{kN}
$$

Zunächst muß der Nachweis der statischen Tragfähigkeit gemäß Gleichung (4.12) gewährleistet werden. Der Bemessungswert der zentrischen Zugrichtung $\mathrm{N}_{\mathrm{Rd}}$ kann dem Bemessungsbeispiel 5.5 entnommen werden. Der Bemessungswert $V_{x R d}$ und $V_{y R d}$ können aus den Versuchsergebnissen in Kapitel 4 abgeleitet werden.

$\mathrm{N}_{\mathrm{Rd}}=26,2 \mathrm{kN} \quad \mathrm{V}_{\mathrm{xRd}}=26,2 \mathrm{kN} \quad \mathrm{V}_{\mathrm{yRd}}=39,3 \mathrm{kN}$

Die zugehörigen Formfaktoren in der jeweiligen Beanspruchungsebene sind dem Bild $4.81 \mathrm{zu}$ entnehmen:

$\alpha_{\text {stat, } \mathrm{N}-\mathrm{Vx}}=2,0 \quad \alpha_{\text {stat, } \mathrm{N}-\mathrm{Vy}}=1,2 \quad \alpha_{\text {stat, } \mathrm{Vx}-\mathrm{Vy}}=1,7$

Somit lautet die Nachweisgleichung:

$$
\begin{aligned}
& \left(\frac{\mathrm{N}_{\mathrm{Sd}, \text { tot }}}{\mathrm{N}_{\mathrm{Rd}}}\right)^{\alpha_{\mathrm{stat}, \mathrm{N}-\mathrm{V}_{\mathrm{x}}}}+\left(\frac{\mathrm{V}_{\mathrm{xSd}, \text { tot }}}{\mathrm{V}_{\mathrm{xRd}}}\right)^{\alpha_{\text {stat } \mathrm{N}-\mathrm{V}_{\mathrm{x}}}}=\left(\frac{9}{26,2}\right)^{2}+\left(\frac{9}{26,2}\right)^{2}=0,24 \leq 1 \\
& \left(\frac{\mathrm{N}_{\mathrm{Sd}, \text { tot }}}{\mathrm{N}_{\mathrm{Rd}}}\right)^{\alpha_{\mathrm{stat}, \mathrm{N}-\mathrm{V}_{\mathrm{y}}}}+\left(\frac{\mathrm{V}_{\mathrm{ySd}, \text { tot }}}{\mathrm{V}_{\mathrm{yRd}}}\right)^{\alpha_{\text {stat }, \mathrm{N}-\mathrm{V}_{\mathrm{y}}}}=\left(\frac{9}{26,2}\right)^{1,2}+\left(\frac{11,25}{39,3}\right)^{1,2}=0,50 \leq 1 \\
& \left(\frac{\mathrm{V}_{\mathrm{xSd}, \text { tot }}}{\mathrm{V}_{\mathrm{xRd}}}\right)^{\alpha_{\text {stat }, \mathrm{V}_{\mathrm{x}}-\mathrm{V}_{\mathrm{y}}}}+\left(\frac{\mathrm{V}_{\mathrm{ySd}, \text { tot }}}{\mathrm{V}_{\mathrm{yRd}}}\right)^{\alpha_{\text {stat }, \mathrm{V}_{\mathrm{x}}-\mathrm{V}_{\mathrm{y}}}}=\left(\frac{9}{26,2}\right)^{1,7}+\left(\frac{11,25}{39,3}\right)^{1,7}=0,28 \leq 1
\end{aligned}
$$

$\Rightarrow$ Alle statischen Nachweise sind erfüllt.

Im nächsten Schritt kann nun der Ermüdungsnachweis (bei $2 \cdot 10^{6}$ Schwingspielen) unter kombinierten ruhenden und nichtruhenden zentrischen Zug- und Querbeanspruchungen mit Hilfe der Interaktionsgleichung (5.32) erfolgen. Zuvor müssen jedoch in Abhängigkeit von den einwirkenden, vorwiegend ruhenden Lasten, die spezifischen Formfaktoren $\alpha$ für jede Beanspruchungsebene bestimmt werden. Dazu sollte das Diagramm mit den zugehörige Formel in Bild 5.12 herangezogen werden. Die neben den Formfaktoren für ,vorwiegend ruhende Beanspruchung $(R=1)^{\text {“ }}$ benötigten Werte für ,vorwiegend nichtruhende Beanspruchung $(\mathrm{R}=0)^{“}$ können dem Bild 4.81 entnommen werden und lauten: 
$\alpha_{\text {dyn, N-Vx }}=1,3 \quad \alpha_{\text {dyn, N-Vy }}=1,2 \quad \alpha_{\text {dyn, Vx-Vy }}=1,0$

Nun werden in Abhängigkeit von einem Faktor $\eta_{\text {stat }}$, welcher aus dem Quotienten der einwirkenden Kraft (ohne nichtruhenden Anteil) zum Bemessungswert der statischen Tragfähigkeit für jede Beanspruchungsebene bestimmt wird, die Formfaktoren für eine kombinierte ruhende und nichtruhende Beanspruchung bestimmt.

$\underline{\mathrm{N}-\mathrm{V}_{\mathrm{x}}}$-Interaktion:

$\mathrm{F}_{\mathrm{Sd}, \mathrm{N}-\mathrm{V}_{\mathrm{x}}}=\sqrt{\mathrm{N}_{\mathrm{Sd}}^{2}+\mathrm{V}_{\mathrm{xSd}}^{2}}=\sqrt{6^{2}+6^{2}}=8,5 \mathrm{kN}$

$\eta_{\text {stat }, \mathrm{N}-\mathrm{V}_{\mathrm{x}}}=\frac{\mathrm{F}_{\mathrm{Sd}, \mathrm{N}-\mathrm{V}_{\mathrm{x}}}}{\min \left(\mathrm{N}_{\mathrm{Rd}} ; \mathrm{V}_{\mathrm{xRd}}\right)}=\frac{8,5}{26,2}=0,32$

$\Rightarrow \quad \alpha_{\mathrm{N}-\mathrm{V}_{\mathrm{x}}}=\left(\alpha_{\mathrm{stat}, \mathrm{N}-\mathrm{V}_{\mathrm{x}}}-\alpha_{\mathrm{dyn}, \mathrm{N}-\mathrm{V}_{\mathrm{x}}}\right) \cdot \eta_{\mathrm{stat}, \mathrm{N}-\mathrm{V}_{\mathrm{x}}}+\alpha_{\mathrm{dyn}, \mathrm{N}-\mathrm{V}_{\mathrm{x}}}=1,52$

${\underline{\mathrm{N}}-\mathrm{V}_{\mathrm{y}}-\text { Interaktion: }}$

$\alpha_{\mathrm{N}-\mathrm{V}_{\mathrm{y}}}=1,2$ unabhängig von der einwirkenden $\mathrm{Kraft} \mathrm{F}_{\mathrm{Sd}, \mathrm{N}-\mathrm{V} \mathrm{y}}$

$\underline{\mathrm{V}}_{\underline{x}}-\underline{V}_{\mathrm{y}}-$ Interaktion:

$\mathrm{F}_{\mathrm{Sd}, \mathrm{V}_{\mathrm{x}}-\mathrm{V}_{\mathrm{y}}}=\sqrt{\mathrm{V}_{\mathrm{xSd}}^{2}+\mathrm{V}_{\mathrm{ySd}}^{2}}=\sqrt{6^{2}+7,5^{2}}=9,6 \mathrm{kN}$

$\eta_{\text {stat }, \mathrm{V}_{\mathrm{x}}-\mathrm{V}_{\mathrm{y}}}=\frac{\mathrm{F}_{\mathrm{Sd}, \mathrm{V}_{\mathrm{x}}-\mathrm{V}_{\mathrm{y}}}}{\min \left(\mathrm{V}_{\mathrm{xRd}} ; \mathrm{V}_{\mathrm{yRd}}\right)}=\frac{9,6}{26,2}=0,37$

$\Rightarrow \quad \alpha_{\mathrm{V}_{\mathrm{x}}-\mathrm{V}_{\mathrm{y}}}=\left(\alpha_{\text {stat }, \mathrm{V}_{\mathrm{x}}-\mathrm{V}_{\mathrm{y}}}-\alpha_{\mathrm{dyn}, \mathrm{V}_{\mathrm{x}}-\mathrm{V}_{\mathrm{y}}}\right) \cdot \eta_{\text {stat }, \mathrm{V}_{\mathrm{x}}-\mathrm{V}_{\mathrm{y}}}+\alpha_{\mathrm{dyn}, \mathrm{V}_{\mathrm{x}}-\mathrm{V}_{\mathrm{y}}}=1,26$

Anschließend ist im letzten Schritt der maßgebende Bemessungswert $(\mathrm{p}=5 \%$ Fraktile, $\mathrm{W}=90 \%$ ) für das Zusammenwirken einer kombinierten ruhenden und nichtruhenden Beanspruchung für jede Belastungsrichtung zu ermitteln. Hierzu sind neben den Bemessungswerten der statischen Tragfähigkeiten zusätzlich die Bemessungswerte bei vorwiegend nichtruhender Beanspruchung notwendig. Diese müssen aus Versuchen vorliegen und lauten:
$\Delta \mathrm{N}_{\mathrm{Rd}, 0}=6,03 \mathrm{kN}$
$\Delta \mathrm{V}_{\mathrm{xRd}, 0}=10,15 \mathrm{kN}$
$\Delta \mathrm{V}_{\mathrm{yRd}, 0} \approx 10,15 \mathrm{kN}$

Wirkt bereits eine statische Last, so muß die ertragbare Schwingbreite gemäß dem Goodman-Diagramm in Bild 5.11 reduziert werden.

$\Delta \mathrm{N}_{\mathrm{Rd}, \text { tot }}\left(\mathrm{N}_{\mathrm{Sd}}\right)=\frac{\Delta \mathrm{N}_{\mathrm{Rk}, 0} \cdot \gamma_{\mathrm{M}}}{\gamma_{\mathrm{Mf}} \cdot \mathrm{N}_{\mathrm{Rk}}} \cdot\left(\frac{\mathrm{N}_{\mathrm{Rk}}}{\gamma_{\mathrm{M}}}-\frac{\mathrm{N}_{\mathrm{Sk}}}{\gamma_{\mathrm{F}}}\right)=\frac{6,03}{26,2} \cdot(26,2-6)=4,65 \mathrm{kN}$ 


$$
\begin{aligned}
& \Delta \mathrm{V}_{\mathrm{xRd}, \text { tot }}\left(\mathrm{V}_{\mathrm{xSd}}\right)=\frac{\Delta \mathrm{V}_{\mathrm{xRk}, 0} \cdot \gamma_{\mathrm{M}}}{\gamma_{\mathrm{Mf}} \cdot \mathrm{V}_{\mathrm{xRk}}} \cdot\left(\frac{\mathrm{V}_{\mathrm{xRk}}}{\gamma_{\mathrm{M}}}-\frac{\mathrm{V}_{\mathrm{xSk}}}{\gamma_{\mathrm{F}}}\right)=\frac{10,15}{26,2} \cdot(26,2-6)=7,83 \mathrm{kN} \\
& \Delta \mathrm{V}_{\mathrm{yRd}, \text { tot }}\left(\mathrm{V}_{\mathrm{ySd}}\right)=\frac{\Delta \mathrm{V}_{\mathrm{yRk}, 0} \cdot \gamma_{\mathrm{M}}}{\gamma_{\mathrm{Mf}} \cdot \mathrm{V}_{\mathrm{yRk}}} \cdot\left(\frac{\mathrm{V}_{\mathrm{yRk}}}{\gamma_{\mathrm{M}}}-\frac{\mathrm{V}_{\mathrm{ySk}}}{\gamma_{\mathrm{F}}}\right)=\frac{10,15}{39,3} \cdot(39,3-7,5)=8,21 \mathrm{kN}
\end{aligned}
$$

Somit können alle erzielten Werte in die Interaktionsgleichung (5.32) mit drei Kraftgrößen eingesetzt werden und der Nachweis für eine kombinierte ruhende und nichtruhende Beanspruchung kann erfolgen. Um jedoch den Vergleich zu einer Interaktionsgleichung mit lediglich zwei Kraftgrößen aufzuzeigen, wird dieser zuvor durchgeführt.

$$
\begin{aligned}
& \left(\frac{\Delta \mathrm{N}_{\mathrm{Sd}}}{\Delta \mathrm{N}_{\mathrm{Rd}, \text { tot }}\left(\mathrm{N}_{\mathrm{Sd}}\right)}\right)^{\alpha_{\mathrm{N}-\mathrm{V}_{\mathrm{x}}}}+\left(\frac{\Delta \mathrm{V}_{\mathrm{xSd}}}{\Delta \mathrm{V}_{\mathrm{xRd}, \text { tot }}\left(\mathrm{V}_{\mathrm{xSd}}\right)}\right)^{\alpha_{\mathrm{N}-\mathrm{V}_{\mathrm{x}}}}=\left(\frac{3}{4,65}\right)^{1,52}+\left(\frac{3}{7,83}\right)^{1,52}=0,74 \leq 1 \\
& \left(\frac{\Delta \mathrm{N}_{\mathrm{Sd}}}{\Delta \mathrm{N}_{\mathrm{Rd}, \text { tot }}\left(\mathrm{N}_{\mathrm{Sd}}\right)}\right)^{\alpha_{\mathrm{N}-\mathrm{V}_{\mathrm{y}}}}+\left(\frac{\Delta \mathrm{V}_{\mathrm{ySd}}}{\Delta \mathrm{V}_{\mathrm{yRd}, \text { tot }}\left(\mathrm{V}_{\mathrm{ySd}}\right)}\right)^{\alpha_{\mathrm{N}-\mathrm{V}_{\mathrm{y}}}}=\left(\frac{3}{4,65}\right)^{1,2}+\left(\frac{3,75}{8,21}\right)^{1,2}=0,98 \leq 1 \\
& \left(\frac{\Delta \mathrm{V}_{\mathrm{xSd}}}{\Delta \mathrm{V}_{\mathrm{xRd}, \text { tot }}\left(\mathrm{V}_{\mathrm{xSd}}\right)}\right)^{\alpha_{\mathrm{V}_{\mathrm{x}}-\mathrm{V}_{\mathrm{y}}}}+\left(\frac{\Delta \mathrm{V}_{\mathrm{ySd}}}{\Delta \mathrm{V}_{\mathrm{yRd}, \text { tot }}\left(\mathrm{V}_{\mathrm{ySd}}\right)}\right)^{\alpha_{\mathrm{V}_{\mathrm{x}}-\mathrm{V}_{\mathrm{y}}}}=\left(\frac{3}{7,83}\right)^{1,26}+\left(\frac{3,75}{8,21}\right)^{1,26}=0,67 \leq 1
\end{aligned}
$$

$\Rightarrow$ Alle Nachweise mittels der Interaktion von zwei Kraftgrößen sind erfüllt.

Die räumliche Interaktion aus Gleichung (5.32) von drei Kraftgrößen ergibt:

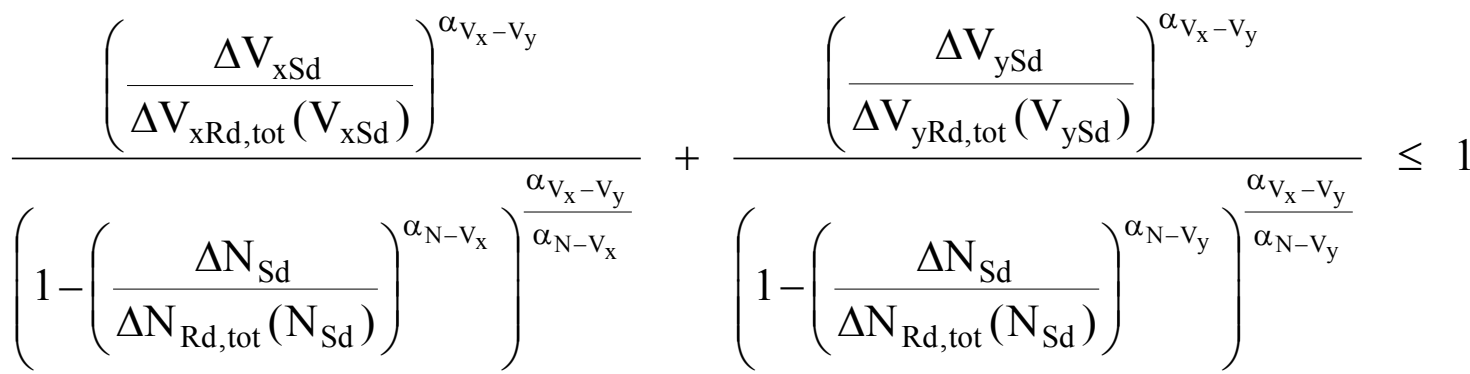

$$
\begin{aligned}
& \frac{\left(\frac{3}{7,83}\right)^{1,26}}{\left(1-\left(\frac{3}{4,65}\right)^{1,52}\right)^{\frac{1,26}{1,52}}}+\frac{\left(\frac{3,75}{8,21}\right)^{1,26}}{\left(1-\left(\frac{3}{4,65}\right)^{1,2}\right)^{\frac{1,26}{1,2}}}=1,48 \geq 1
\end{aligned}
$$

$\Rightarrow$ Nachweis wird nicht erfüllt. 


\section{Zusammenfassung und Ausblick}

Das Ziel der vorliegenden Arbeit ist es, das Tragverhalten von Ankerschienen unter ermüdungsrelevanten Einwirkungen experimentell $\mathrm{zu}$ untersuchen und mittels der erzielten Ergebnisse ein umfassendes Sicherheits- bzw. Bemessungskonzept herzuleiten. Die experimentellen Untersuchungen sind unter Berücksichtigung der Einflüsse auf das Tragverhalten von Lastniveau, Lastrichtung, unterschiedlichen Laststellungen und der Stützwirkung des umgebenden Betons konzipiert und durchgeführt worden. Dabei werden die Einwirkungen in eine zentrische Zuglast N, eine Querlast in Richtung der Schienenlängsachse $V_{x}$ bzw. quer dazu $V_{y}$ und eine Schräglast aufgeteilt. Die ausschließlich periodisch wirkende Beanspruchung ist stets im Zugschwellbereich erfolgt. Derzeitige Bemessungsansätze für nichtruhende Beanspruchungen berücksichtigen lediglich eine zentrische Zugschwellbelastung.

Bei allen Untersuchungen ist ausschließlich Stahlversagen maßgebend gewesen. Als hauptsächliche Versagensursache haben sich Risse im Übergangsbereich des Schienenrückens zum Schienenschenkel und des Schienenschenkels zur Schienenlippe erwiesen. Bei Beanspruchungen quer zur Schienenlängsrichtung haben sich zusätzlich Brüche im Ankerfuß eingestellt. Ein Betonversagen ist in keinem Fall aufgetreten.

Maßgebend für eine Bemessung unter ermüdungsrelevanten Beanspruchungen ist die Höhe der ertragbaren Schwingbreite, die vom Tragsystem dauerhaft aufgenommen werden kann. Diese wird als die Dauerfestigkeit bezeichnet und bei Stahlbauteilen nach etwa $2 \cdot 10^{6}$ Schwingspielen erreicht. Die Gültigkeit dieser Dauerfestigkeit ist für Ankerschienen anhand von vorliegenden Versuchsdaten experimentell bestätigt worden. Weitergehende Untersuchungen und Auswertungen sind im Rahmen dieser Arbeit stets auf Grundlage dieses Ergebnisses erfolgt.

Zulässige Schwingbreiten sind im Rahmen der aktuellen Bemessungsvorschrift an nicht einbetonierten Ankerschienen mit symmetrischen und ausreichenden Endüberständen ermittelt worden. Im Falle der untersuchten Ankerschiene betragen die Endüberstände jeweils $50 \mathrm{~mm}$, obwohl nach aktueller Bemessung Einbauzustände mit mindestens $25 \mathrm{~mm}$ Endüberstand vorgeschrieben sind. Somit ist eine zentrale Fragestellung in dieser Arbeit, welchen Einfluß der umgebende Beton und die Laststellung auf das Ermüdungstragverhalten von Ankerschienenbefestigungen ausüben.

Bei Versuchen mit einbetonierten Ankerschienen unter zentrischem Zug sind sowohl unter Ausnutzung von Mindestendüberständen, als auch bei günstigeren Lasteinleitungskonfigurationen im Vergleich zum nicht einbetonierten Zustand, stets höhere ertragbare Schwingbreiten erzielt worden. Der umgebende Beton bewirkt eine Stützwirkung, welche zu geringeren Dehnungen an den maßgebenden Versagensstellen führt. Mit Hilfe geeigneter meßtechnischer Methoden zur Erfassung der Spannungen und Dehnungen ist dieser Einfluß erfaßt und in Form eines Faktors quantifiziert worden.

Bei Beanspruchungen in Schienenlängsrichtung haben sich ebenfalls Einflüsse auf das Ermüdungstragverhalten durch den umgebenden Beton gezeigt. Mit Zunahme der 
Betonfestigkeit zeigt sich eine Erhöhung der Tragfähigkeit unter Ermüdungsbeanspruchung. Eine meßtechnische Erfassung von Spannungs- bzw. Dehnungszuständen in Bereichen der unmittelbaren Versagensstellen ist nicht vorgenommen worden. Jedoch kann angenommen werden, daß mit steigender Betonfestigkeit die Stützwirkung auf die Ankerschiene zunimmt und somit die Verformungen an den Versagensstellen reduziert werden. Die schadensäquivalenten Spannungen nehmen somit ab.

Belastbare Aussagen zum Einfluß des umgebenden Betons bei Beanspruchungen quer zur Schienenlängsrichtung können aufgrund $\mathrm{zu}$ weniger Versuchsergebnisse nicht gemacht werden.

Experimentelle Untersuchungen mit variierenden Endüberständen (Laststellungen) bei zentrischem Zug haben sowohl im einbetonierten, als auch im nicht einbetonierten Fall, Einflüsse auf die Ermüdungstragfähigkeit gezeigt. Anhand von Spannungs- und Dehnungsmessungen in unmittelbarer Nähe der maßgebenden Versagensstellen wird der Versagensmechanismus verdeutlicht. Hier haben sich deutliche Schädigungszunahmen an den Versagensstellen durch instabiles Rißwachstum erst im unmittelbaren bruchnahen Bereich gezeigt. Unter Ausnutzung von Mindestendüberständen werden stets geringfügig kleinere ertragbare Schwingbreiten erreicht als bei günstigeren Lasteinleitungskonfigurationen. Auf der sicheren Seite liegend sind nachfolgende Untersuchungen ausschließlich für Laststellungen unter Ausnutzung von Mindestendüberständen erfolgt.

Wesentlich für eine möglichst allgemein gültige Bemessung ist die Erkenntnis, daß die ertragbare Schwingbreite eine Abhängigkeit vom Lastniveau aufweist. Gemäß dem Ermüdungsfestigkeitsdiagramm nach Goodman zeigt sich mit Zunahme der statischen Einwirkung eine Abnahme der ertragbaren Schwingbreite. In der aktuellen Bemessung von Ankerschienen hingegen bleibt dieser Einfluß unberücksichtigt. Die ertragbare Schwingbreite wird hierbei unabhängig vom Lastniveau bis zum Bemessungswert der statischen Tragfähigkeit als konstant angesetzt. Anhand experimenteller Untersuchungen unter zentrischem Zug kann der Einfluß des Lastniveaus verdeutlicht werden. Durch eine Vereinfachung des Ermüdungstragfähigkeitsdiagramms nach Goodman wird eine modifizierte Bemessung vorgeschlagen. Dieses vereinfachte Verfahren beruht auf Schwingversuchen auf niedrigen Lastniveaus $(\mathrm{R} \approx 0)$ und statischen Versuchen zur Tragfähigkeit $(\mathrm{R}=1)$. Durch eine lineare Verbindung dieser beiden Punkte wird der Bemessungsbereich bestimmt. Er deckt die derzeitige Bemessung voll ab und erlaubt sogar eine Gesamtbelastung oberhalb der aktuellen statischen zulässigen Last.

Ein weitere Charakteristik der derzeitigen Bemessung liegt darin, daß sie für nichtruhende Beanspruchungen lediglich den Bereich des reinen zentrischen Zugs abdeckt. Experimentelle Untersuchungen zur Interaktion von kombinierten ruhenden und nichtruhenden Zug- und Querbeanspruchungen von einbetonierten Ankerschienen zeigen jedoch, daß eine allseitige nichtruhende Beanspruchung erfolgen kann. Dabei zeigt sich der zentrische Zug stets als kritische Beanspruchungsrichtung. Zur Beschreibung der Abhängigkeit zwischen den jeweiligen Beanspruchungsrichtungen ist ein nichtlinearer Interaktionsansatz nach den Vorgaben der ETAG zugrunde gelegt 
worden. Dieser wird in den überwiegenden Bereichen der Befestigungstechnik zur Beschreibung des Tragverhaltens von Kraftgrößen herangezogen. In Abhängigkeit von einem Formfaktor $\alpha$ im Exponenten dieser Exponentialfunktion gelingt es die erzielten Versuchsergebnisse abzubilden. Beträgt der Formfaktor $\alpha=1$, so entspricht die Form der Interaktion einer Geraden, wo sich hingegen bei $\alpha=2$ eine Kreisgleichung ergibt. Die im Rahmen dieser Arbeit ermittelten Formfaktoren für die drei unterschiedlichen Beanspruchungsebenen beschreiben Verläufe zwischen beiden Grenzfällen. Eine Interaktion für vorwiegend nichtruhende Querbeanspruchungen konnte nicht durch Versuchsergebnisse belegt werden, weshalb auf der sicheren Seite liegend, ein geradliniger Verlauf angenommen wird.

Mit zunehmendem Beanspruchungswinkel zeigen sich deutliche Tragreserven im System, die in der derzeitig gültigen Bemessungsrichtlinie nicht angesetzt werden. Bei der Annahme einer kugelförmigen Interaktionsbeziehung, mit dem nach aktueller Bemessungsrichtlinie anzusetzenden zulässigen Last der zentrischen Zugschwellbelastung als Radius, liegen alle Versuchsergebnisse deutlich oberhalb des Kugelvolumens und somit auf der sicheren Seite. Die Versuchsergebnisse zeigen mit zunehmendem Lastniveau eine Vergrößerung des Formfaktors. Lediglich für die $\mathrm{N}-\mathrm{V}_{\mathrm{y}}$-Interaktion stellt sich ein konstanter Formfaktor ein. Durch die Annahme einer linearen Funktion zur Beschreibung der Abhängigkeit beider Variablen wird eine einfache Darstellungsweise erzielt.

Die Erkenntnisse der experimentellen Untersuchungen finden Eingang in ein neues, umfassendes Bemessungskonzept, welches auf der Methode der Grenzzustände beruht und im Rahmen dieser Arbeit verwendet worden ist. Im Rahmen dieses Stufe II Verfahrens nach Eurocode 1 wird der Sicherheitsindex $\beta$ als Maß für die Zuverlässigkeit eines Tragsystems betrachtet. Der Eurocode 1 bietet die Möglichkeit, durch eine auf der sicheren Seite liegende Vereinfachung, die Sicherheitsbetrachtung bei der Herleitung von Bemessungsansätzen auf der Basis von Versuchen durchzuführen. Dabei wird der Wichtungsfaktor $\alpha_{R}$, welcher den Einfluß der Verteilungsfunktion der Beanspruchbarkeit beschreibt, mit einem konstanten Wert von 0,8 belegt. Als Verteilungsfunktion, zur Beschreibung der ertragbaren Schwingbreite an der maßgebenden Bemessungsschwingspielzahl von $2 \cdot 10^{6}$ Schwingspielen, ist nach Überprüfung der Anpassungsfähigkeit sowohl die Standardnormalverteilung, als auch die logarithmische Normalverteilung zugrunde gelegt worden.

Im ersten Schritt der Sicherheitsbetrachtung werden bisherige Auswerteverfahren nach SVA, EC 3 und ETAG gegenübergestellt. Diese unterscheiden sich in den verwendeten Fraktilwerten und Aussagewahrscheinlichkeiten. Im Streubereich der erzielten Versuchsergebnisse gibt es Überschneidungen zwischen den verschiedenen Auswerteverfahren und es zeigen sich vergleichbare Ergebnisse. Nach dem direkten Verfahren zur Bestimmung des Bemessungswertes im Eurocode 1 können in Abhängigkeit von der Versuchsanzahl und Belastungsrichtung, Teilsicherheitsbeiwerte bestimmt werden. Je nachdem, welcher Verteilungstyp zur Beschreibung der Versuchsergebnisse zugrunde gelegt wird, ergeben sich Unterschiede in den Teilsicherheitsbeiwerten. Ab einer Anzahl von etwa acht Versuchen auf mindestens drei unter- 
schiedlichen Lastniveaus zeigen sich Teilsicherheitsbeiwerte unter 1,35. Als maximale errechnete Teilsicherheit für die Ermüdungsfestigkeit ergibt sich dabei, unter der Annahme einer logarithmischen Normalverteilung, ein Wert von 1,29. Um in Einklang mit europäischen Regelwerken zu stehen, wird im Rahmen dieser Arbeit ein Teilsicherheitsbeiwert für den Grenzzustand der Ermüdung von 1,35 empfohlen. Der 5\%-Fraktilwert der Grundgesamtheit wird in Anlehnung an die europäischen Normung als charakteristischer Wert angesetzt. Die Wahl einer Aussagewahrscheinlichkeit von $90 \%$ erfolgt in Anlehnung an die Vorgaben der EOTA (ETAG).

Auf der Basis dieser sicherheitstheoretischen Erkenntnisse wird anschließend eine umfassende und variable Bemessung für Ankerschienen unter kombinierten ruhenden und nichtruhenden Zug- Querbeanspruchungen vorgestellt. Dabei werden zunächst die jeweiligen Kraftkomponenten der beliebig einwirkenden Gesamtkraft auf die unterschiedlichen Beanspruchungsebenen projiziert. Zur Gewährleistung der statischen Tragfähigkeit muß im ersten Schritt der Nachweis der ruhenden Belastungen für jede Beanspruchungsebene separat erfolgen. Sind zusätzlich zu den ruhenden Lasten die Kraftkomponenten der nichtruhenden Beanspruchung bekannt, so kann anschließend der Nachweis der Tragfähigkeit für eine kombinierte ruhende und nichtruhende Zugund Querbeanspruchung erfolgen. Dazu werden zunächst in Abhängigkeit von der einwirkenden Last, die charakteristischen Formfaktoren der jeweiligen Beanspruchungsebenen bestimmt. Mit Hilfe der Bemessungswerte der Beanspruchbarkeiten, den einwirkenden Lasten und den für jede Beanspruchungsebene ermittelten Formfaktoren kann schließlich der Nachweis der Tragfähigkeit im Grenzzustand der Ermüdung erfolgen. Zur Gewährleistung der Tragfähigkeit bei räumlicher Beanspruchung darf der Betrag der räumlichen Interaktionsgleichung nicht den Grenzwert 1 (Grenzzustand der Ermüdungstragfähigkeit) überschreiten.

Anhand von Beispielen wird das im Rahmen dieser Arbeit entwickelte Bemessungsverfahren vorgestellt. Zunächst erfolgt der Vergleich dieses Verfahrens zur derzeitigen Bemessung von Ankerschienen. Anschließend wird an einem Anwendungsfall der Nachweise der Ermüdungstragfähigkeit demonstriert.

\section{Ausblick}

Die vorgestellten experimentellen Untersuchungen für gezahnte Ankerschienen erfassen hauptsächlich den Bereich der nichtruhenden Zugschwellbelastung bei Einzelbefestigung. Dabei sind Einflüsse auf das Ermüdungstragverhalten infolge des umgebenden Betons, der unterschiedlichen Endüberstände, des Lastniveaus und der verschiedenen Belastungsrichtungen untersucht worden. In Hinblick auf weiterführende Arbeiten erscheinen insbesondere die folgenden Punkte als interessant:

- In den überwiegenden Fällen wird bei Ankerschienenbefestigungen eine reine Zugschwellbeanspruchung vorherrschen. Jedoch sollte die Kenntnis des Tragverhaltens unter nichtruhenden Wechselbeanspruchungen in allen Beanspruchungsrichtungen angestrebt werden. 
- Eine Interaktion zwischen den Kraftgrößen $\mathrm{V}_{\mathrm{x}}$ und $\mathrm{V}_{\mathrm{y}}$ in Querrichtung wird auf der sicheren Seite liegend abgeschätzt. Durch weitere experimentelle Untersuchungen ist diese Beanspruchungsebene zu belegen.

- Das Tragverhalten von Mehrfachbefestigungen unter nichtruhenden Lasten ist bisher nicht untersucht worden. Daher erscheint es sinnvoll, die Trageigenschaften bei paarweiser oder mehrfacher Befestigung zu ermitteln.

- Die Untersuchungen sind an Ankerschienen mit einem angeordneten Ankerbolzen und Laststellungen über dem Ankerbolzen erfolgt. Untersuchungen zum Ermüdungstragverhalten mit variierenden Laststellungen zwischen zwei oder mehreren Ankerbolzen begründen weiteren Forschungsbedarf.

- Die Ermittlung der Trageigenschaften von weiteren unterschiedlichen Profiltypen sollte auf Basis der in dieser Arbeit wiedergegebenen Methodik durchgeführt werden.

- Ankerschienen werden neben der Herstellung aus Normalstahl auch in verschiedenen Edelstahlvarianten für spezielle Anwendungsbereiche im Bauwesen angefertigt. In Bereichen des Brücken- und Straßenbaus sind korrosionsbeständige Stähle unabdingbar. Das Tragverhalten von Ankerschienenbefestigungen aus Edelstahl bedarf weiterer Forschungsarbeiten, weshalb o.g. Untersuchungen hierzu sinnvoll erscheinen. 


\section{Literaturverzeichnis}

[1] Ang, A. H.-S., Tang, W. H.: Probability Concepts in Engineering Planning and Design - Volume I - Basic Principles. John Wiley \& Sons, Inc., 1975

[2] Ang, A. H.-S., Tang, W. H.: Probability Concepts in Engineering Planning and Design - Volume II - Decision, Risk, and Reliability. John Wiley \& Sons, Inc., 1984

[3] BAM: Bericht über die Ergebnisse von Zug-Schwell-Versuchen mit JORDAHL-Ankerschienen. Verkürzter Bericht, Dezember 1981

[4] Betonkalender 1997. Teil I und II, Ernst \& Sohn Verlag für Architektur und technische Wissenschaften $\mathrm{GmbH}$, Berlin

[5] Bidmon, W.: Befestigung von Aufzugsführungsschienen. (8/2000)

[6] Block, K.: Dübelverbindungen im Kraftwerksbau - Eine Bestandaufnahme. Bautechnik 76 (1999), H. 11, S. 976-982

[7] Block, K., Dreier, F.: Das Ermüdungsverhalten von Dübelbefestigungen. DAfStb, Heft 541, Beuth Verlag GmbH, Berlin, 2003

[8] Block, T.: Zur Interaktion von nichtruhenden Zug- und Querbeanspruchungen bei einbetonierten Ankerschienen. Diplomarbeit, Lehrstuhl für Stahl- und Verbundbau, Bereich Konstruktionsteilprüfung, Ruhr-Universität Bochum, $2 / 2003$

[9] Blumenauer, H.: Technische Bruchmechanik. VEB Deutscher Verlag für Grundstoffindustrie, Leipzig, 1987

[10] Bolotin, V. V.: Mechanics of Fatigue. Mechanical Engineering, CRC Press, 1999

[11] Bourgund, U.: Sicherheitskonzept im Bauwesen - Ermittlung und Beurteilung von Teilsicherheitsbeiwerten. Uni Insbrück, Institut für Mechanik, 5/1986

[12] Bourgund, U. , Ammann, W.: Ein Bemessungskonzept für Befestigungen in Beton auf der Basis von Teilsicherheitsbeiwerten. Bauingenieur 66 (1991), S. 401-411 
[13] Breloh, R.: Befestigen von Lasten an Beton mit Ankerschienen. Die Bautechnik 58 (1981), H.1, S. 28-31

[14] Buxbaum, O.: Betriebsfestigkeit - Sichere und wirtschaftliche Bemessung schwingbruchgefährdeter Bauteile. Verlag Stahleisen mbH, Düsseldorf, 1988

[15] CEB-FIP: Model Code 1990. Thomas Telford Services Ltd., 1993

[16] CEN/TC 250: Design of fastening for use in concrete - Final Draft - Part 3: Anchor channels. 6/2004

[17] Christ, H.-J.: Ermüdungsverhalten metallischer Werkstoffe. WerkstoffInformationsgesellschaft mbH, 1998

[18] Comité Euro-International du Béton (CEB) (1995): Design of Fastenings in Concrete. CEB Bulletin 226, pp 1-144, Lausanne, 1995, Thomas Telford Services Ltd., 1997

[19] Cornelsen, H. A. W., Reinhardt, H. W.: Uniaxial tensile fatigue failure of concrete under constant amplitude and programme loading. Magazin of Concrete Research, Vol. 36, No. 129, December 1984

[20] Corten, H. T., Dolan, T.: Cumulative Fatigue Damage. Proceedings of the International Conference on Fatigue Metals, pp. 235-246, London/New York, 1956

[21] DIBt: Allgemeine bauaufsichtliche Zulassung für Halfenschienen des Typs HZA 29/20 und HZA 38/23, Nr. Z-21.4.1691, Berlin 27. November 2000

[22] DIBt: Allgemeine bauaufsichtliche Zulassung für Halfenschienen HTA, Nr. Z-21.4.34, Berlin 19. Juli 1978

[23] DIBt: Allgemeine bauaufsichtliche Zulassung für Jordahl Ankerschienen des Typs JXA-W 29/20 und JXA-W 38/23, Nr. Z-21.4.1690, Berlin 26. Juli 2002

[24] DIBt: Bemessungsverfahren für Dübel zur Verankerung im Beton. Anhang zum Zulassungsbescheid, 6/1993

[25] DIBt: Sachverständigen-Ausschuss für Ankerschienen und Dübel (SVA), Berlin

[26] DIN 1045, Beton und Stahlbeton, Bemessung und Ausführung, Deutsches Institut für Normung Beuth Verlag, Berlin, 7/1988 
[27] DIN 1055-100: Einwirkung auf Tragwerke - Teil 100: Grundlagen der Tragwerksplanung, Sicherheitskonzepte und Bemessung. Beuth Verlag, Berlin, 3/2001

[28] DIN 4132: Kranbahnen Stahltragwerke. Deutsches Institut für Normung, Beuth Verlag, Berlin, 2/1981

[29] DIN 50100: Dauerschwingversuch. Deutsches Institut für Normung, Beuth Verlag, Berlin, 2/1978

[30] DIN 50125: Prüfung metallischer Werkstoffe - Zugproben, Beuth Verlag, Berlin, 1/2004

[31] DIN EN 10002-1: Metallische Werkstoffe - Zugversuch - Teil 1: Prüfverfahren bei Raumtemperatur, Beuth Verlag, Berlin, 12/2001

[32] DIN V ENV 1992-1-1: Planung von Stahlbeton- und Spannbetontragwerken. Teil 1: Grundlagen und Anwendungsregeln für den Hochbau. Beuth Verlag, Berlin, 6/1992

[33] Eligehausen, R.: Bemessung von Befestigungen in Beton mit Teilsicherheitsbeiwerten. Bauingenieur 65 (1990), S. 295- 305

[34] Eligehausen, R., Mallée, R.: Befestigungstechnik im Beton- und Mauerwerksbau - Bauingenieur-Praxis. Ernst \& Sohn, 2000

[35] Entwurf prEN 1990-Eurocode: Grundlagen der Tragwerksplanung. Anhang D Versuchsgestützte Bemessung (Informativ), S. 73-88

[36] ENV 1991-1: Grundlagen der Tragwerksplanung und Einwirkungen auf Tragwerke - Teil 1: Grundlagen der Tragwerksplanung. Beuth Verlag, Berlin, 1994

[37] ENV 1993-1-1: Bemessung und Konstruktion von Stahlbauten - Teil 1-1: Allgemeine Bemessungsregeln für den Hochbau. Beuth Verlag, Berlin, 4/1993

[38] EOTA: Bemessungsverfahren für Metalldübel zur Verankerung im Beton. Leitlinie für die europäische technische Zulassung, Anhang C, (ETAG 001), $6 / 1997$

[39] Fießler, B., Hawranek, R., Rackwitz, R.: Numerische Methoden für probabilistische Bemessungsverfahren und Sicherheitsnachweise. Berichte zur Zuverlässigkeitstheorie, H. 14, TU München, 1976 
[40] Fischer, L.: Bestimmung des 5\%-Quantils im Zuge der Bauwerksprüfung Bezugnahme auf DIN-Normen und Eurocodes. Bautechnik 72 (1995), H. 11, S. $712-722$

[41] Fischer, L.: Charakteristische Werte - ihre Bedeutung und Berechnung Diskussion einiger aktueller Sachverhalte zur neuen Normengeneration. Bauingenieur 78 (2003), S. 179-186

[42] Fischer, L.: Das neue Sicherheitskonzept im Bauwesen - Ein Leitfaden für Bauingenieure, Architekten und Studenten. Bautechnik Spezial, Ernst \& Sohn Verlag für Architektur und technische Wissenschaften GmbH, Berlin, 2001

[43] FMPA Bauwesen: Ermüdungsversuche mit Halfeneisen-Ankerschienen HTA 40/22Q und 50/30Q. Prüfbericht, April 1977

[44] Freudenthal, A., M., Gumbel, E. J.: On the statistical interpretation of fatigue tests. Proceedings of the Royal Society of London, Series A. Mathematical and Physical Sciences, Vol.216, pp. 309-343, Cambridge University Press, $2 / 1953$

[45] Fuchs, W., Eligehausen, R.: Das CC-Verfahren für die Berechnung der Betonausbruchlast von Verankerungen. Beton- und Stahlbetonbau 90 (1995), H. 1, S. 6-9

[46] Fuchs, W.; Eligehausen, R.: Das CC-Verfahren für die Berechnung der Betonausbruchlast von Verankerungen (Fortsetzung aus Heft 1/1995). Betonund Stahlbetonbau 90 (1995), H. 2, S. 38-44

[47] Fuchs, W., Eligehausen, R., Breen, J.: Concrete Capacity Design (CCD) Approach for Fastening to Concrete. ACI Structural Journal, V. 92, No. 1, Title no. 92-S2, pp. 73-94, January/Februrary 1995

[48] Gaede, K.: Versuche über die Festigkeit und die Verformung von Beton bei Druckschwellbeanspruchung. DAfStb, Heft 144, Ernst \& Sohn, Berlin, 1962

[49] Grusibau: Grundlagen zur Feststellung von Sicherheitsanforderungen für bauliche Anlagen. NABau, Deutsches Institut für Normung e.V., Beuth Verlag GmbH, Berlin/ Köln, 1981

[50] Güreş, S., Hanenkamp, W.: Dauerschwingversuche an einbetonierten Ankerschienen -Profil HZA 38/23 St und JXA-W 38/23 St-. Bericht Nr. A02/2001-1, Ruhr-Universität Bochum, Konstruktiver Ingenieurbau, Bereich Konstruktionsteilprüfung, Februar 2001, nicht veröffentlicht 
[51] Güreş, S., Hanenkamp, W.: Untersuchungen zur Tragfähigkeit von Ankerschienen -Profil 29/20 A4 und 38/23 A4-. Bericht Nr. A-08/2002, RuhrUniversität Bochum, Konstruktiver Ingenieurbau, Bereich Konstruktionsteilprüfung, Februar 2002, nicht veröffentlicht

[52] Güreş, S., Hanenkamp, W.: Untersuchungen zur Tragfähigkeit von Ankerschienen -Profil 29/20 St und 38/23 St-. Bericht Nr. A-04/2000, RuhrUniversität Bochum, Konstruktiver Ingenieurbau, Bereich Konstruktionsteilprüfung, Juli 2000, nicht veröffentlicht

[53] Haibach, E.: Betriebsfestigkeit - Verfahren und Daten zur Bauteilberechnung. VDI Verlag mbH, Düsseldorf, 1989

[54] Halfen GmbH: Hochhausfassaden - sicher mit Ankerschienen. Bauingenieur 76 (2001), A10

[55] Halfen GmbH \& Co. KG: Anwendungsbereiche von Ankerschienen, Bilder$\mathrm{CD}$, unveröffentlicht

[56] Halfen-Deha: Halfenschienen. Produktinformationen B-P 04, www. HalfenDeha.de

[57] Halfen-Deha: Aufzugsbau. Technisches Merkblatt, Lift 5/00, www. HalfenDeha.de

[58] Halfen-Deha: Dynagrip-Halfenschienen. Produktinformationen BD 03, www. Halfen-Deha.de

[59] Halfen-Deha: Halfenschienen für den Aufzugsbau. Produktinformationen BDA 02, www. Halfen-Deha.de

[60] Halfen-Deha: Halfenschienen und Zubehör. Produktinformationen B 04, www. Halfen-Deha.de

[61] Hanenkamp, W., Güreş, S.: Zum Tragverhalten von Ankerschienen unter ermüdungsrelevanten Einwirkungen. Stahlbau 73 (2004), H. 9, S. 668-675

[62] Hanenkamp, W., Schmidt-Schleicher, H.: Neuzeitliche Prüftechnik im Bauwesen - zerstörende Werkstoff- und Bauteilprüfung. Konstruktiver Ingenieurbau Berichte, H. 18, Vulkan-Verlag, Essen, 12/1973

[63] Hanswille, G.: Zum Nachweis der Ermüdung von Verbundträgern nach Eurocode 4 Teil 1-1. Stahlbau 63 (1994), H. 9, S. 284-289 
[64] Hartung, J.: Statistik - Lehr- und Handbuch der angewandten Statistik. R. Oldenbourg Verlag GmbH, München, 1995

[65] Hashin, Z.: A Reinterpretation of the Palmgren-Miner rule for Fatigue life Prediction. Transactions of the ASME, Journal of Applied Mechnics, Vol. 47, pp.324-328, June 1980

[66] Hegger, J., Beutel, R., Hoffmann, S.: Statistische Auswertung von Versuchen - Beurteilung von Bemessungsansätzen. Beton- und Stahlbetonbau 94 (1999), H. 11, S. 457-464

[67] Holzapfel, F.: Das Verhalten von Zementstein unter dynamischer Beanspruchung. Dissertation, RWTH Aachen, 1970

[68] John, B.: Statistische Verfahren für Technische Meßreihen, Carl Hanser Verlag, München, 1997

[69] Jordahl: Komplettordner Befestigungstechnik, Deutsche Kahneisen Gesellschaft mbH, Berlin, 8/2000

[70] Kindmann, R.: Verbundträger Schwingungsverhalten Ermüdung/ Betriebsfestigkeit - Unterlagen zur Lehrveranstaltung Brücken und Hochbauten in Stahl- und Verbundbauweise. Schriftenreihe des Lehrstuhls für Stahl- und Verbundbau, Band 10, Ruhr-Universität Bochum, 10/1999

[71] Kindmann, R., Frickel, J.: Elastische und plastische Querschnittstragfähigkeit - Grundlagen, Methoden, Berechnungsverfahren, Beispiele. Ernst \& Sohn Verlag für Architektur und technische Wissenschaften GmbH, Berlin, 2002

[72] Kindmann, R., Hanenkamp, W.: Untersuchungen zur Tragfähigkeit von Ankerschienen -Profil 29/20 St und 38/23 St-. Gutachterliche Stellungnahme, Ruhr-Universität Bochum, Lehrstuhl für Stahl- und Verbundbau, Juli 2000, nicht veröffentlicht

[73] Kindmann, R., Hanenkamp, W.: Untersuchungen zur Tragfähigkeit von Ankerschienen -Profil 29/20 A4 und 38/23 A4-. Gutachterliche Stellungnahme, Ruhr-Universität Bochum, Lehrstuhl für Stahl- und Verbundbau, Februar 2002, nicht veröffentlicht

[74] Kindmann, R., Schweppe, H., Schleich, J.-B., Cajot, L.-G.: Zum Sicherheitskonzept für den baulichen Brandschutz. Bauingenieur 73 (1998), S. $455-461$ 
[75] Klausen, D., Weigler, H.: Betonfestigkeit bei konstanter und veränderlicher Dauerschwellbeanspruchung. Betonwerk + Fertigteil-Technik (3/1979), S. $158-163$

[76] Klingmüller, O., Bourgund, U.: Sicherheit und Risiko im Konstruktiven Ingenieurbau. Friedr. Vieweg \& Sohn Vertragsgesellschaft mbH, Braunschweig/ Wiesbaden, 1992

[77] König, G., Danielewicz, I.: Ermüdungsfestigkeit von Stahlbeton- und Spannbetonbauteilen mit Erläuterungen zu den Nachweisen gemäß CEB-FIP Model Code 1990. DAfStb, Heft 439, Beuth Verlag GmbH, Berlin, 1994

[78] König, G., Hosser, D., Schobbe, W.: Sicherheitsanforderungen für die Bemessung von baulichen Anlagen nach den Empfehlungen des NABau eine Erläuterung. Bauingenieur 57, (1982), S. 69-78

[79] Kraus, J.: Tragverhalten und Bemessung von Ankerschienen unter zentrischer Zugbelastung. Dissertation, Institut für Werkstoffe im Bauwesen, Universität Stuttgart, 2003

[80] Lewandowski, D.: Zur Bemessung von Ankerschienen unter ermüdungsrelevanten Einwirkungen bei zentrischer Zugschwellbeanspruchung. Diplomarbeit, Lehrstuhl für Stahl- und Verbundbau, Bereich Konstruktionsteilprüfung, Ruhr-Universität Bochum, 4/2004

[81] Lind, N. C.: Formulation of Probabilistic Design. Journal of the Engineering Mechanics Devision, pp. 273-285, April 1977

[82] Lotze, D.: Tragverhalten und Anwendung von Dübeln unter oftmals wiederholter Belastung. Dissertation, Universität Stuttgart, IWB Mitteilungen, $2 / 1993$

[83] Maenning, W.-W.: Das Abgrenzungsverfahren, eine kostensparende Methode zur Ermittlung von Schwingfestigkeitswerten - Theorie, Praxis und Erfahrungen. Materialprüfung 19 (1977), Nr. 8, S. 280- 289

[84] Mehlhorn, G.: Der Ingenieurbau - Tragwerkszuverlässigkeit - Einwirkungen. Ernst \& Sohn Verlag für Architektur und technische Wissenschften GmbH, Berlin, 1996

[85] Miner, M. A.: Cumulative Damage in Fatigue. Journal of Applied Mechanics, pp. A159-A164, September 1945 
[86] Monien, M.: Einfluss der Laststellung und der Stützwirkung des umgebenden Betons auf die Tragfähigkeit von Ankerschienen des Profils 38/23 St unter nichtruhender Belastung. Diplomarbeit, Lehrstuhl für Stahl- und Verbundbau, Bereich Konstruktionsteilprüfung, Ruhr-Universität Bochum, 9/2002

[87] Naubereit, H., Weihert, J.: Einführung in die Ermüdungsfestigkeit - Ein Lehrund Übungsbuch mit Beispielen und Aufgaben. Carl Hanser Verlag, München/Wien, 1999

[88] Nause, P.: Brandverhalten von einbetonierten Halfen- und JordahlAnkerschienen. Bauingenieur 78 (2003), S. A6-A9

[89] Neuber, H.: Theory of stress concentration for shear-strained prismatical bodies with arbitrary nonlinear sress-strain law. Transactions of the ASME. Journal of Applied Mechanics, Vol. 28, pp. 544-550, 1961

[90] Oh, B. H.: Cumulative damage Theory of Concrete under Variable-Amplitude fatigue Loadings. ACI Materials Journal, V. 88, No. 1, Title no. 88-M6, pp. 41-48, January/Februrary 1991

[91] Oluokun, F. A.; Burdette, E. G.: Behavior of Channel Anchors in Thin Slabs under Combined Shear and Tension (Pullout) Loads. ACI Structural Journal, V. 90, No. 4, Title no. 90-S43, pp. 407-413, July/August 1993

[92] Owen, D.-B.: Handbook Of Statistical Tables. 5.3 One-Sided Tolerance Limit Factors for a Normal Distribution, S. 117-126, Addison-Wesley Publishing Company, 1962

[93] Palmgren, A.: Die Lebensdauer von Kugellagern. Zeitschrift der VDI (1924)

[94] Paris, P., Erdogan, F.: A critical analysis of crack propagation laws. Journal of Basic Engineering, pp. 528-534, December 1963

[95] Parlak, H.: Untersuchungen zum Tragverhalten von einbetonierten Ankerschienen unter dynamischer Beanspruchung. Diplomarbeit, Lehrstuhl für Stahl- und Verbundbau, Bereich Konstruktionsteilprüfung, RuhrUniversität Bochum, 9/2001

[96] Plate, E. J.: Statistik und angewandte Wahrscheinlichkeitslehre für Bauingenieure. Ernst \& Sohn Verlag für Architektur und technische Wissenschaft, Berlin, 1993 
[97] Powell, J. B., Burdette, E. G, Oluokun, F. A.: Prediction of Pullout Capacity of Channel Anchors. ACI Structural Journal, V. 88, No. 5, Title no. 88-S65, pp. 624-630, September/October 1991

[98] Powell, J. B.; Burdette, E. G. Oluokun, F. A.: Prediction of Shear Capacity of Channel Anchors in Thin Slabs. ACI Structural Journal, V. 89, No. 5, Title no. 89-S53, pp. 564-568, September/October 1992

[99] Rackwitz, R.: Practical Probabilistic Approach to Design. Comité Européen du Béton, Bulletin d'Information No. 112, pp. 13-17

[100] Radaj, D.: Ermüdungsfestigkeit - Grundlagen für Leichtbau, Maschinen- und Stahlbau. Springer-Verlag Berlin Heidelberg, 1995

[101] Reppermund, K.: Konzepte zur Berechnung der Versagenswahrscheinlichkeit bei Ermüdungsbeanspruchung. Dissertation, Berichte zur Zuverlässigkeitstheorie, TU München, LKI, H. 64/1981

[102] Rilem: International Symposium on Connections between Steel and Concrete. Vol. 1 \& 2, University of Stuttgart, Stuttgart, September 2001

[103] Roik, K.: Vorlesungen über Stahlbau - Grundlagen. Verlag von Wilhelm Ernst \& Sohn, 1983

[104] Rosenblatt, M.: Remarks on a Multivariate Transformation. The Annals of Mathematical Statistics, Vol. 52, pp. 470-472, 1952

[105] Schneider, J.: Sicherheit und Zuverlässigkeit im Bauwesen - Grundwissen für Ingenieure. vdf Hochschulverlag AG an der ETH Zürich und B.G. Teubner, Stuttgart, 1996

[106] Schott, G.: Werkstoffermüdung. VEB Deutscher Verlag für Grundstoffindustrie, Leipzig, 1985

[107] Schuëller, G. I.: Einführug in die Sicherheit und Zuverlässigkeit von Tragwerken. Verlag von Wilhelm Ernst \& Sohn, 1981

[108] Sedlacek, G., Hegger, J., Hanswille, G., Brozetti, J., Müller, C., Schneider, R., Kraus, O.: CEN/TC 250 - Report on $\gamma_{M^{-}}$Values and Quality Management for products compatible with EN 1990 -Basis of Structural Design. Institute of Steel Construction, RWTH, 7/2001 
[109] Smeets, W.: Ankerschienen für justierbare Befestigungen an Betonkonstruktionen. Bauingenieur (Forum) 72 (1997), S. A12

[110] Smeets, W.: Die richtige Verankerung von Betonfertigteil-Fassaden. Bauingenieur 75 (2000), S. A6-A10

[111] Smeets, W.: Entwicklung der Befestigungstechnik mit Ankerschienen. Bauwirtschaftliche Informationen, 1991

[112] Spaethe, Gerhard: Die Sicherheit tragender Baukonstruktionen. SpringerVerlag, Wien, 1992

[113] Seeger, T.: Grundlagen der Betriebsfestigkeitsnachweise, Stahlbau Handbuch 1 Teil B. Stahlbau-Verlagsgesellschaft mbH, Köln, 1996

[114] Struck, W.: Zur Berechnung von einseitigem, unteren Grenzwerten (Fraktilen) bei der statischen Auswertung von Meßergebnissen. Sonderausdruck aus "Materialprüfung" Bd. 9, Nr. 6, S.218-222, VDI-Verlag GmbH, Düsseldorf, 1967

[115] Suresh, S.: Fatigue of materials - Cambridge Solid State Science Series. Cambridge University Press, 1991

[116] Tauscher, H.: Dauerfestigkeit von Stahl und Gußeisen - Werkstoffverhalten, Gestalteinfluß und Berechnungsgrundlagen, Erläuterungen, Diagramme, Tabellen, Zahlenbeispiele. Archimedes Verlag, VEB Fachbuchverlag, Leipzig, 1971

[117] Tepfers, R.: Tensile fatigue strength of plain concrete. Journal of the ACI, Proceedings Vol. 76, No. 8, pp. 919-933, August 1979

[118] Tilly, G. P.: Fatigue of steel reinforcement bars in concrete: a review. Fatigue of Engineering Materials and Structures, Vol. 2, 1979

[119] Turan, T.: Interaktion von periodisch veränderlichen Zug- und Schubbeanspruchungen bei Ankerschienenbefestigungen. Diplomarbeit, Lehrstuhl für Stahl- und Verbundbau, Bereich Konstruktionsteilprüfung, Ruhr-Universität Bochum, 9/2004

[120] Wascheidt, H.: Dauerschwingfestigkeit von Betonstählen im einbetonierten Zustand. DAfStb, Heft 200, Beuth Verlag, Berlin, 1968 
[121] Weibull, W.: Fatigue testing and analysis of results. Advisory Group for Aeronautical Research and Development. North Atlantic Treaty Organisation. Pergamon Press, 1961

[122] Wohlfahrt, R.: Tragverhalten von Ankerschienen ohne Rückhängebewehrung. Dissertation, Universität Stuttgart, IWB Mitteilungen, 4/1996

[123] Wöhler, A.: Über die Festigkeitsversuche mit Eisen und Stahl. Verlag von Ernst \& Korn, Berlin, 1870

[124] Zilch, K., Staller, M., Brandes, Ch.: Anwendung statistischer Verfahren für die Auswertung von Versuchsdaten. Bauingenieur 74 (1999), S. 8-16 


\section{Anhang}

\section{Tabellarische Darstellung der Versuchsergebnisse}

Tabelle A1 Versagensfälle unter nichtruhenden Beanspruchungen

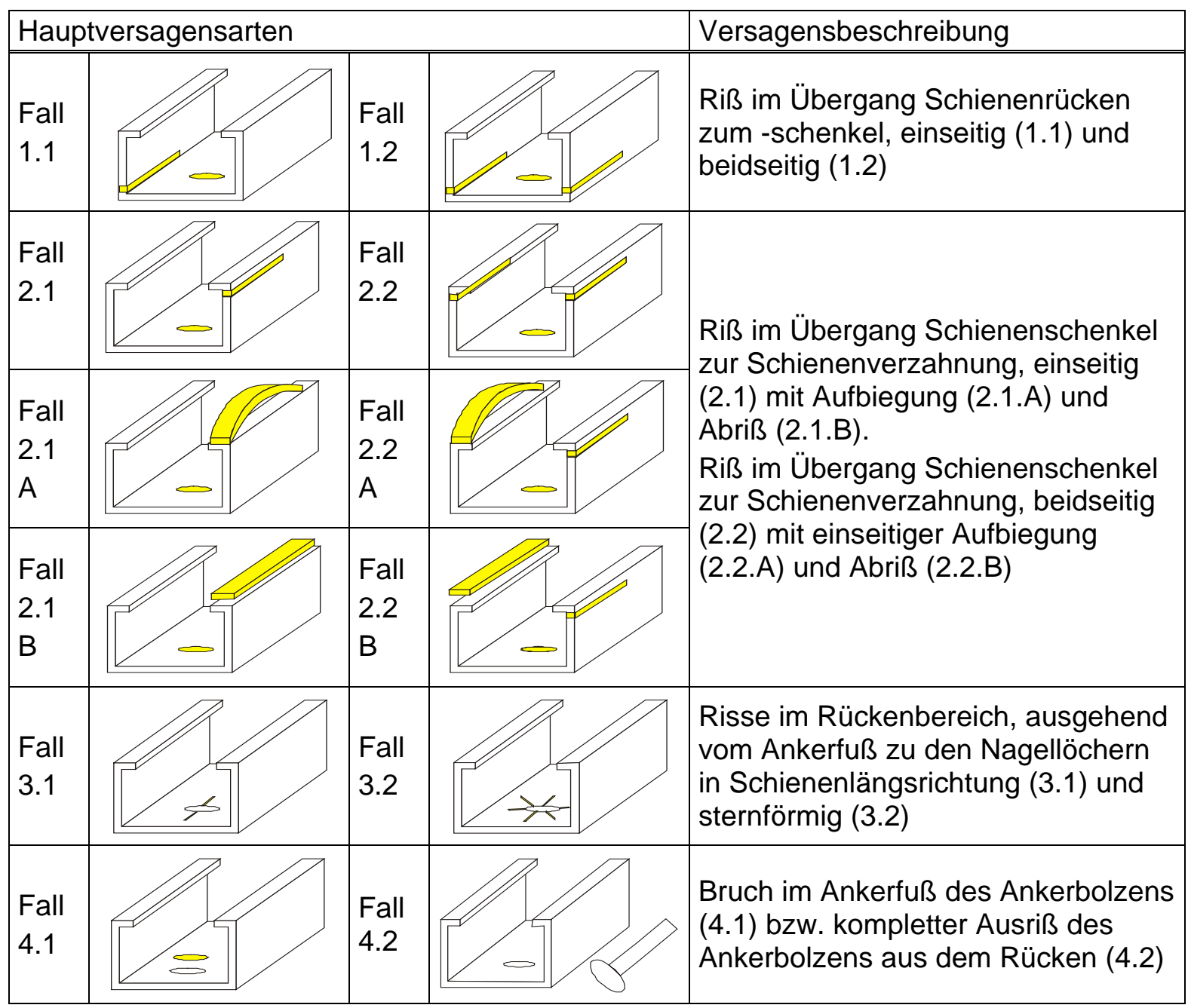




\section{Zentrische Zugschwellversuche}

Tabelle A2 Profil 38/23 St - Variante 25/50-OB Serie A - konst. Oberlast 17 kN

\begin{tabular}{|l|l|l|l|l|l|l|l|}
\hline $\begin{array}{l}\text { Ifd. } \\
\text { Nr. }\end{array}$ & $\begin{array}{l}\mathbf{F}_{\mathrm{O}} \\
{[\mathbf{k N}]}\end{array}$ & $\begin{array}{l}\mathbf{F}_{\mathbf{U}} \\
{[\mathbf{k N}]}\end{array}$ & $\mathbf{R}$ & $\begin{array}{l}\Delta \mathbf{F} \\
{[\mathbf{k N}]}\end{array}$ & $\mathbf{N}_{\mathbf{f}}$ & $\begin{array}{l}\Delta \mathbf{F}\left(\mathbf{2} \cdot \mathbf{1 0}^{6}\right) \\
{[\mathbf{k N}]}\end{array}$ & $\begin{array}{l}\text { Versagen } \\
\text { Fall }\end{array}$ \\
\hline 1 & 17,0 & 11,0 & 0,65 & 6,0 & 428.151 & 4,022 & $1.2+3.2$ \\
\hline 2 & 17,0 & 12,0 & 0,71 & 5,0 & 489.336 & 3,470 & $1.2+3.2$ \\
\hline 3 & 17,0 & 13,0 & 0,76 & 4,0 & 1.347 .277 & 3,610 & $1.2+3.1$ \\
\hline 4 & 17,0 & 11,0 & 0,65 & 6,0 & 394.606 & 3,937 & $1.2+3.2$ \\
\hline 5 & 17,0 & 13,0 & 0,76 & 4,0 & 1.586 .275 & 3,766 & $1.2+3.2$ \\
\hline 6 & 17,0 & 11,0 & 0,65 & 6,0 & 363.223 & 3,854 & $1.2+3.1$ \\
\hline 7 & 17,0 & 13,0 & 0,76 & 4,0 & 1.381 .527 & 3,634 & $1.2+3.2$ \\
\hline 8 & 17,0 & 12,0 & 0,71 & 5,0 & 480.869 & 3,454 & $1.2+3.1$ \\
\hline 9 & 17,0 & 12,0 & 0,71 & 5,0 & 1.082 .293 & 4,263 & $1.2+3.1$ \\
\hline 10 & 17,0 & 12,0 & 0,71 & 5,0 & 739.378 & 3,862 & $1.2+3.2$ \\
\hline 11 & 17,0 & 11,0 & 0,65 & 6,0 & 409.337 & 3,975 & $1.2+3.1$ \\
\hline 12 & 17,0 & 13,0 & 0,76 & 4,0 & 1.879 .129 & 3,936 & $1.2+3.1$ \\
\hline
\end{tabular}

Tabelle A3 Profil 38/23 St - Variante 25/50-OB Serie C - konst. Oberlast 17 kN

\begin{tabular}{|l|l|l|l|l|l|l|l|}
\hline $\begin{array}{l}\text { Ifd. } \\
\text { Nr. }\end{array}$ & $\begin{array}{l}\mathbf{F}_{\mathbf{O}} \\
{[\mathbf{k N}]}\end{array}$ & $\begin{array}{l}\mathbf{F}_{\mathbf{U}} \\
{[\mathbf{k N}]}\end{array}$ & $\mathbf{R}$ & $\begin{array}{l}\Delta \mathbf{F} \\
{[\mathbf{k N}]}\end{array}$ & $\mathbf{N}_{\mathbf{f}}$ & $\begin{array}{l}\Delta \mathbf{F}\left(\mathbf{2} \cdot \mathbf{1 0}^{\mathbf{6}}\right) \\
{[\mathbf{k N}]}\end{array}$ & $\begin{array}{l}\text { Versagen } \\
\text { Fall }\end{array}$ \\
\hline 1 & 17,0 & 10,0 & 0,59 & 7,0 & 353.271 & 4,481 & $1.1+3.1$ \\
\hline 2 & 17,0 & 10,0 & 0,59 & 7,0 & 327.435 & 4,394 & 1.1 \\
\hline 3 & 17,0 & 10,0 & 0,59 & 7,0 & 404.392 & 4,639 & 1.1 \\
\hline 4 & 17,0 & 10,0 & 0,59 & 7,0 & 346.508 & 4,459 & 1.1 \\
\hline 5 & 17,0 & 11,0 & 0,65 & 6,0 & 494.995 & 4,189 & 1.1 \\
\hline 6 & 17,0 & 11,0 & 0,65 & 6,0 & 539.406 & 4,283 & 1.1 \\
\hline 7 & 17,0 & 12,0 & 0,71 & 5,0 & 1.915 .167 & 4,945 & 1.1 \\
\hline 8 & 17,0 & 12,0 & 0,71 & 5,0 & 1.349 .558 & 4,519 & $1.1+3.1$ \\
\hline
\end{tabular}




\begin{tabular}{|l|l|l|l|l|l|l|l|}
\hline $\begin{array}{l}\text { Ifd. } \\
\text { Nr. }\end{array}$ & $\begin{array}{l}\mathbf{F}_{\mathrm{O}} \\
{[\mathbf{k N}]}\end{array}$ & $\begin{array}{l}\mathbf{F}_{\mathbf{U}} \\
{[\mathbf{k N}]}\end{array}$ & $\mathbf{R}$ & $\begin{array}{l}\Delta \mathbf{F} \\
{[\mathbf{k N}]}\end{array}$ & $\mathbf{N}_{\mathbf{f}}$ & $\begin{array}{l}\Delta \mathbf{F}\left(\mathbf{2} \cdot \mathbf{1 0}^{\mathbf{6}}\right) \\
{[\mathbf{k N}]}\end{array}$ & $\begin{array}{l}\text { Versagen } \\
\text { Fall }\end{array}$ \\
\hline 9 & 17,0 & 11,0 & 0,65 & 6,0 & 617.936 & 4,435 & 1.1 \\
\hline 10 & 17,0 & 12,0 & 0,71 & 5,0 & 1.062 .058 & 4,249 & $1.1+2.1$ \\
\hline 11 & 17,0 & 10,0 & 0,59 & 7,0 & 354.418 & 4,485 & 1.1 \\
\hline 12 & 17,0 & 11,0 & 0,65 & 6,0 & 467.602 & 4,128 & 1.1 \\
\hline 13 & 17,0 & 12,0 & 0,71 & 5,0 & 948.307 & 4,127 & 1.1 \\
\hline 14 & 17,0 & 12,5 & 0,74 & 4,5 & 1.468 .847 & 4,156 & 1.1 \\
\hline 15 & 17,0 & 13,0 & 0,76 & 4,0 & 2.535 .636 & 4,252 & 1.1 \\
\hline
\end{tabular}

Tabelle A4 Profil HZA 38/23 St - Variante 25/50-OB Serie C - konstante Unterlast 15 kN

\begin{tabular}{|c|c|c|c|c|c|c|c|}
\hline $\begin{array}{l}\text { Ifd. } \\
\mathrm{Nr} .\end{array}$ & $\begin{array}{l}F_{0} \\
{[k N]}\end{array}$ & $\begin{array}{l}F_{U} \\
{[k N]}\end{array}$ & $\mathbf{R}$ & $\begin{array}{l}\Delta \mathrm{F} \\
{[\mathrm{kN}]}\end{array}$ & $\mathbf{N}_{\mathrm{f}}$ & $\begin{array}{l}\Delta \mathrm{F}\left(2 \cdot 10^{6}\right) \\
{[\mathrm{kN}]}\end{array}$ & $\begin{array}{l}\text { Versagen } \\
\text { Fall }\end{array}$ \\
\hline 1 & 20,0 & 15,0 & 0,75 & 5,0 & 1.070 .262 & 4,197 & $1.1+3.1$ \\
\hline 2 & 21,0 & 15,0 & 0,71 & 6,0 & 460.051 & 3,976 & $1.1+4.2$ \\
\hline 3 & 22,0 & 15,0 & 0,68 & 7,0 & 383.428 & 4,409 & $1.1+3.1$ \\
\hline 4 & 22,0 & 15,0 & 0,68 & 7,0 & 272.201 & 4,005 & 1.1 \\
\hline 5 & 19,0 & 15,0 & 0,79 & 4,0 & 2.271 .844 & 4,145 & 1.1 \\
\hline 6 & 21,0 & 15,0 & 0,71 & 6,0 & 534.061 & 4,146 & $1.1+4.2$ \\
\hline 7 & 20,0 & 15,0 & 0,75 & 5,0 & 1.304 .323 & 4,436 & $1.1+3.2$ \\
\hline 8 & 21,0 & 15,0 & 0,71 & 6,0 & 665.320 & 4,409 & $1.1+4.2$ \\
\hline 9 & 22,0 & 15,0 & 0,68 & 7,0 & 303.946 & 4,131 & $1.1+4.2$ \\
\hline 10 & 19,0 & 15,0 & 0,79 & 4,0 & 1.817 .030 & 3,894 & 1.1 \\
\hline \multicolumn{8}{|c|}{ Parameter der Regressionsgeraden $y=a \cdot x+b$ aus $\log N_{f}$ und $\log \Delta F$} \\
\hline \multicolumn{8}{|c|}{ Faktor $\mathrm{a}=-0,2799$, Faktor $\mathrm{b}=2,3840$, Steigung $\mathrm{m}=3,57$} \\
\hline \multicolumn{8}{|c|}{ Statistische Parameter der Ermüdungsfestigkeit $\Delta F$ bei $N_{f}=2 \cdot 10^{6}$} \\
\hline \multicolumn{8}{|c|}{$\mathrm{m}_{\Delta \mathrm{F}}=4,17 \mathrm{kN}, \sigma_{\Delta \mathrm{F}}=0,19 \mathrm{kN}, \mathrm{V}_{\Delta \mathrm{F}}=4,58 \%$} \\
\hline \multicolumn{8}{|c|}{ Charakteristische Ermüdungsfestigkeiten $\Delta F_{R K}$ bei $N_{f}=2 \cdot 10^{6}$} \\
\hline \multicolumn{8}{|c|}{$\Delta \mathrm{F}_{\mathrm{Rk}, \mathrm{SVA}}=3,78 \mathrm{kN}, \Delta \mathrm{F}_{\mathrm{Rk}, \mathrm{EC} 3}=3,63 \mathrm{kN}, \Delta \mathrm{F}_{\mathrm{Rk}, \mathrm{ETAG}}=3,67 \mathrm{kN}$} \\
\hline
\end{tabular}


Tabelle A5 Profil HZA 38/23 St - Variante 25/50-OB Serie C - konst. Unterlast 10 kN

\begin{tabular}{|l|l|l|l|l|l|l|l|}
\hline $\begin{array}{l}\text { Ifd. } \\
\text { Nr. }\end{array}$ & $\begin{array}{l}\mathbf{F}_{\mathbf{O}} \\
{[\mathbf{k N}]}\end{array}$ & $\begin{array}{l}\mathbf{F}_{\mathbf{U}} \\
{[\mathbf{k N}]}\end{array}$ & $\mathbf{R}$ & $\begin{array}{l}\Delta \mathbf{F} \\
{[\mathbf{k N}]}\end{array}$ & $\mathbf{N}_{\mathbf{f}}$ & $\begin{array}{l}\Delta \mathbf{F}\left(\mathbf{2} \cdot \mathbf{1 0}^{\mathbf{6}}\right) \\
{[\mathbf{k N}]}\end{array}$ & $\begin{array}{l}\text { Versagen } \\
\text { Fall }\end{array}$ \\
\hline \hline 1 & 16,0 & 10,0 & 0,63 & 6,0 & 689.492 & 4,544 & $1.1+2.1$ \\
\hline 2 & 16,0 & 10,0 & 0,63 & 6,0 & 698.653 & 4,560 & 1.1 \\
\hline 3 & 15,0 & 10,0 & 0,67 & 5,0 & 1.072 .619 & 4,250 & $1.1+2.1$ \\
\hline 4 & 15,0 & 10,0 & 0,67 & 5,0 & 809.216 & 3,948 & 1.1 \\
\hline 5 & 14,5 & 10,0 & 0,69 & 4,5 & 1.605 .790 & 4,249 & $1.1+2.1$ \\
\hline 6 & 14,5 & 10,0 & 0,69 & 4,5 & 2.007 .182 & 4,504 & 1.1 \\
\hline 7 & 14,5 & 10,0 & 0,69 & 4,5 & 2.120 .861 & 4,569 & $1.1+2.1$ \\
\hline 8 & 16,0 & 10,0 & 0,63 & 6,0 & 478.739 & 4,131 & $1.1+2.1$ \\
\hline 9 & 17,0 & 10,0 & 0,59 & 7,0 & 353.271 & 4,452 & 1.1 \\
\hline 10 & 17,0 & 10,0 & 0,59 & 7,0 & 327.435 & 4,365 & 1.1 \\
\hline 11 & 17,0 & 10,0 & 0,59 & 7,0 & 404.392 & 4,612 & 1.1 \\
\hline 12 & 17,0 & 10,0 & 0,59 & 7,0 & 346.508 & 4,430 & 1.1 \\
\hline \hline
\end{tabular}

Parameter der Regressionsgeraden $y=a \cdot x+b$ aus $\log N_{f}$ und $\log \Delta F$

Faktor $a=-0,2610$, Faktor $b=2,2861$, Steigung $m=3,83$

Statistische Parameter der Ermüdungsfestigkeit $\Delta \mathrm{F}$ bei $\mathrm{N}_{\mathrm{f}}=\mathbf{2} \cdot 10^{\mathbf{6}}$

$\mathrm{m}_{\Delta \mathrm{F}}=4,38 \mathrm{kN}, \sigma_{\Delta \mathrm{F}}=0,20 \mathrm{kN}, \mathrm{V}_{\Delta \mathrm{F}}=4,64 \%$

Charakteristische Ermüdungsfestigkeiten $\Delta F_{\mathrm{RK}}$ bei $\mathrm{N}_{\mathrm{f}}=\mathbf{2} \cdot \mathbf{1 0}^{\mathbf{6}}$

$\Delta \mathrm{F}_{\mathrm{Rk}, \mathrm{SVA}}=3,98 \mathrm{kN}, \Delta \mathrm{F}_{\mathrm{Rk}, \mathrm{EC} 3}=3,84 \mathrm{kN}, \Delta \mathrm{F}_{\mathrm{Rk}, \mathrm{ETAG}}=3,88 \mathrm{kN}$

Tabelle A6 Profil HZA 38/23 St - Variante 25/50-OB Serie C - konst. Unterlast 0,5 kN

\begin{tabular}{|l|l|l|l|l|l|l|l|}
\hline $\begin{array}{l}\text { Ifd. } \\
\mathbf{N r} .\end{array}$ & $\begin{array}{l}\mathbf{F}_{\mathbf{O}} \\
{[\mathbf{k N}]}\end{array}$ & $\begin{array}{l}\mathbf{F}_{\mathbf{U}} \\
{[\mathbf{k N}]}\end{array}$ & $\mathbf{R}$ & $\begin{array}{l}\Delta \mathbf{F} \\
{[\mathbf{k N}]}\end{array}$ & $\mathbf{N}_{\mathbf{f}}$ & $\begin{array}{l}\Delta \mathbf{F}\left(\mathbf{2} \cdot \mathbf{1 0} \mathbf{}^{\mathbf{}}\right) \\
{[\mathbf{k N}]}\end{array}$ & $\begin{array}{l}\text { Versagen } \\
\text { Fall }\end{array}$ \\
\hline 1 & 10,5 & 0,5 & 0,05 & 10,0 & 276.003 & 7,575 & 1.1 \\
\hline 2 & 9,0 & 0,5 & 0,06 & 8,5 & 535.643 & 7,066 & $1.1+2.1$ \\
\hline 3 & 10,5 & 0,5 & 0,05 & 10,0 & 438.946 & 8,085 & 1.2 \\
\hline 4 & 9,0 & 0,5 & 0,06 & 8,5 & 713.545 & 7,356 & $1.1+2.1$ \\
\hline 5 & 10,5 & 0,5 & 0,05 & 10,0 & 347.424 & 7,824 & 1.2 \\
\hline 6 & 8,0 & 0,5 & 0,06 & 7,5 & 653.361 & 6,411 & $1.1+2.1$ \\
\hline 7 & 8,5 & 0,5 & 0,06 & 8,0 & 1.085 .048 & 7,343 & $1.1+2.1$ \\
\hline 8 & 8,5 & 0,5 & 0,06 & 8,0 & 686.625 & 6,886 & $1.1+2.1$ \\
\hline 9 & 8,0 & 0,5 & 0,06 & 7,5 & 918.975 & 6,725 & $1.1+2.1$ \\
\hline 10 & 8,0 & 0,5 & 0,06 & 7,5 & 1.893 .881 & 7,443 & $1.1+2.1$ \\
\hline 11 & 10,5 & 0,5 & 0,05 & 10,0 & 265.687 & 7,535 & 1.2 \\
\hline 12 & 9,5 & 0,5 & 0,05 & 9,0 & 1.234 .666 & 8,411 & 1.2 \\
\hline
\end{tabular}




\begin{tabular}{|c|c|c|c|c|c|c|c|}
\hline $\begin{array}{l}\text { Ifd. } \\
\text { Nr. }\end{array}$ & $\begin{array}{l}\text { Fo } \\
{[\mathrm{kN}]}\end{array}$ & $\begin{array}{l}F_{U} \\
{[k N]}\end{array}$ & $\mathbf{R}$ & $\begin{array}{l}\Delta \mathrm{F} \\
{[\mathrm{kN}]} \\
\end{array}$ & $N_{f}$ & $\begin{array}{l}\mathrm{F}\left(2 \cdot 10^{6}\right) \\
{[\mathrm{kN}]}\end{array}$ & $\begin{array}{l}\text { Versagen } \\
\text { Fall } \\
\end{array}$ \\
\hline 13 & 12,5 & 0,5 & 0,04 & 12,0 & 87.559 & 7,739 & 1.1 \\
\hline 14 & 12,5 & 0,5 & 0,04 & 12,0 & 81.953 & 7,667 & 1.1 \\
\hline 15 & 9,5 & 0,5 & 0,05 & 9,0 & 273.389 & 6,809 & 1.2 \\
\hline 16 & 12,5 & 0,5 & 0,04 & 12,0 & 81.555 & 7,662 & 1.1 \\
\hline 17 & 9,5 & 0,5 & 0,05 & 9,0 & 276.526 & 6,820 & 1.2 \\
\hline 18 & 8,0 & 0,5 & 0,06 & 7,5 & 2.514 .529 & 1) & kein Bruch \\
\hline 19 & 8,5 & 0,5 & 0,06 & 8,0 & 2.752 .102 & 1) & kein Bruch \\
\hline 20 & 9,0 & 0,5 & 0,06 & 8,5 & 1.901 .155 & 1) & kein Bruch \\
\hline 21 & 10,5 & 0,5 & 0,05 & 10,0 & 517.590 & 1) & kein Bruch \\
\hline 22 & 7,5 & 0,5 & 0,07 & 7,0 & 6.586 .948 & 1) & kein Bruch \\
\hline 23 & 8,0 & 0,5 & 0,06 & 7,5 & 2.895 .441 & 1) & kein Bruch \\
\hline 24 & 8,0 & 0,5 & 0,06 & 7,5 & 2.399 .220 & 1) & kein Bruch \\
\hline 25 & 8,5 & 0,5 & 0,06 & 8,0 & 5.319 .243 & 1) & kein Bruch \\
\hline 26 & 12,5 & 0,5 & 0,04 & 12,0 & 734.997 & 1) & 1.2 \\
\hline 27 & 8,0 & 0,5 & 0,06 & 7,5 & 4.550 .931 & 1) & kein Bruch \\
\hline 28 & 8,5 & 0,5 & 0,06 & 8,0 & 2.277 .933 & 8,147 & $1.1+2.1$ \\
\hline 29 & 8,5 & 0,5 & 0,06 & 8,0 & 1.014 .672 & 7,274 & $1.1+2.1$ \\
\hline 30 & 8,5 & 0,5 & 0,06 & 8,0 & 5.339 .470 & 1) & kein Bruch \\
\hline 31 & 9,0 & 0,5 & 0,06 & 8,5 & 492.892 & 6,984 & 1.2 \\
\hline 32 & 9,0 & 0,5 & 0,06 & 8,5 & 1.061 .251 & 7,777 & $1.1+2.1$ \\
\hline \multicolumn{8}{|c|}{${ }^{1)}$ nicht in der Auswertung berücksichtigt } \\
\hline \multicolumn{8}{|c|}{ Parameter der Regressionsgeraden $y=a \cdot x+b$ aus $\log N_{f}$ und $\log \Delta F$} \\
\hline \multicolumn{8}{|c|}{ Faktor $\mathrm{a}=-0,1402$, Faktor $\mathrm{b}=1,7521$, Steigung $\mathrm{m}=7,13$} \\
\hline \multicolumn{8}{|c|}{ Statistische Parameter der Ermüdungsfestigkeit $\Delta \mathrm{F}$ bei $N_{f}=2 \cdot 10^{6}$} \\
\hline \multicolumn{8}{|c|}{$\mathrm{m}_{\Delta \mathrm{F}}=7,41 \mathrm{kN}, \sigma_{\Delta \mathrm{F}}=0,52 \mathrm{kN}, \mathrm{V}_{\Delta \mathrm{F}}=6,99 \%$} \\
\hline \multicolumn{8}{|c|}{ Charakteristische Ermüdungsfestigkeiten $\Delta F_{R K}$ bei $N_{f}=2 \cdot 10^{6}$} \\
\hline \multicolumn{8}{|c|}{$\Delta \mathrm{F}_{\mathrm{Rk}, \mathrm{SVA}}=6,50 \mathrm{kN}, \Delta \mathrm{F}_{\mathrm{Rk}, \mathrm{EC} 3}=6,19 \mathrm{kN}, \Delta \mathrm{F}_{\mathrm{Rk}, \mathrm{ETAG}}=6,26 \mathrm{kN}$} \\
\hline
\end{tabular}

Tabelle A7 Profil JXA-W 38/23 St - Variante 25/50-OB Serie D - konst. Oberlast 17 kN

\begin{tabular}{|l|l|l|l|l|l|l|l|}
\hline $\begin{array}{l}\text { Ifd. } \\
\text { Nr. }\end{array}$ & $\begin{array}{l}\mathbf{F}_{\mathrm{O}} \\
{[\mathbf{k N}]}\end{array}$ & $\begin{array}{l}\mathbf{F}_{\mathbf{U}} \\
{[\mathbf{k N}]}\end{array}$ & $\mathbf{R}$ & $\begin{array}{l}\Delta \mathbf{F} \\
{[\mathbf{k N}]}\end{array}$ & $\mathbf{N}_{\mathbf{f}}$ & $\begin{array}{l}\Delta \mathbf{F}\left(\mathbf{2} \cdot \mathbf{1 0}^{\mathbf{6}}\right) \\
{[\mathbf{k N}]}\end{array}$ & $\begin{array}{l}\text { Versagen } \\
\text { Fall }\end{array}$ \\
\hline 1 & 17,0 & 11,0 & 0,65 & 6,0 & 1.989 .773 & - & 1.2 \\
\hline 2 & 17,0 & 8,0 & 0,47 & 9,0 & 857.177 & 4,616 & 1.2 \\
\hline 3 & 17,0 & 9,0 & 0,53 & 8,0 & 412.632 & 4,908 & 1.2 \\
\hline 4 & 17,0 & 10,0 & 0,59 & 7,0 & 460.808 & - & 1.2 \\
\hline
\end{tabular}




\begin{tabular}{|c|c|c|c|c|c|c|c|}
\hline $\begin{array}{l}\text { Ifd. } \\
\text { Nr. }\end{array}$ & $\begin{array}{l}\text { Fo } \\
\text { [kN] }\end{array}$ & $\begin{array}{l}F_{U} \\
{[k N]}\end{array}$ & $\mathbf{R}$ & $\begin{array}{l}\Delta \mathrm{F} \\
{[\mathrm{kN}]}\end{array}$ & $\mathbf{N}_{\mathrm{f}}$ & $\begin{array}{l}\Delta \mathrm{F}\left(2 \cdot 10^{6}\right) \\
{[\mathrm{kN}]}\end{array}$ & $\begin{array}{l}\text { Versagen } \\
\text { Fall }\end{array}$ \\
\hline 5 & 17,0 & 11,0 & 0,65 & 6,0 & 1.460 .231 & 5,443 & 1.1 \\
\hline 6 & 17,0 & 11,0 & 0,65 & 6,0 & 1.101 .214 & 4,988 & 1.1 \\
\hline 7 & 17,0 & 11,0 & 0,65 & 6,0 & 1.780 .962 & 1) & 1.2 \\
\hline 8 & 17,0 & 8,0 & 0,47 & 9,0 & 347.921 & 5,238 & 1.2 \\
\hline 9 & 17,0 & 12,0 & 0,71 & 5,0 & 1.939 .685 & 4,953 & 1.1 \\
\hline 10 & 17,0 & 8,0 & 0,47 & 9,0 & 220.584 & 4,549 & 1.2 \\
\hline 11 & 17,0 & 8,0 & 0,47 & 9,0 & 205.171 & 4,448 & 1.1 \\
\hline 12 & 17,0 & 12,0 & 0,71 & 5,0 & 1.999 .390 & 5,000 & 1.1 \\
\hline 13 & 17,0 & 11,0 & 0,65 & 6,0 & 892.206 & 4,673 & 1.1 \\
\hline 14 & 17,0 & 8,0 & 0,47 & 9,0 & 316.934 & 5,089 & 1.2 \\
\hline 15 & 17,0 & 8,0 & 0,47 & 9,0 & 299.159 & 4,998 & 1.2 \\
\hline 16 & 17,0 & 12,0 & 0,71 & 5,0 & 1.694 .191 & 4,750 & 1.1 \\
\hline 17 & 17,0 & 8,0 & 0,47 & 9,0 & 293.984 & 4,972 & 1.2 \\
\hline 18 & 17,0 & 12,0 & 0,71 & 5,0 & 1.486 .120 & 4,561 & 1.1 \\
\hline 19 & 17,0 & 12,0 & 0,71 & 5,0 & 1.444 .570 & 4,521 & 1.2 \\
\hline 20 & 17,0 & 11,0 & 0,65 & 6,0 & 810.079 & 4,536 & 1.2 \\
\hline 21 & 17,0 & 12,0 & 0,71 & 5,0 & 1.802 .981 & 4,842 & 1.2 \\
\hline 22 & 17,0 & 11,0 & 0,65 & 6,0 & 834.399 & 4,578 & 1.2 \\
\hline 23 & 17,0 & 11,0 & 0,65 & 6,0 & 1.251 .015 & 4,324 & 1.1 \\
\hline 24 & 17,0 & 11,0 & 0,65 & 6,0 & 1.108 .042 & 4,998 & 1.1 \\
\hline \multicolumn{8}{|c|}{ 1) nicht in der Auswertung berücksichtigt } \\
\hline \multicolumn{8}{|c|}{ Parameter der Regressionsgeraden $y=a \cdot x+b$ aus $\log N_{f}$ und $\log \Delta F$} \\
\hline \multicolumn{8}{|c|}{ Faktor $a=-0,3095$, Faktor $b=2,6317$, Steigung $m=3,23$} \\
\hline \multicolumn{8}{|c|}{ Statistische Parameter der Ermüdungsfestigkeit $\Delta \mathrm{F}$ bei $N_{f}=2 \cdot 10^{6}$} \\
\hline \multicolumn{8}{|c|}{$\mathrm{m}_{\Delta \mathrm{F}}=4,81 \mathrm{kN}, \sigma_{\Delta \mathrm{F}}=0,28 \mathrm{kN}, \mathrm{V}_{\Delta \mathrm{F}}=5,93 \%$} \\
\hline \multicolumn{8}{|c|}{ Charakteristische Ermüdungsfestigkeiten $\Delta F_{R K}$ bei $N_{f}=2 \cdot 10^{6}$} \\
\hline \multicolumn{8}{|c|}{$\Delta \mathrm{F}_{\mathrm{Rk}, \mathrm{SVA}}=4,31 \mathrm{kN}, \Delta \mathrm{F}_{\mathrm{Rk}, \mathrm{EC} 3}=4,14 \mathrm{kN}, \Delta \mathrm{F}_{\mathrm{Rk}, \mathrm{ETAG}}=4,18 \mathrm{kN}$} \\
\hline
\end{tabular}

Tabelle A8 Profil JXA-W 38/23 St - Variante 25/50-OB Serie D - konst. Unterlast 0,5 kN

\begin{tabular}{|l|l|l|l|l|l|l|l|}
\hline $\begin{array}{l}\text { Ifd. } \\
\text { Nr. }\end{array}$ & $\begin{array}{l}\mathbf{F}_{\mathrm{O}} \\
{[\mathbf{k N}]}\end{array}$ & $\begin{array}{l}\mathbf{F}_{\mathbf{U}} \\
{[\mathbf{k N}]}\end{array}$ & $\mathbf{R}$ & $\begin{array}{l}\Delta \mathbf{F} \\
{[\mathbf{k N}]}\end{array}$ & $\mathbf{N}_{\mathbf{f}}$ & $\begin{array}{l}\mathbf{\Delta} \mathbf{( 2 \cdot 1 0 ^ { 6 } )} \\
{[\mathbf{k N}]}\end{array}$ & $\begin{array}{l}\text { Versagen } \\
\text { Fall }\end{array}$ \\
\hline 1 & 7,0 & 0,5 & 0,65 & 6,5 & 4.089 .041 & $1)$ & kein Bruch \\
\hline 2 & 8,0 & 0,5 & 0,65 & 7,5 & 7.160 .296 & $1)$ & kein Bruch \\
\hline 3 & 10,0 & 0,5 & 0,47 & 9,5 & 745.349 & 8,145 & 1.2 \\
\hline 4 & 9,0 & 0,5 & 0,53 & 8,5 & 1.389 .159 & 8,031 & $1.1+2.1$ \\
\hline 5 & 8,5 & 0,5 & 0,59 & 8,0 & 1.358 .759 & 7,532 & 1.2 \\
\hline
\end{tabular}




\begin{tabular}{|l|l|l|l|l|l|l|l|}
\hline $\begin{array}{l}\text { Ifd. } \\
\text { Nr. }\end{array}$ & $\begin{array}{l}\mathbf{F}_{\mathrm{O}} \\
{[\mathbf{k N}]}\end{array}$ & $\begin{array}{l}\mathbf{F}_{\mathrm{U}} \\
{[\mathbf{k N}]}\end{array}$ & $\mathbf{R}$ & $\begin{array}{l}\Delta \mathbf{F} \\
{[\mathbf{k N}]}\end{array}$ & $\mathbf{N}_{\mathbf{f}}$ & $\begin{array}{l}\Delta \mathbf{F}\left(\mathbf{2} \cdot \mathbf{1 0}^{6}\right) \\
{[\mathbf{k N}]}\end{array}$ & $\begin{array}{l}\text { Versagen } \\
\text { Fall }\end{array}$ \\
\hline 6 & 8,5 & 0,5 & 0,65 & 8,0 & 866.818 & 7,022 & 1.2 \\
\hline 7 & 8,5 & 0,5 & 0,65 & 8,0 & 1.202 .875 & 7,390 & 1.2 \\
\hline 8 & 8,0 & 0,5 & 0,65 & 7,5 & 4.374 .799 & 8,473 & 1.2 \\
\hline 9 & 8,5 & 0,5 & 0,47 & 8,0 & 2.316 .432 & 8,185 & 1.2 \\
\hline 10 & 9,0 & 0,5 & 0,71 & 8,5 & 2.858 .504 & 8,987 & 1.2 \\
\hline 11 & 9,5 & 0,5 & 0,47 & 9,0 & 418.269 & 7,052 & 1.2 \\
\hline 12 & 9,5 & 0,5 & 0,47 & 9,0 & 216.581 & 6,364 & 4.1 \\
\hline 13 & 9,5 & 0,5 & 0,71 & 9,0 & 437.752 & 7,102 & 1.2 \\
\hline 14 & 9,0 & 0,5 & 0,65 & 8,5 & 2.932 .439 & 9,022 & 4.1 \\
\hline 15 & 9,5 & 0,5 & 0,47 & 9,0 & 556.203 & 7,372 & 1.2 \\
\hline 16 & 9,0 & 0,5 & 0,47 & 8,5 & 341.845 & 6,454 & 4.1 \\
\hline 17 & 10,5 & 0,5 & 0,71 & 10,0 & 601.252 & 8,291 & 1.2 \\
\hline 18 & 16,5 & 0,5 & 0,47 & 16,0 & 36.931 & 8,588 & 1.2 \\
\hline 19 & 15,5 & 0,5 & 0,71 & 15,0 & 68.819 & 8,871 & 1.2 \\
\hline 20 & 17,0 & 0,5 & 0,71 & 16,5 & 39.555 & 8,951 & 1.2 \\
\hline 21 & 9,5 & 0,5 & 0,65 & 9,0 & 743.793 & 7,714 & 1.2 \\
\hline 22 & 12,5 & 0,5 & 0,71 & 12,0 & 187.072 & 8,294 & 1.2 \\
\hline 23 & 14,5 & 0,5 & 0,65 & 14,0 & 64.826 & 8,203 & 1.2 \\
\hline 24 & 8,5 & 0,5 & 0,71 & 8,0 & 2.512 .010 & 8,289 & 1.2 \\
\hline 19
\end{tabular}

Tabelle A9 Profil HZA 38/23 St - Variante 50/50-OB Serie A - konst. Oberlast 17 kN

\begin{tabular}{|l|l|l|l|l|l|l|l|}
\hline $\begin{array}{l}\text { Ifd. } \\
\text { Nr. }\end{array}$ & $\begin{array}{l}\mathbf{F}_{\mathbf{O}} \\
{[\mathbf{k N}]}\end{array}$ & $\begin{array}{l}\mathbf{F}_{\mathbf{U}} \\
{[\mathbf{k N}]}\end{array}$ & $\mathbf{R}$ & $\begin{array}{l}\Delta \mathbf{F} \\
{[\mathbf{k N}]}\end{array}$ & $\mathbf{N}_{\mathbf{f}}$ & $\begin{array}{l}\mathbf{\Delta} \mathbf{F}\left(\mathbf{2} \cdot \mathbf{1 0}^{\mathbf{6}}\right) \\
{[\mathbf{k N}]}\end{array}$ & $\begin{array}{l}\text { Versagen } \\
\text { Fall }\end{array}$ \\
\hline 1 & 17,0 & 11,0 & 0,65 & 6,0 & 376.070 & 3,629 & $1.2+3.2$ \\
\hline 2 & 17,0 & 12,0 & 0,71 & 5,0 & 1.060 .526 & 4,131 & $1.2+4.2$ \\
\hline 3 & 17,0 & 13,0 & 0,76 & 4,0 & 2.000 .592 & 4,000 & $1.2+3.1$ \\
\hline 4 & 17,0 & 13,0 & 0,76 & 4,0 & 1.802 .502 & 3,877 & $1.2+4.2$ \\
\hline 5 & 17,0 & 12,0 & 0,71 & 5,0 & 1.010 .118 & 4,071 & $1.2+3.2$ \\
\hline 6 & 17,0 & 13,0 & 0,76 & 4,0 & 1.638 .999 & 3,767 & $1.2+3.2$ \\
\hline
\end{tabular}




\begin{tabular}{|c|c|c|c|c|c|c|c|}
\hline $\begin{array}{l}\text { Ifd. } \\
\text { Nr. }\end{array}$ & $\begin{array}{l}\text { Fo } \\
\text { [kN] }\end{array}$ & $\begin{array}{l}F_{U} \\
{[k N]}\end{array}$ & $\mathbf{R}$ & $\begin{array}{l}\Delta F \\
{[k N]}\end{array}$ & $N_{f}$ & $\begin{array}{l}\Delta \mathrm{F}\left(2 \cdot 10^{6}\right) \\
{[\mathrm{kN}]}\end{array}$ & \begin{tabular}{|l} 
Versagen \\
Fall \\
\end{tabular} \\
\hline 7 & 17,0 & 13,0 & 0,76 & 4,0 & 1.306 .361 & 3,519 & $1.2+3.2$ \\
\hline 8 & 17,0 & 12,0 & 0,71 & 5,0 & 897.360 & 3,929 & $1.2+3.1$ \\
\hline 9 & 17,0 & 11,0 & 0,65 & 6,0 & 494.615 & 3,941 & $1.2+4.2$ \\
\hline 10 & 17,0 & 11,0 & 0,65 & 6,0 & 495.191 & 3,942 & $1.2+4.2$ \\
\hline 11 & 17,0 & 11,0 & 0,65 & 6,0 & 554.882 & 4,080 & $1.2+3.1$ \\
\hline 12 & 17,0 & 12,0 & 0,71 & 5,0 & 896.510 & 3,928 & $1.2+3.1$ \\
\hline \multicolumn{8}{|c|}{ Parameter der Regressionsgeraden $y=a \cdot x+b$ aus $\log N_{f}$ und $\log \Delta F$} \\
\hline \multicolumn{8}{|c|}{ Faktor $\mathrm{a}=-0,3009$, Faktor $\mathrm{b}=2,4866$, Steigung $\mathrm{m}=3,32$} \\
\hline \multicolumn{8}{|c|}{ Statistische Parameter der Ermüdungsfestigkeit $\Delta \mathrm{F}$ bei $N_{\mathrm{f}}=2 \cdot 10^{6}$} \\
\hline \multicolumn{8}{|c|}{$\mathrm{m}_{\Delta \mathrm{F}}=3,90 \mathrm{kN}, \sigma_{\Delta \mathrm{F}}=0,18 \mathrm{kN}, \mathrm{V}_{\Delta \mathrm{F}}=4,68 \%$} \\
\hline \multicolumn{8}{|c|}{ Charakteristische Ermüdungsfestigkeiten $\Delta F_{R K}$ bei $N_{f}=2 \cdot 10^{6}$} \\
\hline \multicolumn{8}{|c|}{$\Delta \mathrm{F}_{\mathrm{Rk}, \mathrm{SVA}}=3,54 \mathrm{kN}, \Delta \mathrm{F}_{\mathrm{Rk}, \mathrm{EC} 3}=3,41 \mathrm{kN}, \Delta \mathrm{F}_{\mathrm{Rk}, \mathrm{ETAG}}=3,44 \mathrm{kN}$} \\
\hline
\end{tabular}

Tabelle A10 Profil HZA 38/23 St - Variante 50/50-OB Serie C - konst. Oberlast 17 kN

\begin{tabular}{|c|c|c|c|c|c|c|c|}
\hline $\begin{array}{l}\text { Ifd. } \\
\text { Nr. }\end{array}$ & $\begin{array}{l}F_{0} \\
{[k N]}\end{array}$ & $\begin{array}{l}F_{U} \\
{[k N]}\end{array}$ & $\mathbf{R}$ & $\begin{array}{l}\Delta \mathrm{F} \\
{[\mathrm{kN}]}\end{array}$ & $\mathbf{N}_{\mathrm{f}}$ & $\begin{array}{l}\Delta \mathrm{F}\left(2 \cdot 10^{6}\right) \\
{[\mathrm{kN}]}\end{array}$ & $\begin{array}{l}\text { Versagen } \\
\text { Fall }\end{array}$ \\
\hline 1 & 17,0 & 11,0 & 0,65 & 6,0 & 975.178 & 4,791 & $1.2+3.2$ \\
\hline 2 & 17,0 & 12,0 & 0,71 & 5,0 & 1.850 .478 & 4,880 & $1.2+3.2$ \\
\hline 3 & 17,0 & 11,5 & 0,68 & 5,5 & 1.266 .907 & 4,767 & $1.2+3.2$ \\
\hline 4 & 17,0 & 11,0 & 0,65 & 6,0 & 862.635 & 4,610 & $1.2+3.2$ \\
\hline 5 & 17,0 & 11,0 & 0,65 & 6,0 & 989.042 & 4,812 & $1.2+3.2$ \\
\hline 6 & 17,0 & 11,0 & 0,65 & 6,0 & 1.122 .347 & 5,006 & $1.2+3.2$ \\
\hline 7 & 17,0 & 12,0 & 0,71 & 5,0 & 1.450 .366 & 4,521 & $1.2+3.2$ \\
\hline 8 & 17,0 & 11,0 & 0,65 & 6,0 & 924.148 & 4,711 & $1.2+3.2$ \\
\hline 9 & 17,0 & 8,0 & 0,47 & 9,0 & 304.869 & 4,992 & $1.2+3.2$ \\
\hline 10 & 17,0 & 11,0 & 0,65 & 6,0 & 1.229 .577 & 5,152 & $1.2+3.2$ \\
\hline 11 & 17,0 & 8,0 & 0,47 & 9,0 & 300.085 & 4,967 & $1.2+3.2$ \\
\hline 12 & 17,0 & 8,0 & 0,47 & 9,0 & 337.810 & 5,155 & $1.2+3.2$ \\
\hline 13 & 17,0 & 11,0 & 0,65 & 6,0 & 1.004 .025 & 4,835 & $1.2+3.2$ \\
\hline 14 & 17,0 & 1,0 & 0,06 & 16,0 & 50.000 & 5,036 & $1.2+3.2$ \\
\hline 15 & 17,0 & 1,0 & 0,06 & 16,0 & 54.085 & 5,162 & 1.2 \\
\hline 16 & 17,0 & 1,0 & 0,06 & 16,0 & 50.685 & 5,058 & 1.2 \\
\hline 17 & 17,0 & 11,0 & 0,65 & 6,0 & 963.969 & 4,773 & $1.2+3.2$ \\
\hline 18 & 17,0 & 1,0 & 0,06 & 16,0 & 52.143 & 5,103 & 1.2 \\
\hline 19 & 17,0 & 1,0 & 0,06 & 16,0 & 50.146 & 5,041 & 1.2 \\
\hline 20 & 17,0 & 11,0 & 0,65 & 6,0 & 1.122 .849 & 5,007 & $1.2+3.2$ \\
\hline
\end{tabular}


Anhang

\begin{tabular}{|c|c|c|c|c|c|c|c|}
\hline $\begin{array}{l}\text { Ifd. } \\
\text { Nr. }\end{array}$ & $\begin{array}{l}\text { Fo } \\
{[\mathrm{kN}]}\end{array}$ & $\begin{array}{l}F_{U} \\
{[k N]}\end{array}$ & $\mathbf{R}$ & $\begin{array}{l}\Delta F \\
{[k N]}\end{array}$ & $N_{f}$ & $\begin{array}{l}\Delta \mathrm{F}\left(2 \cdot 10^{6}\right) \\
{[\mathrm{kN}]}\end{array}$ & $\begin{array}{l}\text { Versagen } \\
\text { Fall } \\
\end{array}$ \\
\hline 21 & 17,0 & 8,0 & 0,47 & 9,0 & 324.497 & 5,090 & $1.2+3.2$ \\
\hline 22 & 17,0 & 11,5 & 0,68 & 5,5 & 1.688 .024 & 5,215 & $1.2+3.2$ \\
\hline 23 & 17,0 & 8,0 & 0,47 & 9,0 & 333.649 & 5,135 & $1.2+3.2$ \\
\hline 24 & 17,0 & 8,0 & 0,47 & 9,0 & 244.362 & 4,657 & $1.2+3.2$ \\
\hline 25 & 17,0 & 11,5 & 0,68 & 5,5 & 1.191 .057 & 4,675 & $1.2+3.2$ \\
\hline 26 & 17,0 & 8,0 & 0,47 & 9,0 & 285.182 & 4,888 & $1.2+3.2$ \\
\hline 27 & 17,0 & 12,0 & 0,71 & 5,0 & 2.069 .910 & 5,054 & $1.2+3.2$ \\
\hline 28 & 17,0 & 8,0 & 0,47 & 9,0 & 272.851 & 4,821 & $1.2+3.2$ \\
\hline 29 & 17,0 & 12,0 & 0,71 & 5,0 & 2.238 .483 & 5,180 & $1.2+3.2$ \\
\hline 30 & 17,0 & 11,5 & 0,68 & 5,5 & 1.119 .242 & 4,585 & $1.2+3.2$ \\
\hline 31 & 17,0 & 11,5 & 0,68 & 5,5 & 1.424 .111 & 4,945 & $1.2+3.2$ \\
\hline 32 & 17,0 & 11,5 & 0,68 & 5,5 & 1.759 .529 & 5,284 & $1.2+3.2$ \\
\hline 33 & 17,0 & 12,0 & 0,71 & 5,0 & 2.374 .720 & 5,276 & $1.2+3.2$ \\
\hline 34 & 17,0 & 11,5 & 0,68 & 5,5 & 1.634 .384 & 5,163 & $1.2+3.2$ \\
\hline 35 & 17,0 & 11,5 & 0,68 & 5,5 & 1.239 .733 & 4,735 & $1.2+3.2$ \\
\hline 36 & 17,0 & 11,5 & 0,68 & 5,5 & 1.672 .775 & 5,201 & $1.2+3.2$ \\
\hline 37 & 17,0 & 12,0 & 0,71 & 5,0 & 1.895 .100 & 4,916 & $1.2+3.2$ \\
\hline 38 & 17,0 & 12,0 & 0,71 & 5,0 & 2.023 .458 & 5,018 & $1.2+3.2$ \\
\hline 39 & 17,0 & 8,0 & 0,47 & 9,0 & 358.977 & 5,254 & $1.2+3.2$ \\
\hline 40 & 17,0 & 12,0 & 0,71 & 5,0 & 2.284 .641 & 5,213 & $1.2+3.2$ \\
\hline 41 & 17,0 & 12,0 & 0,71 & 5,0 & 2.914 .178 & 5,626 & $1.2+3.2$ \\
\hline \multicolumn{8}{|c|}{ Parameter der Regressionsgeraden $y=a \cdot x+b$ aus $\log N_{f}$ und $\log \Delta F$} \\
\hline \multicolumn{8}{|c|}{ Faktor $\mathrm{a}=-0,3134$, Faktor $\mathrm{b}=2,6715$, Steigung $\mathrm{m}=3,19$} \\
\hline \multicolumn{8}{|c|}{ Statistische Parameter der Ermüdungsfestigkeit $\Delta F$ bei $N_{f}=2 \cdot 10^{6}$} \\
\hline \multicolumn{8}{|c|}{$\mathrm{m}_{\Delta \mathrm{F}}=4,98 \mathrm{kN}, \sigma_{\Delta \mathrm{F}}=0,23 \mathrm{kN}, \mathrm{V}_{\Delta \mathrm{F}}=4,62 \%$} \\
\hline \multicolumn{8}{|c|}{ Charakteristische Ermüdungsfestigkeiten $\Delta F_{R K}$ bei $N_{f}=2 \cdot 10^{6}$} \\
\hline \multicolumn{8}{|c|}{$\Delta \mathrm{F}_{\mathrm{Rk}, \mathrm{SVA}}=4,62 \mathrm{kN}, \Delta \mathrm{F}_{\mathrm{Rk}, \mathrm{EC} 3}=4,50 \mathrm{kN}, \Delta \mathrm{F}_{\mathrm{Rk}, \mathrm{ETAG}}=4,52 \mathrm{kN}$} \\
\hline
\end{tabular}

Tabelle A11 Profil HZA-38/23 St - Variante 50/50-OB Serie C - konst. Oberlast 0,5 kN

\begin{tabular}{|l|l|l|l|l|l|l|l|}
\hline $\begin{array}{l}\text { Ifd. } \\
\mathbf{N r} .\end{array}$ & $\begin{array}{l}\mathbf{F}_{\mathbf{O}} \\
{[\mathbf{k N}]}\end{array}$ & $\begin{array}{l}\mathbf{F}_{\mathbf{U}} \\
{[\mathbf{k N}]}\end{array}$ & $\mathbf{R}$ & $\begin{array}{l}\Delta \mathbf{F} \\
{[\mathbf{k N}]}\end{array}$ & $\mathbf{N}_{\mathbf{f}}$ & $\begin{array}{l}\mathbf{A} \mathbf{( 2 \cdot 1 0 ^ { 6 } )} \\
{[\mathbf{k N}]}\end{array}$ & $\begin{array}{l}\text { Versagen } \\
\text { Fall }\end{array}$ \\
\hline 1 & 8,5 & 0,5 & 0,06 & 8,0 & 1.866 .382 & 7,892 & 1.2 \\
\hline 2 & 8,5 & 0,5 & 0,06 & 8,0 & 1.562 .870 & 7,621 & 1.2 \\
\hline 3 & 10,0 & 0,5 & 0,05 & 9,5 & 505.704 & 7,246 & 1.2 \\
\hline 4 & 16,5 & 0,5 & 0,03 & 16,0 & 50.000 & 7,735 & 1.2 \\
\hline 5 & 16,5 & 0,5 & 0,03 & 16,0 & 54.085 & 7,856 & 1.2 \\
\hline 6 & 16,5 & 0,5 & 0,03 & 16,0 & 50.685 & 7,756 & 1.2 \\
\hline
\end{tabular}




\begin{tabular}{|l|l|l|l|l|l|l|l|}
\hline $\begin{array}{l}\text { Ifd. } \\
\text { Nr. }\end{array}$ & $\begin{array}{l}\mathbf{F}_{\mathrm{O}} \\
{[\mathbf{k N}]}\end{array}$ & $\begin{array}{l}\mathbf{F}_{\mathbf{U}} \\
{[\mathbf{k N}]}\end{array}$ & $\mathbf{R}$ & $\begin{array}{l}\Delta \mathbf{F} \\
{[\mathbf{k N}]}\end{array}$ & $\mathbf{N}_{\mathbf{f}}$ & $\begin{array}{l}\Delta \mathbf{F}\left(\mathbf{2} \cdot \mathbf{1 0}^{\mathbf{6}}\right) \\
{[\mathbf{k N}]}\end{array}$ & $\begin{array}{l}\text { Versagen } \\
\text { Fall }\end{array}$ \\
\hline 7 & 16,5 & 0,5 & 0,03 & 16,0 & 52.143 & 7,799 & 1.2 \\
\hline 8 & 16,5 & 0,5 & 0,03 & 16,0 & 50.146 & 7,740 & 1.2 \\
\hline 9 & 10,5 & 0,5 & 0,05 & 10,0 & 557.267 & 7,774 & 1.2 \\
\hline 10 & 10,0 & 0,5 & 0,05 & 9,5 & 340.944 & 6,704 & 1.2 \\
\hline 11 & 12,5 & 0,5 & 0,04 & 12,0 & 170.000 & 7,383 & 1.2 \\
\hline 12 & 12,5 & 0,5 & 0,04 & 12,0 & 188.758 & 7,537 & 1.2 \\
\hline 13 & 9,5 & 0,5 & 0,05 & 9,0 & 1.361 .353 & 8,343 & 1.2 \\
\hline 14 & 11,5 & 0,5 & 0,04 & 11,0 & 274.025 & 7,435 & 1.2 \\
\hline 15 & 9,5 & 0,5 & 0,05 & 9,0 & 726.302 & 7,372 & 1.2 \\
\hline 16 & 12,5 & 0,5 & 0,04 & 12,0 & 159.121 & 7,288 & 1.2 \\
\hline 17 & 9,0 & 0,5 & 0,06 & 8,5 & 4.018 .975 & $1)$ & kein Bruch \\
\hline 1$)$ & nicht in der Auswertung berücksichtigt \\
\hline
\end{tabular}

Tabelle A12 Profil JXA-W 38/23 St - Variante 50/50-OB Serie D - konst. Oberlast 17 kN

\begin{tabular}{|c|c|c|c|c|c|c|c|}
\hline $\begin{array}{l}\text { Ifd. } \\
\text { Nr. }\end{array}$ & $\begin{array}{l}F_{0} \\
{[k N]}\end{array}$ & \begin{tabular}{|l}
$F_{U}$ \\
{$[k N]$}
\end{tabular} & $\mathbf{R}$ & $\begin{array}{l}\Delta \mathrm{F} \\
{[\mathrm{kN}]}\end{array}$ & $N_{f}$ & $\begin{array}{l}\Delta \mathrm{F}\left(2 \cdot 10^{6}\right) \\
{[\mathrm{kN}]}\end{array}$ & $\begin{array}{l}\text { Versagen } \\
\text { Fall }\end{array}$ \\
\hline 1 & 17,0 & 8,0 & 0,47 & 9,0 & 310.464 & 5,429 & 1.2 \\
\hline 2 & 17,0 & 11,0 & 0,65 & 6,0 & 979.523 & 4,943 & 1.2 \\
\hline 3 & 17,0 & 8,0 & 0,47 & 9,0 & 315.376 & 5,452 & 1.2 \\
\hline 4 & 17,0 & 12,0 & 0,71 & 5,0 & 2.115 .874 & 5,077 & 1.2 \\
\hline 5 & 17,0 & 8,0 & 0,47 & 9,0 & 311.497 & 5,434 & 1.2 \\
\hline 6 & 17,0 & 11,0 & 0,65 & 6,0 & 1.425 .946 & 5,474 & 1.2 \\
\hline 7 & 17,0 & 8,0 & 0,47 & 9,0 & 314.587 & 5,448 & 1.2 \\
\hline 8 & 17,0 & 11,0 & 0,65 & 6,0 & 1.207 .935 & 5,233 & 1.2 \\
\hline 9 & 17,0 & 12,0 & 0,71 & 5,0 & 3.510 .709 & 5,825 & 1.2 \\
\hline \multicolumn{8}{|c|}{ Parameter der Regressionsgeraden $y=a \cdot x+b$ aus $\log N_{f}$ und $\log \Delta F$} \\
\hline \multicolumn{8}{|c|}{ Faktor $\mathrm{a}=-0,2713$, Faktor $\mathrm{b}=2,4392$, Steigung $\mathrm{m}=3,69$} \\
\hline \multicolumn{8}{|c|}{ Statistische Parameter der Ermüdungsfestigkeit $\Delta \mathrm{F}$ bei $N_{f}=2 \cdot 10^{6}$} \\
\hline \multicolumn{8}{|c|}{$\mathrm{m}_{\Delta \mathrm{F}}=5,37 \mathrm{kN}, \sigma_{\Delta \mathrm{F}}=0,26 \mathrm{kN}, \mathrm{V}_{\Delta \mathrm{F}}=4,77 \%$} \\
\hline \multicolumn{8}{|c|}{ Charakteristische Ermüdungsfestigkeiten $\Delta F_{R K}$ bei $N_{f}=2 \cdot 10^{6}$} \\
\hline \multicolumn{8}{|c|}{$\Delta \mathrm{F}_{\mathrm{Rk}, \mathrm{SVA}}=4,82 \mathrm{kN}, \Delta \mathrm{F}_{\mathrm{Rk}, \mathrm{EC} 3}=4,61 \mathrm{kN}, \Delta \mathrm{F}_{\mathrm{Rk}, \mathrm{ETAG}}=4,67 \mathrm{kN}$} \\
\hline
\end{tabular}


Tabelle A13 Profil JXA-W 38/23 St - Variante 50/50-OB Serie D - konst. Unterlast 0,5 kN

\begin{tabular}{|c|c|c|c|c|c|c|c|}
\hline $\begin{array}{l}\text { Ifd. } \\
\mathrm{Nr} .\end{array}$ & $\begin{array}{l}F_{0} \\
{[k N]}\end{array}$ & $\begin{array}{l}F_{U} \\
{[k N]}\end{array}$ & $\mathbf{R}$ & $\begin{array}{l}\Delta \mathrm{F} \\
{[\mathrm{kN}]}\end{array}$ & $N_{f}$ & $\begin{array}{l}\Delta \mathrm{F}\left(2 \cdot 10^{6}\right) \\
{[\mathrm{kN}]}\end{array}$ & $\begin{array}{l}\text { Versagen } \\
\text { Fall }\end{array}$ \\
\hline 1 & 9,0 & 0,5 & 0,06 & 8,5 & 667.568 & 7,462 & 1.2 \\
\hline 2 & 9,5 & 0,5 & 0,05 & 9,0 & 688.033 & 7,929 & 1.2 \\
\hline 3 & 8,5 & 0,5 & 0,06 & 8,0 & 1.716 .337 & 7,856 & 1.2 \\
\hline 4 & 9,0 & 0,5 & 0,06 & 8,5 & 1.003 .426 & 7,832 & 1.2 \\
\hline 5 & 9,5 & 0,5 & 0,05 & 9,0 & 827.332 & 8,105 & 1.2 \\
\hline 6 & 8,5 & 0,5 & 0,06 & 8,0 & 1.934 .090 & 7,968 & 1.2 \\
\hline 7 & 8,5 & 0,5 & 0,06 & 8,0 & 953.092 & 7,326 & 1.2 \\
\hline 8 & 9,5 & 0,5 & 0,05 & 9,0 & 2.065 .931 & 9,035 & 1.2 \\
\hline 9 & 9,0 & 0,5 & 0,06 & 8,5 & 1.339 .278 & 8,105 & 1.2 \\
\hline 10 & 8,5 & 0,5 & 0,06 & 8,0 & 4.448 .221 & 8,796 & 1.2 \\
\hline 11 & 9,5 & 0,5 & 0,05 & 9,0 & 839.670 & 8,119 & 1.2 \\
\hline 12 & 9,0 & 0,5 & 0,06 & 8,5 & 9.715 .136 & 1) & kein Bruch \\
\hline 13 & 8,5 & 0,5 & 0,06 & 8,0 & 1.146 .647 & 7,489 & 1.2 \\
\hline 14 & 12,5 & 0,5 & 0,04 & 12,0 & 150.360 & 8,826 & 1.2 \\
\hline 15 & 10,5 & 0,5 & 0,05 & 10,0 & 251.857 & 7,820 & 1.2 \\
\hline 16 & 8,5 & 0,5 & 0,06 & 8,0 & 1.011 .305 & 7,378 & 1.2 \\
\hline 17 & 11,5 & 0,5 & 0,04 & 11,0 & 232.571 & 8,521 & 1.2 \\
\hline 18 & 9,5 & 0,5 & 0,05 & 9,0 & 874.824 & 8,159 & 1.1 \\
\hline \multicolumn{8}{|c|}{ 1) nicht in der Auswertung berücksichtigt } \\
\hline \multicolumn{8}{|c|}{ Parameter der Regressionsgeraden $y=a \cdot x+b$ aus $\log N_{f}$ und $\log \Delta F$} \\
\hline \multicolumn{8}{|c|}{ Faktor $\mathrm{a}=-0,1187$, Faktor $\mathrm{b}=1,6525$, Steigung $\mathrm{m}=8,43$} \\
\hline \multicolumn{8}{|c|}{ Statistische Parameter der Ermüdungsfestigkeit $\Delta F$ bei $N_{f}=2 \cdot 10^{6}$} \\
\hline \multicolumn{8}{|c|}{$\mathrm{m}_{\Delta \mathrm{F}}=8,04 \mathrm{kN}, \sigma_{\Delta \mathrm{F}}=0,51 \mathrm{kN}, \mathrm{V}_{\Delta \mathrm{F}}=6,35 \%$} \\
\hline \multicolumn{8}{|c|}{ Charakteristische Ermüdungsfestigkeiten $\Delta F_{R K}$ bei $N_{f}=2 \cdot 10^{6}$} \\
\hline \multicolumn{8}{|c|}{$\Delta \mathrm{F}_{\mathrm{Rk}, \mathrm{SVA}}=7,11 \mathrm{kN}, \Delta \mathrm{F}_{\mathrm{Rk}, \mathrm{EC} 3}=6,79 \mathrm{kN}, \Delta \mathrm{F}_{\mathrm{Rk}, \mathrm{ETAG}}=6,87 \mathrm{kN}$} \\
\hline
\end{tabular}

Tabelle A14 Profil HZA 38/23 St - Variante 25/50-B Serie A - konst. Oberlast 17 kN

\begin{tabular}{|l|l|l|l|l|l|l|l|l|}
\hline $\begin{array}{l}\text { Ifd. } \\
\text { Nr. }\end{array}$ & $\begin{array}{l}\mathbf{F}_{\mathbf{O}} \\
{[\mathbf{k N}]}\end{array}$ & $\begin{array}{l}\mathbf{F}_{\mathbf{U}} \\
{[\mathbf{k N}]}\end{array}$ & $\mathbf{R}$ & $\begin{array}{l}\Delta \mathbf{F} \\
{[\mathbf{k N}]}\end{array}$ & $\mathbf{N}_{\mathbf{f}}$ & $\begin{array}{l}\left.\mathbf{\Delta} \mathbf{( 2 \cdot 1 0 ^ { 6 }}\right) \\
{[\mathbf{k N}]}\end{array}$ & $\begin{array}{l}\text { Versagen } \\
\text { Fall }\end{array}$ & $\begin{array}{l}\mathbf{f}_{\text {cm,cube }} \\
{\left[\mathbf{N} / \mathbf{m m}^{2}\right]}\end{array}$ \\
\hline 1 & 17,0 & 11,0 & 0,65 & 6,0 & 883.367 & 4,938 & $1.2+3.2$ & 36,10 \\
\hline 2 & 17,0 & 12,0 & 0,71 & 5,0 & 1.746 .623 & 4,841 & $1.2+3.2$ & 38,24 \\
\hline 3 & 17,0 & 10,0 & 0,59 & 7,0 & 480.222 & 4,982 & $1.2+3.2$ & 38,16 \\
\hline 4 & 17,0 & 10,0 & 0,59 & 7,0 & 399.005 & 4,767 & $1.2+3.1$ & 38,06 \\
\hline 5 & 17,0 & 12,0 & 0,71 & 5,0 & 2.263 .974 & 5,150 & $1.2+3.1$ & 36,58 \\
\hline
\end{tabular}




\begin{tabular}{|l|l|l|l|l|l|l|l|l|}
\hline $\begin{array}{l}\text { Ifd. } \\
\text { Nr. }\end{array}$ & $\begin{array}{l}\mathbf{F}_{\mathrm{O}} \\
{[\mathbf{k N}]}\end{array}$ & $\begin{array}{l}\mathbf{F}_{\mathbf{U}} \\
{[\mathbf{k N}]}\end{array}$ & $\mathbf{R}$ & $\begin{array}{l}\Delta \mathbf{F} \\
{[\mathbf{k N}]}\end{array}$ & $\mathbf{N}_{\mathbf{f}}$ & $\begin{array}{l}\Delta \mathbf{F}\left(\mathbf{2 \cdot 1 0 ^ { 6 }}\right) \\
{[\mathbf{k N}]}\end{array}$ & $\begin{array}{l}\text { Versagen } \\
\text { Fall }\end{array}$ & $\begin{array}{l}\mathbf{f}_{\text {cm,cube }} \\
{\left[\mathbf{N} / \mathbf{m m}^{\mathbf{2}}\right]}\end{array}$ \\
\hline 6 & 17,0 & 12,0 & 0,71 & 5,0 & 1.647 .191 & 4,774 & $1.2+3.2$ & 36,89 \\
\hline 7 & 17,0 & 10,0 & 0,59 & 7,0 & 725.707 & 5,497 & $1.2+3.2$ & 35,98 \\
\hline 8 & 17,0 & 11,0 & 0,65 & 6,0 & 931.156 & 5,001 & $1.2+3.1$ & 36,73 \\
\hline \hline
\end{tabular}

Parameter der Regressionsgeraden $y=a \cdot x+b$ aus $\log N_{f}$ und $\log \Delta F$

Faktor $a=-0,2383$, Faktor $b=2,1999$, Steigung $m=4,20$

Statistische Parameter der Ermüdungsfestigkeit $\Delta \mathrm{F}$ bei $N_{\mathrm{f}}=\mathbf{2} \cdot 10^{\mathbf{6}}$

$\mathrm{m}_{\Delta \mathrm{F}}=4,99 \mathrm{kN}, \sigma_{\Delta \mathrm{F}}=0,24 \mathrm{kN}, \mathrm{V}_{\Delta \mathrm{F}}=4,81 \%$

Charakteristische Ermüdungsfestigkeiten $\Delta F_{R K}$ bei $N_{f}=\mathbf{2} \cdot 10^{6}$

$\Delta \mathrm{F}_{\mathrm{Rk}, \mathrm{SVA}}=4,46 \mathrm{kN}, \Delta \mathrm{F}_{\mathrm{Rk}, \mathrm{EC} 3}=4,25 \mathrm{kN}, \Delta \mathrm{F}_{\mathrm{Rk}, \mathrm{ETAG}}=4,32 \mathrm{kN}$

Tabelle A15 Profil HZA 38/23 St - Variante 25/50-B Serie B - konst. Oberlast 17 kN

\begin{tabular}{|c|c|c|c|c|c|c|c|c|}
\hline $\begin{array}{l}\text { Ifd. } \\
\text { Nr. }\end{array}$ & \begin{tabular}{|l}
$F_{0}$ \\
{$[k N]$}
\end{tabular} & $\begin{array}{l}F_{U} \\
{[k N]}\end{array}$ & $\mathbf{R}$ & $\begin{array}{l}\Delta \mathrm{F} \\
{[\mathrm{kN}]}\end{array}$ & $\mathbf{N}_{\mathrm{f}}$ & $\begin{array}{l}\Delta \mathrm{F}\left(2 \cdot 10^{6}\right) \\
{[\mathrm{kN}]}\end{array}$ & $\begin{array}{l}\text { Versagen } \\
\text { Fall }\end{array}$ & \begin{tabular}{|l}
$f_{\mathrm{cm}, \text { cube }}$ \\
{$\left[\mathrm{N} / \mathrm{mm}^{2}\right]$}
\end{tabular} \\
\hline 1 & 17,0 & 11,0 & 0,65 & 6,0 & 850.282 & 5,260 & $1.1+3.1$ & \multirow{6}{*}{$35,2-38,3$} \\
\hline 2 & 17,0 & 12,0 & 0,71 & 5,0 & 2.700 .296 & 5,236 & $1.1+2.1$ & \\
\hline 3 & 17,0 & 12,0 & 0,71 & 5,0 & 1.730 .537 & 4,890 & $1.2+3.1$ & \\
\hline 4 & 17,0 & 11,5 & 0,68 & 5,5 & 1.384 .386 & 5,197 & $1.1+2.1 . \mathrm{A}$ & \\
\hline 5 & 17,0 & 11,5 & 0,68 & 5,5 & 959.457 & 4,912 & $1.1+2.1 . \mathrm{B}$ & \\
\hline 6 & 17,0 & 11,0 & 0,65 & 6,0 & 755.847 & 5,166 & $1.1+2.1$ & \\
\hline \multicolumn{9}{|c|}{ Parameter der Regressionsgeraden $y=a \cdot x+b$ aus $\log N_{f}$ und $\log \Delta F$} \\
\hline \multicolumn{9}{|c|}{ Faktor $\mathrm{a}=-0,1538$, Faktor $\mathrm{b}=1,6774$, Steigung $\mathrm{m}=6,50$} \\
\hline \multicolumn{9}{|c|}{ Statistische Parameter der Ermüdungsfestigkeit $\Delta \mathrm{F}$ bei $N_{f}=2 \cdot 10^{6}$} \\
\hline \multicolumn{9}{|c|}{$\mathrm{m}_{\Delta \mathrm{F}}=5,11 \mathrm{kN}, \sigma_{\Delta \mathrm{F}}=0,17 \mathrm{kN}, \mathrm{V}_{\Delta \mathrm{F}}=3,24 \%$} \\
\hline \multicolumn{9}{|c|}{ Charakteristische Ermüdungsfestigkeiten $\Delta F_{R K}$ bei $N_{f}=2 \cdot 10^{6}$} \\
\hline \multicolumn{9}{|c|}{$\Delta \mathrm{F}_{\mathrm{Rk}, \mathrm{SVA}}=4,70 \mathrm{kN}, \Delta \mathrm{F}_{\mathrm{Rk}, \mathrm{EC} 3}=4,53 \mathrm{kN}, \Delta \mathrm{F}_{\mathrm{Rk}, \mathrm{ETAG}}=4,59 \mathrm{kN}$} \\
\hline
\end{tabular}


Tabelle A16 Profil HZA 38/23 St - Variante 25/50-B Serie C - konst. Oberlast 17 kN

\begin{tabular}{|l|l|l|l|l|l|l|l|l|}
\hline $\begin{array}{l}\text { Ifd. } \\
\text { Nr. }\end{array}$ & $\begin{array}{l}\mathbf{F}_{\mathrm{O}} \\
{[\mathbf{k N}]}\end{array}$ & $\begin{array}{l}\mathbf{F}_{\mathbf{U}} \\
{[\mathbf{k N}]}\end{array}$ & $\mathbf{R}$ & $\begin{array}{l}\Delta \mathbf{F} \\
{[\mathbf{k N}]}\end{array}$ & $\mathbf{N}_{\mathbf{f}}$ & $\begin{array}{l}\mathbf{\Delta} \mathbf{F}\left(\mathbf{2} \cdot \mathbf{1 0}^{\mathbf{6}}\right) \\
{[\mathbf{k N}]}\end{array}$ & $\begin{array}{l}\text { Versagen } \\
\text { Fall }\end{array}$ & $\begin{array}{l}\mathbf{f}_{\mathrm{cm}, \text { cube }} \\
{\left[\mathbf{N} / \mathbf{m m}^{\mathbf{2}}\right]}\end{array}$ \\
\hline 1 & 17,0 & 11,0 & 0,65 & 6,0 & 951.220 & 5,224 & 1.2 & \multirow{2}{*}{42,0} \\
\hline 2 & 17,0 & 10,0 & 0,59 & 7,0 & 366.770 & 5,102 & 1.2 & \\
\hline 3 & 17,0 & 11,5 & 0,68 & 5,5 & 1.264 .560 & 5,049 & 1.2 & \\
\hline
\end{tabular}

Tabelle A17 Profil HZA 38/23 St - Variante 25/50-B Serie C - konst Unterlast 1 kN

\begin{tabular}{|c|c|c|c|c|c|c|c|c|}
\hline $\begin{array}{l}\text { Ifd. } \\
\mathrm{Nr} \text {. }\end{array}$ & \begin{tabular}{|l}
$F_{0}$ \\
{$[k N]$}
\end{tabular} & $\begin{array}{l}F_{U} \\
{[k N]}\end{array}$ & $\mathbf{R}$ & $\begin{array}{l}\Delta \mathrm{F} \\
{[\mathrm{kN}]}\end{array}$ & $\mathbf{N}_{\mathrm{f}}$ & $\begin{array}{l}\Delta \mathrm{F}\left(2 \cdot 10^{6}\right) \\
{[\mathrm{kN}]}\end{array}$ & $\begin{array}{l}\text { Versagen } \\
\text { Fall }\end{array}$ & \begin{tabular}{|l}
$\mathbf{f}_{\mathrm{cm}, \text { cube }}$ \\
{$\left[\mathrm{N} / \mathrm{mm}^{2}\right]$}
\end{tabular} \\
\hline 1 & 13,0 & 1,0 & 0,08 & 12,0 & 157.680 & 8,797 & 1.2 & \multirow{10}{*}{42,0} \\
\hline 2 & 11,0 & 1,0 & 0,09 & 10,0 & 503.040 & 8,448 & 1.2 & \\
\hline 3 & 9,0 & 1,0 & 0,11 & 8,0 & 3.580 .300 & 1) & kein Bruch & \\
\hline 4 & 10,0 & 1,0 & 0,10 & 9,0 & 1.968 .760 & 1) & kein Bruch & \\
\hline 5 & 13,0 & 1,0 & 0,08 & 12,0 & 169.070 & 8,872 & 1.2 & \\
\hline 6 & 10,5 & 1,0 & 0,10 & 9,5 & \begin{tabular}{|l|}
897.260 \\
\end{tabular} & 8,613 & 1.2 & \\
\hline 7 & 10,5 & 1,0 & 0,10 & 9,5 & 855.160 & 8,563 & 1.2 & \\
\hline 8 & 10,0 & 1,0 & 0,10 & 9,0 & 1.401 .790 & 8,617 & 1.2 & \\
\hline 9 & 9,75 & 1,0 & 0,10 & 8,75 & 2.179 .030 & 8,842 & $1.2+3.1$ & \\
\hline 10 & 12,0 & 1,0 & 0,08 & 11,0 & 217.120 & 8,385 & 1.2 & \\
\hline \multicolumn{9}{|c|}{ 1) nicht in der Auswertung berücksichtigt } \\
\hline \multicolumn{9}{|c|}{ Parameter der Regressionsgeraden $y=a \cdot x+b$ aus $\log N_{f}$ und $\log \Delta F$} \\
\hline \multicolumn{9}{|c|}{ Faktor $\mathrm{a}=-0,1222$, Faktor $\mathrm{b}=1,7067$, Steigung $\mathrm{m}=8,18$} \\
\hline \multicolumn{9}{|c|}{ Statistische Parameter der Ermüdungsfestigkeit $\Delta \mathrm{F}$ bei $N_{f}=2 \cdot 10^{6}$} \\
\hline \multicolumn{9}{|c|}{$\mathrm{m}_{\Delta \mathrm{F}}=8,64 \mathrm{kN}, \sigma_{\Delta \mathrm{F}}=0,18 \mathrm{kN}, \mathrm{V}_{\Delta \mathrm{F}}=2,09 \%$} \\
\hline \multicolumn{9}{|c|}{ Charakteristische Ermüdungsfestigkeiten $\Delta F_{R K}$ bei $N_{f}=2 \cdot 10^{6}$} \\
\hline \multicolumn{9}{|c|}{$\Delta \mathrm{F}_{\mathrm{Rk}, \mathrm{SVA}}=8,24 \mathrm{kN}, \Delta \mathrm{F}_{\mathrm{Rk}, \mathrm{EC} 3}=8,08 \mathrm{kN}, \Delta \mathrm{F}_{\mathrm{Rk}, \mathrm{ETAG}}=8,13 \mathrm{kN}$} \\
\hline
\end{tabular}


Tabelle A18 Profil HZA 38/23 St - Variante 25/50-B Serie B \& C - konst. Oberlast 17 kN

\begin{tabular}{|c|c|c|c|c|c|c|c|c|}
\hline $\begin{array}{l}\text { Ifd. } \\
\text { Nr. }\end{array}$ & \begin{tabular}{|l}
$F_{0}$ \\
{$[k N]$}
\end{tabular} & $\begin{array}{l}F_{U} \\
{[k N]}\end{array}$ & $\mathbf{R}$ & $\begin{array}{l}\Delta \mathrm{F} \\
{[\mathrm{kN}]}\end{array}$ & $\mathbf{N}_{\mathrm{f}}$ & $\begin{array}{l}\Delta \mathrm{F}\left(2 \cdot 10^{6}\right) \\
{[\mathrm{kN}]}\end{array}$ & $\begin{array}{l}\text { Versagen } \\
\text { Fall }\end{array}$ & $\begin{array}{l}f_{\mathrm{cm}, \text { cube }} \\
{\left[\mathrm{N} / \mathrm{mm}^{2}\right]}\end{array}$ \\
\hline 1 & 17,0 & 11,0 & 0,65 & 6,0 & 850.282 & 5,150 & $1.1+3.1$ & \multirow{6}{*}{$35,2-38,3$} \\
\hline 2 & 17,0 & 12,0 & 0,71 & 5,0 & 2.700 .296 & 5,275 & $1.1+2.1$ & \\
\hline 3 & 17,0 & 12,0 & 0,71 & 5,0 & 1.730 .537 & 4,872 & $1.2+3.1$ & \\
\hline 4 & 17,0 & 11,5 & 0,68 & 5,5 & 1.384 .386 & 5,150 & $1.1+2.1 . \mathrm{A}$ & \\
\hline 5 & 17,0 & 11,5 & 0,68 & 5,5 & 959.457 & 4,824 & $1.1+2.1 . B$ & \\
\hline 6 & 17,0 & 11,0 & 0,65 & 6,0 & 755.847 & 5,043 & $1.1+2.1$ & \\
\hline 7 & 17,0 & 11,0 & 0,65 & 6,0 & 951.220 & 5,255 & 1.2 & \multirow[t]{3}{*}{42,0} \\
\hline 8 & 17,0 & 10,0 & 0,59 & 7,0 & 366.770 & 5,171 & 1.2 & \\
\hline 9 & 17,0 & 11,5 & 0,68 & 5,5 & 1.264 .560 & 5,068 & 1.2 & \\
\hline
\end{tabular}

Parameter der Regressionsgeraden $y=a \cdot x+b$ aus $\log N_{f}$ und $\log \Delta F$

Faktor $a=-0,1785$, Faktor $b=1,8313$, Steigung $m=5,60$

Statistische Parameter der Ermüdungsfestigkeit $\Delta \mathrm{F}$ bei $N_{\mathrm{f}}=\mathbf{2} \cdot 10^{\mathbf{6}}$

$\mathrm{m}_{\Delta \mathrm{F}}=5,09 \mathrm{kN}, \sigma_{\Delta \mathrm{F}}=0,16 \mathrm{kN}, \mathrm{V}_{\Delta \mathrm{F}}=3,08 \%$

Charakteristische Ermüdungsfestigkeiten $\Delta F_{R K}$ bei $N_{f}=\mathbf{2} \cdot 10^{6}$

$\Delta \mathrm{F}_{\mathrm{Rk}, \mathrm{SVA}}=4,75 \mathrm{kN}, \Delta \mathrm{F}_{\mathrm{Rk}, \mathrm{EC} 3}=4,62 \mathrm{kN}, \Delta \mathrm{F}_{\mathrm{Rk}, \mathrm{ETAG}}=4,66 \mathrm{kN}$

Tabelle A19 Profil HZA 38/23 St - Variante 50/50-B Serie A - konst. Oberlast 17 kN

\begin{tabular}{|c|c|c|c|c|c|c|c|c|}
\hline $\begin{array}{l}\text { Ifd. } \\
\mathrm{Nr} \text {. }\end{array}$ & \begin{tabular}{|l}
$F_{0}$ \\
{$[k N]$}
\end{tabular} & $\begin{array}{l}F_{U} \\
{[k N]}\end{array}$ & $\mathbf{R}$ & $\begin{array}{l}\Delta \mathrm{F} \\
{[\mathrm{kN}]}\end{array}$ & $N_{f}$ & $\begin{array}{l}\Delta \mathrm{F}\left(2 \cdot 10^{6}\right) \\
{[\mathrm{kN}]}\end{array}$ & $\begin{array}{l}\text { Versagen } \\
\text { Fall }\end{array}$ & $\begin{array}{l}\mathbf{f}_{\mathrm{cm}, \text { cube }} \\
{\left[\mathrm{N} / \mathrm{mm}^{2}\right]}\end{array}$ \\
\hline 1 & 17,0 & 11,0 & 0,65 & 6,0 & 1.255 .999 & 5,456 & $1.2+3.2$ & 38,61 \\
\hline 2 & 17,0 & 11,0 & 0,65 & 6,0 & 1.776 .851 & 5,857 & $1.2+3.2$ & 37,06 \\
\hline 3 & 17,0 & 12,0 & 0,71 & 5,0 & 3.603 .283 & 5,640 & $1.2+3.2$ & 37,80 \\
\hline 4 & 17,0 & 10,0 & 0,59 & 7,0 & 579.176 & 5,433 & $1.2+3.2$ & 37,60 \\
\hline 5 & 17,0 & 12,0 & 0,71 & 5,0 & 3.103 .987 & 5,470 & $1.2+3.2$ & 39,27 \\
\hline 6 & 17,0 & 11,0 & 0,65 & 6,0 & 1.639 .974 & 5,761 & $1.2+3.2$ & 39,13 \\
\hline 7 & 17,0 & 10,0 & 0,59 & 7,0 & 708.538 & 5,662 & $1.2+3.2$ & 38,76 \\
\hline 8 & 17,0 & 12,0 & 0,71 & 5,0 & 2.831 .168 & 5,368 & $1.2+3.2$ & 38,86 \\
\hline \multicolumn{9}{|c|}{ Parameter der Regressionsgeraden $y=a \cdot x+b$ aus $\log N_{f}$ und $\log \Delta F$} \\
\hline \multicolumn{9}{|c|}{ Faktor $\mathrm{a}=-0,2045$, Faktor $\mathrm{b}=2,0349$, Steigung $\mathrm{m}=4,89$} \\
\hline \multicolumn{9}{|c|}{ Statistische Parameter der Ermüdungsfestigkeit $\Delta \mathrm{F}$ bei $N_{f}=2 \cdot 10^{6}$} \\
\hline \multicolumn{9}{|c|}{$\mathrm{m}_{\Delta \mathrm{F}}=5,58 \mathrm{kN}, \sigma_{\Delta \mathrm{F}}=0,18 \mathrm{kN}, \mathrm{V}_{\Delta \mathrm{F}}=3,13 \%$} \\
\hline \multicolumn{9}{|c|}{ Charakteristische Ermüdungsfestigkeiten $\Delta F_{R K}$ bei $N_{f}=2 \cdot 10^{6}$} \\
\hline \multicolumn{9}{|c|}{$\Delta \mathrm{F}_{\mathrm{Rk}, \mathrm{SVA}}=5,19 \mathrm{kN}, \Delta \mathrm{F}_{\mathrm{Rk}, \mathrm{EC} 3}=5,04 \mathrm{kN}, \Delta \mathrm{F}_{\mathrm{Rk}, \mathrm{ETAG}}=5,18 \mathrm{kN}$} \\
\hline
\end{tabular}




\section{Schubversuche}

Tabelle A20 Profil HZA 38/23 St - Variante 25/50-B Serie C (B25) - konst. Unterlast 1 kN (Beanspruchung in Schienenlängsrichtung, $\alpha=90^{\circ}$ )

\begin{tabular}{|c|c|c|c|c|c|c|c|c|c|}
\hline $\begin{array}{l}\text { Ifd. } \\
\text { Nr. }\end{array}$ & $\begin{array}{l}\alpha \\
{\left[{ }^{\circ}\right]}\end{array}$ & $\begin{array}{l}F_{0} \\
{[k N]}\end{array}$ & $\begin{array}{l}\mathrm{Fu}_{\mathrm{U}} \\
{[\mathrm{kN}]}\end{array}$ & $\mathbf{R}$ & $\begin{array}{l}\Delta \mathrm{F} \\
{[\mathrm{kN}]}\end{array}$ & $\mathbf{N}_{\mathrm{f}}$ & $\begin{array}{l}\Delta \mathrm{F}\left(2 \cdot 10^{6}\right) \\
{[\mathrm{kN}]}\end{array}$ & $\begin{array}{l}\text { Versagen } \\
\text { Fall }\end{array}$ & $\begin{array}{l}\mathbf{f}_{\mathrm{cm}, \text { cube }} \\
{\left[\mathrm{N} / \mathbf{m m}^{2}\right]}\end{array}$ \\
\hline 1 & \multirow{4}{*}{90} & 22,5 & 1,0 & 0,04 & 21,5 & 445.180 & 16,677 & 1.2 & \multirow{4}{*}{42,0} \\
\hline 2 & & 22,5 & 1,0 & 0,04 & 21,5 & 423.540 & 16,537 & 1.2 & \\
\hline 3 & & 20,0 & 1,0 & 0,05 & 19,0 & 1.425 .670 & 17,943 & 1.2 & \\
\hline 4 & & 17,0 & 1,0 & 0,06 & 16,0 & 1.816 .400 & 15,742 & 1.2 & \\
\hline \multicolumn{10}{|c|}{ Parameter der Regressionsgeraden $y=a \cdot x+b$ aus $\log N_{f}$ und $\log \Delta F$} \\
\hline \multicolumn{10}{|c|}{ Faktor $\mathrm{a}=-0,1691$, Faktor $\mathrm{b}=2,2882$, Steigung $\mathrm{m}=5,91$} \\
\hline \multicolumn{10}{|c|}{ Statistische Parameter der Ermüdungsfestigkeit $\Delta \mathrm{F}$ bei $N_{\mathrm{f}}=2 \cdot 10^{6}$} \\
\hline \multicolumn{10}{|c|}{$\mathrm{m}_{\Delta \mathrm{F}}=16,72 \mathrm{kN}, \sigma_{\Delta \mathrm{F}}=0,91 \mathrm{kN}, \mathrm{V}_{\Delta \mathrm{F}}=5,45 \%$} \\
\hline \multicolumn{10}{|c|}{ Charakteristische Ermüdungsfestigkeiten $\Delta F_{R K}$ bei $N_{f}=2 \cdot 10^{6}$} \\
\hline \multicolumn{10}{|c|}{$\Delta \mathrm{F}_{\mathrm{Rk}, \mathrm{SVA}}=13,93 \mathrm{kN}, \Delta \mathrm{F}_{\mathrm{Rk}, \mathrm{EC} 3}=12,49 \mathrm{kN}, \Delta \mathrm{F}_{\mathrm{Rk}, \mathrm{ETAG}}=13,05 \mathrm{kN}$} \\
\hline
\end{tabular}

Tabelle A21 Profil HZA 38/23 St - Variante 25/50-B Serie C (B55) - konst. Unterlast 1 kN Hauptversagensfall 1.2 (Beanspruchung in Schienenlängsrichtung, $\alpha=90^{\circ}$ )

\begin{tabular}{|c|c|c|c|c|c|c|c|c|c|}
\hline $\begin{array}{l}\text { Ifd. } \\
\mathrm{Nr} .\end{array}$ & $\begin{array}{l}\alpha \\
{\left[{ }^{\circ}\right]}\end{array}$ & $\begin{array}{l}\text { Fo } \\
{[k N]}\end{array}$ & $\begin{array}{l}\mathrm{F}_{\mathrm{U}} \\
{[\mathrm{kN}]}\end{array}$ & $\mathbf{R}$ & $\begin{array}{l}\Delta \mathrm{F} \\
{[\mathrm{kN}]}\end{array}$ & $\mathbf{N}_{\mathrm{f}}$ & $\begin{array}{l}\Delta \mathrm{F}\left(2 \cdot 10^{6}\right) \\
{[\mathrm{kN}]}\end{array}$ & $\begin{array}{l}\text { Versagen } \\
\text { Fall }\end{array}$ & $\begin{array}{l}\mathbf{f}_{\mathrm{cm}, \text { cube }} \\
{\left[\mathrm{N} / \mathrm{mm}^{2}\right]}\end{array}$ \\
\hline 1 & \multirow{7}{*}{90} & 25,0 & 1,0 & 0,04 & 24,0 & 1.365 .780 & 22,708 & $1.2+2.1+3.1$ & \multirow{7}{*}{61,30} \\
\hline 2 & & 25,0 & 1,0 & 0,04 & 24,0 & 890.560 & 21,341 & $1.2+3.1$ & \\
\hline 3 & & 20,0 & 1,0 & 0,05 & 19,0 & 3.253 .870 & 20,390 & $1.2+3.1$ & \\
\hline 4 & & 22,5 & 1,0 & 0,04 & 21,5 & 1.089 .800 & 1) & 1.1 & \\
\hline 5 & & 22,5 & 1,0 & 0,04 & 21,5 & 2.647 .330 & 1) & $1.1+2.1$ & \\
\hline 6 & & 27,5 & 1,0 & 0,04 & 26,5 & 385.060 & 20,865 & $1.2+3.1$ & \\
\hline 7 & & 27,5 & 1,0 & 0,04 & 26,5 & 401.380 & 20,991 & 1.2 & \\
\hline \multicolumn{10}{|c|}{ 1) nicht in der Auswertung berücksichtigt } \\
\hline \multicolumn{10}{|c|}{ Parameter der Regressionsgeraden $y=a \cdot x+b$ aus $\log N_{f}$ und $\log \Delta F$} \\
\hline \multicolumn{10}{|c|}{ Faktor $\mathrm{a}=-0,1451$, Faktor $\mathrm{b}=2,2416$, Steigung $\mathrm{m}=6,89$} \\
\hline \multicolumn{10}{|c|}{ Statistische Parameter der Ermüdungsfestigkeit $\Delta \mathrm{F}$ bei $N_{\mathrm{f}}=2 \cdot 10^{6}$} \\
\hline \multicolumn{10}{|c|}{$\mathrm{m}_{\Delta \mathrm{F}}=21,26 \mathrm{kN}, \sigma_{\Delta \mathrm{F}}=0,88 \mathrm{kN}, \mathrm{V}_{\Delta \mathrm{F}}=4,13 \%$} \\
\hline \multicolumn{10}{|c|}{ Charakteristische Ermüdungsfestigkeiten $\Delta F_{R K}$ bei $N_{f}=2 \cdot 10^{6}$} \\
\hline \multicolumn{10}{|c|}{$\Delta \mathrm{F}_{\mathrm{Rk}, \mathrm{SVA}}=18,89 \mathrm{kN}, \Delta \mathrm{F}_{\mathrm{Rk}, \mathrm{EC} 3}=17,80 \mathrm{kN}, \Delta \mathrm{F}_{\mathrm{Rk}, \mathrm{ETAG}}=18,19 \mathrm{kN}$} \\
\hline
\end{tabular}


Tabelle A22 Profil HZA 38/23 St - Variante 25/50-B Serie C (B55) - konst Unterlast 1 kN Alle Versagensfälle (Beanspruchung in Schienenlängsrichtung, $\alpha=90^{\circ}$ )

\begin{tabular}{|c|c|c|c|c|c|c|c|c|c|}
\hline $\begin{array}{l}\text { Ifd. } \\
\text { Nr. }\end{array}$ & $\begin{array}{l}\alpha \\
{\left[{ }^{\circ}\right]}\end{array}$ & \begin{tabular}{|l|}
$F_{0}$ \\
{$[k N]$}
\end{tabular} & \begin{tabular}{|l|}
$F_{U}$ \\
{$[k N]$}
\end{tabular} & $\mathbf{R}$ & $\begin{array}{l}\Delta \mathrm{F} \\
{[\mathrm{kN}]}\end{array}$ & $\mathbf{N}_{\mathrm{f}}$ & $\begin{array}{l}\Delta \mathrm{F}\left(2 \cdot 10^{6}\right) \\
{[\mathrm{kN}]}\end{array}$ & $\begin{array}{l}\text { Versagen } \\
\text { Fall }\end{array}$ & \begin{tabular}{|l|}
$f_{c m, \text { cube }}$ \\
{$\left[\mathrm{N} / \mathrm{mm}^{2}\right]$}
\end{tabular} \\
\hline 1 & \multirow{8}{*}{90} & 25,0 & 1,0 & 0,04 & 24,0 & 409.630 & 19,909 & 4.1 & \multirow{8}{*}{61,30} \\
\hline 2 & & 25,0 & 1,0 & 0,04 & 24,0 & 1.365 .780 & 22,945 & $1.2+2.1+3.1$ & \\
\hline 3 & & 25,0 & 1,0 & 0,05 & 24,0 & 890.560 & 21,817 & $1.2+3.1$ & \\
\hline 4 & & 20,0 & 1,0 & 0,05 & 19,0 & 3.253 .870 & 20,122 & $1.2+3.1$ & \\
\hline 5 & & 22,5 & 1,0 & 0,04 & 21,5 & 1.089 .800 & 20,015 & 1.1 & \\
\hline 6 & & 22,5 & 1,0 & 0,04 & 21,5 & 2.647 .330 & 22,222 & $1.1+2.1$ & \\
\hline 7 & & 27,5 & 1,0 & 0,04 & 26,5 & 385.060 & 21,823 & $1.2+3.1$ & \\
\hline 8 & & 27,5 & 1,0 & 0,04 & 26,5 & 401.380 & 21,930 & 1.2 & \\
\hline \multicolumn{10}{|c|}{ Parameter der Regressionsgeraden $y=a \cdot x+b$ aus $\log N_{f}$ und $\log \Delta F$} \\
\hline \multicolumn{10}{|c|}{ Faktor $\mathrm{a}=-0,1179$, Faktor $\mathrm{b}=2,0714$, Steigung $\mathrm{m}=8,48$} \\
\hline \multicolumn{10}{|c|}{ Statistische Parameter der Ermüdungsfestigkeit $\Delta \mathrm{F}$ bei $N_{f}=2 \cdot 10^{6}$} \\
\hline \multicolumn{10}{|c|}{$\mathrm{m}_{\Delta \mathrm{F}}=21,35 \mathrm{kN}, \sigma_{\Delta \mathrm{F}}=1,16 \mathrm{kN}, \mathrm{V}_{\Delta \mathrm{F}}=5,44 \%$} \\
\hline \multicolumn{10}{|c|}{ Charakteristische Ermüdungsfestigkeiten $\Delta F_{R K}$ bei $N_{f}=2 \cdot 10^{6}$} \\
\hline \multicolumn{10}{|c|}{$\Delta \mathrm{F}_{\mathrm{Rk}, \mathrm{SVA}}=18,77 \mathrm{kN}, \Delta \mathrm{F}_{\mathrm{Rk}, \mathrm{EC} 3}=17,75 \mathrm{kN}, \Delta \mathrm{F}_{\mathrm{Rk}, \mathrm{ETAG}}=18,07 \mathrm{kN}$} \\
\hline
\end{tabular}




\section{Schrägzugversuche}

Tabelle A23 Profil HZA 38/23 St - Variante 25/50-B Serie B - konst. Oberlast $17 \mathrm{kN}$ (Beanspruchung quer zur Schienenlängsrichtung, $\alpha=30^{\circ}$ )

\begin{tabular}{|c|c|c|c|c|c|c|c|c|c|}
\hline $\begin{array}{l}\text { Ifd. } \\
\text { Nr. }\end{array}$ & $\begin{array}{l}\alpha \\
{\left[{ }^{\circ}\right]}\end{array}$ & $\begin{array}{l}\text { Fo } \\
{[k N]}\end{array}$ & $\begin{array}{l}F_{U} \\
{[k N]}\end{array}$ & $\mathbf{R}$ & $\begin{array}{l}\Delta \mathrm{F} \\
{[\mathrm{kN}]}\end{array}$ & $N_{f}$ & $\begin{array}{l}\Delta \mathrm{F}\left(2 \cdot 10^{6}\right) \\
{[\mathrm{kN}]}\end{array}$ & $\begin{array}{l}\text { Versagen } \\
\text { Fall }\end{array}$ & $\begin{array}{l}f_{\mathrm{cm}, \text { cube }} \\
{\left[\mathrm{N} / \mathrm{mm}^{2}\right]}\end{array}$ \\
\hline 1 & \multirow{10}{*}{30} & 17,0 & 11,0 & 0,65 & 6,0 & 1.283 .193 & 5,723 & $1.1+2.1+4.1$ & \multirow{6}{*}{$\begin{array}{l}26,3- \\
29,3\end{array}$} \\
\hline 2 & & 17,0 & 12,0 & 0,71 & 5,0 & 7.348 .033 & 1) & kein Bruch & \\
\hline 3 & & 17,0 & 10,5 & 0,62 & 6,5 & 1.023 .535 & 6,053 & $4.1+3.1$ & \\
\hline 4 & & 17,0 & 10,0 & 0,59 & 7,0 & 451.235 & 5,975 & $1.1+2.1$ & \\
\hline 5 & & 17,0 & 11,0 & 0,65 & 6,0 & 960.446 & 5,550 & $1.1+3.1+4.1$ & \\
\hline 6 & & 17,0 & 10,5 & 0,62 & 6,5 & 619.252 & 5,738 & $1.1+3.1+4.1$ & \\
\hline $7^{2)}$ & & 17,0 & 11,5 & 0,68 & 5,5 & 638.381 & 4,871 & $1.1+2.1 . B$ & \multirow{4}{*}{$\begin{array}{l}34,3- \\
36,6\end{array}$} \\
\hline $8^{2)}$ & & 17,0 & 11,5 & 0,68 & 5,5 & 683.118 & 4,906 & $1.1+2.1 . B$ & \\
\hline 9 & & 17,0 & 11,5 & 0,68 & 5,5 & 1.733 .492 & 5,417 & $1.1+2.1 . B$ & \\
\hline 10 & & 17,0 & 11,5 & 0,68 & 5,5 & 1.313 .929 & 5,260 & $1.1+2.1 . B$ & \\
\hline \multicolumn{10}{|c|}{ 1) nicht in der Auswertung berücksichtigt } \\
\hline \multicolumn{10}{|c|}{ 2) Vorspannung gegen den Beton } \\
\hline \multicolumn{10}{|c|}{ Parameter der Regressionsgeraden $y=a \cdot x+b$ aus $\log N_{f}$ und $\log \Delta F$} \\
\hline \multicolumn{10}{|c|}{ Faktor $a=-0,1060$, Faktor $b=1,4090$, Steigung $m=9,41$} \\
\hline \multicolumn{10}{|c|}{ Statistische Parameter der Ermüdungsfestigkeit $\Delta F$ bei $N_{f}=2 \cdot 10^{6}$} \\
\hline \multicolumn{10}{|c|}{$\mathrm{m}_{\Delta \mathrm{F}}=5,50 \mathrm{kN}, \sigma_{\Delta \mathrm{F}}=0,43 \mathrm{kN}, \mathrm{V}_{\Delta \mathrm{F}}=7,75 \%$} \\
\hline \multicolumn{10}{|c|}{ Charakteristische Ermüdungsfestigkeiten $\Delta F_{R K}$ bei $N_{f}=2 \cdot 10^{6}$} \\
\hline \multicolumn{10}{|c|}{$\Delta \mathrm{F}_{\mathrm{Rk}, \mathrm{SVA}}=4,59 \mathrm{kN}, \Delta \mathrm{F}_{\mathrm{Rk}, \mathrm{EC} 3}=4,24 \mathrm{kN}, \Delta \mathrm{F}_{\mathrm{Rk}, \mathrm{ETAG}}=4,34 \mathrm{kN}$} \\
\hline
\end{tabular}

Tabelle A24 Profil HZA 38/23 St - Variante 25/50-B Serie B - konst. Oberlast $17 \mathrm{kN}$ (Beanspr. quer zur Schienenlängsrichtung, $\alpha=30^{\circ}$ - bereinigte Ergebnisse)

\begin{tabular}{|c|c|c|c|c|c|c|c|c|c|}
\hline $\begin{array}{l}\text { Ifd. } \\
\text { Nr. }\end{array}$ & $\begin{array}{l}\alpha \\
{\left[{ }^{\circ}\right]}\end{array}$ & $\begin{array}{l}F_{0} \\
{[k N]}\end{array}$ & $\begin{array}{l}\mathrm{F}_{\mathrm{U}} \\
{[\mathrm{kN}]}\end{array}$ & $\mathbf{R}$ & $\begin{array}{l}\Delta \mathrm{F} \\
{[\mathrm{kN}]}\end{array}$ & $\mathbf{N}_{\mathrm{f}}$ & $\begin{array}{l}\Delta \mathrm{F}\left(2 \cdot 10^{6}\right) \\
{[\mathrm{kN}]}\end{array}$ & $\begin{array}{l}\text { Versagen } \\
\text { Fall }\end{array}$ & \begin{tabular}{|l}
$f_{\mathrm{cm}, \text { cube }}$ \\
{$\left[\mathrm{N} / \mathrm{mm}^{2}\right]$}
\end{tabular} \\
\hline 1 & \multirow{8}{*}{30} & 17,0 & 11,0 & 0,65 & 6,0 & 1.283 .193 & 5,545 & $1.1+2.1+4.1$ & \multirow{6}{*}{$\begin{array}{l}26,3- \\
29,3\end{array}$} \\
\hline 2 & & 17,0 & 12,0 & 0,71 & 5,0 & 7.348 .033 & 1) & kein Bruch & \\
\hline 3 & & 17,0 & 10,5 & 0,62 & 6,5 & 1.023 .535 & 1) & $4.1+3.1$ & \\
\hline 4 & & 17,0 & 10,0 & 0,59 & 7,0 & 451.235 & 5,371 & $1.1+2.1$ & \\
\hline 5 & & 17,0 & 11,0 & 0,65 & 6,0 & 960.446 & 5,266 & $1.1+3.1+4.1$ & \\
\hline 6 & & 17,0 & 10,5 & 0,62 & 6,5 & 619.252 & 5,276 & $1.1+3.1+4.1$ & \\
\hline $7^{2)}$ & & 17,0 & 11,5 & 0,68 & 5,5 & 638.381 & 1) & $1.1+2.1 . \mathrm{B}$ & \multirow{2}{*}{$\begin{array}{l}34,3- \\
36,6\end{array}$} \\
\hline $8^{2)}$ & & 17,0 & 11,5 & 0,68 & 5,5 & 683.118 & 1) & $1.1+2.1 . B$ & \\
\hline
\end{tabular}




\begin{tabular}{|c|c|c|c|c|c|c|c|c|c|}
\hline $\begin{array}{l}\text { Ifd. } \\
\text { Nr. }\end{array}$ & $\begin{array}{l}\alpha \\
{\left[{ }^{\circ}\right]}\end{array}$ & $\begin{array}{l}\text { Fo } \\
\text { [kN] }\end{array}$ & $\begin{array}{l}F_{U} \\
{[k N]}\end{array}$ & $\mathbf{R}$ & $\begin{array}{l}\Delta \mathrm{F} \\
{[\mathrm{kN}]}\end{array}$ & $\mathbf{N}_{\mathrm{f}}$ & $\begin{array}{l}\Delta \mathrm{F}\left(2 \cdot 10^{6}\right) \\
{[\mathrm{kN}]}\end{array}$ & \begin{tabular}{|l} 
Versagen \\
Fall \\
\end{tabular} & $\begin{array}{l}\mathbf{f}_{\mathrm{cm}, \text { cube }} \\
{\left[\mathrm{N} / \mathrm{mm}^{2}\right]}\end{array}$ \\
\hline 9 & \multirow{2}{*}{30} & 17,0 & 11,5 & 0,68 & 5,5 & 1.733 .492 & 5,362 & $1.1+2.1 . B$ & \multirow{2}{*}{$\begin{array}{l}34,3- \\
36,6\end{array}$} \\
\hline 10 & & 17,0 & 11,5 & 0,68 & 5,5 & 1.313 .929 & 5,104 & $1.1+2.1 . \mathrm{B}$ & \\
\hline \multicolumn{10}{|c|}{ 1) nicht in der Auswertung berücksichtigt } \\
\hline \multicolumn{10}{|c|}{ 2) Vorspannung gegen den Beton } \\
\hline \multicolumn{10}{|c|}{ Parameter der Regressionsgeraden $y=a \cdot x+b$ aus $\log N_{f}$ und $\log \Delta F$} \\
\hline \multicolumn{10}{|c|}{ Faktor $\mathrm{a}=-0,1779$, Faktor $\mathrm{b}=1,8468$, Steigung $\mathrm{m}=5,62$} \\
\hline \multicolumn{10}{|c|}{ Statistische Parameter der Ermüdungsfestigkeit $\Delta F$ bei $N_{f}=2 \cdot 10^{6}$} \\
\hline \multicolumn{10}{|c|}{$\mathrm{m}_{\Delta \mathrm{F}}=5,32 \mathrm{kN}, \sigma_{\Delta \mathrm{F}}=0,15 \mathrm{kN}, \mathrm{V}_{\Delta \mathrm{F}}=2,74 \%$} \\
\hline \multicolumn{10}{|c|}{ Charakteristische Ermüdungsfestigkeiten $\Delta F_{R K}$ bei $N_{f}=2 \cdot 10^{6}$} \\
\hline \multicolumn{10}{|c|}{$\Delta \mathrm{F}_{\mathrm{Rk}, \mathrm{SVA}}=4,96 \mathrm{kN}, \Delta \mathrm{F}_{\mathrm{RK}, \mathrm{EC} 3}=4,80 \mathrm{kN}, \Delta \mathrm{F}_{\mathrm{Rk}, \mathrm{ETAG}}=4,86 \mathrm{kN}$} \\
\hline
\end{tabular}

Tabelle A25 Profil HZA 38/23 St - Variante 25/50-B Serie B - konst Oberlast 17 kN (Beanspruchung quer zu Schienenlängsrichtung, $\alpha=45^{\circ}$ )

\begin{tabular}{|c|c|c|c|c|c|c|c|c|c|}
\hline $\begin{array}{l}\text { Ifd. } \\
\text { Nr. }\end{array}$ & $\begin{array}{l}\alpha \\
{\left[{ }^{\circ}\right]}\end{array}$ & $\begin{array}{l}F_{0} \\
{[k N]}\end{array}$ & $\begin{array}{l}\mathrm{Fu}_{\mathrm{U}} \\
{[\mathrm{kN}]}\end{array}$ & $\mathbf{R}$ & $\begin{array}{l}\Delta \mathrm{F} \\
{[\mathrm{kN}]}\end{array}$ & $\mathbf{N}_{\mathrm{f}}$ & $\begin{array}{l}\Delta \mathrm{F}\left(2 \cdot 10^{6}\right) \\
{[\mathrm{kN}]}\end{array}$ & $\begin{array}{l}\text { Versagen } \\
\text { Fall }\end{array}$ & $\begin{array}{l}\mathbf{f}_{\mathrm{cm}, \text { cube }} \\
{\left[\mathrm{N} / \mathbf{m m}^{2}\right]}\end{array}$ \\
\hline 1 & \multirow{10}{*}{45} & 17,0 & 9,0 & 0,53 & 8,0 & 293.035 & 5,956 & $1.1+2.1 . \mathrm{A}$ & \multirow{5}{*}{$\begin{array}{l}36,1- \\
37,9\end{array}$} \\
\hline 2 & & 17,0 & 10,0 & 0,59 & 7,0 & 321.862 & 5,287 & $1.1+2.1 . B$ & \\
\hline 3 & & 17,0 & 11,0 & 0,65 & 6,0 & 1.435 .236 & 5,702 & $1.1+2.1 . B$ & \\
\hline 4 & & 17,0 & 10,0 & 0,59 & 7,0 & 511.832 & 5,677 & $4.1+2.1 . B$ & \\
\hline 5 & & 17,0 & 9,0 & 0,53 & 8,0 & 351.612 & 6,125 & $1.1+2.1 . B$ & \\
\hline 6 & & 17,0 & 12,0 & 0,71 & 5,0 & 4.796 .004 & 5,719 & $1.1+2.1 . B$ & \multirow{5}{*}{$\begin{array}{l}35,7- \\
38,5\end{array}$} \\
\hline 7 & & 17,0 & 10,0 & 0,59 & 7,0 & 614.961 & 5,840 & $1.1+2.1 . B$ & \\
\hline 8 & & 17,0 & 11,0 & 0,65 & 6,0 & 4.229 .990 & 1) & kein Bruch & \\
\hline 9 & & 17,0 & 9,0 & 0,53 & 8,0 & 1.138 .553 & 7,337 & $1.1+2.1 \mathrm{~B}$ & \\
\hline 10 & & 17,0 & 8,0 & 0,47 & 9,0 & 434.338 & 7,118 & $1.1+2.1 \mathrm{~B}$ & \\
\hline \multicolumn{10}{|c|}{ 1) nicht in der Auswertung berücksichtigt } \\
\hline \multicolumn{10}{|c|}{ Parameter der Regressionsgeraden $y=a \cdot x+b$ aus $\log N_{f}$ und $\log \Delta F$} \\
\hline \multicolumn{10}{|c|}{ Faktor $a=-0,1537$, Faktor $b=1,7501$, Steigung $m=6,51$} \\
\hline \multicolumn{10}{|c|}{ Statistische Parameter der Ermüdungsfestigkeit $\Delta F$ bei $N_{f}=2 \cdot 10^{6}$} \\
\hline \multicolumn{10}{|c|}{$\mathrm{m}_{\Delta \mathrm{F}}=6,08 \mathrm{kN}, \sigma_{\Delta \mathrm{F}}=0,69 \mathrm{kN}, \mathrm{V}_{\Delta \mathrm{F}}=11,32 \%$} \\
\hline \multicolumn{10}{|c|}{ Charakteristische Ermüdungsfestigkeiten $\Delta F_{R K}$ bei $N_{f}=2 \cdot 10^{6}$} \\
\hline \multicolumn{10}{|c|}{$\Delta \mathrm{F}_{\mathrm{Rk}, \mathrm{SVA}}=4,61 \mathrm{kN}, \Delta \mathrm{F}_{\mathrm{Rk}, \mathrm{EC} 3}=4,04 \mathrm{kN}, \Delta \mathrm{F}_{\mathrm{Rk}, \mathrm{ETAG}}=4,21 \mathrm{kN}$} \\
\hline
\end{tabular}


Tabelle A26 Profil HZA 38/23 St - Variante 25/50-B Serie B - konst Oberlast $17 \mathrm{kN}$ (Beanspr. quer zu Schienenlängsrichtung, $\alpha=45^{\circ}$ - bereinigte Ergebnisse)

\begin{tabular}{|c|c|c|c|c|c|c|c|c|c|}
\hline $\begin{array}{l}\text { Ifd. } \\
\text { Nr. }\end{array}$ & $\begin{array}{l}\alpha \\
{\left[{ }^{\circ}\right]}\end{array}$ & \begin{tabular}{|l}
$F_{0}$ \\
{$[k N]$}
\end{tabular} & $\begin{array}{l}F_{U} \\
{[k N]}\end{array}$ & $\mathbf{R}$ & $\begin{array}{l}\Delta \mathrm{F} \\
{[\mathrm{kN}]}\end{array}$ & $\mathbf{N}_{\mathrm{f}}$ & $\begin{array}{l}\Delta \mathrm{F}\left(2 \cdot 10^{6}\right) \\
{[\mathrm{kN}]}\end{array}$ & $\begin{array}{l}\text { Versagen } \\
\text { Fall }\end{array}$ & \begin{tabular}{|l}
$f_{c m, c u b e}$ \\
{$\left[\mathrm{~N} / \mathrm{mm}^{2}\right]$}
\end{tabular} \\
\hline 1 & \multirow{10}{*}{45} & 17,0 & 9,0 & 0,53 & 8,0 & 293.035 & 5,913 & $1.1+2.1 . A$ & \multirow{5}{*}{$\begin{array}{l}36,1- \\
37,9\end{array}$} \\
\hline 2 & & 17,0 & 10,0 & 0,59 & 7,0 & 321.862 & 5,251 & $1.1+2.1 . B$ & \\
\hline 3 & & 17,0 & 11,0 & 0,65 & 6,0 & 1.435 .236 & 5,695 & $1.1+2.1 . B$ & \\
\hline 4 & & 17,0 & 10,0 & 0,59 & 7,0 & 511.832 & 5,648 & $4.1+2.1 . B$ & \\
\hline 5 & & 17,0 & 9,0 & 0,53 & 8,0 & 351.612 & 6,085 & $1.1+2.1 . B$ & \\
\hline 6 & & 17,0 & 12,0 & 0,71 & 5,0 & 4.796 .004 & 5,738 & $1.1+2.1 . B$ & \multirow{5}{*}{$\begin{array}{l}35,7- \\
38,5\end{array}$} \\
\hline 7 & & 17,0 & 10,0 & 0,59 & 7,0 & \begin{tabular}{|l|}
614.961 \\
\end{tabular} & 5,814 & $1.1+2.1 . B$ & \\
\hline 8 & & 17,0 & 11,0 & 0,65 & 6,0 & 4.229 .990 & 1) & kein Bruch & \\
\hline 9 & & 17,0 & 9,0 & 0,53 & 8,0 & 1.138 .553 & 1) & $1.1+2.1 B$ & \\
\hline 10 & & 17,0 & 8,0 & 0,47 & 9,0 & 434.338 & 1) & $1.1+2.1 \mathrm{~B}$ & \\
\hline \multicolumn{10}{|c|}{${ }^{1)}$ nicht in der Auswertung berücksichtigt } \\
\hline \multicolumn{10}{|c|}{ Parameter der Regressionsgeraden $y=a \cdot x+b$ aus $\log N_{f}$ und $\log \Delta F$} \\
\hline \multicolumn{10}{|c|}{ Faktor $\mathrm{a}=-0,1574$, Faktor $\mathrm{b}=1,7500$, Steigung $\mathrm{m}=6,35$} \\
\hline \multicolumn{10}{|c|}{ Statistische Parameter der Ermüdungsfestigkeit $\Delta \mathrm{F}$ bei $N_{f}=2 \cdot 10^{6}$} \\
\hline \multicolumn{10}{|c|}{$\mathrm{m}_{\Delta \mathrm{F}}=5,74 \mathrm{kN}, \sigma_{\Delta \mathrm{F}}=0,26 \mathrm{kN}, \mathrm{V}_{\Delta \mathrm{F}}=4,52 \%$} \\
\hline \multicolumn{10}{|c|}{ Charakteristische Ermüdungsfestigkeiten $\Delta F_{R K}$ bei $N_{f}=2 \cdot 10^{6}$} \\
\hline \multicolumn{10}{|c|}{$\Delta \mathrm{F}_{\mathrm{Rk}, \mathrm{SVA}}=5,13 \mathrm{kN}, \Delta \mathrm{F}_{\mathrm{Rk}, \mathrm{EC} 3}=4,88 \mathrm{kN}, \Delta \mathrm{F}_{\mathrm{Rk}, \mathrm{ETAG}}=4,97 \mathrm{kN}$} \\
\hline
\end{tabular}

Tabelle A27 Profil HZA 38/23 St - Variante 25/50-B Serie C - konst. Unterlast 1 kN (Beanspruchung in Schienenlängsrichtung, $\alpha=45^{\circ}$ - Versagensfall 1.2)

\begin{tabular}{|c|c|c|c|c|c|c|c|c|c|}
\hline $\begin{array}{l}\text { Ifd. } \\
\mathrm{Nr} .\end{array}$ & $\begin{array}{l}\alpha \\
{\left[{ }^{\circ}\right]}\end{array}$ & $\begin{array}{l}F_{0} \\
{[k N]}\end{array}$ & $\begin{array}{l}F_{U} \\
{[k N]}\end{array}$ & $\mathbf{R}$ & $\begin{array}{l}\Delta \mathrm{F} \\
{[\mathrm{kN}]}\end{array}$ & $N_{f}$ & $\begin{array}{l}\Delta \mathrm{F}\left(2 \cdot 10^{6}\right) \\
{[\mathrm{kN}]}\end{array}$ & $\begin{array}{l}\text { Versagen } \\
\text { Fall }\end{array}$ & $\begin{array}{l}\mathbf{f}_{\mathrm{cm}, \mathrm{cube}} \\
{\left[\mathrm{N} / \mathrm{mm}^{2}\right]}\end{array}$ \\
\hline 1 & \multirow{4}{*}{45} & 11,0 & 1,0 & 0,09 & 10,0 & 817.680 & 8,098 & 1.2 & \multirow{3}{*}{38,95} \\
\hline 2 & & 10,0 & 1,0 & 0,10 & 9,0 & 1.506 .530 & 8,418 & 1.2 & \\
\hline 3 & & 14,0 & 1,0 & 0,07 & 13,0 & 319.800 & 8,436 & 1.2 & \\
\hline 4 & & 18,0 & 1,0 & 0,06 & 17,0 & 94.900 & 8,283 & 1.2 & 38,0 \\
\hline \multicolumn{10}{|c|}{ Parameter der Regressionsgeraden $y=a \cdot x+b$ aus $\log N_{f}$ und $\log \Delta F$} \\
\hline \multicolumn{10}{|c|}{ Faktor $\mathrm{a}=-0,2359$, Faktor $\mathrm{b}=2,4057$, Steigung $\mathrm{m}=4,24$} \\
\hline \multicolumn{10}{|c|}{ Statistische Parameter der Ermüdungsfestigkeit $\Delta \mathrm{F}$ bei $N_{\mathrm{f}}=2 \cdot 10^{6}$} \\
\hline \multicolumn{10}{|c|}{$\mathrm{m}_{\Delta \mathrm{F}}=8,31 \mathrm{kN}, \sigma_{\Delta \mathrm{F}}=0,16 \mathrm{kN}, \mathrm{V}_{\Delta \mathrm{F}}=1,88 \%$} \\
\hline \multicolumn{10}{|c|}{ Charakteristische Ermüdungsfestigkeiten $\Delta F_{R K}$ bei $N_{f}=2 \cdot 10^{6}$} \\
\hline \multicolumn{10}{|c|}{$\Delta \mathrm{F}_{\mathrm{Rk}, \mathrm{SVA}}=7,83 \mathrm{kN}, \Delta \mathrm{F}_{\mathrm{Rk}, \mathrm{EC} 3}=7,58 \mathrm{kN}, \Delta \mathrm{F}_{\mathrm{Rk}, \mathrm{ETAG}}=7,68 \mathrm{kN}$} \\
\hline
\end{tabular}


Tabelle A28 Profil HZA 38/23 St - Variante 25/50-B Serie C - konst. Unterlast $1 \mathrm{kN}$ (Beanspruchung in Schienenlängsrichtung, $\alpha=45^{\circ}$ - Versagen $1.1+2.1$ )

\begin{tabular}{|c|c|c|c|c|c|c|c|c|c|}
\hline $\begin{array}{l}\text { Ifd. } \\
\text { Nr. }\end{array}$ & $\begin{array}{l}\alpha \\
{\left[{ }^{\circ}\right]}\end{array}$ & $\begin{array}{l}F_{0} \\
{[k N]}\end{array}$ & $\begin{array}{l}F_{U} \\
{[k N]}\end{array}$ & $\mathbf{R}$ & $\begin{array}{l}\Delta \mathrm{F} \\
{[\mathrm{kN}]}\end{array}$ & $N_{f}$ & $\begin{array}{l}\Delta \mathrm{F}\left(2 \cdot 10^{6}\right) \\
{[\mathrm{kN}]}\end{array}$ & $\begin{array}{l}\text { Versagen } \\
\text { Fall }\end{array}$ & $\begin{array}{l}f_{\mathrm{cm}, \text { cube }} \\
{\left[\mathrm{N} / \mathrm{mm}^{2}\right]}\end{array}$ \\
\hline 1 & \multirow{5}{*}{45} & 18,0 & 1,0 & 0,06 & 17,0 & 81.810 & 11,348 & $1.1+2.1$ & \multirow{5}{*}{38,95} \\
\hline 2 & & 16,0 & 1,0 & 0,06 & 15,0 & 106.930 & 10,358 & $1.1+2.1$ & \\
\hline 3 & & 14,0 & 1,0 & 0,07 & 13,0 & 167.670 & 9,502 & $1.1+2.1$ & \\
\hline 4 & & 14,0 & 1,0 & 0,07 & 13,0 & 275.760 & 10,119 & $1.1+2.1$ & \\
\hline 5 & & 12,0 & 1,0 & 0,08 & 11,0 & 1.655 .100 & 10,740 & $1.1+2.1$ & \\
\hline
\end{tabular}

Parameter der Regressionsgeraden $y=a \cdot x+b$ aus $\log N_{f}$ und $\log \Delta F$

Faktor $a=-0,1260$, Faktor $b=1,8135$, Steigung $m=7,91$

Statistische Parameter der Ermüdungsfestigkeit $\Delta \mathrm{F}$ bei $\mathrm{N}_{\mathrm{f}}=\mathbf{2} \cdot 10^{\mathbf{6}}$

$\mathrm{m}_{\Delta \mathrm{F}}=10,41 \mathrm{kN}, \sigma_{\Delta \mathrm{F}}=0,69 \mathrm{kN}, \mathrm{V}_{\Delta \mathrm{F}}=6,62 \%$

Charakteristische Ermüdungsfestigkeiten $\Delta F_{R K}$ bei $N_{f}=2 \cdot 10^{6}$

$\Delta \mathrm{F}_{\mathrm{Rk}, \mathrm{SVA}}=8,56 \mathrm{kN}, \Delta \mathrm{F}_{\mathrm{Rk}, \mathrm{EC} 3}=7,70 \mathrm{kN}, \Delta \mathrm{F}_{\mathrm{Rk}, \mathrm{ETAG}}=8,01 \mathrm{kN}$

Tabelle A29 Profil HZA 38/23 St - Variante 25/50-B Serie C - konst. Unterlast 1 kN (Beanspruchung in Schienenlängsrichtung, $\alpha=45^{\circ}$ - Alle Versagensfälle)

\begin{tabular}{|c|c|c|c|c|c|c|c|c|c|}
\hline $\begin{array}{l}\text { Ifd. } \\
\text { Nr. }\end{array}$ & $\begin{array}{l}\alpha \\
{\left[{ }^{\circ}\right]}\end{array}$ & $\begin{array}{l}\mathrm{F}_{\mathrm{o}} \\
{[\mathrm{kN}]}\end{array}$ & $\begin{array}{l}F_{U} \\
{[k N]}\end{array}$ & $\mathbf{R}$ & $\begin{array}{l}\Delta \mathrm{F} \\
{[\mathrm{kN}]}\end{array}$ & $\mathbf{N}_{\mathrm{f}}$ & $\begin{array}{l}\Delta \mathrm{F}\left(2 \cdot 10^{6}\right) \\
{[\mathrm{kN}]}\end{array}$ & $\begin{array}{l}\text { Versagen } \\
\text { Fall }\end{array}$ & $\begin{array}{l}\mathbf{f}_{\mathrm{cm}, \text { cube }} \\
{\left[\mathrm{N} / \mathrm{mm}^{2}\right]}\end{array}$ \\
\hline 1 & \multirow{13}{*}{45} & 19,0 & 1,0 & 0,05 & 18,0 & 26.810 & 1) & $1.1+2.1$ & \multirow{7}{*}{38,95} \\
\hline 2 & & 18,0 & 1,0 & 0,06 & 17,0 & 81.810 & 9,816 & $1.1+2.1$ & \\
\hline 3 & & 16,0 & 1,0 & 0,06 & 15,0 & 106.930 & 9,069 & $1.1+2.1$ & \\
\hline 4 & & 14,0 & 1,0 & 0,07 & 13,0 & 167.670 & 8,491 & $1.1+2.1$ & \\
\hline 5 & & 14,0 & 1,0 & 0,07 & 13,0 & 275.760 & 9,249 & $1.1+2.1$ & \\
\hline 6 & & 11,0 & 1,0 & 0,09 & 10,0 & 817.680 & 8,576 & 1.2 & \\
\hline 7 & & 10,0 & 1,0 & 0,10 & 9,0 & 1.506 .530 & 8,572 & 1.2 & \\
\hline 8 & & 12,0 & 1,0 & 0,08 & 11,0 & 210.130 & 1) & 1.2 & \multirow{4}{*}{38,00} \\
\hline 9 & & 10,5 & 1,0 & 0,10 & 9,5 & 5.226 .660 & 1) & kein Bruch & \\
\hline 10 & & 18,0 & 1,0 & 0,06 & 17,0 & 94.900 & 10,070 & 1.2 & \\
\hline 11 & & 11,5 & 1,0 & 0,09 & 10,5 & 1.310 .530 & 9,764 & $2.2 \mathrm{~A}$ & \\
\hline 12 & & 12,0 & 1,0 & 0,08 & 11,0 & 1.655 .100 & 10,648 & $1.1+2.1$ & \multirow{2}{*}{38,95} \\
\hline 13 & & 14,0 & 1,0 & 0,07 & 13,0 & 319.800 & 9,488 & 1.2 & \\
\hline \multicolumn{10}{|c|}{ 1) nicht in der Auswertung berücksichtigt } \\
\hline \multicolumn{10}{|c|}{ Parameter der Regressionsgeraden $y=a \cdot x+b$ aus $\log N_{f}$ und $\log \Delta F$} \\
\hline \multicolumn{10}{|c|}{ Faktor $\mathrm{a}=-0,1720$, Faktor $\mathrm{b}=2,0534$, Steigung $\mathrm{m}=5,82$} \\
\hline \multicolumn{10}{|c|}{ Statistische Parameter der Ermüdungsfestigkeit $\Delta \mathrm{F}$ bei $N_{\mathrm{f}}=2 \cdot 10^{6}$} \\
\hline \multicolumn{10}{|c|}{$\mathrm{m}_{\Delta \mathrm{F}}=9,37 \mathrm{kN}, \sigma_{\Delta \mathrm{F}}=0,72 \mathrm{kN}, \mathrm{V}_{\Delta \mathrm{F}}=7,66 \%$} \\
\hline \multicolumn{10}{|c|}{ Charakteristische Ermüdungsfestigkeiten $\Delta F_{R K}$ bei $N_{f}=2 \cdot 10^{6}$} \\
\hline \multicolumn{10}{|c|}{$\Delta \mathrm{F}_{\mathrm{Rk}, \mathrm{SVA}}=7,89 \mathrm{kN}, \Delta \mathrm{F}_{\mathrm{Rk}, \mathrm{EC} 3}=7,32 \mathrm{kN}, \Delta \mathrm{F}_{\mathrm{Rk}, \mathrm{ETAG}}=7,49 \mathrm{kN}$} \\
\hline
\end{tabular}


Tabelle A30 Profil HZA 38/23 St - Variante 25/50-B Serie B - konst. Oberlast $17 \mathrm{kN}$ (Beanspruchung quer zur Schienenlängsrichtung, $\alpha=60^{\circ}$ )

\begin{tabular}{|c|c|c|c|c|c|c|c|c|c|}
\hline $\begin{array}{l}\text { Ifd. } \\
\text { Nr. }\end{array}$ & $\begin{array}{l}\alpha \\
{\left[{ }^{\circ}\right]}\end{array}$ & \begin{tabular}{|l|}
$F_{0}$ \\
{$[k N]$}
\end{tabular} & \begin{tabular}{|l}
$F_{U}$ \\
{$[k N]$}
\end{tabular} & $\mathbf{R}$ & $\begin{array}{l}\Delta \mathrm{F} \\
{[\mathrm{kN}]}\end{array}$ & $\mathbf{N}_{\mathrm{f}}$ & $\begin{array}{l}\Delta \mathrm{F}\left(2 \cdot 10^{6}\right) \\
{[\mathrm{kN}]}\end{array}$ & $\begin{array}{l}\text { Versagen } \\
\text { Fall }\end{array}$ & \begin{tabular}{|l|}
$f_{c m, c u b e}$ \\
{$\left[\mathrm{~N} / \mathrm{mm}^{2}\right]$}
\end{tabular} \\
\hline 1 & \multirow{11}{*}{60} & 17,0 & 9,0 & 0,53 & 8,0 & 436.462 & 7,145 & $\begin{array}{l}1.1+2.1 . B \\
+3.1+4.1 \\
\end{array}$ & \multirow{3}{*}{$\begin{array}{l}32,6- \\
34,0\end{array}$} \\
\hline 2 & & 17,0 & 8,0 & 0,47 & 9,0 & 453.759 & 8,061 & $1.1+4.1$ & \\
\hline 3 & & 17,0 & 8,0 & 0,47 & 9,0 & 453.837 & 8,061 & $1.1+4.1$ & \\
\hline 4 & & 17,0 & 9,0 & 0,53 & 8,0 & \begin{tabular}{|l|}
750.972 \\
\end{tabular} & 7,439 & $1.1+4.1$ & \multirow{2}{*}{$\begin{array}{l}32,6- \\
34,0\end{array}$} \\
\hline 5 & & 17,0 & 8,0 & 0,47 & 9,0 & 430.627 & 8,030 & $1.1+4.1$ & \\
\hline 6 & & 17,0 & 5,0 & 0,29 & 12,0 & 348.414 & 10,539 & $1.1+2.1 . B$ & \multirow{4}{*}{$\begin{array}{l}35,7- \\
36,7\end{array}$} \\
\hline 7 & & 17,0 & 7,0 & 0,41 & 10,0 & 642.934 & 9,192 & $1.1+2.1 . B$ & \\
\hline 8 & & 17,0 & 9,0 & 0,53 & 8,0 & 3.281 .802 & 8,300 & $1.1+2.1 . B$ & \\
\hline 9 & & 17,0 & 7,0 & 0,41 & 10,0 & 662.276 & 9,212 & $1.1+2.1 . B$ & \\
\hline 10 & & 17,0 & 8,0 & 0,47 & 9,0 & 359.242 & 7,922 & $1.1+4.1$ & \multirow{2}{*}{$\begin{array}{l}35,2- \\
38,3\end{array}$} \\
\hline 11 & & 17,0 & 9,0 & 0,53 & 8,0 & 450.058 & 7,161 & $1.1+4.1$ & \\
\hline
\end{tabular}

Parameter der Regressionsgeraden $y=a \cdot x+b$ aus $\log N_{f}$ und $\log \Delta F$

Faktor $a=-0,0743$, Faktor $b=1,3882$, Steigung $m=13,46$

Statistische Parameter der Ermüdungsfestigkeit $\Delta \mathrm{F}$ bei $\mathrm{N}_{\mathrm{f}}=\mathbf{2} \cdot 10^{\mathbf{6}}$

$\mathrm{m}_{\Delta \mathrm{F}}=8,28 \mathrm{kN}, \sigma_{\Delta \mathrm{F}}=1,02 \mathrm{kN}, \mathrm{V}_{\Delta \mathrm{F}}=12,28 \%$

Charakteristische Ermüdungsfestigkeiten $\Delta F_{\mathrm{RK}}$ bei $N_{\mathrm{f}}=\mathbf{2} \cdot 1 \mathbf{0}^{\mathbf{6}}$

$\Delta \mathrm{F}_{\mathrm{Rk}, \mathrm{SVA}}=6,23 \mathrm{kN}, \Delta \mathrm{F}_{\mathrm{Rk}, \mathrm{EC} 3}=5,46 \mathrm{kN}, \Delta \mathrm{F}_{\mathrm{Rk}, \mathrm{ETAG}}=5,68 \mathrm{kN}$

Tabelle A31 Profil HZA 38/23 St - Variante 25/50-B Serie B - konst. Oberlast $17 \mathrm{kN}$ (Beanspr. quer zur Schienenlängsrichtung, $\alpha=60^{\circ}$ - Versagen $1.1+2.1$ )

\begin{tabular}{|c|c|c|c|c|c|c|c|c|c|}
\hline $\begin{array}{l}\text { Ifd. } \\
\text { Nr. }\end{array}$ & $\begin{array}{l}\alpha \\
{\left[^{\circ}\right]}\end{array}$ & $\begin{array}{l}\text { Fo } \\
{[\mathrm{kN}]}\end{array}$ & $\begin{array}{l}F_{U} \\
{[k N]}\end{array}$ & $\mathbf{R}$ & $\begin{array}{l}\Delta \mathrm{F} \\
{[\mathrm{kN}]}\end{array}$ & $N_{f}$ & $\begin{array}{l}\Delta \mathrm{F}\left(2 \cdot 10^{6}\right) \\
{[\mathrm{kN}]}\end{array}$ & $\begin{array}{l}\text { Versagen } \\
\text { Fall } \\
\end{array}$ & $\begin{array}{l}\mathbf{f}_{\mathrm{cm}, \text { cube }} \\
{\left[\mathrm{N} / \mathrm{mm}^{2}\right]}\end{array}$ \\
\hline 1 & \multirow{4}{*}{60} & 17,0 & 5,0 & 0,29 & 12,0 & 348.414 & 8,940 & $1.1+2.1 . B$ & \multirow{4}{*}{$\begin{array}{l}35,7- \\
36,7\end{array}$} \\
\hline 2 & & 17,0 & 7,0 & 0,41 & 10,0 & 642.934 & 8,260 & $1.1+2.1 . B$ & \\
\hline 3 & & 17,0 & 9,0 & 0,53 & 8,0 & 3.281 .802 & 8,696 & $1.1+2.1 . B$ & \\
\hline 4 & & 17,0 & 7,0 & 0,41 & 10,0 & 662.276 & 8,301 & $1.1+2.1 . B$ & \\
\hline
\end{tabular}

Parameter der Regressionsgeraden $y=a \cdot x+b$ aus $\log N_{f}$ und $\log \Delta F$

Faktor $a=-0,1680$, Faktor $b=1,9930$, Steigung $m=5,94$

Statistische Parameter der Ermüdungsfestigkeit $\Delta \mathrm{F}$ bei $N_{\mathrm{f}}=\mathbf{2} \cdot \mathbf{1 0}^{\mathbf{6}}$

$\mathrm{m}_{\Delta \mathrm{F}}=8,55 \mathrm{kN}, \sigma_{\Delta \mathrm{F}}=0,33 \mathrm{kN}, \mathrm{V}_{\Delta \mathrm{F}}=3,82 \%$

Charakteristische Ermüdungsfestigkeiten $\Delta F_{R K}$ bei $N_{f}=\mathbf{2} \cdot 10^{\mathbf{6}}$

$\Delta \mathrm{F}_{\mathrm{Rk}, \mathrm{SVA}}=7,55 \mathrm{kN}, \Delta \mathrm{F}_{\mathrm{Rk}, \mathrm{EC} 3}=7,03 \mathrm{kN}, \Delta \mathrm{F}_{\mathrm{Rk}, \mathrm{ETAG}}=7,23 \mathrm{kN}$ 
Tabelle A32 Profil HZA 38/23 St - Variante 25/50-B Serie B - konst. Oberlast $17 \mathrm{kN}$ (Beanspr. quer zur Schienenlängsrichtung, $\alpha=60^{\circ}$ - Versagen $1.1+4.1$ )

\begin{tabular}{|c|c|c|c|c|c|c|c|c|c|}
\hline $\begin{array}{l}\text { Ifd. } \\
\text { Nr. }\end{array}$ & $\begin{array}{l}\alpha \\
{\left[{ }^{\circ}\right]}\end{array}$ & \begin{tabular}{|l|}
$F_{0}$ \\
{$[k N]$}
\end{tabular} & $\begin{array}{l}F_{U} \\
{[k N]}\end{array}$ & $\mathbf{R}$ & $\begin{array}{l}\Delta \mathrm{F} \\
{[\mathrm{kN}]}\end{array}$ & $\mathbf{N}_{\mathrm{f}}$ & $\begin{array}{l}\Delta \mathrm{F}\left(2 \cdot 10^{6}\right) \\
{[\mathrm{kN}]}\end{array}$ & $\begin{array}{l}\text { Versagen } \\
\text { Fall }\end{array}$ & \begin{tabular}{|l|}
$f_{c m, c u b e}$ \\
{$\left[\mathrm{~N} / \mathrm{mm}^{2}\right]$}
\end{tabular} \\
\hline 1 & \multirow{6}{*}{60} & 17,0 & 8,0 & 0,47 & 9,0 & 453.759 & 7,046 & $1.1+4.1$ & \multirow{4}{*}{$\begin{array}{l}32,6- \\
34,0\end{array}$} \\
\hline 2 & & 17,0 & 8,0 & 0,47 & 9,0 & 453.837 & 7,046 & $1.1+4.1$ & \\
\hline 3 & & 17,0 & 8,0 & 0,47 & 9,0 & 430.627 & 6,985 & $1.1+4.1$ & \\
\hline 4 & & 17,0 & 9,0 & 0,53 & 8,0 & 750.972 & 6,806 & $1.1+4.1$ & \\
\hline 5 & & 17,0 & 8,0 & 0,47 & 9,0 & 359.242 & 6,780 & $1.1+4.1$ & \multirow{2}{*}{$\begin{array}{l}35,2- \\
38,3\end{array}$} \\
\hline 6 & & 17,0 & 9,0 & 0,53 & 8,0 & 450.058 & 6,255 & $1.1+4.1$ & \\
\hline \multicolumn{10}{|c|}{ Parameter der Regressionsgeraden $y=a \cdot x+b$ aus $\log N_{f}$ und $\log \Delta F$} \\
\hline \multicolumn{10}{|c|}{ Faktor $a=-0,1650$, Faktor $b=1,8731$, Steigung $m=6,06$} \\
\hline \multicolumn{10}{|c|}{ Statistische Parameter der Ermüdungsfestigkeit $\Delta \mathrm{F}$ bei $N_{f}=2 \cdot 10^{6}$} \\
\hline \multicolumn{10}{|c|}{$\mathrm{m}_{\Delta \mathrm{F}}=6,82 \mathrm{kN}, \sigma_{\Delta \mathrm{F}}=0,30 \mathrm{kN}, \mathrm{V}_{\Delta \mathrm{F}}=4,40 \%$} \\
\hline \multicolumn{10}{|c|}{ Charakteristische Ermüdungsfestigkeiten $\Delta F_{R K}$ bei $N_{f}=2 \cdot 10^{6}$} \\
\hline \multicolumn{10}{|c|}{$\Delta \mathrm{F}_{\mathrm{Rk}, \mathrm{SVA}}=6,08 \mathrm{kN}, \Delta \mathrm{F}_{\mathrm{Rk}, \mathrm{EC} 3}=5,76 \mathrm{kN}, \Delta \mathrm{F}_{\mathrm{Rk}, \mathrm{ETAG}}=5,87 \mathrm{kN}$} \\
\hline
\end{tabular}

